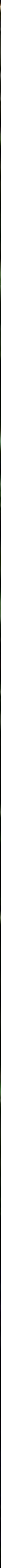




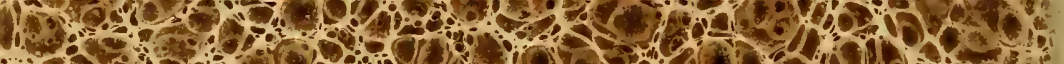

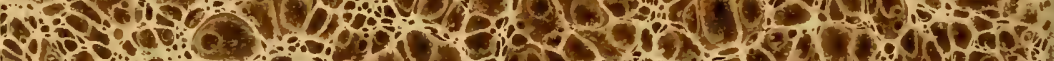

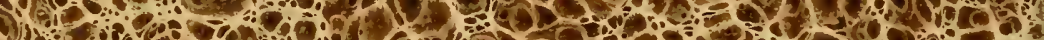

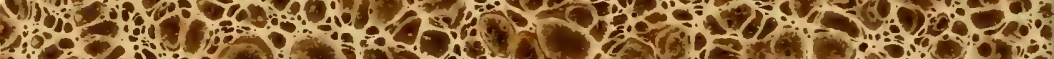

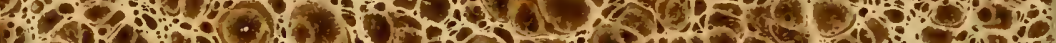

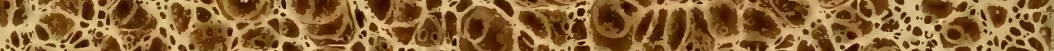

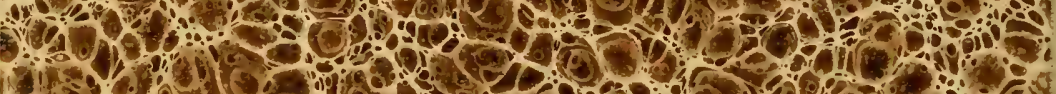

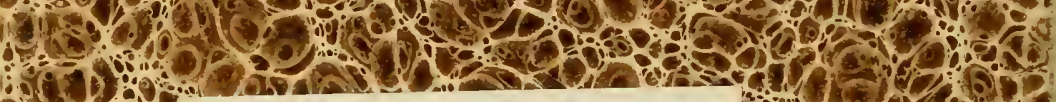

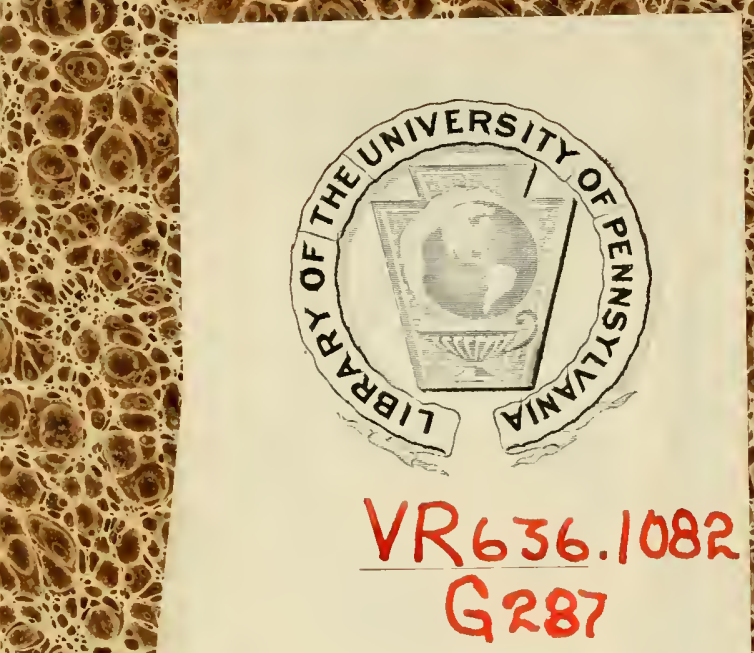

LIBRARY

OF

LEONARD PEARSON

VETERINARIAN

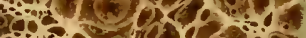

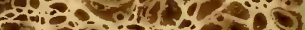

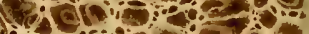
6. Co:

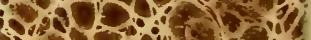

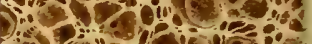

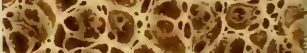
i.

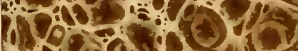

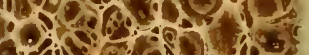

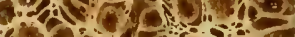
$137=40.92$

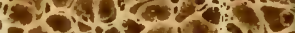

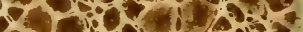

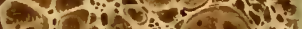

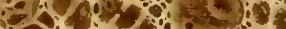
ic 일

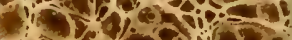
\%

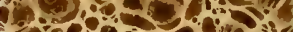

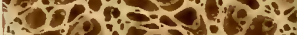

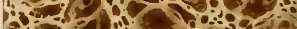

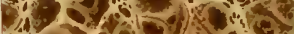
in 1 .

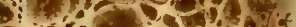

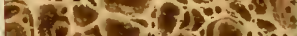

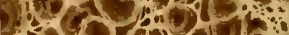

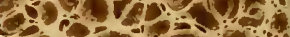

(1) $\gamma=90$

\section{v. 4}

6.

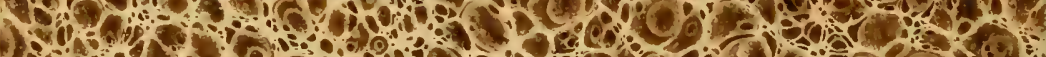
30 by

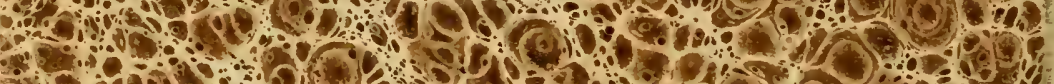
1.198,

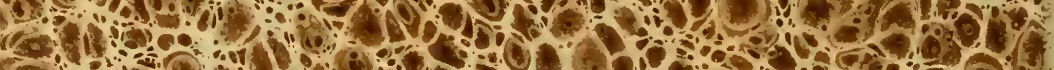

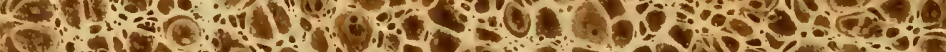

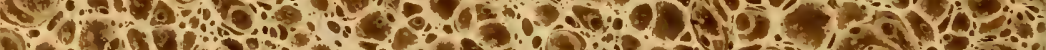

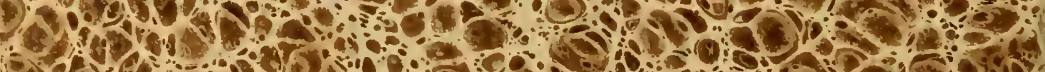
(E) 


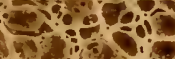

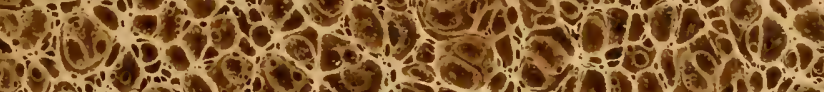

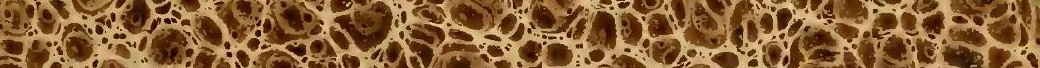

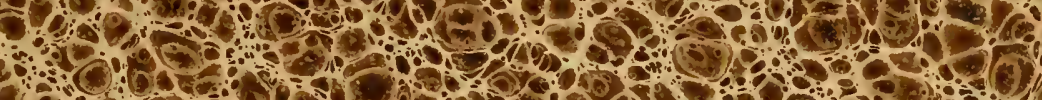

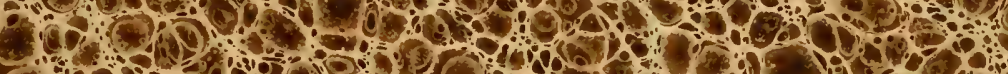

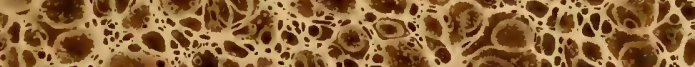

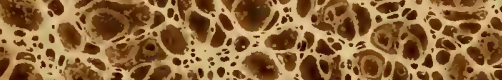

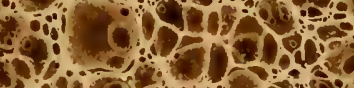

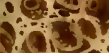

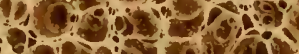

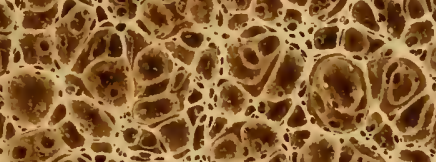

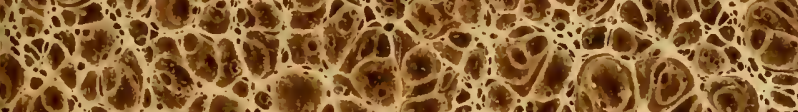

sis.

(ivisent

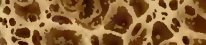

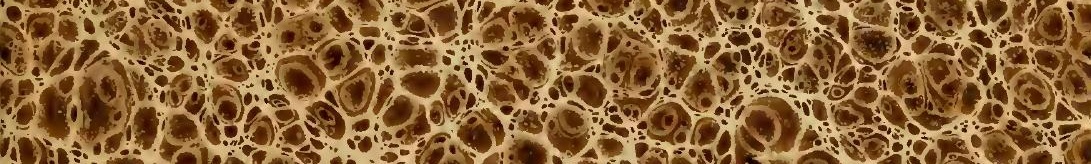
$\therefore-201090$

(o.

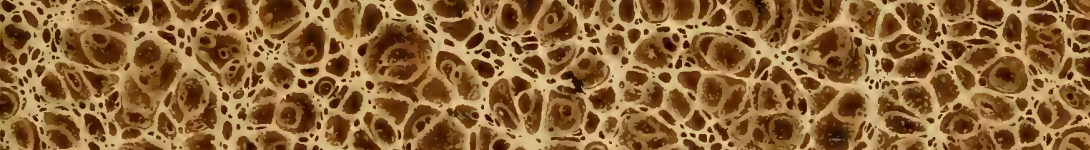

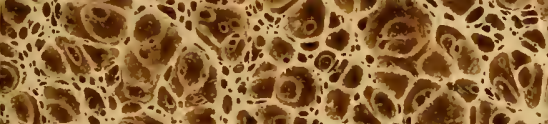

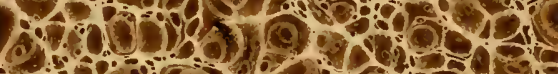

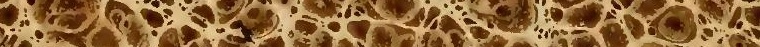

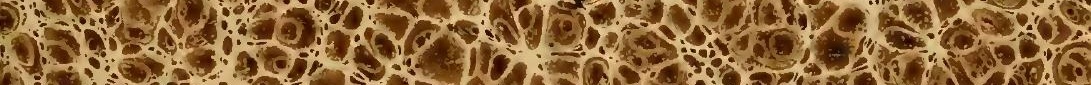

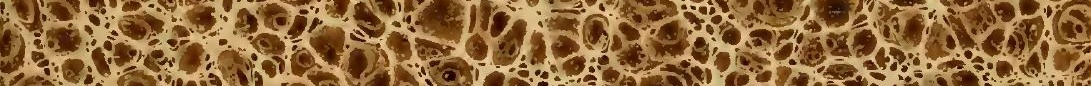

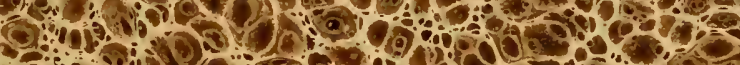

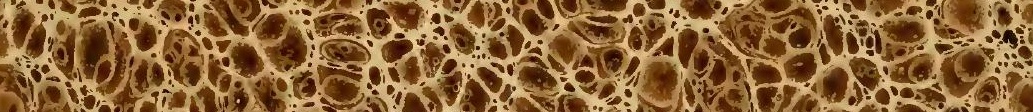

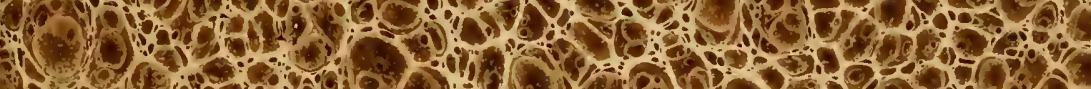
S.0.

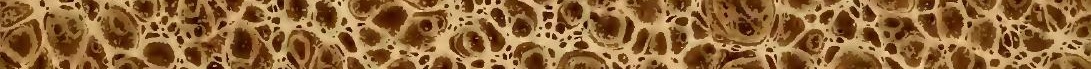

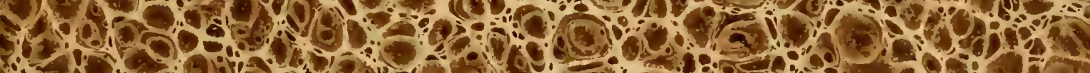

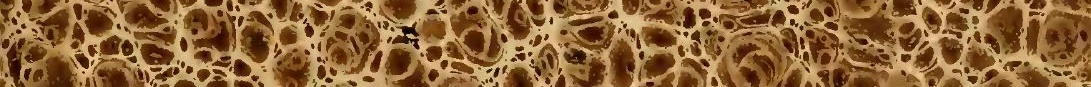

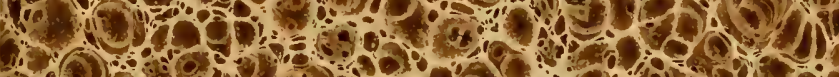

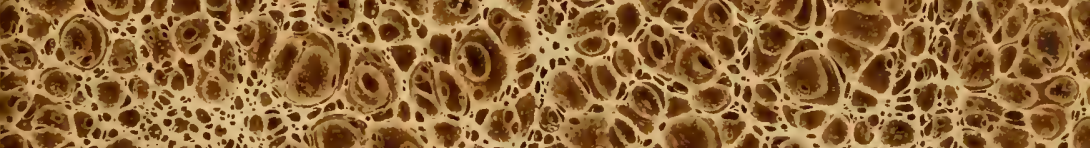

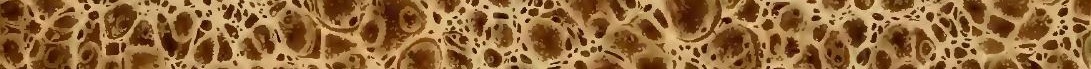

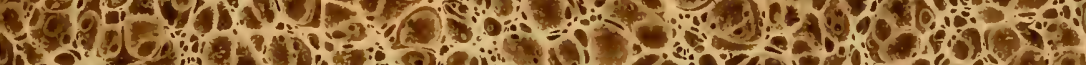

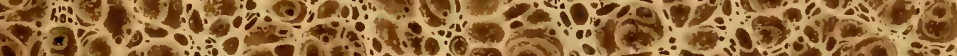

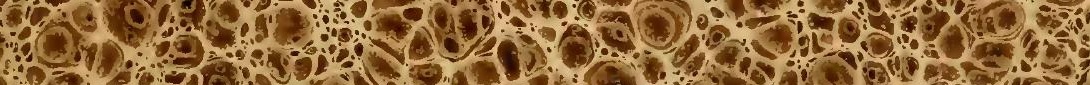




\section{Digitized by the Internet Archive in 2009 with funding from Lyrasis Members and Sloan Foundation}


THE

\section{GENERAL STUD BOOK.}

$$
\text { VOL. IV. }
$$



TH E

\title{
(boneral
}

\section{S T U D B O O K,}

$\operatorname{covTAINING}$

\section{PEDIGREES}

\author{
of

\section{RACE HORSES,}

\&c. \&c.

From the earliest Accounts to the Year 1855, inclusive.

IN FOUR VOLUIES.

VOL. IV.

\section{Brussels :}

PRINTED FOR MELINE, CANS AND C॰, botievard de waterloo, $\mathbf{5 4}$.

II DGGG XINIX. 
VI

$$
\begin{aligned}
& 636.1022 \\
& \text { G. } 8.87 \\
& \text { v. } 4
\end{aligned}
$$




\section{ADVERTISEMENT.}

TuE Publishers cannot suffer another volume of the Stud Book to appear without offering their acknowledgments to the numerous correspondents, with whose assistance it has been compiled. They did not intend to bring it down later than the year 1855 , but the unaroidable delay which has occurred in the publication, has enabled them to include several produces of the present year. Notices of many others arrived too late for this volume; they will be carefully registered, and similar communications will at all times be thankfully received. Indeed, the Publishers would feel greatly obliged, if proprictors of brood mares would make an annual return of their produce, or failure of produce, by which means the work might be kept constantly in a state of preparation, and additional volumes might be published when required, at shorter notice than hitherto, with less labour, and less liability to error. No exertion has been spared to render the present volume as complete as possible; and though some errors and imperfections will doubtless be perceived in it, the Publishers hope they are not more than may be excused in a work, the materials for which are collected from such a great number of sources, and of which the subject matter is, for the most part, in a varying and progressive state up to the moment of publication.

Oxenden Strect,

IIay 4 th, 1856. 



\section{OBITUARY OF STALLIONS.}

Their ages when

they died.

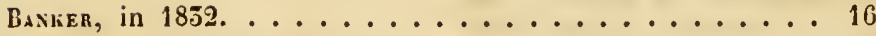

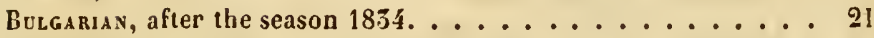

Cannon Ball, in 1852. . . . . . . . . . . . 22

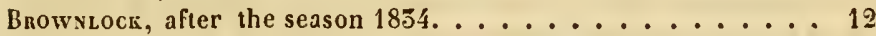

CArron, at Tickhill, December 1835. ............ 24

Hompinet Clinker, after the seasun 1854.......... 12

Longwalst, late in April 1855. ............. 14

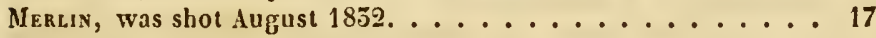

Mivos, after the season 1834 , from a kick. .......... 18

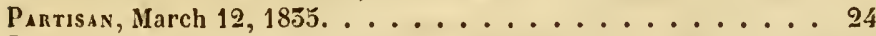

Picton, in Ireland, in $1834 \ldots \ldots \ldots \ldots$

Sober Robix, was destroyed July 11,1855 , not having got a foal in that or the two preceding years. ......... 19

Tramp, was shot at Howbridge, in Yorkshire, December 185̄5; the covercd only eight mares the preceding season, which all

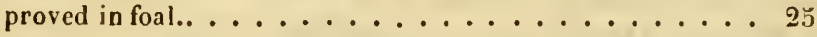

Waxderer, Feb. 1830 , in Ireland, of an enlargement of the heart. 19

\section{STALLIONS SENT ABROAD.}

Albany, to Germany.

Alington, by Gustavus, to France, in 1832.

Alteroter, to France, in 1835.

Cuateau Margaux, to Virginia, U. S. in 1854.

Clinker, by Humphrey Clinker, to Barlen, in 1855 .

Y. Corrector, to Mecklenberg, in 1852.

Caurcil died on the passage to Germany, in 1854. He covered only one or two mares in England.

Daxgerous, to France, in 1855 .

Emilianus, to Germany, in 1834.

Enancipation, to Virginia, after the season $1855^{\circ}$ 
Enysus, to IIolstein, in the spring of 1832 , having covered a few mares it

England that season.

Figaro, to Mecklenberg, in 1831 .

Frlde, to Virginia, in 185 ?.

Gaberlunzie, to Prussia, in February 1856.

Gustavos, to ditto, in March 1850.

Halston, to Germany.

Helexus, to Germany, in 1835.

Horea POKe , to the D. of Holstein, after the season 1835.

liscubus, to Holstein, in January, 1854.

JoceliNe, to Baron Biel, in 18.34 or 55 .

Jonsw , by Little John, to India, in 1850.

Logıc, to Holstein, in 1828. He was brought back to England in 1854, covered a few mares here that season, and the whole of the next, and was re-purchased by the Duke of Holstein.

Luzmorodgh, to Virginia. in June 1852 .

Lapdog, to Alabama, U.S. in August 1835.

Naresfifld, to Sardinia, before the season 1835 ; he died there about three weeks after his arrival.

Margrave, to Virginia, after the season 1835.

Mraman, to ditlo, ditto.

Middeton, to Russia, in 1830.5.

Pericles, to Russia, whe re he died in 1855.

Phantom, to Mecklenburg, in 1852 .

Predictor, to Mecklenburg, in 1834 or 55.

Prime Minister, to Russia, in the spring of 1834 .

Pick pocket, to France, late in 1855.

Rubens Junior, to Mecklenberg, in 1832.

Sarpedon, to Virginia, 1854.

Sir Benjamin, to France, in 1854.

Shatspeare, to Virginia, in September 1855.

Straitivalst, to Germany.

Skylark, by Waxy Pope, to America, in 1856.

Swiss, to the United States, in 1855.

Trandy, to Virginia, in February 1855.

Vicrorr, by Waterloo, to America, 1834.

Varro, to Mecklenberg, after the season 1855.

Windcliffe, to France, late in 1835.

Woruz, to Prussia, in the autumn of 1852 . 


\section{INDEX TO THE WHOLE.}

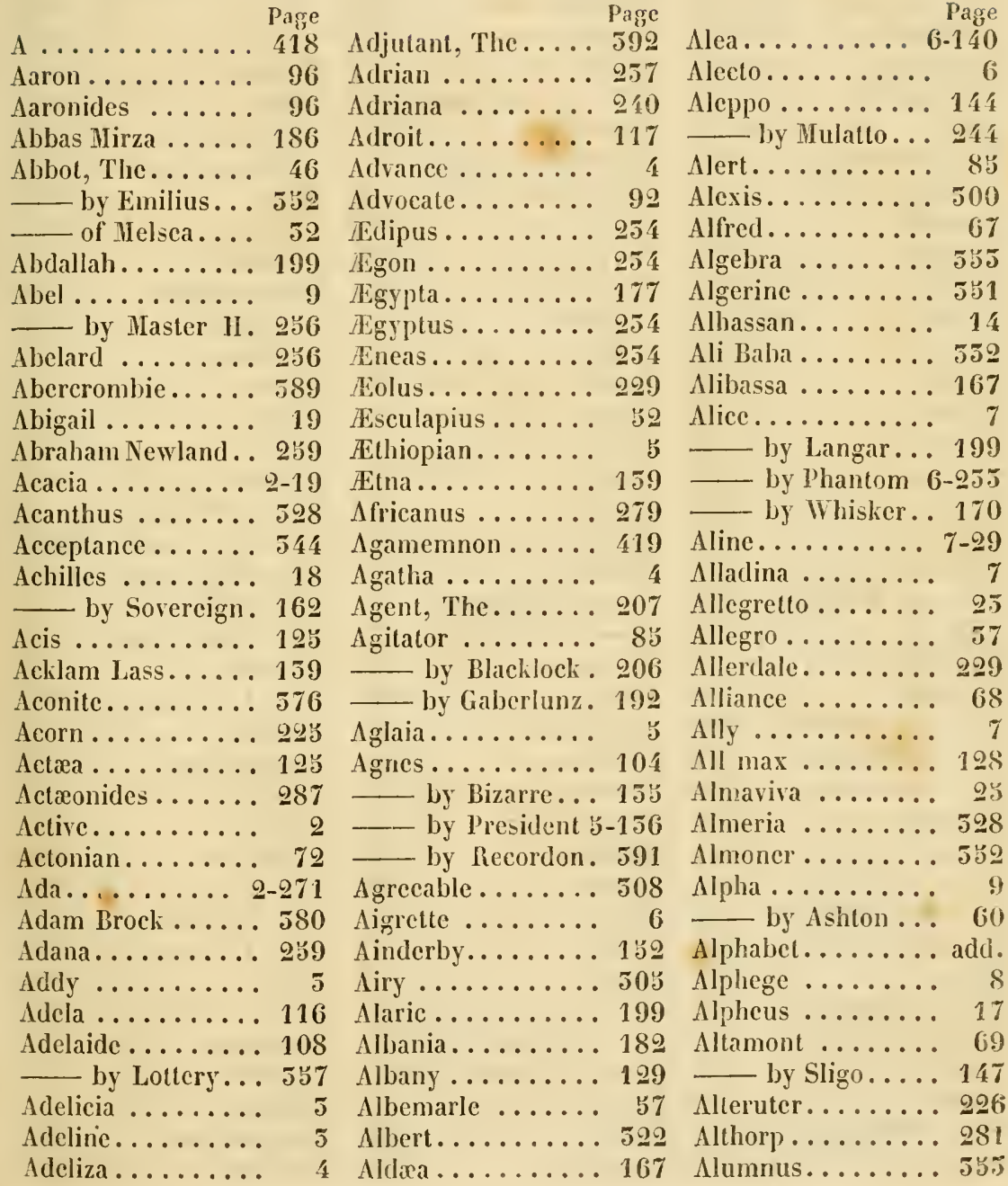


Amadillo........ 5.55

Amadine. ....... 558

Ainadou ........ 177

Amana ........ 8

Amaryllis ........

Amateur........ 270

Amato......... 144

Amazon (Young) . . 150

Ambassador ...... 418

Amber ........ 9

— - by Ambo .... 391

Ambiguity ...... 8

Ambrosio . . . . . 151

Ambuscade . . . . 9. 9-117

Amelia ........ 57

- by Raphacl... 83

Amesbury....... 108

Amethyst ....... 220

Amiable .........

Amidon..... 591-592

Amima ...... 19-5ร8

$\Lambda \operatorname{mina} \ldots \ldots \ldots .171$

Aminta ........ 1-9

Amoret ......... 9

Amphion....... 12

Amphitrite ...... 105

Amril ......... 195

Amulet........ 102

Amurath....... 18

— by Whisker.. 579

Amy .......... 246

Andallah....... : 150

Angelica....... 10

— by Sir II. D.. 592

ly Fyldener. .

10

- by Rubens... 11

Auglesea........ 209

Anna.......... 11

— by Godolp. 11-23

by Jerry Sne. 592

Annaline....... 180

Anne ......... 143

- ol'Geierstein.. 12

Anne Grey ...... 12

Annette....... 417

Annic ........ 10

— by Wand.. 51-5392

Annot Lyle.... 19-160
Anonymous ...... 592

Antar ......... 187

Antelope....... 151

596

Anthony........ 19

Antic ......... 197

Antigone:

9

— by Angustus. 419

Antiope........ 12

Antiphus ...... 199

Antwerp....... 12

— by Waterloo.. 97

Apollo......... 13

- by Whitworth 290

Apollonia .... 15.214

Apparition ...... 15

Apuntador ...... 550

Arab......... 582

—- by Zealot.... 591

Arabella ....... 13

Arachne....... 14

Araminta ......4 417

Aranda ........ 167

Aratus ........ 511

Arbaces ........ 9.

Arbis ......... 14

Arcadian....... 219

Archduchess..... 1 b

Archer ........ 559

— by Sagittarius. 59.5 by Y. Blachl. . 553

Archibald ....... 12y

$A r \cos . \ldots \ldots . .67$

Arcot Lass....... 13

Ardelia ........ 167

Arethissa ....... 17

Argante ....... 18

Argantes....... 589

Argent ......... 290

Argirio ....... 406

Aricia ........ 17

Aricl.

264

591

536

Arinette ....... 17

Ariosto ....... 268

Arlington ....... 524
Page

Armadillo ....... 228

Armida........ 17

Arrillerator...... 419

Arsena ........ 17

Arsenic........ 419

Arthingworth .... 579

Artichoke ....... 215

Artist . . . . . . . 5852

Ascot.......... 11

by I'eterboro. 591

Aspasia........ 18

Associate....... 7

Astracan ........ 222

Astræa......... 171

Atalanta ........ 18

Athenian, The..... 216

Atlas.......... 18

Attaché........ 555

Auburn. ........ 19

Auchencruive . . . . 558

Auctioneer ...... 19

Auditor......... 124

Audrey........ 19

Augur.......... 990

— by Interpreter 305

Augusta ........ 199

- by Tiresias... 19

by Woful. 19-271

Augustina. 545 and err.

Augustus........ 19

__ by Gustavus. . 53

Aurelius ........ 19

— by Emilius. . . 199

Aurora ........ 19

Aurungzebe..... . 56 z̆

Autocrat ....... 105

Areline........ 3

Avon Lass. . . . . . . 3 ระ

Ayesha ........ 98

— by Sultan.... 182 by Drone.... 555

Azalia ......... 12

Babel.......... 20

Bacchanal...... 555

Bacchus........ 7

Bachelor........ 417

Backgammon .... $5 ! 9$

Bad Lebt . . . . . . . . 924 
Bags......... 409

Baguley......... 75

Baillie Jarvic. . . . . 146

Balance......... 195

Baleine (bay). .... . 20

Baleine (roan). . . . 299

Balkan, The... 21-571

Ballad-singer.... 20-68

Dallochmyle ..... 61

Ballyroan .......4 415

Bamboozle ......4 418

Bamfylde ....... 940

Bang-up ........ 420

Bank of Ireland. . . . 416

Banquet ........ 578

Banqueter....... 244

Banquo......... 19 :

Banshee ........ 21

— by Y. Sorcerer 21

Banter..........

Barabbas (aft. Caledonian)........ by Banker... 216

Barataria....... 152

Barbara...... 22-214

Barbara Bell..... 22

— by Middleton. 509

Barbarina...... 342

Barbary......... 226

—_by Jenkins... 578

by $A$ rdrossan. 578

Barber, The..... 516

Barbiche....... 107

Barcarolle....... 132

Barcelona ..... 29-152

- by ג. Henry.

Barclay......... 511

Bard, The...... \$2

Bardolph........ 152

Barebones....... 596

Barefoot ....... 59. 59

Barnacles ........

Barney Bodkin ....

Baron, The.......

- by Blacklock. Bowes......

Baronct......... 95

Baroness.........
Barrister........ Page

Barrosa......... 22

Bartolozzi ....... 389

Bartonia ....... 23

Barytes........ 70

Bashful........ 50을

Bassano........ 515

Bassellaw . . . . . . 299

Bassilia ...... 25-580

Basto.......... 584

Batiste......... 185

Battledore....... 260

Bavarian....... 594

Bawgundee...... 289

Bay Malton....... 260

- Middleton... 68

Baylock........ 52

Bazaar......... 29

Beagle........ $40 \mathrm{~s}$

Beatrice........ 24

Beauchamp...... 16

Beaufort (dead).... 159

Beauty ........ 57

— by Catton.... . 550

Becassine ...... 46

Beda......... 598

Bedershin...... 403

Bedouin ....... 47

— by Harlequin . add.

Bee-in-a-bonnet.... 24

Bee's-wing ...... 16

Beggarman...... 419

Beggar-my-neigh-

bour ........ 419

Beguin ........ 507

Beiram ........ 198

Belinda ....... .24-250

Bella......... 24

- loy Actron... 25

Bella Donna ...... 28

57 Bellows....... 409

57 Belluno........ 179

46 Belphobe....... 25

158 Belsay........ 549

262 Belvidera........ 205

255 Belvoirina...... 26

22 Belus......... 20
Patse

Belzona........ 228

Benedici....... 129

Benefit ......250-5อ8

Benerolence..... 288

Ben Lomond...... 5.50

Bentley........ 206

Верро......... 595

Bequest........ 27

Bequine ....... S8

Berengaria ......

Beresford ...... 164

Beresina ....... 5929

Bertha......... 28

— by Reveller.. 2S

Bertram ........ 160

Bess ......... 28

Bessy Bedlam. . 172-538

Betty Martin..... . 28

Beverley....... 154

Beverlonian...... 7 it

Bhurtpore....... 28s

Biclskoi........ 160

Bigottini....... 262

Bijou, The...... 599

Billesden....... 417

Billinge........ 568

Billingsgate...... 99

- by Filho..... 167

Billy Lack-a-day. . . 418

— Watson.... 241

Birdcatcher...... 141 by Sir Hercules 400

Birdline....... 141

Birkenhead...... 566

Birthday....... gy

Bish.......... 51

Bishop, The..... 581

Bittern ........ 592

Bizarre (the horse). $\quad 99$

- (the mare)... g9

Black-and-all-black . 150

Blackberry . . . . . 198

Blackburn....... 994

Black Daphne .. 50-505 - Diamond.... 59

—_ by Jerry Giant, The.. 40 Limbleton... 512 
Page

Black Polly ... . . 55-88

—- Swan...... 224

Will. . . . . . 59

Blackfoot ....... 402

Blacking ....... 50

Blackleg . . . . . . 149

Blacklock (Ioung).. 13

- (Mr Owst's) 519

__- (Irish) ... 162

Blaise ......... 248

Blanche..... 157-595

Blanche of Jeren.. 270

Blandford . . . . . . 15

Blassis........ 195

Blemish........ 41

Blitz . . . . . . . 504

Blonde-slceves .... 141

Bloodhound ..... 589

Blossom ........ 215

- by Gustavus. . 257

Blue Beard......2 214

— Bell. . . . . . . 500

Bonnct..... 178

Eyes ...... 5

Peter...... 246

Ruin ...... 579

Stocking .... 54

Stockings ... 55

Blundering Farrier. 182

Blush ......... 401

Boadicea ....... 5

Bobadilla....... ż

Bobbinette ..... 44

Bobinet........ 511

Bol Logic. . . . . . . 168

Boblondeb....... 85

Bodice. . . . . . . 299

- by Langar... 505

Bolero......... 195

Boletas ........ 555

Bolivar ........ 193

- by Blacklock. 517

Bollingion....... 215

Bombarina ..... 29

Bombasine ...... J. J.

Bonassus....... 87

Bonby ........ \$6

Honnet Pet.....5 517
Pagge

56

56

234

215

$20 \%$

159

227

$\begin{array}{rr}\text { Bosphorus........ } & 198 \\ \text { Bostock......... } & 41\end{array}$

Botanist......... 118

Bothschafter, The. . 258

Bounce........ 517

Bowmall. . . . . . 595

Boxer .......... 416

Brace ......... 585

Bracelet........ 57

by Mameluke. 292

Bradley........ 225

Bradwardine...... 219

Braham........ 57

Braithwaite...... 291

Brambilla ...... 195

Bramin . . . . . . 198

Bran ......... 585

Brandy Ann..... 196

Brandy Bet...... $\mathbf{5 9 4}$

Bras de Fer...... 585

Bravo ......... 202

419

The........ 244

Bravura..... 57-254

Brazil ...... 57-583

Brecongill....... 254

Breeze......... 591

Brenda, by Catton 21-5s

Bride, The ...... 217

Bridegroom...... 56

Brien Boru . . . . . 416

Brigand, The...... 298

Brighton....... 167

Brillante...... 58

Brilliant ....... 149

Britannia....... 58

Britannicus..... 59

Broadsword...... 559

Brocard........ 589

- by Whaleb.59-522

Brookland....... 216
Pagi!

Brown Betty..... 59

Duchess.... 40

Brownic, The.... 5 5 ü

Brownlock....... 297

Brown Stout..... 81

Brunette, by Dragon 40

- by Camerton. 196

- by Eryx..... 196

- by Lottery... 517

- by Blacklock . 14:

- by Percy .... 181

by Waxy... 40

—_ by $0^{\prime}$ Fy .... 594

Brussels ........ J04

Bryan .......... 252

Bryn......... 516

Bubastes....... 540

Bubble . . . . . . . 265

Buccancer....... 194

Bucellas ....... 418

Bucephalia ....... 221

Bucephalus...... 74

Bud............ 248

by Y. Black-

lock... . . . . . 598

Budget ........ 409

Bugle ..........275

Bulbridge . . . . . 517

Bull-calf . . . . . . 84

Bull-dog . . . . . . . 502

Bullet . . . . . . . . 286

Bullion ........ 152

Bulow........ 160

Bunter ......... 262

Bupta ....... 41-76

Burden ......... 181

Burgomaster..... 180

Burgsdorf .......214

Burgundy ....... 45

Burlator ....... ร้7

Burletta ....... 70

Burlington ...... 211

— by Brutandorf 517

Burman ........ 193

Burton Lass. . . . . . 41

Busk......... 559

Buskin . . . . . ... 205

Bustle ...... 45-221 
Butcher Boy..... Pagre 96 Cantatrice....... 144

Buttercul) ........ 8

Butterfly . . . . 43-116

— - by Lottery ... 72

Button Park...... . 193

Buy a Broom...... 62

Cabin Boy....... 66

Cacador........ 80

Caccia Piatti..... 550

Cachmere....... 50

Cactus.......... 550

Cacus ......... घ

Cadet .......... 55

by Roller.... 414

Cadiz.......... j

Caifacaratadaddera . 4.5

Caird o'Barhullion.. 79

Cajeput......... 557

Calantha ....... 502

Calapee......... 44

Caldicol......... 18

Caledonian ...... 25

Calendar........ 156

Calendula....... 45

Caliban......... 21

Caligula......... 66

Caliph.......... 55

— by Sultan.... 525

Calista....... 4 4 - 294

- - by Velocipede 149

Caller.......... 290

Callisthenes....... 1 gั

Calmuck......... 274

Calypso........ 45

Camarine ........ 274

Camel (Young).... 102

Camelina........ 46

Camelopard...... 102

Camilla........ 46

- by Tramp... 47

Camillina...... 46-294

Camlet ......... 195

Cannonade ...... 81

Canopy .......

Cant.......... 50

Cantab ......... 128

Cantaloupe...... 50
Canwell........ 177

Caprice (Young) ... 50

Captain, The..... 4

Captain Artliur.... в9

322

๖9

215

75

257

287

237

298

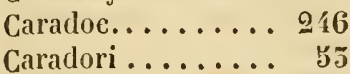

Cararan........ 546

Cardinal, The.... 187

Carew......... 419

Carlton......... 976

Carmelite ....... 290

Carmine ....... 279

Carnaby ........ 200

Carnation ....... 81

- by Blacklock. 219

Carnival ......... 268

Caroline ........ . घ 1

__ by Filho.. 59-588

- by Whalebone 51

- hy Brutandorf 74

— by Chateau M. 506

— by Part. (dead) $2: 37$

— by Sligo..... 194 (Young).....

Carpenter, The.... 61

Carthusian ...... 220

Cartoon......... 210

Cashier........ 153

Cashmere....... 415

Casket......... 418

Caspian........ 574

Cassandra (dam,

Manto) ....... 179 (dam, Zillah) 554

Castanet ........ 129

Castaside........ 970

Castaway........ 270

Castilian........ 977
Castrella 0.5

Castrellina 52-534 and add.

Cat.......... 559

Catalani .......

Catalina......... 277

Catalonian....... 277

Catalpa......... 974

Catehpenny...... 414

Catgut......... 59

- by Catton.... 198

Catherina ....... 6

- by Walton...

Catherine ....... 27

— by Muley.... 417

Catlap......... 418

Cato ........... 295

Catterick, by Oetavian.......... 185

—_ by Bob Logic 52

Cavalier......... 268

Carendish ....... 187

Cauld Lad of Hilton 127

Cecil .......... 247

Cecilia.......... 202

by Filho.... 507

Celandine ....... 561

Celastrus........6. 67

Celeste ........ 69

Cencrentola...... 66

Centaur........ 224

Genturion ....... 202

Ceres.......... 178

Ceresina...... 38-551

Cestus.......... 155

Ceta ......... 160

Cetus......... 160

Chadlington...... 275

Chamois ....... 54

Chance........ 58

— by Patron.... 260

— by K. Corney 589 by Lottery. . . 998

Chancellor....... 10

- (Young) The 590

Changeling....... 5

Chantarclle ...... 215 


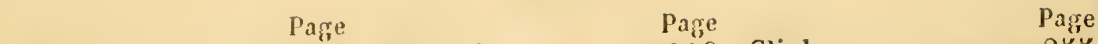

Chanter........409 Chrysalis ...... 116 Clinker........ 25y

Chantilly....... 52:

Chaos .......... 28

Chapeau de Paille. 62

Chryseis (Joung)...6 65

Clinkerina .....6.68

Chrystal ......5.594

Cigar .........274

Clinton ........ 75

62 Cimctar ........ 558

Clintz ......... 121

d'Espagne ... 62 Cicely......... 66

Chapman ....... 270

Charissa ....... 56

Charity ......6.62

Charivari ...... 7

Charley........ 206

- Boy ....... 297

Charlotte ....... 573

West ... 6 6J-116

Charmer....... 547

- The, by Y. Go-

hanna....... 589

Charming Molly... 65

Chasm .......6. 6J

Chat ........ 65

Chataigne....... 259

Chatham....... 97

- Lass . . . . . 905

Chatterbox ..... 531

Cheat ........ 595

Chemisetle ..... 79

Chersidamas . . . . 119

Cherub........ 64

The ...... 25

Cherubino...... 58

Chesnut Helen.... 8

Chester....... 206

Chcralier ...... 564

Ghew Bacon ...... 266

Chica ........ 80

Chickeroni ...... 155

Chico ........ $19 \mathrm{~S}$

Chincilla ....... 188

Chiron . . . .... 293

Chloris ..... 64-218

Choaspes ....... 17

Cholera........ 156

Chorister ......6.6\%

Christaluel ....6 6马.140

Christine ....... 7 I

— by Master II. . 178

Chromatica...... 560

- - by Neptune.. 78

Cinderella .....666.83

Clotilde....... 217

Cloudesley ...... 281

Clove ........ 414

Clown........ 69

Cridian ....... 525

Coalition....... 589

Cobweb ......68 68-116

Cockatoo...... 320

Cock-a-whoop ..... 240

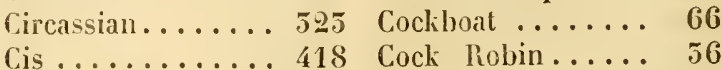

Citron ..... $274560 \longrightarrow$ by Blacklock. 56

Ciudad Rodrigo.... 80 Codicil ......... 68

Clansman ....... 10 Cœur de Lion..... 28

Clara ........ 594 Colly ....... a 1-552

Coloncl, The, by

Abjer ....... 52

liy Selini....

- ly St Patrick. 558

- Nowbray.... 256

Clare .......66-158

Claremont ....... 546

Clarence....... 129

Claret......... 106

- by Chateau

Margaux...... 255

Clari......... 134

- by Smolensko 594

Clarinet .......4 418

Clarion......... 145

Clarionet ....... 274

Clatter .......66-218

Claude ........ 161

L Lorraine .... 60

Claudia.........201

Claw ......... 280

Clear-starcher ..... 414

Clearwell ....... 166

Clematis........ 67

Clementina...... 67

Cleone ......... 268

Cleopatra ....... 599

Clifton ........ 78

- by Cain..... 210

Climber....... 67

_ by Alcaston. . 413

Colossus ........ 197

Colubra........ 67

Colunbia ....... 69

Columbinc ...... 100

_ by Cervan. 69-118

Colverstown ..... 406

Colwick........ 506

Colworth........ 159

Coma ......... 69

Combat......... 202

Comedian ....... 124

Comedy ....... 70

Comcly......... 594

C by Waverley. $\$ 8$

Comet......... 171

—_ by Emilius.. 220

—_ by Lumb. . . . 406

Commandant..... 152

Compton........ 200

Conccalment..... 419

Conciliation...... 560

Confusion ....... 415

Conjuror........ 547

Connoisscur ..... 121

Conquest....... 99 
Conrad, by Whisker 51 Coventry....... 11

Consent........ 41

Conservative..... 46

Conserrator......4 417

Consol.......... 56

Conspiracy ...... 168

Constable, The.... 519

Constance....... 202

- by Leviathan. 220

Constant........ 191

Constantia....... 299

Contentment..... 55

Contest . . . . . . . . 202

Contraband......550

Contrition, by Tiresias 75, 511, and err.

Contriver........ 514

Controller, The.... 107

Conviction....... 75

Copere ........ 257

Copper Captain ...

Coquette....... 7 5

Coquine ........ 280

Cora, by Waxy .... 75

—- by Peruvian. . 76

—_ by M. Bagot. . 595 by Truflle. . . 589

Cordelia ....... 220

Cordial . . . . . . . 594

Cordova........ 59

Corca ........ 80

Corinne........ 77

Corinthian....... 168

Coriolanus ...... 165

Corliscrew ......514

Cornbrough ..... 197

Corncreak....... 550

Cornelia ........ 279

Corneytrowe..... 208

Coronet .......4 418

Corsair . . . . . . 599

Corset........ 507

Corunna ....... 277

Cotillon .......257

Cottage Girl ..... 77

— by Gambler . .

Cottager .........
Counterpart

Count, The...... 55

by Tramp.... 77

D'Orsay .... 230

Ivoll . . . . . . 114

Lauriston . . . 155

Robert...... 99

Robinson.... 272

Countess, by Cat-

ton ...... 77-156

— by Comus 547-589 by The Col. . . 144

Courtesan ....... 148

Cowboy ........ 80

Cowdray ........ 241

Crab.......... 288

Cracker........ 142

Cramer......... ร̌

Crazy Jane ...... 77

Cream ........ 78

—_by Catton.... 549

Credulity ....... 560

Creeper ........ 11

- by Tamworth 67

Creeping Cerus.... 67

Cremona...... ร5

Creole......... 85

Crescent ........ 202

— by Zealot...4 415

Cressida ....... 78

Creusa ........554

Crickict........ 11

_ by Partisan .. 218

Cricketer ...... 150

Crim. Con....... 409

Cripple......... 594

Cripplegate...... 547

Crispin ........5 574

Criterion.......44 417

Crockery....... 86

Crocodile ......4 418

Crocus ........594

Cromarty ....... 265

Crotchet ........ ร.

Crotchet .........

18

77
Croweatcher ..... 64
Creeping Kate..... 78
Page

594

Crumpet . . . . . . . 254

Crusader........ 220

Crutch ........ 552

Young ..... 500

Cuckoo, The..... 52

Cuff Cushion..... 157

Cuirass........ 593

Cupid ......... 2

— by Emilius... 281 by Whisker.. 584

Curioni........ 55

Currency ...... 517

Curtius ........ 257

Cuslınciche . . . . . 405

Custard........ 78

Curiosity....... 555

Curlew........4 411

Cutty Sark ...... 79

Cuyp ......... 161

Cyclops ....... 167

Cymbal......... 274

Cymro ......... 260

Cynthia ........67

— by Comus... 161

Cyprian ....... 121

Cyrus ......... 250

Dabchick..... 18-561

Dacre ......... 25

Janmon ........ 17

—_ by Amadis... 256

by Magic ... 291

Dainty Davie..... 524

Dairy Maid...... 79

Daisy . . . . . . . 150

I)ahlia . . . . . 79-555

by Ilaphazard 257

Dalca......... 565

Damascus ...... 56

_. by Reveller .. 144

Damascene...... 292

Dame Durdan..... 27

Dame Quickly..... 41 b

Dan ......... 111

Dancirg Mlaster... 10

Dandelion...... 556

Dandizette ...... 80

- by W. Pope.. 595 
Dandina ....... Page 170 Dervisc ........ Page 256

Dangerous ...... $\$ 9$

Danicl........ 4

Dante ........ 55

Darfour ....... 219

Dariolctla...... 80

Darius ........ 111

Darlaston ....... 402

Darling........ 146

Dart ......... 404

Datura ........ 89

Daucier ........ 80

Dauntless ....... 82

David ........ 111

Dawdle........ 596

Day and Martin . . . . 50

Deacon . . . . . . . 256

De Beriot . . . . . . 249

Decanter....... 404

Deceit.........

Deceirer ........ 214

Deception ..... 4-561 by่ P'hantom. . 180

Decision ..... 81-262

Decoction ....... 978

Decorator....... 118

Decoy ....... 82-117

Defence ....... 82

Defender ...... 285

Defensive ....... 285

Defiance....... 82

Deformity...... 88

Dejanira........ 107

Delce ......... 419

Delenda ....... 82

Delia ......... 244

Delight ........ 82

Delightful...... 159

Delirium........ 172

J)elphine . . . . . 82-214

Delusion....... 17

Demetrius ...... 570

Democracy...... 581

Denbies ....... 190

Denhall ........ 245

Deposit........ 85

Derby ......... 113

De Roos........ I51
Desdemona (dead). . 85

Design ..... 89-85

Despair....... 418

Despatch ...... 84

218

Desnerate...... 90

Despol ........ 111

Destiay ..... 84-256

_ by Sultan.... 111

Delonator...... 419

Deva.......... 266

Derice ........ 82

Dewdrop........ 555

Diamentina...... 84

Diana, by Teloci-

- by Kill Devil. 84

— by Catlon.... 584

Dice......... 159

Dick.......... 55

by Mulcy.... 74

Dickey Gossip..... $\quad 63$ W'allington.. 71

Diclator ........ 54

Dido ........ 88-201

Dinah ...... 169-361

Jinarzade....... 88

Dirce ......... 12

Dirmid ......... 275

Disaster ........ 118

Discord........ 88

Discovery ... 140-593

Discount........ 566

Dislingué, The... 598

Divan ......... 250

Diversity........ 148

Doctor, The ..... 24

-....... 101

Doctor SIop ...... 184

_ IIalley ...... 191

Dodo ......... 562

Doll Tearshect.... 89

Dolly ......... 116

- Mop....... 89

DoIphin ....... 225

Dolt .......... 596

Domain ....... 83 pede........
Dominie Skelp....4 417

Domino ....... 519

Donald ........ 184

Donation........ 90

Doncaster....... 258

— by Mluley .... 170 by Bustard... 227

Donegani ...... 242

Don Juan ...... 162

Donna Clara ..... 418

— Maria. . . . . . 419

Donzelli ........ 249

Dora......... 182

Dorabella ....... 240

Dorina ........ 91

Doris ......... 17

Dorothy ....... 102

Dorus........ 155

Doska .......... 1 gо

Dottrel ......... 244

Double Entendre 91-519

Doubtful....... 27

Douglas ....... 157

Dovedale....... 77

Dover ......... 17.

Drab......... 9

Draco ......... 557

DragIctail. 540 and add. Dragon, The..... 45

Dramatist...... 85

Dreadnought .... 108

Dream ......... 91

_ - by Waxy Pope 596

Dreanter........ 91

Dromedary...... 278

Dromio........ 553

Drone........ 409

Drowsy......... 91

— by Master Ro-

bert ........ 596

Drudge........ 596

Druid ......... 256

Drummer, The... 1

Dryad ...... 91-158

Dublin ........ 256

Duchess, The, by

Catton ....... 198

— by Card. Y... 562 
Duchess, by loung

Blacklock ..... 598

___ of York..... 553

__ (Young) . . 4 40-91

- - by Cons. 562

Duckling ..... 92-294

—_ by Wlialeb.. 596

Duenna ........ add.

Duke, The......4 418

Dulcamara ...... 92

Dulcinea ........ 92

Dunois ........ 97

Durliam ....... 541

Dust.......... 593

Dust ......... 405

Duvernay ....... 48

Duxbury ........ 596

Eagle .......... 288

Eaglet........ 1 . 1

Earl, The.......64

Earl of Percy.... 64

Earwig....... 95-287

Easton ........ 125

Ebberston....... 955

Ebony, by Jerry... 142

—_ by Trufile... 554

Ebury......... 56

Ecarté........ 5.54

Eccentricity ..... 158

Ecclefechan...... 259

Fclio......... 281

Eclat......... 504

Edgar ......... 105

Edie Ochiltree.... 55 s

Editl ......... ýt

—. by Swap.... 103

—— by Fitzjames. 94

Edmund ........ 104

— by Viscount. . 181

Elivard........ 46

—- by Edmund. . 289

Eel .......... 595

Ellie .......... 94

—- by Catton... 95

Eflie Deans...... 9y

Egbert......... 147

Egremont .......510

Elcanor........ 96
Page

97

Eleanor, by Election

- by Middleton. $\quad 99$

- by Whalebone 229

- by Comus... 227

Electa ...... 96-205

Electra ........ 256

Electress....... 98

Elephant........ 288

Elephanta ....... 288

EIf .......... 127

Elfi Bey ....... 219

Elfin .......... 504

Elfrid.... 98 and add.

Elfrida. . . . . . 98-99

Elighé. . . . . . . 596

Elinor........ 112

Elis... . . . . . . 225

Elisena ....... 80

Eliza......... 100

— by Rubens . . . 99

- by ditto .... 99

- by IVhalebone 224

- by Filho.... 100

__ by Edmund. . 504

- Leeds....... 100

Elizabeth, by

Rainbow ... 26-101

- by Soothsayer 101 by Walt. 100-584

- by Waverley. 288

— by Mango... 101

Elizanne....... 102

Elizondo....... 165

Elk .......... 595

Ella......... 417

Ellen.......... 102

by Peter I Iely 109

by Orville... 157

Ellerton Lass. . . . . 539

IIm. . . . . . . . 595

- by Roller.... 415

Eloisa........ 46

El Pastor. . . . . . . 20 20 .

Elvas......... 163

Elve ......... 96

Emancipation ..... 16

- by Blacklock. I17
Page

Emerald, by Emil. 554

— by Blacklock. 28

Emery.........248

Emigrant ....... 54

— by Figaro.... . $28 S$

Emilia......... 102

Emilina ....... 195

Emiliana....... 559

Emilianus . . . . . . 559

Emilius (Young)... 68 (Mr Sowerby's) 189

Emily .......... 65

- by Inticipat. 178 by Stamford. 105 by Pantaloon. 101

Eminch........ 13 0

Emir.......... 554

Emma, by Tramp.. 124 by Fitz-Emily 104 - by Don Coss. 103 by Orville... 105 - by Whis. 105-127 - by Comus... 56

Emmelina ...... ว้-104

Emmeline....... 104

Emmett........ 595

Emmy . . . . . 104-10'

Emperor....... 252

Emu ......... 554

—. by Picton.... 595

Encliantment .... 589

Enchantress ..... 20 ร

— by Sorcerer. . 569

— by Reveller.. 563

- by Overseer. . 409

Endeavour ...... 94

Energy ........ 150

Englemere ...... 528

Ennui......... 222

Enterprise...... 415

Enville ........ 29

Envoy ..........

Epaulette ...... 252

Epicure........ 251

Equator........ 107

Equiroque ...... 117

Eric . . . . . . . . 287

Ermine . . . . . . . 200 
Payse

— by Langar... 18

lirnest ........ 10y

Error ......... 981

Ersce ......... 406

Erymus........ 100

Escape ....... 10马

Eslar.......... 529

Esmeralıa ... 106-286

_. by Zingance. . 134

Espagnolle.... 22-106

- (Young). 106-106

Esperance ....... 106

Esplandion ...... 267

Esprit ........ 174

Estrella....... 16:

Etching, The..... 107

Ethilda ........ 98

Eliquette ....... 107

Etona ......... 91

Eva.......... 100

by Camelopa. 404

Evadne, by W. Pope 205

- by Gren.... 173

Eve......... 157

—_ by Banker... 410

Eveleen........ 105

Evelina....... 228

Evens ......... 108

Everilda ........ 29

Everton ........ 259

Euanthe ........ 50 马

Eucharis ....... 562

Eucrosia ....... 107

Eugene......... 145

- $\operatorname{Aram}$. . . . . 292

Euphemia...... 564

Euphrasia ....... 108

Euphrosyne ..... 108

Europa...... 109-285

Euryouc..... 285-562

Euxion ........ 27

Exchange .......417

Exile.......... 245

Expectation...... 50

Eycbrow....... 417

Eyesore ....... 109

Fabius ........ 158
Page

109

Fadladecu. . . . . 596

109

109

$2: 44$

\begin{tabular}{l} 
Fairy, The ....... 547 \\
\hline by Woful.... 547
\end{tabular}

by Partisan... 113

by Magis. 110-197

by Figaro....

2

l)y Filho.. 58-110

Ouecn, The. 110

Faith.......... 220

Faithless ....... 597

Falconlıridge..... 34

— by Manfred.. 117

Falconer .......5 515

Falconet ....... 54

Falernia ....... 579

Falstaff ........ 40

Fame ......... 27 ž

— by Calcb $0 . . .565$

Fanatic........ 172

Fanc̀non ....... 419

Fancy ....... ร̌ร -110

— by Moses 110-112 by Phant. 111-204 by Wanderer. 196

Fanny, by M. Henry 190

—_ hy Jerry.... 109

- ly Champion. 597 by Regent... 597

— by Whisker.. 47 Davies ..... 111 Grey ...... 249 Kemble .... 170 Legh . . . . . 111

Fantasima....... 111

Fantoccino ......

2

Farce...... 70-112

Farlington ...... 107

Fatality........ 17 i

Father Barney . . . . 184

Father Long-legs...

Fashion ........ 204

— by Starch.... 258

Fatima ........ 112

Fannus . . . . . . 158
Page

Favori.......... 94

Favorite ....... 112

Faust ......... 282

Faustina ........ 255

liawn, by Smol.... 115

- by Dinmont.. 115

Fear ......... 141

Fearnhurst ...... 75

Fedora ........ 62

Felicity ........ 178

Felix......... 121

Felucea ........ 214

Fenclla, by IVoful

565-385

by Milo .... 113

Ferdinand....... 568

— by Cervantes. 418

Fergus ........ ๖ร

Feruex ........ 551

Festival.......5 515

Fetua ........ 8 b̆

$\mathrm{Fez} \ldots \ldots \ldots \ldots . \ldots 40$

Fickle . . . . . . . 56

Fib........... 187

Fidalgo ........ 415

— by Sultan.... 515

Fidalnu...... 88-115

Fiddle Faddle.... 112

—- (dead) . .... 586

Fiddlestring ... ร.5-114

Fidelio . . . . . . . 588

Fidelity........ 121

__. by Worthy ... 573

Fidget......... 219

Ficnd, The...... 288

Fieschi ........ 210

Fifer......... 16 g

Filcher ........ 18

Filho da Puta (Y.). . 550

Filagree ....... 116

Fille de Joie ..... 116

Finance .......5 515

Finesse ....... 117

Fingal. . . . . . . 189

Finikin ........ 117

Firc-fly . . . . . . 142

—_ by Velocipede 172

Firmaı ........ 158 
Fisher Lass. Page 117.9 Forester Lass .... Page

Fisherman........190

Fitzcloddy ...... 157

Fitzdictor ....... 113

Fitz-Emma ...... 101

- Langton..... 550

Flambeau ....... 172

Flame......... 299

Flamingo ...... 187

Flamock ....... 44

Flare-up ....... 45

Flatterer ........ 66

Fleur de Lis . . . . . 118

Flight . . . . . . . . 206

- (Irish). . . . . . 597

Flighty ........ 84

Flirt .......... 204

- by Blacklock . 324

Flora.......... 112

_ by Camillus.. 118 H'Ivor ...... 355 (Irish). . 598

Floranthe ...... 119

Florence ......... 201

- by Partisan .. 119

Florentia....... 64

Florestine.... 118-119

Florian ........ 119

Florimel ........ 14

- by Gr. Arab. . 119

Florin........ 513

Florine ........ 22

Florio ......... 16

Flounce......... 119

(Young) ..... 119

Flourish. . . . 120-254

Flush. . . . . . 120-167

Fly . . . . . . . . . 598

Flycatcher....... 242

Foam......... 598

Folly, by Waxy. ... 398

Foolman........ 96

Foozool........ 41

Fop.......... 118

.. by Fungus... 316

Forester ........ 121

by Fitz-James. 190

by Gustavus. . 247
Foresail......... 275

Fortitude....... 120

Fortuna........ 120

Fortunatus ...... 20

Fortune-teller .... 46

Forward ....... 269

Foxcote....... 11

Fracture .......4 406

Fra Diavolo...... 1 зЈ

Frail ......... 107

Frailty ....... $5-121$ (Col. P.'s).... 116

Frances........ 121

.. by Emilius... 522

Francesca..... 14-121

_- by Partisan.. 226

Frank ......... 149

—_ by Banker... 578

Frankenstein..... 214

- - by Manfred... 545

Frantic.......

Freckle ....... 570

Frederica........ 121

- by Sultan.... 121

- - by Moses. 122-151 by Little John

122-241

Frederick ....... 55

__ by Edmund.. 187

—— by Filho..... 137

— by Jiltle John 241

— by Watcrloo.. 141

Free-Agent ..... 105

Freedom....... 105

Frecholder...... 105

Freewill........ 103

Freischutz...... 23

Freney.........4 418

Frenzy ....... ร.

Friar......... 595

Friar Tuck....... 989

- - by Little Joln 586

Friday........ s1

Friendship . . . . . $\mathbf{3 9 9}$

Friseur........ .

Frisk. . . . . . 566

Frolicsome ...... 122
Page

57

Fun............. 294

Funny......... 261

Furfau......... 1 ร้ 1

Fury..........61

— by Soothsayer. 506 by Tramp ... 419

Fusilcer........ 536

Gab.......... 123

Gabrielle..... 76-123

Gadabout........ 124

Gaffer Grey....... 72

Gaiety. . . . . . . . 124

Galata.......... 4

Galatea... 124 and add.

Gale Middleton.... 518

Galena....... 70-125

Galliard......... 265

Gallivanter ...... 111

Galopade........ 47

—_ by Reveller .. 266 by Dr Syntax . 94

Galway ......... $39 y$

Gambol......... 45

—— by Filho..... 90

Gamelia........ 12g

Ganges ........ 86

Gantlorpe....... 256

Garcia........ 125

Garlic ........ 176

Garrick........ 65

Gas.......... 89

Gavotte . . . . 76-126

Gawky . . . . . . 99-126

Gazcbo ....... 500

Gem ......... 76

Gemappe........ 74

General........ s0

_- The....... 285

—_ - by Conf . . 509

__ (Irish). . . 594

— Chassé..... 156

$$
\text { Mina ...... } 545
$$

Geuista ........ 155

Geoffrey Crayon... 279

George........4 400

Georgian....... 525

Georgiana....... 21 
Georgiana . by Ten. 259 by IIoful. . . 126

Georgina........ 25

Gerard Dow...... 162

Gershom........ 160

Gertrude..... . 1 $19-574$

Ghost, The....... 247

Gibside Fairy .... 127

Gift .......... 127

Giglet ......... 128

Gilbert ........ 208

Giles......... 410

Gimmer, The. . . 4y-128

Gin........ 128-252

Gingarella.. 128 and add.

Ginnums........115

Giovanni........ 90

Gipsy ......... ร

- by Whalebone 98

by Tramp . 68-159 by Sir Hercu.. 549

Giraffe......... 596

Girouctte..... 76-129

Gitana........ 579

Gladiator........ 198

-_ by Parisan .. 256

Glama, The...... 18

Glamour ....... 79

Glance.......... 599

Glantivy ....... 418

Glaucus......... 216

Glee.......... 108

Glec-singer...... 63

Glenalmond...... 49

Glenamour ....... 505

Glencoe........ 518

Glenfinlas....... 509

Glenlce......... פั0

Glenmore ....... 518

Glenvarlock...... 106

Glider........ 256

Goblin......... 547

Godfrey........ 158

Goldentrop...... 587

Goldtinch......... 225

- by Mamcluke. 558

Goldfringe...... 152

Goldpir......... 151
Pagre Page

Goldwire....... 152 Guard, The...... 206

Gondolier....... 76 Guardian........ 104

Goodluck........ 77 Gudewife o'Tullos-

Goodtidings...... ร 5 hill, The..... $2 y_{4}$

Goshawk........76 Guerdon....... 256

Gossip......... 565 Guerilla........ 76

Gourd, The....... 265 Guiccioli .... 598-599

Governante..... 152 Guinea Chick .... 154

Governess....... 152 Guitar........ 275

Governor, The ... 74 Gulistan ....... 155

Gracchus........ 178 Gulnare..... 15ร-187

Graceful ....... 196 Gutty......... 15马

Gramaric....... 565 Guy Faux......

Grammont...... 127 Gypsiana....... 59

Grampian ....... 99 Gypsy ......... $296^{\circ}$

Grampus....... 262 — by Tramp ... 227

Granby ........ 286 Haidéc........ 570

Grand Cairo ...... 124 Haldon ........ 5 5ั0

Grand Duke, The.. 187 Halyards....... 156

Grand Falconer.... 2 Hambletonia..... 156

Grasshopper...... 196 Hambletonian (1.) . 166

Gratis ......... 160 Hamilcar....... 182

Grapeskin....... 250 Hamlet........ 415

Gravity........ 155 Hampton....... 151

Great St Michael... 191 Hamptonia ....... 26

Grecian Queen .... 134 Handmaiden..... 157

Gregson........ add. Hannah........ 194

Green Leighton... 548 - by Tramp.... 554

Grcen Mantle... 92-154 Hard-bargain..... 152

Grenada ........ 27 Hardwiclie...... 87

Grenoble....... 176 - - by Polygar... 417

Gretna Green...... 150 Harlequin ....... 118

—_Lass....... 221 Harlot......... 65

Greybone........ 69 Harmonia...... 265

Grey Fairy (now Harmony .... 158-226

Coma..... .699 Harpalice ....... 158

—— Rosamond ... 8 Harpham Lass..... 159

Harrict, by Filho.. 207

Grilurig........267 — - by Cannon 13.. 564

Griselda ....... 522 — by Pericles... 140

—. by Lory. . . . 565 — - by Stripling. . 159

Grisette ......76.154 _. (Young) .... 159

—— La ....... 155 Harriette Wilson. .. 589

Grisi......... 419 Harry ......... 65

Grog......... 128 _- by Sir Harry.. 111

Gros de Naples.... 56 Harrington..... 403

Grotesque....... 261 Hartlepool....... 241

Guadaloupe......420 IIartonian...... 157 


\begin{tabular}{|c|c|c|}
\hline Page & & \\
\hline IIartpury........ 16 & Her Ladyship...... . & \\
\hline$\ldots \ldots 240$ & ajesty....... & \\
\hline arum Scarum . . . 116 & Icrmione . . . . . . & \\
\hline Hassan.......... 250 & Hermit, The...... & Ilurdy-gurdy...... \\
\hline Hatficld. . . . . . . 131 & — by Mr Lowe.. & Hussar.......... \\
\hline $\operatorname{ldar} . . . \ldots \ldots .15$ & nani......... & n Pacha..... \\
\hline awk........... 20s & $\ldots \ldots$ & Hussine.......... \\
\hline Iawker......... 270 & 209 & Ilyacinth........ 67 \\
\hline lawk's-eye ....... 281 & $\ldots \ldots$ & Hybla....... 145-162 \\
\hline dam .. 589 & $\ldots \ldots$ & $r \ldots \ldots . . . .15$ \\
\hline$\ldots \ldots 564$ & 82 & 1 \\
\hline$r \ldots \ldots .148$ & 419 & Hydrogen ....... 144 \\
\hline$\ldots \ldots \ldots .266$ & $\ldots \ldots$ & Hylas........... \\
\hline$\ldots \ldots \ldots 140$ & $c \ldots .$. & $\ldots \ldots \ldots \ldots 517$ \\
\hline iils..... 2 & $\ldots \ldots 13$ & $\ldots \ldots \ldots 169$ \\
\hline ase... 161-564 & us, The. 217 & .......291 \\
\hline$\ldots \ldots \ldots \ldots 146$ & le ..... 85 & tern.... 417 \\
\hline$\ldots \ldots \ldots \ldots 120$ & $\ldots \ldots$ & cet...... 141 \\
\hline$\ldots \ldots \ldots 140$ & $s \ldots .$. & $k \ldots \ldots .150$ \\
\hline m.... 194 & $\ldots \ldots$ & ot ..... 500 \\
\hline — by Master H.. 418 & 556 & - Sutton ...... \\
\hline Iccuba ........ 548 & 532 & r....... 580 \\
\hline ....... 186 & 127 & _ and-all-Jack. . \\
\hline Heiress . . . . . . . 69 & 540 & $\mathrm{Jac}$ \\
\hline$\ldots \ldots \ldots 400$ & $\ldots \ldots \ldots$ & 155 \\
\hline $0 n \ldots \ldots .141$ & $\ldots \ldots .87$ & _ by Veloc. 274 \\
\hline с....... 142 & се..... 215 & $\ldots \ldots \ldots \ldots 244$ \\
\hline na......... 598 & 191 & $\ldots \ldots \ldots 184$ \\
\hline s......... 5 ร้ 6 & $\ldots \ldots \ldots \quad 15$ & ane $\ldots \ldots \ldots \ldots$. \\
\hline$\ldots \ldots \ldots \ldots 98$ & ......295 & — by $\Lambda$ utocrat.. 75 \\
\hline$\ldots \ldots \ldots \ldots$ & 185 & - - by Moses.... 144 \\
\hline ...... $79-142$ & 408 & - by Hiddleton. 250 \\
\hline ian....... 81 & bin....... & —— Shore .... 2:-144 \\
\hline$\ldots \ldots \ldots 524$ & $\ldots \ldots \ldots$ & c...... 401 \\
\hline ........ 275 & -363 & ibjer..... $27 \pi$ \\
\hline ...... 295 & eveller.. 947 & - by II hislier. . 134 \\
\hline ...... 142 & tlery... 575 & $\ldots \ldots 108$ \\
\hline$\ldots \ldots \ldots 145$ & Zubens... & $\ldots \ldots \ldots \ldots 189$ \\
\hline st. Rob. 408 & 213 & ........ 255 \\
\hline Jerry & $\ldots \ldots 156$ & ..... 289 \\
\hline 65-364 and err. & $\ldots \ldots 136$ & \\
\hline Henry Masterton. . . 274 & ........ 103 & - by Sultan.... 242 \\
\hline 4 Lacy....... 94 & 57 & $\ldots \ldots \ldots \ldots 144$ \\
\hline 292 & 413 & . . 526 \\
\hline & & \\
\hline — by Carlton... 554 & $\ldots \ldots .401$ & Jdalia.......... 145 \\
\hline $\begin{array}{l}\text { IIer Ilighness. . 232-563 } \\
\text { rol. } 1 \text {. }\end{array}$ & Ilumphrey....... & Idiot .......... 245 \\
\hline
\end{tabular}


Paģe

Jeanue d'Arc..... 145

Jeannetton....... 143

Jeanny Baptiste.... 142

Jemima . . . . . . . 208

Jenny Mills..... ร7-146

— Rintherout . . . 146

Sutton..... 146

—— Vertpré... 23-147

— Wren...... 56

Jereed. . . . . . . . 214

Jeremiah. . . . . . 190

Jericho ....... 171

Jeloboam . . . . . 190

Jerry . . . . . . . . 44

—_ by Smolensko. 168

Jessamine. . . . . . 549

Jessica......... 75

Jessy, by Whalch.. 127

—- by Comus ... 169

Jest. . . . . . . . . 147

Jewcss...... 4:-147

Jilt ..........

Ildegarda........ 401

Ileen.......... 147

Iman.......... 198

Imbar . . . . . . . . 281

Imp. . . . . . . 110

I'm sure he shan't. . 563

Ina, by Smolensko. 147

Ince... . . . . . . 103

Incest. . . . . 147-262

Inch . . . . . . . \& 411

Incognita . . . . . 148

— by Whaleb... 122

Incubus........ 152

Independence .... 506

Independent ..... 51

Indulgence ...... 8 s

Industry . . . . . 1 . 19

Infant Lyra. . . . . 565

Inflexible....... 101

Ingleborough . ... 40

Inheritor ....... 157

Ink .........4 411

Insanity........ 85

Intrigue. . . . . . . 279

Intriguer........ 279
Page

154

- by Paul Pry,

or Belzoni.... 158

Joanna. . . . . 116-565

Job . . . . . . . . . 255

- - by Middleton . 589

Joccline........ 543

Jocko . . . . . . . 198

Johanna . . . . . . . 565

—_ Southcote.... 148

—__- Joung. . 551

John . . . . . . . . . 128

—— o'Gaunt .... 418

Johnny . . . . . . . 405

—— Watson .... 585

Iole.......... 153

Jolly Roger . . . . . 157

Jonas........... 250

—_ by Whalcb... 262

Jonathan. . . . . . . 555

— Bradford ... 54

Ione ......... 148

Joseph.......... 51

Ipsala ......... 2

Iron Founder . . . . 229

Ironsides....... 185

Irvinite . . . . . . 560

Irwell . . . . . . . 105

Isa. . . . . . . . . 250

Isaac. . . . . . . . . 501

Isabel. . . . . . 5 51-552

- by Emilius... 21 by Catton.... 101

Isabella......... 148

— by Smolensko. 148

—_ by Lottery... 259

Ishmael. . . . . . . . 242

Isidora. . . . . . . . 555

Ismene . . . . . . . 168

Isse . . . . . . . . . 527

Itinerant . . . . . 121

Itty Pet. . . . . . . 99

Ivanhoe........ 44

— - by Moses . . . 266

Juba . . . . . . . . 55

Jubilce . . . . . . . 149

by Y. Pipator. 205
Page

Judge, The . . . . . 25 5

Judy Callaghan . . . 571

Jule........... 58

Julia . . . . . . . . 53

Juliana . . . . . . 53

by Gohanna.. 149

by Partisan.. 67

by Pyramus

108-56

Juliet. . . . . . . . . 566

— by Rowlston.. 419

Julius . . . . . . . 158

Jungle Admee..... 403

Juniper (Young) . . 417

Jungfrau. . . . . . . 299

Jupiter . . . . . . . 254

Justice... . . . . . ร4

Justitia . . . . . . 151

Kakistos ....... 5

Kalmia . . . . . . . 5 s4

Kamschatka...... 15้1

Kangaroo........ 256

liate .......... 95

—— hy Frolic.... 507

- - by Callon 1s1-201

by Corinthian. 146 Kearuey.....

57

Katherina (late Pro-

spective)... 152-161

Katherine.... 152-257

Kathleen ........ 105

-_ by Blacklock . 551

by Phantom. . 555

Katinka ........ 417

Keepsake....... 552

Keyiugham . . . . . 518

Kean.........254

Ketchup........ 213

Khylan ......... 999

Kilnocky ........ 54

King Catton....... 288

— Cole....... 22

- Corney... . . 589

- Fisher ...... 266

Lear........ 67

- by Bed-

lamite....... 546 
Page

King of Clubs..... 192 — William..... 248 Kirochtrec...... 50 Kiislar Aga....... 260 Kiss..........401 401 Kit .......... 293 Kitchen Maid . . . . 596 Kite ....... 185-225 Kitten.......... 155 — by W. Pope. 401 Kittums......... 115 Kitty Fisher ..... 295 — Flanagan.... 155 — of Coleraine.. 151 Klepper......... 598 Knobstick....... 8 Lace . . . . . . . . . 132 Ia Bayadere..... 79 Lacerta . . . . . . . . 155 La Danseuse..... . 154 La Meprisée ..... 5 5 La Mode........ 118 La Sonnambula.... 277 Lady $A$ gnes....... 87 Albert . . . . . . 155 - Anna....... 150 Anne....... 586 - Barbara..... 214 - Bec....... 417 - Bell....... 272 - Betty...... 77 - Berners, The. 501 - Bird....... 40 - Blanche, by

Borodino...... 80 — - by Stum. 388 by Boba . 272 - Blessington .. 293 - Bluefoot..... 134 - Brownlock... 58 - Canford..... 51 - Caroline..... 588 - Charlotte.... 15ร - Clapham .... 585 Day....... 348 de Gros.... 57 Ji....... 512
Parge

Lady Easby....... 1:4 Elizabeth.... 206 Eliza ...... 100 by Wh. 15 s Emily ..... 12 Emmeline 13ว-296 Fanny ..... 512 Fly ....... 40 Foot ...... 149 Fractious.... 524 Frances.... 115 Fulford ..... 15y Georgiana ... 156 Grey...... 156 - Harrington .. 214 Henry ...... 1 ä6 Heron... 402-411 Jane ...... 94

Noy St

Nicholas ....... 223 Jersey ...... 418 Leg. . . . . . 149 Louisa...... 78 Maltby..... . 208 - Marcia..... . 158 - Margery.... . 158 - Mary ....... 124 - Ilaud....... 138 Moore Carew. 153 - Mowbray 156-158 - Neville ... 87-157 Newton .... . 1987 of the Lake. . 157 of the Tees.. 158

— of the Vale.. 158 - Olivia ..... 175 Ormond..... 417 - Paul....... 581 - Racket..... 258 - Sarah ... 1 189-206

—— - by Leop. 159 —_ Stafford..... 555

—- Stepney ..... 1 รั

—— Stumps..... 1989

— Thigh ..... 149

- - Vane (dead). . 555 Lady's Slipper. 139-272
Pagge

Læna.......... 268

Lafitte......... 575

La fille mal gardée. 575

La Grace....... 173

Lambkin........ 143

Lambtonian ...... 165

Lamia......... 160

L'Amitić. . . . . . . 289

Lamplighter..... 505

Lancastrian...... 20!

Lancer ......... 70

Lander........ 520

Landgravine..... 98

Landlady ....... 160

Landrail . . . . . . . 29.3

Landscape....... 160

Land's-end ...... 170

Languid ....... 179

Languish ....... 179

Lanstlowne...... 269

Laocoon ....... 509

Laodice......... 14 !

La Pucelle ...... 141

Lara .......... 285

Larissa........ 162

- - by Rub.... 162

Lark ....... 40-566

—— by Rub.. 506-566

Larkspur........ . 79)

Lassie......... 567

Laundress....... 161

Laura... . . . . 149-163

—— by Beningbro. 162 by Don Cossa. 165 by Champ. 162-16.5

Laureola. . . . . . . 16 165

Laud ......... add.

Laurel.......... . 250

Lauretta....... 504

La Valiere ....... 2.

Lawnsleeres..... 16

Lazaronc........ . 519

Lead ...... 152-567

Lcander ........ 8.

Lechmere ....... 190

Leda, by Lott. 159-569 —- by Filho.... 164 
Leeway ........ Paghe

Legacy ........ 567

Legend........ 164

Leila.......... 164

Lcinster . . . . . . . 410

Lelevo.......... 345

Lemonade....... 567

Lemon-squeezer. . . 567

I.ena......... 545

Lent.......... 102

Lentulus....... . 59

Leo......... 16az

Leonella....... 168

Lcopard ........ 240

Leopoldine...... 16 s

Lepanto ........ 71

Lepida . . . . . . . . 279

Lesinka......... 226

Letty....... 127.557

Liberator ....... 543

—- by Feramorz. 595

Lichfield........ 20

Lieutenant ...... 501

Lightning...... 598

Lilias . . . . . . . . 405

Lilka. . . . . . . . . . 164

Lilla ........ .

Lily Lass ....... 156

- of the Valley. 16

Limner.......... 216

I.incoln........ 511

Lincroft . . . . . . 549

Linda ......... 163

by Mameluke,

or Tarrare .... 165

Lindley ......... 566

Linkboy ....... 89

Linnet......... 506

- - by Paulowitz. 506

Linton Loch. . . . . . 512

Linx.......... 58

Lion, The....... 105

— by Brutandorf 290

Lioness.... . . . . . 10.

Iisheth......... 101

Lisette . . . . . . 166

Liskia ........ 118
Page

Lot .......... Page 265

Liston........ 217

Lithuanian ...... 128

Little Bird, The... 52 Cassino .... 584 Charlotte.... 26

Dick........ 22

Duchess ... 5 5- 568

Ecstacy .... 69

Fauny ...... 115

by $\operatorname{Sir}$

Peter......... 166

- Finger . . 150-166

Folly . . . . . 166

Gift....... 520

Go....... 142

John ...... 586

Johnny Myers 545

Red Riding

Ilood . . . . . . . . 568

— Rover ..... 992

Zan....... 250

Liverpool . . . . . . 559

Livonia........ 167

Lizzy.......... 40.

Lochinvar....... 1 g8

Locket ......... 568

Y. Black-

lock ........ 412

Lockington...... 590

Locomotive...... 194

Lodola ....... 410

Logic ......... 200

- by Epperston. 505

Lolotte ........ 171

Longinus ...... 91

Longshanks ..... 90

Longwaist....... 215

_- (Young)..... 289

Lonicera........ 174

Loo........... 167

loo Choo....... 167

Lord Collingwood. . 16

—_ Francis ..... 115

_ John ...... g

- - Stafford..... 55 5

Lorenzo ....... 402

Loretta . . . . . 168
- - by Brutandorf 284

Lotchin........ 157

Lothario........ 265

Lothringer ....... 207

Lotteria......... 1

Lottery......... 403

Lotto......... 529

Loudon........ 575

Louisa......... . 259

- - by Orville... 168

- - by ditto. 169-257

—— by Filho .... 169

Louis d'Or ..... 576

Louise......... 151

Loyalty......... 169

Lubentia........ 571

Lucerne ........ 2 290

Lucetta........ 170

- - by Sarpedon. 561

Lucia .......... 149

Lucifer......... . 945

- - by Peter Lely 254

Lucinda ........ 170

- - by Corvantes. 170

Lucius......... 68

Luck's-all ...... 62

- - by Tramp... 118

—- by Y. Blackl. 412

Lucretia ....... 260

Lucy.......... 42

__ by Tramp... 159

—_ by Mulcy.... 171

_. Kemble .... 58

Ludford ....... 14 ö

Ludlow........ 295

Ludorico........ 169

Lumber......... 547

Luminary ....... 140

Luna, by Wand. 49-171

_— by IIal. . . . . 127

Lunacy. . . . . . . 171

Lunaria... . . . . . 171

Lunatic........ 179

Junette........ 156

Lunettes. . . . . . . 172

Lupin. . . . . . . . 417 
Page

Lupus. ......... 216

Lurcher.......... 158

Lusher . . . . . . . 146

Lustre. . . . . . . . 179

Luther......... 528

Luzborough . . . . . 86

Lycastus. . . . . . . 537

J.ycurgus........ 183

Jydia ........ 179

Lydian . . . . . . . . 202

Jyric. . . . . . . . . 175

Lyrnessa........ 175

Habooba....... 576

I'Adam, by Tramp 250

II'Arab........ 57

Macleth ........ 124

Madame de Jeck . . . 288

— Pelcrine..... 20

— Saqui...... 570

Madcap......... 70

—— by Dinm..... 570 by Tramp, or

Blacklock ...... 15y

Madelina........ 174

Hadoc......... 8 8

Mad Tom....... 65

$\ldots \ldots \ldots . . \ldots 75$

Mrcenas........ 5y

Hronides ....... . 2ร

Macscrygic ....... 182

Magawiska... . 175-293

MIagic ......... 55

Magician. . . . . . 306

MLagister . . . . . . . 168

Hagma ......... 571

Magnate, The..... 1 s

Jagnet. ........ 910

— by Merlin.... 987

Magnolia........ $2 S$

Magus.......... 18

Mahmoud . . . . . . .

IIahomet........ 68

IIaia .......... 113

JLaid-of-all-Work... 370

—— of Ardec... . . 599

— of Cadiz..... 581

of Kent..... 178

of Lorn...... . 17 s
Pagge

Naid of Lune . . . 127

_- of Mclrose... 541

of Ilonor . . . . 107

__ of Mansficld

176-525

- - of Milan.... 175 of the West.. 65 of Underley .. 88

—— of Wirral.... 178

Maiden ......... 176

—- by Orville.... 176

Major, The...... 409

—_ Naltby. . . . . 208

Makanna....... 252

Malcolm ........ 141

Maldonia........ 265

Malck . . . . . . 150

Malgwyn....... 8

Malibran....... 195

— - by IIuley.... 249

- - by Rubens... 176

Malice.......... 176

IIalton ......... 157

Malvina....... 305

Mambrino...... 152

Маmmon........ 183

Mammoth, The.... 217

Mamscl $0 t z \ldots \ldots .540$

Manby......... 590

Manca. . . . . . . . 581

Manchester...... 559

_ - by Whisker .. 214

Mandadinc̀...... 177

Mandeville ..... 402

ITandolinc....... 177

Manes ... . . . 4 48-40J

Manfreda ....... 508

Mangel Wurzel.... 178

MLango......... 213

Maniac (Young) . . . 178

Manille...... 178-584

Manœuvre ... 117.178

- - by The Col . 151

Mansficld Lass. 525-40J

Manta.......... 973

Mantilla......... 9.

Manto......... 179

—— by Tiresias... 44
Manto, by Tir.. 179-555

Mantua ........ 179

—— by Card. Puff. 179

Ianuclla. . . . . . . 179

Maple ......... 248

Maplebeck....... 205

Marathon....... 162

Marceline ....... 180

Marcella ....... 179

March.......... 286

Marchesa........ 179

Marchesiana ..... 180

March Hare...... S 86

Iarchioness..... 25

—_by Catton.... 156

Marcian........ 180

Marciana. ........ 180

Marcus ........ 40

— by Filho .... 260

Maresficld....... 501

Margaret.......404

—— by Pyr... 108-180

- - by Swiss..... 12:

—— by Edmund .. 187

Margarette....... 997

Margcllina ...... 180

Margrave ....... 97

Margravine, The 180-241

Maria, by Whis. 197-181

— by Sir IIcreul. 407

- by Master H. . 170

by Trissy.... 181

by Waterl. 26-18 1

l)arlington. . . 182

Marian ........ 418

Mariana........ 555

Marianne....... :

- - by Edmund.. 124

- by Malck... . $26 ! 3$

- by Sorcerer . . 182

Maridunum. . . . . 18.

Marictte....... 181

Marigold....... 183

Marinella........ 182

IIarion ......... 18.

Mariqueta...... 182

Mlarmalade...... 215

Marmora........ $5 \% 0$ 
Page

Harmoset ....... S0

Maro.......... 261

Marpessa........ 66

Marphisa. ....... 570

Hlarrowfat .... IS 1-957

IIarshal, The ..... 169

_- Blucher ..... 2 gั

Jartello........ 147

Martha..... 184-289

Martinet ........ 2 . $_{1}$

Martyr......... 157

Marrel......... 1รั5

ILary, by Edmund.. 124

— by ligaro .... 146

—— by Friday ... 184 (Young) . . . . 184 Ann...... 18 Anne ...... 229

- by Mlas-

ter Robert. . . . . 40? by IV.P. 404 Horner. . . . 590 IIasaniello....... 574 II asetto........ 296 Naster Burke .... 574 - Charles...... 524 - Christopher.. 253 —— Johı ...... 159 - - of the Cerc... 107

__ of the Rolls .. 187 - Will....... 28

_- Walter. . . . . 253 Ilatador........ 176 Matadore........ 277 Matilda, by Orville. 183 - by Shakspeare 186 - by Ambrosio . 571 by Comus 149-183

- by Whisker . . 262 - by Hemuon.. 515 Hatilde........ 109 Maud ........ 186 Marrocordato .... 162 Maximus........ 73 May-day ....... bs —- by Advent ... 182 - by Lamplight. 274 Nayllower........ 991
Parge

May-fly, by Middlet. 69 294

May-morning..... 141

Mayo......... 176

Nayor, The..... 417

Mazourke....... 589

Mazurka . . . . . . 126

Meaux......... ร8

Medea......... 174

— by Dr Faustus 179

by Whisker.. 220 by Mameluke. 234

Medina........ 186

- by Selim 1 186-384

Medora ........ 187

by Swordsman 187

Medoro........ 182

Mecta ........ 96

Heg.......... 188

- Merrilies.... . ว2

Murdoch ... 187

Melampus....... 179

—- by Soothsayer 536

Meliora........ 157

- - by $\operatorname{Tramp} . . .290$

Mellerstein ....... 296

Melody ... 571 and add.

Mclrose..... 541-571

Memina........ 188

Memnon Junior.... 65

Memplis........ 188

Menas......... 238

Mendicaıt...... 198

Mendizabal ...... 942

Menerer ....... 188

Menivia........ 182

Mercandotti...... 188

Mercury . . . . . . . 167

—— by Merlin ... 282

Mercy ...... 189-287

Meridian....... 177

Merino ........ 526

Merlin..... 591-404

Merlyn Silvester . . 178

Mermaid .... 185-190

—— by Orville.... פ.:

- by Whalebone

189-200
Page

Merman . . . . . . 225

Meroc......... 94

Merry Andrew..... 195

Merrylass ....... 195

Merryınaid ...... 190

Merryman....... 185

Merrytricks...... 100

Mersey . . . . . . 266

Mervinia....... 190

Messenger....... 218

Metcor......... 65

Metheglin ..... 145

Meux......... 84

Mezereon ....... 278

Michael. . . . . . . 526

Michælmas . . . . . 191

Midas ......... 223

Midge . . . . . . . . 58

—- by Whisker.. 191

Midnight....... 148

Midsummer. . . . . 306

Mignon........ 111

Mignonette... 192-526

—_ by Sorcerer.. 192

-_by Vespasian. 192

- by Banker... 216

—_ (Young) . . . . 192

Milanese....... 226

Milcab......... 356

Mildred......... 90

— by Wav. or W. 154

Miletus ........ 996

Miller's-maid...... 176

Miller of Mansfield.. $\mathbf{3 2 5}$

Mlima . . . . . . 195

—— by Gen. Mina. 193

Mimic......... 193

_- by Wauton .. 290

Mimosa....... 536

Mina......... 22

Mine.......... 246

Miner, Tlıc . . . . . 135

Nlinera ......... 291

Minetta..... 195-249

Miniature..... 6. 6ั-195

- - by Teniers... 504

Minikin........ 195

Minima........ 18 
Pagse Minima, by El . 163-194 Minna......... 19. — by Camillus. . 194 Minster.........2.2.5 — by Blacklock. 150 Ilinstrel . . . . . . 194 Minuet ........ 195 Mirabeau........4 417 Miracle....... 571 Mirandola ....... 193 Miriam. . . . . 129-196 Mirth . . . . . . . 195 Mirza ........ 41 by Malek.... 164 — by Roller.... 404 Mischance...... 196 —_ by IIcrlin . . . . 571 Mischief ........ 160 Hisery......... 196 Hisnomer ....... 242 Hisrulc ..... 196-508 IIiss Alice....... 41 - Andrews . . . 197 —- Arnustrong. . . 197 _ Badsley ..... 2.55 - Ball ........ 262 - Bell ....... 24 Bomford .... 404 Bowe...... 575 Brceze..... 197 Cantlcy ..... 197 Catton...... 198 Chantrey.... 198 Charlotte.... 100 Chester .... 508 Clifton...... 199 Crachani. . . 199 Craigic..... 199 Craven .. 199-299 by Canop. 49 Croft... 185-199 Dick ...... 524 Dulwich.... . 324 Eldon....... 2924 Ellen ....... 127 Emma... 200-296 Emily....... 277 Fanny . . 200-226
Page Miss Flareaway ... 420

— Foote...... 408 liriendly..... 113 - For....... 200 - Frill....... 123 Frizzle..... 201

—- Garfortlı.... 201 Gift.... 201-374 Giles.... 13-572

- Golborne.... 219 Gravity ..... 16 J IIarrict Ison. . 203 - Jenry ...... 990

—- Inverarily ... 139 Iris ....... 202

—-Julia ...... 226

- Kershaw .... 291

- L.ely....... 104

-_Lancaster... . 79

- Lora ...... 59

- - Lydia . . 202-226

- Lytham..... 62

- - Maltby . . 202-207

- - Manager.... . 205

- Mlargaret.... 25

- Maria...... 417

- Mary Ann ... 25

Middleton. 205-315 Hilner ..... . 541 0'Ncil ..... 203 Orville .. 196-204 - Patch...... 98 Patrick 204 and err. Percy...... 258 - Potter ...... 294 Pralt... 124-40 Rolson ..... 572 Rush... . 204-246 Sheridan ... 244 Skim . . 204-506 Soutlicote... . 148

- Thomasina... 205

—- Tree... 205-276

- - Walton..... 174

—— Wasp...... 20马

- Wentworth

203-588 West ...... 206
Paģe

Miss Whip ...... 126

— Whitelock ... 128

Wilfred.... 24

Wilkes..... . 206

Windsor .... 580

_- Witch ..... 572

Missy ......... b

Mistletoc........ 198

Mr Bish......... 997

_- Mcrryman ... 10

— - Wags...... 2.51

IIrs Bang....... 510

- Brown..... 515

- - Burdock..... 31

- Clarke...... 207

- Cruikshanks.. 207

— - Fry ....... 579

—— Fulge ..... 207

— Maltby..... 207

—— Nisbett..... 190

—-Oaklcy...... 579

- - Rye.... 138-208

- - Scarlet..... 504

Suggs....... 208

—— 'Touchit..... 208

— Waylett....4 418

Mixbury ....... 140

Nodesty ....... 150

Hoclfamma...... 209

Moggy........ 2

-_- by Canopus. . 575

Mogmousc....... 248

Mohair ........ 556

Mole, The....... 200

Moll Flagon...... 5

Moloch ......... 249

Momus . . . . . . 550

- - by Card. Puff. 193

Mona.......... 209

Mona's Pride...... 208

Monica ........ 136

—_ by Lottery. . . 286

Monimia ........ 209

Monitor........ 290

Monops......... 257

Montrose....... 164

Moonbeam, The... 81

IIoonshine ...... 209 
Hoorhen. . . . . . P. 287 Hopsa . . . . . 209-286 Iopsus......... 179 10р5у ...... 149-572 Wordecai....... 419 Hordelia . . . . . . 156 Worea......... 162 Moredun. . . . . . . $\mathbf{9 8 9}$ Morella........ 215 Morgiana ...... 575 Morisca .... 210.532 Morison........ 158 Moroce . . . . . . 403

Jiorotto........ 184

Morpeth ........ 154

Mlorris Dancer . . . . . 209

Morrison . . . . . . 251

IIoselle . . . . . . . 293

Moses . . . . . . . . 129

Hoslem, The..... 584 Mosquito........ 45

Hoss Rose. . . . . . 1 ว0 Nouche . . . . 189-210 Mountain Maid . . . 5 5 I Nount Eagle . . . . . 147 Mourad . . . . . . . 556 Mouse Deer...... 279 Mouse.........2 210 - (Young).. 211-211

Houser . ....... 508 Mousetrap........ 211 Howbray Hill . . . . 551 Moynalta.......401 Mozart ........ 76 Muezzin (bay) . . . . 198 II fff . . . . . . . 202 Mufti......... 87 Mugger Lass . . . . . 146 Mulatto......... 85 Mulberry....... 267 Mulebird . . . . 21 I-287 Muley (Young) . . . 203 Mulcy IIoloch ..... 215 Mulgrave . . . . . 599 ILuliana........ 213 Mulligatawny .... 246 Mummy, The . . . . 210 Mündig ........ 10 t
Iurillo . . . . . . P. 267

Murpliy . . . . . 206

Hurwari ....... 50 .

Ius ......... 211

IIushroom (licad) . . 215

- by Fungus... 419

Muskham . . . . . 2928

IIusician . . . . . . .

- (Young)....44 416

MIusquito........ 111

Huslapha ....... 2ž

-......2. 257

Mussulman...... 88

Mustard ........ 215

Iustee . . . . . . 540

Iluta......... 214

Muzec......... 174

My Aunt. . . . . . . 246

Iy Cousin ....... 246

My Ineary $0 \ldots \ldots \ldots 146$

Hy Lady ........ s 1

- by Comus.... 214

My Nannic Oh!... 149

Hyra, Jy Soothsay . 214

Myrina . . . . . . . 255

Myrmidon. . . . . . 982

Myrrha ........ 127

— by Malek... 417

Myrtle........ 186

214

290

Myrtilla....... 214

Mysinda . . . . . . . 69

Mystery ........6.65

- by Lottery ... 200 by Marmion .. 418

Nab, The........ 215

Naiad. . . . . 213้-223

Nanine. . . . . 29.216

Nannelte ....2 216-216

Nanny Kilham.... 71

Napoleon (Young).. 598

Nashenden ....... 196

Natty Bumpo..... 415

Nautilus ....... 143

Vararin ....... 155

Navarino . . . . . . 289

- by Blacklock. 75
Parse (aft. Y. Blacki.) 162

Navigator ....... 202

Ned.......... 183

Needle......... 243 by Starch.... 404

Neddums....... 113

Newra........ 81

Nell.......... 71

Nell Gwynne...... 149

- - by Reveller.. $\mathbf{9 4 4}$

—— by Sultan.... 68

— by Tramp ... 916

Nelson......... 213

Nemesis ........ 23

Nemo . . . . . . . 291

Neptune ........ 266

Nerissa ....... 216

Netherby....... 149

Neva ........... 217

Newcastle... . . . 256

Newbrook....... 407

New Fashion...... 408

New Light.. . . . . . 99

Newmarket...... 29

Newton ........ 186

Nicanor........ 207

Nigel.......... 270

Niger ........ 511

Nightshade ...... 2马

Nimble......... 281

Nimrod.......6.64

-- by Bobadil... 185

- by Whalebone 510

- by l'hantom.. 590

Nina ..........217

Niobe . . . . . . . 218

Nisus . . . . . . . 219

Nivalis ..... 297-575

Non Compos..... 556

Nomplus ........20 20

Nonsense ...... 336

Noodle......... 177

Nora Creina...... 406

Norgrove... . . . . 206

Norman........ 270

Norna......... 218

- - by Waterloo. 554

—- by Reveller.. 418 


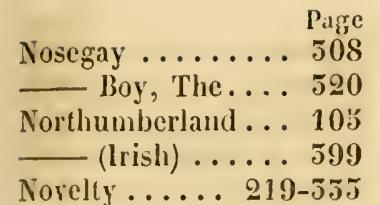

Norelist........ 6

Novicc......... 270

— by Y. Filho.. 219.

Nun, The .... 219-540

Nut-brown Maill ... 574

Nuthurst....... 96

Nutmaid ....... 40 40:

Nutmeg......... 270

Nydia ......... 301

Nymphalin....... 138

Oak.......... 153

Oak-apple ....... 209

Oatlands ........ 264

Oberon ........ 419

Oblivion ......... 263

Obscrvatory...... 89

Occan ......... 59

Occana ........ 574

0'Connell ....... 419

Octave.......... 544

Octavia, by Walton. 219 by Whalebone. 50

Octariaua ....... 220

Octina ......... 221

Oddity .......... 221

Odd Trick, The.... 221

Odessa.......... 242

Ofellus ......... 298

Ogress....... 221-510

Old Bill........ ร58

Oldport........ 83

Old Joe......... 249

Old Swan ....... 350

Oleander........ 222

$0 \operatorname{lga} . . . \ldots \ldots . . \ldots 500$

Olinda......... 163

Olive ....... 84-574

Olive-leaf........ 2925

Olivera ........ 574

Olivia.......... 71

Olympia ........ 225

Olympic ........ 544

Olympus........ 191
Page

584

Omen ........... 544

Omnibus........ 250

Only T'hat.... 281-574

Ontario ........ 556

opal.......... 225

Operator........ 550

Ophelia........ 158

Opodeldoc....... 202

Orangeman...... 155

Orbit......... 544

Orcus ......... 220

Oriana........ 152

Orinda......... 268

Orion ......... 141

— (Young) ..... 141

Oroonok ...... 140

Orphan....... 546

— by Milo.... 408

Orpheline...... 53-225

Orpheus ........ 544

Orthodox....... 21

by Rector.... 157

Orvillia ......... 228

Ostade ......... 161

— by Teniers... $16 \mathrm{~J}$

Osterley ....... 4

Ostrich......... 208

— by Bustard ... 229

Oswald ........ 28

Otć......... 576

olis........... 229

Otho ......... 167

Ottaria ........ 220

Otlowa ........ 79

Outcast........ 84

Outlaw ........ 187

Oxygen...... 344-5 76

Pacific......... 374

Paddy.......... 166

Padshalı ....... 299

Pagan ......... 569

Paganini...... 78 -248

Page, The ...... 156

Pagoda ........ 2.80

Palais Royal ..... 229

Palatine......... 229

Palatine, The..... 13
Page

Palemon....... 156

Palestinc........ 258

palınella........ 162

palmllower ...... 291

—- by Smyrua... 570

Palmyra ........ 250

Pammon........ 으

l'anacea....... 14y

Pancake........ 172

l'andarus........ 160

Pandora ....... 187

Panic.......... 556

l'anopes ........ 250

lantaloon ....... 143

Panteclnetheca... 145

Panthea ........ 250

Pantomime...... 70

Paphian......... 323

Papillote....... 141

Paradigm...... 29-250

Paragon......... 26

Parenthesis...... 418

Paris........... 249

by Whisker.. 288

Parfaite........ 81

Parolles ......... 199

Parson Ilarvey..... 417

Parthenessa ... 182-251

Parthenon....... 28

Parthenope...... $400^{\circ}$

Parthian........ 145

Partner........ 50

-_ Jy W. Pope.. 158

by Whisker.. 160

Partiality....... 112

l'artridge........ 113

l'asithea..... 160-253

Passion ......... 556

l'asta......... 255

—by Catton.... 406

— by Selim.... 254

Pastille........ 254

P'astime...... 254-235

lastora........ 253

l'astorella ....... 250

l'at.......... 287

Patapan ........ 93

Patch........ 407 
Patience ........ 25 Page

l'atrick (Young).... 294

l'atriot

l'atron.......... 271

Patty ........... 204

— Prinrose. 23:5009

Paul Clifford...... 597

Jones . . . . . . 218

paulina....... 576

Pauline...... 25:53.237

I'aulus.......... 117

Jawn ......... 2.56

- - Junior ..... 256

Pawnbroker ..... 12 I

Paymaster....... 106

l'ea-blossom ...... 257

Pea-chick ....... 46

Peacemaker...... 595

Pedestrian....... 250

Pedlar.......... 124

Pedro.......... 78

Реср-0'-day-boy.... 13Ј

Pegasus......... 148

—_ by Tiresias . 578

Peggy ......... 237

Peg-leg . . . . . . . 258

Pelops.......... 218

Pelopia ......... 218

Penance ........ 144

—_ by Oiscau ... 406

Pencil......... 50

Penelope (Young) .. 414

Penhill . . . . . . . 226

Ieninsula ...... 406

Penitence ....... 406

Penitent ....... 407

Penury ......... 2.58

l'epper......... 1:6

—- by 1rone .... 402

Peppermint. . . . . 905

Peradienture..... 515

pera........ 4-258

Jertita....... 82.107

P'erfume ....... 215

Peri . . . . . 258-510

- - The ....... 263

l'erigrine Pickle ... 91

perion......... 80
Pagre

Perkins......... $\mathbf{9 4 6}$

Perpetual Motion .. 258

Perrot........ 529

Perseverance..... 229

Persian ........ 525

Perspective ...... 161

P'eru ......... 168

Peruviana ...... 959

Pestilence ...... 417

Pet........ 259-515

Peter Lely........ 506

—- Liberty . . . . 206

—— by Ama. 150 Pindar..... 255

Protens .... 141 Simple...... 192 - (s. of

Rose)........ 577

Petrel ......... 244

Petuaria (Young)... $\mathbf{2 4 0}$

Petulance.... 240-505

petworth ........ 526

- - Lass........ 510

Pewet....... 45-240

Phantasy ....... 219

Phantom........ 241

—_(Young)..... 104

- Young (late

Virgilius) .... 55

_- Minor. . . . . . 419

Pheasant....... 944

Philip the First.... 408

Philosopher....... 29

Philosopher, The... 294

Philomel .... 407-407

l'hwbe......... 244

— - by Comus ... 2: I

Plicbus........ 214

Pholus......... 95

Physician ....... 2:51

- by Dr Syntax. 418

l'iccolina ....... 106

I'ickninny ....... 145

l'ickpocket ...... 141

Pickle......... 215

Picton......... 86

l'iclure, The..... 160

Picturesque.......
Pigeon ....... 346

Pigmy ......... 245

vilgrim ........ 524

- - by Blacklock . 250

Pilkington ....... 187

pilot......... 47

Pilton ......... 254

Pimlico ......... 245

Pincher........ 110

Pincushion ...... 945

Pinions ........ 145

l'inkic Lass....... 44

Pintail.......... 245

Pioneer. . . . . . . 599

Pippin ......... 248

Pipylina (Young) ... 247

piquet......... $\mathbf{2 4 7}$

Pirate ......... 79

Platiea ......... 162

Plantagenet...... 10

—- by Falcon ... 54

Plaything ....... 514

Pledge........ 947

Pleiad ..........4 407

Plenipotentiary .... 140

Ploughboy....... 55:

Plumber, The.... 567

Plunder......... 997

Pluralist........ 75

Poet, The ....... 175

Point de.Vuc .... 152

lolander ........ 128

Pollio .......... 55

Polly Oliver...... $\mathbf{9 4 7}$

Polly Peachum .... 259

Polly Trashery .... 597

Polonaise ....... 94

Polymnia........ 248

Pomfret........ 156

Pomona......... 58

- - by Vespa.... 2, 8

Pompey, by Lapdog. 221

—- by IVarrior .. 154

Pontefract....... 68

1opanilla........67 67

Popinjay........ 18

Popjoy.........6.65

Popsy ......... 45 
portland ........ 45

Porto Bello...... 250

Portrait......... 195

Possession....... $9 \mathbf{9 7}$

Post-haste....... 249

Postluma..... 187-249

Posthumous ...... 949

Post Obit........ 249

Potentate....... 90

Pouch-box....... 276

poule ......... 167

Pounce......... 508

Poverina....... 175

Powlett..... 200-407

P. P........ 41

Prejudice........ 141

Premicr........ 250

by Priam.... 577

Premium........ 199

Treservative ..... 145

Preserve........ 215

Prestbury ...... 65

P'resto......... 574

Pretender, The.... 154

Priestess....... 570

Prima........ 79

Prima Donna..... 949

Primendorf...... 159

Primette........ 251

Primigena ...... 1-251

Primrose....... 576

Primula......... 231

Primus......... 550

Prince......... 154

— by Figaro.... . 252 - Eugene...... $28 s$

Prince Llewellyn . . . 292 — Nicholas..... 72 Princess......... 187 — by Augustus. 255 by Comus 231-262 Lugusta.... . 255 Jemima.... . уำ Royal...... . 232 Victoria.... 4-25j - by Mlid-

dleton........ 255
P'rincess Victoria, Page

by Langar, or

Falcon........ 136

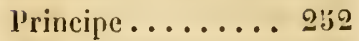

Principessa...... 97

prior.......... 417

Prize ...... 129-408

Problem........ 235

Procne ........ 56

Prodigal ........ 7

Prodigious........ 254

Prodigy........ 4 4

___ by Woful.... 571

Professor, The.... 38

professor........ 93

Profile...... 95-254

profligate ....... 245

Progress........ 4

promise ....... 25:

Prompter...... 254

Proserpine ...... 15

- - by Rhadaman. 254

Prosoly......... 2y5

—_ by Reveller.. 255

Prospective... 152-255

protector........ 48

Protegé........ 219

Protocol ........ 156

Prude......... 255

Prudence........ 255

—— by Wiseacre. . 534

prudish......... 256

Prussian ........ 267

Puce.......... 419

Pucelle...... 187-256

Puff.......... 2:57

punster........ 519

l'upil......... 176

purity .... . . 142-257

Purity, by litho... 2 259

Puss.......... 76

Pussy ......... 529

Puzzlc......... 1 วั0

- - by Irone... 597

—- by Whisker.. 590

Pye Annot....... 12

Quaderoon ...... 168

Quadrillc........ 257
Page

Quadron...... 8 8ร-258

Ouartetto ........ 65

Queen Bathsheba

181-253 and err.

—-Bess....... 232

- Mab....... 417

- of llearts. . . 418 of llearts, 189-238

- of IIcarts, by

Tagus........ 590

--_ of Navarre... 28

- - of Trumps... 253

Queensberry..... 259

—- by Magistrate 519

Quibbler....... 270

Quickly......... 258

Quicksilver...... 990

- - by Memnon. . 419

Quintus......... 1852

Quiver.........4 408

liaby ......... 248

Rachel Ruysch. 60-259

Rachel........ Ti

- - by $\Lambda$ madis... 239

—— by Whal. 129-239

Racket......... 259

Raffle ......... 295

Rail-road ....... 206

lamazan ....... 198

Rambler....... 509

Ramona..... 154-260

Ramsbury....... 295

Randall........ 21

Rance......... 124

Ranger........ 293

Rantipole(Young) . . 260

Rapid .......... 210

_- (Young)..... 263

- - Rhone..... 106

Rarity....... 261-545

Rattle........ 558

- - by Bonassus. 408

Rasselas ........ 2294

Ratcatcher...... 578

Ratler........ 545

liattle........ 266

Raven.........

Raywood....... 256 


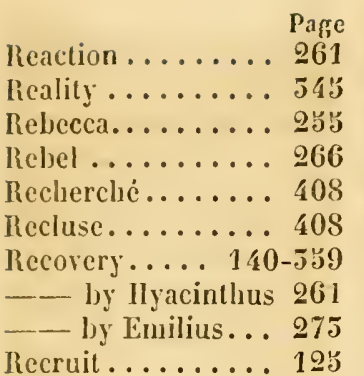

Rectitude...... 81

Rector......... 16

Rectory........262

Redgauntlet..... 92

Redleg ....... 150

Redlock........64 64

Redmantle...... 595

Red Muzzle...... 25

Red Rover, by Mid. 271

— - by Lottery... 203

Red Rose........ 209

- - by Rubens... 418

Redshauk....... 565

Redstart.......263

Reform........ 206

Refugec........ 224

Regatta........ 55

Reginald....... 121

- - by Haphazard 255

Regret......... 87

Regulator...... 409

Regulus........ 64

Reine Canziani.... 598

Reliance....... 545

Rembrandt...... 116

Remember...... 81

Remembrance.... . 262

Remus ......... 118

Repealer....... 169

Repentance...... 111

Reposada....... 265

Resolute....... 543

Reseda ........ 588

Resurrection..... 222

Resemblance..... 545

Retainer....... 126

Retaliator ...... 545

Retreat.........
Pase

Retriever...... 126

Reuben........

Revelry....... 140

Revenge....... 545

Revenue (Young)..

Revolution...... 105

Rex......... 584

Rhagflanied...... 90

Rhapsody ...... 248

Rhoda......... 264

- - (Young)..... 264

Rhodanus ....... 266

Rhodian, The.... 524

Rhodocantha..... 265

Rialto......... 208

Richard........ 550

Richmond....... 200

Riddlesworth..... 116

Ridotto........ 265

Rienzi......... ร9

Rifleman....... 155

Rigadoon........ 154

Righton........ 574

Rigmarol....... 576

Rinaldo......... 68

Ringdove....... 56

_- by Bobadil... 86

Ringlet . . . . 68-266

—_ by The Col... 4

Ringleader ......5 50J

Ringueck ....... 86

Rioter, by Reveller. 260 —_ by ditto.... 519

Ripton.........264

Rip Van Winkle... 159

Risk ......... 268

Rivulet $\ldots \ldots \ldots .266$

Roadster....... 264

Robinetta...... 554

Robin Hlood...... 129

_-___- by Blackl. 185

Robin Roughhead.. 161

Robinson Crusoc... 552

Rocbana........ 201

lockingham ...... 187

—- by Regent... 575

Rodelinda....... 208

4 Roderick......... 236
Page

Rodolph ....... 121

Rocbuck ....... 113

Roger Bacon..... 129

Rokeby........ 5ว̆4

Rolla......... 167

Roman ........ 161

Romeo ........ 134

—— by Sir Herc. . 406

Romer........ 418

Romp.......... 266

__ by Oliver Cr. . 409

Romulus........ 118

Ronald ........ 25้6

Rondo......... 417

Rosabel........ 14

-_ by Shakspeare 98

Rosadelle ....... 177

Rosalba......... 267

Rosalia...... 267-269

Rosalic......... 98

Rosalind....... 267

—- by Paulow... 577

Rosalis....... 56-208

Rosa Mar......... 142

—_Mundi...... 577

Rosanicl........ 268

Rosamond....... 200

- - by Grimaldi. . 268

Rosaune ........26S

Rosario......... 269

Rosary ......... 269

Rosebud ..... 269-556

- - by Rob. Adair 577

Rose......... 58 - by Rub. . 268-577

- - by Waverley. 149

Roscleaf...... 207-269

Rose Roche...... 214

Rosetta ........ 209

Rosette........ 419

Rosina......... 229

Rosinc......... 295

Rossignol....... 263

Rotterdam.... 269-503

Roubillac........ 198

Roué.......... 596

Rough Robin.... 161

Rouncival....... 184 
Page

Roundwaist. ...... 21ว

Rousscau....... 340

Routine........ 415

Rover........294

Rowena..... 25\%-270

by Rubens... 2 270

Rowton........ 152

Roxana........ 409

Royalist........ 268

Royal Gcorge..... 84

William..... 255

Royalty........ 181

Roy's Wife......5 501

Rubens (Young).... 108

Rubicon ........ 208

— by Sult. (dead) 2006

Rubigo........ 45

Rubina ........208

Rubini......... 295

Ruby....... 275-545

— by Reveller... 520 by Interloper. 589

Rufina...... 150-577

Rufus ......... 2รว

Ruinous ........ 265

Runnymede...... 106 - by Little J. 269-556 - by Whalebone 526

Rupert......... $2: 56$

Rushlight ...... 55

— by Whalebone 150

Russell........ 78

Rustan ........ 196

Rustic, The..... 581

Ruth.......... 225

— by Merlin . . . . 256

Rutland........ 26

Sabina......... 366

Saccarina ....... 145

Saddler, The..... 52

Sadek.......... 109

Safeguard ....... 28

Saffi ......... 578

Sagittarius....... 407

St Giles......... 15

St Hilary........ 9

St Ilubert.......448

St John........ 512
Page

St Julien........ 67

St Kevin ......... 225

St Lawrence..... $\quad 24$

— by Tiresias... 260 by llum. Clin. 415

St Leonard........ 148

St Lulie........ 100

St Nicholas ...... 282

St Patrick...... 595

St Vitus........ 117

St Winifred...... 74

Sal........ 169-590 by Scud..... 276 by Revel..... 976

Saladin......... 149

— by Mas. Robt. 408

Salamanca....... 276

Salisbury....... 26

Sally......... 409

- Barlow...... 155

- Maggs ..... 417

Sutton ..... 146

Salmagundi...... 597

Salmonia........ 296

Saltarella....... 1ว

Saltator........ 152

Salvadora...... 24-276

Salute......... 92

Sam............. 167

— by Muley . . . . 260

Sambo......... 2ร0

Samarcand ...... 144

Sandoval....... 180

Sangrado........ 21

Sans Souci....... 418

Saraband....... 263

Sarah, by 'Tramp... 247

_ by Whisker. . 378

- (sister to Swiss)

278-288

- Horner. . . . . 207

Sarcasm ........ 21

Sarpedon........ 145

Sarsaparilla...... 278

Sassafras........ 278

Saturnia..... 168-278

Saucy Dick ...... 500

Saul .......... 207
Page

Sayoyard....... 141

Sawney........ 78

Scamp........ 20

Scandal........ 279

Scarabxus....... 214

Scarbrough...... 257

Scarpa....... 29-279

Sceptre....... 59

Schariar ....... 88

Schedam.... . 279- ว05

Schedule....... 418

Scheik......... 285

Scheme........ 279

Schemer....... 47

- by Speculation 598

Schoolgirl...... 590

Schoolmaster...... 210

Sclioolmistress.... 210

Schumla ........ 280

Sciatica......... 29

Scornful ....... 590

Scotch Kate....... 280

Scramble....... 46

Scratch........ 280

Screw, The...... 27

Scribe ...... 280-280

Scrivener....... 85

Scroll ......... 136

Scuffle,..... 280-282

Scurry...... 280-282

Sea-breeze....... 282

Sea, The....... 225

Seagull ........ 411

Seamew........ 289

Second ........ 59

Sedan ......... 55

Scedling ....... 591

Segar.......... 224

Selina......... 284

- by Fitz-Teazle 284

by Nicolo. $284-380$

— by Delpini. . . 579

Selma......... 284

Sempstress...... 580

Sensitive........ 289

Sentiment....... 285

Sephora........ 215

Sepoy......... 264

d 
Sepoy, by Polygar.. $\begin{gathered}\text { Page } \\ 295\end{gathered}$ Shylock......... 199

Serenade........ 983

Scrpent, The..... 142

Sessions...... 224-579

Seviglia....... 579

Serilla...... 71-579

Sevillian....... 62

Shadrack........ 550

Shadow......... 290

Shah, The...... 25:

Shakspeare...... 65

Shalot......... 176

Shamrock....... 2.3

— by Tancredi. . 104

— by St Patrick. 547

— by Bedlanite. 155

__ by Roller. . . 400

Shark......... 187

— by Whalebone 262

Shararogues ...... 596

Sharpset....... 71

Sharpshooter...... 157

Sheet Anchor .... 575

Sheereen........ 196

Sheik.......... 101

Shclah.......... 211

Sheldrakc ...... 580

Shepherdess, by

Strephon...... 250

- by Y.Phantom 77

Sheriff......... 450

—_ The Iligh.... 178

Sherry....... 51-283

— by Reveller. . . 586

Shilelagh....... 559

Shirine ........ 265

Shirley ........ 112

Shoehorn........ 285

Shorhopes...... 99

Shortwaist. . . 213-286

Shot .......... 286

Shoveler........ 286

Show-lass .... 287-547

Shrew, The...... 287

Shrigley ........ 107

Shrimp........ 155

Shuttlecock, hy Orville..........
Sierra............417

Sigh . ........289

Sigismunda...... 104

Signorina ....... 289

Silenus ........ 518

Silksleeves ...... 141

Silly Billy....... 60

Silverfringe...... 152

Silvertail 224-290 and crr.

Silvio......... 81

Simia ......... 198

Simon......... 208

Sinbad........ 168

—_by Swap..... 177

_ by Priam.... 190

Sinc qua non..... 303

Sir Bamfylde..... 183

—-Benjamin.... 279 Bachbite 279 Edward Cod-

rington ........

- Felix ...... 1

- Hercules . . . . 258 Jacob...... 10

John........ 187 John, by Tramp 353

—_ by Little

John ......... 241 Malachi Mala-

grow ther...... 154 Peter...... 204 Richard.... g

Andrews ...... 257.

- Robert..... 108 Roger...... 550 Thè....... घ้ Thomas ..... 13 by $\Lambda$ bjer 156 by Filho 175 William.... 295

Skeleton....... 409

Slictch......... 161

Sketch-book...... 44 580
- Isaac....... 14
Page

Skimmer....... 27

Skim Milk ....... \& 1

Skirmisher....... 171

Skylark (mare) .... 409

— (horse)..... 410

Sladeland ...... 557

Slander........ 82

Slanc.......... 225

Slang.......... 99

Skyrocket....... 65

Slashing IIarry..... 17

Sledmere........ 105

- by Y. Phant. 48

Slight ......... 292

Slime ......... 559

Slinker........ 57

Sluggard....... 53

Slut.......... 528

Slyboots ........ 130

Smallhopes...... 198

Smallwaist ...... 589

Smash.......... 293

Smedley ........ 254

— Lely....... 526

Smoke........ 517

— by Oiscau.... 410

Smoker........ 277

Smolt, The ...... 296

Smuggler ....... 41

— by Shaver.... 259

Smutta ........ 240

Snacks ........ 418

Snail......... 296

Snare ........ 297

Sneaker........ 390

Snowball........ 41

— by. Alington. . 579 by. . Prime.

Minister...... 297

Snowdrop....... 298

Soho........... 299

Sola........... 298

Solace......... 92

Soldan......... 283

Solicitor ....... 250

Solitaire......... 284

Solomon ....... 590

Solon.......... 204 
Page

Somerset....... 524

Somnambule..... 91

Sontag.......... 298

Soot ......... 410

Soubahdar....... 509

Souter Johnny..... 142

Souvenir..... 92-501

Sovereign ....... 550

spaewile.... 79-501

Spaniard........ 106

Sparkier....... 58

Sparrow........ 56

Sparrowhawk.... 579

Sparina.... 19. 19302

Spec.......... 598

Specic......... 581

speck ......... 262

Spectacles....... 1836

Spectre........ 419

Speculation....... 531

Speculator....... 559

Speneer......... 92

Spermaceti... 129-502

Spider......... 149

Spinettc........ 581

Spinning Jenny. . . . 502

Splendor........ 81

Sptinter......... 280

Splitpost....... 366

Spondec ........ 2ย้

Sporting Mag...... 404

Spotless......... 505

Sprec......... 295

Sprat....... 2\$0-505

Spray ......... 598

Sprig.......... 505

Spring-cautle .... 588

Springe......... 505

Spring-kell ...... 503

Sprite, by Comus.. 547

— by l'hantom.. 504

Spruce ........ 11

Spume ........ 598

Squat......... 77

Squil)......... 142

by Soothsayer. 304

Squire, The..... 401

Squire Western.... 65
Paire

Squirrel ....... 9. 95

— by Caill.... 304

Stallord....... 419

Stag, The...... 2!) 1

Stain......... 156

Stainton ....... 51

Stapleton ....... S5

— by Magistrate. 149

by Strephon. . 590

Stargazer....... 186

Startle........ 551

Statcly......... 251

Stays.......... 505

- Dy W. Pope. . 410

Staysail........ 31

Steam........ 411

Steamer........ 276

522

Stella ......... 506

Stiff.......... 405

Stingo......... 388

Stirling........210

Stockwell Beauty... 40

— Lass....... 548

Stockport .......

Stork.......... 411

Stradbally...... 502

Straitwaist....... 218

Stratagem...... 117

Stratherne....... 289

Strathtay........ $\mathbf{8 9}$

Strawberry...... 506

Stream....... 411

Streamlet.... 50-582

Strength...... 419

Striking Beauty. . . 506

Strumpet....... 506

Sublime ........ 502

Suffolk Punch..... 580

Suke ....... 288-507

Sultan (Young).... 2299

Sultana........ 507

Sultancss..... 132.508

Summerhill...... 258

Sunbcam....... 528

Sunflower....... 508

Superb ........ 251

Surprise....... 508
Superstition ..... $5 \mathbf{3}^{360}$

Susan......... 590

Susanna ........ 574

—by Swap..... 535

Susanne ....... 95

Sussex......... 221

Swagger........ 99

Swallow ........ 125

Sweetbriar....... 269

Sweetlips ....... 501

—- by Roller.... 401

Sweet 110 oggy . . . . 6 60

Swect-pea...... 2.57

— (Young) .... .508

Marjorum.... 6 65

Sweno......... 168

Swilt.......... git

Swing........ 551

Swiss .........288

Sybil......... 106 by Interp. 125-308

Sycorax........ 50 9

Sylph....... 111-309

by Number

Nip.........580

— by Swap..... . 989

Sylvan......... 989

Syma......... 265

Symmetry.......501

Syphon ........ 60

Syren......... :

Tabby......... 565

Tabitha Bramble... 17

Tağ.......... 176

Taglioni ........ 54

— by Camel.... 267

Taishteer....... 2

Talma......... jut

Tamburine...... 177

Tamborini....... 516

'Tan o' Shanter. ... 280

Tamworth (Young) . 284

Tancredi........ 106

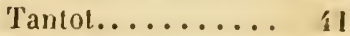

Tanworth....... 彺

Tar........... 110

Tarantella....... 152

Tarick........ 508 
Tarquin ........ Page

_- by Smolensko 354

Tarquinia ....... 516

Tarragon (Young).. 274

Tartan......... 56

Tartar........ 84

Tartarina. . 555 and err.

Taunton........ 85

Tauntonian...... 85

Taunton Lass ..... 85

Taurus ........ 152

— by Ardrossan. 996

Tears ......... 510

Teddy the Tiler.... 5 5 0

Tell-tale . . . . . . 585

Tempè......... 162

Temperance ..... 283

Tempest....... 26

Tennis-ball..... 577

Terapia.......5 547

Teresa........ 188

Termagant ...... 285

__ by Pollio.... 565

Terror......... 84

-- - by Magistrate. 316

Terry Alt........ 195

Tertia......... 206

Tesane...... 158-583

'Tetotum ........ 294

Thalestris ...... 510

Thalia........ 419

Thatcher........ 545

Theano........ 64

Theban, The..... 8

Thebes........8-811

- - by Conductor. 186

Themesis ....... 351

Theodore........ 58

Theodoric....... 23

Theodosia .... 159-511

Theophania... 511-584

Theresa.........277

Theresa Panza. 124-311

Therese........

Thermometer ..... 191

Thomasina ...... 101

Thoresby........ 516

Thorngrove...... 111
Page

Thump........ 56

- - by Il. Clinker. 401

Tiara.......... 513

by Waxy Po. . 346

Tib.......... 515

Tiber.......... 106

Tiberius ........ 516

Tiby-tight...... 409

Ticket........ 546

—- by Lottery... 419 (Mr Riddell's). 94

Tiffany ......... 158

Tightboy........ 247

Tigress........ 74

514

Tillah ......... 75

Tim.......... 417

Timothy ....... 207

Timour........ 555

Tint ........ 161

Tintoretto...... 515

Tippet......... 515

Tippitywitchet.... 514

Tipple Cider..... 85

Tippoo Saib...... 125

Tipsy, by Reveller. 285

- - by Election .. 411

_- (Young). . 411-411

Tiptoe......... 290

Tirailleur....... 4

Titania ........ 79

Titian ......... 161

Titlark......... 244

Tittle Tattle ..... 46

583

Titus.......... 516

Tivoli .......... 106

Toaster........ 285

Toby......... 547

Toga......... 419

Tom ......... 169

Tom Basford. .... 77

— Bowling..... 516

— Brown...... 217 Jones....... 165

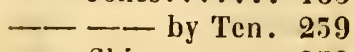
Shipman .... 259 Thumb ..... 130
Pagre

Tom Tit....... by Firm. 115 the Towter... 174

Tomboy ....... 16

Tom Cat ....... 142

Tommy Whitehead. 590

Tontine......... 518

Tony Lumpkin.... 66

Toquay........ 18

Torelli......... 516

Tormentor ..... 549

Torvaldo....... 552

Toso...... 40-516

Touchstone...... 21

Toughskill....... 504

Toujours....... 41

Tourist ........ 155

Tournament..... 25:

Toy......... 516

Tragedy...... 85-517

Trampina ....... 585

Trampoline...... 318

Tranby ........ 375

Trapes $262-519$ and add.

Trap-ball ...... 188

Traveller....... 315

Treadmill (dead)... 78

Treasure....... 519

Tredrille....... 584

Tremaine ...... 81

Trepidation....... 218

Trick........ 19-519

Trickery ........ 221

Trickster....... 280

Trictrac........ 519

Trident........ 516

Trim......... 184

— by Laurel . . . 222

Trinket........ 19

- by Godolphin. 116

— by Gr. W. 77.520

— by Whalebone 412

- by Tramp 313-320

Tristram Shandy... 418

Triton.......... 129

Tr by IIemnon.. 190

Tritonia ........ 128

__ by Peter Lely. 190 
Tritonia, by Mr Altwood's Ch. Arab 278 Troubadour...... 144

Trout ......... 412

Trouville

60

True Blue....... 15.5

Trull.......... 584

Trulla .......... 44

by Sorcerer... 584

Trumpery....... 28

Trumpeter...... 25

'Trustce......... 104

Truth......... 45

Tuberose........ 584

Tulip, The...... 65

Tullia........ 516

Turban......... 156

- by Sultan... . 515

Turf.......... 158

Turfcutter....... 278

Turk......... 507

Turncoat........ 85

Turquoise....... 320

Tutor, The...... 291

Twalty ......... 520

Tweedledec....... 248

Tweedledum...... 248

Twin ......... 81

Twinkle ....... 521

Twist ......... 588

Typhon........ 546

Tyrant ........2 28z

Tyrrel......... 75

Vacuna......... 15

Vagabond....... 124

Vagary ......... 124

Vagrant.........

Valencia ..... 47-521

Valentine....... 117 by Soothsayer 321

Valentinian...... 257

Valentissimo...... 144

Valet.......... 271

Valiant........ 62

Valparaiso........ 149

Valve ......... 521

Vamp......... 412

Vampyre....... 592
Page

192

Van.............

Vandenhoff...... 220

Vanessa ....... 522

Vanguard.......412

Vanity ........ 412

Vanloo ........ 304

Vapid .......... 290

Varennes....... 522

Varia........ 51-52J

Variella..... 241-52J

Variety ........ 523

Varlet......... 221

— by Spec.... . 413

Varna......... 525

Varnish........ 524

Vat......... 415

Vathek ........ 523

Vauban........ 98

Vaulter........ 524

Vaultress........ 524

Veil ......... 324

Velocipede....... 150

Velocity ....... 150

Velvet........ 585

Venilia........6.62

Venison ........ 115

Venus, by Lottery.. 80

80

— by Langar ... 526 by North Star 52s by Smolenstio 82

Verandah...... 42

Verbena....... 267

— by Comus... 525

Verderer........ 88

— by Tiresias... 161

Verdure....... 161

Vermilion....... 41.5

Vernal ........ 541

Veronica........6 62

Versatility.....15-32

Vertigo......... 281

Vertumnus...... 263

Verulam....... 413

Vespa......... 20.

Vesper........ 78

Vespers......... 415

Vesta, by Arbulus.. 225
Pesta, pye

\section{Vesta, by Gorernor. 525}

- (Young)..... 326

Vestment....... 92

Vestris........ 522

Viator........ 152

Vicarage....... 526

Vicissitude...... 148

Victim ......... 94

— by Blacklock . 117

Victoire ........ 326

Victoria...... 2y-527

-by Whalebone,

or Little John... 160

— by Bay Malt.. 247

Victorine ....... 527

Victory........ 4

Vidocq......... it

Vigo.........4 418

Vigornia....... 521

Villosa ........ 527

Vinegar........ 418

Viola.......... 590

Violante.......

— by Gustarus.. 552

Violetta........ 168

Virago ......... 172

-_ by Reveller.. 324

Virgilius........ 55

Virginia......... add.

Virginius........ 151

Vision......... 12

— by Vanish... 528

Vitellina....... 327

Vittoria........ 18

Vivaude........ 562

Vivian Grey...... 18

Ulick.......... 520

Ulrica ...... 206-527

Ultima . . . . . . . . 528

Ulysses........ 532

Vltimalum ...... 208

Uncle Bol....... 5

__ John......2 207

— Toby....... 184

Unicorn ........ 282

Unfortunate Youth,

The........ 70

Unit......... 207 
Page Page Page

Vocabulary...... 7 Water-witch...... 218 Wigan Lass..... 229

Volaut ........6.62

Voluey .......... 242

Voltaire ........ 241

Voluptuary...... 140

Vortigern....... 270

Vourncen...... $\mathbf{5} \mathbf{2 8}$

Vulcan ........ 556

Vulpes . . . . . . . . 208

Vulturc........ 135

Vyvyan........ 183

Urganda..... 290-415

—— by Hilo..... 528

Ursula......... 528

Usury ... . . . . . . 188

Wagtail, by Whisk. 529

-_ by Orville... 250

- - by Y. Wood-

pecker....... 529

Wait-a-while .... 412

Wait still ....... 412

Walfruna ....... 116

Wallace ........ 169

Wallflower...... 579

Walter........ 98

- by Waverley. 375

Waltram....... 552

Waltz........ 352

Wandercr....... 52

- by Wanderer. 97 - (Young).....

Wandering Boy... 557

Wanton, by Frolic. 224

Warbler, The.... 205

Waresti....... 559

Warlock....... 18

Warrencr....... 297

Warter (Young)... $\mathbf{3 9 0}$

Warwick........ 74

Washerwoman.... 553

The...... 401

Wasp .......... 142

- by Wamba.. 190

— by Golianna.. 555

Wassailer....... 12

Wastrel........ 96

Waterman ...... 266

Water-nymph.....
- by Sir Herc.. 404

Wat Tyler....... 187

Warerer....... 523

Waslight ...... 150

Wcasel......... 297

IVeathercock..... 523

Wedge ........415

Wedlock....... 295

IVeed ..........5 501

Weeper.........218

— by Woful... 511

Wegenkorb .....4 414

Weighton ...... 57

Welch Pool...... 559

Welcome ...... 527

Well-a-day ...... 312

Wellington...... 78

- by CannonBall.......... 590

by Champion: 418

Wentworth...... 20

Werner......... 168

Westbury....... 109

Western Lass . . . . 187

Westport.......4 410

Whale.......... 269

Whalebone...... 50

Whaley........ 45

Wharncliffe...... 54

Whim........ 23

—- by Comus... รु0

— by Droue.... 401

Whimper ....... 29

Whimscy..... 50-557

Whirlpool........ 559

Whisk ......265-358

Whiskerandos.... 68

Whiskey....... 279

Whisper....... 180

- by The Col.. 279

Whitefoot....... 194

by Y. Blackl. 402

White Rose...... 543

Whizgig....... 544

Whynot ....... 162

Wicket........ 578

Widow, The...... 109 widgeon..... 87-344

Wilberforce ..... 180

Wildair........ 183

Wildboy, by Whis. 261

Wild-duck ...... 287

Wildfire ....... 504

—- by Marnion.. 418

WVildgoose ...... 26

Wilfrida ....... 270

Wilful Will...... 511

Wilhelmina...... 125

Wilibert....... 521

Will Scarlet..... 125

Willesden....... 81

—_ by Velocipede 132

William ....... 289

William Tell. .... . 59

William the Fourth 150

Will o' the Wall... 5ss

Will o' the Whisp. 116

Wilna......... 297

Windcliffe....... 54

Windermerc..... . . 95

Windfall........ 415

Window Shut, 'The 81

Windrush ....... 98

Winford ........ 528

Wingficld. . . . . . 544

Wings . . . . . 2929-346

Winkley....... 75

Winterficld......26马

Wire......... 415 - by Sir Hulde-

brand........ 297

Wisdom ....... 114

Wish......... 512

Wishful. . . . . 26-387

Witch, The......241

- by Soothsayer 546

Witless........ 270

Wittingham ..... 154

Wizard........ 501

by Y. Blackl. 547

Wodenblock...... 555

Woman of Endor

401-41

Wonford....... 297 
xxxix

\begin{tabular}{|c|c|c|c|}
\hline Page & Page & & \\
\hline Woodbine ........ 549 & Zany.......... 41 & Zillah (afterwards & \\
\hline Woodland Mary. . . . 112 & Zara......... & Barebones) ... & \\
\hline Woodlark ........ 127 & 45 & Zimri ...... & \\
\hline orcester...... . 255 & — by Reveller.. & Zinc. & \\
\hline Worlaby Witch.... 100 & — by Moriseo & be 70 otat & \\
\hline -Baylock...... 325 & $147-552$ & Zingarella........ & \\
\hline thless........ 550 & ... 552 & ingaro... & \\
\hline Worry...... 276-550 & Zebetta.......... & by Zingance. . & \\
\hline Wrangler........ 400 & Zegri Maid, The... 215 & Zirza........... & \\
\hline — by Rajah.... 297 & Zeline........... 555 & Zitclla..... & \\
\hline$\ldots \ldots \ldots 264$ & $\ldots \ldots \ldots 286$ & Zodiac, by $\mathrm{c}$ & \\
\hline$\ldots \ldots \ldots 521$ & ___ by Rubens 5:55-556 & Zoć.... & \\
\hline 415 & co......... 558 & — by Recordon. & \\
\hline 588 & 218 & Zohrab.......... & \\
\hline yndham........ & cнana........ & - by Lottery... & \\
\hline 551 & 535 & ne. & \\
\hline 571 & 553 & Zoolfa & $34.25+3$ \\
\hline$\ldots \ldots \ldots 88$ & 180 & Zora. & \\
\hline$\ldots \ldots, 110$ & 554 & — by Nimrod... & \\
\hline engwyn... 551 & $s \ldots . .$. & -Y. Catton.... & \\
\hline$\ldots \ldots$ & $\ldots \ldots \ldots \ldots$ & & \\
\hline e Lass... . . . & Zethus .......... & & 10 \\
\hline Ipsilan & 556 & & \\
\hline ister to & 297 & - (Young) & \\
\hline Zeal). & _ by Whisker.. 534 & Zulima & \\
\hline Zaire........... ร5. & — by Blacklock. 415 & — by Gullirer.. & \\
\hline & — by Rercller. . 210 & umalacarregui.... & \\
\hline
\end{tabular}



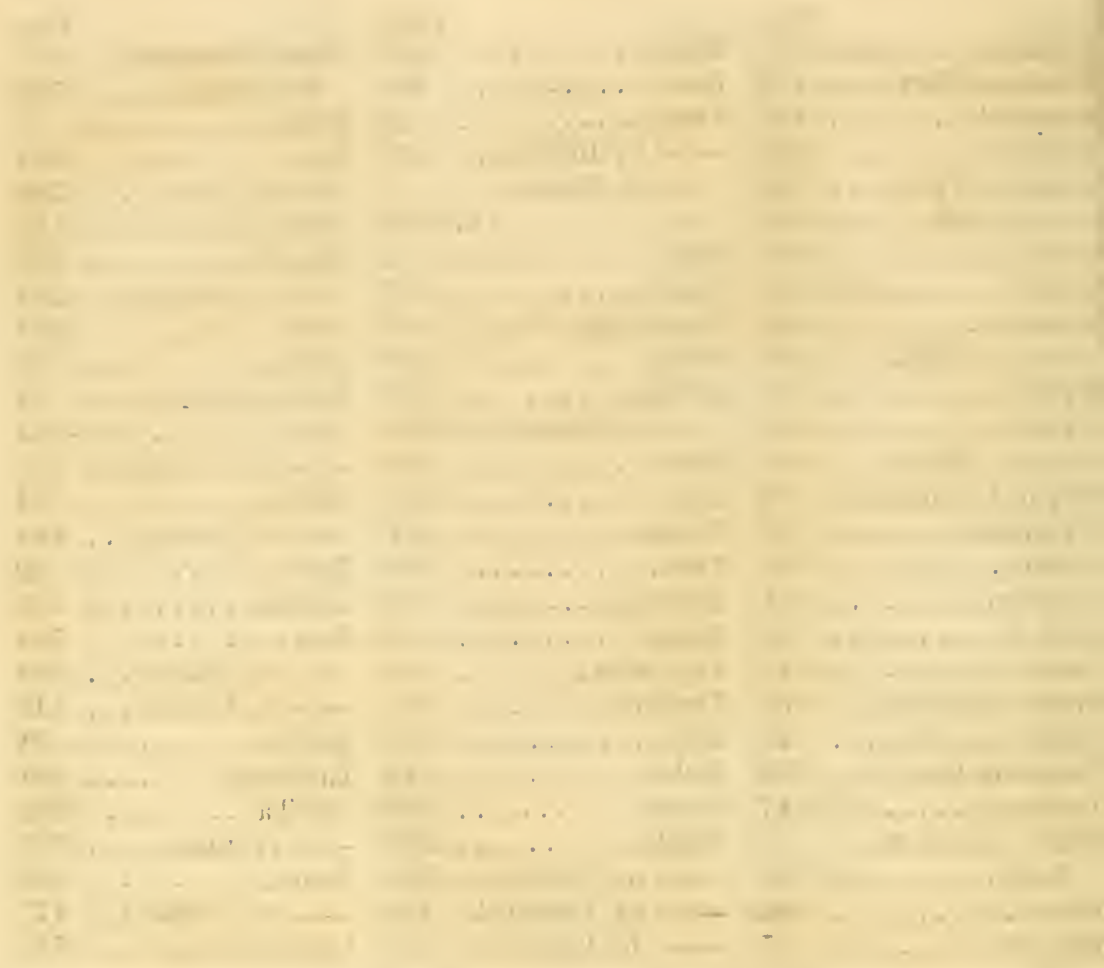


\section{THE}

\section{GENERAL STUD BOOK.}

[X. B. Inder the head of "Owser on Befene," when a liorse has run, or is solil with a probability of his being trained, it has been our endeavonr to seleet the person in whos: name he first appears, or is likely to appear, in the Racing Calendar, in order to facilitate tha seareh for lis periormaneps. - Where the name of the horsi: is enclosed in a parentliesis (as, for example, Mloggy in the 2nd page, and Frailty in the 5th) it siguifies that such namewis given by a subsequent proprietor.]

\section{BROOD MARES, WITH THEIR PRODUCE.}

\section{ABJER MIARE,}

Bred by Lord Dermas, in 1824, her dam, The Duchess, by Cardinal York, out of Hiss Nancy, by Beningbrough.

IEAR.

PRODUCE.

OWYER OR EREEDER.

1828 ch. f. Primigena, by his Grey Arabian.)

1829 ch. c. by ditto (dead )............. IIr Attwood

1850 ch. f. Aminta, by his Chesnut Arabian.

1851 missed to Lottery..............

1852 ch. c. Sir Felix, by Blacklock or Langar. Mr Copeland

1855 ch. c. The Drummer, by The Colonel. Mr Grerille

1854 br. c. by Tranby............. IIr Pettit

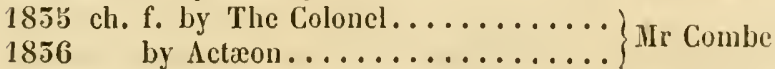

\section{ABJER MARE,}

Bred by Mr Vaxsittart, in 1824, her dam, Slight, wy Selim, grandam by lot8o's, out of Editha, by Herod-Elirila, by Snap.

1851 b. f. Lotteria, by Lottery..........

1852 b. f. by a half-bred horse......... Mr Tansittart

f. by St Nicholas.............

VOL. IT. 


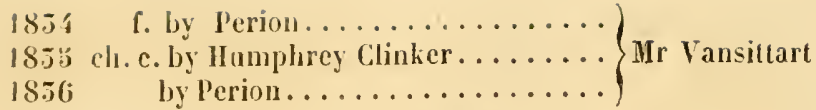

ACACIA,

lired by Lord Exeter, in 1826, got by Phantom, lier dam, Augusta, by Woful-Rubens-Guildford Nan, by GuildfordJustice.

18.55 b. с. Taishteer, by Zinganec, or Whisker

18.56

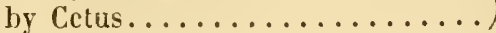

ACTIVE,

Bred by Sir Chanles Bunbury, in 1820, got by Partisan, her dam,

Elcanor, by Whiskey; out of Young Giantess, by Diomed-.

Giantess, by Matchem.

1826 b. f. by Filho da Puta (died a foal)...)

1827 ch. c. Cupid, by Tramp..........

1828 b. f. Fairy, by Figaro............

1829 b. c. The Grand Falconer, by Merlin

(sold to D. of Holstein in Feb. 1855)

$1850 \mathrm{ch}$. f. (Moggy) by Sultan (put to the

stud) Sir G. l'igot

1851 b. f. Heads-or-Tails, by Lottery .....

1852 slipped foal by ditto............

1855 missed to Brutandorf. . . . . . . . .

1854 b. f. hy Filho da Puta...........

1855 b. c. by Emancipation (dead) .......

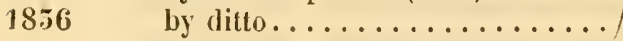

ADA (sister to Augusta)

Bred by Lord ExETER, in 182: got by Woful, her dam by Rubens, out of Guildford Nan, by Guildford (son of Highflyer) Justice.

1826 b.c. (Fantoccino) by Phantom.....

1827 b. f. Ipsala, hy Sultan...........

1828 ch. c. Vagrant, by Tramp....... Ld Exeter

1829 ro. c. by Nountebank. . . . . . . . L Ld Eveter

1850 ch. c. by ditto..............

1851 b. с. by Redgauntlet............

1852 b. c. by ditto (dead).......... His Majesty 
1853 ch. f. Moll Flagon, by The Colonel... \} Ld Lichtield

1854 ch. f. by Emilius..............

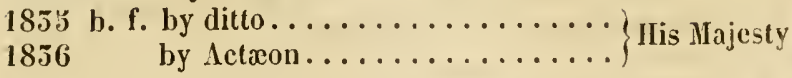

\section{ADDY (sister to Chateau Margaux)}

Bred by Lord Egkenont, in 1820, got by Whalebone, lier dam,

Wasp, by Gohanua--Ilighflyer--Eclipse.

1826 b. f. by Tanered..............

1827 b. c. by Anticipation................ Ld Orford

1850 c. by St Patrick..............

1851 f. by ditto.................

1852 slipped foal by Tiresias..........

1855 b. f. by ditto (dicd young)........ D. of Portland

1854 b. c. by ditto ................

185 b. b. by ditto................

1856

by The Colonel............

\section{ADELICIA (sister to Trinidada)}

Bred by Mr Claridge, in 1817, got by Y. Woodpecker, her dam, Platina, by Mercury - Herod-Y. Hag, by Skim-Hag, by Cral.

1821 b. c. Cadiz, by Ebor............

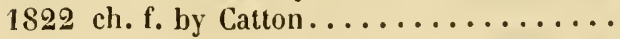

1824 , b. f. by Ardrossan..............

1826 b. c. by Berlin................

1827 ch. f. by Berlin (not trained)........ Mr Claridge

1851 b. c. by Nimrod (son of Whalebone)..

1855 b. f. Aveline, by Jerry . . . . . . . . .

$1854 \mathrm{ch}$. f. by $\Lambda$ ctixon...............

$1855 \mathrm{ch}$. c. by ditto ................

1856 by Jerry................

In 1825 missed to Catton, in 1825 to Blacklock, 1828 to Lottery; 1829 slipped foal by Nimrod, 1850 missed to Chateau Margaux, and not covered in 1831.

\section{ADELINE (sister to Adeliza)}

Bred by the Duke of Ruxusd, in 1821, got by Soothsayer, her dam, Elizabeth, by Orville, out of Tennytrumpet, by Trumpater-Young Camilla, by Woodpecker. 
1823 b. c. (Victory) by Waterloo........

1826 c. by Phantom............... D. of Rutland

1827 b. f. (Princess Victoria) by Middleton.

1828 b. f. by Merlin................

1851 ch. f. by ditto.................Mr T. J. Wood

1852 b. c. Danicl, by St Patrick......... Ld Stradbroke

1855 b. c. The Captain, by Zingance..... Ld Lichficld

1854 f. Ringlet, by The Colonel........ Ld Uxbridge

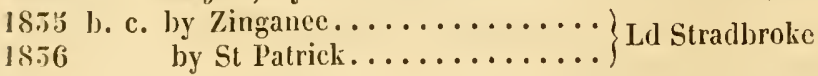

No foal in 1829 or 1850 .

\section{ADELIZA,}

Bred by the Duke of Rutuand, in 1822, got by Soothsayer, her dam, Elizabeth, by Orville.

1829 b. c. Osterly, by Middleton........ Ld Chesterfield

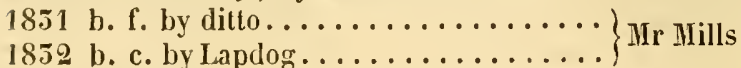

Missed in 1850; sent abroad in Feb. 1855, barren.

\section{ADVANCE,}

Bred by Mr Goodissox, in 1815, got by Pioneer, her dam by Buzzard, out of sister to Champion, by Pot80's--Huncamunca, by llighflyer-Cypher, by Squirrel.

1821 br. c. Progress, by Pericles......... 1822 br. c. (Retreat) by Comus.......... 182.3 ch. c. Tirailleur, by Captain Candid. . 1824 b. c. by Soothsaycr...............

1893 br. c. Father Long-legs, by Filhoda Puta

$1 \$ 26$ b. f. Pera, by Sultan........... Ld Excter

1827 b. c. Mahmoud, by ditto..........

$1 \$ 29 \mathrm{br}$. f. Galata, by ditto ...........

1850 cl. f. Deception, by Mountebank....

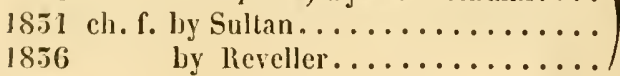

Slipped foal by Sultan in 1852, missed to him in 1828, 1855. 1854 , and 185 .

\section{AGATHA,}

Bred by Mr JAQves, in 1814, got by Orville, her dam by Star Y. Marskic-Emma, by Tclemachus-A-la-Grecque, by Regulus. 
1821 b. f. (Frailty) by Filho da P'uta......

1822 br. c. Lord John, by Interpreter.....

1823 ch. f. Missy, by Catton........... Mr Petre

$1823 \mathrm{br}$. f. Emmelina, by Blacklock.......

1827 ch. f. Good Tidings, by Tiresias......

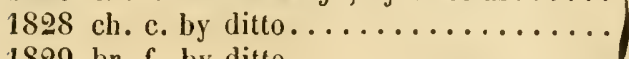

1829 br. f. by ditto ...............

1850 br. f. by ditto ............... D. of Portland

1851 ch. c. Cacus, by ditto...........

1855 br. c. Kalistos, by ditto ..........

1855 b. f. by ditto................

In 1824 missed to Filho da Puta, in 1826 to Sultan, in 1852 and 1854 to Tiresias.

AGLAIA,

Bred by $\mathrm{Mr}$ Wightwick, in 1821, got by Fyldener, her dam Ly

Walton-Gipsy, by Guildford-America, by Imperator.

182马 ch.c. by Bobadil.............. Ir Denhain

1826 ch. c. lyy ditto................ Mr Wightwick

1827 b. f. Little Duchess (aft. Lilla) by ditto Mr Massey

1850 b. f. Marianne, by Champion....... Mr T. Critchley

1831 b. f. Syren, by Sligo ............ Gen. Yates

1855 b. c. Ethiopian, by Memnon....... Ld Lichfield

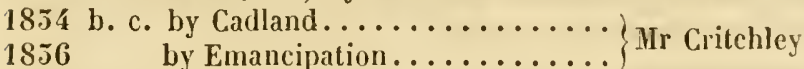

In 1828 missed to Bobadil, in 1829 to Champion, in 1852 to Pantaloon, in 185 to Emaucipation.

\section{AGNES,}

Bred by Lord Lowther, in 1821, got by l'resident. her dam by

IIambletonian, out of Marcia, by Coriander - Faith, by Pacolet.

1825 ch. c. Sir Richard, lyy Woful. . . . . . Mr Cockburn

1827 gr. l. by Carbon.............. Mr Turner

1828 b. c. by Whalebone............ Mr IIunter

1851 ch. f. by Middleton............. Mr Maberly

1852 gr. f. by Camel............... Mr Stephenson

$1835 \mathrm{gr}$. c. by Wrangler............ Mr R. Wilson

1854 gr. c. by Albany $\ldots \ldots \ldots \ldots \ldots \ldots \ldots$. Wr Ridge

Yot covered in 1825 or 1828 ; missed to Sober Robin in 1850 , to Shakespear in 185. 
AIGRETTE,

Bred by Lord Grosvevon, in 1821, got by Rubens, her dam, Opal, by Sir Pcter, out of Olivia, by Justice.

1826 b. f. by Hedley ............... 1827 b. c. by Anticipation.......... Mr L. Charlton

1829 b.c. Novelist, by Waverley........

1851

1855

1853

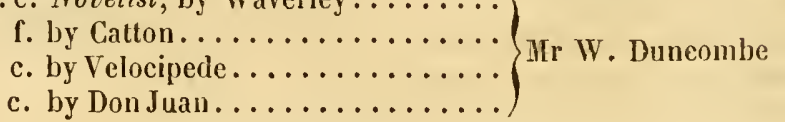

Barren in $1828,50,52$, and 54 .

\LEA,

Bred by Lord Egrenowt, in 1828, got by Whalebone, her dan,

Hazardess, by liaphazard, graudam by Orville, out of Spinetta, by Trumpator.

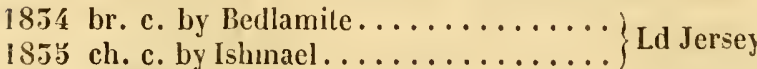

\section{ALECTO,}

Bred by Mr Houldsworte, in 1820, got by Filho da Puta, or Hetman, her dam, sister to Agnes Sorrel, by Stamford, out of Remnant, by Trumpator-Fancy, by Florizel.

1828 c. by Banker (not trained)........

1850 b. f. Catherina, by Whisker........

1831 f. by Tramp (put to the stud) .....

1852 b. c. by Langar................ Mlr Barrow

1853

$185:$

1856

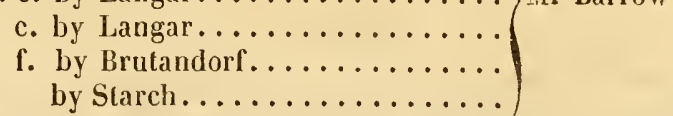

In 1829 missed to Whisker, in 1854 to Brutandorf.

The ALEXANDER MARE (Mayfly's dam, Vol. III, p. 6)

Was barren in 1852 and 55, and was destroyed. IIer produce of 1831 was poisoned when a yearling.

\section{ALICE,}

Bred by Mr TAYloR, in 1827, got by Phantom, her dam, Pasithea, by Rubens, out of Lamia, by Gohanna-Certhia. 
1852 b. c. Procligal, by Master IIenry.....

1853 b. c. by Hiddleton.............

1834 slipped foal by Minos............ Mr Corbet

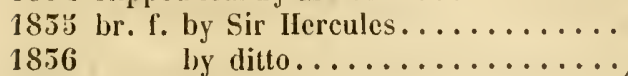

\section{ALINE,}

Bred by Lord Lowtrer, in 1822, got by Woful, her dam, Bizarre, by Peruvian, out of Violante, by John Bull.

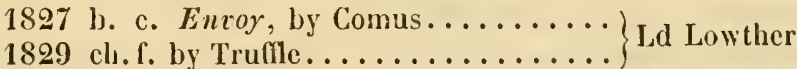

1850 b. f. Alice, by ditto............ Mr Stephenson

1851 ch. e. Charivari, by Reveller (sent to

1852 b. c. by Partisan.................

1854 ch. c. by ditto.............. Sir II. Wood

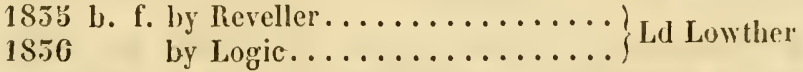

Missed to Partisan in 1828 and 1853.

\section{ALLADINA,}

Bred by II. R. If. the Duke of York, in 1820, got by Aladdin, lier dam by Young Whiskcy, out of Duchess, Sir C. Cockerell's Arabian mare.

1828 b. f. by Whalcbone........... Ld Egremont

1829. gr. c. Bacchus, by ditto.......... Mr Batson

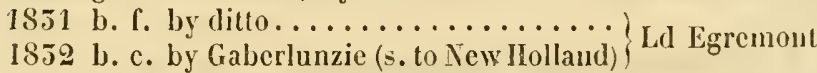
Missed to Whalebone in 1850 , and shot in 1835 .

\section{ALLT,}

Bred by Mr Walker, in 1818, got by Partisan, her dam, Jest, by

Waxy-Scotia, by Delpini-King Fergus-Calia, by Herod.

1825 b. foal (dicd from a kick). . . . . . . .

1824 bl. f. Vocabulary, by litterpreter....

1826 ro. c. Associate, by Little John (sent Mr Walker

1828 b. f. by Straitwaist (sent to Jamaica in 1855)

1829 gr. f. by Little John.............. Wr Grant 


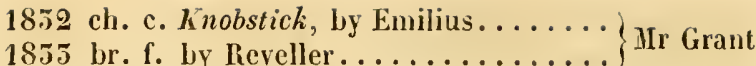

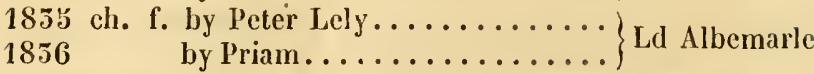

Barren in 1825, missed to Richard in 1827 , to Little John in 1850.

\section{ALPHEGE,}

A Black Mare, bred by Mr Brunt, in 1826, got by Buffalo, her dam, Grey Helen, by Teasdale-Election-Fair ILelen, by Hambletonian - Ifelen, by Delpini.

1855 missed to Camel...............

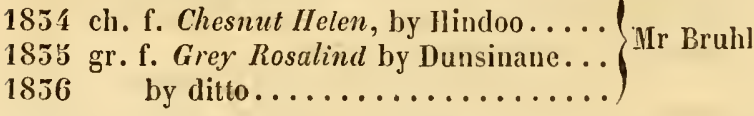

AMLN L,

Bred by Mr Barndrigge, in $181 \%$, got by Sir Oliver, her dam,

Strumpet, by IIambletonian, out of Moss Rose, by Sir Peter.

1852 ch. f. by Langar.............. Mr J. S. Burgess

1854 ch. c. by Granby.............. Mr Lacey

In 1853 missed to Langar, in 1855 to Vanish.

\section{AMARYLLTS,}

Bred by Ifr Chr. Sykes, in 1826, got by Cervantes, out of Lady

Rachel, by Stamford-Y. Rachel, by Volunteer-Rachel, by Highllyer.

1853 f. by Tramp (foaled a cripple and lilled)

1854 b. f. by Tramp............... Mr Ridsdale

1853 b. f. by St Nicholas.............

1856 by The Saddler.

\section{AMBIGUITY,}

Bred at IIampton-Court, in 1818, got by Election, or Blucher, her dam, Selim's dam, by Alexander-Highflyer-Alfred--Eugineer -Bay Malton's dam.

1825 ch. f. (Buttercup) by Tiresias......

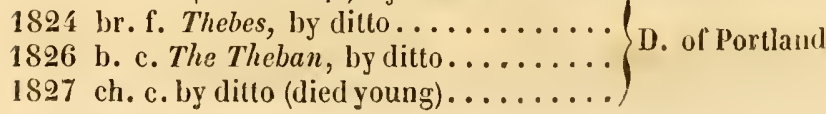




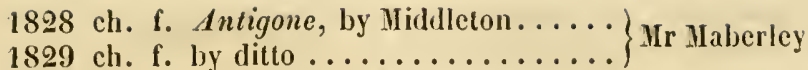

1851 ch. c. by ditto ...............

$1852 \mathrm{ch}$. f. Drab, by Reveller ..........

1855 ch. c. by Emilius ............. Ld Chesterfield .

1854 b. c. Zimri, by Zingance.........

1853 b. c. by Priam................

1856 ly Priam................

In 1823 missed to Tiresias, in 1850 to Middleton.

\section{AMBUSCADE,}

Bred by Lord Grosveron, in 1826, got by Master Henry, her dam, Finesse, sister to Bizarre, by Peruvian, out of Violante, by John Bull-liightlyer.

1851 b. c. St Hilary, by Camel..........

1852 missed to Peter Lely ..............

1853 b. f. by Teniers.............. Idd Westminster

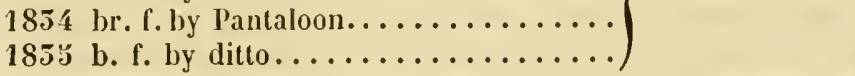

\section{A MIIABLE,}

Bred by Mr Cinanpron, in 1818, got by Orville, her dam, Sprite, by Bobtail, out of Catherine, sister to Colibri, by Woodpecker.

1825 ch. c. Alpha, by Magistrate.........

1826 b. c. Abel, by Filho da Puta...........

1852 ch. c. Amber, by Langar............. IIr Ilouldsworth

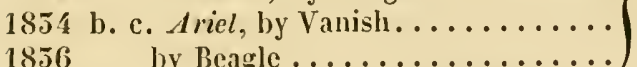

In 1827 missed to Whalebone, 1828 to Comus, 1829 slipped foal by Filho, 1850 and 55 missed to Sultan, 1851 to Velocipede, and 185:5 to Brutandorf.

\section{AHINTA,}

Bred by Mr ATrwoop, in 1850, got by his Chesnut Arabian, her dam by Abjer, out of The Duchess, by Cardinal York.

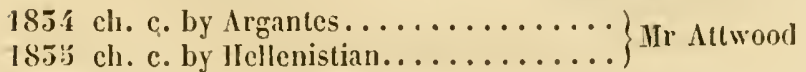

\section{AMORET,}

Bred hy Col. LActock, in 1897, got by Abjer, out of sister to Woodcock, by Y. Woodpecker. 
1854 slipped twins by $A$ ugustus.........
1855 ch. f. Annie, by ditto ......... Mr Sowerby

\section{ANDREW MARE,}

Bred by Mr Gouning, in 1824, her dam by Quiz, out of the Alexander mare, dam of Rubens, Selim, and Castrel.

1828 b. c. Clansman, by Partisan....... D. of Rutland

1829 b. c. Sir Jacob, by Truffle.......... Mr Arnold

1852 ch. c. by Buzzard (son of Blacklock)....

1855 b. f. by Partisan .............. D. of Rutland

1854 b. c. by Shakespear............

1853 b. f. by Buzzard

1836

by Wiseacre............... Mr Golding

In 1850 missed to Camel, 51 to Middleton.

\section{ANDREW MARE (Bay)}

Bred by Mr Henlex, in 182, her dam, Governess, by Governor,

grandam by Young Marske, out of Gentle Kitty, by Silvio.

1850 b. c. Plantagenet, by Camel........ Mr Sowerby

1831 br. c. by Middleton, or Stainborough

1852 missed to Filho da Puta........... (dead) Mr R. Wilson

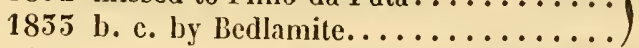

1854 b. c. by Shakespear ..............

185 b. b. by Sir Benjamin ............ Mr Carter

1856

by Rowton .............

\section{ANGELICA,}

Bred by Major Bower, in 1817, got by Anadis, her dam, Miss Craigie, by Orville, out of Marchioness, by Lurcher.

$1826 \mathrm{gr}$. f. by Minos ............... Mr R. Bower

1829 f. by Figare (dead) ............. Major Bower

1856 by The Saddler........... Mr Allen

Barren in $1830,52,5,4$, and 3.

\section{ANGELICA,}

Bred by Mr West, in 1819, got by Fyldener, her dam by Rubens, out of Streatlam Lass, by Pipator-Beatrice, by Sir Peter. 
1826 b. c. Foxcote, by Fitz-Orville (son of $\begin{array}{r}\text { Sheba's Queen) } \\ \text { Sr Tomes }\end{array}$

1827 ch. f. by Claude Lorraine ..........

1829 ch. c. Creeper, by ditto...........

1850 ch. c. Cricket, by ditto........... Mr West

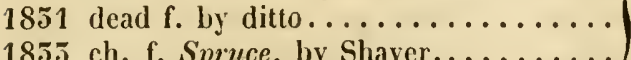

1855 ch. f. Spruce, by Shaver..........

Missed to Claude Lorrainc in 1828, to Shaver in 1852, and was sent to Germany in Oct. 1855 , covered by him.

\section{ANGELICA,}

Bred by Lord Grosvenon, in 1821, got by Rubens, her dam, Plover, by Sir Peter-Boudrow-Escape's dam, by Squirrel.

1826 b. f. by Master IIenry (died 5 days old)

1827 b. c. Coventry, by Tramp..........

1828 missed to Ld Dartmouth's Manec.....

1829 f. by Perchance (died a foal).......

1850 ch. c. by ditto ................

1851 b. c. by St l'atrick.............. Ld Orford

1852 b. c. Ascot, by Reveller...........

1835 c. by Mulatto (died a foal)........

1834 b. f. by ditto.................

1855 f. by Zinganee...............

1856

by Reveller

\section{ANNA (sister to Flexible)}

Bred by Lord EGrenont, in 1826, got by Whalebone, out of Themis, by Sorcerer-Ilannah, by Gohanna.

1850 gr. c. by Little John (sent to France). )

1831 gr. c. by ditto (sent to Van Diemen's Ld Egremont

Land)

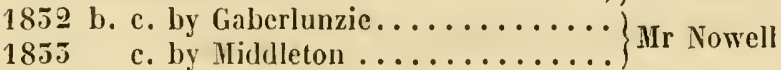

1854 ch. c. by Mulcy ............... Mr Tattersall

Sold to go to France in 1834 , covered by Logic.

ANNA,

Bred by lord G. H. Carexdish, in 1826, got by Godolphin, out of Parrosa, by Vermin-Nikè, by Alexander.

1855 b. c. by Defence.............. Mr Blandy

1834 ch. f. by Middleton ............ MIr Tattersall 
The mare was sent to France in June 1854.

\section{ANNE OF GEIERSTEIN,}

Bred by Mr T. O. Powlett, in 1829, got by Calton, her dain, Rebecea, by Soothsayer, out of Prudence, by Waxy.

1834 b. f. Anne Grey, by Belzoni........

183 . b. c. Antwerp, by ditto........... Ir Carter 1856

\section{ANNOT LYLE,}

Bred by Mr Bigks, in 1819, got by Ashton, her dam, Lamia, by Gohanna-Certhia, by Woodpecker-Trentham-Cunegonde.

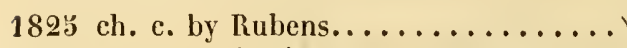

1826 b. f. by Tiresias ..................

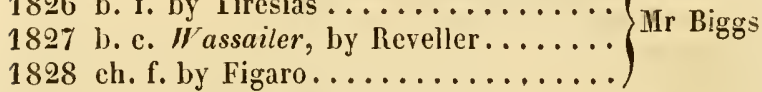

1855 ch. c. Pye Annot, by Langar....... Ld Derby

1854 ch. f. Azalia, by Catton ..........

185 b. f. by Langar................. Mr F. L. Savile 1856 by Langar.................

She was ridden as a charger between 1828 and 1835.

\section{ANTIOPE,}

Bred by Lord Egremont, in 1817, got by Whalebone, her dam,

Amazon, by Driver-Fractious, by Mereury-WoodpeckerEverlasting.

1824 b. c. (Amphion) by Partisan (s. abroad) Mr Udny 1825 was trained ............... Ld Clarendon 1826 b. f. Lady Emily, by Emilius (put to the stud) Mr Udny

1827 foal to ditto (died 2 days old)....... Ld Clarendon

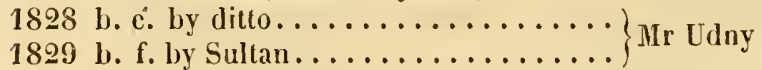
1850 b. f. Dirce, by Partisan (put to the stud) Ld Chesterfield 1851 b. c. Zellus, by Mameluke......... Mr Greville 1852 b. c. by Reveller .............. D. of Portland

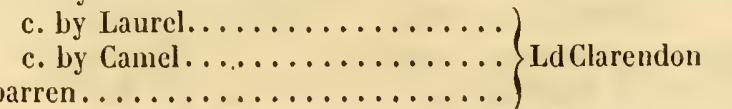




\section{MPOLI,ONIA,}

Bred by Mr Petne, in 1826, got by Whisker, her dam, My lady, hy Comus, out of 'The Colonel's dam, by Delpini-Tipple Cider, by King Fergus.

1855 ch. c. A pollo, by Partisan..........

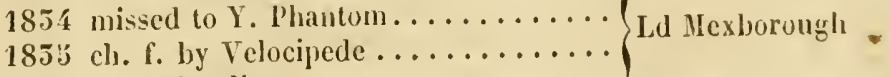
1856

\section{APPARITION,}

Bred by Lord Exeter, in 1820, got by Comus, out of Phantom, by Hambletonian - Precipitate-Highflyer-Tiffany.

1831 bl. f. rision, by Rajah ..........

1852 b. c. by Luzborough........... Mlr Taunton

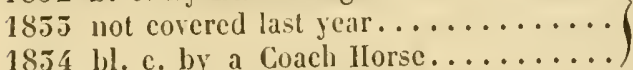

1853 b. c. by Firman................ Mr E. V. Fox

1856 by Coronet........... Mr Taunton

\section{MRABELLA,}

Bred by Mr Fletcuer, in 1811, got by Williamson's Jitlo, her dau, Esther, sister to Staveley, by Shuttle.

1817 b. c. (Sir Thomas) by Whitworth.... Mr Yates

1818 b. с. by Comus..............

1819 b. f. by Caliban (dead) .........

1820 b. f. by Comus...............

1821 b. f. by Smolenslio (dead).........

1822 c. by $\operatorname{Ardrossan} \ldots \ldots \ldots \ldots \ldots \ldots$

1825 f. by ditto..............

$1823 \mathrm{br}$. c. (Y. Blacklock) by Blacklock.... Hr II. Fleteher

1826 br. f. (Versatility) by ditto........

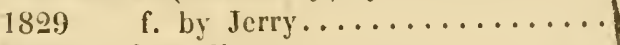

1850 b. f. by ditto...............

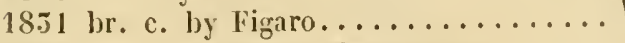

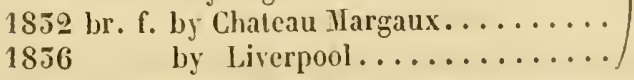

In 1824 missed to Whislier, 1827 to Tramp, 1828 to Waverley; missed also in 1855,54 , and 55. 


\section{ARABIAN MARE,}

A natural Arabian, prescuted, when a foal, by the Imaum of Muscat to Capt. Collier, of II. MI. S. The Liverpool, and reared in India.

1827 b. c. by Mozart................ Mr Scruton

$1829 \mathrm{ch}$. or ro. f. Francesca, by ditt......

1852 gr. c. Alhassan, by his Grey Arabian..

1854 b. c. by a black Turk or Barb....... IIr Attwood

1853 gr. f. by Argantes ............

1856 by his Chesnut Arabian........

Barren in 1828, 50, 51, and 55.

\section{ARABIAN MARE (Rosabel)}

A Bay Mare, bred by Mr WALker, in 1824, got by Mr Altwoot's cliesnut Arabian, her dam by Smoleusko, grandam by Luck's-all -Toby-Maguet--Dolly, by Snap.

$1829 \mathrm{gr}$. ro. or ch. f. Florimel, by his Grey Arabian

1851 cl.. c. by his Grey Arabian (dead) ....

$1852 \mathrm{br}$. c. by a black Turk or Barb (dead). Nr Attwood

1855 bl. or gr. f. by ditto

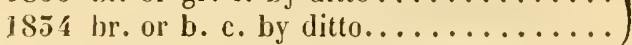

No produce in 1850 or $185 \%$.

\section{ARACHNE,}

Bred by Mr Houbosworth, in 1822, got by Filho da Puta, her lam, Treasure, by Camillus-Hyacinthus-Flora, by King Fergus.

1851 br. c. (Sir Isaac) by Camel........ Ld Wilton

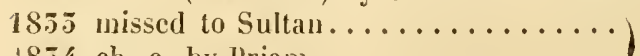

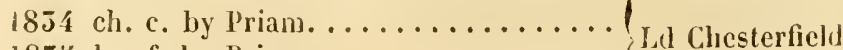

1855 br. f. by Priam. . . . . . . . . . . . . . $1856 \ldots \ldots$

\section{ARBIS,}

Bred by Lord Stradroke, in 1819, got by Quiz, luer dam, Persepolis, by Alexamter-sister to Tickle Toby, by Alfred-Herod - Proserpine, sister to Eclipse. 
1825 b. c. IIindostan, by Whalebone .....

1826 b. e. Ilindoo, by ditto ..........

1827 f. by Tiresias...............

1829 ch. c. IJyler, by Whalebone; or Moses

1851 ch. c. Holkar, by Helenus........ D. of Richmond

$1852 \mathrm{ch}$. c. Ifyderabad, by ditto.........

$1855 \mathrm{ch}$. c. IIaveldar, by ditto.........

185 : ch. e. by The Colonel ..........

1856 by Rubini.............

In 1825 missed to Phantom, in 24 to Octarius, in 28 to Hidlleton, in 1850 to Moses, and barren also in 5 i.

\section{ARCHDUCIESS,}

Bred by Sir Tuomas Mostry, in 1821, got by Rubens, or Articlıohe, out of Queen of Dianonds, by Diamond, grandan by Sir H'eter, out of Lucy, by Florizel.

1850 b. f. hy Teniers................ Mr IIuntingdon

1851 br.f. Vittoria, by Camel (put to (hestud)

1852 b. c. The Magnate, by Rattledore....

1855 eh. c. The Palatine, by ditto....... Mr Mostyn

1855 br. f. by Camel..............

1856 by Emancipation ...........

Missed to Reveller in 1829, to Felt in 1854.

\section{ARCOT LASS,}

Bred by Mr Storer, in 1821, got by Ardrossan, her dam by Cramlington, out of Floyerkin, by Stride-Jitte England's dlam, by Javelin-sister to Toby, by Highflyer.

1828 b. f. by Alexander............. Mr Cattle

1829 ch. c. St Giles, by Tramp......... Mr Ridsdale

1850 br. c. by Lottery, or leter Lely ......)

1851 b. f. Miss Giles, by Lottery (put to the Mr Cattle stud) )

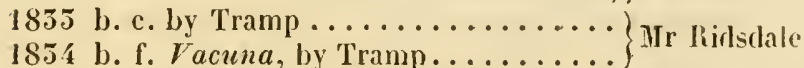

1836 by Tramp, or Mulatto......... Mr Cattle

In 1827 missed to Figaro, 52 to Comus, 53 to Velocipede.

Sr Giles was sent to Alabama. in the United States, in September 18.75. 
ARDROSSAN MIARE,

Bred by Mr RindezL, in 1817, her dam, Lady Eliza, by Whitworth - Spadille-Sylvia, by Y. Marske.

1822 b. c. by Ebor (died in 182:3) . . . . . .

1824 cl. c. II artpury, by $\Lambda$ bjer.........

1825 b. c. Rector, by Doctor Syntax...... Hr Riddell

1826 ch. c. Lawnsleeves, by ditto........

1827 b. c. Fimancipation, by Whisker....)

1829 b. c. Tomboy by Jerry ............

1830 ch. c. The Dancing MI aster, by Felton.

1851 ch. f. Lily of the Talley, by Doctor Syntax

1852 ch. c. Lord Collingwood, by Shamrock Mr Orde

(an Irish horse)

$185 \pi$ b. f. Beeswing, by Doctor Syntax....

1855 ch. c. by Actxon..............

1856

by Jerry................

Missed to Soothsajer in 1825 ; barren also in 1828 and 54.

\section{ARDROSSAN MARE,}

Bred by Lord Kelderse, in 1825, her dam by Rubens, out of Queen of Hearts, by Sorcerer-Tooee, by Buzzard-Violet, by Slark.

1855 ch. f. by $\Lambda$ ctæon . . . . . . . . .

1834 bl. c. by Jerry ................

$\left.\begin{array}{c}1833 \text { br. c. by Jerry } \ldots \ldots \ldots \ldots \ldots \ldots \ldots \ldots \\ 1856 \quad \text { by Retainer } \ldots \ldots \ldots \ldots \ldots \ldots\end{array}\right\}$ Idd Kelburne

\section{ARDROSSAN MARE (sister to May-day)}

Bred by Mr Bunlett, in 1820, her dam by Sir Peter, oul of Mliss Gunpowder, by Gunpowder--Y. Marshe.

1829 b. c. Florio, by his Chesnut Arabian.)

1850 b. c. Beauchamp, by ditto ......... Hr Attwood

1851 b. c. by his Grey Arabian..........

1854 b. f. by Caccia P'iatti, or Vollaire....

185 ' b. f. by llumphrey Clinker......... Ir J. Wood

18.56 by Plysician.............

She had no produce before 1829 , and missed in 1832 and 53. 


\section{ARETIHSSA,}

Bred by Lord SrRanenoke, in 1824, gol by Quiz, her dam, Persepolis, by Alexander-sister to Tickle Toby, by Alfred.

*1828 ๖. f. Arsena, by Morisco ..........

1829 clı. c. Choaspes, by Trumle ........

1850 ch. c. Alpheus, hy Sultan .........

1851 ch. f. by Middleton ............

1852 missed to Partisan............. Ld Stradbroke

1855 ch. f. Water-nymph, by Bedlamite...

1834 ch. f. Doris, by The Colonel.......

1853 ch. c. IIelicon, by ditto..........

1836 by Taurus ...............

* Arsena produced a ch.f. by The Colonel in 185.5, was not covered that year", missed to The Colonel in 1855, and died sudelenly Sep1. 25. 1855.

\section{ARICIA (sister to Hampden)}

Bred by Mr Newtox, in 1824, got by Rubens, lier dam, Jiana, by Stanford, grandam by Whiskey, out of Grey Dorimant, by Dorimant.

1851 ch. f. Delusion, by Comus ......... Mr II alkel

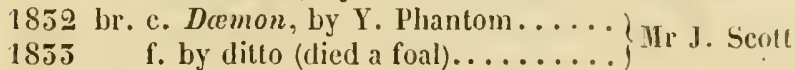

1854 br. c. by ditto...............

185 b. f. by Partisan .............. Ld Chesterdield

1856

by Rubini ...............

\section{MRINETTE,}

Bred by Mr H. Eowands, in 1827, got by Wanton, her dam by Ardrossan, out of Vieissitude, by Pipator-Beatrice, by Sir Peter.

1855 b. f. by Jerry

1831 br. c. Stashing Hawy, by Völaire...

1835 ch. f. Tabitha Bramble, by Humplırey Mr Slipsey Clinker)

\section{ARIIIDI,}

Bred by Lord Derey, in 1819, got by Rinalılo, her dam, sister to Bos, lyy Buzzard-liennington's dam. Iy the Perey ArabianUcrod-Suap-Shepherd's Crab. 
$1823 \mathrm{br}$. f. by Filino da Puta .......... 1826 br. f. by Master IIeury .......... 1897 b. f. by Whisker (dead).......... 1828 ch. c. Filcher, by Tramp.......... 1829 b. c. Warlock, by Whisker........

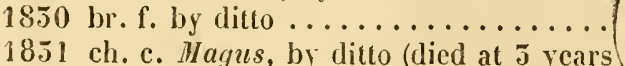
1851 ch. c. Magus, by ditto (died at 5 years 0 Ld Derby

1852 ch. c. Amurath, by Langar........ 1855 ch. f. Erminia, by ditto.........

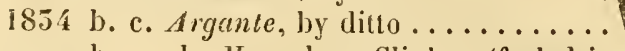
br. c. by Ilumphrey Clinker (foaled in

1856 by Telocipede.............. December)

\section{ASPASIA,}

Bred by Lord Exeter, in 1817, got by Pericles, her dam, Bess, by Waxy, out of Vixen, by Pot8o's.

1328 br. c. by Sultan.............. Mr Poyntz 1529 b. c. by Wanderer.............. Ld Tavistock $1850 \mathrm{br}$. f. Minima, by Sultan......... Ld Exeter 1852 ch. c. The Glama, by Camel........ Sir M. Wood 1855 br. c. Cottager, by Orville Junior. . . . Mr Marson She had no foal till 1828, missed to Sultan in 1851, slipped a colt foal by Augustus in December 1855, and died a few days afterwards.

\section{ATAL $\Lambda$ NT $\$,}

Bred by Mr BLake, in 1808, got by Walton, her dam, sister to

Tup, by Javelin-Flavia, by Plunder-.- Hiss Eustou, by Suap.

1818 b. c. Atlas, by IIedley........... Mr Beechey

$1821 \mathrm{ch}$. f. by Usquebaugh . . . . . . . .

1822 ro. c. P'opinjay (once called Tokay) by Mr Sadter ditte 1824 ch. c. Vivian Grey, by Anticipation (s.) Mr MIolony

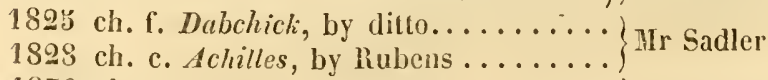
1850 ch. c. Caldicot, by Doctor Eady, or

1855 b. c. by Doctor Eady ............ Virgilius Mr Pryse

Barren in 1819, 25, 26, 27, and 29 ; in 1820 slipped her foal by Usquebaugh, in 1851 slipped twins by Doctor Lady, in 1832 missed to Cardinal 1'uff, in 1854 to Doctor Eady, and was shot. 
AUBURN, by Blacklock (Vol. III, p. 20) died in 1852, in foal to Emilius.

\section{AUDREY,}

Bred by Lord Lowtier, in 1814, got by Marmion, her dam, Ignes, by Sorcerer, out of $\Lambda$ melia, by Highflyer-Miss Timms.

* 1818 clı. f. Abigail, by Haphazarl...... . +1826 br.f. Augusta, by Tiresias (put to the

1828 b. c. Auctioneer, by Tancred .......

1850 b. c. by Pacha (dead)............ Col. Lowther

1852 b. f. by Y. Juniper (brother to Oscar).

1855 f. c. (dead) $\}$ by Neptunc

185 ๖ b. f. by Y. Juniper . . . . . . . . .

Not covered from 1818 till 1895; barren in 1827 and 51 ; slipled a foal it 1829; missed to Y. Juniper in 185 í.

* Adigall was sent to France, in 1829 , having had but one foal that lived, viz. in 1827, b. f. Trinket, by Woldsman (son of Sir Peter).

† Augosta produced in 1850 , a b. c. by Pacha, missed to him in the next year, and has since been sent to Sardinia.

\section{$\triangle$ UGUSTA,}

Bred by Mr Lucas, in 1818, got by Woful, her dam by Rubens, out of Guildford Tan-Justicc-Parsley, by Pot8o's-Larly Bolingloroke, by Squirrel.

1826 ch. f. Acacia, by l'hantom........

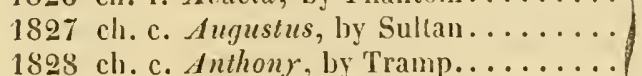

IS29 h. f. Trick, by Mountebank ........ Ld lixeter

1830 b. f. Amima. by Sultan (put to the stud)

1852 ch. c. Aurelius, by Sultan.........

$1854 \mathrm{ch}$. c. by ditlo ..............

Missed to Redgauntlet in 1851, to Sultan in 1853, and died in lugust 1834 of a ruptured blood-ressel in the head.

\section{ALROR 1,}

Bred by Mr Porxtz, in 1826, got by Sultan, her dam, Phantom, ly

Hambletonian-Precipitate-Ilighnyer-Tiffany, by Eclipsc. 
1852 b. f. by Rasping

1855 b. c. by Royal Oak...............

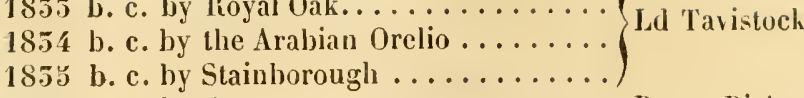

1856 by $A$ ctæon............. Baron Biel

Sent to Germany.

BABEL,

Bred by Mr R. W. WaLKER, in 1825, got by Interpreter, out of Fair Ellen, by the Wellesley Grey Arabian-Maria, by Highflyer-Nutcracker, by IIatchem.

1850 missed to Middleton.

1851 b. c. Fortunatus, by Sultan . $\left.\begin{array}{l}\text { sold to } \\ \text { the D. of } \\ \text { Holstein }\end{array}\right\}$

1855 b. с. by Zingance............. Ld Chesterfield

1854 b. c. by Y. Phantom.............

1853 b. f. by Tramp ...............

1856

by Zinganee.

\section{BALEINE,}

Bred by Mr Snarn, in 1825, got by Whalebone, her dam, Vale Royal, by Sorcerer, out of Orangeade, by Whiskey-Orangebud, by Highllyer.

1852 b. f. Madame Pelerine, by Velocipede.

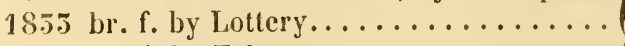

. Hr Watt

1855 b. f. by Humphrey Clinker ..........

1856

by Belshazzar

Mr Allen

\section{BALLAD SINGER,}

Bred by I.ord Fıtzwılııม, in 1825, got by 'Tramp, out of Clinke rina, by Clinker-l'ewet, by 'Tandem.

1851 b. c. by Young Phantom............
1852 b. f. by Confederate (died a foal) . . . . Ld Fitzwilliam

1855 b. c. Wentworth, by ditto........ Mr T. 0. Powlett

1854 slipped colt by $\Lambda$ madis ............. Wd Fitzwilliau 
TILE BILKAN,

Bred by Mr Fox, in 1827, got hy Blacklock, her dam. Mrs Fry, by

Walton, out of Vourneen, by Sorcerer-Tooee, ly Buzzard.

1852

c. hy Whisker ............. Mr Batson

1854 br. c. by Peter Lely ........... Ld Albemarle

18.53 ch. f. hy ditto ............. Mr Grant

1856

ly Langar............... Mr Gratwicke

B.NSIFEL, hy Sorcerer, died in 1852, laving had no foal in the preceding Spring (see Vol. III, p. 25). Her filly by Master Henry. was sent to Germany.

\section{BANSIIEE,}

Bred by Mr Naxrey, in 1815 , got by Y. Sorcerer, her dam, Podagra, by Gouty, out of Jet. by Magnet-Jewel, by SquirrelSoplia, by Blank - Lord Leiglı's Diana.

1821 br. c. Otthodox, by Fillio da Puta....

1822 br. c. Randall, by Cliampion.......

1825 br. c. Sangrado, by Filho da Puta... Sir WV. Wynn

1824 b. f. Brenda, by Catton..........

1825 br. c. by Hobgoblin............

1827 bl. f. Georgiana, by Welbeck.......

1829 b. f. Zorilla, by Trufle..........

1850 br. c. Caliban, by Camel......... Mr F. R. Prica

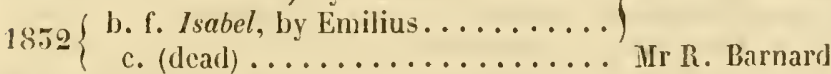

Missed to Sherwood in 1828; in 1851 had produce by Camel, which was maliciously destroyed; and was sent to Germany in 1855 , in foal to Zingance.

\section{B.INTER,}

Bred by Lord Grosvexon, in 1826, got by Naster Hesry, her dau.

Boarlicea, by Alexander, out of Brunette, by Amaranthus-

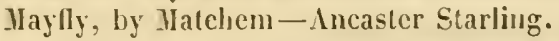

18.51 b. c. Touchstone, by Camel.......

18.52 br. с. Yorick, by Peter Lely . . . . . .

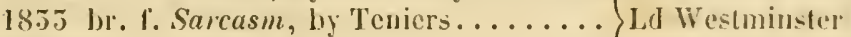

1854 br. f. by Pantaloon............

18.35 b. f. by ditto.............. 


\section{BARBARA,}

Bred by Mr Petre, in 1894, got by The Laird, her dam, My Lady, by Comus, out of 'The Colonel's dam, by Delpini.

1829 b. f. Florine, by Polygar.......... Mr Whecler

1850 and 51 her foals died young........ 1832 Mr J. Scott

1855 b. f. by Young Phantom.......... Hr Leedlrain

1854 ch. c. Newmarket, by Priam.......

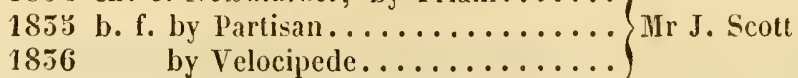

by Velocipede.

\section{BARCELONA,}

Bred by Lord Fitzwillial, in 1827, got by Cervantes, her dall.

Governante, by Governor-Beningbrough-Precipitate.

1852 b. c. by Confederate............

1855 ch. f. by ditto ................

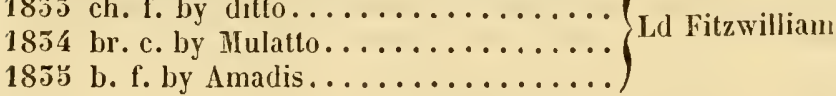

\section{BARONESS,}

Bred by Lord Durnam, in 1822, got by Leopold, her dam, The Duchess, by Cardinal York, out of Miss Nancy, by Beningbrough.

1850 b. c. Philosopher, by Astrologer....)

1851 b. c. Enville, by ditto.......... Ld Grey

1835 b. c. King Cole, by Memnon.......

1854 b. c. Pammon, by Priam......... Sir T. Stanley

1856 by The Colonel........... Mr R. Mostyn

She had no foal before 1850 , missed to $\Lambda$ strologer in 1852 , and was not covered in 1854 .

\section{BARROSA,}

Fred by Lord Grosteror, in 1808, got by Fermin, her dam, Nihi. by Alexander, out of Nimble, by Florizel.

1814 b. c. Little Dick, by Dick Andrews...

1816 br. f. Espagnolle, by Orville........

1817 b. f. by Middlethorpe........... 1818 bd G. II. Cavendish

1819 br. c. by ditto................

1820 br. c. Hina, by Orville.......... 
1821 br. f. (Georgina) by ditto.........

1825 b. c. (Allegretto) by Allegro (sent to

Hungary)

1824 br. f. Young Barrosa, by l'artisan...

1895 b. f. by Godolphim............. Ld G. II. Cavendish

1826 b. f. $($ Anna) by ditto.............

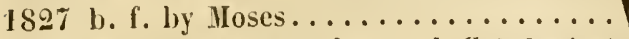

1828 br.c. (Barabbas, afterwards Caledonian)

by Partisan

1829 b. c. by Bizarre..............

1850 b. f. (Ih him) by ditto........... Ld Burlington

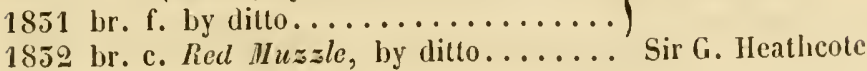

In 1815 missed to Mliddlethorpe, in 1829 to Orrille.

\section{YOUNG BARROSA,}

Bred by Jord G. H. Cavexnisn, in 1824, out of the preceding marc.

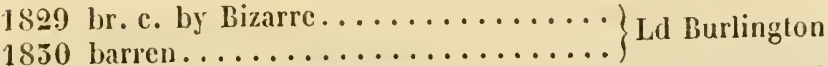

1831 br. c. (Dacre) by Bizarre........... D. of Rutland

1852 br. f. by ditto............... Ld Southampton

\section{BARTONI ,}

Bred by Sir Charles Bexeery, in 1819, got by Haphazard, out of sister to Orange-llower, by Trumpator-Orange-bud, by Highflyer-Orange-girl, by Hatchem.

$1828 \mathrm{ch}$. c. by Middleton.............

1829 b. c. by Emilius (sent to Florence in Ld Orford 1851)

1850 b. c. Shamrock, by St Patrick....... Mrr Gardnor

Not covered in 1825, missed to Centaur in 1827 , to St Patrick in 18.51 , and was sold to Baron Bicl in that year covered by him.

\section{BASSILEA,}

Bred by Mr R. Wilsox, in 1823, gol by Snolensko, her dam by Shuttle, out of sister to Dowager, by Hambletonian.

1851 br. c. Theodoric, by Luzborough.... Sir L. Glyn

$18 \pi$ b. f. by Rereller...............

1855 ch. f. by The Colonel............ Mr Morgan

1854 ch. f. by ditto............... 
BEATRICE,

Bred by Mr R. Harrisox, in 1826, got by Blacklock, out of Royal Oak's dam, by Smolensko-Lady Mary, by Beningbrough Highflyer.

1851 ch. f. by Y. Whisker (brother to Cat-)

1852 ch. f. by Wanton.............. Mr R. Harrison

1854 ch. f. by Action...............

In 1855 missed to Wanton, in 1855 to Actæon.

The br. f. by Lottery, out of Beatrice's dam, foaled in 1850 , is now called Miss Wilfret. -See Vol. lII, p. 580.

\section{BEE IN A BONNET,}

Bred by Mr EuLus, in 1825, got by Dlacklock, her dam, Maniac, by Shuttle, out of Ariticipation, by Beningbrough-Expcetation, by Herod-Skim.

1850 b. c. by Emilius.................. Hr Thornhill

1852 b. c. by ditto............... D. of Cleveland

1855 ch. f. by Emilius..............

1854 b. c. by ditto............... Mr Thornhill

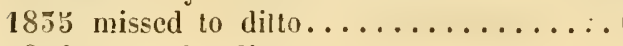

1856

by ditto.................

\section{BELIDA (sister to Laurel)}

Bred by Major YAReungI, in 1825, got by Blacklock, out of Wagtail, by Prime Bhinister-Orville-Mliss Grimston.

1832 br. f. Miss Bell, by Lottery ( sold abroad) at 2 years old)

1855 b. c. St Lawrence, by St Nicholas... Major Yarburgh

1854 b. c. The Doctor, by Doctor Syntax..

1836 by Starch..............

In 1851 missed to Figaro, in 1853 to St Nicholas.

\section{BELLA,}

Bred by , in 1811, got by Beningbrough, her dam, Peterea, by Sir Peter, out of Mary Grey, by Friar.

1819 b. f. by Stamford (died a foal)..... S Sir R. K. Dick 
1821 ch. c.(Freischutz) by Outery....... Jajor Cuninghame

1824 b. c. The Cherub, by Champignon....

1827 b. c. Almaviva, by Figaro.......... Mr S. Stonehewer

1828 b. f. Marchioness, by Eglinton...... . Mr S. Day

1829 b. C. Miss Mary Ann, by Truflle (put) to the stud )

1850 b. f. by Champignon (died a foal)..... Sir R. K. Dick

1851 b. f. Miss Margaret (afterwards Bella) by $\Lambda$ ctron

1834 ch. c. Master Will, by Brunswick....

1853 b. f. by Beagle............... Sir Jas. Boswell

1836 by Corinthian.............

In 1816 missed to Caleb Quotem, in the following year wits trained; was not covered in 1821, missed to Prime Minister i:1 1825, to Comus in 1825, to Benedict in 1852, and to Caccia Piatti in 1855 .

\section{BELLA DONNA,}

Bred by H. R. H. the Duke of Yonk, in 1816, gol by Seymour, her dam, Gramarie, by Soreerer-Sir Peter-Deceit, sister to tise Yellow Filly, by Tandem, out of Perdita, by IIerod.

1820 b. f. Jane Shore, by Woful........ D. of York

182.3 b. f. by Shuttle Pope (dead).......

1824 b. c. Trumpeter, by Waxy Pope..... Mr Iaffenden

1825 b. f. by Boloadil (dead) ...........

1826 b. f. Nightshade, by ditto......... Mr Molony

1827 b. f. Jenny Vertpré, by ditto....... IIr Corbet

1828 b. f. Nemesis, by Manfred.......... Mr Molony

1829 b. f. by Bobadil.............. Mr Payne

1850 slipped foal by Waxy Pope........

1851 b. f. Trumpery, by ditto...........

1855 b. f. Jane, by Bobadil............ Mr Ilaffenden

Not covered in 1820, missed to Waxy Pope in 1852, and to Bobadil in 183 .

\section{BELPHOEBE,}

Bred by Mr ATTw0op, in 1829 , got by his Chesnut $\Lambda$ rabian, her dam by Remembrancer, out of Mary, by Y. Marske. 
18.53 ch. c. by his Grey Arabian........

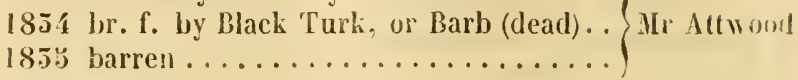

\section{BELTOIRINA,}

Bred at Ilanpton Court, in 1815, got ly Stamford, her dam, sister to Silver, by Mercury-Ilerod-1. Iag, by Skim.

$1 S 18$ b. c. by Rubens.............. Mr Thornhill

1819 b. c. by Blucher.............. Mr Bouveric

1820 ch. c. by ditto............... Mr Prendergast

1822 ch. c. (Paragon) by Soothsayer...... His Majesty

1825 ch. f. Elizabeth, by Rainbow...... Mr Greville

1828 b. 1. by Mustachio (sent to Germany). Iis Majesty

1829 b. f. Little Charlotte by Waierloo... Mir J. H. Peel

1951 b. c. Rutland, by Sultan.......... Mr E. Peel

185 \& b. f. II amptonia, by The Colonel.... D. of Leeds

1856 by Actron............. His Majesty

In 1821 missed to Selim, 26 and 27 to Moses, 50 to Waterioo. 32 to Sultan and Waterloo, 55 and 58 to The Colonel, and in 25 slipped foal by Waterloo.

\section{BENINGBROUGH HARE.}

Bred by Mr Moss, in 1808, her dam, Blacklock's dam, by Curiander, out of Wildgoose, sister to Hyperion, by Ilighllyer.

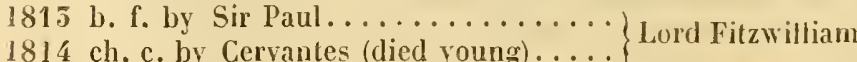

1816 gr. c. Tempest, by Caliban (died in 1822)

1817 b. f. Wishful, by Walton...........

1819 b. f. by ditto (died, leaving no produce)

1821 ch. c. Salisbury, by Soothsayer......

1822 br. f. Wildgoose, by ditto........

1823 L. f. by Comus (died 2 years old) . . . .

$1824 \mathrm{ch} . \mathrm{f}$. by Soothsayer............. Mr liyan

1825 b. f. by Woful................

1826 b. f. by ditto (dearl).............

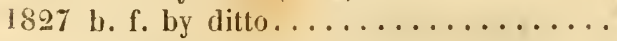

1829 b. f. by Sligo...............

$18.50 \mathrm{~b}$. c. by Skiff (sold to Mr Lichtwald) for Germany) 
1851 b. c. Slimmer, by Sliff. . . . . . . . Mr W. M. Stanley

1852 b. f. by Canuon-ball............. Mr Turner

II 181: missed to Castrel, 18 to Cerrantes, 20 to Comus, 28 to Tiresias, 5.5 to Zinganec, and died that year.

\section{BENINGBHOUGII MARE,}

Bred by Sir Wuluau Gerarn, in 1811, her dam, Hary Aun, by Sir l'eter-Y. Marske - Matchem - sister to Antelope, by Young Belgrade.

1817 b. c. by Langton (dead)...........

1818 bl. c. lyy Sir Thomas.............

1819 b. f. by Da Capo.............. Mir Anderton

1821 bl. f. by Rinaldo..............

1822 br. f. by ditto..............

1825 br. c. Euxton, by ditto.......... Sir iW. Gerard

182: gr. c. by The Marshal (son of Comus). Sir T. Stanley

1826 b. c. by ditto................ Mr Anderton

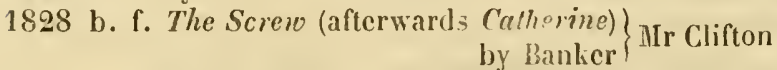

1850 b. f. Dame Durdan, by ditto........ Sir J. Gerard

Barren in 1827 and 29 , had no foal after 1830 , and is dead.

Catherise was sent to France in October 185.

\section{BEOUEST,}

Bred by Mr Shand, in 1819, got by Election, her dam, Legacy. by Beningbrough--Roxana, by Sir Peter-Tulip, lyy DamperEclipse-Rarity, by Matchem.

1824 bl. c. by Smolensko (dieda few daysold) Mr Day

1825 b. f. by Buffalo...............

1826 b. c. by Walton............... Mr Nowell

1827 b. f. by Bustard...............

1828 br. f. by Mluley............... Mr Sowerby

1829 b. f. by ditto............... Mrr Giffarı

1850 missed to ditto............. Mr Nowell

1851 b. c. by ditto................. D. of Clereland

1852 ch. c. Doubtful, by Mulcy.......... MIr Nowell

1855 b. f. Grencada, by ILuley........... Ld Wilton

1854 h. c. by MIuley............... Ld Albemarle

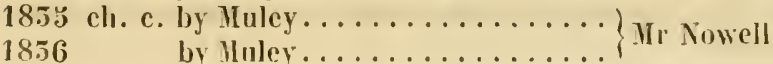




\section{BERENGARIA,}

Bred by Mr Lautour, in 1822, got by Quizzer, her dam, Sleightof-hand, by Sorcerer, out of Trollop, by Waxy-Trull, by Volunteer.

1831 ch. c. Cour de Lion, by Nigel......

1855 gr. f. Queen of Navarre, by ditto.... Mr Lautour 1856 by ditto................

In 1852 missed to Wiseacre, 54 to Nigel, 53 to Agreeable.

\section{BERTHA,}

Bred by Lord Grosvevor, in 1821, got by Rubens, her dam, Boadicea, by Alexander, out of Brunette, by Amaranthus.

1827 c. by Hymettus............ Ld Grosvenor

1828 ch. f. by Champion (dead)......... Ld Westminster

1829 br. c. Parthenon, by Conductor..... . Mr Smith

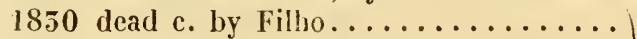

1851 dead foal by Camel..............

1852 br. c. Oswald, by Conductor........

1853 br. f. by ditto (given away young).... Ld Westminster

1854 ch. f. by Pantaloon..............

1855 ch. f. by ditto................

18.56

by Camel...............

\section{BERTHA,}

Bred by Mr SHARD, in 1827, got by Reveller, her dam, Legacy, by Beningbrough, out of Roxana, by Sir Peter.

1852 ch. f. Chaos, by Emilius.......... IIr F. Craven

1855 ch. f. by ditto.............. Mr 'Turner

1854 br. f. Magnolia, by ditto..........
1855 b. b. by Laurel................ Heathcote

BESS, by Waxy (Vol. III, p. 55)

Was barren in 1852, and ordered to be destroyed.

\section{BETTY MARTIN (first called Emerald)}

Bred by Dr Ввонетт, in 1823, got by Blacklock, her dam, by Fitho da Puta, grandam by Smolensko, out of Farnsfield's dam, by Stamford. 
1832 b. f. Everilda, by Y. Phantom......

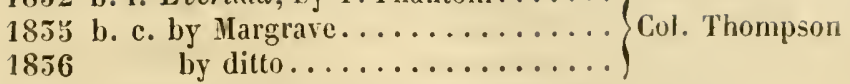

Not covered in 1852 , missed in 1854 .

\section{BILLINGSGATE,}

Bred by Mr Crockforn, in 1819 , got by Selim, her dam, Palma, by Sir Peter, out of Palmflower, by Weasel-Columba, by Alfred.

1823 b. f. by Smolensko (died in 1828).... Mr Brubl
1827 b. f. Bombarina, by Sober Robin...

1828 b. c. by Acorn .............. Ld Egremont

1829 b. c. Swagger, by Sober Robin...... Mr Bruhl

1851 b. c. Slang, by ditto........... Ld Egremont

1833 br. f. Sciatica, by Chateau Margaux. Mr Bruhl

Missed to Sober Robin in 1826, 50, and 52, slipped a filly by Hindoo in December 1855, and died in January 1854.

\section{BIRTIDAY,}

Bred by Mr Twasley, in 1822, got by Blucher, her dam, Belinda, by Beningbrough, out of Petrina, by Sir Peter.

1832 b. c. by Lory (died young) ........

1855 ch. c. by Bedlanite.............

1834 b. c. by Shakespear ............ Ir Haxell

1855 missed to Sir Benjamin..........

1836

by 4 grecable..............

She had no foal before 1852.

BIZARRE (sister to Finesse),

Bred by Lord Grosveror, in 1811, got by Perurian, her dam.

Violante, by John Bull, out of a sister to skyscraper, by Highflyer.

1815 b. f. by Waxy (dam of Larly Vane). . Mr Paync

1818 ch. f. Scarpa, by Crispin.......... Mr T. Thoruhill

1819 cl. f. Paradigm, by Partisan....... Mr J. Walkcl

1820 br. c. Bizarre, by Orville......... Ld C. H. Carendish

1822 br. f. Aline, by Woful...........

1825 ch. f. Nanine, by Selim (d. of Glaucus).

1824 br. f. Whimper, by Woful (put to the

stud) $\}$ Ld Lowther

1825 ch. f. by Tiresias. ..................
1826 ch. c. Bazaar, by ditto (sent to Vienna

in Dec. 1852) 
1828 b. f. Streamlet, by Tiresias......... Mr Wagstaff 1829 b. f. Whimsey, by Partisan.........
1850 b. f. by Truffle (. to Germany)..... Lowther 1851 b. c. Pencil, by Partisan.......... Ld Pembroke 1855 ch. c. Partner, by ditto.......... Col. Peel

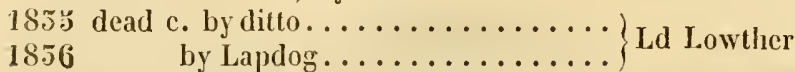
Barren in 1821 and 27, missed to Partisan in 52 and 54.

\section{BLACK DAPHNE,}

Bred by Col. Wilsor, in 1820, got by Juniper, her dam, Spotless, by Walton-Trumpator-IIighllyer-Otheothëa.

1828 br. c. Expectation, by Merlin....... Mr W. Chifney

$1899 \mathrm{ch}$. f. by ditto.............. Col. Wilson

1850 br. c. by Emilius.............. Mr Gardnor

1851 br. c. by Merlin ............. ל Col. Wilson

1852 br. c. by Filho da Puta..........

18.55 ch. c. by IIerchant............

1854 ch. c. by ditto............... Ld Berners

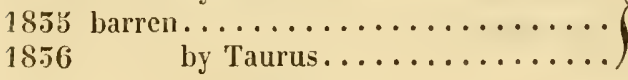

BLACKING,

Bred by Lord Esneuovt, in 1821, got by Octavius, out of Recruit's dam, by Teddy the Grinder-Gohanna-Catherine, by Woodpecker.

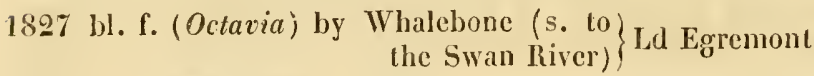

1828 br. c. Whalebone, by ditto........ Capt. G. Bulkeley

1829 bl. c. Dar and Martin, by ditto..... Ld Orford

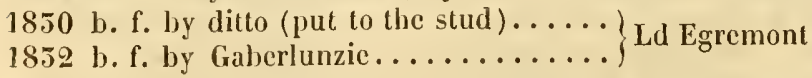

Missed in 1851, and sold to Baron Biel in 1852 with her foal, and stinted to Chateau Margaux.

\section{BLACKLOCK MARE,}

Bred by Mr Hıckes, in 1820, her dam by Orville, grandam by IIambletonian-Shuttle-0verton-Beningbrough's dam. 
1851 ch. f. Lady Canford, hy Catton (put to

the stud)

1855 b. f. Stay-sail, by Sailor (lialf-bred).. Mr Allies

1854 br. f. by ditto.

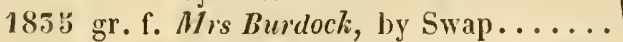

1836

by Safeguard.............

\section{BLACKLOCK MARE,}

Bred by Mr WATt, in 1821, her dam, Altisidora, by Dick Andrews, out of Mandane, by Pot8o's-Y. Camilla, by Woodpecker.

1827 ch. c. (Independent) by Jack Spigot..)

1828 ch. c. by Whisker................. IIr T. 0. Powlett

1829 ch. c. (Con rad by ditto..........

1851 b. f. by Jerry (died 2 days old).......

1855 b. c. by Whisker, or Cetus (s. to D. of

IIolstein) Sir Mark Wood

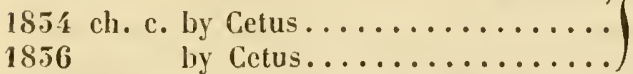

In 1850 missed to Chateau Margaux, in 52 to Partisan, in 55 to Cetus.

\section{BLACKLOCK MARE,}

Bred by Mr WArt, in 1824, her dam by Cerberus, out of Miss

Cranfield, by Sir Peter-Pegasus-Paymaster-Pomona, by

Ilerod.

1828 b. f. Varia, by Lottery, or Arbutus.)

1851 b. c. Wyndham, by Chateau Margaux. MIr Mlleu

$1855 \mathrm{br}$. c. by Hulatto (died young)......

In 1829 missed to Lottery, 30 to Humphrey Clinker, 32 to Grey Walton, and died in 185.5 .

\section{BLACKLOCK MARE,}

Bred by Mr Onbler, in 182:, her dam, Camerton's dam, hy Precipitate, out of Magnolia the Younger, by Pegasus - Young Magnolia, by Ilighflyer.

1827 f. by Grenadier (son of Blacklock). $1 S 29$ b. c. Joseph (afterwards Stainton) by
ditto 
1853 br. c. Wanderer, by Tramp........ Mr E. V. Fox

Not covered in 1829, missed to Lotlery in 1831 and 32 , and has since been sent abroad.

\section{BLACKLOCK MIARE,}

Bred by Mr Russeli, in 182y, lıer dam, Selina, by Delpini-Beningbrough-Highflyer.

1899 no produce....................

1850 ch. c. by Figaro (died a foal) .......

1851 br. c. by ditto ................

1852 gr. f. by Actæon.............. Hr Russell

1855 missed to Master Richard.........

1854 b. c. by Caccia Piatti............

1855 ch. f. by a country horse ......... by Dr Syntax...

\section{BLACKLOCK MARE (sister to Brownlock)}

Bred by Mr Richardson, in 1824, her dam, Diana, by Kill Devil, grandam by PotSo's, out of Maid-of-all-Work, by Hightlyer.

1829 b. c. Baylock, by Young Phantom...

1850 b. f. by ditto................

1831 b. c. by ditto (died a foal)......... Mr W. Richardson

1852 b. c. The Abbot of Melsea, by ditto, or Mr Best's Tinker)

Missed to Y. Phantom in 1833, and died in the spring of 1854 without produce.

BLACKLOCK MARE,

Bred by Mir Herwoon, in 1825, her dan, Marchesa, by Comus, out of The Colonel's dam, by Delpini-Tipple Cider, by King Fergus.

1851 b. f. by Jerry ( sent to Germany)....

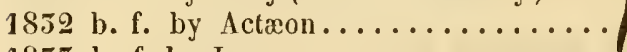

1853 b. f. by Jerry.............. Hr W. Hope

1834

1855

b. f.

1856

b. c. by

y Actieon

by Liverpool $\ldots \ldots \ldots \ldots \ldots$

\section{BLACKLOCK MARE,}

Bred by Mr Husbaxd, in 1826, her dam, Primette (Physician's (lam) by Prime Minister, out of Miss Paul, by Sir Paul. 
1852 b. c. by Borodino............ $\left\{\begin{array}{l}\text { Mr Walker, of } \\ \text { Huntington }\end{array}\right.$

1855 b. c. by ditto (died young)......... Mr Geo. Crompton

1854 missed to St Nicholas............ Mr Walker

$185: 5$ ch. c. Contentment, by Medoro..... IIr J. Briskham

1856 hy St Nicholas............ Mr Walker

\section{BLACKLOCK MARE,}

Bred by the Duke of Grafton, in 1898, her dam, Pope Joan, by Waxy, out of Prunella, by IIightlyer.

1852 bl. l. by Lamplighter (died in 1855 )...)

1855 br. c. by Taurus (died 2 days old).... D. of Grafton

1856 by St Patrick.............

In 1853 missed to Sultan, in 1854 to Shakspeare.

\section{BLACKLOCK MARE,}

Brel by Mr Gilbert Crompton, in 1822, her dam by Cerberus, out of Rosamond, by Buzzard.

1854 br. f. by Falcon............. Mr Hall

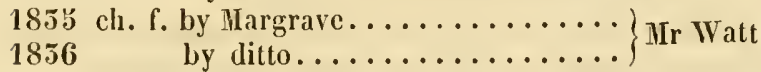

BLACK POLLY (sister to Schahriar)

Bred by Mr Haffevder, in 1895, got by Shuttle Pope, her dam, Dinarzade, by Selim-Princess, by Sir. Peter-DungannonTurf-IIerod-Golden Grove, by Blank-Widdrington mare, by l'artuer.

1828 br. f. by Manfred.............. Mr Maffenden 1829 br. c. Sluggard, by Bobadil (died in MIr Mills March 1855) $\}$ Mr Mills

1850 br. f. by ditto . . . . . . . . . . . Ir Haffenden

1851 b. f. Juliana, by Waxy Pope....... Mr Mills

1852 missed to ditto................ Mr Haffenden

185.5 br. f. Julia, by Trumpeter........ IIr Haffenden

1834 b. c. by Bobadil.............. Col. Pecl

$1855 \mathrm{br}$. c. by ditto $\ldots \ldots \ldots \ldots \ldots \ldots \ldots$
$1856 \quad$ by ditto $\ldots \ldots \ldots \ldots \ldots$

\section{BLUCHER MARE,}

Bred by Mr Urres, in 1822, lier dam, Jervinia, by Walton, out of Phantasmagoria, by l'recipitate. 
1851 br. c. by Doncaster (son of Blacklock) Mr Mott

1855 ch.f. by Anticipation. . . . . . . . . .

1855 br. f. by Doncaster............ Mr Norcop

1856 by Emancipation..........

Barren in 1852 and 54.

\section{BLUCHER MARE,}

Bred by Lord Grosvevor, in 1824, her dam, Opal, by Sir Peter, out of Olivia, by Justice-Cypher, by Squirrel.

1828 b. f. by Champignon (put to the stud). Mr Carey

1829 b.c. by Brutandorf (died at 2 yrs old). Mr S. L. Fox

1852 c. by Lottery (died young)........

1854 b. f. by ditto (dicd a foal)..........

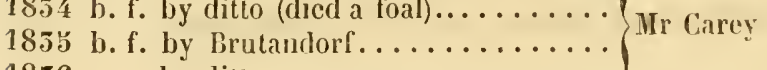

1856

by ditto ...............

Not covcred in 1829, missed to Lottery in 51 , to Brutandorf in 55.

\section{BLUCHER MARE,}

Bred by Mr LumLey, foaled in September 182:, her dam by Camillus, grandam by Gabriel-Pot8o's-Flyer, by Sweetbriar.

1829 gr. c. Falconbridge, by Falcon...... Ld Derby

1851 br. c. Emigrant, by Tramp......... Col. Cradock

1832 gr. c. Plantagenet, by Falcon...... Mr Lumley Savile

$185.5 \mathrm{ch}$. f. by Langar.............. Ld Chesterfield

1854 ch. c. by ditto ..............

185 . hr. f. by ditto .............. Mr F. Lumley Savile 1856

by ditto...............

\section{BLUCHER MARE,}

Bred lyy Sir Tatrox Sykes, in 1825, her dam, Leicester Lalss, by

Young Imperator-Alexander-Kiss my Lady, by Highflyer.

1852 b. c. by Chateau Margaux (died a foal)

185.5 b. c. by Y. Phantom
1854 b. c. by Comus....

185 b. f. by Comus..............

BLUF STOCKING, by John Bull (Vol. III, p. 41)

Yissed in 18.52, and was shot. 


\section{BLUE STUCKINGS,}

Pred by Gen. Grostevor, in 1816, got by Popinjay, her dan, Briseis, by Beningbrough-Lady Jane, by Sir Peter-Paulina, by Florizel-Captive, by Hatclien-Calliope, by Slouch.

1821 b. c. Virgilius (also called Y. Phanlom) by Phantom

1822 b. f. The Brownie, by ditto....... Gen. Grosvenor

1825 b. c. Pollio, by Orville.............
1825 br. c. Mocenas, by Waterloo......

1826 b. c. Augustus, by Gustavus........ Mr W. Chifney

$1828 \mathrm{gr}$. c. Sedlan, by ditto........... Ld Tavistock

1829 b. c. by Truffle.............. IIr Udny

1850 b. f. Blue Eyes, by ditto..........

1851 b. c. Dick, by Lamplighter (sent to

France in $0 \mathrm{cl} .1854)$

1852 b. f. Rushlight, by ditto.......... Gen. Grosvenor

1855 b. c. The professor, by Zingance....

1855 f. by Tantiry . . . . . . . . . .

Barren in 1824, 27, and 34 .

BOADICEA, by Alexander ( $\mathrm{T}$ ol. III, p. 42)

Had no foal after 1851, and is dead. Her br. f. by Camel (fualed in 1851) was called Rcyatta.

\section{IROBADILLA,}

Bred by Mr Harrexder, in 1823, got by Bobadil, her dam, l'ythoness, by Sorcerer, out of P'rincess, by Sir Peter-Dungannou.

1852 b. c. by Mameluke (died a foal)......

18.56

Missed to Camel in 1855 and 53.

\section{BOMBASINE,}

Bred by Lord Grostenor, in 1817, got by Thunderbolt, her dan, l)elta, by Alexanter-Isis, sister to Osiris, by Sir l'eler-Ibis, by Woodpecker-Isabella, by Eclipse. 
1822 b. f. by Sovereign.............

1823 b. f. Cachmere, by Blucher.........

1824 b. c. Gros de Naples, by ditto.......

1823 br. c. by Blacklock (died at 2 yrs old) Ld Grosienor

1826 b.c. Ebury, by Master Henry.......

1827 b. f. Tartan, by 'Tramp...........

1828 br. c. Damascus, by Whisker.......

1829 b. f. Barcelona, by Haster Henry....

1850 b. c. Bridegroom, by Filho da Puta...

1851 c. by Camel (dead)............

1852 missed to Peter Lely........... Ld Westminster

1855 b. c. by Teniers............... Ld Westminster

1854 br. f. by Pantaloon............

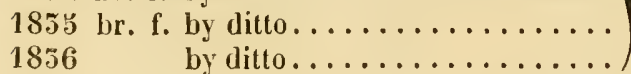

BONBY,

Bred by Mr C. Enpsox, in 1824, got by Mr Attwood's Chesnut Aralian, her dam by Pilgrim (son of Restless)-CorianderAlfred-Tuberose.

1851 ch. f. Procne, by his Grey Arabian.. 1852 ch. f. Charissa, by ditto (put to the stud)

1855 barren ...................... Mr Attwood

1854 bl. or br. f. by Mr Astley's Black Turk, or Barb

183 . ch. c. by Callisthenes............

\section{BONNY BESS (sister to Y. Corrector)}

Ired by Mr Sinpsov, in 1818, got by Corrector, her dam, Lady Abbess, by Cardinal York, out of Miss Nancy, by Beningbrough.

1826 b. c. Cock Robin, by Constable..... Mr Walker

1827 slipped foal, by ditto............

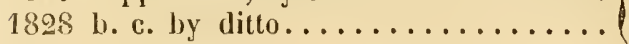

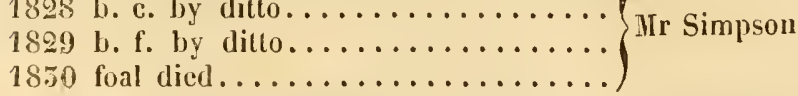

1851 br. f. by Y. Corrector........... Mr Scarisbrick

1852 b. f. by Peter Lely.............. Mr Scarisbrick 


\section{BOURBON MARE,}

Bred by Mr Stokes, in 1821, her dam, Tobosa, by Don (Quisutebungannon-Lady 'Teazle.

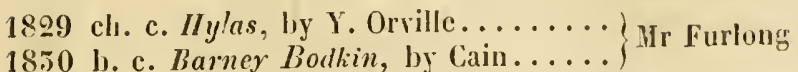

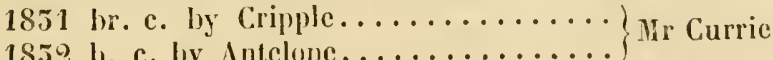

1852 b. c. by Antelepe... ........... Mr Andrew

185.4 b. c. by Doctor Faustus................. Mr Curric

18.56 by 1 intelope, or Wamba.....

\section{BRACELET,}

Bred by Lord Winaxcrifre, iu 1821, got by Filho da l'uta, hee dam, Bistirpa, by Cervantes (or Sir Charles) out of Fanny, by Sir Peter-Diomed-Deslemona, by Marske.

1828 br. c. by Ecarté................ Mr Badams

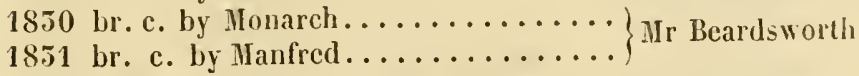

Missed to Monarch in 1852, and sent to Prussia.

\section{BR IVURA,}

Bred by Mr G. Dawson, in 1821, got by Outery, her dam, Irodigious, by Calcb Quotem-Fair Forester, by Alexander-sir Peter-Maid of Ely, by Tandem.

1827 ch. c. Braham, by Centaur........ Mr R. Pettit

1828 gr. f. by Eglinton............. Sir R. l. Dick

1899 ch. f. Fuga, by Partisan.......... Ir Pettit

$1850 \mathrm{gr}$. c. Allegro, by Jock..........

$1851 \mathrm{gr}$. C. Mcdrab, by the Ilumanich Sir R. K. Dick Arab

1852 b. f. Kate Kearney, by Benedict..... Mr Pettit

1855 b. f. by Emilius............... Sir S. Graham

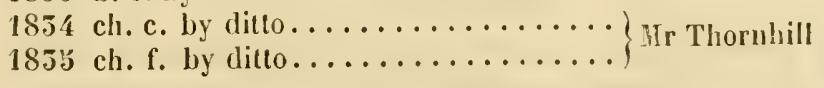

\section{BRAZII,}

Bred by Jord Stigo, in 1829, got by Iranhoe, out of Velret, loy Oiscau, ont of Wire, sister to Whalebone. VOL. IV. 
15.5.5 br. f. Lucy Komble, by Camel (or Mameluke)

185í ch. f. Linx. by Mamelulic.......... Col. White

$18.5 \%$ b. c. by Camel................

18.56

by $\operatorname{Logic} . \ldots \ldots \ldots \ldots \ldots$.

BRENDA,

Bred by Sir W. Wrxw, in 1894, got by Catton, her dam, Banshee, by Young Sorcerer, out of Podagra, by Gouty-Jet, by Hagnet.

1850 barren...................

1851 br. c. Cherubino (afterwards Chance)
by Figaro Mr Leicester

18.52

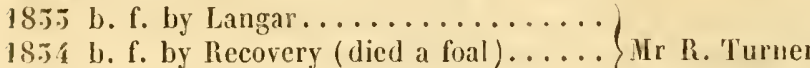

18.5:5 b. c. by Olympus..............

\section{BRILLANTE,}

Rired by Sir W. Maxwete, in 18פ0, got by Viscount, her dam, Brillante, by Whiskey-Dioned-Imperator-Othëothea.

1824 b. c. Sparkler, by Blacklock....... Gen. Grosvenor

1826 br. f. by Tiresias..............

1829 br. c. by Merlin.............

$1850 \mathrm{ch}$. f. by ditto (died in Germany).... Mr New ton

$18 \pi 2$

18.54

18.56

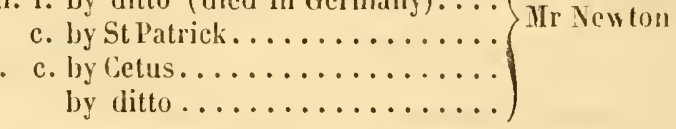

Jot covered in 1824, missed to Comus and Y. Juniper in $182 \pi$, to Woful in 28, to Lamplighter in 51 , and to Rereller in 35 ; barren also in 55.

\section{BRITANNIA,}

Bred by Mr Warserond, in 1815, got by Orville, her dam, Rovedino's dam, by Coriantler - sister to Fanny, by Weasel-Turk.

18 I b. f. Midge, by Milo (never ran).....

$1892 \mathrm{ch}$. f. by Ardrossan............. $\left.\begin{array}{r}1823 \text { br. f. (Rose) afterwards Fairy, by } \\ \text { Filho da Puta }\end{array}\right\}$ Sir T. Mostyn 
1827 cl. c. William Tell, by Swiss....... Col. Yates

1828 ch. f. by St Patrick............ Ld Verulam

1829 ch. c. Ocean, by Comus........... Mr Fox

1850 b. f. by ditto (put to the stud)...... Ld Orford

1852 br. f. by Waverley............. Mr S. L. Fox

1855 b. f. Miss Lora, by Lottery .........

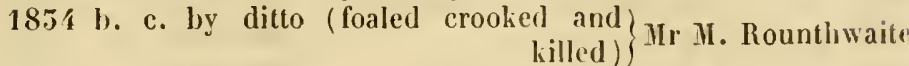

1853 b. c. Britannicus, by Ifumphrey Clinker D. of Leeds

1856 by Brutandorf............ Mr M. Rounthwaite

Missed to Teniers in 1824, 25, and 26, to Y. Whisker in 51.

\section{BROCARD,}

Ered by Lord Verubsu, in 1824, got by Whalebone, her dam. Varennes, by Selim, out of Canary-bird, by Whiskey, or Sorcerer

- Canary, by Coriander.

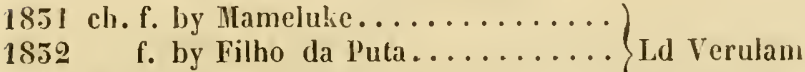

1855 b. f. by Emilius...............

1854 ch. c. Cordova, by The Colonel...... Mr Ridsdale

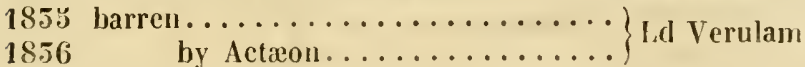

\section{BROIVN BETTY,}

Bred by Sir Charbes Krighrter, in 1814, got by Williamson's Ditto, her dam, Boadicea, by Alexander, out of Brunette.

1825 b. c. Sceptre (afterwards Second) by)

1824 b. f. by a half-bred horse.......... Mr Haywood

1823 b. c. by Master IIenry (dead).......

1826 b. c. Black \#ill, by Gainsborough. .

1827 br. f. by ditto (died 5 years old)..... Mir Langstun

1828 br. c. by ditto................

1829 br. c. Black Diamond, by ditto......

$1831 \mathrm{gr}$. c. by Buckfoot............. Mr Thornhill

1852 br. c. Lentulus, by Emilius.........

1855 b. c. by Fungus............ Nir Haywood

1854 dead $\mathrm{c}$. by ditto..............

Missed to Manfred in 1822, not covered in 1829. 


\section{BROWN DUCIIESS,}

Bred by his Royal Highness the Duke of Yonk, in 1816, got by

()rville, her dam, Sagana, by Sorcerer-Woodpecker - IIerod.

1821 b. c. by Aladdin............. D. of York

1822 b. . Lark, by Partisan........... Ld Huntingficld

1825 b. c. (Ingleborough) hy Mladdin..... Mr Nowell

1824 br. f. Toso, by Rainbow.......... Mr Irby

1826 b. f. (Y. Buchess) by Walton....... Mr Nowell

* 1897 b. f. Larly Birl, by Bustard (Castrel) Mr Payne

1829 br. f. Lady Fly, by ditto.......... Mr Dilly

18.50 b. c. Zone, by Loingwaist......... Mr Yales

1851 b. c. by ditto (sent to Constantinople).
1854 b. f. by dilto (s. to Virginia, 1855).. Mr Nowell

Barren in 1825, 28, 52, 55, 55, and 56 .

* Ladr Bind was sold to the Duc de Guiche in June 1854, with a !. by Camel, and covered by Cain.

\section{BRUNETTE,}

Bred by the Dulie of Graftox, in 1814, got by Waxy, her dam,

Charcoal, by Sir Peter-Dioned-Desdemona, by Marske.

1819 ch. f. by Osiris..............

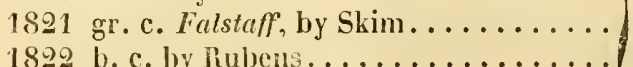

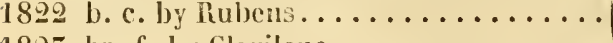

1825 br. f. hy Clavileno.............. Mr Molbrouk

1823 b. f. by Rubens Junior...........

1896 bl. f. by Comus..............

1828 bl. f. by Smolensko.............

1829 bl. c. The Black Giant, by ditto..... Mr Lawrence

1850 b. f. by Phantom............. Mr Holbrook

1851 b. f. Stockivell Reauty, by Mameluke. Ir Theobald

1852 b. c. by Camel, or Phantom.......

18.55 b. f. by Camel............... IIr Holbrook

$185: 5$ b. c. by Laurel..............

Barren in 1820, 24, 27, and $\overline{7} 4$.

\section{BRUNETTE,}

Got hy Marmion, her dam by Dragon, out of sister to Minimus, by Durgannon-Matchen.

1826 ch. f. by Y. Haphazard..........

1829 ॐ. c. by I. Filho (Miniature)....... Dr Willis

18.55 ro. f. by Hountebank...........

Barren in $1827,28,50,51,54$, and $5 \dot{s}$. 
BHUTANIOORF MARE,

Bred by Mr Honner, in 1829, lier dam Scancataldi, by Sancho, grandam, Miss Teazle-llornpipe, by Sir Peter.

1855 br. f. Miss Alice, by St Nicholas.... . Mr WV. Crompton
1854 b. c. Bostock, by ditto (died young).

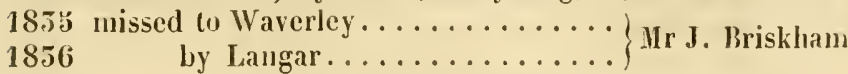

1856

\section{BUPTA,}

Bred by the Duke of Graftov, in 1824, got by Partisan, her dam,

Coquette, by Dick Andrews, out of Vanity, hy Ruzzard-Dahchick, by pot8o's.

1828 b. c. Zany, by Morisco........... Mr Peltit

1829 b. c. by Trufle................. Mr S. Stonehew er

1830 b. c. Blemish, by Camel.......... Mr Grove

1831 ch. c. by Emilius (dead).......... Mr S. Stonehewer

1852 ch. c. by ditto (dead)............ Ir Gireene

1853 b. c. Foozool, by ditto............ Mr Greville

1854 b. c. by Cetus (died a foal) ........

1855 barren.................. Mr Greene

1856 by Wiseacre $\ldots \ldots \ldots \ldots \ldots$.

Zany was sent to Germany, and ran there.

\section{BURTON LASS (sister to Toplass)}

Bred by Mr James IHLL, in 1811, got by Dick Andrews, her dam. sister to Repeater, by Trumpator-Demirep, by llighflyerBrim, by Squirrel-Ilelen, by Blank.

1818 b. c. Snowball, by Sir Paul. . . . . . . Ir Jrage

1825 b. c. Smuggler, by Tiresias ........ Mr Nevill

1824 ch. c. Tantot, by ditto........... Ir Platel

1825 ch. f. Mirza, by Pacha .......... Mr Treasham

1826 b. c. by Cannon-ball............

1827 clı. f. Toujours, by Smyrna........

1828 b. f. Consent, by Nolens Volens.....

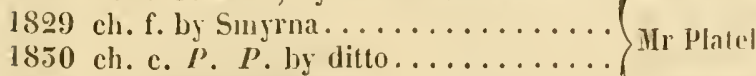

1851 ch. f. by Bedlamite............

1852 missed by The Flyer...........

18.5 .5 1. f. by Nolens Volens. . . . . . . . 


\section{BUSTARD MARE,}

Bred by Mr Woov, in 1819, got by Bustard (son of Buzzard) her dam, Harpalice, by Gohanna, out of Amazon, by DriverFractious, by Mercury.

1854 b. f. by Harry (son of Sir IIarry)..... )

1853 b. c. by Musquito (son of Master IIenry) Mr•P. Thonipson 1856 by ditto...............

\section{BUSTARD MARE,}

Bred by Mr Wigntwick, in 1822, got by Bustard (son of Castrel) her dam by Walton, out of Gipsy, by Guildford.

1828 ch. f. by Bobadil..............

1829 b. f. Lucy, by Cain .............

1950 missed to Edmund ............. Mr Bolton King

1851 ch. e. Tanworth, by Outlaw.......

1852 foal by ditto (died)............

1855 ch. f. Verandah, by Sir Gray.......

1834 ch. c. by Cain (died) ............. Mr E. Peel

183 . ch. c. by Bedlamite (dead)........

1856 by l'riam.

\section{BUSTARD MARE,}

Bred by Mr Bodevian, in 1824, got by the Castrel Bustard, her dam, Fillikins, by Gouty-King Fergus-Herod.

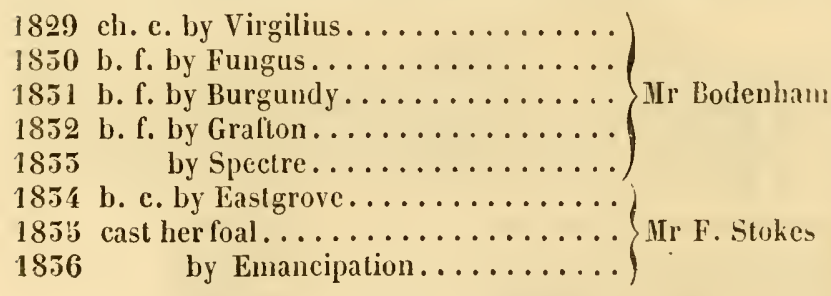

\section{BUSTALD MARE,}

Bred by Mr NoweLr, in 1827, got by the Castrel Bustard, her dam, Bequest, by Election, out of Legacy, by Beningbrough--Roxana, by Sir Peter.

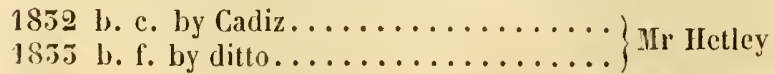

1854 br. f. by Defence............. Mr C. Finch

1853 ch. c. by ditto.............. Ifr ILetley 
BUSTLE,

Bred by Lord Egrenont, in 1827, got by Whalebouc, ther dam, The Odd Trick, by Quiz, out of Grey Duchess, by Pot8o'sDuchess, ly Herod.

1855 br. f. by Partisan.............

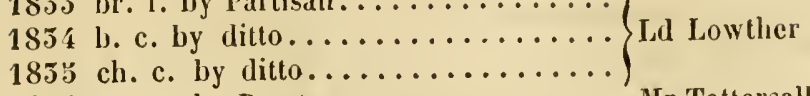

1856 by Rowton............ Mr Tattersall

\section{BUTTERFISY,}

Bred by Lord Jersey, in 1825, got by Magistrate, her dam, Filagree, by Soothsayer, out of $\mathrm{Wcb}$, sister to Whalebone, by Waxy - Penclope, by 'Trumpator.

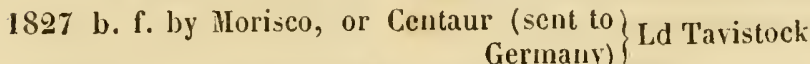

$1828 \mathrm{ch}$. f. (Zarina) by Middleton.......

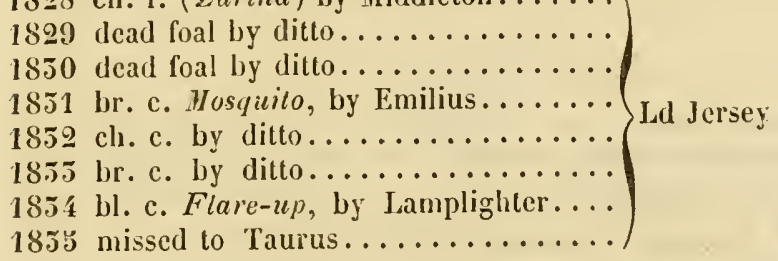

\section{CIIFACARATAD ADDERA,}

Bred by Mr T. O. Powletr, in 1816, got by Walton, her dam hy Pipator-Delpini-Tuberose, by Ilerod-Grey Starling, by Starling-Coughing Polly.

1821 ch. c. by Conius..............

1822 ch. f. by $\operatorname{Ardrossau} \ldots \ldots \ldots \ldots \ldots \ldots$

1825 ch. c. (Truth) by Catton..........

1824 b. c. Popsy, by Blacklock.............. Tr. O. l'owlett

1825 b. c. by Jack Spigot...........

1826 b. f. by Magistrate............

1828 lor. f. by Lottery, or Jack Spigot....

1829 b. f. by Figaro............... Nr IIotchkin

1850 b. c. ly Lottery .............. Mr Orde Powlett

18.51 by Jack Spigot...........

1855 l. f. by Olympus...................... Ir R. Turner

1854 ch. c. Portland, by Recorery........

Barren in 1827 and 1355. 


\section{4}

\section{CALAPEE,}

Bred by Sir Joun Cope, in 181 כ̌, got by 'Turtle, her dam, Roxana, by Alexander, out of Princess, by Eclipse.

1821 b. c. Flamock, by Vivaldi.......

1822 ch. f. by Anticipation..........

1825 ch. f. by Copenhagen ..........

$1823 \mathrm{gr}$. c. by Acupuncturer...........

1826 b. c. by Hercules (died 2 years old)..

1827 ch. e. by Snadon............. Sir J. Cope

1828 b. f. by Reveller..............

1829 gr. c. by Acupuncturer.........

1850 b. c. by Reveller............

1852 ch. c. by Cardinal Wolsey........

$1854 \mathrm{ch}$. f. by ditto ..............

$1855 \mathrm{ch}$. f. by ditto..............

Barren in 1824, 51, and 55 .

\section{CALEB QUOTEM MARE,}

Bred by Lord Montgonerie, in 1809, her dan, Scotia's dam, by

King Fergus-Cælia, by Herod-Proserpine, by Eclipse.

1825 f. by Anticipation (dead)......

1826 br. f. Prodigy (aft. MIanto) by Tiresias.

1827 b. c. Sketch-book, by Rubens.......

1828 b. f. Bobbinette, by Robin Adair (sent Mr Goddard to Jamaica in May 1855)

1852 b. f. by Emilius..............

In 1829 missed to Reveller, in 1851 to Bobatil, had no lual after 1852 , and was destroyed.

\section{CALEB OUO'TEM MARE,}

Bred by Mr G. Dawsor, in 1814, her dam by Weasel. grandam by MIfred, out of Wakefield Lady, by Lot.

1850 b. c. Ivanhoe, by Canteen........

1851 ch. c. Jerry, by Corinthian.......

1832 b. f. Iinkie Lass, ly Canteen...... D. of Buccleugh

1855 ch. 1. Trulla, lyy ditto..........

1853 b. c. by Scarbrough............

1 issed to Canteen in 1854 , and died in 1855 after foaling. 


\section{CALENDULA,}

Bred by Mr C. Dar, in 181\%, got by Camerton, her dam, Snowdrop, hy highland Fling-Daisy, by Buzzard - Tulip, by Damper.

1820 l. f. F'ewet, by Bustard (Castrel)....

1822 ch. c. Burgundy, by Usquebaugh...

182.5 b. c. by Anticipation............ IIr C. Ilay

1823 b. f. Manes, by Spectre.......... IIr C. Ilay

1826 b. f. Rubigo, by Rubens ..........
1827 b. f. Jexess, by Moses..........

1829 1. c. Gambol, by Bobadil. ........ IIr Mott

18.31 b. c. by Godolphin.............

1832 f. by Bobadil............. Mr C. Day

1853 ch.c. Protector, by Defence.......

Not covered i'l 1820, missed to Anticipation in 1824, to Swap in 1828 , to Luzhorough in 1850 , and died in 1853 in foal to Fungus.

* Rubigo had no living produce to 1855 inclusive, and was covered that year by Defence.

\section{CALISTA,}

Bred by Mr Rosrsos, in 1828, got by St Patrick, her dam ly

Smolensko, grandam by Sir Peter-Mambrino-Marigold.

1852 f. liy Jangar (died young) ......

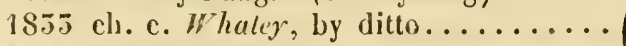

1854 not covered last jear.................. Mr Jobinsou

$183:$ b. c. by Humphrey Clinker.........

18.56 by Langar..............

\section{CALIPSO,}

Bred by Sir Jonv Shelcer, in 1816, got by Sorcerer, her dam, Houghton Lass, by Sir Peter-Alexina, by King Fergus- Lardella, by Y. Marske-Cade-Beaufremont's dan, by brother to Fearnought-Miss Wyndham, by Wyndham-Belgrade Turk.

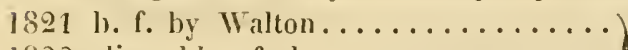

1822 slipped her foal..................
1893 br. c. The Dragon, by Cervantes ( sent Hr Stuart Wortley

to ludia in 1829)

182 if br. f. (The Gimmer) by Filho da Pula.

1825 b. c. by Whisker.............

1826 b. f. by ditto.............. Ld Wharocliffe

1827 b. f. by Cervantes (sent to Florence in 
1828 ๖. I. Peachick, by Whisker....... Mr Peacock

I829 c. by Whisker............. Ir Forth

1850 dead c. by IIuley.............. Mr Nowell

1852 b. c. Conservatice, by Longwaist.... Mr Lushington

1853 br. f. Scramble, by ditto......... Mr Buckley

1854 br. c. loy Inuley ..............

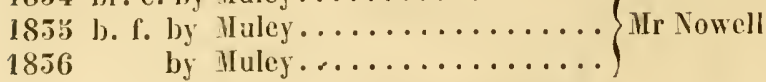

Barren in 1831.

\section{CAMELINA (sister to Camel)}

Bred by Lord Egreuont, in 1825, got by Whalebone, her dam

by Selim, out of Maiden, by Sir Peter-Phænomenon-Matron.

by Florizel-Maiden, by Matchem.

1828 b. c. by Bustard, or Aladdin....... Mr Nowell

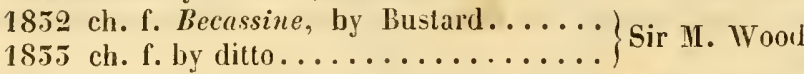

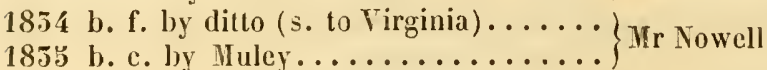

She had no foal that lived between 1828 and 1852 .

\section{CAMILLA,}

Bred by Lord Fitzwiluiar, in 1815, got by Camillus, her dam.

Paulina, sister to Sir Paul, by Sir Peter-Pewet, by Tandem.

1818 gr. c. The Baron, by Cervantes.....
1820 gr. c. by ditto (died a yearling)..... Fd Fitzwilliam

1821 lor. c. Edward, by Comus......... Sir J. Byng

1824 gr. f. Tittle Tatile, by Blacklock.... Ld Muncaster

1825 gr. c. The Abbot, by Oiseau....... Mr G. Peters

1826 b. c. Fortune-teller, by Tramp..... Sir J. Shelley

1827 ch. c. by Sam............... Mr Thornhill

1828 Jr. c. Harcus, by Emilius......... Ld Cleveland

1850 cl. c. Iy ditto ................ Jd Chesterfield

$1851 \mathrm{gr}$. f. Eloisa, by ditto (put to the stud))

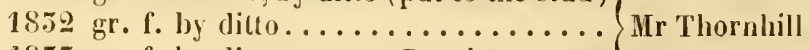

$1855 \mathrm{gr}$. f. by ditto (sent to Russia)...... . . . . Hr Thornhill

Missed to Cervantes in 1819 and 25 , to Emilius in 1829 and 54 .

barren also in 1822, and died in 1855 in foaling.

\section{CAMILLINA,}

Bred by Mr Potter, in 1822, got by Canillus, her dam by Smolensko, out of lliss Cannon, lyy Orrille--Weathercock-Cora. by Matchem. 
1828 gr. f. Galopade, by Catton......... Col. hing 1829 b. c. (Pilot) by Catton........... Mr Uppleby 1850 gr. c. Schemer, by Lottery........ Ld Derby

She was not covered in 1850 , had no produce that lived to 18.55 inclusive, when she was put to a half-bred horse.

The CAMILLUS MARE (sister to Orphan, Vol. III, p. :36)

Was barren in 1852 and 55, and turned out of Sir Tatrox Sries's stud the following year.

\section{CAMILLUS IIARE,}

Bred by Messrs Srkes and Hall, in 1811, her dam by GabrielI'ot 80 's-Flyer.

1824 b. f. by Cervantes ( put to the stud).. Sir Tatton Sykes

1823 br. f. by Blucher (foaled in September) Mr Lunley

1827 ch. l. Camilla, by Tramp......... Mr Sadler

1829 b. c. Bedouin, by ditto.......... Ir Cookes

1855 ch. c. Patriot, by Langar.......... Col. Cradock

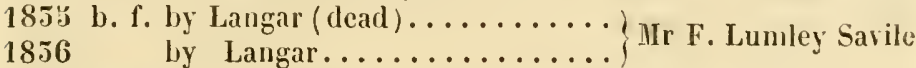

She slipped foal in 1828 and 1850 , missed to Tranı in 1851 , to Langar in 1852 , and was barren also in 1834 .

\section{CAMILLUS HARE (chesmut)}

Bred by Mr Drvconee, in 1815 , her dam by Precipitate, out of Hendoza's dam, by Paymaster-P'omona, by IIerod.

1894 c. by Cervantes (died a foal)...... Mr Kinapton

1827 ch. f. (Fanny) by Whisker (put to the )

$$
\left.\begin{array}{c}
\text { to the } \\
\text { stud) }
\end{array}\right\} \text { Mr lidisdale }
$$

In 1820, 1821, and 1892, had foals by common horses, in 182.; missed to Amadis, in 1826 to Tramp, in 1828 slipped twins by II hisker, in 1829 and 1850 missed to Peter Lely, 1851 to a common horse, had no foal afterwards, and died about the spring of 1853 .

\section{CANILLUS MARE (sister to Claudius)}

Bred by Mr Tartox Srkes, in 1818, her dam by Sancho, grandam by Ilighllyer, ont of Junn, by Spectator. 
1826 br. f. by $\|$ avertey . . . . . . . . . . )

1828 gr. f. by Lottery............. Sir Tatton Sykes

1851 ch. c. by Comus................

1853 gr. c. by ditto ................

In 1827 missed to Dinmont, $1829,50,55$, and 54 to Young Phantom, and in 1852 to Velocipede.

\section{CAHILLUS MARE (sister to Speaker)}

Bred by Hir Tatron Srkes, in 18ı7, her dam, sister to Prime Miinister, by Sancho, out of Miss Ifornpipe Teazle, by Sir Peter.

1827 b. c. by Dinmont..............

1828 produce died................

1829 b. c. Sledmere, by Young Phantom. .

1850 b. c. by ditto.................

18.51 ch. f. by Comus.............. Sir Tatton Sykes

1832 br. f. by Young Phaniom..........

1853 b. f. by ditto ...............

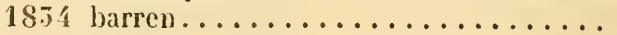

185: ch. e by Comus..............

\section{CAIIILLUS MARE (}

Bred by Sir Mark Sykes, in 1820, her dam, Lady Rachel, ,

Stamford, out of Y. Rachel, by Voluntecr-Rachel, by lighflyer.

1850 b. f. by Young Phantom...........

1851 ch. f. by ditto, or Mr Best's Tiuker. .

1852 b. c. by Young Thantom.......... Sir Tatton Sykes

$1853 \mathrm{gr}$. or ch. c. by Comus............

Slipped foal in 1855 and 54.

\section{CANNON BALL MARE.}

Bred hy , in 1825, her dam, Miss Hap, by Shuttle, ont of sister to Haphazard, by Sir Peter-Miss IIervey, by Eclipse- I oung Cade.

1899 c. by Champignon...........

1852 b. f. Duxernay, by Rossini........
1855 b. f. by Swap................. Hr R. Harris 
She had no produce that lived in 1850 or 51 , and missed to Swap in 1854.

The CANOPUS MARE, dam of Lapdog and Spaniel (Vol. III, pp. 59,60$)$ died in 1855 . She had a filly in 1852, which died young. Spaniel died in 1855.

\section{CANOPUS MARE (Miss Craven)}

Bred by Lord Egremont, in 1814, her dam, Fraetious, by Mereury-Woodpecker, etc.

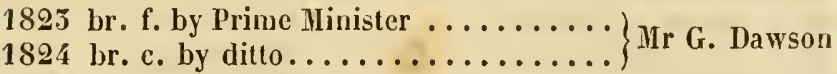

1897 b. c. Glenalmond, by Stratherne..... Ld Lynedoch

1829 gr. c. by Oulcry ..............

1850 b.f.

dead $e$.$\} by Champignon .........$

1851 b. c. by Cleveland...............

1852 b. f. by Percy ................ Mr G. Dawson

1855 b. e. by Percy................

1854 b. c. by ditto.................

1855 slipped foal by Telegraph .........

1856 by Perey $\ldots \ldots \ldots \ldots \ldots \ldots$.

Missed to Prime Minister in 1825 and 26.

\section{CANOPUS MARE,}

Bred by Mr Brereton, in 1814, her dam by Teddy the GrinderPrecipitate-Pumpkin-Flea-cateher, by Goldfinder.

1824 b. f. Luna, by Wanderer......... Mr Gardnor 1823 gr. e. by Little John............ Ld Egremont 1826 el. f. by ditto............... Mr Jas. Moon $18 \Omega 8$ gr. c. by ditto............... Ld Lynedoch

1829 b. c. Tarquin, by Moses ......... Mr Gardnor

1850 ch. f. by Tarrare...............

1851 ch. f. by Skim............... Mr Jas. Moon

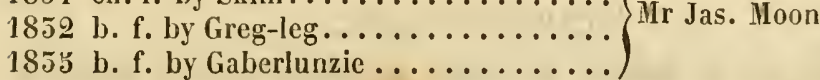

Barren in 1827 and 34 , slipped foal in 1855 , and died in 1853 shortly after foaling. 


\section{CANOPY,}

Hreil by Sir (i. Hesticute, in 1826, got by Blacklock, her dam, Corna, by Waverley, out of Margarelta, by Sir Peter.

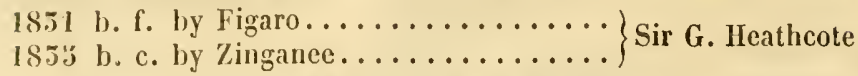

In 1852 missed to Mameluke, 55 to Cydnus, 34 to Lottery.

\section{CANTALOUPE,}

lired by Lord G. II. CavernisH, in 1S18, got by Soothsayer, her dam, sister to Whalebone, by Waxy, out of Penelope, by Trumpator-Prunella, by Highflyer.

1826 b. c. by Robin Hood........... Mr Ingram

1828 ch. c. by ditto .................

1852 br. c. by Scipio (Filho)......... Mr Burgess

185.5 b. c. by ditto.

18.54 b. c. by ditto

Hissed to Phantom in 1829 , to Scipio in 55 , not corered in 50 .

\section{YOUNG CAPRICE,}

Bred by the Duke of Graftor, in 1817, got by Waxy, her dam, Caprice, by Walton - Vanity, by Buzzard - Dabchick, by Pot8o's.

1825 missed to Rasping...........

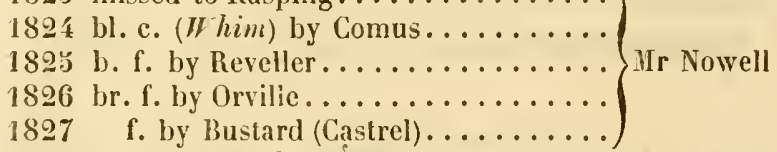

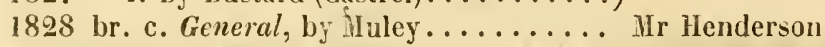

1829 b. f. by IIuley ................ Sir J. Gerard

18.50 b. f. by ditto............. Mr Dunkley

1851 b. f. The Jilt, by ditto.......... Mr Tobin

1852 slipped foal by ditto............

1855 b. f. by ditto (s. to Mr Corbin, of

Virginia) Mr Nowell

1854 b. f. by ditto................

18.55 b. c. by Longwaist ............

\section{CAPSICUM MARE,}

Bred by Mr R. IIunsos, in 1825, her dam, Acklam Lass, by Prime Hinister, out of Young Harrict, by Camillus-llarriet, by i'recipitate. 


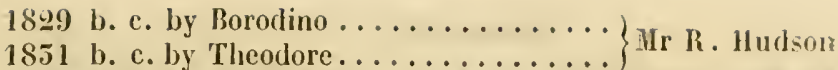

1855 b. f. by St Nicholas.............

185 4 b. f. by Ilumphrey Clinker..........

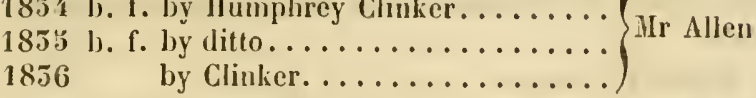

In 1828 missed to Arbutus, in 1850 to Y. Phantom.

\section{CAROLINE (a bay mare)}

Foaled in 1808, got by Trombone, her dam by Sir Peter.

1815 dead twins.

1816 b. f. Y. Caroline, by Orville........

1817

f. by ditto

1818

c. by Walton

1819

1821

c. by Phantom (died).

1822 ch. f. My Lady, by Anacreon...... .

1825 br. f. by Vandyke Junior.

1825 br. f. Sherry, by Pendulum.......

1826 bl. f. Parfaite, by Eryx..........

1827 b. f. by Eryx (died)

\section{CAROLINE,}

Bred by Lord Egrenorr, in 1817, got by Whalebone, her dam.

Marianne, by Mufti-Maria, by Telamachus- $\Lambda$-la-Grecque, by

Regulus.

1824 b. f. (Isabel) by Wanderer ......... 1826 br. f. by ditto (sent to Sweden in June 1851 , with a filly foal by Partisan)

1827 br. f. (Colly, afterwards Annie) by ditto 1828 gr. c. by Shim............... Ld Egremont

1851 b. f. (Stim Milk) by Little John, or Skim (foaled April 9)

Barren in 1825 , produced a lead foal in 1825, missed to skim in 1829 , to Sober Robin in 1850, to Greyleg in 1832, to Chatean Margaux and Gaberlunzie in 1855, and to Gaberlunzie in $185:$. - N.B. She was covered by Little Jolın for the last time February 2:5, 1850, and by Skim on the 14th of $\Lambda$ pril. 


\section{CAROLINE,}

Bred by Mr Orusby Gore, in 1826, got by Filho da Puta, her dam, Lady Caroline, by Partisan, out of sister to Orange-flower, by Trumpator.

1851 ch. c. Deceit, by Langar...........

1855 b. f. by ditto................ Mr Ormsby Gore

1856 by Zinganee.............

Barren in 1852,54 , and 53.

\section{CASTRELLINA,}

Bred by Mr L. Charltow, in 1893, got by Castrel, her dam by

Waxy, out of Bizarre, by Peruvian-Violante, by John Bullsister to Skyscraper, by Highflyer-Everlasting, by Eclipse.

1826 b. c. The Colonel, by Abjer........ Mr Martindale

1828 br. c. The Saddler, by Waverley.....

1829 br. c. Esculapizus, by Lottery ........

1832 b. f. Violante, by Waverley (put to the

1835 b. c. The Bard, by ditto........... Iir Skipsey

1854 b. f. The Cuskoo, by ditto.........

1853 b. f. The Little Bird, by ditto.......
1856 by Physician.............

Missed to Grey Walton in 1827, to Lottery in 1851 ; not covered in 1829.

\section{CATALANI,}

Bred by Mr Beaunont, in 1827, got by Tiger (son of Sir Paul) out of IVilna, by Smolensko-Horgiana, by Coriander-Fairy by Highflyer.

1853 br. f. Meg Merrilies, by Waverley...

1854 she was trained...................

1833 b. e. Catterick, by Bob Logic (late Col. Cookson

1856 Werner)

\section{CATGUT,}

Bred by the Duke of Graftox, in 1816, got by Comus, ro Juniper, her dam, Vanity, by Buzzard-Dalschick, by Pot8o's-Drab, by Highflycr-IIebe, by Chrysolite. 
1822 b. c. Cramer, by Election.........

1825 ch. f. Cremona, by Partisin........

1824 b. f. Crotchet, by ditto (s. to France) - D. of Graftou

1825 b. f. (Fiddlestring) by Woful.......

*1826 b. f. Caradori, by Centaur.........

1827

by ditto..................

1850 bl. c. Juba, by Jerry ............

1852 b. c. Caliph, by Lottery............ Mr S. L. Fox

1855 br. c. Curioni, by Waverley........

1856 by ditto................

In 1828 and 29 missed or slipped foal ; barren in 1834 and 5.5.

* Caradorı had a b. f. by Shakspeare in 1855 , her first foal.

\section{CATIIERINA,}

Bred by Mr lioulnsworti, in 1819, got by Walton, her dam,

Catherine, by Castrel-Alexander-Ivy, by Woodpecher.

1826 b. f. by Swap ............... IIr E. Peel

1828 br. c. Changeling, by ditto, or Paulowitz Mr Davis

1829 b. f. by Paulowitz............. Mr Wyatt

1850 ch. f. Frensy, by Bedlamite........ Mr E. Pcel

1851 ch. f. Frantic, by ditto ...........

1852 b. c. by Filho da Puta (dead) ........

185.3 b. c. Cadet, by ditto ............ Mr Lacey

1854 b. c. by ditto .................

1853 ch. c. by ditto, or Vanish .........

\section{CATO MARE,}

Bred by Lord Stawel., in 1819, her dam, Omphale, sister to

Blucher, by Waxy-Pantina, by Buzzard-Trenthan--sister (1)

Drone, by Herod.

1824 ch. f. by Waxlight ............ Ir E. F. Heynell

$182 \%$ b. f. by Walton ...............

1826 b. f. by Orville ...............

1827 b. f. (Orpheline) by ditto.......... Ir Nowell

1828 b. c. by Mulcy................

1829 b. f. by ditto...............

1850 br. c. (Fredericli) by dilto......... Mr J. Smith

1855 f. by Bustard................ W Mr Nowell

In 1851 missed to Huley, in 52 to Bustard. 


\section{CATTON MIARE,}

Bred by Sir W. Munver, in 1818, her dam, Hannah, by Sorcerer, out of Amelia, by IIighflyer-Miss Timms, by Matchem.

1829 ch. c. (Justice) by Magistrate ....... Ld Scarbrough

$1825 \mathrm{ch}$. f. (Edith) by ditto (s. to Hungary). Mr Davis

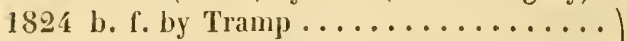

1826 br. f. by Waverley $\ldots \ldots \ldots \ldots \ldots$

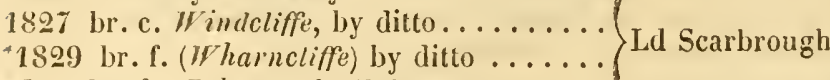

1850 br. f. (Falconet) by Falcon .........

$1851 \mathrm{br}$. or gr. f. by ditto ............

In 1825 missed to Tramp, in 23 to Waverley, in 52 to Langar, and sent to America that year, covered by Waverley.

* Wuarcliffe was sent to Charleston, in the United States, in the summer of 1855 , covercel by Tramp.

\section{CATTON MARE,}

Bred by Mr Moss, in 1821, her dam by Sancho, out of Blachloch's dam, by Coriander.

1824 ch. f. by Alexander (Don Cossack) ... Mr Armstrong

1826 br. c. Kilnocky, by Columbus....... Mr Bogue

1828 b. c. hy Lottery (died young) .......

1899 b. f. by ditto (died a foal) .........

1831 b. f. by ditto................ Ir Moss

1852 b. c. by ditto........................

1854

$185:$

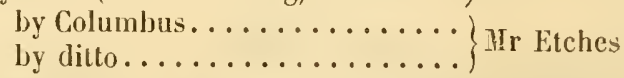

In 1827 missed to Figaro, in 1850 to Lottery.

\section{CATTON MARE (sister to Coronation)}

Bred by Mr S. Kıxa, in 1822, her dain by paynator, out of Violet, by Shark.

$1 \$ 27$ ch. f. (Taglioni) by Whisker........ Mr Macnamara 1898 b. c. Dictuotr, by Antelope (son of )

${ }^{*} 1899$ b. f. Chamois, by ditto.......... Mr Perren

1850 c. by Sprite (son of Overseer)..... . Mr Macnamara

$18 \pi 1$ b. c. Jonathan Bradfurd, by Tamworth Mr Mew

- Casmors produced a f. by Emilianus in 185/, and was sent to Belgium. 
1852 foal by a half-bred horse.........

1835 br. c. Sir The, by Theodore.......

1854 barren........................ E. l'eel

1855 ch. c. by Bedlamite...........

\section{CATTON MARE,}

Bred by Mr W WTT, in 1825, her dam, Altisidora, by Dick Andrews, out of Mandane, by Pot8o's.

1850 b. f. by Blacklock............. 1851 b. c. by Figaro ............. Dennison

1852 ch. f. by Velocipede............ IIr Shard

1854 ch. f. Magic, by Reveller........... IIr Shard

IIad dead twins in 1828 ; barren in 1829,55 , and 55 .

\section{CATTON MARE (sister to Countess)}

Bred by lord Lovdoverrry, in 1824, her dam by Hambletonian -Shutlle-Drone.

1828 b. f. Fancy, by Osmond..........

1829 ch. f. by Ben Ledi..............

1831 ch. 1 . by Lottery (dead)........... Ir Blakelock

18.35 bl. c. The Blach Diamand, by Jerry...

1855 ch. f. by Actæon ..............

Barren in 1850, 52, and 54 ; not covered in 5.5.

\section{CATTON MARE (sister to Tarrare)}

Bred by Lord Scarerocgin, in 1824, her dam, Henrietla, by Sir

Solomon, out of sister to Olive, by Woodpecker-Trentham.

$1829 \mathrm{gr}$. c. (Uncle Bob) by Falcon........ Ld Scarbrough

1852 b. c. Fergus, by Waverley (sent to)

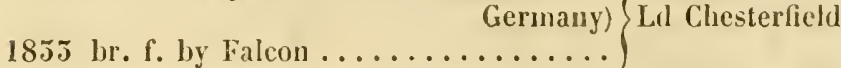

Barren in 1850 and 51 , and died in $185 j$, ten days after foaling.

\section{CATTON MARE,}

Bred by Lord Exeter, in 1828, her dam, Dulcinea, hy Cervantes. out of Regina, by Moorcoch.

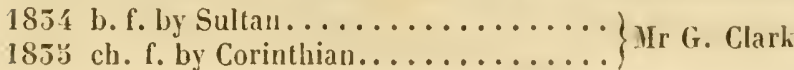


CENTAUR MARE,

Bred by Mr Batson, in 1826, her dam, Cecilia, by Worthy, out of

Miss Furey, by 'Trumpator-Mark Anthony-Signora.

1851 b. с. Thump, by Hal...........

1854 b. c. by Scipio .............. Mr Burgess

1855 b. f. by llal.................

Not covered in the intermediate years.

CERBERUS MARE,

Bred by Mr Best, in 1813, her dam, Merlin's dam, by Delpini-

Tipple Cyder, by King Fergus-Sylvia, by Y. Marske.

1819 br. f. by Comus................. Mr J. Perren

1821 b. c. (Guy Faux) by Welbeck. . . . . . Mr Jackson

1822 b. f. by Catton (dead) ...........

1823 b. f. by Ardrossan.............

1824 b. f. Rosalis, by Blacklock ........ Mr Hall

1825 b. f. by Macduff. . . . . . . . . . .

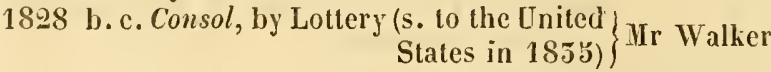

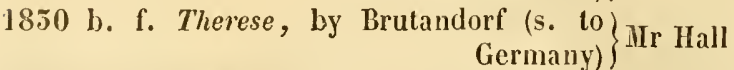

1852 ch, f. Gipsy, by Best's Tinker (s. to $\left.\begin{array}{r}\text { Germany } 1855)\end{array}\right\}$ Ld Lichfield

1855 ch. f. by Hargrave............ Mr Hall

Not covered in 1819 , and had no foal in $1826,27,29,51,55$, and 34 .

\section{CERBERUS MARE,}

Bred by Mr WAtr, in 1817 or 1818, her dam, Alfana, by Dick Andrews-Saİtram-Matchem-Regulus.

1822 b. c. Cock Robin, by Blacklock..... Mr Ridsdale

1821 br. c. by ditto............... Mr Bookless

1826 b. c. Sparrow, by Waverley........

1828 cl. f. Ringdove, by Comus.........

1829 cls. c. Musician, by ditto.........

1850 b.f. Emma (aft. Jenny Wren) by ditto. Hr Ridsdale

* 1831 br. f. by Figaro..............

1832 b. l'. by Velocipede (sent to Germany).

1854 b. c. by Tramp (foaled in Germany)...)

Missed to Tramp in 1825, to Waverley in 1827, to Brutandorf in

1855 ; was not covered in 1824, and sent to Germany in 1835.

* The Figaro mare produced a b. c. by St Nicholas in 1855, and wis covered by Mulatto. 


\section{CERBERUS MARE,}

Bred by Mr Cromptos, in 1818, her dam, Rosamond, by Buzzard - Roseberry, by Phœnomenon - Miss West, by Matchem.

1822 b. f. by Blacklock............. Mr Crompton

1823 ch. f. Amelia, by Amadis.......... Mr Russell

1824 b. c. Burlador, by Cerrautes.......

1825 b. f. by Blacklock..............

1826 b. f. by Waverley.............

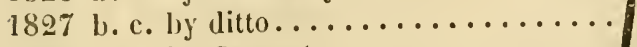

1828 b. c. by Cervantes................

${ }^{*} 1829$ ch. f. Beauty, by Blacklock........

1850 b. c. Stinker, by IIumphrey Clinker.. Mr G. Crompton

1851 ch. f. by Comus (s. abroad) ..........

1852 ch. f. hy Velocipede............

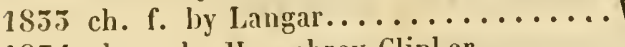

1854 ch.. c. by ILumphrey Clinker........

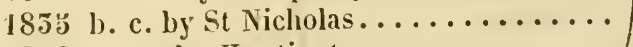

1856 by Huntington............

* Beaurr produced a ch. c. by Humphrey Clinker in 1835 , and was covered by Iluntingion.

\section{CERBERUS MARE (sister to Duport)}

Bred by Mr WatT, in 1820, lier dam, Miss Cranficld, by Sir Peter -Pegasus-Paymaster-Pomona, by Herod.

1824 ch. f. by Blacklock...............

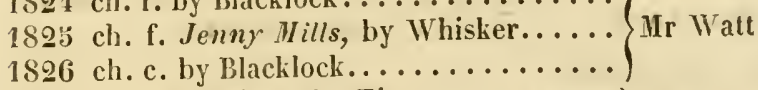

1827 b. f. Hermione, by Figaro.........

1829 b. c. Ilercules, by ditto.......... Mr W. Richardson

1851 b. f. Ladly de Gros, by Y. Phantom...

1852 cl. c. IIornsea, by Velocipede......

1853 b. c. Weighton, by Brutandorf...... Ld Chesterficld

1834 b. f. Lady Agnes, by Y. Phantom....

1855 br. c. Albemarle, by ditto......... Mr W. Richardson

1856 by Brutandorf............

In 1828 missed to Lottery, 50 to Y. Phantom.

\section{CERBERLS MLRE,}

Bred by Mr W. Richarinsor, in 1820, lser dam, Diana, by Kill Devil, grandam by Pot8o's, out of Maid-of-all-W'ork. 


\section{8}

1827 b. c. (died a foal) by Grenadier (son of

Blacklock)

1828 ch. f. by ditto ...............

1829 b. f. May-duy, by Blacklock.......

1850 ch. c. Theodore, by Comus.........

1851 ch. f. Lady Brownlock, by Blacklock.. Mr W. Richardson

18.52 b. f. Diana, by Velocipede.........

1855 b. с. Meaux, by Y. Phantom.......

1854 b. c. loy ditto .................

1853 barren $\ldots \ldots \ldots \ldots \ldots \ldots \ldots \ldots$

1856

by $\operatorname{Priam} . . . \ldots \ldots \ldots \ldots \ldots$

\section{CERESINA,}

Bred by Mr WiLEr, in 1826, got by Blacklock, out of Clarendon's dam, by Wortly-Tiny, by Sir Peter.

${ }^{*} 1851$ br. f. Pomona, by Lottery.........

1852 missed to Chateau Margaux.........

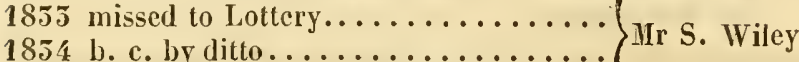

185 bl. f. Comely, by Waverley .........

1856 by Starch................

* Posiona produced in 1855 a ch.f. by Margrave, and was covered by Mulatto.

\section{CERVANTES MARE,}

Bred by Mr Funtian, in 1816, her dam by The Cryer-Mambrino-Cricket, by Herod-Sophia, by Blank.

1824 ch. f. by Cannon-ball.............

1826 ch. f. Jule, by Sinyrna.............

1827 ch. c. by ditto (dead)..............

1828 b. c. by Nolens Volens, or The Flyer

18.51 ch. c. by Pacha....................

(dead) Mr Platel

18.52 b. f. by Amadis, or Sandbeck.......

$1855 \mathrm{ch}$. f. by Nolens Volens...........

Missed to Smyrna in 1829 and 50 , and is dead.

\section{CERVANTES MARE,}

Bred by Mr Haffenden, in 1819, her dam, Miss Brocket, by Sir Peter-Spaniard's dam, by Alfred-ILerod-Engineer-Bay Malton's dam. 
1825 b. f. by Shuttle Pope............

1824 ch. e. Tom Tit, by Waxy Pope..... Ir IIaffenden 1825 ch. c. by Bobadil (died young).......

1826 br. e. by Shuttle Pope (died a yearling)

1827 ch. c. Captain Arthur, by Bobadil... Ld Sefton

1828 ch. c. Caplain Bob, by ditto........ Mr Giffard

1829 ch. c. Copper C'aptain, by ditto..... Ld Worcester

1855 ch. f. by ditto (s. to Spain)......... Mr Haffenden

1854 b. c. by Trumpeter.............. Col. Peel

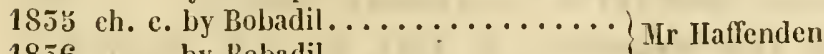

1856

by Bobadil.

In 1850 missed to Bobadil, 51 to Waxy Pope, 52 to both.

Captain Artuur was sent to Hungary in 185̃2, and Coppro Captain to France in Oclober 1834.

\section{CERVANTES MLARE,}

Bred by Mr Garforti, in 1925, her dam, Marciana, by Stamford, out of Marcia, by Coriander.

1831 b. c. by Lottery (died a foal)........ IIr Garforth

1852 gr. f. Gypsiana, by Tramp...........

1853 ch. c. by Tramp (dead) ........... IIr Ridsdale

1834 b. c. Rienzi, by Tramp...........

1855 missed to Tramp and Brutandorf..... IIr Foljambe

\section{CERVANTES MIARE,}

Bred by Sir Tatron Sikes, in 1824, her dam by CamillusGabricl-PotSo's-Flyer, by Sweetbriar.

1852 b. f. by Y. Phantom............

1835 b. c. by ditto............... Sir Tatton Sykes

1834 ch. f. by Comus...............

1855 ch. f. by Comus...............

\section{CERTANTES MARE,}

Bred by Sir Tatrox Srkes, in 1825, her dam, Emma, by Don Cossack, out of Vesta, by Delpini.

1852 br. f. by Chateau Margaux ........

1855 missed to Y. Phantom........... Sir Tatton Sykes

1854 ch. f. by Comus..............

1855 ch. c. by ditto.............. 


\section{CERVANTES MARE,}

Bred by Mr S. ReED, in 1825, her dam by Golumpus-Paynator - sister to Zodiac, by St George-Abigail, by Woodpecker.

1834 br. c. by IIumphrey Clinker........

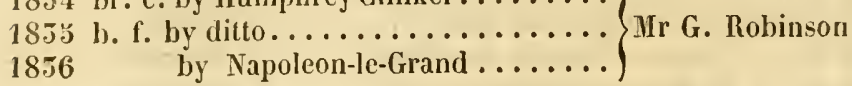

\section{CERVANTES MARE (sister to Crusader)}

Bred by Lord Winaraliffe, in 1827, her dam, Octaviana, by Octavian-Shuttle-Zara, by Delpini.

1852 br. f. by Woful (put to the stud).....

1855 ch. f. by Varro...............

1854 b. с. by ditto.............. Mr E. F. Meynell

1855 ch. c. by $\operatorname{ditt0} \ldots \ldots \ldots \ldots \ldots \ldots \ldots$

1856

by ditto.................

\section{CESARIO MARE,}

Bred by Mr LAve, in 1812, her dam, Miss Holt, by Buzzard Camilla, by Highflyer-Matchem, etc.

1816 br. c. Alpha, by Ashton..........

$1817 \mathrm{ch}$. f. by llaphazard (dicd at 2 yrs old)

1819 b. f. by Marmion...............

1820 ch. c. Claude Lorraine, by Rubens...

1821 b. c. by Crecy ................

1825 b. c. Syphon, by Smolenslio........ IIr West

1824 b. f. Rachael Ruysch, by Rubens.....

1825 bl. c. by Smolensko...........

1827 br. c. Trouville, by Rubens.......

${ }^{*} 1828$ ch.f. Sweet Moggr, by Shaver......

1850 dead f. by ditto...............

Missed in $1818,22,26,29,51,52$, and was shot the following Christmas.

* Sweet Mogar missed in 1855 , had a dead foal in 34 , a b. c. by Stumps in 55, which is dead, and she was covered that year by Exilc.

\section{CESARIO MARE,}

Bred by Mr Binen, in 1815, her dam by Sir Solomon, out of the twin sister to Lilliputian, by Young Marske-Phœenomenon.

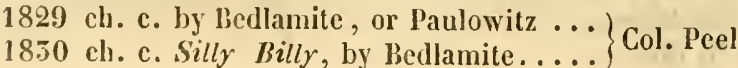


$185 \mathrm{I}$ ch. c. by Bedlamite............

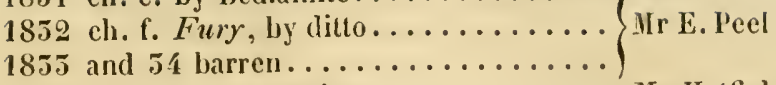

1853 ch. c. by Bedlamite............ Mr Hatfield

CESTRIAN MARE,

Bred by Sir Jonx Grey ligertov, in 1829, her dam, Paulina, by Orville-Shuttle-Sir Peter-Herod.

1828 b. c. by Whittington............. Mr R. Spencer

1829 b. f. by Menclaus (Whisker)......... IIr l'ainter

$1850 \mathrm{ch}$. c. by Champion (Selim) .........

1851 b. c. by P'eter Lely..............

1832 ch. f. by Champion (son of Selim)......
1853 b. c. by Olympus.............. James Lea

Sent to the United States in $\mathbf{1 8 5 5}$, in foal to Felt.

\section{CHAMPIGNON MARE,}

Bred by Mr Alexander, in 1894 , her dam, Chromatica, by Stamford, out of Pecunia, by l'ensioner-Honica, by Sir Peter.

1828 b. c. Ballochmyle, by Peter Lely..... Mr Wilkin

1829 b. c. by Epperston.............. Sir James Boswell

1830 b. f. by Monreith..............

1851 b. c. by ditto............... Mr Wilkin

1852 barren....................

1855 b. c. by Percy................ Mr Campbell

1854 b. f. by Malek...............

1858 b. c. by Corinthian............ Mr Wilkin

1856

\section{CHAMPIGNON MARE,}

Bred by MIr Carey, in 1898, her dam by Blucher, out of Opal, by Sir Peter-Olivia, by Justice.

1852 b. f. by Brutandorf (s. to Germany)... Mr Carey

1853 b. c. The Carpenter, by Lottery...... Mr S. L. Fox

1854 b. c. by ditto (died a foal) .........

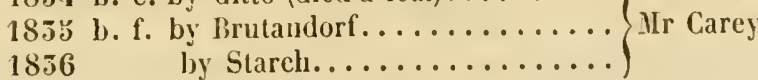

CIIANCE MARE,

Bred by Lord Eardeex, in 1816, got by Chance (son of Lurcher) her dam by Orville-Falcon-old Pumphin-Bagatelle, lyy Chrysolite-Playthiug, by Matchem. 
1824 b. f. by Cardinal Wolsey.......... Mr W. L. Fox

1826 ch. 1. by ditto ................ Mr Knapton

1827 ch. f. by Peler Lely.............. Mr W. Fox

I82S br. f. by Borodino.............

1851 b. c. by Comus (dead)...........

$1852 \mathrm{ch}$. f. by $\operatorname{Sir}$ Walton............ Mr John Moiser

185 b. f. by Brutandorf .............

1856 by Sandbeck ............

Barren in 182y, slipped foal to Borodino in 29 , missed to Comus in 50 , to St Nicholas in 55 and 54 .

\section{CHAPEAU DE PAILLE,}

Bred by Mr Cumpon, in 1821, got by Rubens, her dan, Fadladinida, by Sir Peter, out of Fanny, by Diomed.

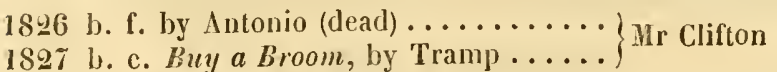

1829 b. f. Miss Lytham, by Brutandorf... Sir T. Stanley

1850 b. f. by Whisker (dierl young)..... Mr Clifton

1851 b. c. Luck's-all, by Lottery ......... Mr Brown

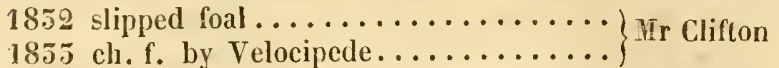

1854 b. f. Chapeau d'Espagne, by Dr Syntax Mr Mills

185 b. c. by Flexible.............. Mrr Tattersall

In 1828 missed to Catton, 56 to Camel.

\section{CIIAPEAU DE PAII.LE,}

Bred by Col. Wisson, in 1824, got by Iiubens, her dam, 'Tippilywitchet, by Waxy, out of Hare, by Sweetbriar.

185 与 b. f. by Cetus..............

1856 b. f. by Buzzard .............. Mr Foljambe

1857 by Langar ...........

CHARITY (a twin)

lired by Mr Lurey, in 1894, got by Tramp, out of Euphrosync's dam, by Shuttle-Drone.

1899 b. f. Fedora, by Figaro..........

1850 b. c. Sevillian, by ditto (s. abroad) ...

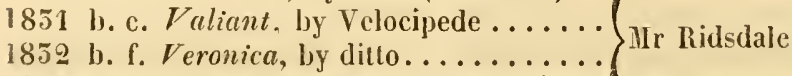

1855 b. f. Volant, by ditto...........

1854 b. . Venilia, by ditto........... 


\section{3}

1854 b. f. by Brutandorf(foaled Dec. 1854).

18.56

f. by I.angar .............. MIr Ridsdale

\section{CHARLOTTE WEST,}

Bred by Lord Jersey, in 1827, got by Tramp, her dam, Fillagrec, by Soothsayer, out of Web, by Waxy.

1855 foal by The Colonel (killed by accident) $1854 \mathrm{ch}$. f. Maid of the West, by The Colonel

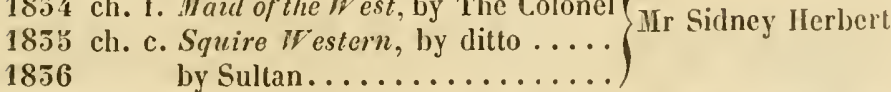

\section{CHARMING MOLLY,}

Bred by , in 1815, got by Rubens, her dam, Comedy, by Beningbrough - Mrs Jordan, by IIighflyer.

1822 b. f. Mystery, by Phantom........

182.5 br. c. Shakspeare, by Smolensko..... Mr West

1824 missed to Orville.............

1825 ch. c. Garrick, by Blacklock....... Mr Pickernell

1826 cast her foal by Tiresias..........

1827 b. f. Harlot, by Fitz-Orville .......

${ }^{*} 1828$ b. f. Siveet IIIarjomun, by Shaver...

1829 ch. . Meteor, by Mcrlin........... Mr West

$1850 \mathrm{ch}$. f. by Claude Lorraine (s. to Germany in 185z)

1851 b. c. Popjoy, by Rereller.........

1852 b. c. Mad Tom, by Bedlamite.......

Died of the dropsy in October 1832.

" Siveet Marjonun produced in 1834 a gr. f. by Stumps, was Iarren in 35. and sent to Germany in that year, covered by Exile.

CIISSI, by Thunderbolt (Vol. III, p. To) is dead, and had no produce that lived after 1851 .

\section{CHМT,}

Bred by Lord Rows, in 1815, got by Quiz, her dam, Paleface, hy

Y. Woodpecker, out of I'latina, by Mercury-Herod.

1827 ch. c. by Sovereign............. Mr J. Smith

1828 b. c. Dicler Gossip, by Waverley.... Najor Wood

$1829 \mathrm{ch}$. c. by Ilumphrey Clinker........ Mr A. Wood

1831 b. f. Henrietta, by ditto.......... Mr J. Snith 
1852 ch. c. Nimrod, by Actxon.......... Major Wood

1853 br. f. Florentia, by Jerry . . . . . . .

18.54 ch. c. by Velocipede............ MI. J. Smith

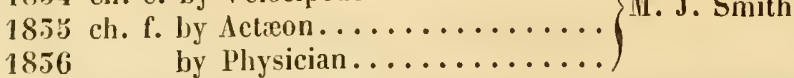

She was used as a hack till 1826 , and missed in 1850.

\section{CHERUB,}

Bred by Mr Waltov, in 1816, got by Hambletonian, her dam, Spitfire, by Pipator-Farewell, by Slope-Y. Marske-brother

to Silvio - sister to Stripling, by Hutton's Spot.

1824 b. c. Earl of Percy, by Percy....... Mr T. Sykes

1825 b. c. The Earl, by ditto...........

1827 b. f. by Swiss (died immediately)....

1828 b. f. by ditto................ Mr Walton

1899 b. c. by Wauton..............

1831 b. c. by Perey, or Agricola (died in)

1852 b. f. by Waverley, or Wanton....... $\}$ Mr T. Hudson

1834 b. c. by I.0.U...............

$185:$ b. f. by Leonardo.............. Ir T. Hudson

1856

by Bob Logic.............

Iu 1826 missed to Percy, in 50 to Bob Logic.

\section{CIILORIS,}

Bred by the Duke of Grafton, in 1824, got by Partisan, her dam,

Niobe, by Sir David-Buzzard--Totterella, by Dungannon.

1850 b. f. by Sober Rolin............. Mr Champion

1851 b. c. Regulus, by Emilius.......... Mr Houldsworth

1855 ch. c. by ditto............... Mr Champion

Missed to Centaur in 1899 , to Emilius in 52, and sent to France in 53 .

\section{CHORUS MARE,}

Bred by Mr Horver, in 1813, her dam by Orville, out of Anticipation, by Beningbrough--Expectation, by Herod-Skim.

1822 b. c. Croweatcher, by Blacliock..... Mr J. Smith

182.5 ch. f. Redlock, by ditto.......... Wr Jarnell

1824 b. c. by Walton............... Mr Jarnell

1823 ch. c. by Jack Spigot (died a foal) . . . Mr J. Smith 
1828 b. c. Chorister by Lottery (died in 1855 ) Ld Cleveland 1829 b. c. (Quartetto) by ditto...........
1850 b. f. by Jerry (died 2 yrs old) ........ Mr G. Walker 1851 b. c. Glee-singer, by Lottery ........ 1852 b. c. Memnon Junior, by Memnon... 1855 b. c. by Lottery (broke his back at Mr J. Smith

1854 b. c. by Jerry (died a foal).........

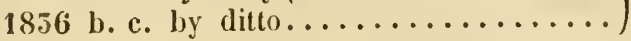

Missed to Jack Spigot in 1826 and 27, to Voltaire in 5 .

\section{CIIRISTABEL,}

Bred by Mr Cooper, in 1824, got by Woful, her dam, Ilarrict, by Pericles--Selim-Pipylina, by Sir Peter.

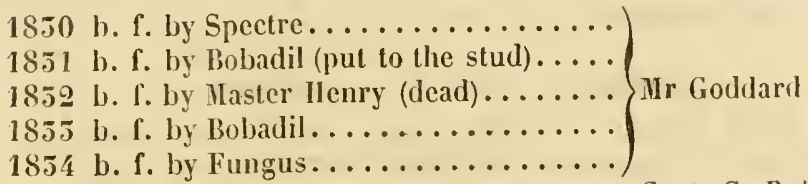

1855 f. by Bulgarian............. Capt. G. Bulkeley

\section{CHRYSEIS (Young)}

Bred by Mr Crockford, in 1814, got by Dick Andrews, her dam, Chryscis, by $\Lambda$ sparagus.

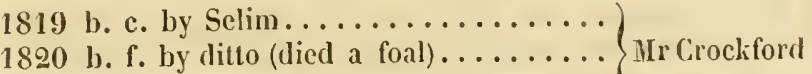

1821 b. c. by ditto................

1822 ch. f. Miniature, by Rubens........ Id Anson

182.5 b. c. Styrocket, by Selin......... Mr Forth 1824 b. c. Prestbury, (afterwards Sir Edward)
Codrington) by Rubens (s. to Ireland)

1823 b. f. (Emily) by Sasenagh, or Rubens. IIr L. Charlton

1827 b. c. IIarry, by IIaster Henry..... . Mr Thorne

1899 br. f. by ditto............... Mr I. Charlton

1831 cl. c. The Tulip, by Wamba....... Mr F. R. P'rice

1852 by Wamba

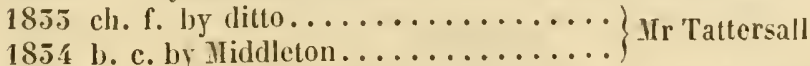

In 1826 missed to Xaster lienry, 50 to Manfred, 55 to I.ogic, not covered in 27. 


\section{CICELY (sister to Cain)}

Bred by Mr Yates, in 18थ6, got by Paulowitz, her dam by

Pajuator-Delpini-Young Marske-Gentle Kitty, by Silvio.

1852 br. c. Tony Lumpkin, by Bedlamite..

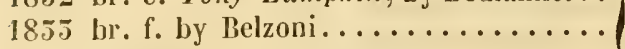

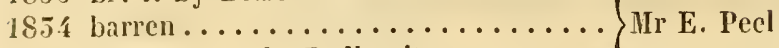

1855 br. or gr. c. by Bedlamite.........
1856 b. c. by Emancipation...........

\section{CINDERELLA,}

Bred by Mr Stelfax, in 1822, got by Walton, her dam by Dick Andrews - Shuttle-Sir Peter - Play or-pay's dam, by Ilerod.

1855 br. c. by Bay Malton...........

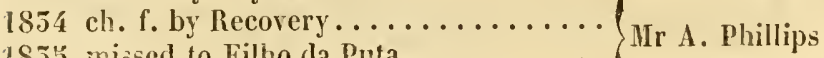

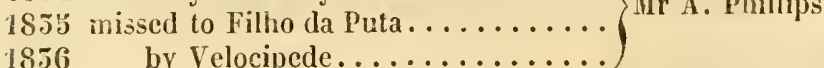

\section{CINDERELLA (sister to Lamplighter)}

Bred by Lord Berners, in 1824, got by Merlin, out of Spotless,

by Valton-Trumpator-Highflycr-Otheothëa, by Otho.

1855 b. c. Cabin Boy, by Skiff..........

1354 ch. c. Cockboat, by ditto....................... Sowerby

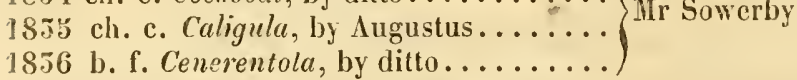

\section{CLARE,}

Jired by Lord Egrmovt, in 1824, got by Marmion, her dam, IIarpalice, by Golıana, out of Amazon, by Driver-Fractious.

1850 b. f. Marpessa, by Muley........ Mr Forth

1851 b. c. Flatterer, by ditto.......... Sir M. Wood

1852 b. c. by Bustard............. IIr Nowell

1855 b. f. by Bustard.............. Sir M. Wood

1854 b. c. by Muley .............. Mr Etwall

183 b. f. by ditto.............. Mr Nowell

In 1827 missed to Bustard, 28 to Bustard and Aladdin, 29 and 56 to Muley.

Flatterer was sent to the United Slates in 1855.

\section{CLATTER,}

Bred by Lord Lowtuer, in 1824, got by Clinker, her dam, Niua, by Selim, out of Pennytrumpet, by Trumpator-Y. Camilla. 
1829 b. f. by Master IIenry. . . . . . . .

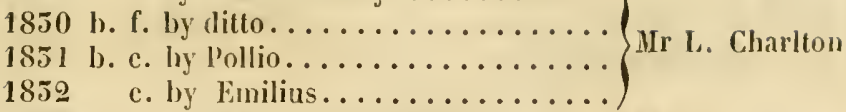

1855 ch. c. Cicero, by ditto........... Mr Greville

1854 b. f. by Pollio............... Ir Tattersall

Sent to France October 1834, in foal to Sir Ilercules.

\section{CLEIIATIS,}

Bred by Mr Capel, in 1824, got by Waxy Pope, her dam, Cobbëa, by Skyscraper-Woodpecker-Heinel, by Squirrel.

1850 b. c. Creeper, by Tamworth........

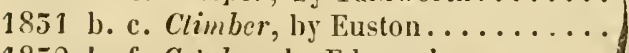

1852 b. f. Colubra, by Edmund..........

1855 missed to Cardinal Puff........... Hr Bowen Dasics

1834 ch. c. Celastrus, by Gambler........

1855 b. f. Creeping Cerus, by Adventurer..

1856

by Wamba................

\section{CLEMENTINA (chesnut)}

Bred by lord Yarrorocgil, in 1820, got by Discount, out of Maiden, by Quichsilver-Adamant-Paymaster.

1829 b. f. Cynthia, by Don Juan. . . . . . .

1851 ch. c. King Lear, by ditto, or a half-

1855 ch. f. Hyacinth, by Memnon....... ITr Ilarding

In 1850 slipped foal by Bedlamite, in 52 missed to him, and has been used as a hack since $\check{\jmath}$.

CLINKER MARE (sister to Busto)

Bred by Mr Butler, in 1816, her'dam, Bronze, by BuzzardAlexander-llighflyer.

182 s. ch. c. Popanilla, by Thantom......

$1826 \mathrm{ch}$. f. by Sherwood.............

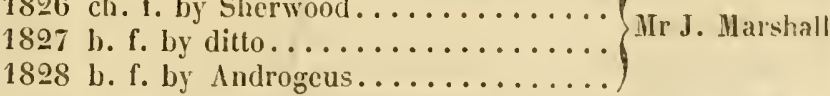

1829 c. by Figaro (died a foal) ........

18.50 br. c. St Julien, by Chateau Margaux. Id Low ther

1851 b. f. by Partisan..............

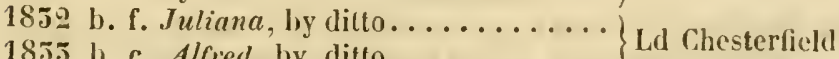

1853 b. c. Alfred, by ditto........... 
1854 ch. c. by Partisan (dead) .........

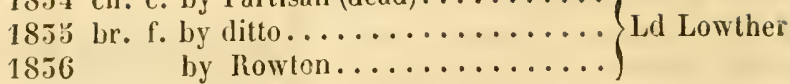

\section{CLINKERINA,}

Bred by Lord Fitzwiliax, ii 1812, got by Clinker, her dam, Pewet, by Tandem, out of Termagant, by Tantrum.

1818 b. f. by Amadis (died a foal) ........

$1819 \mathrm{br}$. c. Rinaldo, by ditto ...........

1820 missed to Comus..............

1821 b. c. Whiskerandos, by Whisker....

1829 b. c. II umphrey Clinker, by Comus. .

1825 b. f. by Partisan (dead)...........

1824 b. c. by Comus (dicd young)........

1823 b. f. Rallad-singer, by Tramp...... Ld Fitzwilliam

$1826 \mathrm{br}$. f. Ringlet, by Whisker.........

1827 br. c. Pontefract, by Cervantes......

1828 b. f. Gipsy, by Tramp (put to the stud)

1829 bl. f. by Confederate............

1850 b. c. by ditto.................

$1831 \mathrm{bl}$. f. Alliance, by ditto (put to the stud)

1832 b. c. by ditto (died a yearling).......

1855 b. f. by ditto................ Mr Bowe

1854 br. f. by Mambrino.............
1853 b. c. by Mulatto...........

COBWEB,

Bred by Lord Jersey, in 1821, got by Phantom, out of Filagrec,

by Soothsayer - Web, by Waxy-Penelope, by Trumpator.

1827 ch. f. by Comus................

1828 b. c. $Y$. Emilines, by Emilius (s. to France in Oct. 1854)

1829 br. c. by Master Henry . . . . . . . . .

1850 ch. c. Lucius, by Emilius...........

1851 ch. f. Nell Gwovine, by Sultan......

1852 b. c. Mahomet, by ditto .......... Ld Jersey

1855 b. c. Bay Middleton, by ditto.......

1854 b. с. by ditto.................

$185 z$ b. c. by Buzzard..............

1856 c. by Sultan................

1857

CODICIL,

Fred by Mr Suard, in 1890, got by Smolensko, her dam, Legacy, by Beningbrough, out of Roxana, by Sir Peter. 
1826 b. f. Little Ecstacy, by Phantom....

1827 f. by Reveller.

1829 b. f. May-fly, by Middleton (s. to Ger-\}Mr Shard

1855 ch. f. Ileiress, by The Colonel.....

1854 b. c. by Tranby...............

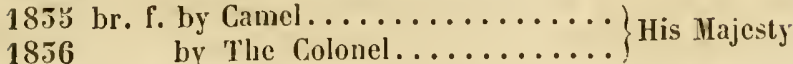

Missed to Middleton in 1828 , to Reveller in 50 , barren also in 51 and 52 .

\section{COLUMBIA,}

Bred by Sir George Pigot, in 1824, got by Filho da Puta, her dam, Sunflower, by Castrel-Alexander-Nelly, by Otho Syphon.

1852 b. c. Altamont, by Sligo.........

1855 c. by Tramp (dead) ...........

1854 b. f. by Patron............... Sir G. Pigot

1855 slipped twius by Emancipation......

1856 by Zinganee, or Priam.......

\section{COLUMIBINE (sister to Hariequin)}

Bred by Mr Garforti, in 1824, got by Cervantes, her dam, Flora, sister to Oiseau, by Camillus-Ruler-Woodpecker.

1828 missed to Lottery . . . . . . . . . .

$1829 \mathrm{gr}$. c. Clown, by ditto . . . . . . . .

1850 br. f. Mrsinda, by ditto. p put to the

1851 b. f. Celeste, by ditto... stud

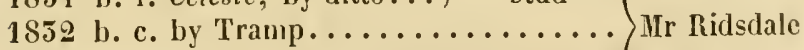

1855 b. f. by ditto...............

1854 picked a c. by ditto............

1853 clı. f. by ditto .............

1856

by Brutandorf.

COIIA,

Bred by Mr N. Hongsor, in 1818, got by Comus, her dam, Priscilla, by Delpini, out of Eliza, by Alfred-Eclipse.

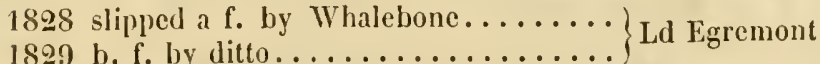

18.50 b. f. Cinderella, by ditto......... Mr Gardnor

$18.51 \mathrm{gr}$. f. Greybone, by ditto......... Ld Egremont

1852 missed to Sober Robin........... Mr R. Wilson 
1855 ch. f. Malcap, by Bedlamite........ Mr Gardnor

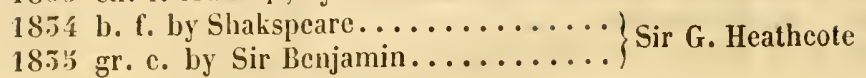

\section{COMEDY,}

Bred by Mr JaQues, in 1817 , got by Comus, her dam by StarY. Marske-Emma, by Telemachus.

1822 ch. c. Barytes, by Walton.........

182.5 br. f. Galena, by ditto................... Mr Jaques

1826 ch. f. by St Patrick...............

1828 ch. f. Farce, by ditto............ Mr Maberly

1850 ch. f. Pantomime (dead) by ditto....

1852 ch. f. Burletta, by Actæon.........

1855 br. c. by Wavcrley............. Mr Jaques

1854 b. c. by Voltaire..............

185 ch. f. by Action..............

In 1824 missed to Walton, 25 to Whisker, 29 to Swiss, and 31 to Velocipede.

\section{COMUS MARE,}

Bred by Mr Wratner, in 1816, her dam, Plumper's dam, by

Delpini, out of Miss Muston, by King Fergus-Espersykes.

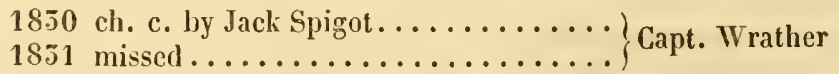

1852 gr. f.by Columbus............ Dr Husband

1855 b. c. by ditto ...............

1834 b. c. by The Lion (son of Brutandorf) . Capt. Wrather.

$1853 \mathrm{ch}$. c. by Acteon.............. Capt. Wrather.

1856

by Physician.............

\section{COMUS MARE (sister to Torchbearer)}

Bred by Col. Cranock, in 1817, her dam by St George-Pontac -Syphon-Nliss Wilkinson, by Regulus-Lodge's Roan mare, by Partucr.

1821 b. f. by Whisker (dead) ........

182.5 br. c. Illussar, by ditto............

1824 b. c. Lancer, by Grey Walion ...... Col Cradock

1823 c. and f. by Whisker (died foals)..

1826 b. f. loy ditto (dead)............. 
1830

c. by Actæon.

1855 b. c. by Waverley (dead)............ Col. Cradock

Missed to Whisker in 1822, had no thorough-bred foals between 26 and 52 , and died in 55 .

\section{COMLS MARE,}

Bred by Mr Peirse, in 1818, her dam, Lisette, by Ilambletonian, out of Constantia, sister to Gamenut, by Walnut-Contessina, by Y. Marske-Tuberose, by Herod.

$\left.\begin{array}{r}1825 \text { gr. c. Dicky Walkington (afterwards } \\ \text { Y. Revenue) by Revenue }\end{array}\right\}$ Ir Skipsey

$1826 \mathrm{gr}$ f. by ditto.............. Mr Heseltine

185 I gr.f. Nell, by Blacklock..........

1852 gr. f. Olivia, by Waverley (put to the Mr Skipsey stud)

$1855 \mathrm{bl}$. c. by Waverley (dead).........

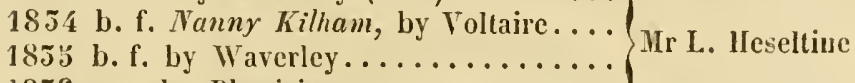

1856

by Physician.

Barren in $1827,28,29$, and 30 .

\section{COMUS MLARE,}

Bred by Mr Garronti, in 1820 or 1821, her dam, Marciana, by Stamford, out of Marcia, by Coriander-Faith, by PacoletAtalanta, by Natchem.

1825 ch. c. Sharpset (afterwards Lepanto) by Cervantes Mr Ridsdale

1826 gr. f. by Peter Lely.............

1827 b. c. by Theodore (died a foal) ...... Mr Garforth

1828 not corered last year..............

$1850 \mathrm{ch}$. f. Sevilla, by ditto............

1851 ch. c. Vidocq, by Velocipede....... . Mr Ridsdale

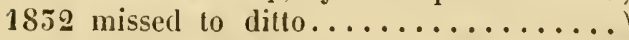

$1855 \mathrm{ch}$. f. by ditto (dead) .............

$1854 \mathrm{gr}$. c. by his Y. Filho da Puta....... Mr Garforth

1855 b. c. by Tramp, or Waverley......

$18 \pi 6$ by Brutandorf............

COIUS MARE (sister to the preceding)

Bred by Mr Garforti, in 1822, out of Marciana.

1851 f. by his Y. Filho da Puta....... Mr Garforth 


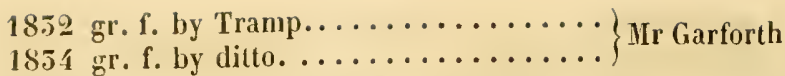

In 1850 missed to Lottery, 55 to Velocipede, 55 to St Nicholas, and sold.

\section{COMUS MARE,}

Bred by Mr Garrorth, in 1820, her dam, Flora, by CamillusRuler.

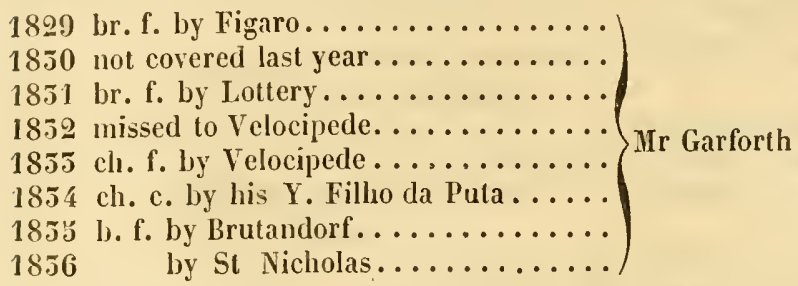

COMUS MARE,

Bred by Mr Fuetcher, in 1820, her dam, Arabella, by Williamson's Ditto, grandam, Esther, sister to Staveley, by ShuttleDrone-Matchem.

1852 b. f. Butterfly, by Lottery......... Ld Eglinton

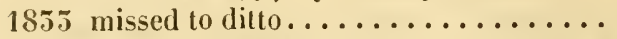

$\left.\begin{array}{l}1854 \text { br. c. Actonian, by Jerry ........... } \\ 1853 \text { br. c. Prince Nicholas, by Sir Hercules }\end{array}\right\}$ Mr Weatherley

She was not trained, but was used as hack or hunter till 1851.

\section{COMUS MARE,}

Bred loy Mr Garforth, in 1820, her dam, by Sancho, grandam, Vesta, by Delpini, out of Faith, by Pacolet.

1828 gr. f. by Sir Malagigi............

1829 gr. f. by Truth (son of Rubens)........... Mr S. Duncombe

1850 barren...................

1851 gr. c. Gaffer Grey, by Buzzard ...... Col. Westenra

1855 b.c. by Solyman (Sir J. Malcolm's Arab) His Majesty

1854 b. f. by The Colonel............. Mr J. W. Patten

1855 cl. c. by ditto (dead)............ Mis Majesty 1856

\section{COMUS MARE,}

Bred by Sir W. W. Wrns, in 1820, her dam by Beningbrough, out of Constantia, by Sir Peter. 
1826 br. c. Pluralist, by Ambu.......... Mr Williams

1827 b. f. Jessica, by Alderman Wood.... Mr Stelfax

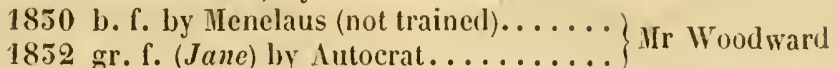

1855 ch. c. Reuben, by Bay Malton....... Mr Birch

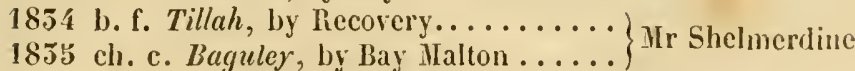

Missed in 1828, 29, 51 , and 56 .

\section{COMUS MARE,}

Bred by Mr Lıтом, in 1819 , her dam by Mowbray-Beningbrough

- Mercury-Mary Ann, by Florizel.

${ }^{*} 1828$ b. f. by Coronation............. Mr Sturdy

1829 b. c. by Brutandorf............ Ld Scarbrough

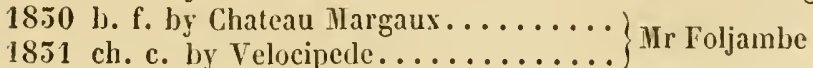

1855 ch. c. by Langar (dead)...........

185 y br. c. by St Nicholas ........... Mr potter

$1856 \quad$ by Big Ben.............

Missed in 1852 and 54.

* This Coronation mare produced in $1852 \mathrm{Mr}$ Wilson's b. c. Winkley. by Velocipede, and died about a month after he was foaled.

\section{CONUS MARE (sister to Sophy)}

Bred by Mr Garfortn, in 1820, her dam by Camillus, out of Helen, by Delpini-Rosalind, by Phoenomenon-Atalanta.

1823 b. c. Clinton, by Blacklock........ 1826 b. c. Fararino, by ditto ......... 1829 b. c. by Figaro ..............

1850 b. c. Captain Ross, by ditto.......

1851 b. c. Fearnhurst, by Peter Lely...... Mr R. Turner

$1832 \mathrm{ch}$. c. by Teniers (died a foal).......

1855 ch. f. by l'eter Lely............

$1856 \quad$ f. by Olympus.............

Slipped foal by Tramp in 1827 and 28 , barren in 54 and 5 s.

\section{COIIUS MARE,}

Bred by Sir W. W. Wrxx, in 1820 , her dam by Alexander, out of sister to Haphazard, by Sir Peter-Miss Herrey, by Eclipse. 1826 ch. c. by Piscator ............. Sir W. W. Wynn 1827 ch. f. by The Grand Dukc......... Mr Wadlow 
1828 b. c. Warwick, by Filho da Puta..... Mr Beardsworth 1899 b. f. St Winifred, by Master Henry.. Mr R. Mostyn 1830 b. c. The Governor, by Filho da Puta. Mr F. R. P'rice 1832 ro. c. Bucephulus, by Timour....... Mr A. Bower

Hissed in 1851 and 54 , slipped foal in 55 and 53 , and was shot.

\section{COIIUS MARE (sister to Swinton)}

Bred by Mr Riddels, in 1820, her dam by Shuttle-DelpiniTubcrose, by IIcrod.

1850 b. f. Tigress, by Brutandorf. ....... Col. King

1851 b. в. (dead) by Loticry ............ Mr Greenwoorl

1852 b. f. Caroline, by Brutandorf ........ Mr Marson

185.5 b. f. by ditto ................

1854 b. c. by ditto...................... Gr Greenwood, of 185. br. f. by Margrave............ Beverley

1856

by Belshazzar.............

\section{COIIUS MARE (grey)}

Bred by Mr Garfortu, in 1821, her dam, Flora, sister to Oiseau. by Camillus.

1828 b. f. by Waverley ..............

1829 b. c. Beverlonian, by Blacklock ..... IIr Haworth

1850 gr. f. by Waverley (put to the stud) ..

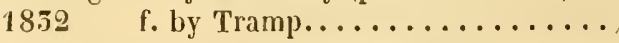

Sold to the D. of Holstein in Sept. 1832 in foal to Brutandorf.

\section{COMUS MARE,}

Bred by Mr Nowell, in 1824, her dam, Nargrave's dam, by Election, out of Fair Ifelen, by Hambletonian-Helen, by Delpini.

1850 c. by Muley..............

1852 ch. c. by Bustard ............. Mr S. L. Fox

1855 br. c. Dick, by Muley ............ . Mr Buckley

1854 ch. f. Rachel, by ditto........... Mr G. Cooke

18.5: ch. f. by ditto $\ldots \ldots \ldots \ldots \ldots \ldots \ldots \ldots \ldots \ldots \ldots$ ) Mr Nowell

\section{COMUS MARE,}

Bred by Lord Jersey, in 1827, her dam, Cubweb, by Phantom, out of Filagrec, by Soothsayer-Web, by Waxy.

1855 ch. c. Gemappe, by Waterloo ....... Ld Uxbridge 
$\left.\begin{array}{l}1854 \text { ch. f. by ditto (s. to South Carolina). } \\ 1853 \text { ch. f. by ditto, or The Colonel...... }\end{array}\right\}$ His Majesty

1856

by Rubini.

COHUS HARE (own sister to Voluna *)

Bred by Mr Horster, in 1828, her dain, sister to Acklam Lass, by Prime Minister, out of Y. Ilarriet, by Camillus.

1832 ch. c. Tyrrel, by Velocipede........

1855 and 54 missed to Y. Phantom....... Mr Horsley

1855 br. c. by Waverley ................

* Voluna is a bay mare foaled in 1829 , and first ran in the name of Mr Etty.

CONSUI, MARE,

Bred by Mr Yates, in 1824, her dam, Isidora, by Blucher, out of Zora, by Selim-Zoraida, by Don Quixote-Lady Cow.

1828 gr. f. by Johnny Raw ........... Mr Willock 1830 gr. c. by Roderick.............. Mr E. Peel

1831 gr. or ro. c. Mlad Tom, by Bedlamite. Mr Willock 1852 b. f. by Langar............... Mr E. Peel Missed in 1855, and sent abroad.

\section{CONTRITION,}

Bred by Mr B. Surm, in 1824, got by Tiresias, her dam, Theresa Panza, by Cervantes, out of Gadabout, by Orville-Minstrel.

$\left.\begin{array}{r}1852 \text { b. c. MIaximus, by Y. Whisker (son of } \\ \text { Sir Paul mare) }\end{array}\right\}$ Mr Dickinson

1853 b. c. Treadmill, by Tramp......... IIr Greville

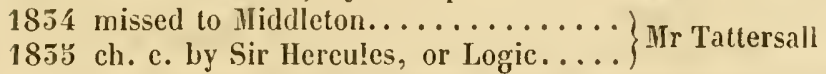

Sent to France in January 1856 , in foal to Logic.

\section{CONVICTION,}

Bred by Mr Platel, in 1822, got by Cannon-ball, her dam, Chasm, by Thunderbolt, out of Enchantress, by Voluntecr.

1854 ch. e. by Confederate............ Mr R. Richardson

\section{COQUETTE,}

Bred by the Duke of Graftor, in 1814, got by Dick Andrews, her dam, Vanity, by Buzzard, out of Dabehick, by 'ot 80 's. 


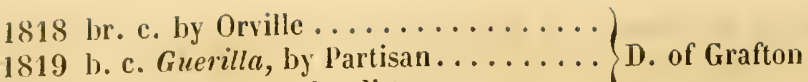

1820 ch. f. Gabrielle, by ditto..........

1821 b. f. (Girouette) by ditto........... D. of Portland

1822 ch. f. Garotte, by Election..........

1825 ch. c. Goshawk, by Merlin (s. to Flo- D. of Grafton rence in 1831)

1824 b. f. (Bupta) by Partisan.......... I.d Wharncliffe

$182 y$ ch. f. Grisette, by Merlin..........

1826 br. f. Gem, by Wrangler.......... D. of Grafton

1827 ch. c. Gondolier, by Herlin.........

1898 ch. f. by ditto...............

1899 b. f. by Centaur.............. Mr Harmar

1831 br. f. ly Figaro..............

1852 b. c. Mozart, by ditto............

18.55 ch. f. by Partisan.............. Idd Lowther

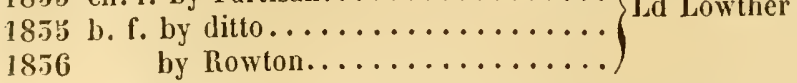

Barrell in 1850 , missed to Partisan in 1854 .

CORA,

Bred by Mr Gascoigne, in 181\%, got by Waxy, her dam, Vourneen, by Sorcercr, out of Tooec, by Buzzard-Violet, by Shark.

1824 br. or bl. f. hy Grey Walton........

1825 b. f. ly Tramp (put to the stud)......

1826 cl. c. by ditto................

1827 ch. c. by Blacklock............. Mr Gascoigne

1828 b. c. by St Patrick..............

1829 ch. f. by Figaro . . . . . . . . . .

1851 b. f. by Figaro................

1852 b. c. by Actxon..................... Mr Spalding

1856 by Big Ben.............. Mr Spalding

Missed to Lottery in 1850,55 and 54, to Brutandorf in 55.

\section{CORA (a chesnut mare)}

Bred by Sir E. Ltorn, in 1814, got by Peruvian, her dam loy Alexander, out of Berrington, by Sweet William-Herod-Flora.

1829 ch. f. P'uss, by Tenicrs...........

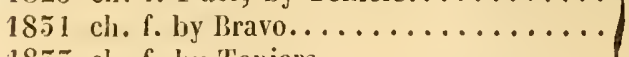

1855 ch. f. by Tenicrs.............. Mr Mostyn

1853 ch. f. by ditto...............

18.56 by birdcatcher...........

In 1830 missed to Teniers, 52 to Bravo. 
CORINNE, by Waxy (Vol. III, p. 87) was barren in 1852, and sold to Baron Biel that year, covered by Partisan. Her produce of 1829 and 50 were also sent to Germany.

\section{COTTAGE GIRI,}

Bred by Mr WATT, in 181 g, got by Thunderbolt, her dam by Worthy, out of Y. Camilla, by Woodpecker-Camilla, by Trentham.

1825 b. c. Squat, by Blacklock, or Tramp.. Ir Wise

1824 br. c. Cottager, by Blacklock........ Mr Burton 1'eters

1825 f. by ditto .............. Mr Wise

1826 b. c. Barrister, by Minos ........... Mr Bower

1827 br. f. Lady Betty, by Figaro........

$1829 \mathrm{gr}$. f. by Arbutus...............

1850 b. f. Shepherdess, by Young Phantom.

1851 b. f. by ditto................ Ir Wise

1855 b. c. by Mrr Best's Tinker .........

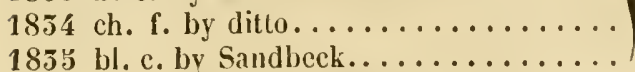

No foal in 1828 or 52.

\section{THE COUNTESS,}

Bred by Lord Loxnoxderry, in 1822, got lyy Catton, her dam by Hambletonian-Shuttle-Drone-Catherine, by Y. Marske.

1828 b. c. The Count, by Tramp.........

1852 br. c. Tom Basford, by Timour......

1835 br. f. Doredale, by ditto.......... Mr A. Bower.

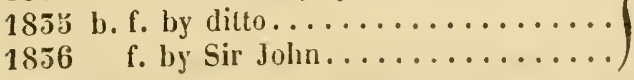

Slipper foal in 1850 , and not covered; missed in 29 and 54.

\section{CRAZY JANE,}

Bred by Mr Fergusox, in 1815, got by Remembrancer, her dam by Beninglorough, out of Fraxinclla, by Trentham-Woodpecker.

1824 gr. f. Trinket, by Grey Walton...... Mr II. Wormald

1823 b. f. ly a coach-horse........... Ir Peacock

1826 b. f. by Rhadamanthus........... Ir W. Armitage

1898 b. c. Goodluck, by Lottery.......... Ir Petre

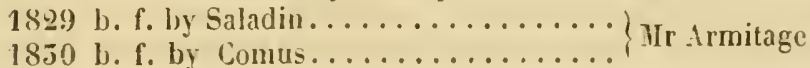




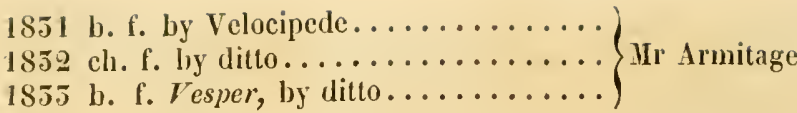

Missed to Octavius in 1827, and died in 1855.

\section{CREEPING KATE,}

Bred by

in got by Sunderland, her dam by Druggist, out of Nelly, by Otho.

1824 b. c. Sawner, by North Star (son of $\begin{gathered}\text { Daffodil) } \\ \text { Mr Bretherton }\end{gathered}$

1823 b. f. Cottage Girl, by Gambler...... Mr Hardy

1826 b. c. Wellington (aft. Russell) by Corin-

1827 b. c. (Clifton) by Blucher........... Mr Steele

1829 b. f. Lady Louisa, by Corinthian.... Mr Woolmer

1851 b. c. by ditto................. Sir T. Stanley

She had dead foals in $\mathbf{1 8 5 2}$ and $\mathbf{5 5}$, and is dead.

\section{CREHONA,}

Bred by the Dule of Grafton, in 1825, got by Partisan, her dam,

Catgut, by Comus, or Juniper, out of Vanity, by Buzzard.

1828 ch. c. by a lialf-bred horse........

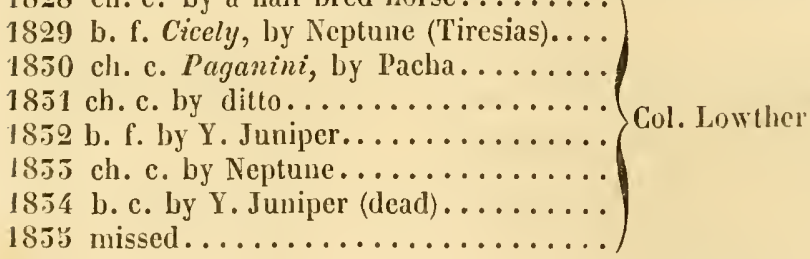

CRESSIDA (sister to Elcanor) died August 14, 1834, aged 27, having had no foal since 1828 .

\section{CUSTARI,}

Bred by Mr Elwes, in 1821, got by Soothsayer, her dam, Miss Sophia, by Stamford-Sophia, by Buzzard-Huncamunca.

1827 ch. c. by Middleton.............

1828 br. c. Pedro, by Enilius............

1829 b. c. by Partisan.............. Ld Tavistock

1850 missed to Sultan...............

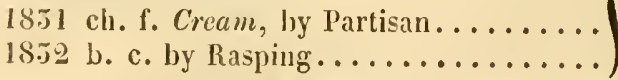

Sold to the I). of Holstein in 1832, in foal to Royal Oak. 


\section{CUTTY SARK,}

Bred hy Sir Wibliam Maxwell, in 1815, got by Haphazard, her dam, Brillante, by Whiskey-Diomed-Imperator.

1817 b. f. Ilell Cat, by Cerberus ........

1820 b. c. Pirate, by Comus ..........

1821 ch. f. by Epperston............

1822 b. c. by Viscount (died young)

$1825 \mathrm{ch}$. f. Spaewife, by Soothsayer.

189 b. f. Glamour, by Viscount ......

1823 b. c. by Reveller (lamed young) ..... Sir W. Maxwell

1826 b. c. by Viscount..............

1897 gr. c. by Champignon..........

1828 br. c. Caird-o'-Barhullion, by Ben Ledi

1850 ch. f. Chemisette, by ditto..........

1851 b. f. by ditto................

1832 gr. f. by ditto (killed when 5 yrs

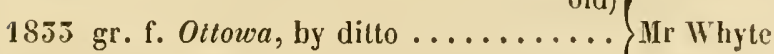

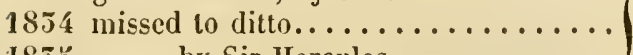

1855 by Sir Hercules. ..........

\section{DAHLIA,}

Bred by II. R. II. the Duke of York, in 1822, got by Phantom, her dam by Waxy, out of Moses' dam, by Gohanna.

1828 b. f. Titania, by Merlin .........

1829 dead c. by Sultan . . . . . . . . .

1850 barren.

1851 b. f. La Bayadere, by Leviathan.....

1852 c. by Sultan (dead) ........... Ld Chesterfield

1855 b. c. by Velocipede.

1854 b. f. Prima, by Priam ..........

1853 b. f. by ditto.

1856

by Colwick.

\section{DIIRYM $\Lambda$ ID.}

Bred by Mr Braxdixg, in 1819, got by Woful, her dam, Tiny,

by Sir Peter, out of Wren, by Woodpecker-Sir Peter's dam.

1829 b. f. Miss Lancaster, by Coronation.. Mrs Dawson

1850 b. c. Larkspur, by Lollery.......... IIr S. L. Fox 
1851 br. f. by ditto $\ldots \ldots \ldots \ldots \ldots \ldots \ldots$
1852 br. c. by Waverley................ Mrs Dawson

1854 b. c. Cow-boy, by Voltaire........... IIr Skipsey

1856 by Physician.............. Mrs John Jawson

Barren in 1853 and $5 \%$.

\section{DANDIZETTE,}

Bred by Mr WatKer, in 1820, got by Whalebone, her dam, Fair

Ellen, by the Wellesley Grey Arabian-Maria, by Hightlyer.

1827 gr. f. Corea, by Emilius

1828 gr. c. Cindad Rodrigo, by ditto........

1829 gr. c. Caçador, by Middleton......... D. of Richnond

1851 f. Chica, by Helenus...........

1852 gr. c. Marmoset, by ditto......... Mr Greville

1855 c. by ditto (broke its leg)........ D. of Richmond

1856 by Rubini ... .

Barren in $1826,50,54$, and 55.

\section{DARIOLETTA,}

Bred by Mr Vaxsittart, in 1822, got by Amadis, her dam, Selima, by Selim-Pot8o's-Editha, by Herod.

1827 ch. c. by Middleton.............

1828 f. by Grey Middleham (dead) ......

1829 ch. c. Perion, by Whisker........... Mr Vansittart

$1850 \mathrm{br}$. f. Elisena, by ditto............

1851 missed to ditto..................

1832 b. c. by Catton.............. D. of Rutland

1853 c. by Waverley (dead)............

1854 b. c. by Catton ................ Ir Vansittart

185 a

1856

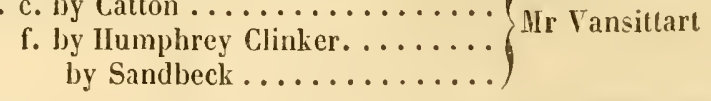

\section{DAUCIER,}

Bred by Mr G. BamLetr, in 1821, got by Waltou, her dam, Stella, by L'Orient-Ruler-Highllyer.

1827 b. f. by Figaro (dead) ............ Mr W. Storry

1899 b. f. Lady Blanche, by Borodino.... Ir Tate

1850 br. f. Venus, by Lottery............ W Mr G. Oates
18.51 b. f. by ditto................ 
1853 b. c. Willesden, by ditto, or Jerry ... Mr Ramsay

1854 b. c. by Count Porro............ Mr Brown

1855 ch. f. The Moon Beam, by ditto...... Mr Ramsay

Missed to Lottery in 1828 and 52 ; not covered in 53.

\section{DECEIVER MARE,}

Bred by . Mr Varsitrart, in 1818 , out of Slight's dam, by Iot80's -Editha, by IIerod-Elfrida, by Snap.

1827 ch. c. Splendor, by Sovereign. ...... Mr Hebden

1828 b. c. Tremaine, by Tramp ..........

1829 ch. f. Carnation, by Comus.........

1850 ch. c. Cannonade, by Comus........

1851 ch. f. by Blacklock ..............

$1852\left\{\begin{array}{l}\text { b. c. Tivin ....... } \\ \text { b. f. (dicd a foal) . }\end{array}\right\}$ by Tramp...... Mr Singleton

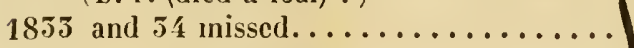

1855 b. f. by Sandbeck .............

1856 by MIulatto.

\section{DECEIVER MARE,}

Foaled in , out of a natural Arabian mare, imported by the late Duke of Northumberland.

1851 br.c. IIellenistian, by Mr Astley's Black Turk or Barb

$1852 \mathrm{ch}$. f. Necra, by his ch. Arabian....

1855 gr. f. by his Grey Arabian.......... Mr Attwood

1854 b. f. by Argantes...............

$185 \mathrm{~s}$ ch. c. by Callisthenes ............

$18 \tilde{3} 6$

by ditto................

\section{DECISION,}

Bred by the Trustees of Lord Stratmione, in 1825, got by JIagistrate, her dam, Remembrance, by Sir Solomon.

1828 br. c. Brown Stout, by Y. Phantom, or $\begin{array}{r}\text { Jack Spigot } \\ \text { Mr J. Smith }\end{array}$

1829 br. c. $X X X$, by Jack Spigot......... Mr Ramsay

1850 br. f. Remember, by Jerry .........

1852 b. f. Rectitude, by Lottery.......... Ld Eglinton

1855 b. c. Silvio, by Jerry............. Sir J. Boswell

1854 br. c. by Jerry (died a foal)......... Mr J. Smith

Died in August 1854, with a colt in her by Voltaire. 
DECOY,

Foaled in 1850 , got by Filho da Puta, out of Finesse.

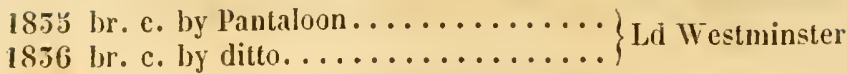

\section{DEFIANCE,}

Bred by Mr SADLer, in 1816, got by Rubens, her dam, Little Folly, by Highland Fling-Harriet, by Volunteer-Alfred.

1822 b. c. Dauntless, by Whalebone...... Mr R. Benson

1824 b. c. Defence, by ditto ...........

1826 ch. f. Derice, ly Tramp ..........

1827 ch. f. Design, by ditto...........

1828 b. f. Delight, by Reveller.......... Mr Sadler

1850 ch. c. Dangerous, by Tramp........

1855 ch. c. by Reveller.............

1854 b. f. by Fungus...............

Hissed in all the intermediate years, and in 1855.

* Delignt was sent to the United States in 1855 , with a c. by Windcliffe, and covered by St Patrick.

\section{DELENDA,}

Bred by Lord Egremovt, in 1808, got by Gohanna, her dam,

Carthage, sister to Ilannibal, by Driver, out of Fractious.

1819 b. f. IIilline, by Dominie Sampson... Mr Portman

1820 ch. c. by Aladdin.............. Mr Williamson

1821 b. f. by Iuley................ Mr R. Wilson

1822 br. c. by ditto.............. Mr Blagrave

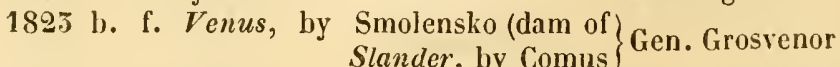

1824 b. f. by ditto .................... Tr. Tarleton

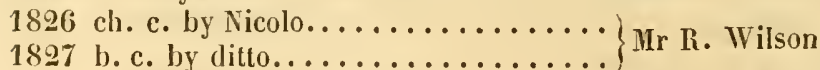

1828 b. f. by Gulliver ............... Mr E. F. Meynell

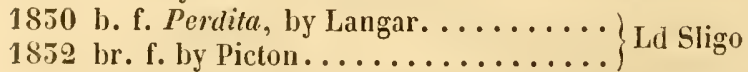

Slipped foal in 1825, missed in 51 and 55 , and is dead.

\section{DELPHINE,}

lired by Mr Perne, in 1823, got by Whisker, her dam, My Lady,

by Comus, out of 'The Colonel's dam, by Delpini - Tipple Cider. 
1851 ch. c. Stapleton, by Velocipede...... Mr Gully

1852 ch. c. Leander, by Langar.......... Mr Goring

1855 br. c. by Velocipede............ Ld Lichfield

1854 b. c. by Priam (s. to South Carolina)..)

$1855 \mathrm{ch}$. f. by Priam.............. His Majesty

1836 slipped foal by Sultan..........

\section{DEPOSIT (chesnut)}

lired at Farnsfield, in 1825, got by Blacklock, her dam by Comus, out of sister to Zodiac, by St George-A bigail, by Woodpecker.

1851 br. f. Taunton Lass, by Fillo da Puta (put to the stud)

1852 b. c. Tauntonian, by Lambtonian....

1855 ch. c. Tipple Cider, by Defence...... Mr Fox

I854 not corered last year.

1853 h. c. Turncoat, by Swap...........

1856 b. c. Taunton, by Lambtonian......

\section{DESDEMONA,}

Bred by Lord Fitzwillair, in 1811, got by Orville, her dam, Fanny, by Sir I'eter-Diomed-Desdemona, by Marske.

1818 b. f. Amelia, by Raphael...........

1819 bl. f. by Comus............... Ld Fitzwilliam

1820 br. c. by Filho da Puta (died a foal)..

1821 bl. f. Tragedy, by Smolensko...... Sir J. Byng

1822 br. c. Iramatist, by Comus........

1825 b. c. Mulatto, by Catton..........

1824 b. c. by Partisan..............

1826 b. f. Quadron, by Catton.......... Id Fitzwilliam

1828 b. c. Creole, by ditto.............

1829 b. c. by ditto................

$1830 \mathrm{bl.}$ or gr. c. by Chateau Margaux....

$1852 \mathrm{gr}$. f. by ditto (put to the stud)....... Mr Allen

1854 sipped foal by Sandbeck......... Ld Fitzwilliam

Missed in 1825, 27, 51, 55, and $5 \%$.

\section{DESIGN (sister to Dangerous)}

Bred by Mr Sadeer, in 1827, got by Tramp, out of Defiance.

1854 f. by Reveller............. Mr Sadler

185 ร. by Windclife........... Hr Boardman of the

1856 by $A c t x o n \ldots \ldots \ldots \ldots \ldots$ Lnited States 


\section{4}

\section{DESPATCII,}

Bred by Lord Grosvexor, in 1822, got by Blucher, her dam, Iris, by Sir Peter-Isabella, by Eclipse-Squirrel.

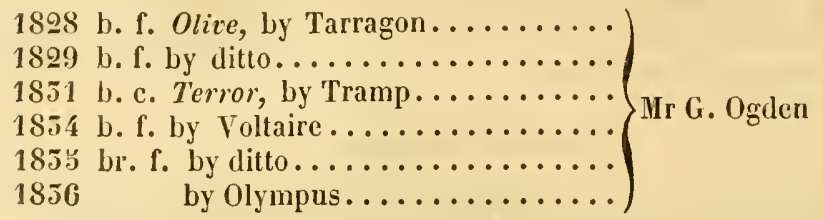

In 1850 missed to Chateau Margaux, 52 to Timour.

\section{DESTINY,}

Bred by the Duke of Graftor, in 1829, got by Centaur, out of Pawn. Junior, by Waxy-Pawn, sister to Penelope.

1855 br. c. Royal George, by Royal Oak....

1834 b. c. by Augustus (died rising 2 yrs old)

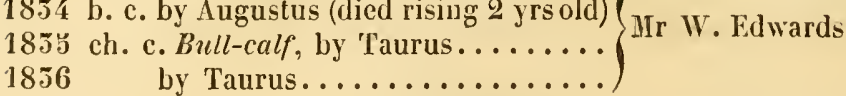

\section{DIAMENTINA,}

Bred by Mr Goldixg, in 1824, got by Rubens, her dam, Canarybird, by Whiskey, or Sorcerer, out of Canary, by Coriander.

1829 ch. c. by General Mina.......... Mr Mostyn

1854 b. c. by Belzoni..............

1855 c. by Priam.............. Mr Poyntz

1856

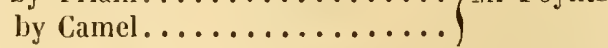

She missed or slipped foal every year from 1829 till 54.

DIANA, by Kiill Devil (Vol. III, p. 99)-add the names Meux to her b. c. by Chorus in 1816, and Flighty to her b. f. by Y. Phantom in 1850 . Flighty is put to the stud.

To the DICK ANDREWS IIARE, St Patrick's dam (Vol. III, p. 100) add.

1852 b. c. (Outcast) by Mulatto......... Sir E. Dodsworth

Iso the name Tartar to her b. c. by Brutandorf in 1829. She is still living, but has had no foal since 1852 .

\section{DICK ANDREWS MARE,}

Bred by Mr Diwson Lambton, in 1812, her dam by ShuttleSir P'eter-l'lay-or-Pay's dam, by Herod-Regulus. 
1813 b. c. by Marnion (killed in 1816)....

1816 a foal, which died ..............

1817 b. c. Alert (afterwards Domain) by Bu-

cephalus (sent to Russia) Mr loftus

1818 b. f. by Whitworth .............

$1819 \mathrm{ch}$. f. by Octavian .............

1821 b. f. by Walton..............

1829 ch. f. Cinderella, by ditto......... Mr Paulden

1824 ch. f. by Sir Oliver $\ldots \ldots \ldots \ldots \ldots \ldots$
1825 gr. f. Fetna, by the D. of Devonshire's

Arabian

1827 b. c. Oldport, by Whisker.........

1898 dead foal, by Sherwood ........... IIr Ormsby Gore

1829 b. c. Agitator, by Waxy Pope.......

1850 ch. f. Indulgence, by ditto ........ Mr Griffiths

1831 ch. f. by The Duke.............

1852 ch. f. by ditto...................

1855 ch. f. by ditto .............. Mr Ormsby Gore

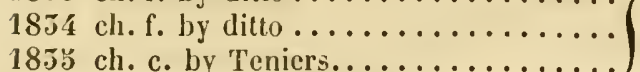

Slipped foal in 1820, missed in 26 , and is dead.

\section{DICK ANDREIVS MARE (sister to Rosanne)}

Bred by Mr Pelrse, in 1812, her dam, Rosette, by Beningbrough

- Rosamond, by Tandem-Tuberose, by Herod.

1819 b. c. Malgwyn, by Sir Oliver.......

1820 b. c. Macloc, by Comus............

${ }^{*} 1824 \mathrm{ch}$. f. Fodlondeb, by Teniers......... Sir T. Mostyn

$182 \mathrm{~b}$ br. f. by Magistratc..............

1826 slipped her foal, by Filho da I'uta....

1829 ch. c. Scrivener, by Banker ....... Mr Turner

1850 b. c. by Waxy l'ope (died)......... Sir T. Mostyu

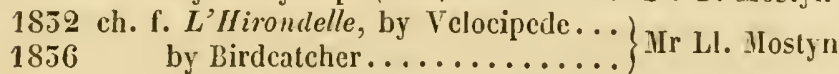

Barren in 1821, 22, and 51 ; missed to Teniers in 27, to Emilius in 28 , to Battledore in 55 , to Velocipede in $\mathbf{5 4}$ and 53.

* Bodloxder missed to Comus in 1829, had a c. by Waxy Pope in 1850 , which died young, and she was sent to Hungary in 1851 with a b. $f$. foal by Lottery.

The DICK ANDREWS MARE, Ferdousi's dam (Vol. III, p. 102) died at Christmas 1852, with a colt in her by Voltaire. The ch. f. by Bedlamite, foaled in 1829, was called Insanity, and sent to Russia in 1834, corcred by Middleton.

vol. 1 . 
DICK ANDREWS MARE,

Bred by Mr Goobrssox, in 181\%, her dam, Hare, by SweetbriarJustice-Chymist.

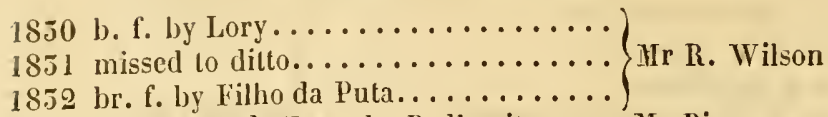

1855 ch. f. March Hare, by Bedlamite .... Mr Biggs

$1854 \quad$ c. by Shakspeare..............

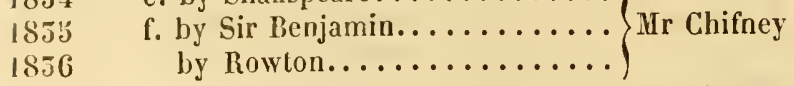

This mare had several foals prior to 1850 , by half-bred horses.

\section{DICK ANDREWS MARE,}

Bred by Sir C. Bunbury, in 1814, her dam, Eleanor, by Whiskey, out of Y. Giantess, the dam of Sorcerer.

1819 br. c. Picton, by Smolensko........ Mr R. Benson

1820 br. c. (Luzborough) by Williamson's $\begin{array}{r}\text { Ditto } \\ \text { MIr Heynell }\end{array}$

1851 b. c. Ganges, by Tigris (s. 10 Germany in 1855$)$ ) Mr Payne

1855 b. f. Ringdore, by Bobadil..........

1854 b. f. Ringneck, by ditto........... Mr Haffenden

1856 by ditto $\ldots \ldots \ldots \ldots \ldots \ldots$. . .

In 1852 missed to Captain Candid, 5y to Bobadil.

This mare was purchased of Sir C. Bunbury by the French Government in 1820, re-purchased by Mr Haffenden early in the year 1851 , in foal to Tigris, and remained in France to foal and be covered by Captain Candid.

DICK ANDREWS MARE (sister to Rob Roy)

Bred by Mr Crockford, in 1814, her dam, sister to Remembrancer, by Pipator, out of Queen Mab, by Eclipse.

1820 br. c. by Pioneer.............. 1821 b. c. by ditlo............. Ur Crockford

1822 b. c. Crockery, by Rubens (sent to $\}$ Gen. Grosvenor

1825 b. c. Iy Comus (dead) ........... Mr Crockford

1824 b. c. by Rubens................

1823 b. c. by Waxlight.................... Mr E. F. Meynell

1826 b. c. by Walton.................

1827 b. c. by Wolul (died young)........ 
*1828 b. f. by Woful..............

1829 b. с. by Gulliver..............

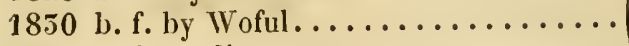

1831 b. f. by ditto............... Mr E. F. Meyneil

1852 b. f. Regret, by $\operatorname{ditto} \ldots \ldots \ldots \ldots \ldots$

1835 br. c. by ditto..............

1854 b. f. by Varro ................

1855 b. c. by ditto (dead)............. Mr Nowell

Shot.

- This Woful mare died at Underley in 1855 , having produced that year a ch. c. by Varro. She had colts by Varro in 55 and 54 , both of which are dead.

\section{DICK ANDREWS MARE,}

Bred by Mr Crockforn, in 1814, her dam, Desdemona, by Sir Peter, out of Heroine, by Phœenomenon-Princess, by Eclipse.

1821 b. c. Bonassus, by Orville, or Castrel.)

1922 b. f. (Ladr Neville) by Orville...... Mrr Russell

1823 b. с. Hardwicke, by ditto .........

1823 br. c. by Abjer ................

1827 b. f. Widgeon, by Whisker........ Col. Peel

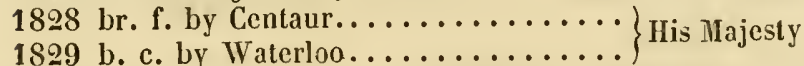

1850 b. f. by ditto (s. to Germany)........ Sir S. Grahau

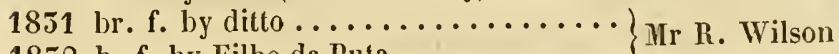

1852 b. f. by Filho da Puta . . . . . . . . . M Mr Biggs

$1854 \quad c$. by Albany .............. Mr Kingston

1856 by Don Cossack ........... Whr Woodin

No foal in 1824 or 26.

The DICK ANDREWS MARE, out of Jemima (Vol. I11, p. 104) had a b. c. in 1852, and died in 55 in foaling.

\section{DICK ANDREWS MARE (sister to Troubadour)}

A Bay Mare, bred by Lord S.Avnwich, in 1815, her dam, Donna Clara, by Cesario-Nimble, by Florizel-Rantipole, by Blank.

1822 b. f. by Partisan.............. Mr Newton

1824 b. f. by Merlin .............. Mr Nowell

1825 b. c. Mufti, by $\operatorname{ditto} \ldots \ldots \ldots \ldots \ldots$. . . Ir Payne 
1826 b. f. by Orville, or Walton .........

1827 f. by Bustard................. IIr Nowell

1828 f. by Muley (put to the stud)......

1829 b. c. by Bustard...............

1830 br. c. Mussulman, by Mlulcy........ Mr Walker $\left.\begin{array}{r}1851 \mathrm{br} \text {. f. Maid of Underley, by ditto (s. to } \\ \text { Berlin in 1854) }\end{array}\right\}$ Hr Etwall

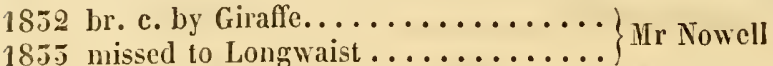

1854 b. c. by Muley............... Mr Osbaldeston

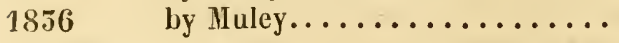

\section{DIDO,}

Bred by Mr Burtox, in 1850, got by Whisker, out of Miss Garforth, by Walton-Hyacinthus-Zara, by Delpini.

1854 b. f. by The Lion..............

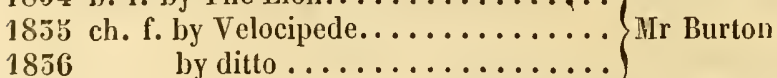

\section{DINARZADE,}

Bred by Mr Haffendev, in 1811, got by Sclim, her dam, Princess, by Sir Peter-Dungannon-Turf-Herod.

1816 b. c. by Election ...............

1818 ch. c. by Juniper (s. to India)....... Mr Haffenden

1820 b. c. by Bramshill...............

1821 b. c. Schahriar, by Shuttle Pope....

1822 br. c. Verderer, by ditto.......... Ir Sadler

1825 br. f. Black Polly, by ditto .........

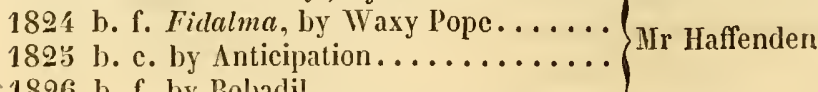

1826 b. f. by Bobadil................

1827 ch. f. Deformity, by ditto......... Mr Molony

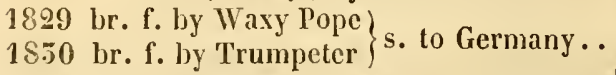

1852 ch. f. (Bequine) by Waxy Pope (put to Mr Haffenden

1855 br. f. by Trumpeter (s. to Spain) ....

1854 br. c. by ditto.................

Not covered in 1816, missed to Poulton in 19, to Manfred in 28, to Waxy Pope in 51, and dicd in Scpt. 1854.

DISCORD, by Popinjay (Vol. III, p. 106) has had only one thorough-bred produce from 1829 to 56 inclusive-viz.

1855 b. c. by Cardinal Puff........... Mr W. Henderson 


\section{DOCTOR SYNTAX MARE,}

Bred by Mr RidDeLL, in 1826, her dam by Shuttle-OvertonBeninglorough's dam, by Ilerod.

1851 b. f. by Remnant...............

1852 b. c. by Malck................

1855 b. f. by Voltaire ............. Mr Chilton

1854 b. f. by ditto (dead).............

185 . b. c. by ditto.................

1856

\section{DOLL TEARSHEET,}

Bred by Lord FoLex, in 1815, got by Sorcerer, her dam, Blowing, by Buzzard-Pot80's - Ilaid-of-all-Work, by Ilighflyer.

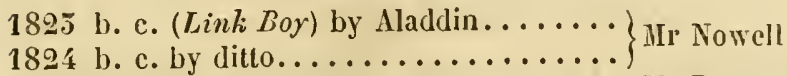

1826 bl. c. Gas, by Walton. ........... Mr Payne

1850 br. c. by Mulcy ................... Ir Nowell

1852 b. f. (Observatory) by Bustard....... Sir M. Wood

1855 b. f. by Longwaist. . . . . . . . .

1854 b. c. by ditto................ Mr Nowell

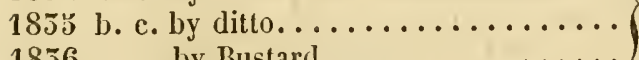

1856 by Bustard .............

Hissed scveral years prior to 1825 ; barren in $25,27,28$, and 29.

\section{DOLLY MOP,}

Bred by Mr Goodlake, in 1828, got by Tramp, her dam by Waxy, out of Thomasina, by Timothy - Violet, by Shark.

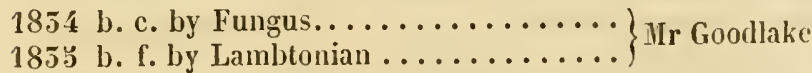

\section{DON COSSACK MARE,}

Bred by Mr Villiers, in 181, her dam by Soreerer-JusticeParsley, by Pot8o's.

1824 b. c. by Vampyre (s. to Van Dieman's Land)

1826 ch. f. by Clavileno............. Ld Clarendon

1827 br. f. by Centaur.

1829 ch. f. Datura, by Reveller.

18.31 b. f. by Tarrare. 


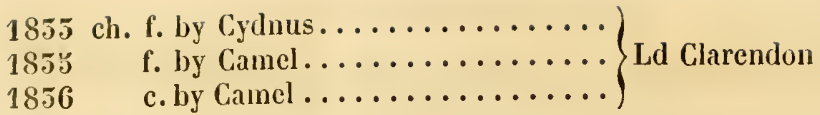

Barren in $1828,50,52$, and 54 .

\section{DON COSSACK MARE,}

Bred by Mr Viluiens, in 1818, her dam, Iris, by Brush. 1897 b. c. Longshanks (aft. Rhag flanied) by $\begin{array}{r}\text { Anticipation } \\ \text { Mr Gully }\end{array}$

1829 b. f. by Manfred .............. Mr L. Charlton

Barren in 1828, and sent to Germany in 29 , covered by Sober Robin.

\section{DON COSSACK MARE,}

Bred by Mr Pryse, in 1821, her dam, Mistake, by Waxy, out of Woodcot, by Mentor - Macaria, by IIerod.

$1850 \mathrm{ch}$. f. Mildred, by Middleton........

1851 b. c. Desperate, by Defence ........

1852 ch. f. by ditto............... Mr Sadler

1855 ch. f. by ditto. ................

1854 missed to ditto...............

1855 b. f. by ditto...............

1856

by $\operatorname{ditt}(\ldots \ldots \ldots \ldots \ldots \ldots \ldots$ Baron Biel

\section{DON JUAN MARE,}

Bred by Iir Watron, in 1895, her dam, Moll-in-the-Wad, by Ilambletonian-Spitfire, by Pipator-Farewell, by Slope.

1828 br. c. Giovanni, by Filho da Puta....

1829 br. c. Gambol, by ditto............ Mr Houldsworth

1850 b. c. by Sherwood.............

1852 b. c. The Polentate, by Langar...... Mr T. 0. Powlett

1854 gr. or bl. f. by Jack Spigot......... Ld Chesterfield

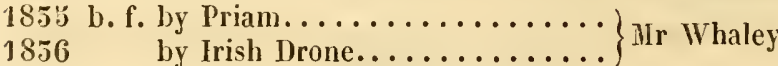

In 1851 missed to Buzzard, not covered in 52.

DONATION,

Bred by Mr Ridsdale, in 1826, got by Borodino, out of Camerton's dam, by Precipitate-Magnolia the Younger, by Pegasus1. Magnolia, by Highnlyer. 
$1832 \mathrm{gr}$. c. by Grey Walton (Peirse) ......

1855 gr. c. by ditto............................... John Newton

1854 gr. f. by ditto................

1836

by Tramp.................

In 1831 missed to $t_{1}$ ottery, $5 \mathrm{~s}$ to Humphrey Clinker.

DORIN.I (Vol. III, p. 110)-add the name Etona to her b. f. by Rubens in 1828. - Etona is put to the stud.

\section{DOUBLE ENTENDRE,}

Bred by Mr Laxe Fox, in 1821, got by Comus, her dam, Trictrac,

by Dick Andrews, out of Pope Joan, by Waxy-Prunella.

1829 ch. c. (Peregrine Pickle) by Rubens...)

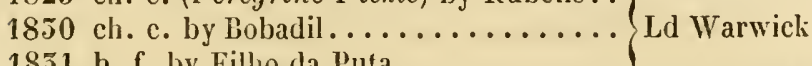

1851 b. f. by Fillıo da Puta...........

Barren in 1852 and 53 , cast lier foal in 54 , and died.

\section{DREAII,}

Bred by Lord Dormay, in 1825, got by Soothsayer, her dam, Wathcote Lass, by Remembrancer-Walnut-Ruler.

1827 ch. c. Dreamer, by Middleton.......

1828 ch. f. Drowsy, by Mloses......... D. of Richmond

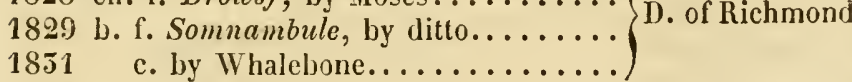

1952 c. by Camel.............. D. of Holstein

Somnambule and her dam were both sold to the D. of Holstein - the former ran in Germany.

\section{DRYAD,}

Bred by I.ord Egreyoxt, in 1829, got by Whalebone, her dam, Harpalice, by Gohanna, out of Amazon, by Driver.

1854 b. c. by Augustus, or Bedlamite .....
1855 b. f. by Shakspeare............. Sir G. Healhcote

\section{YOUNG DUCHESS,}

Bred by Mr Nowell, in 1826, got by Walton, her dam, Browu Duchess, by Orville, out of Sagana, by Sorcerer-Woodpecker.

1851 b. f. by Longwaist (put to the stud)... Mr Nowell

1852 b. c. Longinus, by ditto.......... D. of Leeds 


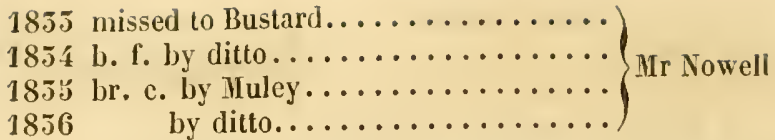

\section{DUCKLING,}

Bred by

in 1821, got by Phantom, her dam by Orville-Hambletonian-Shutlle-Overton.

$\left.\begin{array}{l}1850 \text { br. f. by Emilius.. } \\ 1851 \text { br. f. by Blacklock. }\end{array}\right\}$ put to the stud.. \} Mr Grant

1952 b. c. Advocate, by Partisan..... . . . Ir Biggs

Dead.

\section{DULCAMIRA (the last of Waxy's get)}

Bred by Lord Rous, in 1818, got by Waxy, her dam, Witchery, sister to Sorcery, by Sorcerer, out of Cobbëa, by SkyscraperWoodpecker-IIeinel, by Squirrel.

1825 ch. c. by Quiz............... Mr E. F. Meynell 1824 b. f. Souvenir, by Orville ......... Mr S. Stonehewer

${ }^{4} 1829$ b. f. Salute, by Muley $\ldots \ldots \ldots \ldots \ldots$ b. f. Solace, by Longwaist........ Mr Dilly

1851 b. c. (Vestment) by ditto......... D. of Cleveland

1854 f. by ditto..............

1853 ch. c. loy Muley .............. Mr Nowell

1856

by ditto.................

In 182\% missed to Walton, in 26 to Walton and Orville, 27 to Orville and Bustard, 28 to Muley and Mladdin, 52 to Longwaist, 53 to Muley.

* Salute produced a b. f. by Sultan in 1855 , and was covered again by him.

\section{DULCINEA,}

Bred by Mr Sissor, in 1816, got by Cervantes, her dam, Regina, by Mloorcock-Rally, by Trumpator-Fancy, sister to Diomed.

1821 ch. f. by Interpreter (died 2 yrs old).. 1822 b. c. Redganntlet, by Scud .........

1826 b. f. Green Mautle, by Sultan (put to the stud) $>$ Ld Exeter

1827 b. c. by ditto (died 5 weeks old)..... 1828 b. f. by Catton................ 1829 ch. c. Spencer, by Sultan ......... 
*1850 b. f. Mantilla, by Sultan.........

1851 b. c. by ditto (died young) .........

1832 ch. f. by ditto (put to the stud) .......

1835 b. f. by ditto............... Id Exeter

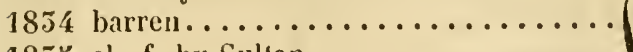

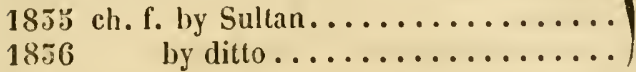

She was used as a hack between 1822 and 26.

* Mavtilla pro:luced a f. by The Colonel in 1855 , and was covered by tetæon.

\section{DUPLICATE MARE,}

Bred by Mr PaLis, in 1S25, got by Mr Tomes's Duplicate, out of Laura, by Beningbrough-Teddy the Grinder.

1828 b. f. by Sherwood .............. MIr Bloor

1850 b. f. by Banker (dead)............

1851 b. f. Zara, by Camel............ Mr Palin

1832 br. c. by Peter Lely.............

1853 ch. f. by ditto ...............

Sent abroad.

The EAGLE MARE, dam of Nessus (Vol. III, p. 114) had no foal after 1851, and is dead. Her two last foals should stand thus :

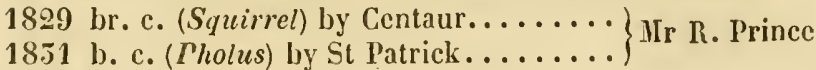

\section{EAR WIG,}

Bred by Mr Tronxurte, in 1828, got by Emilius, out of Shoveler, by Scud-Goosander, by IIambletonian-Rally, by Trumpator.

1855 ch. c. Arbaces, by Merchant........ Ld Lichfield

1854 ch. c. by Merchant.............

1855 missed to Merchant................. Mr Thornhill

1856 missed to St Patrick............

\section{EATON MARE,}

Bred by Mr Nevilee, in 1817, her dam by Sorcerer, out of sister

to Rumbo, by Whiskey -Spinetta, by Trumpator-IIerod.

1819 b. c. by King Richard...........

1820 b. c. by ditto................ 
1826 ch. c. Zodiac, by Centaur........

1827 ch. c. by Doctor Syntax...........

1828 bl. c. Galopade, by ditto (s. to Hungary

1829 ch. c. Favori, by Whisker..........
1850 b. c. Ticket, by Lottery ..............

1854 ch. f. Polonaise, by Doctor Syntax... MIr Greville

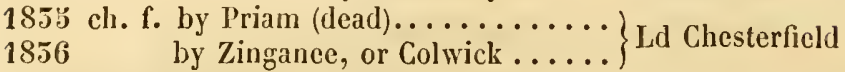

In 1851 missed to Dr Syntax, 52 to Lottery, 53 to Velocipede.

\section{EDITH,}

Bred by Sir Thouns Sranley, in 1819, got by Fitzjames, her dam, Maid of J„orn, by Castrel-Richardson's Marske.

1824 ch. f. by Strephon..............

1823 ch. c. Herbert Lacy, by Sir Oliver ...

1826 b. f. by Master Henry...........

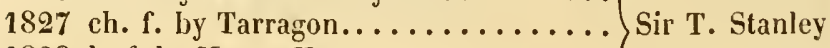

1828 b. f. by Master Henry. . . . . . . . . . . .

1829 barren or foal died.............

1850 br. c. Zohrab, by Filho da Puta .....

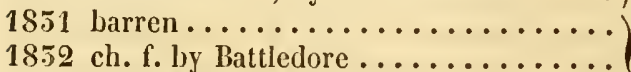

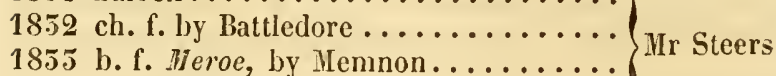

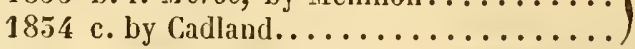

1853 by Flexible..............

\section{EFFIE,}

Bred by Sir Nicliolas Loftes, in Ireland, got by Pickpocket, her dam by Master Bagot, out of Commodore's dam, by the Duke of Kingston's Scaramouch-Blank-Old Traveller.

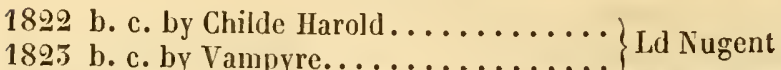

*1824 br. f. Victim, by ditto............ Mr Hervey

1826 by Childe Harold ........... Nr E. Tattersall

1827 ch. c. by Tancred.............

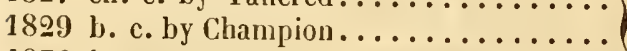

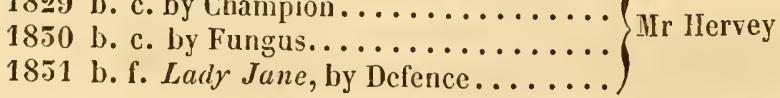

\footnotetext{
* Victim produced Mr Hervey's ch. c. Endeavour, by Phantom, in 1829, and has not been put to horse since.
} 
1852 b. c. by Fungus (dead)............

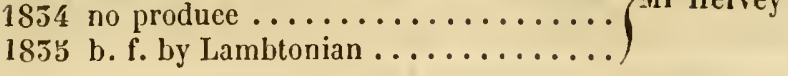

EFFIE,

Bred by Mr Naxxey, in 1824, got by Catton, her dam, Fanina, by Sir Solomon-Fan, by Beningbrough-Miss Judy, by Alfred.

1851 missed to Lottery ..............

1852 ch. f. Ayesha, by Champion..........

1855

IIr Nanney

1855 eh. e. by Belzoni.

\section{EFFIE DEANS,}

Bred by Mr Bronl, in 1816, got by Ashton, her dam, Harriet, by Sir IIarry-Dorimant-II usc, by Herod-Shepherd's Crab.

1823 b. f. Profile, by Rubens ..........

1824 b. c. Windermere, by Whalebone....

1825 b. f. by Don Cossack............. Mr Radclyffe

1826 b. c. Windrush, by Whalebone .....

1828 b. f. Susanne, by Figaro .........

1829 b. f. Kate, by Lapdog............ Mr Mills

1850 b. c. by ditto................. Ir Sloane Stanley

1851 b. c. (Patapan) by ditto ..........

1852 b. f. Hiss Patch, by ditto ...........

1854 br. c. by Peter Lely.............. Mir Wills

1855 b. f. by Lapdog.

1856

by Defence. . . . . . . . . . . . .

In 1827 missed to Reveller; barren also in 55.

\section{EGLINTON MIARE,}

Bred by Sir R. K. Dick, in 1828, her dam, Bravura, by Outery, out of Prodigious, by Caleb Quotem-Fair Forester, by Alexander.

1851 gr. c. by Merlin...............

1853 ch. e. Professor, by Wiseacre........ MIr B. Greene

1855 f. by ditto $\ldots \ldots \ldots \ldots \ldots \ldots$.

She had no produce in 1854 , and was shot in 55 . 


\section{6}

\section{ELEANOR,}

Bred by the Duke of Haxuton, in 1815, got by Governor, her dam, Elizabeth, by Spadille-Dungannon-Pastorella, by Otho.

1821 b. c. Footman, by Filho da Puta, or $\begin{array}{r}\text { Hetman } \\ \text { Mr Burgess }\end{array}$

1822 br. c. Ifis Grace, by Filho da Puta, or Magistrate (s. to Prussia in 1826)

1823 b. f. Meeta, by Filho da Puta........ Mr Houldsworth

1826 br. f. Elve, by Sherwood ..........

1828 b. c. Butcher Boy, by Catton......... Mr Broomhead

1829 b. f. Wastrel, by ditto........... Sir J. Hilton

1852 b. f. by Lottery................ Mr. W. Turner

Not covered in 1826 , missed to Lottery in 1850 , to Filho in 51 , barren also in 55,54 , and 55 .

\section{ELEANOR,}

Bred by Mr Keex, in 1826, gol by Muley, her dam, by SanchoSir Harry-Florizel-Nosegay, by Justice.

1855 b. f. by Memnon. ..............

1854 ch. f. by Patron................ IIr Painter

185 y ch. f. by Emancipation ...........

1856

by ditto

\section{ELECTA,}

Bred by Mr Stinura, in 1817, got by Election, her dam, Miss Manager, by Giles, out of Rosabella's dam, by Diomed-Harriet, by Matchem-Flora, by Regulus.

1826 ch. c. Aaron, by MIoses...........

1828 b. f. by Middleton..............

1850 b. c. by ditto (died 2 yrs old)........ Mr Stirling

1852 ch. f. by Irameluke.............

1854 b. c. Aaronides, by Aaron..........

In 1851 and 53 missed to Phantom, in 55 to $\Lambda$ aron, and not covered that year.

The ELECTION MARE, Gayhurst's dam (Vol. III, p. 118) died in 1852, in foal to Gaberlunzie. The sister to Gayhurst, foaled in 1850, was called Nuthurst, and is now a brood mare. Gayhurst was sent to Jamaica in 1851, and Spree to the D. of Holstein in 1852. 


\section{ELECTION MARE (sister to Maron)}

Bred by II. R. H. the D. of York, in 1820 , her dain by Sorcerer, out of Black Diamond, by Stamford-Louisa, by Ormond.

$\left.\begin{array}{l}1826 \text { b. f. by Moses.... } \\ 1827 \text { ch. f. ly Waterloo. } \\ 1828 \text { ch. c. by ditto.... }\end{array}\right\}$ dead......... IIis Majesty 1850 b. c. by ditto................ Sir M. Wood ${ }^{*} 1851$ b. f. Antwerp, by ditto ............
1852 b. c. Dunois, by Filho da Puta..... Wilson Missed to Waterloo in 1829, and died in 55.

* Astwenp produced, in 1855, b. c. Arcos, by Belzoni.

\section{ELECTION MARE,}

Bred at Hampton Court, in 1815, her dam, Fair Helen, by Hambletonian-Helen, by Delpini-Rosalind, by Phœnomenon.

1820 br.c.(Chatham) by Waterloo, or Blucher Mr Prendergast

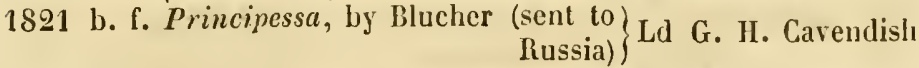

1822 ch. c. by Soothsayer............ His Majesty

1825 foal by Rainbow (dead)...........

1824 ch. f. by Comus .................. Mr Nowell

1825 b. c. by Walton (s. to D. of IIolstein in 1852)

1826 b. c. by Orville............... Mr Payne

1827 b. c. by ditto $\ldots \ldots \ldots \ldots \ldots \ldots \ldots \ldots \ldots \ldots \ldots \ldots \ldots \ldots \ldots$ Mr Nowell

1829 ch. c. Margrace, by ditto......... Mr Dilly

1850 ch. c. by ditto............... Sir Mark Wood

1855 b. c. by Muley ............... Mr Greatrex

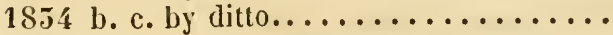

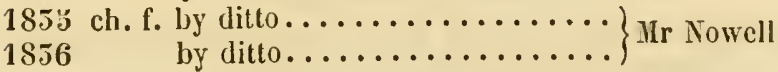

In 1851 missed to Muley, 52 to Bustard.

\section{ELECTION MARE (Eleanor)}

Bred by Mr Rubee, in 1816, her dam, Emily, by Y. Whiskey, out of Amelia, by Highflyer-Miss Timms, by Matchem.

1851 b. c. Wanderer, by Wanderer....... Mr Boulding

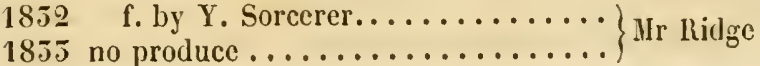

vol. IV. 


\section{YOUNG ELECTION MARE,}

Bred by Mr Stralixg, in 1827, her dam, Miss Manager, by Giles.

1850 b. f. by Phantom .............

1855 b. f. by ditto............... IIr Stirling

1853 ch. c. by Aaron. . . . . . . . . . . .

Not covered in 1850 ; missed in 52 and 54 .

\section{ELECTRESS (sister to Regent)}

Bred at Hampton Court, in 1819, got by Election, her dam by Stamford, out of Miss Judy, by Alfred-Manilla, by Goldlinder.

1827 slipped twin colts by Smolensko..... His MIajesty

1828 br. c. (Walter) by Whalebone....... MIr D. Radeliffe

1829 br. f. Landgravine, by Waterloo, or $M r$ Greville Smolensko (died on the road to Berlin)

1851 b. f. Rosalie, by Whalebone........ Col. Peel

1835 ch. c. by Bedlamite............. Mr R. Wilson

1834 b. f. Rosabel, by Shakspeare........

185 s cl. c. Vauban, by Defence........ Col. Peel

1856 slipped foal by Camel ...........

In 1825 missed to Woful, 26 to Waterloo, 50 to Whalebone, 52 to Filho da Puta.

\section{ELFRID (sister to Gaberlunzie)}

Bred by Lord Egresort, in 1820, got by Wanderer, her dam by Selim, out of Maiden, by Sir Peter.

1827 b. f. (Elfrida) by Whalebone........ 1829 br. f. Gipsy, by ditto (s. to France. October 1854)

1851 b. f. by ditto................ Id Egremont

1852 b. f. Ethilda, by Skim.

1855 br. f. IIelga, by Chateau Margaux ...

1854 ch. c. by Skim .................

Misserl to Whalebone in 1828 and 50. 


\section{ELFRIDA,}

Bred by Lord Egrenovt, in 1827, as above.

1851

1852 ch. c. by Cade (an Arabian)........ His Majesty

1833 b. c. New Light, by Lamplighter . . . . Col. Peel

1854 dead foal, by Helenus............

1855 b. f. by Tranby............... Ilis Majesty

1856

by Priain

\section{ELIZA,}

Bred by II. R. If. the Duke of York, in 1813, got by Rubens, her dam, Aladdin's dam, by Walnut-Javelin .

1817 b. c. by Woful ................

1819 gr. c. by Y. Gohanna ............ H. R. H. D. of York

1820 c. by

................

1821 b. f. Itty Pet, by Blucher.......... Ir Benson

1822 b. f. Conquest, by Waterloo ....... Ld Palmerston

182.5 b. c. by Anticipation. . . . . . . . . IIr Day

1824 b. f. by Rainbow.............. Ld Orford

1823 b. c. Grampian, by Walton ........ Mr Gully

1826

1827

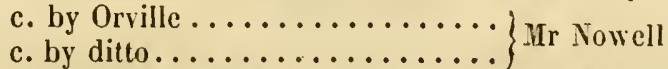

1828 b. f. Gawky, by MIuley........... Sir M. Wood

1829 ch. c. Count liobert, by ditto ....... Mr Cosby

1850 foal, by ditto (died) ............. Mr Noweil

1832 b. c. Shorthopes, by Longwaist ..... Mr Wright

1834 b. f. by ditto ................ Mr Nowell

Hissed to Muley in 1851 and 55 , and died in 54 , in foal to him.

\section{ELIZA, (sister to Defiance)}

Bred by Mr Sadeer, in 1825, got by Rubens, her dam, Little Folly, by llighland Fling-Harriet, by Volunteer.

1829 b. f. Eleanor, by Middleton (s. to Berlin)

1851 br. f. by Tramp...................... IIr Sadler

$1852 \mathrm{ch}$. f. by Defence .............

1855 c.......... $\}$ by ditto, or Windeliffe \} Mr Kirkman

Barren in 1850,55 , and 54 ; sent to Alabama, in the United States, in Aug. 1855, with the colt foal, and covered by Emilius. 
ELIZA,

bred by Mr N. II. SuıтI, in 182.5, got by Smolensko, her dam, Epsom Lass, by Sir Peter-Alexina, by King Fergus.

1828 c. by Figaro (died soon after foaling)

1850 b. f. Mliss Charlotte, by Pantaloon ...

1855 ch. f. Columbine, by ditto......... IIr Giffard

1854 b. f. by Cadland ...............

185 s ch. f. Emancipation ............

1856 by Pantaloon..............

Missed to Emilius in 1829 , to Leviathan in 51 , and not covered that year.

\section{ELIZA,}

Bred by Mr L. Heselrine, in 1825, got by Filho da Puta, her dam by Vermin-Beningbrough - Eustatia, by IIightlyer.

1855 b. f. Worlaby Witch, by Brutandorf. . Mr Skipsey

1854 b. c. by ditto..................

185 f. by Revenue................... Mr L. Heseltine

1856

by Physician..............

\section{ELIZA LEEDS,}

Bred by IIr C. IIARrison, in 1817, got by Comus, her dam, IIclen, by Hambletonian, out of Susan, by Overton-Drowsy, by Drone.

$\left.\begin{array}{r}1823 \text { b. f. Lady Eliza (aft. Merrytricks) by } \\ \text { Filho da Puta }\end{array}\right\}$ Mr Græme

1826 dead foal, by Blacklock..........

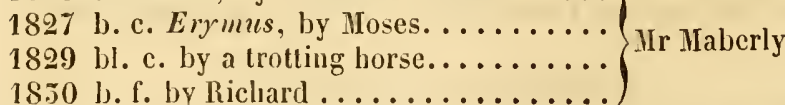

1832 b. f. Eva, by Sultan............ Ld Chesterfield

1855 cl. c. St Luke by Bedlamite........ Mr Sowerby

1854 b. c. by Shakspeare ............

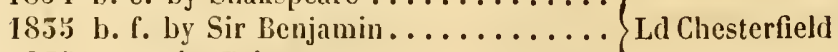

1856

Barren in 1828 and 51.

\section{ELIZABETH,}

Bred by Mr Gascorgne, in 1821, got by Walton, out of Trulla, by Sorcerer, grandam by Weathercock, out of Cora, by Matcliem. 


\section{1}

*1828 br. f. by Lottery ..............

1829 br. f. Isabel, by Catton..........

1851 br. c. Zohrab, by Lottery......... Hr Gascoigne

1855 b. f. Thomasina, by Brutandorf.....

1834 bl. f. by Jerry ...............

1856

by Starch .................

In 1850 missed to Whisker, 52 to Velocipede, 55 to Brutandorf.

* This Lottery mare produced Mr Fox'sbl. c. T\%e Doctor, by Doctor' Syntax, in 1854, missed ia 55 and 55 , and was covered in 55, by Starch.

\section{ELIZABETH,}

Bred by Mr Gisborve, in 1822, got by Mango, her dam Stamfordia, by Stamford, out of Legacy, by King Fergus.

1822 b. c. by Bonassus..............

1830 b. c. by Champion (s. to Constantinople) UIr T. Critchley

1851 b. f. by Bonassus..............

1852 ch. f. Emily, hy Pantaloon ......... Mr Jones

1835 b. c. by Memnon ............. Mr Gritchley

Died Aug. 1855.

\section{ELIZABETH,}

Bred at Ilampton-Court, in 1822, got by Soothsayer, her dam,

Grey Ducliess, by PotSo's, out of Duchess, by Iferod-Blank.

1826 br. f. by The Grand Duke.......... Mr R. Barnard

1829 b. f. by Haster Ilenry . . . . . . . . . . Mr Bach

1850 br. c. Inflexible, by Flexible.......

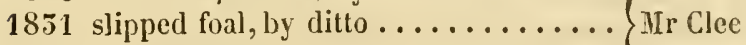

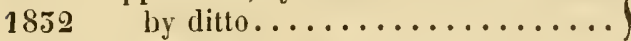

185.5 b. c. by Pollio (s. to Germany, 1S.5.). ' IIr 'Tattersall

1855 b. f. by Moses................. Ir Tattersal

This mare was sold to the D. of Holstein in 1854 , covered by The

Colonel; was barreı, and sent back, covered by Moses-she died in 1855 .

\section{ELIZABETH,}

Bred at Hampton-Court, in 1S9j, got by Rainbow, her dam, Belroiriua, by Stamford, out of sister to Silver, by IIercury.

1828 b. f. Lisbeth, lyy Phantom..........

1829 ch. f. by Merlin (s. to Florence in 1851) His Majesty

1830 b. f. by Waterloo ............. 
1831 b. c. by Phantom ............. Mr Harvey

1853 ch. f. Dorothy, by Whisker......... Ld Orford

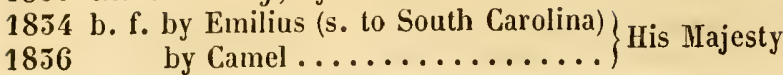

In 1853 missed to Camel, 58 to Sultan.

\section{ELIZANNE,}

Bred by Mr Houndworti, in 1827, got by Filho da Puta, her dam by Stamford, out of Miss Buckle, by Precipitate-Highnyer-Goldfinder.

1832 br. c. Camelopard, by Camel, or

1853 b. c. Y. Camel, by Camel...........

1834 missed to Mameluke ............

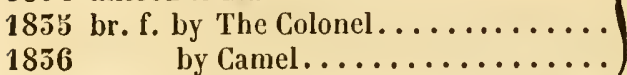

ELLEN, by Y. Woodpecker (Vol. III, p. 125) had no thoroughbred foal after 1829 , and died in 1853 .

\section{EMILIA,}

Bred by Mr Udxy, in 1825, got by Abjer, out of Emily, by Stamford -Whiskey-Grey Dorimant.

1827 ch. c. by Woful

1829 b. f. by Jerry (died at foaling) .......

1850 br. f. by ditto................

1831 br. f. (Amulet) by ditto (put to the stud)

1835 br. f. by Jerry (died a foal)......... Ld Kelburne

1834 b. c. by ditto (died a foal).........

183 b. c. by ditlo....................

In 1828 missed to Whisker, and 32 to Actæon.

\section{EIILIUS MARE (sister to Agrecable)}

Bred by Mr Thornhll, in 1828, her dam, Surprise, by Scud, out of Manfreda, by Williamson's Ditto-Tawny, by Mentor.

1834 b. c. Lent, by Rowton............

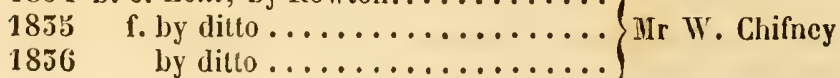


EMILY, by Stamford (Vol. III, p. 125-4). Her last two produce should stand thus :-

1899 ch. f. Amphitrite, by Tiresias (s. to Ger.)

1830 b. c. Autocrat, by ditto Inany) D. of Portland

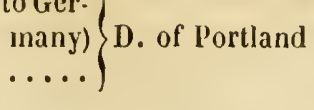

\section{EMIIL,}

Bred by Mr Garforte, in 1817, got by Don Cossack, her dam, Vesta, by Delpini, out Faith, by Pacolet-Atalanta.

1825
1826
gr. f. by Cervantes (put to the stud)... Sir Tatton Sykes

1827 ch. c. Revolution, by Oiseau ....... Mr Shepherd

1851 gr. c. by Comus ...............

1832 ch. c. Sledmere, by Velocipede..... Sir Tatton Sykes

1834 ch. f. by Comus ...............

In 1828 and 50 missed to Y. Phantom, and in 29 had produce by him, which died; barren in 55 and 55 .

\section{EMMA (sister to Master IIenry)}

Bred by Mr Elwes, in 1819, got by Orville, her dam, Miss Sophia, by Stamford-Sophia, by Buzzard-Highflyer-Cypher.

1825 b. f. by Skim (dead) ............

1826 ch. c. The Lion, by Tiresias .......

1827 ch. f. by Woful. ...............

*1828 br. f. Lioness, by Tiresias.........

1829 b. f. (IIornet) by Partisan (put to the stud)

1850 b. f. by ditto (s. to Germany) ....... Mir S. Stonehewer

1851 b. f. Zulima, by Sultan ...........

1852 b. f. by ditto................

1855 b. f. by ditto.................

1854 gr. c. by Gustavus...............

1855 ch. f. by Zinganee.............

1856 by Buzzard ..............

* Liovess is in Lord Lichfield's stud; she produced a ch. f. Kathleen, by Sligo, in 18.34, missed in 33 and 35 , and was covered in the latter year, by Emancipation.

ЕММム,

Bred by the Trustees of Lord Stratunore, in 1824, got by Whisker, her dam, Gibside Fairy, by Hermes, out of Vicissitude, by Pipator-Beatrice, by Sir Peter. 


\section{4}

1829 ch. c. Trustee, by Catton (sold to Mr

Corbin, of Virginia, in 185\%) Mr Ridsdale

$1850 \mathrm{ch}$. f. Agnes, by Blacklock (put to the stud)

1851 b. c. Guardian, by Catton (s. to Hun- D. of Cleveland gary in 1854, and died there) $\}$

1852 ch. c. Mündig, by ditto..........

1855 ch. f. by ditto (died a foal) $\ldots \ldots \ldots$.

1854 ch. c. by ditto................ Mr Bowes

185 . ch. c. by $\Lambda$ ctæon..............

1856 c. by Priam..............

\section{EMIIA,}

Bred by Major Jonssos, in Ireland, in 1817, got by Fitz-Emily, out of Cutlass, by Whiskey-Highflyer-Marske.

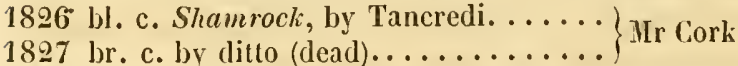

1828 bl. f. by ditto ..............

1829 hi. f. by ditto ............... Hr G. Booth

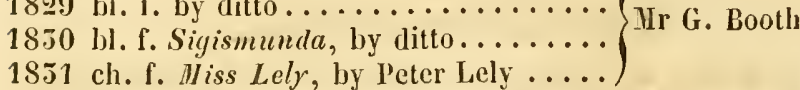

1853 ch. c. Fitz-Emma, by Doncaster.... Mr W. II. Bainbrigge Barren in 1852; hunted in 55 and 54.

\section{EHMELINA,}

Bred by Mr Petre, in 182y, got by Blacklock, out of Agatha, by Orville-Star-Y. Marske-Emma, by Telemachus.

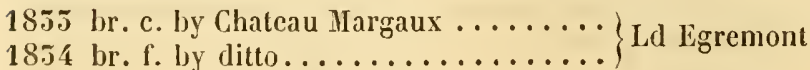

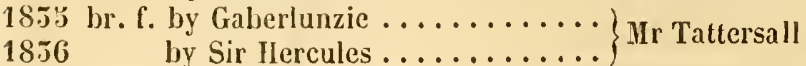

\section{EHMELINE,}

Bred by the Duke of Rotuavo, in 1817, got by Waxy, her dam, Sorcery, by Soreerer, out of Cobbëa, by Skyscraper-Woodpecker-Heinel, by Squirrel-Blauk, etc.

1822 b. c. (Young Phantom) by Phantom.. \}D. of Rutland

1825 l. f. by Partisan..............

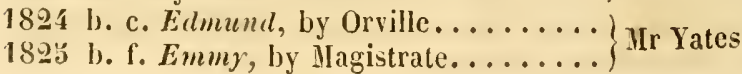


1827 b. c. Edgar, by Paulowitz......... *1828 b. f. Edith, by Swap, (s. to Baron Bicl) Col. Peel 1829 b. c. Ernost, by Paulowitz, or Bedlamite

1850 b. c. Sir Robert, by Sultan......... Ld Exeter

1852 b. f. Evclecn, by Bedlamite, or Belzoni (sold to Baron de Maltzahn)

1854 twins by Priam (dead) ........... Mr E. P'eel

1855 b. f. by Bedlamite..............

1856 br. c. by Belzoni..............

In 1826 missed to Waterloo, 31 to Sultan, barren also in 55.

* Edrru produced a f. by Bedlamite in 185.5, and was sold to Baron Biel, and sent to Germany.

\section{EIIIIY,}

Bred by Mr Yates, in 1825, got by Magistrale, her dam, Emmeline, by Waxy, out of Sorcery, by Sorcerer-Cobbëa, by Skyscraper.

1851 b. c. Ince, by Battledore..........

1852 b. c. Irwell, by ditto......................... Mr Yates

She had no produce that lived in 1854 or 55 , and was sold in the Iatter year to Col. Hampton, of Charleston, covered by Camel.

EOINA, by Haphazard (Vol. III, p. 126) was sent to Vicnna in 1832, covered by Lottery. IIer produce of that year should stand thus :--

1852 br. c. Northumberland, by Percy .... Mr G. Dawson

\section{ESCAPE,}

Bred by Mr WARE, in 1818, got by Colossus, her dam, Columbine, by Skyscraper-Fidget-sister to Pharamond, by Jlightlyer.

1827 b. c. by Anacreon.............

1829 b. โ. by Middleton............. Mr Fellowes

1850 no foal.....................

1851 br. c. Freedom, by Sherwood (s. to Germany in 1855)

1852 b. c. Free will, by Sultan..........

18.5.5 c. Freeholder, by Luzborough...... Mr Wreford

18.54 b. c. Free-agent, by Sultan.........

18.53 b. c. by Defence...............

1856 not covered last year............ 


\section{ESIIERALDA (sister to Granby)}

Bred by Mr Fryat, in 182\%, got by Cannon-ball, her dam, Shoehorn, by Teddy-tle-Grinder, out of Roxana, by $\Lambda$ lexander.

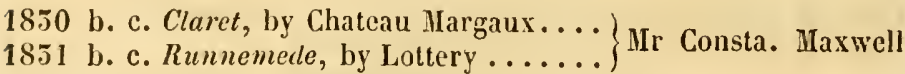

1855 b. f. by Brutandorf, or Y. Phantom...

1855 b. f. by Brutandorf............. Mr S. Templeman

18.36

by Mulatto...............

In 1852 missed to Tramp, 54 to Lottery.

\section{ESPAGNOLLE,}

Bred by Lord G. II. Cavenosm, in 1816, got by Orville, her dam,

Barrosa, by Vermin, out of Nike, by Alexander-Nimble.

1822 b. c. (Paymaster) by Partisan....... Mr Thompson

1825 b. f. by ditto (put to the stud) ......

1824 ro. c. Rapid Rhone, by ditto....... Ld G. H. Cavendish

1826 br. c. Spaniard, by Godolphin......

1828 b. f. Y. Espagnolle, by Partisan.....

1829 dead foal, by ditto .............

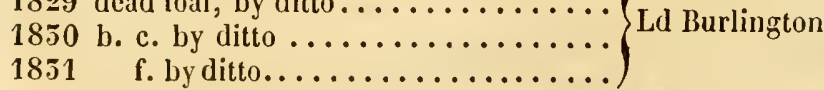

1852 b. c. Tiber, by ditto............ Mr SI. Stanley

1855 b.f. Piccolina, by ditto (put to the stud) Sir G. Heathcote

1853 b. c. Tivoli, by ditto........... D. of Rutland

Barren in 1825 and 27, slipped foal in 1854.

\section{Y. ESPAGNOLLE,}

Bred by Lord Burlixgron, in 1828, out of the preceding mare.

1854 b. f. by Bizarre...............

$185:$ l. c. by ditto.................. Ilis Majesty

18.56

by $\operatorname{Logic} . \ldots \ldots \ldots \ldots \ldots$........

\section{ESPERANCE,}

Bred by Mr Lautour, in 1827, got by Partisan, out of Sleight-ofhand, by Sorcerer-Trollop, by Waxy-Trull, by Voluntecr.

1855 cls. f. Sybil, by Nigel

18.54 ch. c. Glenvarlock, by ditto........

18.5:5 missed to ditto................. Mr Lautour

18.56

by Nigel, or Lugustus.......... 
THE ETCIING,

Bred by Mr Gaunteetr, in 1826, got by Rubens, her dam, Lamas, by Gohanna, out of sister to Chester, by Sir Peter.

1830 ch. c. Farlington, by Luzborough....

*1851 b. f. Barbiche, by Lapdog........ IIr Gauntlett

1852 dead foal, by Waterloo ...........

1835 f. by 'The Colonel (dead) .........

1854 c. by Camel................ Mr Wright

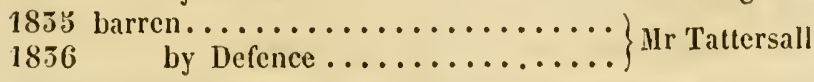

* Barbicue produced a f. Dejanira, by Sir IIereules, in 1855.

\section{ETIOUETTE,}

Bred by Lord Grosveror, in 1820, got by Orville, her dam, Boadicea, by Alexander, out of Brunctle, by Amaranthus - Maylly.

1826 b. f. by Sir Gilbert............ Ld Grosvenor

1827 br. f. Frail, by Filho da Puta....... MIr Bloor

$18 \supseteq 8$ b. c. Master of the Ceremonies, by Cham- $\begin{gathered}\text { pion } \\ \text { Ld Grosvenor }\end{gathered}$

1829 br. f. Maid of Ilonor, by ditto (put to

the stud)

1830 br. c. The Controller, by Filho (s. to Ld Westminster

1851 dead foal, by Camel...............

In 1832 missed to Peter Lely, 35 to Teniers, 54 to Pantaloon, and is dead.

IIr Allen's EVANDER MARE (Vol. III, p. 487) produced a gr. c. in 1852, missed the three following years, and was covered by Physician in 1835 .

\section{EUCROSIA,}

Bred by Mr Massers, in 1825, got by Walton, her dam, Emma, by Ilambletonian, out of Margaret, by Beningbrough-Roxana, by Sir Peter.

1828 b. c. Shrigley, by Macduff..........

1832 ch. c. Equator, by Battedore........ Mr W. Turner

185 s. ch. f. by ditto, or Recorery.........

She was barren in 1850 , and her produce in $29,51,55$, and 54 , were born dead or died very young. 


\section{8}

\section{EVENS,}

Bred by Mr Fergtsox, in 1819, got by Walton, her dam by

Sancho, out of Mliss Furey, by 'Trumpator-Mark Anthony.

1823 ch. c. by Blacklock ............

*1826 b. f. by Waverley ............. Capt. Ramsden

1827 l. f. by ditto...............

1829 b. c. by Brutandorf.............. Ld Mountcharles

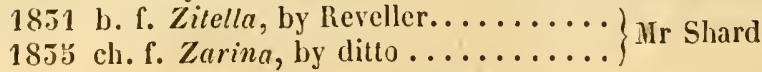

Barren in 1828, $50,51,52$, and 55 .

* This Waverley mare produced in 1855 a br. f. Ianthe, by Belzoni, and was sent to France in Oct.1854, covered by Oppidan. Ianthe was sold to the D. of IIolslein in 1835.

\section{EUPHRASIA,}

Bred by Mr Dundas, in 1815, got by Rubens, lier dam, Witch of Endor, by Sorcerer-Delpini-Ld Carlisle's Squirrel.

1825 ch. f. Jutiana, by Pyramus ....... Mr MIorris

1824 ch. f. Margaret, by ditto.........

1825 br. c. by Robin Adair (s. to France)..

1826 ch. c. IIonest Robiu (aft. Y. Rubens) by MIr Dundas

ditto

*1827 ch. f. Adelaide, by Moses .........

1829 ch. c. (Amesbury) by Phantom......

1850 ch. c. Dreadnought, by Zealot...... Ld Amesbury

1851 b. c. by $\Lambda$ lloany (s. to Germany).....

Missed to Figaro in 1828, to Phantom in 52, and sent to Prussia in the latter year, covered by The Colonel.

* Adelaide was sold to the D. of Holstein in 1852 , with a f. by Robin Adair, and covered by Pyramus.

\section{EUPHROSINE,}

Bred by MIr F. Lumley, in 1819, got by Comus, her dam, sister

to Anna Bella, by Shuttle-Drone-Contessina, by Y. Marske.

1823 b. f. by Tramp (died young)........ MIr F. Lumley

1826 b. c. by ditto ............... Ld Scarbrough

1827 br. f. by ditto (s. to Ireland)........

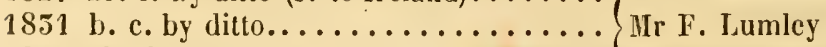

$1852 \mathrm{ch}$. f. by Langar..............

$1835 \mathrm{ch}$. f. Glee, by ditto........... Ld Derby

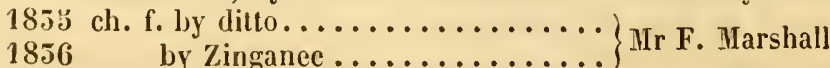

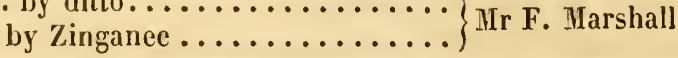

Not covered iu 1827, cast foal by Falcon in 50 , barren in 54 . 


\section{EUROPA,}

Bred by Mr SAder, in 1829, got by Reveller, her dam by Selim, out of Euryone, by Witcheraft.

1855 ch. f. by Defence.............

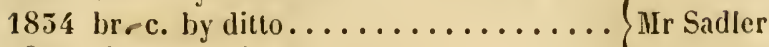

1855 ch. c. by ditto..............

\section{EYESORE,}

Bred by Lord Lowrien, in 182I, got by Castrel, her dam, Sting-

tail, by Gohanna, out of sister to Skysweeper, by Highflyer.

1852 b. c. Westbury, by Sailor (half-bred).. )

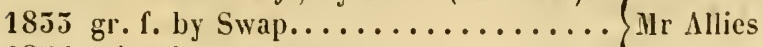

1854 missed to Fungus .............

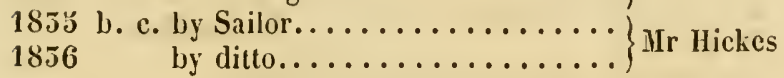

FADI.ADINIDA, by Sir Peter (Vol. III, p. 150) was shot Feb. 14, 1835 , having liad no foal since.

1851 b. f. Ellen, by Peter Lely......... IIr Ricardo

IIer produce of 1825 and 26 are both dead.

\section{FAIR CHARLOTTE (sister to Tarrare)}

Bred by Lord Scarbrougi, in 1819, got by Catton, her dam, Henrietta, by Sir Solomon, out of sister to Olive.

1829 b. c. by Jerry (died in foaling). . . . . Ld Scarbrough

1850 b. f. Fanny, by ditto............ Col. Cradock

1832 twins by Warerley (died a day old)... L Ld Scarbrough

Slipped foal by Comus in September, 1827, missed in 51, 55, and 34, and sent abroad.

\section{FAIR IIELEN,}

Bred by Sir J. H. Maxwelt, in 1817, got by Viscount, her dam, Anna, by Coriander-Highflyer-Tillany, by Eclipse.

1928 gr. c. by Epperston.............. Sir J. II. Maxwell

$1829 \mathrm{gr}$. f. The Hidow, by ditto........ Mr Ramsay

$1850 \mathrm{ch}$. f. by Springkell............ G Gen. Sharpe

$1852 \mathrm{ch}$. f. by Honreith............ Sir P. Maxwell

1853 ch. c. Sadek, by Malek .......... Gen. Sharpe

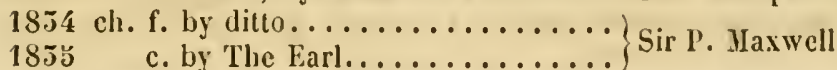




\section{FAIRY,}

Bred by $\quad$, in 1825, got by Magistrate, out of Gibside Fairy, by Hermes-Vicissitude, by lipator-Beatrice.

1832 b. f. by Bachelor, or Theodore......

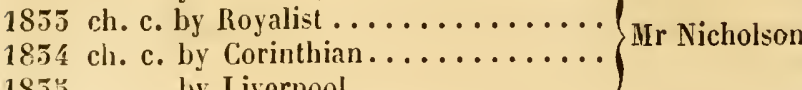

18.53

by Liverpool..............

\section{FAIRY,}

Bred by Sir T. Mlostry, in 1895, got by Filho da Puta, her dam,

Britannia, by Orville, out of Rovedino's dam, by Coriander.

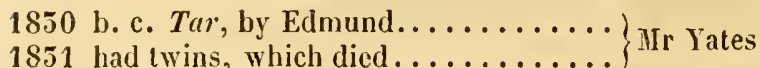

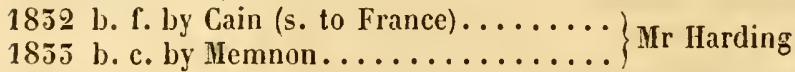

She has since been used as a hack.

TIIE FAIRY QUEEN (sister to the Black Prince)

Bred by Lord Scarbrovgit, in 1817, got by Walton, her dam by Paynator, out of Violet, by Shark.

1829 bl. c. Imp, by Tiresias...........

1830

1851

1854

185 a

1856

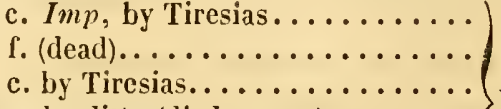

ch. c. by ditlo (died young)........ D. of Portland

ch. c. by ditto

by Akarius..................

In 1852 slipped foal by Tiresias, in 55 missed to him.

FANCY,

Bred at IIampton Court, in 1826, got by Moses, her dam, Favourite, by Blucher, out of Scheherazade, by Selim --Trumpator.

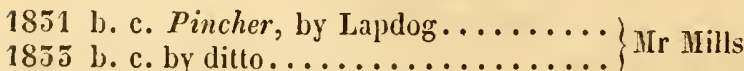

Barren in 1852 , slipped foal in 55 and 56 , and died in 56 .

\section{FANCY,}

Bred by Mr BLAKELock, in 1828, got by Osmond, her dam, sistcr to Countess, by Catton - Ilambletonian-Shuttle-Drone.

1855 b. f. by Waverley

1854 b. f. Yaric, by ditto .............

1855 b. c. by a country horse ........... Mr Blakeloch

$18 \tilde{3} 6$

by Liverpopl.............. 
FANCY,

Bred by Mr Ноцвrook, in 1828, got by Phantom, out of Miss Skim, by Skim-Striking Beauty, lyy Sorcerer-Beningbrough.

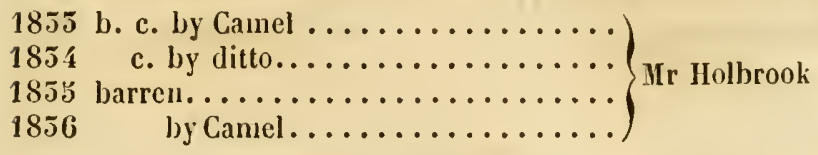

\section{FANNY DAVIES,}

Bred by Mr Ilouldswortil, in 1825, got by Filho da Puta, her dam,

Treasure, by Camillus,-IIyacinthus-Flora, by King Fergus.

1829 b. c. David, by Catton ...........

1850 br. c. Despot, by Sultan..........

1851 b. c. Darius, by Reveller .

1852 b. c. by Sultan (died young) ........

Mr IIouldsworth

1854 br. c. Dan, by Catton............

185 z b. f. by Brutandorf..............

18.36

by Langar ...............

\section{FANNY LEGII (sister to Bustard)}

Bred by Mr Legn, in 1812, got by Castrel, her dam, Hiss Hap, by

Shuttle, out of Haphazard's sister, the dam of Sir Joshua.

1819 b. c. Gallizanter, by Fyldener......

1820 b. f. by ditto (dead) ............ IIr H. Roberts

1821 b. f. by ditto (dead).............

1823 b. c. IIarry, by Sir Ilarry..........

1824 b. f. Sylph, by Spectre.

1825 br. c. Musquito, by Master Henry ...

1826 b. c. by Spectre (died a foal)........ Mr Griffiths

1827 b. c. Thorngrove, by Smolensko (s. to

Sardinia, in 1854)

1828 b. c. by Spectre...............

$1850 \mathrm{ch}$. f. Repentance, by Reveller (s. to Mr E. Griffiths France)

1851 b. c. Mignon, by ditto............ Mr Martyn

Barren in 1829 and 52 , and is dead.

\section{FANTASINA,}

Bred by $\quad$, in 1821, got by Phantom, out of Haid of the Mill, by Zodiac-Tobosa, by Don Quixote-Dungannonsister to Sir Peter. 


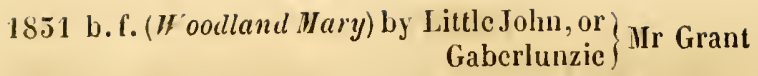

1855 b. c. by Laurcl. ............. Mr Forth

1854 b. c. by Peter Lely............

1855 ch. f. by ditto.............. His Majesty

1856 by Rubini............

\section{FARCE,}

Bred by Mr Jaques, in 1828, got by Swiss, out of Comedy, by Comus-Star-Y. Marske-Emma, by Telemachus.

1854 ch. f. by Emilius............. Sir S. Graham

1855
1856
$\mathrm{ch}$.

\section{FATIMA,}

Bred by Mr СвоскғоRD, in 1820, got by Selim, her dam, sister to Remembrancer, by Pipator, out of Queen Mab.

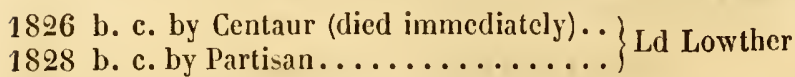

1829 b. f. Flora, by ditto (s. to Baron Bicl)

1850 b. c. by ditto (died 5 weeks old).....

1851 b. f. Fiddle Faddle, by Whalebone (s.to Mr Grant

1852

Spa in 18.54)

In 1855 missed to Bedlamite, and sold to Baron Biel in 55 , covered by Actæon.

\section{FAVOURITE,}

Bred at Ilampton-Court, in 181, got by Blucher, her dam, Scheherazade, by Selim - Trumpator-sister to Postmaster, by IIerod.

182 ch. f. Elinor, hy Gustavus .........

1826 b. f. Fancy, by Moses............... Mr Greville

1827 f. by ditto(deformed and destroyed)

1828 ch. c. Shirley, by Middleton........ D. of Richmond

1899 b. f. by Middleton.............. Mr Maberly

1850 ch. f. P'artiality, by ditto......... Mr W. Ley

1855 ch. f. by Roller, or Snout........ Ir Hunter, Dublin

Barren in 1851 and 52 , and is dead. 


\section{3}

\section{FAWN,}

Bred by Lord Sтradroke, in 1825, got by Smolensko, her dam. Jerboa, by Golıanna, out of Camilla, by Trentham.

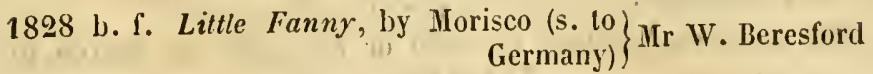

1829 b. c. (Roebuck) by Partisan......... Ld Lowther

1850 b. f. (The Fairy) by ditto ......... Sir II. Wood

1851 br. c. by ditto (dead).............. L Lowther

1852 b. f. by Merlin (s. to Berlin in 1853).
1855 br. c. Venison, by Partisan.......... Mr J. Day

1854 missed to ditto .................

1855 b. f. by ditto............... Ld Lowther

1856 by Lapdog, or Partisan ........

\section{FAWN,}

Bred by , in , got by Dinmont, her dam,

Eliza, by Moorcock, out of Mulespinner, by Guildford.

1828 br. c. by Y. Filho da Puta (Miniature)

1850

c. by ditto..................

1851 b. f. by Sultan.............. Dr Willis

1853 b. f. by his $\mathrm{X}$. Ilaphazard.........

1854 b. f. by Redgauntlet..................

Barren in 1829 and 52.

\section{FENELLA,}

Bred by Sir Thomas Standey, in 1820, got by Milo, her dam. Maid of Lorn, by Castrel-Ricliardson's Marske.

1827 b. f. by Fitz-Langton ............

1850 ch. c. Fitz-dictor, by Predictor. ....

1852 b. c. Lord Francis, by ditto.........

1853 ch. f. Ladr Frances, by ditto....... IIr Thompson

185.4 br. f. by ditto................

185 b̆ br. c. by ditto ................

1836

In 1828 and 29 missed to Fitz-Langton, 51 to Predictor.

FIDALMA,

Bred by Mr IIAfrendex, in 1824, got by Waxy Pope, her dan,

Dinarzade, by Selim, out of Princess, by Sir Peter-Dungannon

- Turf. 


\section{4}

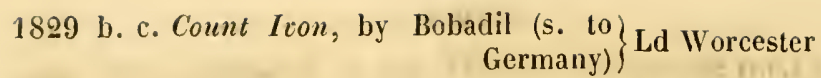

1852 b. c. by ditto $\ldots \ldots \ldots \ldots \ldots \ldots \ldots \ldots \ldots \ldots \ldots \ldots \ldots$ Mr Haffenden

She had no living produce in 1850 or 51 , and was barren in 54 and 55.

\section{FIDDLESTRING,}

Bred by the Duke of Grafton, in 1825, got by Woful, her dam,

Catgut, by Comus, or Junijer, out of Vanity, by Buzzard.

1833 b. f. Wisdom, by Wiseacre........ Mr Greene

1854 barren.................. Mr R. Wilson

1855 b. f. by Shakspeare............. Mr Combe

1856 by Actaon..............Mr Tattersall

\section{FIGARO MARE,}

Bred by Lord Cuesterfield, in 1827, her dam by Waxy, out of Elve, sister to Magic, by Sorcerer.

1851 b. c. Mammoth, by Leviathait. . . . . . Ld Chesterfield

1855 b. f. by Camel ............... Baron de Maltzahn

1854

$185:$

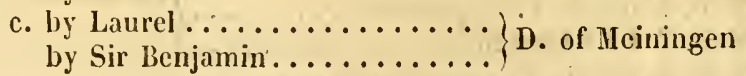

FIGARO MARE,

Bred by Mr Gascolgne, in 1829, her dam, Cora, by Waxy, out of Vourneen, by Sorcerer-Tooee, by Buzzard.

1855 b. f. by Brutandorf. . . . . . . . . .

1854 ch. c. by Conus ..............

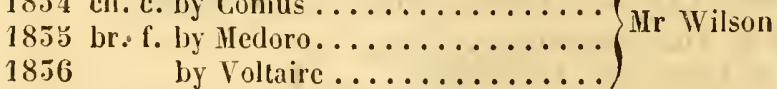

FIGARO MARE,

Bred by Mr T. 0. Powlett, in 1829, her dam by Whisker, out of sister to Bourbon, by Sorcerer.

1853 bl. c. (dead)

1854 b. f. by Voltaire............... Mr T. O. Powlett

185 missed to Tramp .............

N. B. It cannot be stated who was the sire of the foal in 1855 , as it was not known that the mare had been covered; but The Mole stood in the same stable with her in 1852, and on one uccasion was loose. 


\section{5}

FILIIO DA PUTA MARE,

Bred by Lord Scsnerovgn, in 1820, her dam, Miss Caton, by Golumpus, out of Lucy Gray, by Timothy.

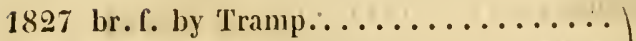

1828 br. c. by Merlin .............

$1829 \mathrm{lor}$. f. Krittums, by $\Lambda$ bjer.........

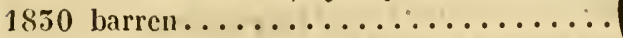

1851 b. c. Neddums, by Leviathan (s. to Ger- Ld Chesterfield nıany)

1852 f. (dead) by Dunsinanc .........

1855 b. c. Ginnums, by Sultan .........

$1834 \quad$ f. by Zingance.............

1855 by Priam..............

\section{FILIIO DA PUTA MARE,}

Bred by , in , her dam, sister to The Clipper, by Sancho - Fidget-Lily of the Valley, by Eclipse.

1832 br. f. Miss Friendly, by Edmund.... Mr Evans

1855 b. f. Maia, by Cain ........... Mr Bristow

Used as a hack since 1855 .

\section{FILHO DA PUTA MARE,}

Bred by Mr L. Charctos, in 1820, her dam, Spindle, by Shuttle, out of Dimple, by Ilighflyer.

1830 b. c. Derby, by Truant ...........

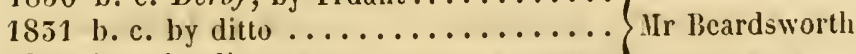

1852 b. c. by ditto..............

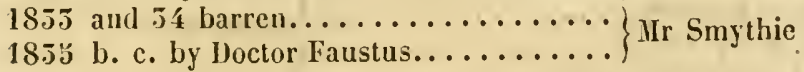

\section{FILHO DA I'UTA MARE,}

Bred at Farnsfield, in 1826, her dam, Deposit's dam, by Comus, out of sister to Zodiac, by St George.

*1851 b. f. Partridge, by Buzzard ....... Mr IIouldsworth

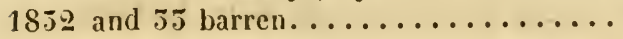

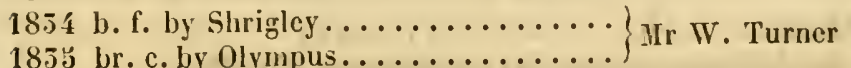

* Partridge produced, in 1856, ch. c. Tom Tit, by Firman. 


\section{FILHO DA PUTA MLARE,}

Bred by Sir George Sitwell, in 1821, her dain by Dick Andrews, out of Miss Watt, by Delpini-Trumpator-Demirep.

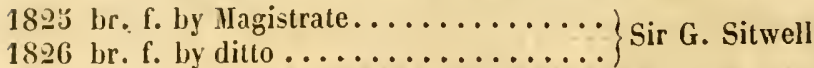

1827 b. f. Dolty, by Figaro............ Ir Arnold

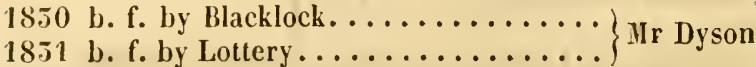

1852 br. f. Walfruna, by Velocipede ...... Mr Fielding

185.5 br. f. by Velocipede ............. Mr Dyson

1853 b. c.by Battledore............... II W. Turner

\section{FILLAGREE,}

Bred by Lord Jerser, in 1815, got by Soothsayer, her dam, Web, sister to Whisker, by Waxy, out of Penelope, by Trumpator.

1819 b. c. (Rembrandt) by Vandyke Junior 1820 ch. f. by Phantom (Donegani's dam).. 1821 b. f. Cobweb, by ditto............ 1825 ch. f. Butterfly, by Magistrate...... 1824 b. c. Chrysalis, by Orville.......... 1826 b. c. W'ill-o'-the-Wisp, by Phantom .. 1827 ch. f. Charlotte West, by Tramp.... Ld Jersey 1828 ch. c. Riddlesworth, by Emilius (s. to

Ir Lichwald, and sent to Germany in 1852) 1829 ch. f. by Partisan. . . . . . . . . . . 18.50 br. f. Joanna, by Sultan (put to the stud)

1851 ch. f. Trinket by Godolphin........ Sir F. Johnstone

1852 f. Adela, by Emilius............

1855 ch. c. by ditto...............

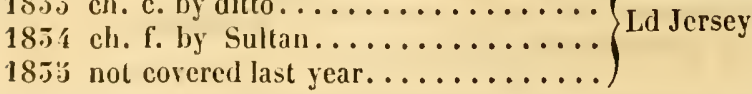

In 1822 missed to Scud, in 25 to Tramp.

FILLE DE JOIE,

Bred by Mr Yates, in 1821, got by Fillo da Puta, her dam by Paynator-Delpini-Young Marske-Gentle Kitty, by Silvio. 1829 barren..................

1850 br. f. Frailly, hy Paul Pry, or Bedlamite Col. Peel

18.51 b. c. II arum Scarum, by Bedlamite.. 
1852 gr. c. Paulus, by Emilius........

$185.5 \mathrm{gr}$. . Zenana, by Sultan .........

$185.4 \mathrm{gr} . \mathrm{f}$. by ditto.............. Col. l'eel

1853 b. c. Sı Vilus, by Bedlamite........

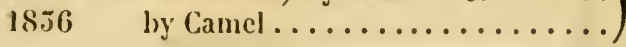

\section{FINESSE (sister to Bizarre)}

Bred by Lord Grosveron, in 1813, got by Peruvian, her dam, Violante, by John Bull, out of a sister to Skyscraper, by llightlyer.

1820 ch. c. Adroit, by The Flyer (s. to Ire-) land, and aft. to the Netherlands)

1821 ch. f. Mancuver, by Rubens.......

1825 deal foal, by Sovereign..................... Ld Grosienor

1823 ch.c.Victim(afterwards Emancipation)

by Blacklock

1826 b. f. Ambuscade, by Master Ilenry. ...

1829 b.f. Stratagem, ly ditto (died 3 yrsold)

1850 1. f. Decoy, by Filho da Puta...... Ld Westminster

1851 dead foal, by Camel............

$185 j$ b.f. by Teniers. ...............

Missed in 1822, 27, 28, 52, 54, and is dead.

\section{FISHER LASS,}

Bred by Mr R. Stephersor, in 1927, got by Osmond, out of Voltairc's dam, by l'hantom-Overton-Gratitude's dam, by Walnut.

1852 b. f. by Velocipede .............
1855 br. f. Falentine, by Voltaire.......

185.4 b. f. by Brutandorf............. Mr W. Chilton

1853 b. c. by Voltairc..............

\section{FITZ-JAMES MLRE,}

Bred by Mr Psuxter, in 1816, her dam by Windle, grandam by Anvil, out of Virago, by Snap.

1824 bl. f. by Piscator..............

1826 b. c. Falconbrielye, by Manfred........... Ir F. I.. Cliariton

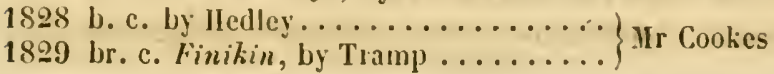




\section{8}

1850 bl.c. Disaster, by Woful .........

1852 gr. c. Fop, by Stumps ........... Mr Cookes

183.5 ch. c. by Velocipede (died young).....
1834 gr. f. La Mode, by Stumps ........

1835 gr. c. Decorator, by ditto................... Mr Dyson

In 1897 missed to IIedley, 51 to Stumps.

FITZ-TEAZLE MARE,

Bred by Mr Belt, in 1816, her dam, sister to Recovery, by IIyacinthus-Overton-Katherine, by Highflyer.

1827 br. c. Remus, by Hazard (s. to Russia) in 1855)

1828 b. c. Romulus, by ditto.......... Mr Bell

1854 b. f. by Humphrey Clinker .........

1853 missed to ditto...............

1856 by Lot, son of Brutandorf. .....

\section{FLEUR-DE-LIS,}

Brell by Mr Sykes, in 1822, got by Bourbon, her dam, 'Lady Rachel, by Stamford, out of Y. Rachel, by Voluntecr-Rachel, by Ilighflyer.

1855 b. c. by The Colonel (died 2 days old)

1834 b. f. by ditto (s. to South Carolina)...

1854 b. f. by ditto (s. to South Carolina)...
$1856 \quad$ by Emilius $\ldots \ldots \ldots \ldots \ldots \ldots \ldots \ldots$ His Majesty

by Emilius

\section{FLORA (sister to Oiseau)}

Bred by Ir Garforth, in 1814, got by Camillus, her dam, by Ruler, out of Treecreeper, by Woodpecker-Trentham.

1820 ch. f. by Comus ............. Mr Garforth

1821 gr. f. by ditto ............... Mr Haworth

1824 gr. f. Columbine, by Cervantes...... Mr Ridsdale

1825 ch. c. IIarlequin, by ditto(s. to France) $\}$ Mr Ridsdale

1827 ro. f. by Theodore ............. Mr Garforth

1829 b. f. Florestine, by Whisker........

*1850 b. f. Liska, by Lottery ......... Mr Ridsdale

1851 gr. c. Botanist, by ditto ..........

1832 gr. c. Luck's-all, by Tramp........

-Lısкa produced ab. f. by Partisan in 185̃ , missed in 35, and was coveredby Mulatto. 
1834 ch. c. Chersidamas, by Priam................. Mr Ridsdale 1856

Not covered in 1827, missed to Trampin 55 and 55.

\section{FLORANTIIE,}

Bred by Lord Fitzwhlin:x, in 1822, got by Amadis, her dam, Orvillina, sister to Orville, by Beningbrough, out of Exelina, by Hightflyer.

1827 b. c. by Camelopard ............. Ld Fitzwilliam

1828 b. c. Florian, by ditto ..........

1831 bl. f. by Confederate (put to the stud). Mr Allen

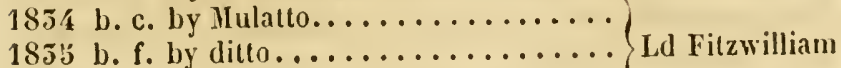

1856

by IIcdoro...............

Not covered in 1828, 29, 51 , or 32.

\section{FLORESTINE,}

Bred by Ilr Garfortil, in 1829, got by Whisker, out of Flora, by Camillus-Ruler-Woodpecker-Treutham.

1854 br. f. by Tramp ..............

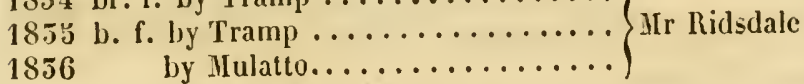

\section{FLORINEL,}

Bred by Mr $\Lambda$ Trwood, in 1829, got by his Grey $\Lambda$ rabian, her dam,

Rosabel, by his Chesnut $\Lambda$ rabian-Smolensko-Luck's-all.

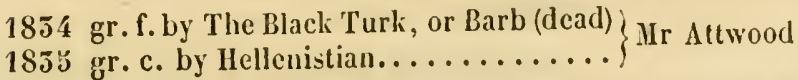

\section{FLOLNCE,}

Bred by Lord Lowrher, in 1824, got by Clinker, her dam by IIambletonian, out of Lady Sarah, by Fidget-Alfred.

*1829 ro. f. Y. Flounce, by Partisan .....)

1850 ro. c. by ditto (died a few days old)... Ld Lowther

1851 ch. c. (Florence) by ditto..........

$1852 \mathrm{ch}$. or ro. c. by ditto........... Mr Pigott

1855 br. f. by ditto............... Ld Chesterfield

1854 b. or ro. f. by ditto (s. to Berlin, 1853))

1853 ch. c. by ditto............... Ld Low ther

1856

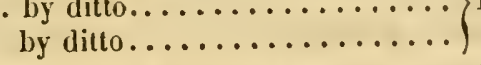

* Y. Flounce produced a ch.f. Ly Wiseacre, in 1855, was not covered that year; missed to Lapdog in 35 , and was corered by Logric. 


\section{FLOURISII,}

Bred by Lord Lowrher, in 1828, got by Partisan, out of Pasta, sister to Langar, by Selim-Walton-Sorcerer's dam, by Diomed.

1855 missed to Chateau Margaux........

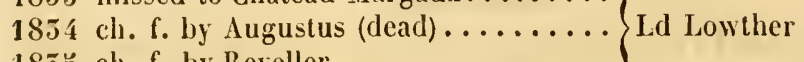

185 ž ch. f. by Reveller .............

Sent to Berlin in 1855, covered by Rowton.

\section{FLUSH,}

Bred by Lord Durnam, in 182y, got by Blacklock, her dam, Loo, by Waxy, out of Piquet, by Soreerer-Prunella, by llighflyer.

1852 b. e. Hectic, by Sultan..........

1853 b. f. by Emilius...............

1854 missed to Filho da Puta........... MIr Houldsworth

185 b. f. by Vauish...............

1836 by Filho da Puta..........

\section{THE FLYER MARE (sister to Icaria)}

Bred by , in 1825, her dam, Parma, by Dick Andrews, out of May, by Beningbrough - Primrose, by Mambrino.

1855 b. f. by Varro................

$1854 \mathrm{ch}$. f. by ditto............... Mr F. F. Meynell

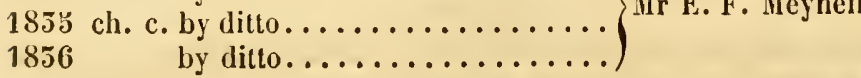

\section{FORESTER IASS,}

Bred by , in 1821, got by Filho da Puta, her dam, Miss Forester, by Diamond-Alexander-Sweetwilliam.

1855 b. c. by Memnon............

1854 br. e. by Priam..............

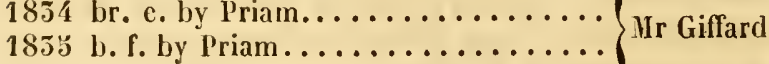

1856

by Zinganee...............

\section{FORTUNA,}

Bred by Mr Watson, in 1818, got by Comus, her dam by Patriot

-Phonomenon-Czarina, by Babraham Blauk-Melpomene.

1823 b. c. by Tramp............... Mr Lambton

^1826 ch. f. Fortitude, by Whisker....... Mr Houldsworth.

- Fortitude produced in 1854 a ch. f. by Vanish, missed to him in 55, and was covered by Lambionian. 
1828 b. f. Frederica, by Sultan.........

1830 ch. f. Fidelity, by Whisker........

1851 b. c. Forester, by ligaro........... Mr Ilouldsworth

1852 br. c. Fetix, hy Sultan ..........

1855 ch. f. by Vanish ..............

Missed in 1827 and 29 , and died in 34, covered by Sultan.

\section{FRAILTY,}

Bred by Mr Petre, in 1821, got by Filho da Puta, out of $\Lambda$ gatha, by Orville-Star-Y. Narske.

1826 b. f. by Phantom (died a foal) ....... MIr J. Scott

1827 br. c. Reginald, by Figaro.......... Mr Petre

1828 br. c. Rodolph, by Der Freischutz....

1829 missed to Comus..............

1830 br. c. Connoisseur, by Chateau Margaux

(died on the road to Vienna)

1851 ch. c. Constant, by Blacklock ........ Mr J. Scott

1852 b. c. Itinerant, by Tramp..........

1853 b. f. Cyprian, by lartisan .........

1855 ch. c. Bretby, by Priam ...........

1856 by Zinganee............

In 1829 missed to Comus, 54 to Tramp.

FRANCES, by Ambrosio (Vol. IlI, p. 140). Add the name Pawnbroker to her ch. c. by Duplicate, foaled in 1827.

\section{FRANCESCA,}

Bred by Mr ArTwood, in 1829, got by Mozart, out of the Arabian mare, Alhassan's dam, p. 14.

1835 gr. f. by a Black Turk, or Barb (died in 1853)

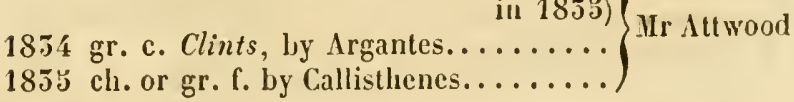

\section{FREDERICA,}

Bred by Lord Axsox, in 1826, got by Phantom, her dam, Elizabeth, by Orville, out of Pennytrumpet, by Trumpator.

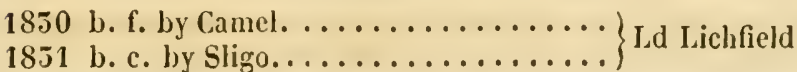

VOL. IV. 


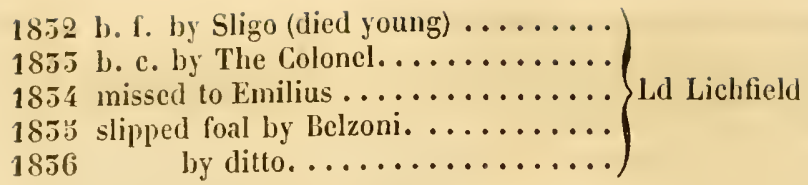

\section{FREDERICA,}

Bred by His MLsusty, in 1827, got by Hoses, her dam, sister to Romana, by Gohanna-Sir Peter-Nerissa, by Volunteer.

1835 ch. c. by The Colonel. .......... Mr Combe

1854 cl. f. by ditto. ............. Mr Greville

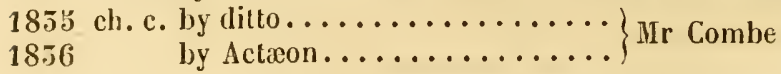

\section{FREDERICA (sister to Frederieli)}

Bred by Mr Gratwicke, in 1827, got by Little John, her dam by Phantom, out of sister to Election, by Gohanna-Chesnut Skim.

1852 ch. f. by Partisan $\ldots \ldots \ldots \ldots \ldots \ldots \ldots$
1855 ch. c. by Aaron................

1854 barren.................... Mr Gratwicke

$185:$ b. f. by The Colonel............

1836 by Hokee Pokec............

\section{FROLIC MARE,}

Bred by Lord Eqremont, in 1819 , her dam by Selim, out of Maiden, by Sir Peter-Phœnomenon.

1824 b. f. (Incognita) by Whalebonc...... Mr King

1826 b. c. (Benedict) by ditto .......... Ld Egremont

1829 b. c. by ditto................ Ld Chesterfield]

1851 b. f. by ditto................ Mr Osbaldeston

1855 br. f. by Chateau Margaux (died 2 yrs old) ) Mr King

185 : b. c. by ditto ............. D. of Richmond

Barren in 1825, 27, 28, 50, and 54 .

\section{FROLICKSOME,}

Bred by Lord Egrenoxt, in 1824, her dam by Stamford, out of Alexina, by King Fergus-Lardella, by Y. Marske. 
1827 ch. f. (Wilhelmina) by Wicked Will, an

1828 gr. f. by Marengo (died a yearling)...

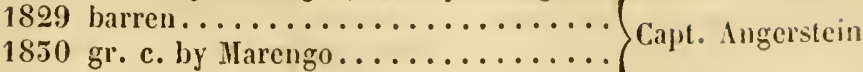

1851 ch. f. by St latrick

1852

1854

1853 by a son of Marengo (dam by Sor-

cerer)

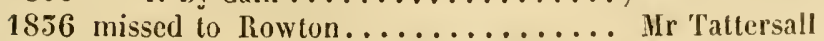

\section{FYLDENER MARE,}

Bred by Mr Cavrixg, in 1817, her dam, Morgiana, by Coriolanus -Lurcher-Phlegon-Merlin-Regulus.

1850 br. c. Easton, by Luzborough. ...... $1852 \mathrm{ch}$. f. by Sailor (son of Candidate)....

1855 ch. c. Amril, by Luzborough....... Mr Sainsbury

1854 bl. c. Tippoo Saib, by Rajah.......

1856 by Priam.

Barren in 1851, not covered in 54 .

\section{FYLDENER MARE (bay)}

Bred by Mr , in 1820, her dam, Guildford Naı's daın,

by Justice, out of Parsley, by Pot8o's-Lady Bolingbroke.

1828 br. c. by Robin Ilood............ Mr Grover 1829 b. c. Will Scarlet, by ditto........ Mr Cooper 1850 b. f. loy ditto.......................... IIr Grover

1853 b. c. Swallow, by ditto.......... Mr Mills

1854 b. с. by Gaberlunzic............ Col. Peel

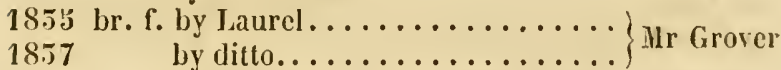

In 1851 missed to Robin Hood, 56 to Scipio.

\section{GABRIELLE,}

Bred by the Duke of Graftox, in 1820, got by Partisan, out of Coquette, by Dick Andrews-Vanity, by Buzzarl.

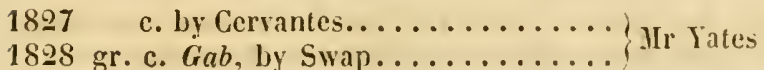


$1 \$ 29$ ch. c. Vagabond, by Cain.........

18.50 slipped foal . . . . . . . . . . . . . . . . . . .

1852 b. f. Vagary, by Cain (s. to Baron de Mr Yates

1855 b. f. Mary, by Edmund...........

1854 c. Grand Cairo, by Camcl........ Gen. Yates

1856 by Camel............. IIr Boardman

Sent to the United States in 1853.

\section{GADABOUT,}

Bred by Lord Fitzwiluan, in 1812, got by Orville, her dam,

Minstrel, by Sir Peter-Matron, by Florizel-Maiden, by

Matchem.

1818 clı. f. Teresa Panza, by Cervantes...

1819 br. f. Larly Mary, by Amadis.......

1820 ch. c. by Comus ..............

1821 br. f. IIannah, by Smolensko......

1822 br. c. Comedian, by Comus......... Sir J. Byng

1824 ch. c. Pedlar, by Tramp..........

1823 b. f. Miss Pratt, by Blacklock......

1827 br. f. by Figaro ...............

1828 br. f. (Emma) by Tramp..........

1829 ch. f. by Humphrey Clinker ........ Mr Hellier

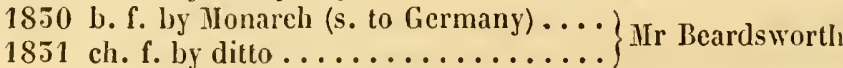

1855 b. f. Ranee, by Harlequin (the Arab)... Wajor Hancock

Missed in 1825, 26, 52, and 55, and is dead.

\section{GAIETY,}

Bred by Lord Egremont, in 1824, got by Frolic, her dam, Mermaid, by Orville-Sir Solomon-Miss Brim, by llightlyer.

18.52 b.c. Macbeth, by Sliakspeare...... Mr Martyn 1855

1854

185

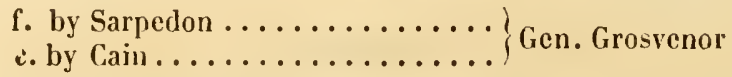

\section{GAl.ATEA,}

Bred by Lord Fitzwiluan, in 1816, got by Amadis, her dan, Paulina, by Sir Peter-Pewel, by Tandem-Tantrum, etc. 
1822 b. f. Sybil, by Interpreter. . . . . . .

1824 br. c. by Tiresias .............. Ld Fitzwilliam

1823 b. с. by Oiseau ...............

1826 br. c. by Blacklock ..............

1827 ch. c. Acis, by Blacklock.......... Ir Young

1828 b. c. Auditor, by Middlleton ......... Mr Maberly

1829 ch. f. Actca, by ditto (sent abroad) .. Mr Young

1852 br. f. Tritonia, by Camel ......... Mr Goodwin

1853 ch. c. Recruit, by The Colonel...... Sir L. Glyn

1854 b. f. by Trauby...............

1855 b. c. by The Colonel. ........... IIis Majesty

by ditto.................

In 1825 missed to Catton, 50 to Middleton, 51 to Lazarus.

\section{GALENA,}

Bred by Mr JaQes, in 1825, got by Walton, her dam, Comedy, by Comus-Star-Y. Marske-Emma, by Telemachus.

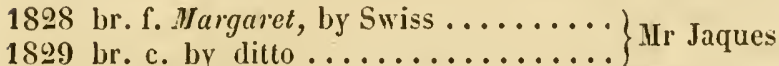

1850 br. c. by ditto............... Mr Chifney

1851 b. f. by Wanton..............

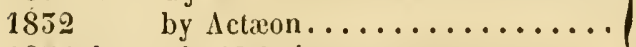

1834 br. c. by Voltaire .............. Mr Jaques

1853

1836

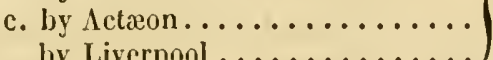

GAMELIA, by Whalebone (Vol. III, p. 145) died in 1851.

\section{GARCII,}

Bred by Lord Muxcaster, in 1825 , got by Octavian, her dam by

Shuttle, out of Katherine, by Delpini-Paymaster-Le Sang.

1829 b. c. Archibald, by l'aulowitz......

*1850 b. f. Malibran, by Whisker........

1853 f. by Êmilius ..............

1854 ch. f. by Bedlanite............. Col. Peel

1853 b. c. by Cain.................

1856 b. f. by $\operatorname{ditt} 0 \ldots \ldots \ldots \ldots \ldots \ldots \ldots$

Missed to Sultan in 1851 and 32.

* Malibrax produced a b.c.by Cain, in 18.5, anil missed to Actæon in 1850 . 


\section{GAVOTTE,}

Bred by the Duke of Graftov, in 1822, got by Election, her dam, Coquette, by Dick Andrews, out of Vanity, by Buzzard.

1827 ch. c. by Merlin.............. His Majesty 1828 b. f. Mazurka, by Mustachio ....... Col. Russell 1829 ch.c. by Waterloo (dead) ......... His Majesty 1850 br. e. by ditto (s. to Germany in 1852) Ld Wilton 1831 b. f. by Partisan ............. Mr Williamson

1852 missed to Filho da Puta.......... Mr R. Wilson 1835 ch. f. Pepper, by Bedlamite (s. to Ger-) $\begin{gathered}\text { many) }\end{gathered}$ Mr Edwards 1854 br. f. by Sliakspeare (s. to Germany)..

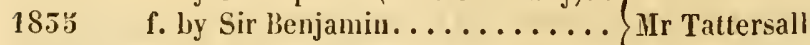

1856 by Shakspeare............

\section{GATKEY,}

Bred by Mr Nowell, in 1828, got by Muley, her dam, Eliza, by Rubens, out of Aladdin's dam, by Walnut-Javelin.

1855 br. f. by Bobadil. .............

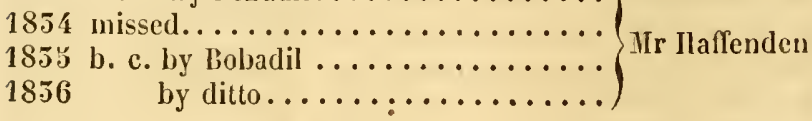

\section{GEORGIANA,}

Bred by Mr Gascoigne, in 1819, got by Woful, her dam, Shepherdess, by Shuttle-Buzzard-Ann of the Forest.

1825 b. f. by Grey Middleham.......... Mr J. Croft

1825 ch. f. by Viscount............

1826 br. c. Retreicer, by Smolensko.....

1828 ch. f. by Comus...............

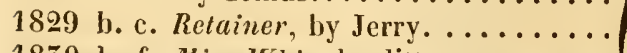

1850 b. f. Miss Whip, by ditto ...........

1851 br. $\{$. by ditto (died 5 yrs old)........ Ld Kelburne

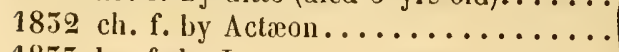

1855 br. f. by Jerry . . . . . . . . . .

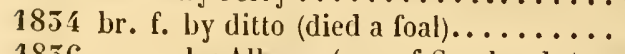

1856 by Albany (son of Smolensko)...

In 1824 missed to Ardrossan, 27 to Osmond, 55 to Jerry.

GERTRUDE,

Bred by Mr Kraptov, got by Amadis, her dam, Oceana, by Cerberus, out of Doctor Syutax's dam, by Beningbrough. 
1850 ch. c. Ilodge, by Sober Robin.......

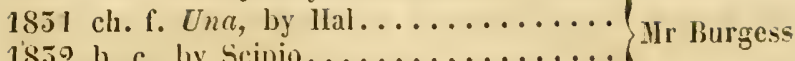

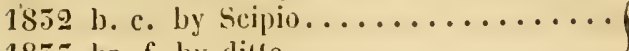

1853 br. f. by dillo..............

Not corered in 1853 and 54 .

\section{GIBSIJ)E FAIRY,}

Bred by Lord Stratnyore, in 1811, got by Hermes, her dam, Vicissitude, by Pipator, out of Beatrice, by Sir Peter.

1816 b. ro. c. Woodlark, by Remembrancer

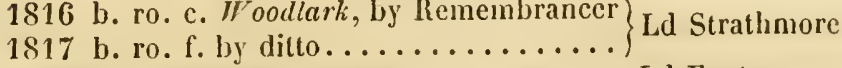

1820 br. c. Elf, by Comus............ Ld Exeler

1825 b. f. Faivy, by Magistrate......... Mr J. Smith

1824 cl. f. Emma, by Whisker......... Mr Russell

1826 b. . by Tramp.............. The Trustees

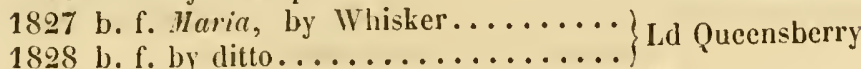

1829 b. c. by ditto............... Mr Ridsdale

1851 ch. f. II aid of Lune, by ditto (put to the stud)

1855 b. c. The Cauld Lad of Ililton: by Mr Bowes

1856 b. f. by lhysician.............

1857

by Curtius...................

Missed in $1818,19,22,25,50,52,54,55$, and was not covered in 1820 .

\section{GIFT,}

Bred by Lord Egremoxt, in 1818, got by Young Gohamna, her dam, sister to Grazier, by Sir Yeter-Trumpator-Ilerod.

1826 gr. f. Letty, by Whalebone........

1827 bl. f. (Jessy) by ditto (sent to the Sivan

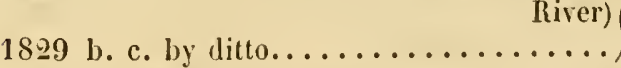

Ld Egremont

1850 b. f. Myrrha, by dilto........... Mr Gardnor

1851 b. f. Miss Ellen, by dillo......... Mr Hills

$1852 \mathrm{gr}$. c. Grammont, by Gaberlunzie.... Gen. Yates

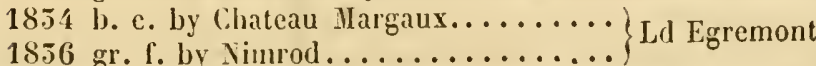

Barren in 1824 and 28, had a dead foal in 25, missed to Chateau Margaux in 1855 and 55. 
GIGLET,

Bred by Jir Metcalfe, in 1826, got by Wanton, her dam, Floranthe, by Octavian, out of Caprice, by Anvil-Madcap, by Eclipse.

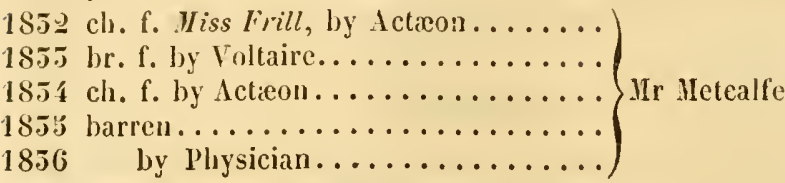

THE GIIIIER,

Bred by Lord Wharxcerfa, in 1824, got by Filho da Puta, her dain, Calypso, by Sorcerer, out of Houghton Lass, by Sir Peter.

$18 \geq 9$ bl. c. Cantab, by Wrangler........

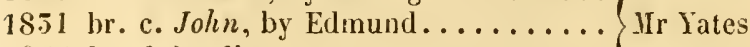

1855 br. f. by ditto...............

Cast her foal in 1850 , barren in 52 and 54 , and sold to the D. of Orleans, in foal to Limilius.

GIN,

Bred by Mr Wnitelock, in 1825, got by Juniper, her dam, Prillcess Jemima, by Remembrancer-Ormond.

1829 b. f. Miss Whitelock, by Swiss...... . Mr Young

1850 b. c. All max, by ditto..........

1851 br. c. (Polander) by Lottery (died 5 yrs Mr Whitelock old)

18.52 ch. c. Grog, by Ictæon.......... D. of Leeds

1855 ch. c. Lithuanian, by Wanton...... IIr Attwood

1854 b. f. by Wavertey..............

1856 h. f. by Jerry................ Mr Whiteloek

1857 by Voltaire.............

In 1828 missed to Swiss, 55 to Leonardo.

GINGARELIA,

By Young Warter, her dam by Cardock, out of Georgiana, sister to Conductor.

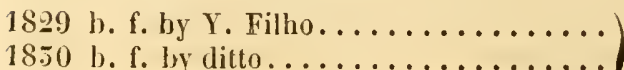

1850 b. f. by ditto...............

1852 ch. с. Ly Y. Haphazard......... Dr Willis

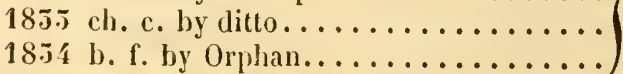

$1856 \mathrm{ch}$. c. by ditto.............. Mr Walton

Barren in 1851 and 55 . 


\section{GIROUE'TE (sister to Bupta)}

Bred by the Duke of Grafrox, in 1821, got by Partisan, her dam, Coquette, by Dick Andrews, out of Vanity, by Buzzarl.

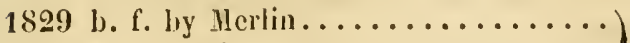

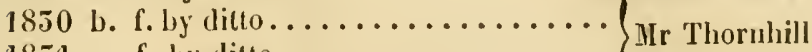

1851

1852

f. loy ditto .

1855 ch. c. Triton, by Zinganec......... Mr Greville

18.54 c. by Augustus...............

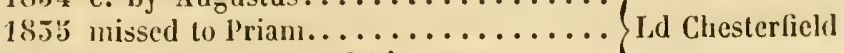

1856

loy Priam and Zingance......

\section{GOIINNA MARE (sister to Castanea)}

Bred by Lord Egremont, in 1807, her dam, Grey Skim, by IVoodpecker-Silver's dam, by Herod-Y. Hag, by Slim-Hag, by Crab-Ebony, ly Childers-Old Ebony, ly Basto.

1812 b. c. Castanet, by Granicus (s. to Russia) 1813 ch. c. Roger Bacon, by Sorcerer (s. to India in 1821)

1816 c. by Sir David (died in foaling)... 1817 b. f. by Waxy .................

1819 b. c. Moses, ly Whalcbone, or Scymour II. R. H. the D. of $1820 \mathrm{ch}$. c. Premium, by Aladdin (sent to York Irance in 1853)

1822 b. f. (Prize) by ditlo (s. to lreland).. 1825 b. f. Rachel, by Whalehone........ 1824 b. f. Miriam, by ditto ..........

1823 b. c. (Albany) by ditto (not trained)...)

1826 b. c. (High Priest) by ditto....... D. of Rutland 1827 slipped a colt, by ditto.......... Ilis Majesty 1850 b. c. Shylock, lyy Waterloo........ D. of liutland 1852 br. c. Clarence, by Camel......... Mr Yates

Barren in 1818 and 21, missed to Whalebone in 28, to Waterloo in 29 and 51 , to The Colonel in 55 , and died that year.

\section{GOHANNA MARE (sister to Wanderer)}

Bred by Iord Egrexoxt, in 1809, her dam, Catherine, sister to Colibri, by Woodpecker, out of Camilla, by Trentiam.

1814 f. by 'Teddy-the-Grinder .........

1817 b. c. Robin Hood, by Octarius................ Is Egremont

1820 b. f. Spermaceti, by Whalebone..... 
1822 b. c. Waxlight, by ditto.........

182 y. b. Rushlight, by ditto ...........

1827 b. c. by ditto (foaled Dec. 1826 , and s.

to Ireland in 1829) Ld Egremont

1850 b. c. by ditto (s. to Germany).......

1855 b. f. by Gaberlunzic ...........

Barren in 1825, 24, 26; in 1829 missed to Wlıalebone, 51 to little John and Whalebone, 32 to Greyleg, 5 it to Chateau Margaux, 35 to Chateau Margaux and Gaberlunzic.

GOIINNA HARE (Young Amazon) Vol. IH, p. 131-has had no foal since 1832. Her two last produce should stand thus :1829 b. f. Gretna Green, by Whalebone... Capt. Bulkelcy 1852 b. f. Redleg, by Greyleg.......... Ld Egremont

\section{GOIIANNA MARE,}

Bred by Lord Egrenovt, in 1810, her dam, Allegretta, by Trumpator, out of Y. Camilla, sister to Colibri, by Woodpecker.

1815 b. f. by Teddy-the-Grinder (sent to

1817 f. by Bustard (son of Buzzard) .... 1818 bl. c. Black-and-all-Black, by Octavius

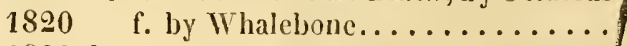
1822 b. с. Criclieter, by Octavius.......

1824 b. c. Tom Thumb, by Whalebone....

1823 b. . Little Finger, by ditto .......

1827 b. c. by ditto (died a few days old)...

*1829 bl. f. Ly ditto................

1851 b. f. by Whalcbone............

1852 b. f. by Greyleg..............

18.55 ch. f. by Chateau Margaux (dicd 5 days

old)

Barren in 1825 and 50, slipped foal in 1826 and 28 , missed to Chateau Margaux in 55, to Gabcrlunzie in 5 $\mathbf{4}$, and dicd in $\mathbf{5} 5$, in loal to Acorn.

- This mare was sent to France in 1854, with a c. by Bedlauite, and covered by Priam.

\section{GOHANML M.IRE (sister to Y. Pitshill)}

Bred by Lord Egrenove, in 1S11, her dam, the Pitshill mare, by Driver, out of Niglıtshade, by PotSo's-Cytherea, by Drone. 
1852 b. f. by Langar.............. Mr J. Robinson

1855 b. c. Mancurre, by The Colonel..... Mr Martyn

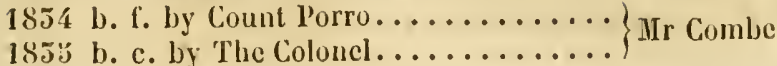

\section{GOHANNA MLRE (sister to Romana)}

Bred by Lord Eghexont, in 1814, her dam by Sir Peter, out of

Nerissa, by Volunteer-sister to Sting, by Herod-Cygnet.

1817 f. by Totteridge............ Mr Farrall

1820 t. by Phantom (died a foal)....... Mr J. Ramsay

1825 b. f. by Carbon...............

1824 f. Louise, by ditto............ IIr Farrall

1825 c. by ditto...............

1826 b. c. Virginius, by ditto..........

1827 ch. f. Frederica, by Hoses.......... Ilis Majesty

1828 b. c. by Mustachio..............

1829 ch. c. Ambrosio, by Waterloo, or Mid- dleton $\}$ Mr Munro

1850 ch. c. by Dunsinane............ His Majesty

1851 cl.. c. IIampton, by Waterloo....... IIr Ramsay

1852 b. f. by ditto................

1855 b. f. Kitty of Coleraine, by The Colonel Mr Copeland

1854 b. f. by Tranby.............. His Majesty

1855 b. f. by Defence.............. Jir Greville

1856

by Rubini ..............

Used as a hack in 1818 and 19, missed to Skim in 22.

\section{YOUNG GOHANNA MARE (grej)}

Foaled in 1825, her dam, sister to Amazon, by Driver, out of Fractious, by Mcrcury.

1828 gr. f. by Figaro.............. MIr Davis

1854. ro. f. Antelope, by Camel......... Ld Lichficld

She was used in harness for sereral years, and has had only these two foals.

\section{GOLDPIN (sister to Goldwire)}

Bred by Lord EgRENoxt, in 1826, got by Whalebone, her dam by Gohanna, out of Amazon, by Driver-Iractious, by MercuryWoodpecker. 
1852 b. f. by Y. Filho (Hiniature).......

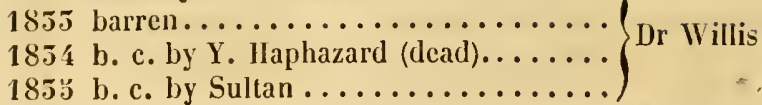

1856 by Y. Haphazard.......... Ir Tattersall

\section{GOLDWIRE (brown)}

Bred by Lord Egremort, in 1825, as the preceding mare.

1851 b. f. Goldfringe, by Phantom, or Wa-

$\left.\begin{array}{l}1852 \text { b. f. Silverfringe, by Helenus......... terloo } \\ 1853 \text { b. c. Bullion, by Emilius......... }\end{array}\right\}$ Capt. F. Berkeley

1856 by Rubini, or The Colonel...... Mr H. Clay

Barren in 1855 and 54 , and sold to go to Kentucky.

\section{GOLUMPUS MARE,}

Bred by Sir. Tatron Srkes, in 1818, her dam by Shuttle, out of Sancho's dam, by Highflyer-sister to Tandem.

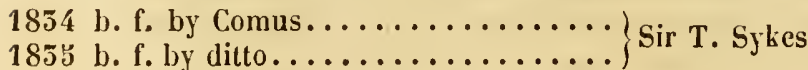

She was used as a hunter till 1855.

\section{GOVERNANTE,}

Bred by , in 181s, got by Governor, her dam

by Beningbrough, out of Camerton's dam, by Precipitate.

1821 ch. c. by Amadis............. Sir J. Byng

1822 bl. c. by ditto (dead)..............

1824 ch. f. Oriana, by Amadis..........

1825 ch. c. Commandant, by ditlo ........

1827 b. f. Barcelona, by Cervantes....... Ld Fitzwilliam

1828 b. f. by Amadis (died 4 days old)......

1829 b. c. Mambrino, by Cervantes.......

1850 b. f. by Confederate.

1851 br. f. by ditto, or Mulatto..........

Barren in 1826 and 52, and died in 1855 , in foal to Amadis.

GOVERNESS, by Milo (Vol. III, p. 156) cast her foal in 1832, was barren in 53, and sold in the spring of $\mathbf{3 4}$ to $\mathrm{Mr}$ Broënenburg, of Germany, covered by St Nicholas. 


\section{GOVERNOR MARE,}

Bred by Mr Cock, in 1816, her dam, Anticipation, by Beningbrough, out of Expectation, by IIerod.

1826 b. f. by Catton................ Mr Wastell

1828 ch. c. Orangeman, by Doctor Syutax. Mr Bradshaw

1829 bl. c. Tourist, by ditto (s. to the 1). de $\left.\begin{array}{r}\text { Guiche) }\end{array}\right\}$ IIr J. Scott

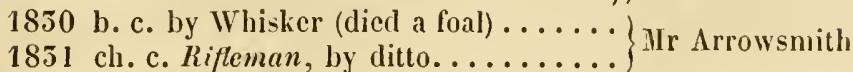

1852 ch. l. by Malek ..............

1855 br. f. by Langar (s. to Baron Biel).... Ld Chesterfield

$1854 \mathrm{ch}$. f. by Priam..............

185 by ditto.............

\section{GRAND DUKE MARE,}

Bred by Sir T. Staxley, in 1823, out of Cowslip, by AlexanderAnvil - Virago, by Snap.

1829 bl. c. by a half-bred horse ........

${ }^{*} 1850$ br. f. by Battledore ............ Sir T. Stanley

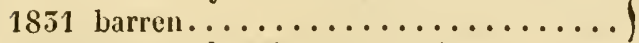

1852 ch. c. The Miner, by Teniers.......

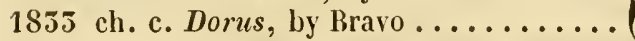

1854 missed to Felt ................. M Mr R. J. Mostyn

- This battledore mare produced, in $1855, \mathrm{~b}$. f. by Joceline.

\section{GRAND DUKE MARE,}

Bred by Sir W. W. Wrrx, in 1827, her dam by Comus, grandam by Alexander, out of sister to Haphazard, by Sir Peter.

1855 ch. f. by Memnon.............

1854 b. c. by Cadland.............. Mr Eyke

1855 and 56 barren..............

\section{GRAVITY,}

Bred by Mr Broнl, in 1824, got by Sober Rol,in, her dam, Risk, by Ilaphazard, out of sister to Horns, by Precipitate.

1829 b. and ro. f. by Acorn (died soon after foating)

1850 b. c. Oak, by ditto ............. Mr M. Henderson

1851 b. c. Zephyrus, by Zadig (died in 1854)

1852 b.f. Chickeroni, by dilto.......... 
1855 b. f. Guinea Chick, by ditto.......

185 4 b. f. Ilindoostanee, by llindoo....... Ir R. Ilenderson

$185:$ not corered last year . . . . . . . . . . .

18.56 by a cart horse.......... Mr Bruhl

\section{GRECIAN OUEEN,}

Iired by Mr T. Ifutcnussox, in 1825, got by Catton, her dam,

Queen Coil, by Sweetwilliam-Remembrancer-Y. Marske.

1829 b. c. Wittingham (alt. Prince) by Figaro Mr Ilope

1850 b. c. by Grey Walton ...........

1851 b. c. The P'retender (aft. Morpeth) by IIr Ross $\Lambda$ ction

She had no living produce after 1851 , and is dead.

\section{GREEN MINTI.E,}

Bred by Lord Exeten, in 1826, got by Sultan, her dam, Dulcinea,

loy Cervantes, out of Regina, by Moorcock-Rally.

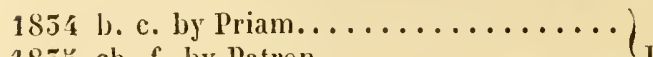

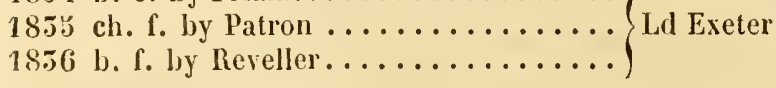

GRENADIER MARF,

Iired by Mr W. Richarnsor, in 1828, got by Mr Watt's Grenadier, her dam by Cerberus, out of Diana, by Kill Devil.

195 I b. c. Beverley, by Y. Phantom......

1855 c. (died a foal) by ditto ................ Wr Wichardson

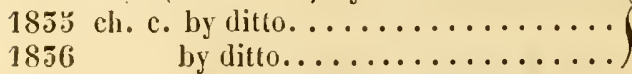

\section{Missed to $\mathrm{Y}$. Phantom in 18.52 and 54.}

GREY MIDDLEHAM MARE,

Bred by Mr Metcalfe, in 1825, her dam, Floranthe, by Oclavian, out of Caprice, by Anvil-Madeap, by Eelipse.

1851 b. f. by Wanton .............. IIr Jaques

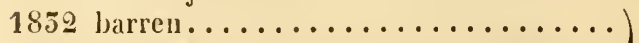

$1855 \mathrm{gr}$. f. Mildred, by Waverley, or Wanton

1854 l.. c. Pompey, by Waverley......... IIr Metcalfe

1853 cl. f. by Actieon ..............

1856 by Physician............

GRISETTE (sister to Goshawk)

Bred by the Duke of Graftox, in 1823, got by Merlin, her dam. Cofpuette, by Dick Andrews, out of Vanity, by Buzzard. 


\section{3:}

18.52 b. c. by Filho da Puta.......... D. of Grafton

1855 b. c. by Emilius .............. Sir S. Graham

1854 b. c. loy ditto................

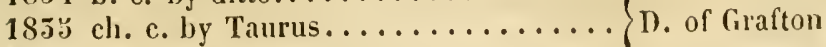

1836 b. f. by Lapdog..............

IA GRISETTE, by Viscount (Vol. III, P. 138) is now used as a hack; her last produce should stand thus :-

$1951 \mathrm{gr}$. f. Miss Annie, by Ionrcith..... . Ld Eglinton

\section{GULNARE,}

Bred by Mr R. Wusov, in 1824, got by Smolensko, her dam,

Medora, by Selim-Sir Harry-Volunteer-Ilerod-Blank.

*1851 br. f. Gulistan, by Whalcbone .....

1853 br. f. Genista, by Camel (s. to Belgium D. of Richmond

$18.5 \%$ in 1853 )

1856 br. c. by Sir IIercules .......... Ilis Majesty

Barren in 52 and 54 .

* Gulistax produced, in 1855 , a b. c. by St Patricli, and was sent $t 0$ Berlin that year, covered by Logic.

\section{GUTTY (sister to Chateau Margaux)}

Bred by Lord Eqreyroxt, in 1824, got by Whalebone, out of Wasp. by Gohanna-Highflyer-Eclipsc-Snap.

*1829 ch. f. Shrimp, by Greyleg ........

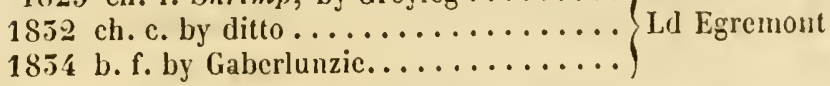

Barren in 1830 and 51 , missed to Gaberlunzie in 55 and $5 \ddot{3}$, and

was sold to Mr Kirkman, of Alabama, U. S. in 1855 , corered by Logic.

* Sunur produced, in 1853 , b. f. by Gaberlunzie, and died the next day; the filly was brought up by hand.

\section{GUY MANNERING MARE (sister to Auta)}

Bred by Mr Taylor, in 1825, her dam, Miss Platoff, by Remenbrancer-Overton-Gratitude's dam, by Walnut-Ruler.

1852 ch. f. Proserpine, by IIclenus. ......

1855 b. f. Agnes, loy Bizarre........... Sir J. 1). King

She was not covered in 55 , and is dead. 


\section{HAMBLETONIA,}

Bred by Gen. Lestie Cuming, in 1817, got by Stamford, out of Harmonica, by IIambletonian-Honica, sister to Mary Ann, by Sir Peter.

1827 b. f. Monica, by Champignon .......

1828 br. f. Mordelia, by Monreith (put to Gen. L. Cuming the stud)

1851 ch. c. General Chassé, by Actæon.... Sir Jas. Boswell

1855 b. f. by Canteen .............. Mrs L. Cuming

1854 b. c. Calendar, by Canteen.........
185 b. c. by Beagle...............

Missed in 1829 to Canteen, 32 to Jerry, 56 to Corinthian; not covered in 29.

\section{HAMBLETONIAN MARE,}

Bred by Mr Garforth, in 1812, her dam, Marcia, by CorianderFaith, by Pacolet - Atalanta, by Matchem.

1818 b. c. by Prime Minister........... Mr Garforth

1821 gr. f. (Agnes) by President ......... Mr l'age

1822 slipped a colt, by Comus . . . . . . . .

1825 gr. c. Turban, by Selim ..........

$\left.\begin{array}{l}1824 \text { b. c. by Clinker } \\ 182 y \text { b. c. by Tiresias }\end{array}\right\}$ died young......... Ld Lowther

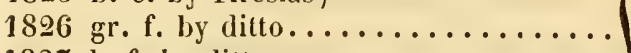

1827 b. f. by ditto.................

1828 gr. c. Protocol, by Partisan (s. to Vienna) Mr Williamson

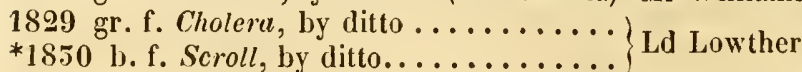

1855 gr. c. by ditto................ Mr J. Day

1854 slipped f. by ditto ............ Ld Lowther

1857 by Maple............. Mr Pigott

Barren in 1851, 52, 55, and 56.

* Scroll produced, in 185́, b. c. Stain, hy Stainborough, missed in 5̆5, and was covered that year by kowton.

\section{HAMBLETONIAN MARE,}

Bred by Sir H. V. Tenpest, in 1815 , her dam by Shuttle-Drone - Catherine, by Y. Marske.

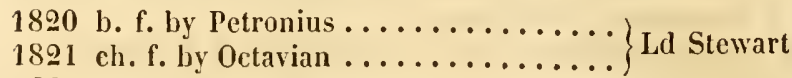

1822 b. f. Countess, by Catton.......... Mr Robinson 
1824 b. f. by Catton................

1823 b. f. by Blacklock (dead) . . . . . . . .

1827 b. f. by Osmond ..............

$1 S 51$ br. c. IIartonian, by Nimrod (son of

Whalebone) s. abroad IIr Blacklock

1852 b. c. by a country horse (dead) ......

1855 missed to ditto..............

1854 b. c. by ditto...............

1833 b. f. by Mac Orville ...........

1856 by ditto

In 1826 missed to Blacklock, 28 to Cedric, 50 to Nimrod.

\section{IIAND MAIDEN,}

Bred by Mr G. Cock, in 18I7, got by Walton, her ham, Anticipation, by Reningbrough, out of Expectation, by Herod.

18 by Palınerin.

1828 b. f. by a coach-horse...........

1829 cast a colt, by Lottery . . . . . . . .

*1850 br. f. Eve, by ditto

1851 bl. c. Inheritor, by ditto.......... Mr G. Cock

1852 slipped foal, by ditto...........

1835 br. c. by Brutandorf (died a yearling).

1854 slipped foal, by Lottery..........

183 ว br. c. Jolly Roger, by Voltaire ......

1856 by Liverpool .............

* Eve produced a br. c. by Voltaire, in 1855 , and was covered by Mulatto.

\section{HAPIIAZARD IIARE (sister to Perchance)}

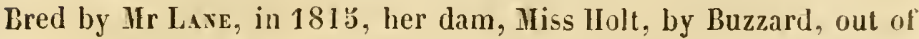

Camilla, by Highflyer-Hatchem-Regulus-Blaze-Fox, elc.

1825 br. f. by Iuley...............

182 i br. f. by ditto ...............

1826 ch.f. by ditto....................... Ir Lane

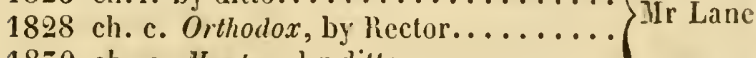

1850 ch. c. Martyr, by ditto ..........

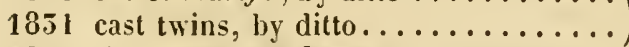

1852 ch. c. Cuff Cushion, by ditto........ Mr Sowerby

185.5 ch. c. by ditto (died soon after foaling)

185.4 ch. f. by ditto ................ Mr Lane

Barren in 1825, 27, and 5y. 


\section{HAPHAZARD MARE,}

Bred by Lord Lowtner, in 1819, her dam, Miss Furey, by Trumpator-Nark Anthony-Signora, by Snap-Miss Windsor.

$1825\left\{\begin{array}{l}\text { b. c. Partner, by Waxy Pope..... } \\ \text { and another foal . . . . . . . . . }\end{array}\right.$

1824 b. c. by $Y$. Warter ........... Dr Willis

1823 b. f. by Dinmont (died 2 days old) ...

1826 b. c. loy Tiresias ...............

1827 ch. c. Firman, by Sultan.......... Ld Lowther

1829 b. f. by Mountebank (dead)........

1850 b. f. by ditto ................

1851 b. f. by ditto............. Dr Willis

1852 b. f. by Y. Filho (son of Miniature)...

185.5 ch. c. by ditto ..............

In 1828 missed to Partisan, 1854 and 55 to Sultan.

\section{IIARMONY,}

Bred by Mr SHarn, in 1826, got by Reveller, her dam by Orville, out of Mirth, by Trumpator.

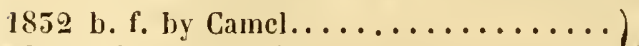

1855 missed to Pacha..................... Mir Shard

1854 b. c. Morison, by Tranby..........

\section{HARP ALICE,}

Bred by Lorl Egrenont, in 1814, got by Gohanna, her dam, Amazol, by Driver, out of Fractious, by Mercury.

1819 b. f. by Bustard (Buzzard)......... Mr Wood

1821 b. f. by Teddy-the-Grinder ......... Mr Thompson

1825 b. f. by Marmion. . . . . . . . . . . Mr Wood

1824 b. f. (Clare) by ditto............

1895 dead foal ......................... Ed Egremont

1828 b. f. by Whalebone (put to the stud)..

1829 b. f. (Dryad) by ditto ........... D. of Richmond

18.30 b. f. by ditto (put to the stud)....... Ld Egremont

1851 b. c. Faunus, by ditto ........... Mr Greville

1852 b. c. Lurcher, by Greyleg ........ Mr Mills

1853 dead f. by Chateau Margaux ........

Barren in 1826 and 27 , missed to Chatcau Margaux in 54 and 55. 


\section{HARPIIAI LASS,}

Bred by Sir M. M. Srkes, in 1817, got by Camillus, her dam, Statira, by Beninghrough-Stella, by Plıcnomenon-Skypecper.

1821 ch. f. Gipsy, by Tramp (put to the stud) Mr Tarleton $1822 \mathrm{lr}$. f. by ditto (sent to France in 1827) Mr B. leters 1824 ch. f. by a half-bred horse.........

1826 ch. f. Lucy, by Tramp............ Mr F. Dickson

1828 b. f. Leda, by Lottery...........

1829 b. f. by ditto ............... Mr Critchley

1850 b. f. by Brutandorf............ Mr Ilicks

1851 b. c. Dice, by Lottery............ Mr Ijickson

1852 b. c. Borghese, by Brutandorf....... Sir R. W. Bulkeley

1855 ch. f. by Theodore................ Mr Magniac

No produce in 1825,25 , or 27 .

\section{HARRIET (Young)}

Bred by Mr Tatтon Srkes, in 1812, got by Camillus, her dan,

Harriet, by Precipitate, out of Ioung Rachel, by Voluntecr.

1818 br. c. by Prime Minister..........

1819 b. f. (Acklam Lass) by ditto........ Mr Tatton Sykes

1820 b. f. by ditto................

1821 b. c. by ditto................ Col. Cradock

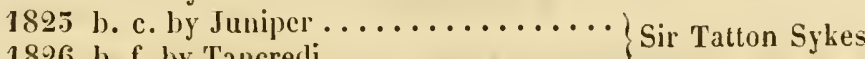

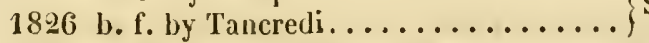

1835 bl. f. Lina, by Velocipede.......... Mr J. S. Barber

1854 b. f. by Tramp.............. Mr E. Barlow

Barren in 1822, 24, 25,53 , and from 26 to 55 , having been used as a hack during that time; not covered in $18.5 \mathrm{~s}$.

\section{IIARRIET (sister to St Hlelena)}

Bred by Mr Elus, in 1816, got by Stripling, her dam, Maniac,

by Shuttle-Anticipation, hy Beningbrough - Expectation.

$1820 \mathrm{ch}$. c. Rip Van Winkle, by Raphael.. Mr Kirby

1822 ch. c. by Bourbon (died a foal)....... Mr Ellis

1823 b. f. Theodosia, by Tramp......... Ld Fitzwilliam

1824 b. f. by Blacklock (died a foal).......

1823 ch. f. by ditto (dicd a foal)........ Ir Ellis

1827 b. f. by Figaro (died a foal) ........

1829 b. c. Primendorf, by Brutandorf..... 
$18 \pi 1$ b. f. by Figaro............... Mr Orde

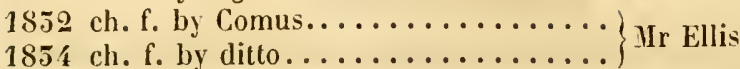

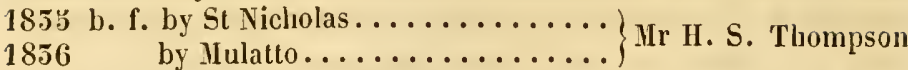

Missed in 1821, 26, 28, and 55, and slipped foal in 50.

\section{HARRIET,}

Bred by Mr Hanmoxp, in 1819, got by Pericles, luer dan by Selim, out of Pipylina, by Sir Peter-Rally, by TrumpatorFancy, by Florizel.

1824 b. f. Christabel, by Woful.......... Mr Thornhill

1825 b. c. (Hector) by ditto .......... Ld G. H. Cavendish

1826 br. f. Discovery, by Tíresias......... Mr Batson

1827 br. f. Recovery, by Centaur......... Mr G. Boast

1829 b. c. Mixbury, by Catton...........

1850 ch. f. Revelry, by Reveller..........

1851 ch. c. Plenipotentiary, by Emilius....

1854 ch. f. Voluptuary, by Reveller......

1856 by Emilius...............

Missed to Emilius in 1828 and 55 , to Peter Lely in 55.

\section{HAZARDESS,}

Bred by Mr Williassov, in 1818, got by Haphazard, her dam

by Orville, out of Spinetta, sister to Peggy, ly Trumpator.

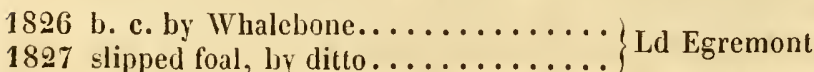

1828 br. f. Alea, by ditto ............ Ld Jersey

1829 bl. c. Oroonoko, by ditto.......... Sir G. Heathcote

1850 b. f. by ditto (died a foal) ......... Ld Egremont

1851 b. f. by ditto................. Mr M. Gray

1852 b. f. by Gaberlunzie.................

1855 missed to Chateau Margaux......... L Ld Egremont

1854 b. c. by ditto (died 6 months old) .....

1853 b. f. by ditto, or Gaberlunzie ....... .Ir Tattersall

\section{HEDLEY HARE (sister to Prince Leopold)}

Bred by II. R. H. the Duke of Yonk, in 1817, her dam, Gramarie, by Sorcerer-Sir Peter-Deceit, by Tandem-Perdita, by Herod. 
1821 b. c. Orion, by Phantom .........

1822 br. c. Peter Proteus, by Partisan.... H. R. H. the D. of 1825 b. f. by ditto............... York

1824 b. c. by Waterloo..............

1825 b. c. Frederick, by ditto.......... Mr Beardsworth

1826 b. f. hy Moses............... H. R. H. D. of York

1827 b. c. Young Orion, by Master Henry.)

1828 b. f. (Papillote) by IIustachio (s. to Mr Delmè Radcliffe Baron Biel) )

1850 ch. f. Fear, by Phantom, or Dunsinanc Mr E. Peel

1831 gr. c. Malcolm, by Dunsinane....... Mr Sowerby

1832 b. f. La Pucelle, by Filho da Puta... Mr R. Wilson

1855 by Sir Benjamin .......... Mr Green

Slipped foal in 1829, missed in 55 and 54 .

\section{IIEDLEY MARE (sister to Luss)}

Bred by Mr Barson, in 1822, her dam, Jessy, by Totteridge, out of Cracker, by High/fyer-Nutcracker, by Matchem.

1827 b. c. Sarorard, by Swiss ......... Sir T. Mostyn

1828 b. c. Pickpocket, by St Patrick......

1850 b. c. Jack Faucet, by Jack Spigot (s. to

$\left.\begin{array}{l}1851 \text { b. f. Birdlime, by Comus............ } \\ 1852 \text { ch. f. by IIalek (dicd a yearling).... }\end{array}\right\}$ Mr Mostyn

The mare died in the Spring of $\mathbf{1 8 5 5}$.

\section{IIELEN AROON,}

Bred by Sir J. H. Maxwel, in 1820 , got by Epperston, her dan, Anna, by Coriander-Ilighflyer-Tiffany, by Eclipse.

1826 ch. f. Silk-sleeves, by Doctor Syntax. Mr Gilmour

1851 br. f. by Richard.............. MIr Gibson

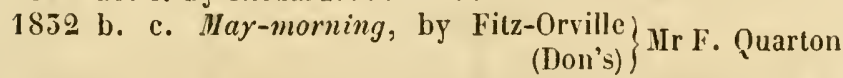

1855 br. f. Prejudice, by Brunswick (son of

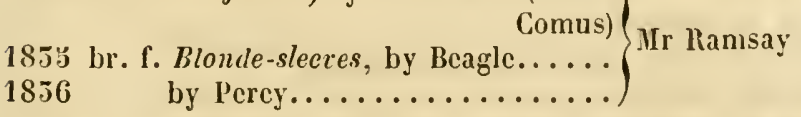

She was not put to horse in $1826,27,28$, or 29 , and missed to Brunswick in 34. 
HELEN MAR,

Bred by Sir J. II. Inaxwel., in 1819, got by Viscount, out of Amma, by Coriander-IIighllyer-Tilfany, by Eclipse.

1824 br. C. Jeannie Baptiste, by Epperston. 1826 br. f. by Smolensko.............

1827 br. f. by Epperston.............. Sir J. II. Maxwell

1828 ch. f. by ditto ................

1850 b. f. Rosa Mar, by Springkell.......

1852 gr. c. Squib, by Rochet (son of Muley)

1855 gr. c. Cracker, by ditto.......... b. of Buccleugh

1834 b. f. Nell Gwynne, by Canteen......

$185:$ gr. f. by Scarbrough ............

18.56 by ditto................

Missed to Canteen in 1829 and 51.

\section{HELL CAT,}

Bred by Sir W. Maxwert, in 1817, got by Cerberus, her dam,

Cutty Sark, by Haphazard-Brillante, by Whiskey-Diomed.

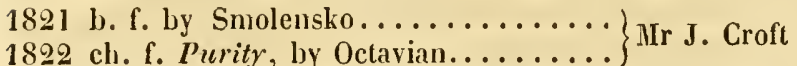

1825 br. c. (Souter Johnny) by Reveller... Ld Kelburne

1827 b. f. Brunette, by Blacklock ........ Mr Critchley

1828 br. c. The Serpent, by Doctor Syutax.. Mr Dickinson

1829 br. f. Ebony, by Jerry............

1850 b. f. Little-go, by Lottery ........

1851 ch. f. Fire-fly (aft. Wrasp) by Honreith.

1859 b. c. Tom Cat, by Fitz-Orville (Don's) MIr W. Mlexander

185.5 ch. f. by Percy ................

18.5.5 ch. c. by Beagle..............

18.36 by Jack Spigot............

Barren in 1825,21 , and 26 ; not corered in 25.

\section{HENRICU,}

Bred by Mr Ezwes, in 1895, got by Woful, out of Miss Sophia.

1829 b. c. by Hiddleton............. Mr Elwes

18.50 f. by ditto (died soon after foaling)

18.92 b. f. by Partisan ............... Ld Jersey

18.5 .5 b. c. loy Emilius................ Mr Elwes

Missed in 1851, and sent to France in 55. 
IIENRIETTA, the dam of Tarrare (Tol. III, p. 175) was destroyed in Nor. 1851. IIer last two produce should stand thus :-

1828 b. c. Clarion, by Calton (s. to Francé

b. f. (Anne) by ditto (s. to Ilungary Ld Scarbrough in 1854 , covered by l'artisan)

The IIIT-OR-HISS IIARE, flora's dam (Vol. III, p. 17j) was sold to go abroat in 1855, with a filly foal by Belzoni.

\section{IIOI'E,}

Bred by Mr Rawlirsox, in 1826, got by Rubens, her dam by IIaphazard, out of Promise, by Walton-l'arasol, by l'ot8o'sl'runclla.

1851 gr. f. Pickininy, by Buckfoot (put to

1955 b. f. by Eimilius..

1853 b. c. liy l'riam................

Not covered in 1851 or 55.

\section{HUMPIIREY CLINKER HARE,}

Bred by in 1829, her dam, Gadabout, by

Orville, out of Minstrel, by Sir l'eter-Matron, by Florizel.

1854 ch. c. by Winton (son of Mulcy)......
1858 b. f. by Bryth-yones, Newcross

IIIBL.A,

Bred by Lord Grosvexon, in 1821, got by lubeus, her dam, Larissa, by Trafalgar, out of Meteora, by Meteor.

1828 b. c. Metheglin, by Filho da Puta.... I.d Grosicnor

1829 ch. f. Saccarina, by Champion......

1850 b. f. IIoneymoon, by Filho rla I'uta (put to the stud)

1851 and 52 missed............... Ld Westminster

185.5 br. c. by Conductor (hilled a yearling)

1854 ch. f. by Pantaloon..............

185 s ch. c. by ditto.................

18.56

by Camel................ 


\section{HYDROGEN,}

Bred by Mr Dunderdale, in 1820, got by Comus, her dam, Nitre, by Precipitate, out of Grey Skim, by Woodpecker.

1827 ch. f. by Cervantes ............

1828 ch. f. by ditto ...............

1850 b. f. by Chateau Margaux.........

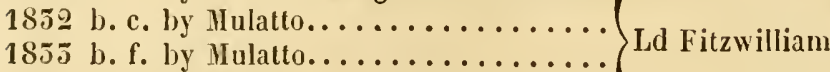

1854 b. f. by Mambrino.............

1855 ch. f. by Amadis ..............

1856 by Medoro..............

In 1829 missed to Cervantes, in 31 to Mulatto.

\section{JANE,}

Bred by Mr Hereert, in 182:, got by Hoses, out of IIarrict, by Sclim-Precipitatc-Woodpecker.

1850 ch. c. Samarcand, by Blacklock.....

1851 ch. c. by Lottery (dead) ............

1852 ch. c. Valentissimo, by Velocipede...

1855 barren........................ Sir G. Ileathcote

1854 ch. f. Countess, by The Colonel

1855

f. by Buzzard (dead)...........

\section{JANE SIIORE,}

Bred by H. R. H. the D. of Yonk, in 1820, got by Woful, her dam, Bella Donna, by Seymour, out of Gramarie, by Sorcerer.

1826 b. c. by Centaur..............

1827 b. f. Cantatrice, by Comus (put to the

1828 f Penance, by Emilius .......

1829 br. c. Damascus, by Reveller....... Sir G. Heathcote

1852 b. c. Troubadour, by Comus........

1854 br. c. Aleppo, by Revelter..........

1853 br. c. Amato, by Velocipede.........

Missed to Lottery in 1850 and 51 , barren also in 55 .

$\operatorname{IC} \Lambda \mathrm{RI} \Lambda$,

Bred by Gen. Grosveror, in 1824, got by The Flyer, her dam, larma, by Dick Andrews, out of May, by BeningbroughPrimrose, by Mambrino. 
1828 br. c. Sarperlon, by Emilius........

*1829 lor. f. Pinions, by Trultle.........

1852 b. c. Nantilus, by Skifl' (s. to Germany

1854 b. f. Laodice, hy Priam......... in 1853) Gen. Grosvenor

1853 clı. c. by Buzzard $\ldots \ldots \ldots \ldots \ldots \ldots \ldots$

by kowton ..............

Barren in 1850 and 51 .

- Pinions produced a colt by The Colonel in 1854, was barren in 55. anddied.

ID ALIS,

Bred by Lord Grosvexon, in 1815, got by Peruvian, her dam, Iusidora, sister to IIeteora, by Meteor, out of Maid-of-all- Work.

1820 f. by Ambo (dam of Mayday, by

1821 by Lismahago............. Manfred) Mr W. Wood

1822 ch. f. by Manfred (died from spaying).

1823 ch. f. Panacea, by Castrel (lamed at a Mr L. Charlton year old, and died at 4 or 3 yrs old $)$ )

1824 ch. c. Pantaloon, by Castrel......... Mr Giffard

1826 b. f. Pantechnetheca, by Master Henry \} Mr L. Charlton

1829 b. c. by Ilaster llenry, or Wamba ... Mr Ileywood

1850 ch. c. Parthian, by Champion ....... Mr L. Charlton

1851 ch. c. Preservative, by Rossini....... Mr Mackintosh

1852 ch. c. Ludford, by Wamba......... Mr F. R. I'rice

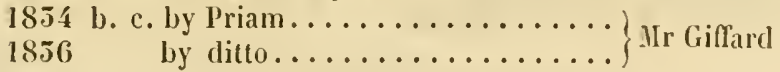

Barren in 1825, 28, 53, and 53.

JEANNE D'ARC, by Comus (Vol. III, p. 181), produced a bl. c. in 1852 which died, and the mare died the next year; her produce of 1851 should stand thus :-

1851 b. c. Lambkin, by Y. Phantom...... II. T. O. Powlett

\section{JEANNETTON,}

bred by Mr Prexdergast, in 1825, got by Rainbow, her dam, Jannette, by Camillus, out of Helen, by Delpini.

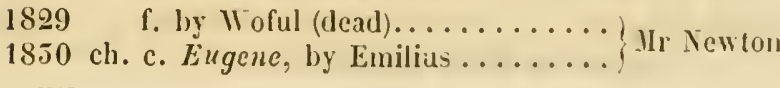

rol. Ir. 


\section{6}

$1852 \mathrm{ch}$. f. IIeberlé, by ditto (s. to Baron de

Maltzahn in 1856 , covered by Cetus)

1855 b. f. by ditto............... Jur Newton

1854 b. f. by Shakspeare.............

1853 ch. c. by Cetus...............

1856

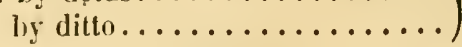

JENNY HILIS,

Bred by Mr Watr, in 182:, got by Whisker, her dam, Hornsea's dam. by Cerberus, out of Miss Cranfield, by Sir Peter-Pegasus.

$18.5 \mathrm{~s}$ ch. c. by Malek, or Champion........

18.4 ch. f. Darling, by Actron......... D. of Leeds

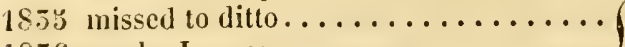

1856 by Langar $\ldots \ldots \ldots \ldots \ldots \ldots$

\section{JENNY RINTHEROLT,}

Bred by Mr Ballie, in 1815, got by Orion (son of Star) dam by Sir Peter-King Fergus-llary Anne, by Florizel.

1825 cl. f. Mr Dearie Oh, by Ardrossan...

1896 b. c. (Baillie Jarvis) by Champignon. .

1827 br. f. by Stainborough ........... Mr Baillie

1850 b. f. by Cleveland...............

$18 \pi 1$ ch. f. Kate, by Corinthian. ..........

1855 b. f. Hugger Lass, by Canteen.......

185: b. c. hy Scarbrough ............. D. of Bucclcuch

1856 by Canteen..............

In 1828 missed to Stainborough, 29 to Nicolo, 52 and 54 to

Canteen.

\section{JENNY SUTTON (Chesnut)}

Bred by Mr Goomissos, in 1817, got by Soothsayer, out of Johı of Paris' dam, by Buzzard-Rose, by Sweetbriar.

1 \$2j clı. c. Jack Sutton, by Carlton....... Mr Day

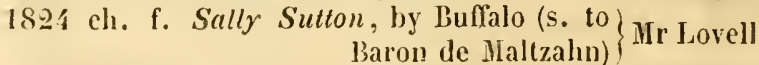

1827 ch. c. Lusher, by Reveller........

1828 ch. c. lyy dilto (dead)............ Mr Day

1829 f. by taprlog (died in foaling).....

1850 b. f. loy Reveller..............

1851 b. f. (Her Ladyship) aft. Mary, by Figaro Ld Wilton

1854 ch. f. by Bay Malton............

1856 by Lirerpool ............ Mr J. Etty

Missed in 1859,55 , and 55. 


\section{JLNNY VERTPRÉ,}

Bred by Mr Ilaffevdex, in 1827, got by Bobadil, her lam. Bella Doma, by Seymour, out of Gramarie, by Sorcerer.

1855 slipped foal by The Colonel.........

1854 f. ly Emilius (died a foal)...... Sir L. Glyn

$185:$

1856

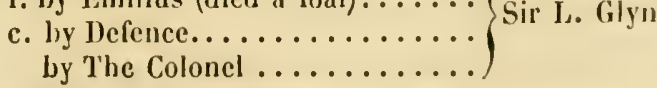

JEST, by Waxy (Vol. III, p. 185) produced a ch. c. in 18j2, which died immediately; she missed in 55 and 54 , and was shot.

\section{JEWESS,}

Bred by Mr C. DAY, in 1827, got by Moses, her dam, Calendulx, by

Camerton-Snowdrop, by Highland Fling-Daisy, by Buzzard.

185 2 b. f. by Godolphin ............. Mr C. Ilay

1855 b. c. Martello, by Defence ......... Mr Greville

1854 b. f. by Fungus............... Mr Mott

1856 by Defence.............. Mr Tattersall

In 1855 missed to Emancipation.

INA,

Bred by Sir Joms Brxg, in 1821, got by Smolensko, lier dan, Morgiana, by Coriander, out of Fairy, by Highflyer-Fairy Qucen, by Young Cade-Routh's Black-eyes, by Crah.

1827 ch. f. Zarina, loy Morisco..........

1828 b. c. Egbert, by ditto (s. to D. of Hol- Lal Anson

$1829 \mathrm{ch}$. f. by Sligo.................

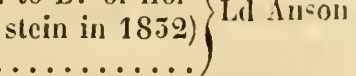

1850 ch. c. Mounteagle, by ditto........

1851 b. c. Altamont, by ditto (s. to France in Oct. 1854)

1852 b. f. Ileen, by ditto............ Lal Lichfich

1853 b. c. by Emilius................

1834 missed to Sligo ...............

1853 br. f. by P'riam

1856 not covered last year.

IXCEST (Vol. III, p. 185) produced a b. f. by Gaherlunzie, in 1852 , and was sold to Baron Biel that year with her foal. 
INCOGNITA (Vol. III, p. 185) produeed a eh. f. by Grey-leg in 1852, and was sold to Baron Biel in that year with her foal.

JOIIANNAH SOUTHCOTE, Variation's dam (Vol. III, p. 187) died in 1S54, having had no produce since 1851. ,fler last three produce should stand thus :-

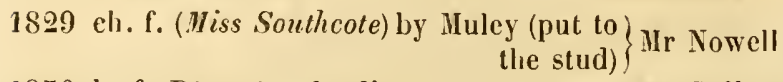

1850 b. f. Ditersity, by ditto .......... Mr Gully

1851 b. f. Vicissitude, by Longwaist....... Mr Nowell

Varation is in the Riddlesworth stud, and prorluced a b.c. by Emilins in 1835.

\section{IONE (sister to Duncan Grey)}

Bred by Sir M. W. Rinley, in 1827, got by Peirse, her dam by IIambletonian, out of Lady Caroline, by Buzzard-Calash, by Herod.

1854 gr. c. Midnight, by Caceia Piatti..... Mr Holtby

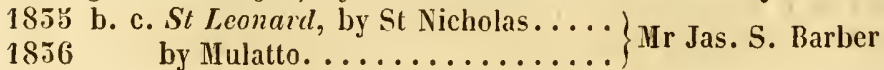

\section{ISABELLA,}

Bred by Mr Gascorgve, in 1820, got by Comus, her dam, Shepherdess, by Shuttle-Buzzard - Ann of the Forest, by K. Fergus.

1825 slipped foal, by Slim........... Mr R. Wilson

1826 b. e. by Nicolo............... Mr Williamson

1827 dead foal, by Wrangler.......... H Mr R. Wilson

1829 b. e. II urmaker, by Wrangler...... Ld Woreester

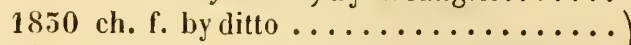

1831 br. c. by Stainborough .............

1832 b. f. Courtesan, by Filho da Puta (put MIr R. Wilson

1855 b. e. by Bedlamite..........................

1854 b. e. Pegasus, by Shakspeare ....... Mr Holmes

1855 eh. e. by Sir Benjamin .......... His Majesty

1856

by The Colonel ............

IS $A B E L L A$,

Bred by Mr Atusos, in 1822, got by Smolensko, her dam, Annabella, hy Shuttle-Drone-Contessina. 


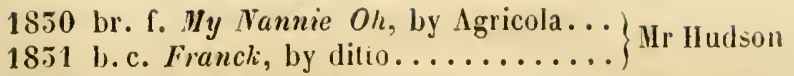

1852 b. f. Calista, by Velocipede........ Mr Altwood

1855 bl. f. by Waverley..............

1834 bl. f. by ditto .............. Hr T. Hudson

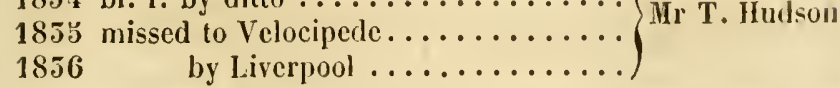

\section{JUBILÉE (sister to Coronet)}

Bred by Mr S. Kıse, in 182i, got by Catton, her dam by Paynator, out of Violet, by Shark -Syphon.

1829 b. f. by Waverley (died in 1850)..... Mr S. King

1850 br. c. Spider by Y. Phantom........ Mr Gully

1832 b. f. Rose, by Waverley........... Mr Houldsworth

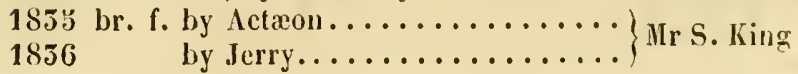

Barren in 1831, 35, and 54.

\section{JULIANA (late Lady Thigh)}

Bred by Lord Egrenoxt, in 1810, got by Gohanna, her dam, Platina, by Mercury-IIcrod-Y. Hag, by Skim.

1816 b. f. Lady Leg, by Fyldener........

1817 ch. f. Lady Foot, by Rubens (died on the passage to India) Mr I. Sadlet

1818 b. c. Black-leg, by Fyldener........

1819 ch. c. Brilliant, by Usquebaugh......

1822 b. c. Saladin, by Selim...........

1825 b. c. by Ardrossan (dead)..........

1824 b. f. Matilda, by Comus...........

1825 b. c. Stapleton, by Magistrate.......

1826 b. c. Netherby, by Cervantes (s. to IIr Petre

1827 ch. f. Laura, by Figaro. ricmu in 1850$\rangle$

1828 ch. f. Iopsy, by Comus ..........

1829 ch. c. by ditto................

1831 ch. c. Valparaiso, by Velocipede..... D. of Leeds

1852 ch. f. Lucia, by Granby........... Ir Petre

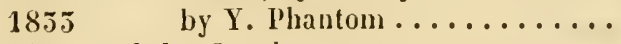

1854 b. f. by Granby ................ Mr B. Eddison

1836 by Langar .............. Mr L. Savile

In 1820 missed to Soothsayer, and was not covered that season, in 50 missed to Whisker. barren also in 5.5 . 


\section{JUNIPER MIARE,}

Bred by Col. Wrisox, in 1814 , her dam, Trimbush, by Teddy-theGrinder-P'rincess, by Sir l'eter-Dungannon-Turf, etc.

1822 ch. c. by The Flyer............ Mr Randolph

1826 ch. c. Jack Junk, by Nicolo........ Col. Wilson

182 T cl. c. by Wrangler............. Mr R. Wilson

1828 b. f. Modesty, by Fungus.......... Mr Connor

1829 ch. c. Andallah, by Nigel......... Mr Nevill

1850 ch. f. Doska, by ditto .

1851 b. c. Puszle, by Reveller...........

1852 ch. 1. Daisy, by Tarrare ..........

1855 br. f. Lady Anna, by Camel........ Mr W. R. Phillimore

1834 b. c. Sheriff, by Laurel............

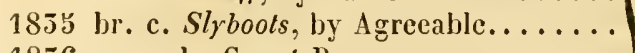

1856 by Count Porro ...........

\section{JUNIPER MARE,}

Bred by Mr , in $181 \%$, her dam by Sorcerer, out of Virgin, by Sir Peter-Pot8o's-Editha, by Herod.

1821 ch. f. Rufina, by Blacklock........ Sir W. Wynn

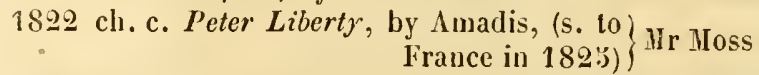

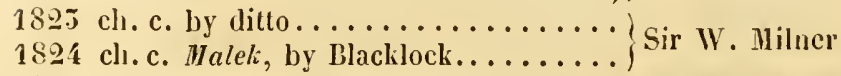

1895 ch. c. Velocipede, by ditto ......... Mr Armitage

1826 ch. c. Minster, by ditto..........

1827 ch. f. Moss Rose, by ditto (put to the Mr Clifton stud))

1828 b. f. by Theodore (died a foal)....... Mr Moss

1829 ch. c. William the Fourth, by llacklock Mr Ridsdale

1850 ch. f. Energy, by ditto (in Ld Chester-

(1851 field's stud)

1831 b. f. Velocity, by ditto ...........

1855 ch. f. Emineh, by Sultan........... Whr G. Walker

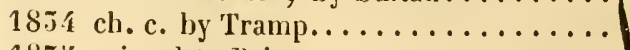

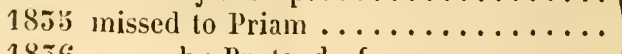

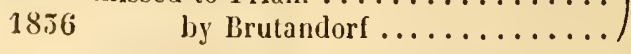

\section{JUNIPER MARE,}

Bred by the Dule of Graftox, in 1819, out of Caprice. 


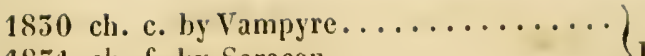

$1851 \mathrm{ch}$. f. by Saracen............. D. of Grafton

1852 ch. f. by ditto (s. to France)........

185.5 ch. c. IIalfield, by Bcdlanite......... IIr W. Edwards

185 . ch. f. by ditto...............

1855 missed to Cian..................... of Gralton

1856

by Lapdog. ..............

\section{JUNIPER MARE,}

Bred by Col. Wrusor, in 1819, her dam (Spotless and Bolter's dam) by 'Trumpator-Highflyer-Othcothëa.

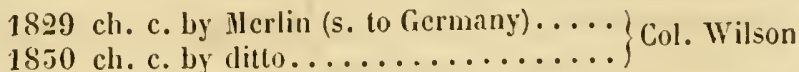

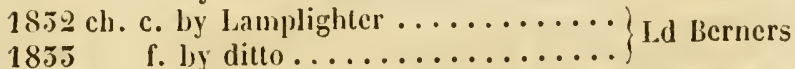

In 1851 missed, and sent to Germany in 55 , with her foal.

\section{JUSTITIA,}

Bred by Mr Srkes, in 1824, got by Cervantes, lıer dam, lady

Rachel, by Stamford, out of Young Rachel, by Voluntecr.

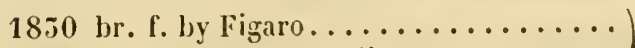

1851 br. c. Furfan, by ditto.............

1852 br. c. De Roos, by Velocinede...... Wr Foulis

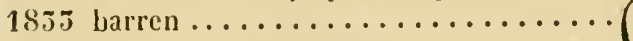

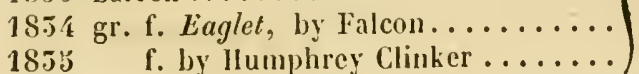

\section{KAMSCHLTKA,}

Bred by Lord Westunster, in 18:6, got by Haster Heury, her dam Passamaquoddi, by lignum Vitæ, out of Ilind, by Sir l'eter.

1851 br. c. by Camel............... Ir T. Evans

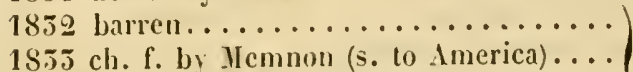

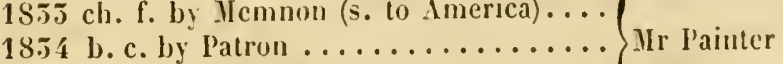

185 : b. c. by Emancipation ..........

1336

by ditto...................

\section{K.ITE,}

Bred by Mr Buntor, in 1825, got by Catton, luer dam, Miss Garforth, by Walton-liyacinthus-Zara, by Delpini. 
1828 ch. f. by Oetavius............. Mr Burton

1829 b. f. Sultaness, by Saladin .........

1851 ch. f. by Velocipede (put to the stud). Mr John Scott

1852 ch. c. Ainderby, by ditto......... Capt. Taylor

185.5 ch. c. Willesden, by ditto........ Sir G. Heatheote

Died in 1855 .

\section{KATHERINA (late Perspective)}

Bred by Lord Lowther, in 1817, got by Woful, her dam, Landscape, by Rubens, out of Iris, by Brush-Herod.

1821 b. f. Point de Vue, by Navigator.....

1822 ch. f. Lace, by Oiseau............. Ld Sligo

1825 b. f. Lead, by ditto .............

1824 ch. f. Prospective, by ditto ........ Ir Allen

182 s ch. c. IIardbargain, by Langar...... . Mr Allen

1826 ch, c. Rowton, by Oiscau.......... Mr Petre

1827 b. f. by Figaro (died 2 yrs old)...... Ir Allen

1828 missed to Der Freischutz..........

1829 ch. c. Beaufort, by Comus (died rising)

5 yrs old) IIr Petre

1850 ch. r. by Blacklock (died rising 2 yrs

1851 br. c. Bordolph, by ditto.......... Mr E. Peel

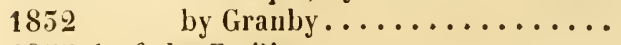

1853 b. f. by Emilius...............

1854 missed to Priam ............... Ld Chesterfield

$\left.\begin{array}{ll}1855 & \text { f. by Priam } \ldots \ldots \ldots \ldots \ldots \ldots \ldots \ldots\end{array}\right\}$ Ld Chesterfield

\section{KATHERINE,}

Bred by the Duke of Rutbaxn, in 1821, got by Soothsayer, her dam, Quadrille, sister to Varennes, by Selim, out of Canary-bird.

1826 ch. c. Taurus, by Phantom, or Morisco Ld Tavistock

1827 b. f. by Hoses (died young)........ Ld Warwick

1828 h. c. Incubus, by Phantom (s. abroad).

1899 barren.....................

1850 ch. f. Tarantella, by Tramp........

1851 gr. c. Viator, by Stumps ..........

1852 b. c. Quintus, by Emilius........... Jir Cookes

183.5 b. c. Saltator, by Stumps ..........

18.54 b. f. Saltarella, by ditto . . . . . . .

185.5
1856 b. f. Barcarolle, by ditto........... 


\section{3}

KITE,

Bred by Lord Stınfokn, in 1821, got by Bustard (son of Castrel) her Iam, Olympia, by Sir Oliver, out of Scotilla, by AnvilScola, by Eclipse.

1850 b. f. Lady Moore Carew, by Tramp...

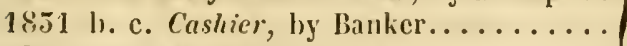

18.5 .5 clı. f. Fulture, ly langar. ......... Mr Allanson

1854 br. c. Sir Bamfylde, lsy Trausp...... .

1853 h. c. Fra Diavolo, by Recovery.......

Had twins in 1828; not covered in 28, 51, or 5:5.

KITTEN, by Beringbrough (Vol. III, p. 194). Her last produce should stand thus :--

1829 b. f. Sally Barlow, by IIumphrey Clinker Mr G. O. Smith

\section{KITTY FJ $A N \Lambda G \Lambda N$,}

Bred by Mr Inwsor Lıuetox, in 1814, got by Orville, her dam, by Stamford, out of Belle Fille, by Weasel-Y. Marske.

1821 b. f. by Raphacl ..............

183 2 b. f. by Leopold..............

1825 b. c. True Blue, by Catton ........ Mr Loftus

1824 b. c. by ditto ..... . . . . . . . .

1826 b. c. by 1 hjer (shot by accident in 1826)

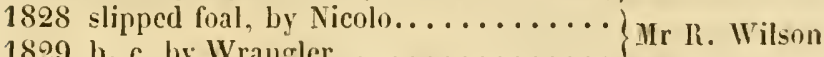

1829 b. c. by IVrangler.............

1855 br. c. Shamrock, by Bedlamite...... Mr Sowerby

1854 c. by Shakspearc................. R. Wilson

Missed in 1827, 50, and 52, and died about Christmas 51 .

\section{I.ACERT $\Lambda$,}

Bred by Lord Rocs, in 1816, got by Zodiac, her dam, Jerboa, by Gohamna, out of Camilla, by Trentham.

1826 b. c. Tacarin, by Orville ......... Ir Forth

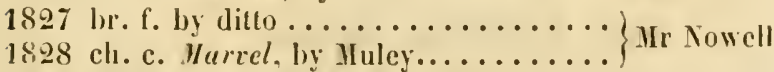

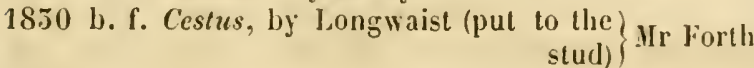

1851 b. c. by Longwaist ............. D. of Cleveland 
1852 f. by Longwaist (dead) $\ldots \ldots \ldots \ldots \ldots$.

1834 b. f. by Longwaist.............. IIr Nowell

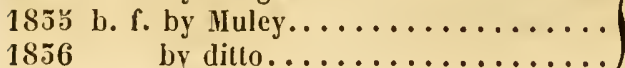

Missed in 1825 and 29. - She was ridden before being put to the stud.

\section{LA DANSEUSE,}

Bred by Mr Cuftox, in 182y, got by Blacklock, her Jam, Madame Saqui, by Remembrancer, out of Fadladinida, by Sir Peter.

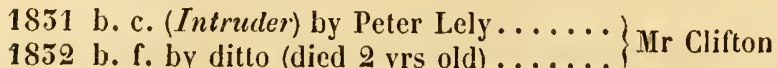

1835 not covered last year............

1854 b. f. Rigadoon, by Jerry ........... Gen. Yates

1855 c. by Recovery (died a foal)...... Mr Yates

1836 b. f. by Camel.............. His Majesty

LADY BLUEFOOT, sister to Spectre (Vol. III, p. 19马) produced a b. f. by Wamba in 1832, and was sold to Baron Biel that year in foal to him.

\section{LADY CRAMFEAZER,}

Bred by Sir W. Maxwelu, in 1814, got by Stamford, her dam,

IIrs Barnct, by Waxy-Woodpecker-Heinel, by Squirrel.

1820 ch. f. by Comus.............. Sir W. Maxwell

1822 b. f. Clari, by Ardrossan..........

1823 ch. c. Sir Malachi Malagrowther, by

1824 br. c. Romeo, by ditto....................... Mr Baird

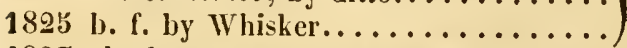

1827 ch. f. Ramona, by Whisker........

1850 b. c. by ditto................ Sir D. Baird

1853 c. by Partisan................. Mr Grant

1855 ch. f. by Emilius.........................

Barren in $1826,28,29,51$, and 32 .

\section{LADY EASBY (late Jannette)}

Bred by , in 1821, got by Whisker, her dam, Y. Woodpecker - Beningbrough - Nercury - Mary Ann, by Florizel. 


\section{5}

$\left.\begin{array}{r}1850 \text { b. f. Lady Charlotte, by Catton (s. to } \\ \text { [Baron IBicl) }\end{array}\right\}$ Mr Spalding

1851 b. f. by Velocipede............ Mr G. Clark

1832 b. f. Lady Albert, by Langar....... Mr Spalding

1855 ch. c. Jacob Faithful, by ditto....... Col. P'ecl

1854 br. f. Lady Stepney, by Jerry.......

185 y br. c. Count Lauriston, by Brutandorf Mr Watson

1856 missed to Canteen............

\section{LADY ELIZA,}

Bred by Mr RıDvel., in 1815, got by Whitwortl, her dam, X Y Z's dam, by Spadille, out of Sylvia, ly Y. Marske.

1817 ch. f. by Ardrossan............ Mr Riddell

*1820 b. f. by Lcopold................ Mr G. Davidson

1824

f. by $A$ bjer (died 2 days old) .....

1825 ch. f. ly Ardrossan...........

1826 b. e. Godfrey, ly Dr Syntax .......

1827 b. c. by ditto (lead) ............

1850 b. c. Blandford, ly his Grey Arabian. Ilr Attwood

1851 ch. c. Callisthenes, by ditto ........

$1852 \mathrm{ch}$. f. Iole, by his chesnut Arabian...

1854 ch. f. by Argantes..............

1855 b. f. by Ariosto (son of Rosalind).....

Barren in 1818, 19, 21, 22, 25, 28, 29, and $5 \tilde{5}$.

- This Lcopold mare produced a b. f. by Alexander in 1825, missed in 26 . and was afterwards ridden.

\section{ADY EIIMELINE,}

Bred by Mr T. O. Powlett, in 1827, got by Y. l'lantom, her dam by Orville, out of Golden-leg's dam, by Buzzard-Hornpipe, ly Trumpator.

1855 b. f. ly Jack Spigot............

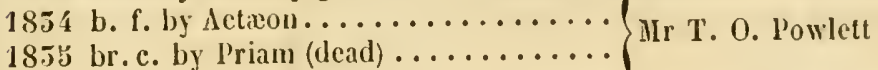

18.56

\section{LADY FULFORD,}

Bred by Mr Elus, in 1820, got by Walton, her dain, Maniac, loy Shuttle, out of Anticipation, by Beningbrough-llerorl.

1826 ch. c. Madcap, by Tramp, or Blacklock Col. King 
"1829 ch. f. Lunette, by Figaro.........

1850 b. c. The Page, by ditto.............. Mr J. Ir. Ellis

1851 ch. f. by ditto (s. to Germany, 1835)..

1855 b. c. by St Nicholas.............. Mr H. S. Thompson

$1854 \mathrm{eh}$. f. by Velocipede ............ Mr J. W. Ellis

18.56

by Duke (son of Champion).... Mr II. S. Thompson

Hissed in 1825, 27, 28, 52, and 35.

- Lunette was sent to Ireland, and produced

1855 br. f. Spectacles, by Picton

Ld Sligo

\section{LADY GEORGIANA,}

Bred by Lord Scarerovgr, in $\mathbf{1 8 2 5}$, got by Catton, her dam by Paynator, out of Violet, by Shark.

1852 cl. e. Pomfret, by Langar ...........
18.55 b.f.Princess Victoria, by ditto, or Falcon Mr Gutly

She had some foals before 1852 , which died very young, and she was sold to the D. of Ilolstein in July, 53.

LADY GREY (Fol. III, p. 197) missed in 18.52 and 35 , and was destroyed.

\section{LADY HENRY (sister to Master Henry)}

Bred by Mr Ecwes, in 1816, got by Orille, her dam, Miss Sophia, by Stamford-Sophia, by Buzzard-Huncamunea, by Ilighllyer - Cypher, by Squirrel.

1824 b. f. by Vampyre ............. Mr Pell

1825 b. e. Palemon, by ditto (sent abroad). Col. Lautour

1897 b. f. by Reveller................

1828 b. c. Sir Thomas, by $\Lambda$ bjer........

1899 b. c. by Partisan (dead)...........

1850 b. f. Lilly Lass, by Partisan, or Skiff. .

1855 ch. c. Ilalyards, by Slim .......... Mr Sowerby

1854 b. f. Iloratia, by Y. Emilius (brother to 1855 ch. c. Horace, by ditto............

In 1826 missed to Tiresias, 51 to Camel, 52 to Skiff.

\section{LADY MOWBRAY,}

Bred by the Duke of LeEDs, in 1827, got by Blacklock, her dant, Lady of the Vale, by Mowbray -- Shuttle--Hopeful, by Sir Peter. 
1853 b. f. Lotchin, by Lottery..........

1854 b. f. Meliora, by Waverley................ D. of Leeds

$\left.\begin{array}{rr}1855 & \text { missed to } \Lambda \text { ctacon............... } \\ 1856 & \text { by Physician, or Winterfield ... }\end{array}\right\}^{\text {D. of Leeds }}$

\section{ADY NEIVTON,}

Bred by Mr Howard, in 1826, got by Oiseau, her dam by Haphazard, grandam by Stamford, out of Alexina.

1850 b. c. by Theodore............ Mr Newton

1851 ch. c. by ditto...............

1852 b. f. loy Y. Phantom (put to the stud) Mr Allen

1855 b. c. by Victory (son of Waterloo)....

1854 ch. c. Malton, by llumphrey Clinker.. Mr Finch

1853 ch. f. by ditto ......................... IIr Allen

\section{LADY NEVILLE,}

Bred by Mr Russel., in 1822, got by Orville, her dam by Dick Andrews, out of Desdemona, by Sir Peter-lleroine.

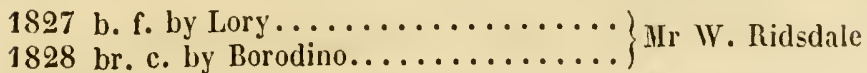

1851 b. c. by Lottery ............... Mr Greatrex

1852 br. c. by ditto............... Mr Kirby

1854 b. f. by ditto............... Mr Lee, of Gardhan

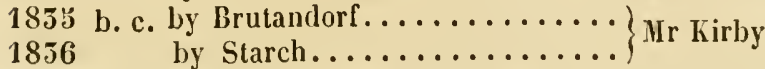

Missed to Lottery in 1829 and 50 , barren also in 55 .

\section{LADY OF THE LAKE,}

Bred by his H. R. H. the Duke of Tonk, in 1809, got by Sorcerer, her dam by Saltram-Herod-Thisbe, by Latham's Snap.

181 b b. c. Fit zcloddy, by Election, or Seymour 1818 br. f. by ditto................ 1819 b. f. by Iledley............... 1820 b. f. by Catton (dead) ........... H. R. H. the D. of

1822 b. f. by Octavius .............. York

1825 b. f. Ellen, by Orville........... 1824 br. c. Sharpshooter, by l'aulowitz ....

1825 b. c. Frederick, by Filho da Puta .... IIr Yates

1826 b. c. Douglas, by ditto ..........

1827 b. f. Blanche, by ditto .......... 
1828 b. c. Lochinvar, by Swap..........
1829 br. f. Eccentricity, by Bedlamite ... Col. Peel

$18.30 \mathrm{br}$. c. Tuft, by Whisker..........

1831 b. f. Ophelia, by Bedlamite......... HIr E. Peel

1832 b. c. Intruder, by Paul l'ry, or Belzoni

1853 b. c. Fabius, by Emilius...........

In 1816 slipped her foal by IIedley, 21 ditto by Seymour; barren in 1817,54 , and 53 .

\section{LADY OF THE TEES,}

Bred by Mr Fergusox, in 1822, got by Octavian, her dam by

Sancho, out of Miss Furey, by Trumpator-Mark Anthony.

1829 b. c. Julius, by Jerry . . . . . . . . .

*1850 b. f. Tesane, by Whisker .........

1852 b. f. Nymphalin, by Langar (died rising

3 yrs old)

1855 b. f. Tiffany, by Jerry ............ Ir S. L. Fox

1854 b. f. Teresa, by Langar............

1858 b. c. by Ilumphrey Clinker.........

1856 by Langar...............

In 1828, missed to Cedric, 51 slipped foal by Lottery.

- Tfsaxe produced, in 1855 , b. f. by Medoro, and was covered by Langar.

\section{LADY OF THE VALE,}

Bred by Mr JaQues, in 1817, got by Mowbray, her dam by Shuttle, out of Ilopeful, by Sir l'eter-Play or l'ay's dam, by Herod.

1825 ch. f. by phantom..............

$1824 \mathrm{gr}$. f. by Grey Hiddleham.........

1825 ch. f. Mrs Rye, by Octavian ........

1826 b. f. by Wanton (dead) ...........

1827 ch. f. Ladly MIowbray, by Blacklock. .

1828 b. f. Lady Margery, by Wanton (put

1829 b. f. Ladly Maud, by Jerry.......... D. of Leeds

b. f. Lady II arcia, by Whisker (foaled

Dec. 23)

1831 br. c. The Baron, by Blacklock......

1852 ch. f. by Actæon (dead)...........

Died in 1853.

* Lady Nancia produced a ch. c. Arcturus, by 1 ctxon, in 1855 , and was covered that year, by Physician. 


\section{LADY SARAII,}

Bred by Lord Strutunore, in 1819, gol by Lcopold, her dam ly Wizard, out of Remembrance, by Sir Solomon-Queen Mab, by Eclipse.

1827 ch. f. Miss Inverarity, by Mozart....

1828 ch. f. by ditto $\ldots \ldots \ldots \ldots \ldots \ldots \ldots$

1829 ch. f. by ditto ...............

1830 b. f. Industry, by Agricola ......... Dr Chifton

1832 b. c. by litto.................

1835 b. c. by Lucerne.............

1834 ch. c. by Wildfire

Died in June, 1854.

\section{LADY SARAII,}

Bred by Mr Gieeeson, in 1826, got by Tramp, her dam, Mliss Wentworth, by Cervantes.

18.52 b. c. Master John, by Emilius. ....... Mr F. Richardson

1854 b. c. by St Nicholas ............. Mr Greville

1856 by Actæon............. Ld Chestcrfield

Missed to St Nicholas in 1855 , to Priam in 53.

\section{LADY'S SLIPIER,}

Bred by Mr Inaffexden, in 1824, got by Waxy Pope, her dam by Rubens, out of Slipper, by Precipitate-Catherine, by Woodpecker.

1850 b. f. by Bobadil (s. to Germany) ......

1831 cast her foal................. Mr Haffenden

183 a b. c. by Bohadil (died a foal) ........

1853 b. f. by ditto (s. to Spain)..........

1854 b. c. by ditto................ Mr Cooper

1855 b. c. by ditto $\ldots \ldots \ldots \ldots \ldots \ldots \ldots \ldots$
1856 by ditto $\ldots \ldots \ldots \ldots \ldots \ldots$ Ir IIaffenden

\section{LADY STUMPS,}

Bred by , in , got by Tramp, dam by Cervantes, out of Fanny, by Sir Peter.

1831 ch. f. Delightful, by Defence........

1852 b. f. hy ditto .................

18.55 b. c. by ditto................ Mr Sadler

1854 b. f. by ditto................

$1953 \mathrm{ch}$. f. by ditto.............. 


\section{LAMIA (sister to Qquail)}

Bred by Mr Bıges, in 1809, got by Gohanna, her dam, Certhia, by Woodpecker-Trentham-Cunegonde, by Blank.

1817 ch. f. by Camerton.............

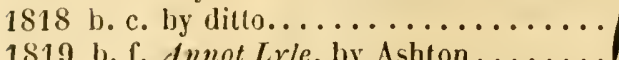

1819 b. f. Annot Lyle, by Ashton.........

1820 br. c. Bertram, by Smolensko (died in Mr Bigors

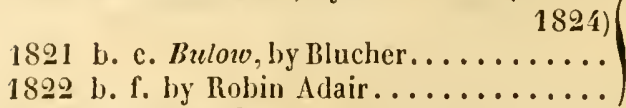

182.5 b. f. Pasithea, by Rubens.......... HIr H. Jones

1824 b. c. Pandarus, by Whalebone....... H Mr Taylor

1826 ch. c. The Picture, by Rubens ...... Mr Gauntlett

1827 b. c. Cetus, by Whalebone .........

1828 ch. c. Gershom, by Itoses......... Mrr Dilly

1829 b. c. Gratis, by Middleton..........

1850 missed to ditto .............. His Mlajesty

$\left.\begin{array}{r}1851 \text { b. c. (Victoria) by Whalebone, or Little } \\ \text { Jolnn (s. to Germany, 1853) }\end{array}\right\}$ Mr Sloane Stanley

1852 b. f. Mischief, by Reveller.......... Mr Kent

1853 ch. f. Ceta, by The Colonel......... Ld Exeter

llad no foal after 1855, and is dead.

\section{LANDLADY,}

Bred by Mr T. IIurcursos, in 1821, got by Bigot, her dam by

Remembrancer, out of Mary, by Y. Marske-Gentle Kitty, by Silvio.

1828 br. f. by Grey Malton............. Mr T. Hutchinson 1850 b. c. Partner, by Whisker (s. to Holland) Col. Cradock

$\left.\begin{array}{l}1851 \text { br. f. by Figaro } \\ 1852 \text { bl. f. by Waverley }\end{array}\right\}$ s. to ditto, 1853

1855 b. f. by Waverley ............. Ur T. ILutchinson

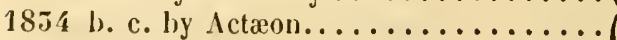

$185 z$ missed to Lconardo .............

1856 by Liverpool $\ldots \ldots \ldots \ldots \ldots \ldots$

\section{LANDSCAPE (sister to Raphacl)}

Bred by Gen. L. Gower, in 1815, got by Rulsens, her dam, Iris, by Brush-Herod-Doctor's sister. 
1817 b. f. Perspectice, (aft. Katherina) by t Id Slign

1818 b. c. Cuyp, by Haphazard $\ldots \ldots \ldots . . .$.
1819 b. c. Claude, hy ditto (sent to lirance) .Ir W. Chifuey

1820 b. f. Tint, hy ditto ............. L.d Low ther

182.5 ch. f. Cynthia, hy Comus.......... Hr Ormsby Gore

182.4 ch. c. by Clinker...............

-1825 b. f. Laundress, hy Tiresias........ ld lowther

1826 ch. c. Verderer, by ditto .........

1827 br. c. Zucharelli, ly ditto......... II Ramsbottom

$18 \pm 8$ ch. f. by ditto .................. I I L Iowther

1850 ch. c. Titian, by ditto ........... Mr Wood

1851 b. c. Ostade, by ditto............ Mr G. Walker

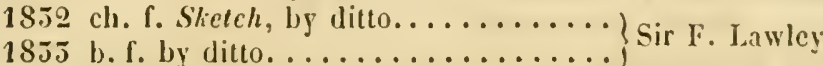

In 1821 missed to Castrel, 22 to Scud, and died in 1854 .

- Latexdess producel, in 1828, Col. Lowiher's b. f. Odclity. by Lapdog. missed the next year, and was sent abroad.

$\dagger$ Vendere was sent abroad in 1852 , eovered by Celus.

\section{I.ANGTON UARE,}

Bred by , in 181:, out of Passion-flower, by sir

Peter, out of Peggy Bull, sister to John Bull, by Fortitude-

Xantippe, liy Eclipse.

1824 b. f. by Sober Robin (died a foal) ....

1823 ch. c. Rough Robin, lyy ditto........

1826 b. f. by Filho da Puta ............ IIr Champion

1829 br. f. Ileart's-ease, by ditto.........

1850 b. c. Robin Roughhead, hy Sober Robin

1851 ch. c. Roman, hy Emilius.......... Ir Irouldsworth

18.32 br. f. hy ditto (put to the stud) ...... Mr Champion

Missed in $1827,28,55,54,53$, slipped foal in 56 , and was shot.

L.NGTON MARE (sister to the preceding)

Bred loy , in 1816, out of lassion-llower.

1826 b. f. by Mr Tomes's Duplicate (s. to India)

1827 barren................. Ir Champion

1828 b. c. by Fitho jlied young from acci-

1829 br.c. by ditto $\}$ dents 


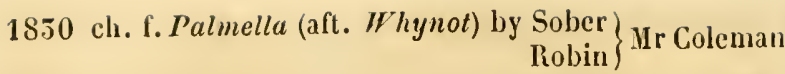

185 ! b. f. by Emilius................

1852 b. c. by ditto............... Jr Champion

1855 ch. c. by Merlin ..............

LARISS $\Lambda$,

Bred by Lord Grosvevor, in 1815 , got by Trafalgar, her dam,

Metcora, by Meteor, out of Maid-of-all-IV ork, by Highlyer.

1818 br. f. by Thunderbolt ............

1819 br. f. Tempè, by ditto...........

1820 br. c. Hymettus, by ditto .........

$1821 \mathrm{ch}$. f. IIybla, by Rubens..........

1822 b. c. Achilles, by Sovereign .........

1825 b. f. by ditto (died in breaking)...... Ld Grostenor

1824 b.c. Mavrocordato, (aft. Don Juan) by

Blucher

$182:$ b. c. Navarino, by Blacklock (s. to Ire-

land in 1829 , and there called Y. Blacklock)

1826 b. f. Plateca, by Haster Henry .......

1827 ch. c. Marathon, by Blacklock...... Capt $\Lambda$ ngerstein

1828 dead foal, by Filho da Puta........ Ld Grosvenor

1829 b. f. Laura, by Champion..........

1850 b. c. by Filho da Puta (died rising

5 yrs old)

1852 b. c. Gerard Dow, by Peter Lely..... Ld Westminster

1855 br. f. Horea, by Teniers (put to the stud)

1854 br. f. by Pantaloon .............

In 1851 missed to Camel, and is dead.

\section{LARISSA,}

Bred by Mr Hervey, in 1825, got by Rubens, her dam by Squire Teazle-Sweeper-Dungannon-Barbiniola, by Herod.

1827 ch. c. by Orville Junior..........

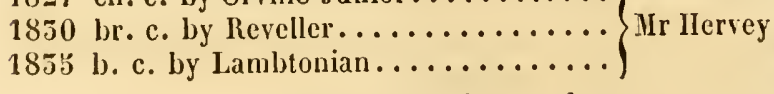

She had no other produce.

L.ILRA, by Beninglbrough (Vol. III, p 205) produced a ch. f. by Battledore, in 1852, and died. 


\section{3}

\section{I.1LRA,}

Bred by Mr Garporta, in 1817, got by Don Cossack, out of Marciana, by Stamford-Marcia, by Coriander-laith, by Pacolet.

1850 missed to Chateau Margaux.........

1851 bl. f. by his Y. Fillo da l'uta .......

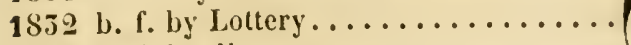

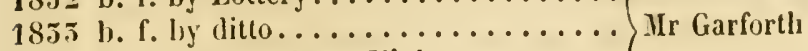

1854 b. f. by llumphrey Clinker ........

185 b. c. by Brutandorf.............

1856 by St Nicholas ...........

\section{LAURA,}

Bred by Mr Perre, in 1827, got by Figaro, her dam, Juliana, by Gohanna, out of Ilatina, by Mercury-Ilerod-Y. Hag, by Skim.

1852 b. c. by Blacklock, ${ }^{*}$ or Tinker......
1855 missed to Y. I'hantom. . . . . . . .

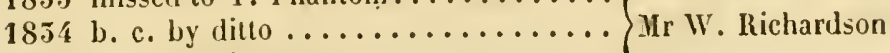

$185 ;$ c. by ditto................

1836 by Langar ..............

- Brack lock dropped down imınedialelyafter covering Laura, and died in an hour, Feb. 24, 1851 .

\section{LAURA,}

Bred by Lord Westmisster, in 1829, got by Cliampiou, her dam, I.arissa, by Trafalgar, out of Meteora, by Meteor.

1835 b. c. Ostade, by Tenicrs .........

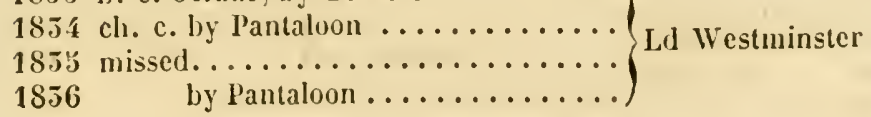

\section{L.IUREOLA,}

Bred by Lord Verciax, in 1825, got by Orville, out of Laurelleaf, by Stamford-sister to Uruid, by l'ot8o's.

1850 b. c. by Whalebone ........... Mr Poyntz

1851 br. f. Miss Gravity, by Mameluke.... Gen. Grosrenor

1852 missed to Truflle............. 1). of Holstein

18.55 c. by Jloses (deall)........... JIr Tattersall 


\section{4}

1854 b. f. by Bustard (s. to Virginia) .....

185 : b. f. by Longwaist............. Ir Nowell

18.36 by Bustard ............

This mare was sent to the $\mathrm{D}$. of IIolstein, in $185 \mathrm{l}$, covered by Truffle, and brought back in 52 , stinted to Hloses.

\section{LEDA (sister to Fanny Davies)}

Bred by Mr Hocensworth, in 1824, got by Filho da Puta, out of Treasure, by Camillus-Hyacinthus.

1854 ch. c. Mirza, by Malek.

1855 b. c. Beresford, by Beagle......... Hr IV. Sharpe

1856 missed to Cantecn .............

\section{LEEW AY,}

Bred by Mr Nowelt, in 1825, got by Aladdin, her dam, Johannah Southcote, by Beningbrough, out of Lavinia, by Pipator.

1851 b. c. by Royal Oak (died young) .....

$1855 \mathrm{br}$. f. by ditto ...............

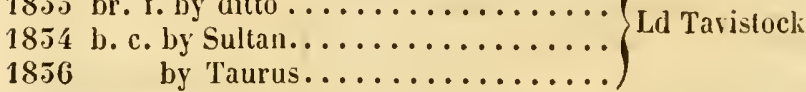

Not covered in 1851 , missed to Taurus in 55.

\section{LEGEND,}

Bred by the Duke of Graftox, in 1825, got by Merlin, out of Piquet, by Sorcerer, out of Prunella.

1850 b. c. by Centaur.

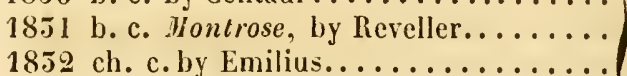

1855 dead foal, by Sultan ........... D. of Grafton

1854 ch. c. by Emilius..............

1855 missed to ditto.............

1856 by Langar $\ldots \ldots \ldots \ldots \ldots \ldots \ldots$

LEILA,

Bred at Hampton-Court, in 1825, got by Waterloo, her dam, Scheherazade, by Sclim, out of Gipsy, by Trumpator-Herod-Snap.

$18 \mathrm{2S} \quad \mathrm{c}$. by Middleton (died young)......

1829 b. f. by ditto ...............

18.31 ch. f. by Reveller.............. Sir II. Wood

18.52 Jr. f. Lilka, by Emilius, or Lamplighter

18.3 .3 c. (died a foal) by Enilius, or Cetus...) 
1854 b. c. by Cetus ............... Sir M. Wood 1836 cast twins, by Shakspeare, Dec. 185 5. Mr C. Stoviu Not covered in 29 , barren in 53.

\section{LEONELLA,}

Bred by Lord Firzwillis, in 1819 , got by Cervantes, her dam, Sally, by Sir Peter-Diomed-Marske.

1828 ch. c. Leo, by Comus............ 1829 b. f. by Humphrey Clinker (put to the

1830 ch. c. by Comus . . . .

1831 b. l'. by IIulatto.............. Ld Fitzwilliam

1832 b. c. by Lottery..............

1855 b. c. by Hulatto...............

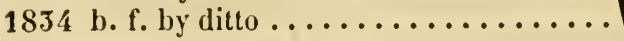

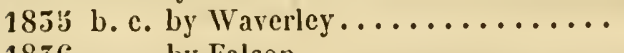

1836

by Falcon.

\section{LEOPOLDINE,}

Bred by Sir Jonv Sheleey, in 1814, got by Walton, her dam,

Cressida, sister to Eleanor, by Whiskey, out of Y. Giantess.

1822 ch. f. Minima, by Election........ Mr Lambton

1824 ch. c. Tom Jones, by $\Lambda$ bjer (s. to Poland) Mr Petre

1825 b. c. Lambtonian, by Filho da Puta .. Ir Ilouldsworth

1826 b. f. Estrella, by Blacklock.........

1828 b. c. Elvas, by Whalebone (s. to Ire-)

land) D. of Richmond

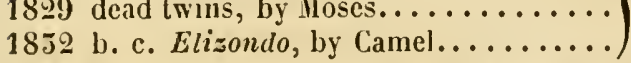

1855 slipped foal ................................

Missed in $1825,27,50,51$, and 35 .

\section{LINDA,}

Bred by Sir Jons Snelter, in 182\%, got by Waterloo, her dam,

Cressida, sister to Eleanor, by Whiskey, out of Y. Giantess.

1851 b. f. Linda, by Mameluke, or Tarrare Mr Grant

1832 ch. c. Coriolanus, by Einilius........

1835 br. f. Olinda, by The Colonel......... IIr Ridsdale

183.4 picked a filly............... 
LISETTE, by IIambletonian (Vol. III, p. 210-11) died in 1852.

Paddy, her foal of 1828 , was sent to Jamaica in 1854 , and her last produce should stand thus :-

1850 gr. c. Clearwell, by Jerry......... Ld Orford

\section{IITTLE FANNY,}

Bred by , in 1805 , got by Sir Peter, her dam, Katharine, by Delpini-Paymaster-Le Sang .

181 b. f. by Hambletonian ...........

181 b. c. by ditto (killed in castration)..... IIr II Donnell

1819 b. c. Y. Ilambletonian, by ditto.....

She had no thorough-bred foal after 1819. Y. Hambletonian was never broke, but used as a stallion in Ireland.

\section{LITTLE FINGER (sister to 'Tom Thumb)}

Bred by Lord Egremoxt, in 1825, got by Whalebone, her dam by Gohanna, out of Allegretta, by Trumpator-Y. Camilla.

1852 b. c. by Gaberlunzie.............

1855 b. c. by ditto................

1854 missed to ditto.............. Ld Egremont

185 . b. f. by ditto................

1856 gr. f. by $\Lambda$ corn................

LITTLE FOLLY (Vol. 1II, p. 211) was barren in 1832, and died that year.

\section{LITTIE JOIIN IIARE,}

Bred by Mr Moox, in 1826, her dam by Canopus-Teddy-theGrinder-Precipitate-Pumpkin-Fleacatchcr.

1851 b. f. by Whalebone.

18.32 b. f. by Camel (dead).............. Mr Moon

18.5.j bl. f. by Chateau Margaux.........

Barren in 1854 and 55. 


\section{LIVONLA,}

Bred by

, in 1824 , got by Smolenskn, her dam, Stella, by L'Orient-Ruler-Highflyer.

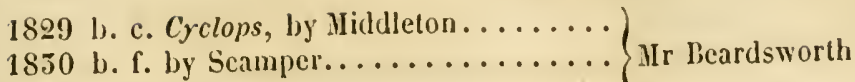

$18 j 1$ missed to Bonassus.............

1832 br. c. by Belzoni (dead)........... Mr Dunkley

1855 bl. f. by ditto................ Mr Beardsworth

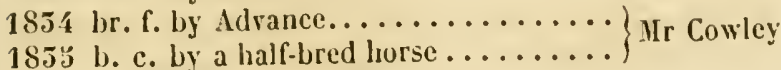

\section{LOO,}

Bred by the Duke of Gisarrox, in 1815, got by Waxy, her dam,

l'iquet, by Sorcerer, out of Prunella, by Hightlyer-P'romise.

1821 b. f. lyy Watton (died a foal) .......

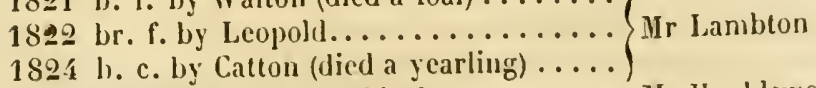

1823 b. f. Flush, by Blacklock ........ Mr Houldsworth

1826 b. f. Aldcea, by Richard ...........

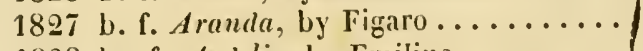

1828 br. f. Ardelia, by Emilius.......... D. of Richmond

1830 br. f. Alibassa (aft. Ponle) by Sultan

(put to the stud)

1852 b. c. by IIameluke...............

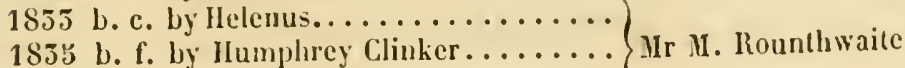

1856 by Brutandorf ............

Missed in $1820,25,29,51$, and 54.

\section{L.00 (1100,}

Bred by Lord Grosiexon, in 1813, got by l'eruvian, her dain, Iris, by Sir Peter-Isabella, by Eclipse - Squirrel.

1820 br. c. Brighton, ly Pavilion ........ Mr Scaile

1822 br. c. Sam, by Rhadamanthus....... Mr Roberts

1925 br. c. Otho, by ditto ............ Mr Houldsworth

1824 br. f. Billinsgate, by Filho da Puta... Mr Wakefield

1823 br. f. by ditto ............... Mr Leigh

1826 br. c. Mercury, by ditto ........... Mr Johnson

1827 b. c. Rolla, by Catton............ Mr P'alin 
1828 b. c. Peru, by ditto (s. to D. of Hol- $\begin{array}{r}\text { stein) } \\ \text { Mr Dudson }\end{array}$

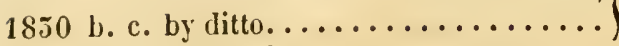

1851 b. f. by Camel............... Ld Wilton

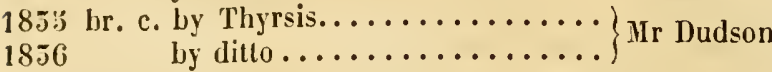

barren in the intermediate years.

\section{LORETTA,}

Bred by Mr Mackale, in 1815, got by Whitworth, her dam, Esther, by Shuttle-Drone-Matchem-Jocasta.

1820 b.c.Sinbad (aft. Conspiracy) by Raphael Mr IIutchinson 1822 cast twins, by Zeno ............ Mr Mackall

1823 b. c. Magister, by Soothsayer (sent to

189 Vienna) Mr Iambton

1825 b. c. by ditto $\}$ died young .........

1826 br. c. Sweno, by ditto...........

1828 gr. f. Saturnia, by his grey Arabian (put

to the stud) (Ir Attwood

1829 b. f. Violetia, by his chesnut Arabian.

1850 b. f. Ismene, by ditto .............

1851 b. c. Lorenzo, by Lottery ..........

1852 ro. f. Quaderoon, by Camcl ........ Sir G. Ileathcote

1855 br. f. by his Chance............

1855 b. f. by Count Porro............ IIr Brown

Barren in 1821,27 , and 54 .

\section{LOTTERY MARE,}

Bred by Sir Tatron Sikes, in 1828, her dam, sister to Claudius,

by Camillus-Sancho-Highflyer-Juno, by Spectator.

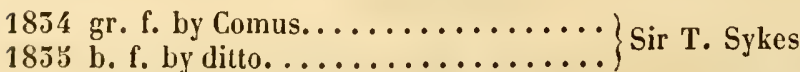

\section{LOUISA,}

Bred by Mr Gascoigne, in 1815 , got by Orville, her dam, Thomasina, by Timothy, out of Violet, by Shark-Syphon.

1819 ch. c. (Corinthian) by Comus ....... Mr Gascoigne

1820 b. c. Werner (aft. Bob Logic) by Walton Mr J. Croft

1821 bl. c. Jerry, by Smolensko......... Mr Gascoigne 
*1825 b. c. Tom, by 11 alton........... Mr Clifton

1824 ch. f. Jessy, by Comus..........

182 b ch. c. by Blacklock ............

1826 br. f. Sal, by Smolensko..........

$1827 \mathrm{ch}$. f. by Whisker (put to the sturl)...

1828 br. f. Dinah, by Champignon...... Mr Gascuigne

1829 b. c. Repealer, by Whisker.........

1851 b. f. by Blacklock (put to the stud)...

1855 br. c. Wallace, by Lottery .........

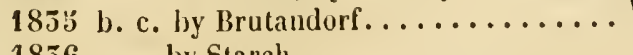

1856 by Starch..............

In 1822 missed to Ardrossan, in 50 and 52 to Lottery, 54 to Brutandorf.

* Tos was sold to the D. of Holstein in January, 1855.

\section{LOUISA,}

Bred ly

, in 1815, got by Orville, her dam,

Quadrille, by Selim, out of Canary-bird.

$1828 \mathrm{ch}$. c. by Phantom.............. Mr Nevill

1829 ch. c. The Marshal, by ditto (died in

1852)

1851 b. c. Ludorico, by Reveller.........

1852 slipped by Whisker............. Sir II. Wood

1854 b. f. by Rereller (s. to Germany) ....

1853 b. c. by Partisan, or Cetus...........

1856

by Cetus ...............

In 1830 missed to Middleton, barren in 55.

\section{LOUISA,}

Bred by Lord Licnfield, in 1825, got by Filho da Puta, her dam, Elizabeth, by Orville, out of Penny-trumpet, by TrumpatorY. Camilla.

1855 b. c. by The Colonel...............

1834 b. c. by Emilius (died young)....... Ld Lichfield

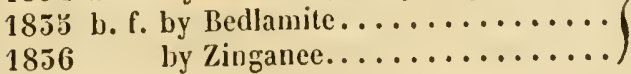

\section{LOYALTY,}

Bred by Sir George Pigot, in 1817, got by Rubens, her dam, Pennyroyal, by Coriander, out of Peppermint, sister to Prunella. vol. Ir. 
1894 b. f. Manfred (dead) .............

1826 b. f. Dardina, by Muley.......... Sir G. Pigot

1827 b. f. Fanny Kemble, by Paulowitz...

1828 b. f. (Maria) by Master Henry........)

1850 b. f. (Alice) by Whisker ...........

1852 ch. c. by Velocipede ............ I Id Derby

185.5 b. c. by Filho da Puta.................... I Derby

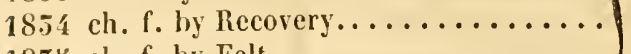

185 a ch. f. by Felt...............

II 1893 missed to Tramp, 31 to Camel, not eovered in 28.

\section{LUCETTA,}

Bred by Mr Barsos, in 1826, got by Reveller, her dam, Luss, by Hedley, out of Jessy, by Totteridge.

1855

1856
c. by Cetus (died a foal)................... Sir M. Mood

\section{LLCINDA,}

Bred by Mr Micnaelsos, in 1817, got by Haphazard, her dam by

Sancho, out of Miss Furey, by Trumpator-Mark Anthony.

1824 gr. f. by Grey Middleham ..........

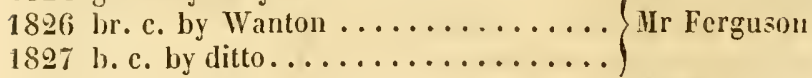

1828 b. c. by Muley................ Mr Nowell

1829 b. c. Doncaster, by ditto......... Ld Ranelagh

1850 bl. f. by ditto (s. to Russia in 1853).. Hr Nowell

1851 b. f. by Jongwaist ............. Ir Beardsworth

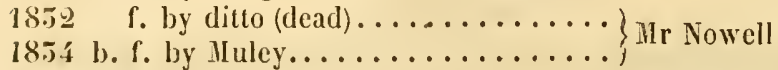

Missed to Whisker in 1855 , to Jongwaist in 55 , and died in 54 .

\section{LUCINDA,}

Bred by Jord Fitzwillias, in 1817, got by Cervantes, her dam, Sally, by Sir Peter-Diomed - Desdemona, by Marske.

1828 b. f. by Whalchone......................... Mr Trelawny

. Ifer produce of 1826 and 27 died, she missed in 29, was not covered in 50, and died in December 1851. 


\section{1}

\section{JUCY (sister to J,eriathan)}

Bred by Mr PAinter, in 1826, got by Mulcy, her dain by Windle - Anvil-Virago, by Snap - Regulus.

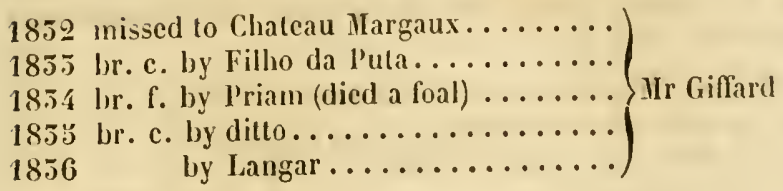

\section{LUNA,}

Bred by Mr Moos, in 1824, got by Wanderer, her dam by Canopus-Teddy-the-Grinder-Precipitate-Punspkin.

1851 br. c. Comet, by Whalcbone.......

1852 b. f. Amina, by Gaberlunzic .......

1855 b. c. Skirmisher, by The Colonel.... IIr Gardnor

1854 missed...................

183 b. c. by Gaberlunzic............

1856

by Actæon................

\section{LUNACY (sister to Bee-in-a-Bonnel)}

Bred by Mr Eusis, in 1824, got by Blacklock, her dam. Ilaniac.

by Shuttle-Beningbrough-Expectation, by Herod.

1851 b. f. Lolotte, by Lottery (put to the) stud)

1852 ch. f. by Tramp.............

1855 ch. c. by ditto.............. Ifr Ridsdale

185 b. c. by ditto...............

1855 missed to Waverley............

1856 slipped a colt, by Mulatto.........

\section{LUNIRIA,}

Bred by Mr Fergesor, in 1824, got by Whisker, her dam lyy Sancho, out of Miss Furey, by Trumpator-Mark InthonySignora, by Snap.

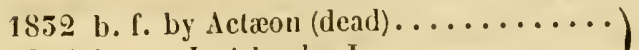

1855 br. c. Jevicho, by Jerry .............. of leeds

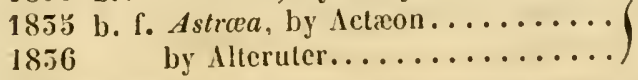

Jissed to Lottery in 1851, to Waverley in 51. 
LUNATIC,

Bred by Mr Elus, in 181S, got by Prime Ninister, her dam, Maniac, by Shuttle, out of Offa's Dyke's dam, by Beningbrough -Expectation, by Herod.

* 1825 br. f. Bessy Bedlam, by Filho da Puta Col. King 1827 b. f. by ditto (died in training) ....... Mr King 1829 b. c. Fanatic, by Filho da Puta.... . Ir Carter 1850 b. c. by Blacklock............ D. of Rutland 1831 b. c. Delirium, by Filho da Puta .... Mr G. Walker $1852 \mathrm{ch}$. f. Virago, by Velocipede....... Mr Raynes 18.55 br. f. Firefly, by ditto.......... Ld Albemarle 1854 ch. c. by ditto............... Sir M. Wood

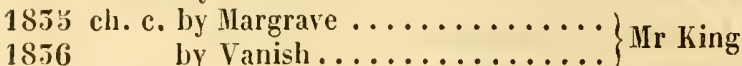

Barren in 1826 and 28.

* Bessy Bfolan prodnced, in 185.4, Sir M. Wood's br. c. by Lotlery, missed in 55 , and was covered that year by Vanish.

\section{LUNETTES (sister to Torchbearer)}

Bred by Col. Cradock, in 1820, got by Comus, her dam by St George-Pontac-Syphon-Regulus.

1826 b. c. Flambeau, by Grey Malton..... Mr Healey

1827 b. c. Pancake, by Swiss.......... Col. Cradock

1829 b. f. by ditto............... Mr J. Scott

1830 b. f. Lustre, by ditto . . . . . . . . MIr IIebden

1851 b. c. by Wanton ..............

1854 b. c. by Grey Viscount........... Mr Leroy

1856

by Liverpool..............

Barren in 1852,53 , and 53 .

\section{LYDIA,}

Bred by Mr R. Joxes, in 1822, got by Poulton, her dam, Variety, by Iyacinthus - Weasel-Turk-Locust.

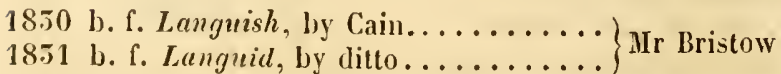

Cast foal in 1828, missed in 29 , had no foal after $\mathbf{3 1}$, and is dead. 


\section{LYRIC (sister to Legend)}

Bred by the Duke of Graftox, in 1826, got by Merliu, her dam, Piquet, by Sorcerer, out of Prunella, by Ilightlyer.

1851 slipped foal, by Mancluke ........

1852 br. c. by Lottery (died a foal) .......

1855 br. f. Zingarella, by Chance....... Sir G. Heathcote

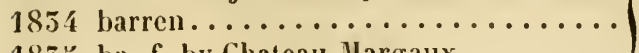

1855 br. f. by Chateau Margaux ........

\section{LYRNESSA,}

Bred by Gen. Grosveror, in 1821, got by The Flyer, her dam, Briseïs, by Beningbrough, out of Lady Jane, by Sir Peter.

1827 cls. f. (Poverina) by Partisan (put to the stud)

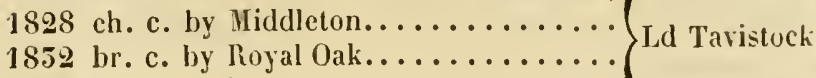

1853 b. f. by ditto...............

1856 by Taurus ...............

Barren in $1829,50,51,54$, and 55.

\section{MACBETH MARE,}

Bred by Mr Cromptos, in 1819, her dam, Margaret, by Hambletonian-Rosamond, by Buzzard-Roseberry, by Phœenomenon.

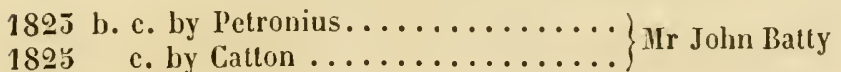

1826 b.c. The Poet, by ditto.......... Mr Dryden

1851 b. f. by Coronation............

1852 c. by Waverley ............. Mr Harrison

1855 b. f. by Duncan Grey............

1854 missed to Ilumphrey Clinker........ Mr R. Johnson

185 y br. f. by Voltaire.............

Not covered in 1825 , had no thorough-bred foal in $27,28,29$, or 50 .

\section{MACBETII MARE,}

Bred by Mr Hopksssox, in , her dam by RemembrancerBeningbrough-Slipton's dam.

1826 b.f. Evadne, by Grenadier (son of)

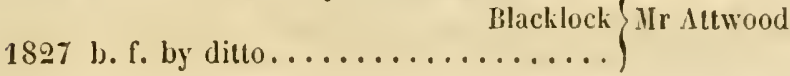


1830 b. c. Tom the Towter, by Y. Phantom Mr Peck

185 I br. f. by Lottery ............. Mr Skipsey

1852 b. f. by Brutandorf............. Mr Peck

1855 br. c. by Waverley ............ Mr Gray

\section{MACDUFF MARE,}

Bred by Mr Best, in 1823, her dan, Merlin's dam, by Delpini, out of Tipple Cider, by King Fergus.

1854 b. f. by Y. Phantom ............

1855 ch. c. by Langar.................. MIr Best

1856 ch. f. by ditto ...............

1857 by ditto.............

\section{MAC ORVILLE MARE,}

Bred by Mr Stовеy, in 1822, her dam by Cramlington-Stride-

the blind Javelin Mare, out of sister to Toby, by Hightlyer.

1827 br. f. Lonicera, by Whitworth . . . . .

1828 b. f. Hiss W'alton, by Lory........

1829 b. f. Erminia, by his grey Arabian (put)

to the stud)

1850 b. f. by his chesnut Arabian (died)... Mr Attwood

1851 br. f. Fatality, by a black Turk, or

Barb (put to the stud)

1852 br. c. by ditto (cut) ...........

1855 b. f. by Argantes...............

This mare was gyiven to Mr Palmer, of Gilling, near Richmond, in Yorkshire, and was stolen from him in the autumn of 1835 . She has not been heard of since.

\section{MIDELINA (sister to Nera)}

Bred by Mr Watsor, in 1819, got by Cervantes, her dam, Mary, by Sir Peter-Dioned-Desdemona, by Marske.

*1827 b. f. Esprit, by Emilius...........

$1851 \mathrm{ch}$. f. by Merlin (s. to Baron de Malt-) zahn in Feb. 1856 , in foal to Emilius)

1855 b. . Muzce, by Merchant ......... Mr Thornhill

1854 b. c. by ditto................

1853 ch. f. by ditto..............

In 1828 missed to Enilius, 29, 50, and 51 to Nierlin, 56 to St Patrick.

* Esrat procinced in $18.52 \mathrm{Mr}$ Greville's b. f. Medea, by Merlin, and was sent to Sardinia in 53, in toal to Mcreliant. 


\section{ULGA WVISKA,}

Bred by Mr Vassitant, in 1896, got bj Whisker, her dam. Slight, by Selim-Pot8o's-Editha, by Herod.

\section{2}

f. by Catton (dead).............

1855 b. c. by Comus...............

1854 ch. c. by Catton .............. Mr Vansittart

1855 br. f. by Humplirey Clinker $\ldots \ldots \ldots \ldots$
1856 by Physician $\ldots \ldots \ldots \ldots \ldots$

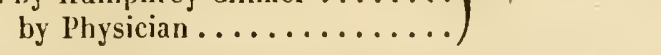

\section{IIAGSTR ATE MARE,}

Bred by Mr Ilovinsworth, in 182y, her dam, Mr's Maltby, by Cervantes, out of Legacy, by King Fergus.

185.5 b. c. by Brutandorf (died young) ....)

1854 b. c. by Ilumphrey Clinker........ Hr John Newton

1835 b. f. by ditto...............

1856 by Starch.............. Mr Benson

MAID OF KENT, by Soothsayer (Vol. III, p. 221) produced in 1852 b. c. Docer, by Patron, and was sold to the D. of IIolstein carly in 1855.

MAID OF L.ORN, by Castrel (Vol. III, p. 222) has had only one living produce since 1828 , viz. :-

1855 ch. f. Maid of Wirral, by Battledore..)

1856 by Birdcatcher, Batledore, or Sir MI. Stanley Joceline

\section{MAID OF MILAN,}

Brel by Sir Tromas Staxley, in 1820, got by Comus, her dam, Cowslip, by Alexander-Anvil-Virago, Saltran's dam, by Snap.

1823 br. c. (Sir Thomas) by Filho da Puta. 1826 b. c. by ditto................ 1827 ch. c. by Strephon (died a yearling)... 1828 b. f. Laily Olivia, by Sir Oliver ..... Sir T. S. H. Stanley 1829 br. f. by Champion ............ $1850 \mathrm{ch}$. f. La Grace, hy Battledore ...... 1851 ch. c. The Iligh Sheriff, loy ditto..... Sent to liussia in 1855 , in foal to Battledorc. 


\section{IIAID OF IINSFIELD,}

Bred by IIr Houldsworti, in 1822, got by Filho da Puta, her dam, Variety, by Selim, or Soothsayer, out of Sprite, by Bobtail - Catherine, by Woodpecker.

1854 b. f. by Cadland $\ldots \ldots \ldots \ldots \ldots \ldots$ )

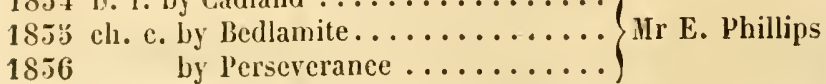

\section{MAIDEN,}

Bred by Mr Owev, in 1819, got by Iledley, her dam, by SelimOsear-Dairymaid, by Diomed.

1826 b. f. Garlic (aft. Miller's IIaid) by Master

1827 br. c. Tag, by ditto.............. Ilenry Onions

1829 br. c. by Manfred, or Wamba.......

1851 b. f. Shalot, by Master Henry....... Mr T. Warren

1852 Ly Wamba.

1854 b. f. lyy The Tutor.............. Mr Tattersall

Missed in 1828, slipped foal in 50, and sent to France October 54 , in foal to Logic.

\section{MAIDEN,}

Bred by Mr Buoss, in 1824, got by Orville, her dam, Merrymaid, by Buzzard - Highflyer-Smallbones, by Justice.

$18 \pi 2$ b. c. by IIameluke............ Mr Grant

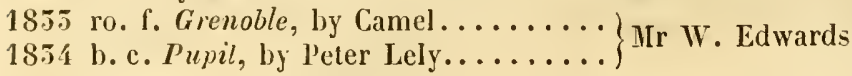

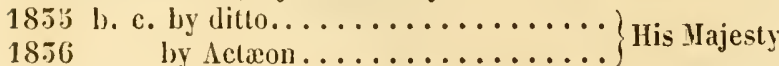

\section{MLLIBRAN,}

Bred by Col. Wrısox, in 1822, got by Rubens, out of Bolter": dam, by Trumpator-JIighllyer-Otheothëa.

1850 b. f. Mayo, by Johnny............ 1851 missed to IJumpligo

1852 b. c. Matador, by Picton .......... Mr Painter

185.5 b. f. Malice, by ditto .......... Ld Sligo

185. c. by ditto $\ldots \ldots \ldots \ldots \ldots \ldots$.

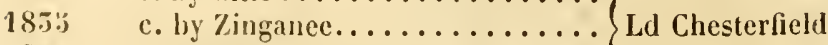

1856 f. hy l'riam............. 


\section{MANDADINE,}

Bred by Mr Onbeen, in 182í, got by Filho da Puta, out of Cardinal Wolsey's dam, by Precipitate-Pegasus.

1827 b. f. by Waverley (died a yearling)...

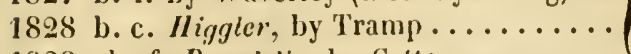

1829 clı. f. Rosadelle, by Catton ......... IIr Nevill

1851 ch. f. Amalou, by Sultan ..........

1856 by Belzoni ...............

She was not covered in 1829 , had no foal in 1852 or $\mathbf{5 5}$, when she was sent to Germany. She was brought back in the syring of 1854 , and put to Sultan, but missed.

\section{INDOLINE,}

Bred by the Duke of Retcasd, in 1818, got by Waxy, her dam,

Pennytrumpet, by Trumpator-Y. Camilla, by Woodpecker.

1823 b. c. Tamborine, by Orville........ Ld Egremont

1826 b. c. Meridian, by Gulliver........

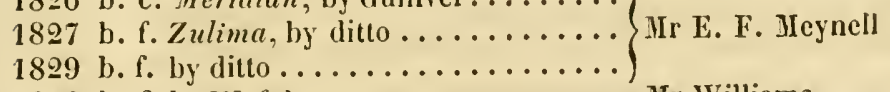

1850 b. f. by Woful................ IIr Williams

1831 b. f. by ditto (s. to Germany in 1853 ,

in foal to Varro)

1852 ch. f. by ditto ............... Mr E. F. Meynell

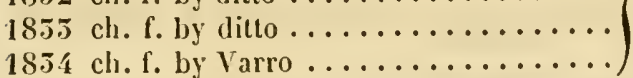

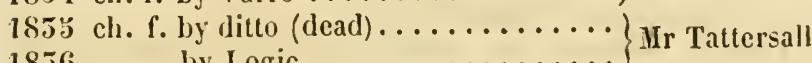

1856

by $\operatorname{Logic} \ldots \ldots \ldots \ldots \ldots \ldots$...........

\section{MANFRED MARE,}

Bred by Sir George Pigot, in 1822, her dam, Sunllower, by Castrel-Alexander-Nelly, by Otho.

1828 b. c. Sinbad, by Swap........... Ir Yates

1829 f. by Paulowitz (died a foal).......

1850 h. c. by Whisker (died rising 2 yrs old)

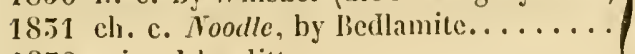

1852 missed by ditto ................. Hr E. Peel

185.5 bl. f. Egrpta, by Belzoni .........

185 4 b. c. Canuell, by Cadland.........

185.5 ch. f. by Bedlamite ............

18.56

ly Cain . 


\section{IANGEL-WURZEL,}

Bred by Mr Thorxull, in 1825, got by Merlin, out of Morel. 1828 ch. c. Merlyn Silvester, by IIcrlin... . Capt. Bulkclcy 1829 missed to Emilius.............. Mr Thornhill 1850 b. f. Ceres, by ditto............ Mr S. Day 1851 ch. c. Gracchus, by ditto ......... Mr E. Peel 1852 b. f. by ditto $\ldots \ldots \ldots \ldots \ldots \ldots \ldots$ 185.5 b. f. by ditto............... 185 i br. f. by ditto............... Mr Thornhill $1853 \mathrm{ch}$. c. by St Patrick 1856 by ditto.................

\section{MANOEUVRE,}

Bred by Lord Grosvevor, in 1821, got by Rubens, her dam, Fincsse, by Peruvian, out of Violante.

1826 b. f. (Christine) by Haster Henry (s. to Guernsey) $\}$ Mr Cosby

1827 b. f. (Blue-bonnet) by ditto.......... Mr Onions 1828 ch.f. Felicity (aft.Emily)byAnticipation Mr Jones

Sold to the Duc de Guiche in Sept. 1829, in foal to IVaxy Pope.

\section{YOUNG MANIAC,}

Bred by Mr Eclis, in 1826, got by Tramp, out of Maniac, by Shuttle.

1850 ch. f. by the Arabian Signal (dead) ...)

*18.51 b. f. by Y. Whisker (son of Sir Paul Mr B. Thompson mare)

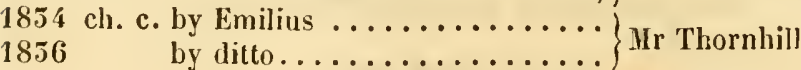

Missed in 1852, 55, to Emilius in 55. This
Mulallo.

MANILLE,

Bred by Lord Verousy, in 1825, got by Orville, out of Tredrille. $18.50 \mathrm{~b} . \mathrm{f}$. by Sultan. 1851 br. f. lyy Reveller ..............

18.32

18.5 .5

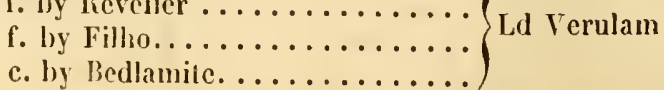

Sold to the I). of Orleans, in foal to Mameluke. 


\section{MANTO,}

Bred by Mr Fousane, in 1824, got by Tiresias, her damby

Walton, out of $\mathrm{Y}$. Noiselte, by Diomed-Noisette, by Squirrel.

1831 br. c. by Tramp..............

1832 b. с. by lieveller...............

1855 br. f. by Sultan (llied a yearling)..... J. of Portland

185 1 br. f. Cassandra, by Priam.........

1855 b. c. by ditto................

1856

by ditto $\ldots \ldots \ldots \ldots \ldots \ldots \ldots$.

\section{MANTO,}

Bred by the Duke of Pontcaxd, in 1825, got by Tiresias, her dam, Zora, by Selim, out of Zoraida, by Don Quixote.

1851 missed to Euston.............

1852 b. c. Mopsus, by Edmund............

1855 b. . Mantua, by Cardinal Puff .... Mr Bowen Dasies

1854 br. f. Medea, by Doctor Faustus.....

1853 b. c. Melampus, bj Adventurer .....

1856 by Wamba..............

MANTLA, by Woful (Vol. III, p. 226) produced b. f. by Langar in 1852 , and was sent abroad with her foal.

MANUELLA, by Dick Andrews (Vol. III, p. 227) is dead. IIer last produce was.

1851 b. c. Belluno, by Blacklock........ Mr Watt

\section{MIARCELLA,}

Bred by Lord Fitzwilliay, in 1826, got by Whisker, her laim. Maritornes, hy Cervantes, out of Sally, by Sir Peter.

1851 b. c. by Mulatto (died a foal).......

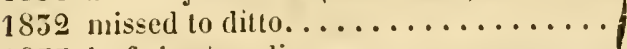

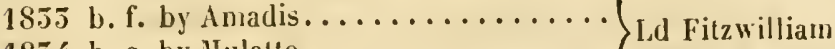

1854 b. c. by Mulatto...............

1856 by ditto..............

\section{MARCHESA (sister to My Lady)}

Bred by Mr Wrviul, in 1819, got by Comus, her dam by Delpini. uut of Tipple Cyder, by King Fergus-Sylvia, hy Y. Marske. 


\section{0}

1824 b. c. by Blacklock.............. Mr Ileywood

182 s. b. f. by ditto................ Lfl Kelburne

1826 b. c. Sandoval, by Cervantes ........ Mr Ileywood

1829 b. c. Burgomaster, by Brutandorf....

1831 b. f. Marceline, by Lottery .........

1852 br. c. Marcian, by Chateau Nargaux.

1855 b. f. Annaline, by lirutandorf ...... M. Mr Ridsdale

1854 b. f. Marchesiana, by Tramp.......

$185: 5$ b. f. by ditto................

1856 by IIulatto............

MARCIANA, by Stamford (Vol. III, p. 227)-produced gr. c. by Lottery in 1852 , missed in 55 and 54 , and was shot. IIcr produce of 1850 should stand thus :-

1850 gr. c. (Grey Wiganthorpe) by Figaro.. Mr Garforth

\section{MARGARET,}

Bred by Mr Duxdas, in 1824, got by Pyramus, her dam, Euphrasia, lyy Rubens, out of Witch of Endor.

1829 ch. c. (Deception) by Phantom.......

1850 br. c. by Robin Adair (dead) ........ IIr Dundas

$18 \pi 1$ b. c. by $\Lambda$ lbany ................

1852 missed to Phantom ............ LI Amesbury

185.) c. by The Colonel ............ Mr Wilson

1854 by Defence..............

1855 br. f. by Pontiff ............ Mr Matthews

\section{MARGELLINA (sister to Memnon)}

Bred by Mr Watt, in 1826, got lyy Whisker, her dam, Manuella,

by Dick $\Lambda$ ndrews, out of Mandane, by l'otSo's-Y. Camilla.

185 I b. c. Whisper, by Blacklock.........

1852 b. c. by Luzborough (broke his leg and

$185.5 \quad$ shot)

1854 b. c. Wy Sultan. ............. Mr Wreford

1855 rlead foal. . . . . . . . . . . . .

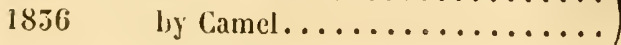

\section{THE MARGRAVINE (sister to Frederick)}

Bred by Mr Gratwicke, in 1850 , got by little John, her dam by Phantom, out of sister to Election, by Gohanna. 


\section{1}

1854 br. c. by a son of Filho da Puta and

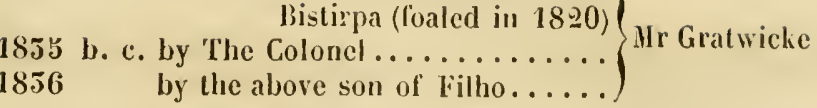

\section{MLIRIA,}

Bred by Sir D. H. Bhalk, in 1818, got by Trissy, her dam lyy Cáleb Quotem, out of Anna Bullen, by John Bull.

1824 br. c. Edward (aft. Zephyr) by Viscount 1825 br. f. by Champignon.............

1826 b.f. (Queen Bathsheba) by P'rime llinister Sir D. H. Rlair

1828 b. c. by Peter Lely .............

1829 br. c. by Epperston..............

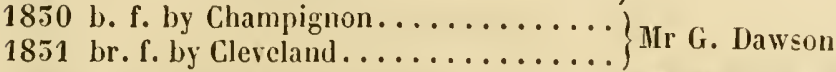

1852 br. c. by Percy .............. Mr Meiklam

1853 br. f. Brunette, by ditto .......... Sir W. Scott

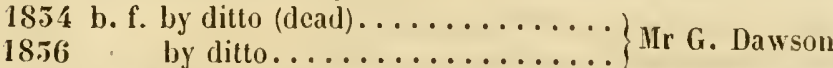

Nissed to Viscount in 1827, not covered in 54.

MARIA,

Bred at Hampton Court, in 1824, got by Watcrloo, her dam, Belvoirina, by Stamford, out of sister to Silver, by MercuryHerod-Y. Hlag.

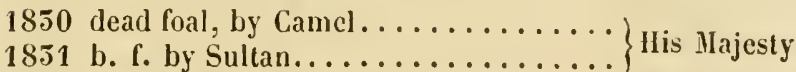

1852 b. f. Burden, by Camel............ Jir W. Edwards

1853 ch. f. Royalty, by Emilius......... Mr Ramsay

1834 ch. f. Mariette, by Priam.......... Mr Greville

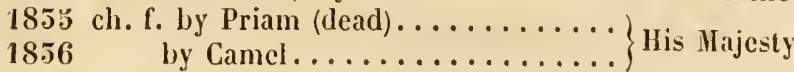

\section{MARIA (sister to Emma)}

Bred by the Trestees of Lord Strathiore, in 1827, got by Whisker, her dam, Gibside Fairy, by Hermes, out of Vicissitude, by Pipator-Beatrice, by Sir Peter.

1854 b. f. by Emilius............... D. of Cleveland

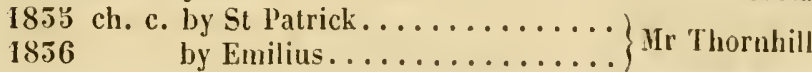




\section{MARIA DARLINGTON.}

Bired by Lorl Chevecard, in 1817, got by Bay Trophonius, out of Monymusk, by John Bull-Maid-of-all-work.

1827 ch. c. by Placeman ............. Mr Price

1829 gr. $\int$. by Lycastes . ............. Mr Lewis

1850 ch. c. Maridmnum, by Cripple.....

18.55 b. c. Maescrygie, by Cardinal Puff ...

1854 cl. f. Menivia, by Climber........ Mr Bowen Davies

1855 b. C. May-day, by Adventurer.......

1856 by Wamba $6 \ldots \ldots \ldots \ldots \ldots$.

IIer foal of IS26 died; she missed in 1828,51 , and 52.

\section{MALIANNE,}

Jired by Mr Gascolgive, in 1816, got by Sorcerer, her dam, Thomasina, by Timothy, out of Violet, by Shark-Syphou.

1821 br.f. Parthenessa, by Cervantes.....

1822 bl. f. by Amadis (s. to Prussia in 1826)

1824 b. c. Medoro, by Cervantes........

182 əै clı. f. Mariqueta, by ditto..........

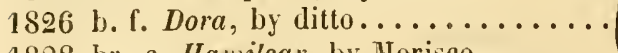

1828 br. c. IIamilcar, by Morisco...... Ld Fitzwilliam

1829 ch. f. by Cervantes...............

1852 b. f. by ditto (s. to America in 1855).

1854 b. c. by Mulatto..............

1855 b. c. by ditto ..............

18.56 by Langar..............

Fot covered in 1822, missed in 27,50 , and 55.

\section{IIARINELLA,}

Bred by Lord Exeter, in 1824, got by Soothsayer, her dam, Bess, by Waxy, out of Vixen.

1850 b. c. by Sultan...............

1851 ch. f. Ayesha, by ditto (put to the stud)

1852 ch. f. Albania, by ditto...........

185.5 b. f. by ditto............... Id Exeter

1854 barren..................

1855 ch. c. by Sultau..............

1856 by ditto $\ldots \ldots \ldots \ldots \ldots \ldots \ldots$ 


\section{MARION,}

Bred by Mr Crouptos, in 1819, got by Tramp, her dam, liosamond, loy Buzzard-Roseberry, by Phoenomenon-Miss West.

1824 br. c. Robin IIood, by Blacklock ..... Mr Golden

182 z ch. c. by Whisker.............

1826 b. c. The Blundering Farier, by dillo. Mr Crompton

1827 ch. f. by Figaro..............

18 .8 b. c. IIonest John, by Whisker...... IIr Ridsdale

1829 missed to Jumphrey Clinker.......

1850 l. f. by Blacklock .............

$1851 \mathrm{ch}$. f. by Figaro.

18.52 b. с. by Langar. . . . . . . . . . .

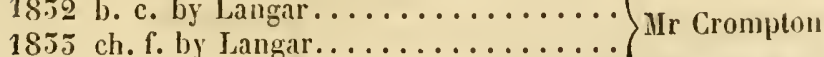

1854 hr. c. by St Nicholas...........

1855 b. f. hy ditto (died a foal) .........

1856 by Huntington...........

\section{IIARMION MARE,}

Bred by Mr Gonnsed, in 1819, her dam, Nymphina, by Gouty, out of Mademoiselle, by Diomed-Belle, by Justice-Marske.

1824 ch. c. Lycurgus, ly Anticipation..... Mr Goddard

1823 b. c. Nimrod, by Hobadil........... Mr Giffarl

1826 missed to Anticipation...........

1827 b. f. I;atiste, by IIaster Henry .......

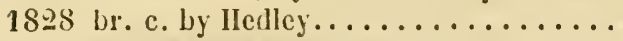

1829 h. f. Master Henry............

1850 b. c. by dillo, or Manfred......... Mr F. I. Charlton

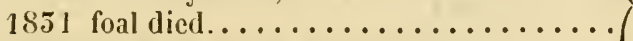

1852 b. с. by Wamba.............

$18.5 \mathrm{~b}$ b. c. by Defence

1854 gr. c. by Stumps.............

$18.5 \%$ c. by a half-bred horse.........

Dead.

\section{MARMION MARE,}

Bred by Ir WooD, in 1825, her dam, llarpalice, by Gohanna, out of Amazon, by Driver.

1850 b. c. by Royalist.............

18.51 b. c. by ditto, or Frolic ..........

$1852 \mathrm{gr}$. f. by Gregson ............... IIr Thompson

185.1 b. f. by IIarry (son of Sir Iarry)..... 


\section{4}

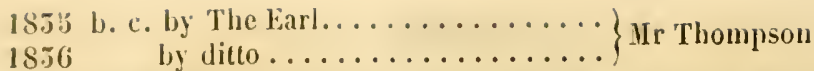

She had two foals previous to 1850 , which died, and she was not corcred in 32.

MARROWFAT,

lired by Mr Huxter, in 1825, got by Orville, her dam, l'eahlossom, by Don (Quixote-l'ipator-sister to Snow, by Slope.

$18 \supseteq 9$ b. c. Rounciral, by Partisan ........

1850 gr. c. loy Gustarus..............

1851 gr. c. Morotto, by ditto (s. to France

185.9 missed to ditlo...........................

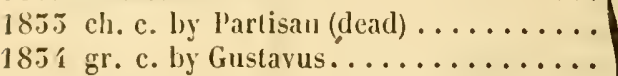

$185.5 \mathrm{ch}$. f. by ditto ..............

1856 gr. c. by ditto .............. Mr Tattersall

М $\Lambda$ RTHL,

Bred by Mr Tuorsnile, in 1824, got by Merlin, her dam, Seamew, by Scud, out of Goosander, by llambletonian.

1829 b. f. by Straitwaist ............ Mr R. W. Walker

1852 b. f. by Camel (sent to France)......

1854 ch. I. by Tarrare, or Camel......... Mr Greatrex

1856 by ditto, or ditto..........

She was trained in 1850 , and has had only the above foals.

\section{MARY,}

Bred by $\quad$ in 1825, got by Friday, out of Luna, by Volunteer-Stargazer, by llighflyer.

1899 b. c. Father Barney, by Champignon. 18.50 ch. c. Uncle Toby, by Cain .........

18.51 b. c. Donall, by Cail ............

$185 \pm$ br. c. Trim (aft. James) by Edmund. . Mr Bristow

$185.5 \mathrm{hr}$. c. Doctor Slop, by Doctor liaustus

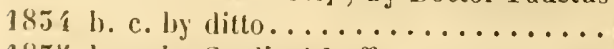

$185{ }^{\circ}$ b. c. lyy Cardinal luff .............

UART (Young)

Bred by the Duke of Leens, in 1816, got by Mowloray, her dam, Mary, by Y.Mar-ke, out of Gentle Kitty, ly. Silvio-Dorimond. 


\section{3}

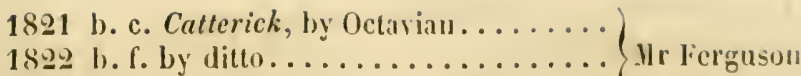

1823 b. ศ. by ditto...............

1826 clı. f. (Miss Croft) by Wanton .......) ur J Croft

1897 br. f. by ditto.................... Jr J. Croft

1828 b. c. by ditto (dead).............. Mr S. I. For

1829 lor. f. by ditto . . . . . . . . . .

1850 br. f. lyy Lottery..............

1851 b. f. loy Whisker.............. 1). of Leed

18.52 br. f. by Waverley ............ 1). of Leerls

18.3 b. c. Wildair, by Winterfield.......

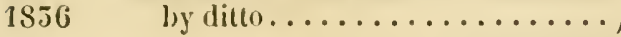

In 1855 missed to Waverley, 34 to Lottery.

\section{MARY ANS,}

Bred by Mr TAxтox, in 1S25, got by Blacklock, out of Allana.

1829 1. c. Vyeyan, by Canteen......... Sir J. Boswell

1850 b. f. ly dilto (dead)

18.5 I missed to ditto, and was trained............ Ir Gilmour

1852 by 1 ctæon .............. Mrr Dawson

1835 bl. c. by Jerry (s. to Baron Biel).....

1854 c. by Iapdog.............. Col. P'ecl

1855 f. by vefence............

MLTILDA,

A Bay IIare, foaled in 1818, got by Orville, her dan by Sorcerer,

out of Matilda, by Whishey-sister to Toby, by Ilighflyer.

1825 ch. f. Mermaid, by Merlin ........

1826 cl. c. by llagistrate (dead) .........

1897 h. с. Merryman, by l'aulowitz...... Ir Yates

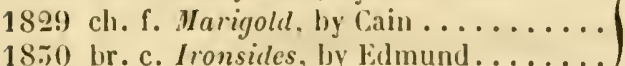

1852 bl. с. Mammon, by Cair........... Mr

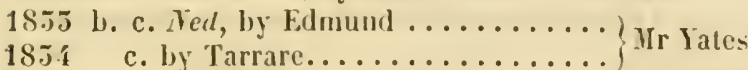

1856 by Camel............. Mr Stirling

Barren iı 1828, 51, and 5..

\section{M.TTILD.I.}

Brel by Mr Petre. in 182i, got by Cumus, out of Juliana, by Gohanna-l'latiua. by Mercurj. 


\section{6}

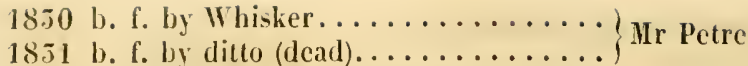

18.52 b. с. Tewolon, by Velocipede .......

185.5 ch. c. ly Langar...............

1851 c. by Voltaire............. D. of Cleveland

$185 y$ b. c. by ditto...............

1856 by Physician.............

\section{M $1 \mathrm{UD}$,}

Bred by Mr BLoss, in 1828, got by Morisco, her dau, Merry-maid,

by Buzzard-Ilighflyer-Smallboues, by Justice.

1852 b. f. Matilda, hy Shakspeare.......

1855 b. f. Hyrtle, by Zinganee................. Hr Bloss

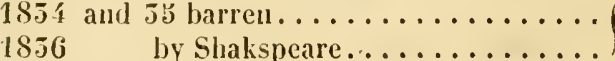

\section{MEDINA,}

Bred by Lord Grosveyor, in 1894, got by Shebdeez, her dam, Passamaquoddi, by Lignum Vitæ-Hind, by Sir Peter.

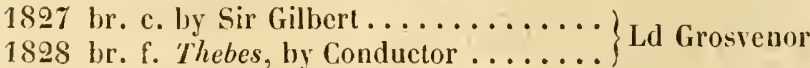

1829 br. f. IIegira, by Champion.........

$18 \% 0$ missed to Filho

1851 bl. c. Abbas Mirza, by Cannel.

1852 b. c. by Peter Lely (born with defective

1855 b. f. by Teniers (died a foal) ........ sight, and shot) $>$ Ld Westminster

1854 c. Ly Pantaloon (bori bliud, and shot)

$1853 \mathrm{bl}$. c. by ditto ...............

18.56 by Camel............

\section{MEDINA (sister to The Hloslem)}

Bred by lord Verulay, in 1822, got by Selim, her dam, Tredrille,

by Walton, out of l'ope Joan, by Waxy-l'runella, by Highllyer.

1827 b. f. hy Centaur .............. Mr Forth

1852 b. c. Stargazer, by Tramp (died on the)

1854 b. c. by Mulatto ..............

She had three foals between 1827 and 52, which all died young; missed 10 Partisan in 55, to Emilius in 55, to Revel]er in 56, and sent to Germany in February in that year. 


\section{7}

MEDOR 1.

Brel by Col. Aneave, in 181 I, got hy Selim, her dam by Sir Harry-Volunteer-Herod-Golden-grove, by Blank.

1818 l. by Junjper............... Mr Cussans

1819 b. f. Posthuma, by Orville........ D. of Grafton

1821 ch. l. Pucelle, by Iluley.............. D. Dof Crafton

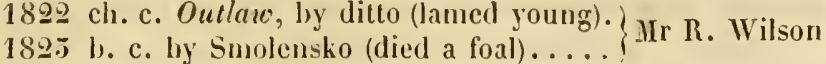

1824 b. f. Gulnare, by ditto .......... D. of Richmond

1828 slipped foal, by Lory............

1826 h. f. by Wrangler (died a foal)....... Mr R. Wilson

1827 ch. c. Wat Tyler, by ditto (s. to India)

1828 br. c. The Grand Duke, by ditto... L Ld Orford

1829 b. f. Pandora, by Wrangler........

1850 br. c. Pillington, by Edmund......

18.51 br. 1. Margaret, lyy ditto (put to the Mr Yates

1855 br. c. Frederick, by Edmund ........

185 4 br. f. by ditto.............. Col. Peel

Barren in 1820, 32, and 55, and died on the passage to Germany in the autumn of $185 \mathrm{y}$.

\section{MEDORA,}

Bred loy Mr Kinwax, in 181;, got by Swordsınan, her dam by Trumpator, out of Peppermint, sister to Prunella, by Highlyer.

1818 b. f. by Waxy Pope ........... K L Sligo

1821 b. f. Western Lass (aft. Fib) by Bohadil,

1822 b. c. SirJohn, by Souvenir ......... Mr Noke

1823 b. c. Flumingo, by Oiseau ........ Mr Ridsdale

1824 b. f. Princess, by Regent......... Ir Toke

1827 l.r. e. The Cardinal, by Waxy Pope... Ir Allen

1828 b. c. Sharli, by Theodore......... Mr Petre

1850 l. e. Rockingham, ly Humphrey Clinker

$1852 \mathrm{gr} . \mathrm{c}$. Cavendish, by l.ottery, or l'eirse Mr Allen

18.5 .5 b. c. The Master of the Rolls, by Lottery)

In 1820 missed to Oiseau, 26 to Langar, 29 and 31 to Lottery, and died in 1855. The lirst seren foals were bred in Ireland.

\section{IIEG MLRDOCK,}

Bred by Ur l'Rres, in 1815 , got by Grimaldi, ont of Jistake. 


\section{8}

$1820 \mathrm{gr}$. f. Meg, by the Malcolm Arabian ..

1851 gr. f. by Doctor Eady ...........

1852 slipped a c. by Doctor Eady ........

$1854 \mathrm{gr}$. f. by Fungus . . . . . . . . . . Mr Pryse

1853 c. by Doctor Eady (dicd soon after

(oaling)

She was ridden from 1820 to 50 , missed in 55 , and died in 55 , immediately after foaling.

\section{MEMINA (sister to Fawn)}

Bred by Lord Stranzroke, in 1824, got by Smolensko, her dam, Jerboa, by Gohanna, out of Camilla, by Trentham.

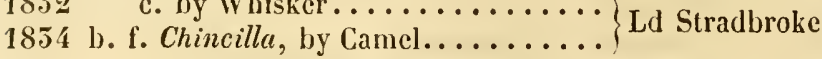

In 1855 missed to Lamplighter, 5 घ̈ to Camel.

\section{MEMPHIS (sister to Memnon)}

Bred by Mr WATt, in 1825, got by Whisker, out of Manuella.

1850 br. f. by Blackilock ............ Mr Watt

$1851 \mathrm{ch}$. f. by Figaro...............

1852 br. f. by Mameluke............

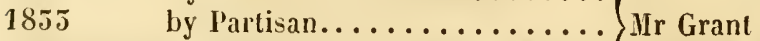

1854 f. by Peter Lely............

1855 by ditto $\ldots \ldots \ldots \ldots \ldots \ldots \ldots$

\section{MENEVER,}

Bred by Mr Tnornille, in 1851, got by Merlin, her dan by Walton, out of Lisette, by Hambletonian-Constantia, by Walnut.

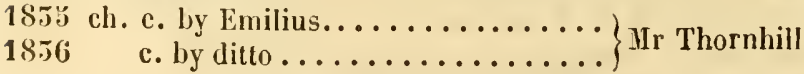

\section{MIERCANDOTTI,}

Bred by Lord Sufriesn, in 1820, got by Muley, out of sister to Petworth, by Precipitate.

1828 ch. f. by Teniers (s. to Ilungary) ....

$1829 \mathrm{ch}$. f. by ditto (s. to Mr Corbin, of

1850 ch. f. by Waxy Pope (dead) ........
1852 ch. f. Usury, by Banker ...........

1855 br. c. Trap-ball, by Battledorc........ Mr Mostyn 
1854 slipped foal, by lielt............

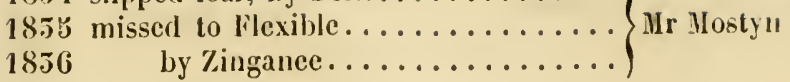

\section{MERCY,}

Bred by Mr Thonvilu, in 1822, got by Merlin, her dam, Shoveler, by Scud, out of Goosander.

1826 b. f. Oucen of Hearts, by Emilius.... Lo Ranelagh

1827 b. f. Mouche. by ditto........... Lul Selton

1828 b. c. ( . Sr Sowerby's $Y$. Emilius) by ditto

1853 b. c. Ermine, by ditto............

1854 ch. c. by ditto.................. Mr Thornhill

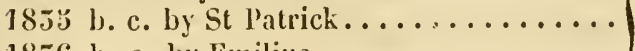

1856 b. c. lyy Emilius.................

Missed to Emilius in 1829,50 , and 52 , to St Patrick in 51.

\section{MERLIN MARE,}

Bred by Col. Wissos, in 1825, her dam by Oscar, out of Dairymaid, by Diomed-Nelly, by Conductor.

1829 b. c. Fingal, by Middleton......... Ld Jersey

1850 b. c. Jason, by Centaur (s. to France, \} Sir S. Graham

October 1834)

1851 ch. f. by Middleton............. Mr R. Curtis

1852 b. f. by lartisan..............

18.3 .5 b. c. by Bedlamite (died young)......

1834 slipped foal, by $\Lambda$ ugustus ..........

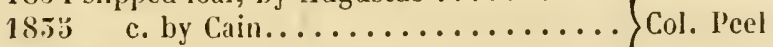

1856 slipped foal by Cain. ............

1857 by Glencoc...............

\section{MERLIN MARE (sister to Goshawk)}

Bred by the Dulie of Graftov, in 1828, her dam, Coquette, by Dick Andrews, out of Vanity, by Buzzard - Pot 80 's.

18.52 b. f. by Figaro (sold abroad) ........

1853 b. c. by Lottery (died a foal).......... .Hr J. Richardson,

1854 f. by Lottery..............

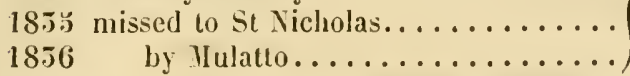

\section{MERMAID,}

Bred by Col. Cratrurb, in 1829, got by Whalebone, her dain, IIiss Emma, by Walton-Orville-Buzzard. 


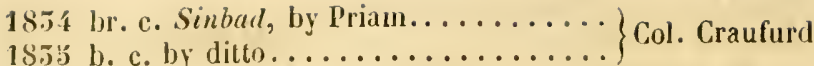

\section{MERIAID,}

Bred by Mr E. Yares, in 182:5, got by Merlin, out of Matilda, by Orville-Sorcerer-Matilda, by Whiskey.

1850 br. c. Denbies, by Filho da Puta..... Mr Wood

1851 ch. f. Tritonia, by Peter Lely.......

185.5 ch. c. Triton, by Memnon .........

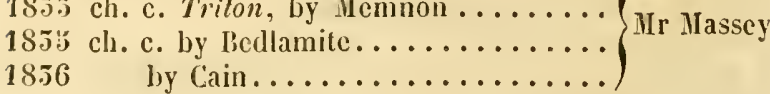

Misserl in 1852 and 54 .

MERRYMAID, by Buzzard (Vol. III, p. 259) was barren from 1828 to 55 inclusive, and was then shot.

\section{MERVINIA,}

Bred by Mr Bocltees, in 1815, got by Walton, her dam, Phantasmagoria, by Precipitate, out of Cerberus's dam, by IIerod.

1821 b. f. by Rubens (died a yearling) .....

1822 br. or b. f. by Blucher............

182.3 br. c. Fisherman, by Bustard (Castrel)

1824 br. c. Lechmere, by Master IIenry, or Mr Mytton

Castrel

1826 br. f. by Filtio da Puta.............

1827 br. f. Fanny, by Master Henry...... Mr Ilenderson

1828 c. by Champion.............

1829 b. c. Jeremiah, by Wamba......... JIr L. Charltou

1850 b. c. Jeroboam, by ditto..........

1831 br. f. WFasp, by ditto............ Ld Portarlington

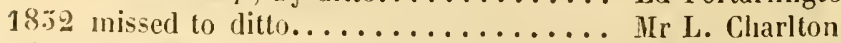

1855 b. f. Mrs Nisbett, by ditto......... Mr Weatherley

185 亿 b. c. by Shakspeare............. Sir S. Graham

185.5 missed to Sir Benjamin.........

\section{METEOR MARE,}

Bred by Lord Grosiexok, in 1810, her dam, Alexandria, by Alexander, out of Esther, sister to Escape, by Highflyer.

1816 gr. c. Forester, by Fitz-James....... Mr Ree 


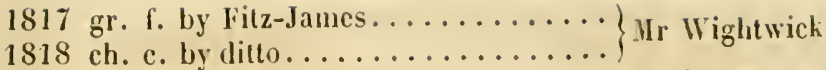

182.5 b. c. by Astrologer (Sir Oliver)..... . IIr Green

1825 b. c. by Master Heury

1826 br. c. by Muley ..... died 2 days old

1828 b. c. by $\Lambda$ strologer...

1850 b. f. by Roderick, or Bedlanite.....

$18 \pi 2$ ch. c. Dr Haller, ly Bedlamite...... Mr Fryer

1855 slipped a colt, by Belzoni.........

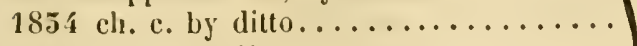

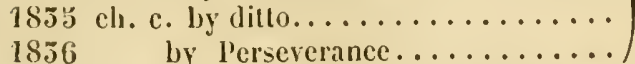

Was barren sereral years after 1818 and in 51 , was not corcred in 25,26 , or 28 .

\section{MICILALLMS,}

Bred by Lord Grosrevon, in 1818, got by Thunderbolt, her dam, I'lover, by Sir Peter-Bouldow-Escape's dam, by Squirrel.

182.5 br. f. by $\Lambda$ bjer..............

1823 br. c. Olympus, by Blachlock.......

1827 b. c. Thermometer, by Whisker.... I Id Grosrenor

1898 br. f. Iloliday, by Conductor.........

1829 b. f. by Haster Henry (died a foad)....

1850 b. f. by Filho (dead) .............

1851 br. c. Sheik (aft. Great St Michael) by

1852 slipped twins, by Peter I.ely.........

1853 br. f. by Teniers.............. Ld Westminster

1854 missed to Pantaloon ............

1855 b. c. by ditto .................

1856 bl. f. by Camel..............

\section{MIDDIETON MARF,}

Bred by Mr Srinuiga, in 1828, her dam, Electa, by ElectionMiss Manager, by Giles.

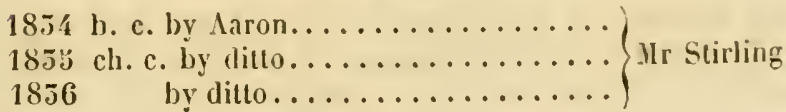

\section{MIJ)GE (bay)}

Bred by Mr Fox, in , got by Whisker, her dam by Cuck Robin, grandam by Precipitate-Colibri, hy Woodpecker. 
1851 b. c. Peter Simple, by Velocipede.... Mr Armitage

1855 b. f. by ditto................ Mr George Oates

1854 ch. c. by ditto...............

185 barren................. Mr Armitage

1856 by Velocipede............

MIGNONETTE, by Sorcerer (Vol. III, p. 242) was barren in $1852,55,54$, and 55.

\section{MIGNONETTE,}

Bred by Lord Egreworr, in 1825, got by Whalebone, her dam, Vicarage, by Octavius - Election-Highflyer.

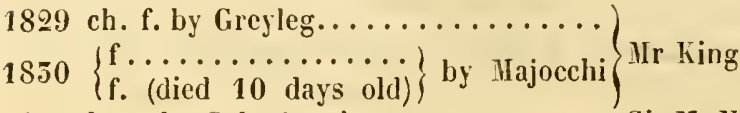

1851 b. c. by Gaberlunzie............ Sir M. Wood

1852 b. c. Agitator, by ditto ........... Mr Balchin

185.5 dead foal, by Chateau Margaux......

1854 missed to Gaberlunzie.............. Hr King

1856 not covered last year............

\section{MIGNONETTE,}

By Vespasian, out of sister to Minimus, by Dungannon.

1852 ch. c. by Y. Filho..............

1855 ch. f. by Y. Haphazard ......... Dr Willis

1854 barren.........................

\section{YOUNG IIIGNONETTE,}

lired by Mr Noweth, in 1829, got by Bustard, her dam, Mignonette, by Sorcerer, out of Symmetry, by Sir Peter - Phantasmagoria.

1855 b. f. by Longwaist .............. IIr Nowell 1854 br. c. King of C'ubs, by IIuley...... Capt. Williamson 1853 br. c. by ditto............... Hir Nowell 


\section{3}

\section{HIIIA,}

Bred by Mr P'RYse, in 1820, got by Grimaldi, out of Frances, by Ambrosio-Hightlyer-Eclipse.

$1829\left\{\begin{array}{l}\text { b. f. } \\ \text { br.f. }\end{array}\right.$ by Multum in Parvo, or Dupli-

1850 b. f. Mimic, by Mallard ..........

1831 b. f. Mirth, by Euston ...........

1852 missed to Edmund.............. IIr Bowen Davies

1835 b. c. Momus, by Cardinal P'uff ......

1834 b. f. Merry Lass, by Dr Faustus.....

1855 b. c. Merry Andrew, by Adventurer. .

1856 by Wamba

\section{MIIIA,}

Bred by Sir T. Stanley, in 1828, got by General Mina, her dam, Maid of Lorn, by Castrel-Richardson's Marske-Rochingham -Eclipse.

1853 ch. f. by Battledore.............

$1834 \mathrm{ch}$. f. by ditto (died young) ....... Sir T. Stanley

1855 ch. f. by ditto $\ldots \ldots \ldots \ldots \ldots \ldots . . .$.

1856

by Birdeatcher............

\section{MINETTA,}

Bred at Euston, in 1828, got by Woful, her dan, Posthuma, by Orville, out of MIedora, by Selim-Sir Harry - Volunteer.

1853 ch. f. Minna, by The Colonel.......

1834 missed to Tranby ............ Mr Martyn

1855 ch. c. by Sir Hercules............

1856

by Logic .

\section{MINIATURE,}

Bred by $\mathrm{Mr}$ Своскғолd, in 1822, got by Rubens, her dan,

Y. Chryseis, by Dick Andrews, out of Chryseis, by Asparagus.

1827 ch. c. Portrait, by Morisco.........

1828 ch. f. by ditto .................. Ld Anson

1822 b. c. Terry Alt, by Sligo .......... 


\section{4}

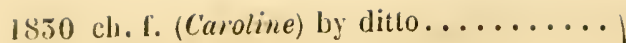

1851 ch. c. Whitefoot, by ditto ........

18.52 ch. c. by ditto ..............

1855 missed to Sultan .............. Ld Lichficld

1854 ch. c. IIector, by Priam .........

1855 ch, c. by Bedlamite...........

1856

\section{MINIMA}

Bred by Lord Durhan, in 1822, got by Election, out of Leopoldine, by Walton-Cressida, by Whiskey.

1850 b. c. by Contest..............

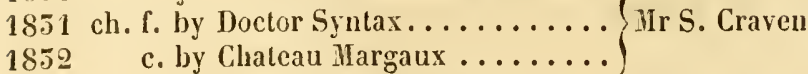

1855 br. c. Locomotive, by Waverley ..... Mr T. Dawson

Died in 1854.

MINNA,

Bred by Mr HawontI, in 18உ0, got by Camillus, out of Miss Zilia Teazle, by Sir Peter-Zilia, by Eclipse.

1850 b. с. by Comus............... Mr Haworth

1851 ch. c. Buccaneer, by Blacklock...... Mr Maxwell

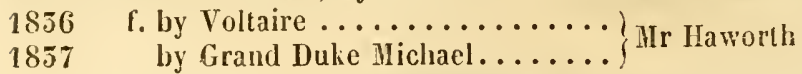

She had two colts by Comus prior to 1850 , which died foats, and all the produce she had between 1851 and 56 , died young.

\section{MINOS MARE (sister to Chancellor)}

Bred by Mr Bower, in 1826, her dam, Angelica, by Amadis, out of Miss Craigie, by Orville-Marchioness, by Lurcher-Phœnomenon.

1855 gr. c. by Y. Phantom ..........

1854 b. f. by dilto............... Mr Bower

1856 by The Saddler............

\section{MINSTREL,}

Bred by Mr R. W. WaLKer, in 1827, got by Little John, out of IIetre, by Waxy-Woodbine, by Woodpecker-Puzzle, by Matchem. 


\section{5}

1851 b. f. by Turtle .............. Mr Bailey

1852 br. c. by Crosus.............. Sir J. I). King

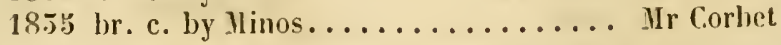

Missed in 1855 , and was not covered that year.

MINIKIN,

Bred by Mir L. Cinarlox, in 1927, got by Manfred, her dam, Morgiana, by Coriolanus - Lurcher-Phlegon.

1855 ch. f. by Emancipation ...........

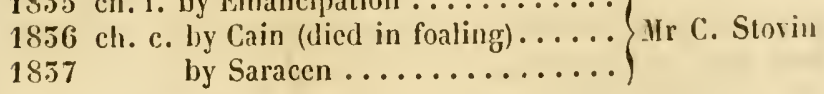

\section{MINUET,}

Bred by the Duke of Graftos, in 1812, got by Waxy, her dam, Woodbine, ly Woodpecker, out of Puzzle, by Matchem-Princess, by Heror-Julia, by Blank-Spectator's dam, hy Partner.

1821 b. c. Banquo, by Phantom.........

1829 b. c. Bolero, by Partisan..........

1895 b. c. Bolivar, by Comus ..........

$1824\left\{\begin{array}{l}\text { b. f. (dicd } 4 \text { days old) } \\ \text { b. f. (died } 5 \text { months old) }\end{array}\right\}$ by Partisan.

$182: 3$ b. c. Bramin, by ditto sent to India in D. of Craftent

1826 b. c. Burman, by dillo 1829

1827 b. f. Brambilla, by ditto..........

*1828 b. f. Blassis (aft. Mistletoe) by Emilius

1829 b. f. by Ilerlin ...............

1852 b. f. Balance, by Emilius...........

In 1829 missed to Quiz, 50 to Centaur, 51 slipped foal, and was shot in $\Lambda$ pril 1855.

* Mistletof (now Capl. Marlyn's) produced a b.f.by The Colonel, in 185.5. and was covered by Aciæon.

\section{JIRANDOLA,}

Bred by Lord Clarexnox, in 1817, got by Haphazard, her dau, Allegretta, by Trumpator, out of Y. Camilla.

1823 b. f. Spavina, by Orville..........
1826 ch. f. Emilina, by Enilius .......

1826 ch. f. Emilina, by Ensilius ....... I I Orford

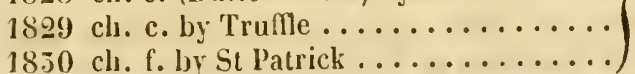


1852 br. c. (Tashenden) by Camel.......

185.5 cl. c. by Bedlamite ....................... Mr Ricardo

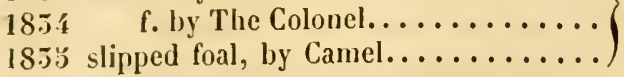

In 1827 missed to Master Henry, 51 to St Patrick.

\section{MIRIAM,}

Bred by Il. R. II. the Duke of York, in 1824, got by Whalebone, out of Moses' dam, by Gohanna.

1829 b. c. by Rosario, or Filho .........

1830 dead colt, by Phantom ...........

1851 ch. f. by Middleton........................... Mr Wigram

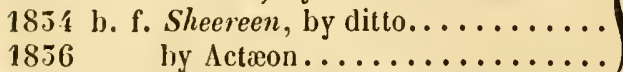

Missed to Mameluke in 1855, to Cain in 55.

\section{MISCHANCE,}

Bred by Mr Tones, in 1825, got by Castrel, or Master Henry, out of Miss Hap, by Shuttle-Sir Peter-Eclipse.

1829 b. f. Graceful, by Sir Gray ......... Mr Tomes

Not covered in 1829 , and died in 32.

\section{MISERY (first called Brunette)}

A Brown Mare, foaled in 1815, got by Camerton, herdam, Tobina, by Toby, out of the Eclipse mare, sister to Crassus.

1823 b. f. Fancy, by Wanderer .........

$182:$ b. f. Miss Orville, by Pendulum .....

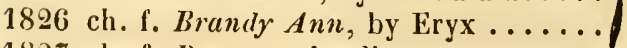

1827 ch. f. Brunette, by ditto......... Mr S. Pearce

1828 b. f. Grasshopper, by ditto ................. Mr S. Pearce

1829 br. f. by ditto

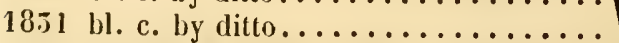

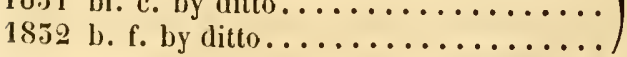

Not covered in 1823 or 29 , and died in 52 , with her foal.

\section{MISRULE (sister to Pounce)}

lired lyy Mr Thornilu, in 1850, got by Merlin, her dam, Surprise, by Scud, out of Manfreda, by Williamson's Ditto-Tawny. 
1854 b. c. by Emilius $\ldots \ldots \ldots \ldots \ldots \ldots \ldots$
1853 b. f. by ditto $\ldots \ldots \ldots \ldots \ldots \ldots$

\section{IIISS ANDREWS,}

Bred by Lord Scarnovar, in 1826, got by Catton, her dam by Dick Andrews-Sir Peter-l'lay or l'ay's dam, by llerod.

1852 b. f. Antic, by Brutandorf ......... Mr Vansittart

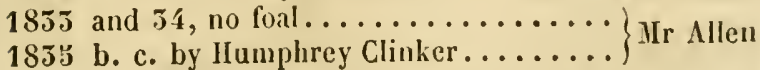

Sold to go to America in $\mathbf{1 8 5 5}$ with her foal.

\section{MISS ARMSTRONG,}

Bred by Mr Aвиsтroxa, in 1825, got by Whisker, her dam, Staveley Lass, by Shuttle, or IIambletonian-Drone-IIatchem -Jocasta.

1850 b. f. by Alexander.............

1851 b. f. by Figaro (put to the stud) ..... MIr . Irmstrong

1852 by Borodino.............

1855 by ditto $\ldots \ldots \ldots \ldots \ldots \ldots$

1854 b. c. Cornbrough, by Tramp........

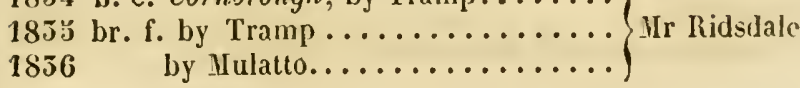

\section{MIISS BREEZE,}

Bred by Lord Foley, in 1S26, got by Phantom, her dam, Breeze, by Soothsayer, out of Blowing, by Buzzard.

1850 b. f. by Trinidad.............. Mr Ilolbrook 1851 ch. c. Colossus, by Mameluke....... Ir Theobald

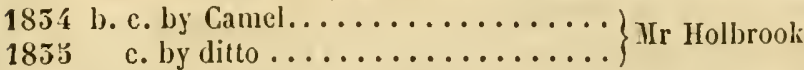

\section{MISS CANTLEY (sister to Burleigh)}

Bred by Col. Cullders, in 1812, got by Stamford, her dam, sister to Silver, by Ilercury-IIerod-Y. IIag, by Skim.

1817 b. c. by Orion................

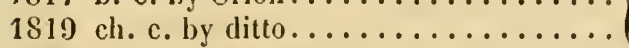

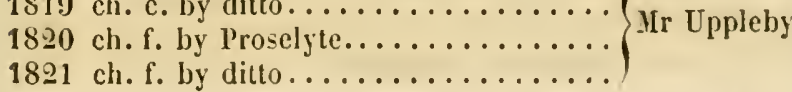




\section{8}

1822 ch. c. by Proselyte............ Sir H. Goodricke

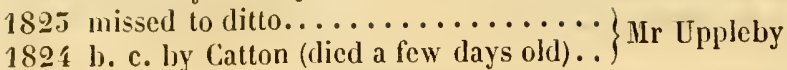

1823 b. c. Chico, by Filho da Puta....... D. of Richmond

1826 b. c. Mendicant, by Tramp.......... Mr G. Walker

1827 b. f. (The Duchess) by Catton (s. to

Germany)

1828 ch. c. Ramazan, by Sultan ........

1829 ch. c. Beiram, by ditto............

1850 b. c. Iman, by ditto.............

1851 b. f. by ditto (died a foal) ......... Ld Exeter

1832 b. c. Gladiutor, by Redgauntlet .....

1855 c. Muezsin, by Sultan.........

1854 b. c. by ditto..................

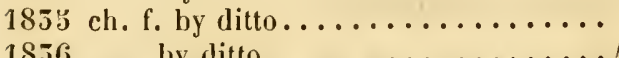

1856

by ditto

IIISS CATTON, *Harmora's dam (Vol. III, p. 249) missed in 1855,54 , and 53 , and was shot. Her last produce was.

1852 b. c. Bosphorus, by Sultan (broke his leg) in running at 3 yrs old, and shot) $\}$ Ld Exeter

* Marmora is in the stud. She produced ch. c. by Redgauntlet in 1854, missed to him in 55, and was covered by Reveller.

\section{MISS CHANTREY (sister to Busto)}

Bred by Mr Butser, in 1818, got by Clinker, her dam, Bronze, sister to Rubens, by Buzzard.

1825 br. c. Jocko, by Filho da Puta ..... . Ld Lowther

1824 br. f. by ditto (hipped) .................... Mr Butler

1826 br. f. Blackberry, by Sherwood ..... Mr IIouldsworth

1828 br. c. Roubillac, by Filho.......... Mr G. Walker

1829 b. f. Smallhopes, (aft. Catgut) by Catton Mr Hudson

1850 c. by Sherwood (died) .......... Mr Butler

1832 slipped a filly, by Partisan......... Ld Lowther

1834 br. or ro. f. Simia, by ditto ........ MIr J. Day

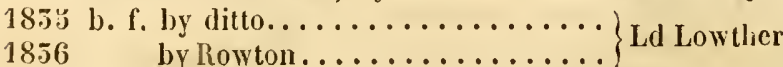

Barren in 51 and 55. 


\section{MISS CLIFTON,}

Bred by , in 1822, got by l'artisan, her dam, Isis, by Sir Peter, out of Ibis, by Woodpecker-Isabella, by Eclipse.

185 I ch. c. by Defence............. Mr Sadler

1852 b. f. by ditto............... Mr Ilowes

1855 b. f. by The Colonel............ . I.d Chesterfield

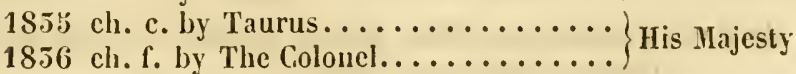

\section{MISS CRACIIAMI,}

Bred by Mr IIouldswortr, in 1829, got by Magistrate, her dam by Ruler, out of Treecreeper.

1826 b. f. by Filho (died a foal) ........

1829 ch. c. Abclallah, by Tiresias........

1850 gr. f. Augusta, by Gustavus........

1951 ch. c. Alaric, by Velocipede........ IIr Walker

1852 ch. f. Alice, by Langar (in Mr Allen's stud)

1855 ch.c. Aurelius, by Emilius ....... Ld Wilton

1954 b. c. Antiphus, by Priam......... Ld Chesterfield

Not corered in 1826 or 27, and sold to Count Ilunyady in September 1854 , covered by Humphrey Clinker.

MIISS CRAIGIE, the dam of Birmingham ( $\mathrm{Tol}, 111$, p. 250) missed to Filho in 1852, 55 , and 54 , and died in February 55, by the rupture of a blood vessel, with a colt in her, by Fillı, or Vanish.

\section{MISS CRAVEN,}

Bred by Mr Gates, in 1824, got by Mr Lowe, her dam by Soothsayer-Buzzard-sister to Bangtail, by Highllyer.

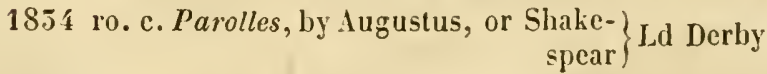

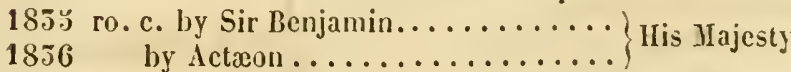

\section{MISS CROFT,}

Bred by IIr Jayes Croft, in 1826, got by Wanton, her dam, Young Mary, by Mowbray, out of Mary, by Y. Marske. 
$18 \geq 9$ ch. c. Richmond, by Jack Spigot.....

1832 ch. f. Rosamond, by ditto..........

1853 ch. c. by ditto............... Mr Edmondson

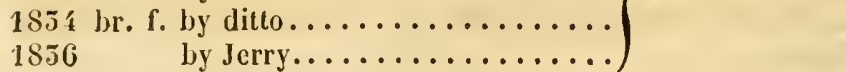

IIissed in 1850 and 51 , and not covered in $\mathbf{5 4}$.

\section{MISS EMHI,}

Bred by Mr T. 0. Powletr, in 1824, got by Walton, her dam by Orville-Buzzard-Hornpipe.

1829 bl. f. Mermaid, by Whalcbone......

1850 bl. c. The Mole, by ditto..........

1831 b. f. Ermine, by Whisker (put to the Col. Craufurd stud)

Jissed to Lottery in 1852 , and died in 1855.

\section{MISS FANNY (sister to Miss Emma)}

Bred by Mr T. 0. Powlett, in 1820, as above.

1826 bl. f. (Powlett) by Jack Spigot......

1828 bl. c. by ditto..................

1829 b. f. by Whisker (put to the stud)....

1855 br. f. The Mystery, by Lottery...... Ur T. 0. Powlett

1835 slipped foal, by Lottery ...........

1854 b. f. by Priam................

185 b. c. by Velocipede.............

In 1827 missed to Tramp, 51 to Lottery, 52 to Sultan.

\section{IIISS FOX,}

Bred by Mr Krsptox, in 1823, got by Glow-worm (son of Brainworm) out of Miss Paul, by Sir Paul-Shuttle.

1828 b. c. by Lottery (died a yearling).....

1829 missed to Brutandorf.............

1830 b. c. Carnaby, by ditlo........... Mr G. Robinson

18.31 b. c. Logic, by Lottery.............

1852 b. c. by Brutandorf (died 2 yrs old)...

1834 b. c. Compton, by Humphrey Clinker. Mr S. Herbert

1853 b. c. by ditto................. 


\section{MISS FRIZ7LLE,}

Bred by , in 1827, got by Whisker, her dam by Cervantes, out of Anticipation, by Beningbrough.

1851 gr. f. by Gustavus.............

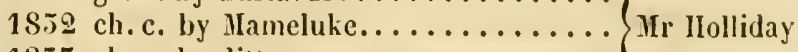

1855 ch. c. by ditto...............

1854 ch. f. by ditto............... Baron Biel

1855 by Camel................ Baron Biel

Sold to Baron Biel in November 18.54.

\section{MISS GARFORTII,}

Bred by Mr Burtox, in 1819, got by Walton, her dam by Ilyacinthus, out of Zara, by Delpini-Flora, by King Fergus.

1825 ch. f. Kate, by Catton........... Ir Eurton

1824 b. c. (Nonplus) by ditto ............. IIr W. Armitage

1826 ch. f. Florence, by Blucher........ Ld Normanby

1827 cl. f. Claudia, by Tramp (dam of Gib-)

1829 b. f. by Saladin................. IIr Armitagc

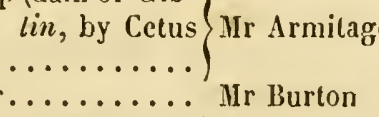

1850 ch. f. Dido, by Whisker............

1851 ch. f. Rocbana, by Velocipede (put the

$1852 \mathrm{ch}$. f. (Her Majesty) by ditto...... IIr Armitage

1855 ch. c. by ditto................

1856

by ditto...............

Barren in 1828, missed to Velocipede in 34.

MISS GIFT,

Bred by IIr Kraptov, in 1825, got by Glow-worm, out of Occana.

by Cerberus - Dr Syntax's dam, by Beningbrough.

1827 b. c. by Peter Lely (dead) . . . . . . . Mr W. Fox

1828 b. c. by Borodino.............

1829 b. f. by Peter Lely ..............

1850 ch. c. by Regalia.............. IIr Burgess

$18 \pi 2$ b. f. by Hal................

185.5 b. f. by Scipio................

Not covered in the intermediate years, or in 1854. 


\section{MISS IRIS,}

Bred by Lord Grosrevor, in 1824, got by Blucher, out of Iris, by Sir Peter-Isabella, by Eclipse.

1829 b. f. by Brutandorf............ Ir Husband

1831 br. c. Opodeldoc, by Comus ........ Col. Cradock

1852 br. c. by Columbus.............. Mr Husband

185.5 b. c. Navigator, by ditto ......... Col. Cradock

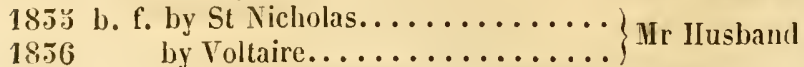

In 1850 missed to Figaro, 34 to Tramp.

\section{MISS LYDI (sister to Miss Fanny)}

Bred by Ir T. O. Powlett, in 1821, got by Walton, her dam by Orville-Buzzard-Hornpipe, by Trumpator.

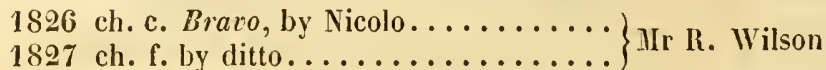

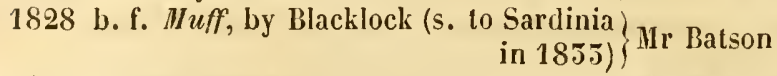

1850 b. c. by Albany............... Mr Willianson

1852 br. c. Lydian, by Filho da Puta.....

1854 b. f. by Albany (s. to Berlin in 1855).

I853 b. c. by Sir Benjamin ........... Ld Chesterfield

1836

by Zinganee.............

Slipped foal in 1825 , barren in 29 and 51 ; slie was sold and sent to Germany in 51 , covered by 1 Ioses in 52 , and repurchased.

\section{MISS MALTBY,}

Bred by Mr Houldsworth, in 1821, got by Filhoda Puta, her dam, IIrs Maltby, by Cervautes, out of Legacy, by King Fergus - Mortonia, by Ilerod.

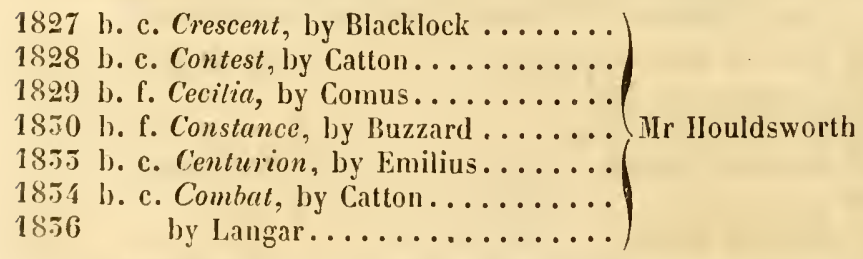

In 1851 and 59 missed to Lottery, and was not covered in 54 . 
MISS MANAGER,

Bred by H. R. H. the Duke of York, in 1807, got by Giles, out of Rosabella's dam, by Diomed.

1812 br. f. by Coriolanus, son of Gohanna) (killed) $\}$ D. of York

1815 b. f. Peppermint, by ditto ......... Mr Turner

1814 b. c. Jubilee, by Y. Pipator......... Ir Grisewood.

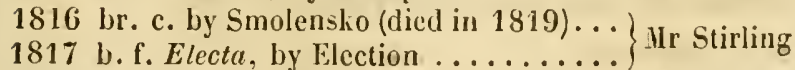

1818 bl. f. Chatham Lass, by Don Cossack.. MIr Farrall

1819 bl. c. by Dominie Sampson.........

1820 br. c. by the above son of Smolensko.

1822 bl. c. by Vandyke Junior (died young)

1824 br. f. by Seymour (s. to Russia) ..... Mr Stirling

1827 br. f. by Y. Election $\ldots \ldots \ldots \ldots \ldots$

1829 b. c. by Rubens Junior . . . . . . . . .

She missed to Phantom in 1830 , and did not breed afterwards.

-Y. Electios, by Election, dam by Lignum Vitx, out of Isis, by Sir Peter.

\section{MHSS MIDDLETON,}

Bred by Mr Hoclioswortu, in 1850 , got by Middleton, her dam,

Tiara, by Soothsayer, or Castrel, out of l'ope Joan, by Waxy.

1855 ch. c. Maplebeck, by Vanish.......

1854 not covered last year.............

1853 br. f. Miss IIarriet Ison, by Gambol Mr Key

1856

(son of Filho)

\section{MISS O'NEIL,}

Bred by Hajor Bower, in 1819, got by Camillus, out of Miss Craigie, by Orville-Marchioness, by Lurcher.

1824 br. f. Belvidera, by Blacklock (sent to Germany in 1817)

1827 gr. f. by Rowlston............. Mr Ormsby Gore

1828 ch. c. Buskin, by Tramp.........

1829 b. f. Evadne, by Waxy Pope........

1850 b. c. by ditto...................... Mr Grant

1855 
1854 b. c. Sir Peter, by Peter Lely....... Mr W. Edwards

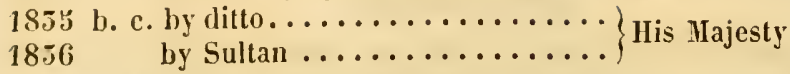
Missed in 1825 and 51 , had a dead foal in 1826 .

\section{MISS ORVILLE,}

Bred by Mr S. Pearce, in 1825, got by Pendulum, her dam, Misery, by Camerton, out of Tobina, by Toby.

1852 b. c. by Mameluke ..............

1855 missed to ditto...............

1854 b. f. by Longwaist............. Mr Nowell

$185 \mathrm{~s}$ ch. c. by Huley ..............

1856 by ditto...............

\section{MISS PATRICK (sister to St Patrick)}

Bred by Sir E. Donswortr, in 1825 , got by Walton, her dam by Dick Andrews-Trumpator-Highflyer.

1829 b. f. by Brutandorf............ Sir E. Dodsworth

1850 b. c. Solon, by Lottery............. Mr J. Robinson

1851 ch. f. Patty, by Whisker.......... Mr Brown

$1832 \mathrm{ch}$. f. by Velocipede............

1854 ch. c. by Battledore............ Sir R. W. Bulkeley 1856

Barren in 1855 and 55.

\section{MISS RUSH,}

Bred by Mr Rusi, in 1828, got by Phantom, her dam, sister to Carthago, by Pioneer, out of Reserve, by Waxy.

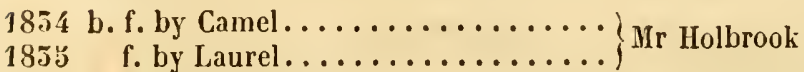

\section{MISS SKIM,}

Ired by Mr Holsrook, in 1821, got by Skim, her dam, Striking Beauty, by Sorcerer-Beningbrough-Sir Peter.

1827 b.f. Fashion, by Phantom (died at 2 yrs old) $\}$ Gen. Grosvenor

1828 b. f. Fancy, by ditto.............

$1829 \mathrm{ch}$. f. by Trinidad.................... Ir Ilolbrook

1850 b. f. Flirt, by Phantom.......... 


\section{5}

1851 ch. c. Bon Ton, by Phantom ....... Gen. Grosvenor

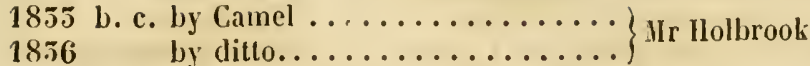

Barren in 1852 and 51 , slipped foal in 35.

\section{MISS TIIOHASINA,}

Bred by Mr Kirex, in 1895, got by Welbeck, her dam, Thomasina, by 'Timothy, out of Violet, by Shark.

1828 b. f. by Champignon (died a foal) .....

1851 br. c. Red Rover, by Lottery........... Mr G. Cooke

1832 br. c. Lichfield, by ditto .......... Mr Baster

1855 b. c. by ditto............... Sir R. W. Bulkeley

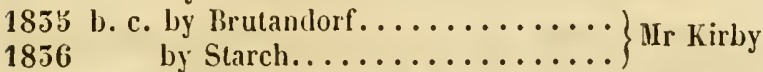

In 1827 missed to Figaro, 50 and 54 to Lottery.

\section{MIISS TREE,}

Bred by Mr ThornhlL, in 1823 , got by Merlin, out of Sal, by Scud-Hyale, by Phœnomenon.

1827 f. by Middleton (died a foal) ......

1828 by Lottery............... MIr Tarleton

$1850 \mathrm{ch}$. f. The Warbler, by Grey Walton..

1831 ch. f. Enchantress, by Gucrilla...... Mr Potts

1852 by Buzzard ..............

185.5 ch. c. El Pastor, by Guerilla ........ Ir Greville

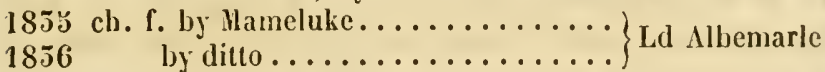

MISS WASP, lyy Waxy (Tol. IlI, p. 261) missed in 18.52 and 55, and died in 1854. Iler two last produce should stand thus :-

1828 bl. c. by Muley (s. to Germany, and $\}$ Ir Nowell

1850 br. f. Vespa, by MIuley (s. to Hungary Oct. 1S34) Sir M. Wood

\section{MISS WENTWORTII,}

Bred by Mr Gibessox, in 1819, got by Cerrantes, her dam by Stamford, out of Wryneck, by Beningbrough - Miss Tippet, by Morwick Ball-Miss Cornforth, by Matchem.

vor, 1 r. 
182.5 b. c. Murphy, by Filho da Puta ..... Mr Corbet

1826 b. f. Lady Sarah, by Tramp........ Ir Gibbeson

1828 b. f. Lady Elizabeth, by Lottery ..... MIr F. Richardson

1829 br. c. Chester, by Filho........... IIr Beardsworth

*1850 br. f. Ulrica, by Sherwood........

1851 ch. c. Benlley, by Buzzard..........

1832 missed to Tramp................ Mr Cookes

1855 cl. f. Tertia, by Emilius...........

1854 b. c. Norgrove, by Stumps.........

Died in Jan. 1853, with a filly foal in her by Buzzard.

* Iilrica produced br. f. by Stumps in 1855 , and was covered by Langar.

\section{MISS WEST (sister to Teniers)}

Bired by Mr C. Dar, in 1822, got by Rubens, her dam, Snowdrop, by Highland Fling, out of Daisy, by Buzzard.

$1829 \mathrm{ch}$. f. by Middleton, or Burgundy ....

1850 ch. c. by Luzborough ............

1851 ch. f. by Godolphin, or Burgundy....

1852 missed to Whisker.......................... Mr Day

1854 b.c. by Fungus...............

1855 b. f. by ditto $\ldots \ldots \ldots \ldots \ldots \ldots \ldots \ldots$
$1856 \quad$ by Cain $\ldots \ldots \ldots \ldots \ldots$

\section{MISS WILKES,}

Bred by Mr T. Hutcurson, in 1818, got by Octavian, her dam

by Remembrancer, out of Mary, by Y. Marske-Gentle Kitty.

1824 b. c. Charley, by Percy ..........

1826 br. c. Agitator, by Blacklock ...........

*1828 b. f. Reform, by Nlexander.........
1829 ch. c. Peter Liberty (aft. The Guard) by Mr. Mounthwaite

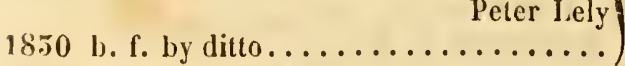

1851 br. f. Flight, by Velocipede ....... Mr G. Crompton

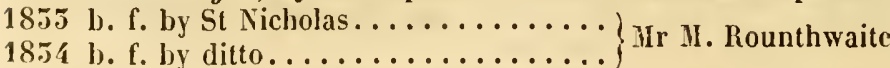

In 1825 missed to Blacklock, 27 to Swiss, 52 to Comus, and died in 54 , in foal to Ilumphrey Clinker.

" lierona produced in 1854 a br. f. by Voltaire, and was sold that year to go abroad. 


\section{MRS CLARKE,}

Bred by Mr IIEsective, in 1816, got by Marmion, ber dim by Sir leter-Florizel-Teresa, by Natchem.

\section{2}

1826

b. f. by Warsaw...............

182 b. f. by Sir Oliver.............

1850 b. c. Unit, by Astbury, or Sir Oliver.. Mr Trafford

1851 b. c. Scamp (aft. Timothy) by Lottery. Mr Giffarl

1855 b. c. Lothringer, by ditto......... Mr Broëucnherg

1833 by St Nicholas.............

Missed in 1852, slipped foal in 54.

\section{MRS CRUICKSHANKS,}

Bred by Mr Kiner, in 1825, got by Welbeck, her dam (Tramp's dam) by Golıanna, out of Fraxinella, by Trentham-Woodpecker.

1829 b. f. by Brutandorf............ Mr J. Wilson

*1850 b. f. (Sarah IIorner) by Lottery..... . IIr Kirby

1852 b. c. by ditto............... Mr Giffart

1854 b. f. by Doctor Syntax..........

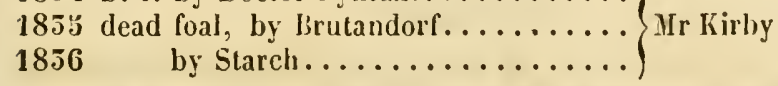

In 1827 slipped foal by Figaro, 51 by Lottery; in 1828 missed tu Champignon, 55 to Lottery.

- Sarail Horner proluced, in 1855 , a b. c. Saul, by Waverley.

IIRS FUDGE, by Williamsou's Ditto (Vol. IlI, p. 500) was shot in the autumn of 1851. She was not corered in 1829.

\section{MRS MALTBY,}

lired by Mr LAmus, in 1816, got by Cervantes, her dam, Legacy, by King Fergus - Mortonia, by IIerod-NorthumberlandReguius.

1820 b. c. The Agent, by Filho da Pula...)

1821 b. . Miss Maltby, by ditto.........

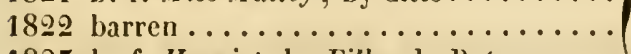

182.5 b. f. IIarriet, by Filho da Puta ..... IIr Houldsworth

$1824 \mathrm{ch}$. f. by Magistrate...........

1823 br. f. by ditto................

1826 b c. Uncle John, by ditto, or Sherwood 
1897 b. c. Gilbert, by Sherwood.........

1828 b. c. Simon, by Comus............

1829 ch. f. by Catton............. Hir Houldsworth

1850 b. c. llawk, by Buzzard...........

1851 ch. c. Vulpes, by Velocipede........

1852 b. c. Comeytrowe, by Lambtonian... Mr Finch

1855 b. f. Lady Maltby, by Swap.........

1854 b. c. by Swap (killed when a foal).... IIr Allies

1850 by Safeguard..............

\section{IIRS RYE,}

Bred by the Duke of Leens, in 1825, got by Octavian, her dam,

Lady of the Vale, by Mowbray-Shuttle-Sir Peter-IIerod.

1831 b. c. Rialto, by Blacklock.........

1852 b. c. by Brutandorf (dead)..........

1855 b. f. Rubina, by Waverley....... D. of Lceds

1854 b. c. Rubicon, by ditto........... D. of Leeds

1855 ch. f. Rodelinda, by Winterfield.....

1856

\section{MRS SUGGS,}

Bred by , in 1817 , got by Crispin, her dam by Totteridge, grandam by Sir Peter, out of Maid of Ely, by Tandem.

1825 ch. c. Ultimatum, by Teniers.......

$1826 \mathrm{ch}$. c. Mona's Pride, by ditto ............ Sir T. Mosty"

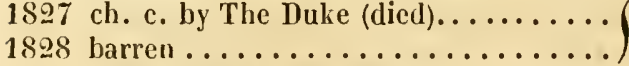

1829 bl. f. Jemima, by Count Porro......

1850 ch. f. by Waxy Pope.............

18.31 b. f. by Peter I.ely...............

1852 ch. f. by Teniers.............. Sir R. W. Bulkeley

1855 ch. f. by ditto ...............

1854 ch. f. by ditto................

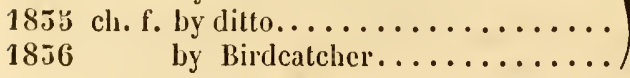

MRS TOUCHIT, by Rubens (Vol. III, p. 265) died in 1852, in foaling. Her produce of 1831 died young, and that of 30 should stand thus :-

1850 b. c. Osirich, by Buzzard........... Mr Houldsworth 
MOEL FAIIMA (Vol. III, p. 267) prodnced a colt in 1852, which is dead, had no living produce in $18550 \mathrm{r}^{\circ} \mathbf{4}$, and was sold.

\section{IIONA,}

Bred by the Duke of Graftov, in 1819, got ly Partisan, her dam,

Miltonia, by Patriot, out of Miss Muston, by King Fergus.

1825 b. c. Lancastrian, by Merlin....... D. of Grafton

1826 b. c. Morris-dancer, by Morisco..... Ld Sefton

$1827 \mathrm{ch}$. c. by Merlin (s. to France in 1850) I). of Grafton

*1828 b. f. Red Rose, by Merlin.........

1829 b. f. by Centaur .............

1850 ch. c. Anglesea, by Sultan........

1852 b. f. Oak Apple, by Royal Oak...... Ld Tavistock

1855 b. f. by ditto...............

1854 b. c. by Àgustus.............

1856

Barren in 1851 and 55 .

- Red Rose produced, in 1833, Mr Molt's b. f. Rosetta, by Royal Oak, and has since been sent to Germany.

\section{MONIMIA,}

Bred by Lord Sufield, in 1821, got by Muley, out of sister to Petworth, by Precipitate.

1827 ch. c. by Flibbertigibbet .......... Mr Theobald

1828 b. c. by Cyduus ............... Mr Gardnor

1829 br. f. by Smolensko (dead) ........ Mr Theobald

1852 br. f. IIester, by Camel.......... Mr Mills

1835 br. c. by ditto...............

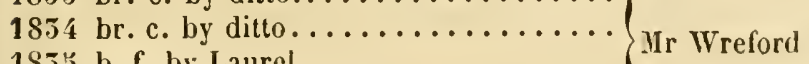

1855 b. f. by Laurel ............... Mr Wreford

Had dead twins in 1830 , and was barren in 51 .

MOONSIINE, by Soothsayer (Vol. III, p. 269) was sold to D. of Holstein in June 1852.

MOPSA (sister to Bullet)

Bred by Mr Wormald, in 1828, got by Cannon Ball, her dam, Shochorn, by Teddy-the-Grinder, out of Roxana, by Alexander. 
1852 ch. c. (Rapid) by Velocipede........

1855 barren................... Hr Wormald

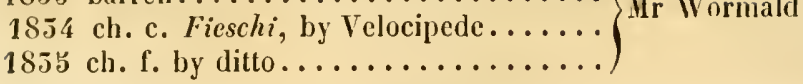

\section{MORISC $\Lambda$,}

Bred by the Duke of Grafton, in 1826, got by Morisco, her dam, Waltz, by Election, out of l'enelope.

1829 ch. c. Schoolmaster, by Rasping.....

1832 ch. c. by ditto.................. Mr Gardnor

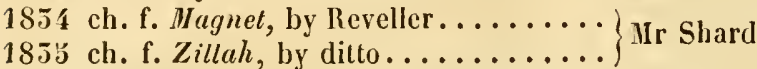

Missed in 1850 and 53 , not covered in 55 , and sold to Baron de Maltzahn in Feb. 1856.

\section{MORISCO MARE (sister to Portrait)}

Bred by Lord Lichғield, in 1828, her dam, Miniature, by Rubens, out of Y. Chryseis, by Dick Andrews-Chryseis, by Asparagus - Lady Jane, by Sir Peter.

1852 b. c. by Emilius (died a foal)........

1855 cli. c. by ditto (died a foal).........

1854 ch. c. Cartoon, by Priam .......... Ld Lichfield

185 z̆ b. c. by Emancipation. . . . . . . . . .

\section{MOUCIIE,}

Bred by Mr Tuonville, in 1827, got by Emilius, her dam, Mercy, by Merlin, out of Shoveler, by Scud-Goosander, by Ilambletoniau.

1855 ch. c. The Mummy, by Mcmnon.....

1854 ch. c. Clifton, by Cain........... Mr E. Peel

1855 ch. c. by Bedlamite.............

1856

by ditto.

\section{MOUSE,}

Bred by Lord George Henry Caverdisn, in 1816, got by Sir

David, her dam, Louisa, by Ormond, out of Orville's dam.

1818 c. by Middlethorpe (died young).... 1819 b. f. by Bourbon.................. Havendish 
1820 br. c. by Selim................

1822 br. f. by Bourbon............. L Ld G. H. Cavendish

1826 b. f. Y. Mouse, by Godolphin.........
1827 b. c. by ditto (s. to Germany, and there called Burlington)

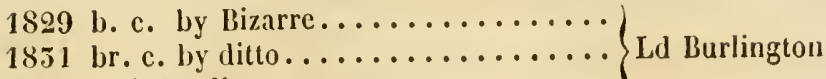

1854 f. by ditto ..............

Barren in $1821,25,93,28,50,52,55$, and 53.

\section{Y. MOUSE,}

Bred by Lord G. H. Caverdisu, in 1826, as above.

1832 br. f. Mousetrap, by Bizarre (dead)... Gen. Grosvenor 1835 b. c. Mus, by ditto............. D. of Richmond

1834 br. c. by ditto................ IIr Sl. Stanley

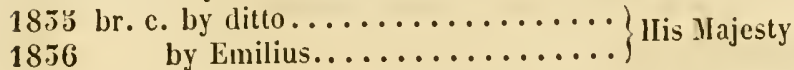

\section{MULEBIRD (sister to Mercy)}

Bred by Mr Tuorxilu, in 1824, got by Merlin, out of Shoveler, by Scud-Goosander, by IIambletonian.

1899 f. by Perchance (died 2 days old).. Ld Orford

1850 missed to Camel and St Patrick...... Ir Jott

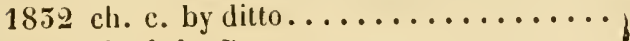

1855 ch. f. by Patron .............. Ld Orford

1834

1855

f. by St Patrick.

f. by Emilius.....

She was sent to Germany in 1855 , with her foal, and corered by Clearwell.

\section{MULEY MARE,}

Bred by Lord Sepfield, in 1821, her dam, Miss Witch, by Sorcerer, out of Rosetta, by Y. Woodpecher-Equity, by Dungannon.

1854 b. c. by Wrangler.............. Sir J. B. Y. Buller 183 br. c. by ditto............... Col. W. R. Gilbert 


\section{2 \\ MULEY MARE,}

Bred by Mr Laxe, in 1825, her dam by Haphazard, out of Miss

Ifolt, by Buzzard-Camilla, by Highflyer-Matchem-Regulus.

1827 missed to IIerlin.............

18 28 ch. c. by Rector (died in foaling from

a hurt)

$1829 \mathrm{ch}$. c. by ditto (died of strangles in

1850 br. c. by ditto................. Mr Lane

1851 br. f. by ditto ...............

1852 ch. f. by ditto................

1855 b. c. by ditto (died soon after foaling).

1854 bl. f. by ditto.................

1835 bl. f. by ditto..............

MULEY MARE (sister to the preceding)

Bred by Mr Lave, in 1824, as above.

1827 b. f. by Merlin

$18 \mathrm{~g}$ ch. c. by Rector................

1829 ch. f. by ditto (s. to Germany)......

1850 ch. f. by ditto................ Ir Lane

1831 b. c. by ditto .................

1852 ch. c. by ditto ..............

1855 ch. c. by ditto.............. Gen. Grosvenor

MULEY MARE (sister to the preceding)

Bred by Mr LaNe, in 1826, as above.

1855 ch. f. by Rector...............

18.54 barren ............................ Ir Lane

1853 ch. f. by Rector (died soon after foaling)

\section{MULEY MIARE (sister to Mussulman)}

Bred by Mr Nowelt, in 1828, her dam by Dick Andrews, out of

Douna Clara, by Cesario-Nimble, by Florizel-Rantipole, by Blauk.

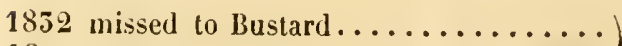

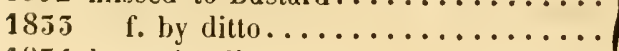

1854 br. c. by ditto................ Hr Nowell

1853 b. c. by Longwaist.............

1856

by Bustard................. 


\section{MULEY MARE,}

Bred by Mr Nowelt, in 1829, her dam, Lady Ern, by Stamford, out of sister to Repeator, by Trumpator-Demirep, by Highflyer.

1855 br. f. The Zegri Maid, by Longwaist. . Mr Bowes

1854 b. c. by ditto (s. to N. America, 1855)

1853 br. c. by ditto.................... Mr Nowell

\section{IIUSIIROON,}

Bred by IIr Axprew, in 1813, got by Dick Andrews, her dam, IIorel, by Sorcerer, out of Hornby Lass, by Buzzard.

1822 b. c. by Sir Harinel.............

1823 b. f. Sephora, by Vampyrc........ Col. Lautour

*1827 gr. f. Articholie, by Skim.......... Mr Gibbs

1850 b. c. Ketchup, by IIoses...........

1851 ch. f. Chantarelle, by IIelcnus....... D. of Richmond

1852

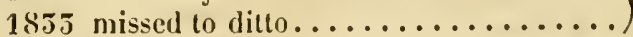

1854 b. f. by Hiddleton.............

1853 br. c. by Sir Ilercules........... Mr Tattersall

1856 b. c. by $\operatorname{Logic} . \ldots \ldots \ldots \ldots \ldots \ldots$

1857 by Glencoe...............

Mr Tattersall

- Anticuoke produced in 1854 a ch.c. The Hermil, by Defence, and was barren the ncxt year.

MUSTARD,

Bred by Mr TnoRxmLL, in 1824, got by Merlin, her dam, Morel, by Sorccrer, out of Hornby Lass, by Buzzard.

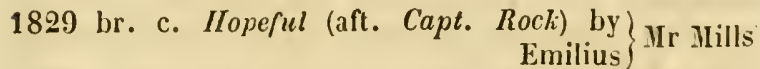

${ }^{*} 1850$ ch. f. (Terfume) by ditto......... Mr Thornhill

+1851 b. f. (Pickle) by ditto .......... II Gully

1852 ch. f. Presere, by ditto..........

1855 ch. f. Marmalade, by ditto ......... Ir Greville

1854 br. c. Mango, by ditto............
1855 b. f. Morella, by ditto ...........

1856 missed to ditto.............. Ir Thornlitl

* Perfene is in Lal Albemarle's stud; she produced in 1835 a ch. c. by Count Porro, and was covered again by him.

+ Pickle was sold to Mr Kirkman, of the Uniled States, in 1855, covered by Taurus. 


\section{MUTA (sister to Lottery)}

Bred by Ir Watr, in 1819, got by Tramp, her dam, Mandane, by Pot8o's, out of Y. Camilla, by Woodpecker.

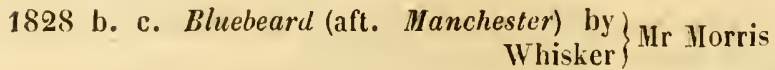

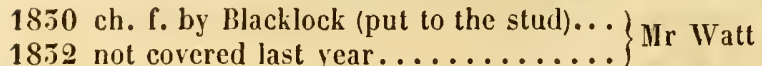

1854 b. c. by Falcon................ Mr Lee

Had a dead foal in 1825 and 27 , missed in $26,29,51,55$, and $5: 5$, and was shot in Sept. 185 5.

\section{IIY LADY,}

Bred by Mr Wrvitu, in 1818, got by Comus, out of The Colonel's dam, by Delpini-Tipple Cyder, by King Fergus.

1824 b. f. Barbara, by The Laird........

1825 br. f. Delphine, by Whisker........

1826 ch. f. Apollonia, by ditto .......... IIr Petre

1829 ch. f. Lally Barbara, by Catton.......

1830 b. c. Frankenstein (aft. Deceiver) by Y.)

Phantom Mr Gully

1852 b. f. Rose Roche, by ditto.............
1855 b. c. Burgstorf, by Brutandorf.... Wr Walker

1854 b. c. Jereed, by Sultan.......... Ld Chesterfield

1856 by Langar.............. Mr Corbin, Virginia

Missed in 1827, 28, 51, and 55 .

IIYRA, by Soothsayer (Vol. III, p. 275) was sold to the D. of Holstein in 1855 , with a filly foal by Wamba, and covered by Tarrare-her b. f. by Master Henry, in 1829, was called Lady IIarrington.

\section{MYRTLE,}

Bred by General Grosvevor, in 1825 , got by Abjer, her dam, Mite, by Meteor, out of Nikè.

1828 b. f. Myrtilla, by The Flyer.........
18.50 br. f. Felucca, by Skiff, or Johnny... Gen. Grosvenor

1831 ch. c. Scarabceus, by Trumle........ Mr Westenra 
1853 br. f. Blossom, by Camel..........

1854 ro. c. Bontibok, by IIelenus ....... Mr Westenra

1856

1857

c. by Camel.

$\int$

by Count Porro ............

She had a dead foal in 1852, and no foal in 29 or 53.

TIIE NAB,

Bred by

, in 1827, got by Sam, out of Lionel

Lincolu's dam, by Sorcerer-Black Diamond, by Stamford-

Louisa, by Ormond.

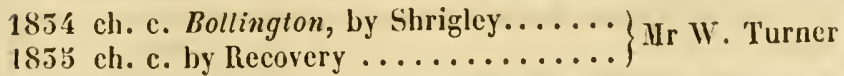

\section{NAIAD (sister to Merman)}

Bred by Lord Egrevost, in 1828, got by Whalebone, out of Mermaid, by Orville-Sir Solomon-Mliss Brim, by llightlyer.

1834 b. c. Nelson, by Lamplighter....... IIr Carter

1855 b. c. by Cain (foaled in France)...... Ld H. Seymour

\section{NANCY,}

Bred by Mr Gooptssox, in 1815, got by Dick Andrews, her dam, Spittire, by Beningbrough-Y. Sir Peter (son of Doge)-Engineer-Wilson's Arabian--Hutton's Spot.

1819 b. f. by Ashton...............

1820 b.c. by Whalcbone (put out his shoulder, Mr Walker and was killed)

1821 b. c. Longwaist, by ditto .......... Ir F. Craven

1822 b. c. by ditto (died a yearling) .......

1825 b. f. Shortwaist, by Interpreter ...... IIr Forth

1824 b. c. Straitzaist, by ditto (lamed in the $M r$ Walker paddock)

1826 br. c. Roundwaist, by Whalebone (sent MIr Nowell to liaron Biel in 1832)

1827 c. by Smolenslio (died the day after) foaling)

1829 br. c. Ilokee Pokee, by Muley....... MIr Giffard 1850 br. c. Muley Moloch, by ditto....... Ld Clereland 1851 br. f. Muliana, by ditto (put to the stud) Mr Biggs 1852 br. c. by Giraffe (s. to Germany in 1852)

1855 b. f. by Bustard (dead) ........... MIr Nowell

$1854 \mathrm{br}$. f. by IIuley (sent to Virginia)..... 


\section{6}

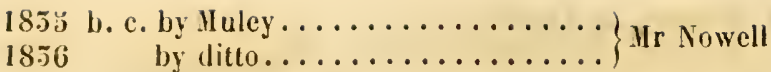

\section{NANINE,}

Bred by Lord Lowtmer, in 1825, got by Selim, her dam, Bizarre, by Peruvian, out of Violante.

1827 ch. c. by Middleton............ Mr Wilson

1828 missed to Tiresias.............. Ld Lowther

1899 ch. f. Nannette, by Partisan........ Mr Maberly

1830 b. c. Glaucus, by ditto ........... Gen. Grosvenor

1831 b. f. by ditto (s. to Baron Biel)...... Mr Goodwin

1852 missed to ditto.............. Ld Lowther

1853 b. c. The Athenian, by ditto....... Mr W. Chifney

1854 ch. c. by ditto............... Sir II. Wood

18.55 ch. f. by ditto .............. Ld Lowther

1836 by Rowton............ liis Majesty

\section{NANNETTE (sister to Glaucus)}

Bred by Lord Lowtiler, in 1829, got by Partisan, out of Nanine. 1854 b. c. Lupus, by Laurel............
1855 b. f. by Shakispeare............

\section{NELI GIVYNNE.}

Bred by , in 1826, got by Tramp, her dam by

Beningbrough, out of sister to Star, by Ilighflyer-Snap-

Riddle, by Matchem.

185 I b. c. (Limner) by Peter Lely........ MIr Legh

1853 br. c. Brookland, by Filho da Puta... Capt. Williamson

1834 br. f. by Velocipede ............ Sir R. W. Bulkeley

Missed to Battledore in 1852 and 35 ; sold to Mr Minor, of the United States, in 35 , covered by Rowton.

\section{NERISSA,}

Bred by Lord Sramrord, in 1819, got by Woful, her dam, Miss Hap, by Shuttle, out of sister to llaphazard, by Sir Peter.

1824 b. c. by $\Lambda$ stbury............. Mr Painter

1826 br. c. Barabbas, by Banker......... Mr Giffard

1827 br. c. Stirling, by ditto.......... Ir Painter

1829 b. f. Mignonette by ditto (s. to Germany $\begin{array}{r}\text { in } 1855) \\ \text { Mr Griffin }\end{array}$ 
1850 br. f. by Banker.............. MIr Peirson

1851 b. f. by Bay Malton............. IIr Boardman

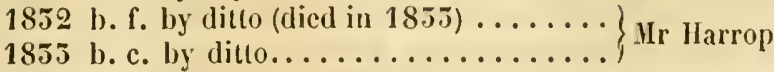

1853 b. c. by Iumphrey Clinker..........
1856 by The Saddler, or Tramp ....

Missed to Banker in 1823 and 28, not covered in 55.

\section{$\operatorname{NEV} \Lambda$,}

Bred by Mr Tinetres, in 1814, got by Cervantes, her dam, Mary,

by Sir Peter-Jiomed-Desdemona, by Marske.

1820 b. c. by Comus...............

1825 b. c. by Catlon ............... Sir W. Milner

1824 b. c. Liston, by Comus...........

1823 br. c. by Tramp (dicd a foal)........

1826 br. f. Clotilde, by ditto (died rising 5 )

1827 c. by Figaro (killed in foaling)....

1829 b. c. by Whisker ............ Mr Ridsdale

1832 b. c. by Tramp...............

1853 b. c. by ditto................

1853 b. c. by ditto, or St Nicholas .......

1856

by St Nicholas

Missed in 1821,$22 ; 28,50,51$, and 54 .

\section{NEWCASTLE MARE,}

Bred by Mr R. Spencer, in 1816, her dain, Vitula, by Alexander.

1827 b. f. by a half-bred liorse.........

1828 b. c. The Mammoth, by Elephant....

1829 b. c. The llippopotamus, by ditto (died ill 1852)

1850 b. c. Tom Brown, by ditto........ Mr E. Phillips

1852 br. f. The Bride, loy ditto ..............

1835 ch. c. by Anticipation...........

1854 b. c. by ditto................

1855 missed to ditto

\section{NINA,}

Bred by the Duke of Rutund, in 1816, got by Selim, her dam, Penny-trumpet, by Trumpator, out of Y. Camilla.

1821 ch. c. by Castrel (died young) ....... Ld Low ther

VOL. IT. 


\section{8}

182.5 ch. f. by Comus............. Ld Orford

1824 bl. f. (Clatter) by Clinker.........

182.5 b. f. Zoé, by Orville ............. Ld Lowther

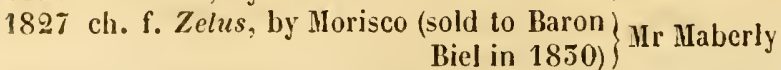

1828 b. c. Despatch, by Partisan.........

1829 b. c. Messenger, by ditto (died in 1853 )

1851 b. c. by ditto (shot 1 year old)..... L Ld Lowther

1853 c. by Chateau Margaux (dead) .....

1855 b. f. by Lapdog . . . . . . . . . . .

1856 by ditto................

In 1822 missed to Tiresias, 26 to Woful, 50, 52, and 54, to Partisan.

AIORÉ,

Bred by Mr Sierbroke, in 1812, got by Sir David, her dam by Buzzard, out of 'Tottcrella, by Dungannon-Marcella.

1818 b. c. by Waxy.............. D. of Grafton

1819 ch. c. (Weeper) by Juniper........ Ld Warwick

1820 ch. f. by partisan ..............

1821 dead foal, by ditto............

1822 missed to ditto.............. D. of Grafton

1825 ch. c. Paul Jones, by ditto.........

1824 ch. f. Chloris, by ditto ...........

1823 ch. c. by ditto...............

1826 ch. c. by Centaur ..............

1827 b. f. (Cricket) by Partisan..........

1828 b. f. Water Witch, by Whalebone (s. to Ld Warwick Virginia in 185\%)

1829 ch. c. by Sligo (died a foal)........

1850 b. c. Trepidation, by Centaur.......

1851 gr. c. lyy Stumps.............. Ld Monson

1852 b. c. Pelops, by Middleton .........

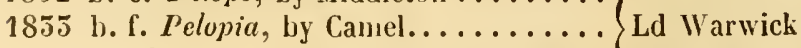

1854 ch. f. by The Colonel ...........

Died in 1854.

\section{NORNA (sister to Waverley)}

Bred by Lord Egremont, in 1818, got by Whalebone, out of Margaretta, by Sir Peter-sister to Cracker, by Highflyer. 1826 b. f. Canopy, by Blaclilock (put to the)

182 \% b. f. by Comus............... stud) Sir G. Heathcote 
1850 b. f. Carnation, by Blacklock.......

1831 b. c. Nisus, by Velocipede.......... Sir G. Heathcote

1853 br. c. Darfour, by Reveller.........

Missed in 1828, 29, 52, 54, and 53 , and sold to Baron de Maltzahn in February 1856 .

\section{NOVELTY,}

Bred by Mr WATt, in 1825, got by Blacklock, out of Washerwoman, sister to Nectar, by Walton-L'Huile de Venus.

$1828 \mathrm{ch}$. f. by Comus (died a foal)......... IIr J. Scott

1829 b. c. Arcadian, by ditto........... Mr G. Walker

1850 b. c. Phantasy, by Y. Phantom......

1831 b. f. by ditto (died a fortnight old).... NIr J. Scott

1832 ch. f. by Comus...............

1855 br. c. Bradicurdine, by Waverley....

1854 br. c. Fidget, hy Y. Phantom........ Mr Ellis

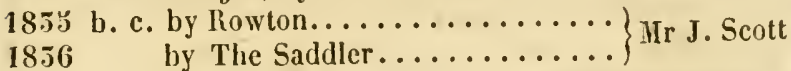

\section{NOVICE,}

Bred by Mr Harrisos, in 1895, got by Y. Filho da Puta, her dam by Walton, out of Two Shoes, by Asparagus-Mercury.

1851 b. f. by Lottery................ Mr Beardsworth

1832 b. c. by Mameluke.............. Mr Watson

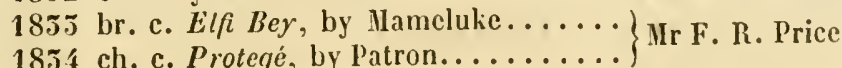

Not covered in 1834 or 33 .

\section{THE NUN,}

Bred by in 1825, got by Blacklock, her dam by Whisker-Orville-Otterington's dam.

$1829 \mathrm{ch} . \mathrm{c.}$ by Sir Oliver............ Ir Legh

1831 br. f. Miss Golborne, by Lottery......)

1832 b. f. by Battledore.................. Tr R. Turner

1855 b. f. by Langar ...............

Not covered in 1829 , barren in $\mathbf{5} 4$ and 53 .

\section{OCTAVIA,}

Bred by Mr Garforth, in 1817, got by Walton, out of Marcia, by Coriander-Faith, ly Pacolct-Atalanta. 
$1827 \mathrm{ch} . \mathrm{c}$. by Wisker (died a foal) ......

${ }^{*} 1828$ b. f. Faith, by ditto............

1829 dun f. by Lottery (died 2 days old)...

1852 ch. f. Ottavia, by Tramp......... MIr Ridsdale

1855 br. f. Meliora, by ditto........... Mr Ridsdale

1854 b. c. by ditto..............

1835 b. f. by St Nicholas............

1856 by Niulatto.

In 1850 missed to Lottery, 51 to Figaro.

${ }^{*}$ Faith died in September 1855, having had only one foal born alive, viz: 18.5. b.c. Vandenhoff, by Brutandorf Mr Ridsdale

\section{OCTAVIAN MARE,}

Bred by Mr T. Fergusor, in 1823, her dam, Young Mary, by

Mowbray, out of Mary, by Young Marske-Gentle Kitty.

1829 ch. c. by Whisker.............

$1850 \mathrm{ch}$. f. by ditto..............

1851 ch. f. Medea, by ditto...........

1832 br. c. by Waverley ................. D. of Leeds

1855 br. c. by ditto.................. of Leeds

1854 b. f. by ditto (dead)............

185 ๖. ๖. . Orcus, by Velocipede.........

1856 by Alteruter ..............

\section{OCTAVIANA,}

Bred by Mr. F. Lensis, in 1815, got by Octavian, her dam by

Shuttle, out of Zara, by Delpini-Flora, by King Fergus.

1820 b. c. by Sootlısayer............

$1821 \mathrm{ch}$. c. by Comus ..............

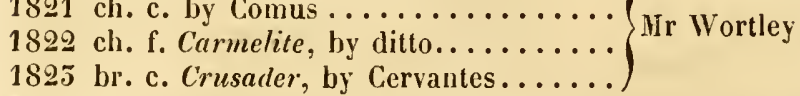

182.4 ch. c. Carthusian, by Comus.......

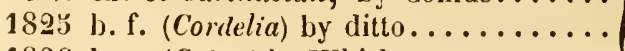

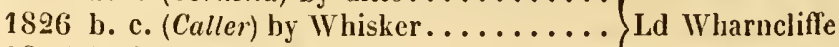

1827 b. f. by Cervantes.............

1828 b. c. by Whisker (died in 1850).....

1829 by ditto ..............

1851 cls. f. Constance, by Leviathan (s, to

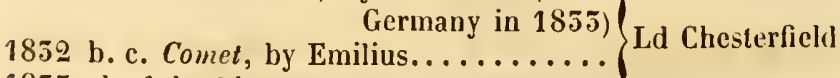


1854 ch. c. by Priam..............

$\left.\begin{array}{l}1855 \\ 1856 \\ \text { by Priam } \ldots \ldots \ldots \ldots \ldots \ldots \ldots \ldots\end{array}\right\}$ Ld Chesterfield

OCTINA,

Bred by Mr Gaunteete, in 18 25 , got by Octavius, out of Vishap, by Haphazard-Nercury-Dorimant.

1827 b. f. by Reveller..............

1829 b. c. Pompey, by Lapdog..........

1853 f. by Chateau Margaux (shot a foal) Mr Gauntlett

1856 by Sir Hercules............

Not covered in 1827 or 35 ; hunted in 50 and 51 .

\section{TIIE ODD TRICK,}

Bred by Lord Rovs, in 1819, got by Quiz, her dam, Grey IJuchess,

by Pot8o's, out of Duchess, by Ilerod-Gaudy, by Blank.

1826 gr. f. by Skim ............. Ld Egremont

1827 b. f. (Bustle) by Whalebone........ Ld Clinton

1828 b. c. Varlet, by ditto............ Ld Orford

1829 missed to ditto............. Ld Egremont

1850 b. f. Trickery, by ditto.................. Capt. G. Bulkeley

1832 ch. f. Bucephalia, by Emilius....... Ir Gardnor

1855 ch. c. by The Colonel ........... 1854 slipped foal ................ Bulkeley

183 b. f. by Tranby............. Gen. Grosvenor

$1856 \quad$ f. by Camel................ Mr Poyntz

\section{ODDITY,}

Bred by Lowtner, in 1828, got by Lapdog, her dam, Laundress, by Tiresias, out of Landscape, by Rubens.

1852 b. f. by Y. Juniper (dead).........

1853 b. f. by Partisan .............. Col. Lowther

1834 b. f. by Y. Juniper............

\section{OGRESS,}

Bred by Lord Egremoxt, in 1825, got by Octavius, out of Thalestris, by Alexander-Rival, by Sir Peter.

1826 b. c. (Y. Wanderer) by Wanderer (sold) to Mr Henty, and sent to the Swan Ld Egremont River) 
*1828 br. f. Herculea, by Whalebone..... Mr Bruhl 1829 bl. c. by ditto.................. +1852 b. f. Eleanor, by ditto (s. to Ireland) 1855 bl. c. liy Chateau Margaux........... Ld Egremont 1854 bl. f. by ditto.................

Missed to Whalebone in 1827,50 , and 51 , and sent to Hungary in October 1854, covered by Chateau Margaux.

* Henculea produced b. f. by Hindoo in 1854 , missed in 55 , and was covered by Dunsinaue that year.

† Eleanon produced, in 1855, Mr Hunter's b. c. Ennui, by Roller.

\section{OISEAU MIRE,}

Bred by Sir Tatton Sykes, in 1826, her dam, sister to Prime Minister, by Sancho-Miss Iornpipe Teazle, by Sir PeterHornpipe.

1855 b. c. Resurrection, by Y. Phantom... Mr Singleton 1854 barren $\ldots \ldots \ldots \ldots \ldots \ldots \ldots \ldots \ldots \ldots \ldots \ldots \ldots \ldots \ldots$ Sir T. Sylkes

\section{OISEAU MARE,}

Bred lyy Sir Tatrox Sykes, in 1826, her dam, Anna Maria, by Stamford-Stella, by Phœnomenon-Skypeeper, by Ilighllyer. 18.54 ch. f. by Comus...............
1853 ch. f. by ditto ............ Sir T. Sykes

\section{OLEANDER,}

Bred by , in 1815 , got by Sir David, her dam by Whiskey, out of Grey Dorimant, by Dorimant-Dizzy, by Blank.

1822 ch. f. Wings, by The Flyer........ Gen. Grosvenor

1823 straw c. by a Norman horse........ 1824 br. c. by Chance............... 1823 bl. f. by ditto (dead) ............ 1826 ch. c. by Blacklock (broke his leg)....

1827 b. c. by Woful................ Sir G. Heathcote

1829 bl. f. Damascene, by Reveller.

1850 b. c. Astracan, by Chateau Margaux..

1854 b. c. Trim, by Laurel.............

Missed to Blacklock in 1828 and 51 , to Velocipede in 32 , to The Colonel in 35, to Logic in 55. 


\section{OLIVE-LLAF (brown)}

Bred by Lord Grosvevor, in 182i, got ly Blucher, out of Olivebranch, by Sir Peter-Olivia, by Justice.

1828 b. f. by Arbutus ...............

* 1829 br. f. Ruth, lyy Borodino, or Peter Lely IIr R. IIudson 1830 gr. f. Vesta, by $\Lambda$ rbutus...........

1851 ch. c. by Comus................ Ir S. L. Fox

185 bl. f. Lady Jane, by St Nicholas..... II J. Briskham

1834 br. c. by ditto ................ Mr E. Peel

1855 b. c. Si Kevin, by ditto .......... Mr J. Briskham

1856 by ditto.

* Rorn produced b. c. by Humphrey Clinker in 1854 .

\section{OLYMPIL,}

Bred by Lord Sramrond, in 1813, got by Sir Oliver, her dam, Scotilla, by Anvil, out of Scota, by Eclipse-Herod, etc.

1821 b. f. Kite, by Bustard........... Ir Roberts

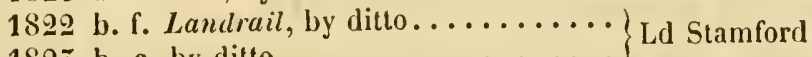

1825 b. c. by ditto................. $189 . \ldots \ldots$ Mr Alanson

1829 b. c. Cato, by Catton.............. Mr Wood

1832 b. c. Stockport, by Langar........ Mr Robinson

1855 ch. c. Elis, by ditto ........... Ir Greville

1854 ch. c. by ditto............... Ir Lumley Savile

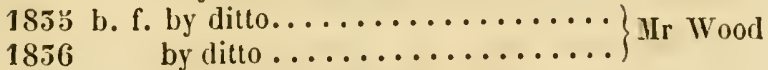

Barren in $1824,26,27,28,50$, and 51 .

OPAL'S produce of 1825 (Vol. III, p. 284; should stand thus :$\left.\begin{array}{r}1825 \text { b. f. Amethyst, by Master Henry, or } \\ \text { Blaclilock (s. to Germany) }\end{array}\right\}$ IIr Attwood

\section{ORPHELINE,}

Bred by Mr Nowelz, in 1827, got by Orville, her dam by Cato, out of Omphale, by Waxy-Pantina, by Buzzard.

1852 b. f. Goldfinch, by Filho da Puta..... Ir Grant

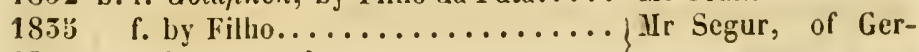
by Buzzard ............ many 
ORVILLE MARE,

Bred by the Duke of Quensserrry, in 1809, out of the Alexander mare, dam of Selim and Rubens.

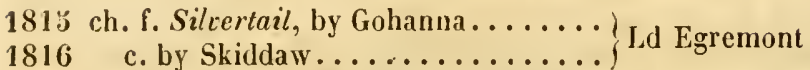

1817 bl. f. Eliza, by Whalebone......... Mr H. Edwards

1818 b. c. Centaur, by Canopus.........

1819 b. c. $W$ anton, by Frolic...........

1820 b. c. Fun, by ditto..............

1822 c. by Whalebone (died a foal) ..... Ld Egremont

1825 bl. c. Black Swan, by ditto ........

182 . b. c. (Rasselas) by Wanderer.......

1826 br. f. (Sessions) by ditto..........

1827 br. f. Refugee, by ditto (s. to the United) States in 185I) D. of Richmond

1828 b. f. by ditto (put to the stud)......

1829 b. c. (Sussex) by Skim . . . . . . . . .

1830 b. f. by ditto.............. Ld Egremont

1832 b. c. by ditto................

$1854 \mathrm{gr}$. f. by ditto (dead) ............

Barren in 1824, 31, 55, and 55, and shot in 1855 .

\section{ORVILLE MARE,}

Bred by , in , her dam by HambletonianShuttle-Overton-Beningbrough's dam.

1820 b. f. by Blacklock (dam of Lady) Mr Hickes

1821 b. f. Duckling, by Phantom........ Mr Bowles

182.3 br. c. Segar, by Waterloo..........

1823 c. by Flibbertigibbet..........

1826 br. f. by ditto................. JIr Messer

1828 br. f. by Smolensko..............

1829 b. f. Miss Ellon, by Flibbertigibbet. .)

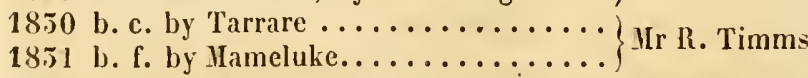

She missed in 1824, had no foal after 51 , and is dead.

N. B. T!ıe note to this mare, at p. 502 of the Third Volume, may be erased, the Erlitor havingascertained since its publication that he peligree is correct, and that it was not the IIambletonian mare, her dam. which Mr Kirby took to Russia in 1818. 


\section{ORVILLE MIRE,}

Bred by Mr Arnatw, in 1819, her dam, Epsom Lass, by Sir Peter, out of Mexina, by King Fergus-Iardella, by Y. Marske.

182.5 b. f. by Filho da Puta........... 1824 ch. c. Musliham, by Magistrate..... 1825 b. f. by ditto................... Wr Houldsworth

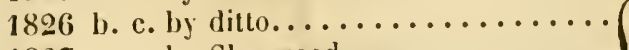
1827 1828 b. c. Bradley, by Filho da Puta..... $\left.\begin{array}{c}1829 \text { b. c. Minster, by Catton (s. to France } \\ \text { in 1854) }\end{array}\right\}$ Id Mountcharles

1850 b. c. by Buzzard...............

1851 b. c. by Waterloo (died 5 weeks old) . Ld Conyngham

1852

1855

1834

1853

1856 by Filho da Puta...........

b. c. Slane, by Royal Oak............

c. by Catton (died a foal)........ Col. Peel missed to Cain..................

by Camel...............

\section{ORVILLE MARE (Mermaid)}

Bred by IIr Alurx, in 1811, her dam by Sir Solomon, out of Miss Brim, by Hightlyer-Brim, by Squirrel.

\section{8}

1819 gr. c. Midas, by Young Gohanna..... Ld Egremont

\section{1}

1822 gr. c. Acorn, by Skin
1824 b. f. Gaiety, by Frolic

1824 b. f. Gaiety, by Frolic ............ Mr Bruhl

1826 br. c. Merman, by Whalebone...... Ir Payne

1828 b. f. Naiall. by ditto........... Ld Orford

1829 b. c. Dolphin, by ditto......... Ld lowther

1850 b. c. The Sea, by ditto.......... Mr Day

1855 slipped foal, by Nimrod............

1855 b. f. by Chateau Margaux, or Gaber- Junzic Ld Egremont

1856 by Gaberlunzic ...........

Barren in 1825 , had a dead foal in 25, missed in $27,51,52$, and $\mathbf{5 . 4}$.

\section{ORVHLLE MARE,}

Bred by Lord Stawelt, in 1815, her dam, Golden-leg's dam, by Buzzard, out of llornpipe, by Trumpator-Iuna, by Herod. 
1819 bl. f. Gipsy, by Juniper (s. to Russia) Mr J. Smith

1820 bl. f. Miss Fanny, by Walton.......

1821 b. f. Lesinka (aft. Miss Lydlia) by ditto

1822 br. f. by $\Lambda$ rdrossan.............

$1825 \mathrm{bl}$. c. by Walton..............

1824 br. f. (II iss Emma) by ditto .......

$182 y$ br. c. by Jack Spigot............

1826 br. c. Penhill, by ditto............

1827 b. f. Lady Emmeline, by Y. Phantom.

1828 b. f. by ditto..................... Hr T. O. Powlett

1850 b. f. by Whisker................... (s. to France in 1855)

1855 b. f. Miss Julia, by Partisan........

1854 b. f. by Priam................

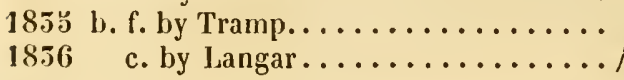

\section{ORVILLE MARE,}

Bred by Gen. Leveson Gower, in 181\%, her dam, Mirth, by Trumpator, out of Hoity-toity, by Highflyer-Goldfinder.

1824 bl. c. by Buffalo................ Mr J. Day

1823 bl. c. by ditto................... Mr Mr Shard

1828 b. c. IIussein, by ditto...........

*1829 b. f. Milanese, by Herlin.........

1850 br. c. Barbary, by Blacklock ....... Sir G. Heathcote

1851 br. c. by Velocipede.............

*1 855 br. f. by Brutandorf............

Missed in 1827 and 54 ; not covered in 51.

* Milanese produced br. f. by Lottery in 1854, was barren in 55 , and sold to Baron Maltzahn in 1855, with a filly foal by Reveller, as was also the Brutandorl' filly.

\section{ORVILLE MARE,}

Bred by Mr Wizliason, in 1822, her dam, sisfer to Tekeli, by Waxy-Highflyer-Squirrel-Sophia, lyy Blank.

1826 b. c. by Phantom.............. Sir II. Goodricke

1827 b. c. by Tramp ................

1828 b. c. by Sir Walton (s. abroad)....... MIr Wood

1829 b. f. by Alexander. 
1850 b. c. Charley Boy, by Tarragon ...... Mr Wood

1851 br. f. Eleanor, by Comus .......... Mr Clegg

1852 br. f. by Borodino .............

1855 br. c. Borough Reeve, by ditto......

1854 bl. f. by Jerry ................. Hr II ood

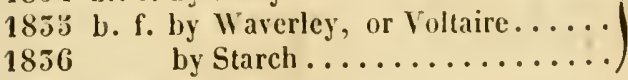

ORVILLE MARE,

Bred by Mr Nowetx, in 1827, her dam, Rosanne, by Jick

Andrews, out of Rosette, by Beningbrough-Rosamond, by

Tandem.

1855 b. c. by Bustard............. Mr Beardsworth

1854 b. c. Doncaster, by ditto......... IIr E. V. Fox

1855 b. c. by Longwaist............. Jir Nowell

1836

\section{ORVILLE MARE,}

Bred by Mr Dobngtos, in 1820 , her dam by Walton, out of Spindle.

1829 b. f. by Reveller (born without eyes)..

$1851 \mathrm{ch}$. f. by Moses, or Nigel.......... Mr Wigram

1836

by Nigel.

Missed in 1826, 28, and 50, had dead twins in 27 and 54 , missed to Camel in 32 , and was not covered in 34 .

\section{ORVILLE MARE,}

Bred by Mr St PAce, in 1826, her dam by Wizard, out of Lisette, by Hambletonian-Constantia, by Walnut-Contessina.

1851 b. f. by Corinthian (died a foal) .....

1832 b. f. (Gipsy) by Tramp............

1855 br. c. by Lottery ............... IIr C. II. St I'aul

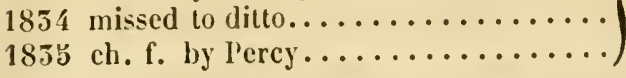

\section{ORVILLE MARE,}

Bred by Mr Ifurter, in 1822, her dam, Sprightly, by Whiskey, out of Romance, by Gouty-Diomed.

1855 b. f. by ditto................ 


\section{8}

ORTIJLE MARE,

Bred by Mr Nower.L, in 1827, her dam, Lacerta, by Zodiac, out of Jerboa, by Gohanna-Camilla, by Trentham.

1851 b. c. Armadillo, by St Patrick......

1852 bl. f. Flame, by Lamplighter .......

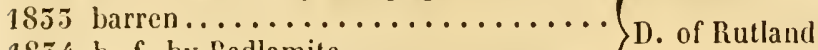

1854 b. f. by Redlamite............

185 . b. с. by Buzzard.............

1856 by Taurus..............

\section{ORVILLE OR WALTON MARE,}

Bred by Mr Nowelt, in 1826, her dam, Mufti's dam, by Dick Andrews, out of Donna Clara, by Cesario-Nimble, by Florizel.

1852 b. f. by Longwaist............. Mr Etwall

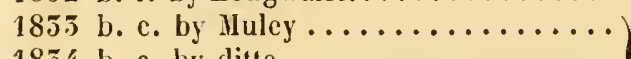

1834 b. с. by $\operatorname{ditto} \ldots \ldots \ldots \ldots \ldots \ldots \ldots \ldots$ Ir Nowell

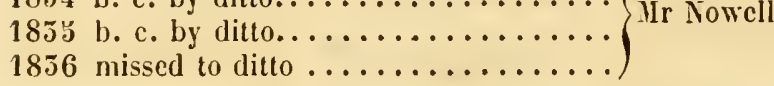

\section{ORVILLIA,}

Bred by Mr March Phillipps, in 1819, got by Orville, her dam, Glauvina, by Moorcock-Matchem-Snap-Cade-Partner.

1823 b. f. Evelina, by Muley (dicd, lcaving no produce)

1825 b. c. by Duplicatc.............

*1827 br. f. by Vandyke Junior.......... Mr C. II. Phillipps

+1828 br. f. by Sir Gilbert..........

1850 b. c. by ditto ..............

ऽ 1851 br. f. Belzona. by Belzoni.......

1855 b. c. by longwaist............

Barren in 1829,54 , and 55 , and not covered in 51 .

- This mare produced b. 'c.by Phantom in 185̄, and is dead. siud.

+ This mare produced br.f. by Belzoni in 1852, which is in Mr Phillipps's

5 Berzova is in the stud, and produced a c. by Priam in 1850 .

\section{OSCAR MARE,}

Bred by Col. Wrisox, in 1829, her dam, Camarine's dam, by

Rubens, out of Tippitywitchet, by Waxy - Hare, by Sweetbriar. 
1854 ch. c. by Lamplighter ...........

1855 b. c. by Sir Benjamin............ His Vajesty

1836 by 'The Colonel. .

\section{OSTRICH,}

Bred by Lord Stanforn, in 1820, got by the Castrel Bustard, out of Olivelta, by Sir Oliver-Scotina, by Delpini.

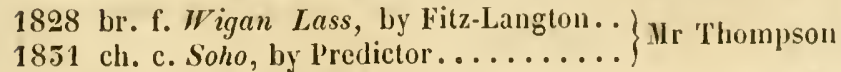

1852 ch. c. Iron Founder, by ditto....... Sir J. Gerard

1855 ch. c. by ditto (dead) ............ Mr Thompson

In 1829 missed to Fitz-Langton, 50 to Predictor, and killed by accident in 54 , in foal to him.

\section{O'TIS,}

Bred by Lord Egremont, in 1890, got by Bustard (son of Buzzard) out of Gayliurst's dam, by Election-sister to Sliysweeper, by llightlyer.

1825 b. f. Mary Anne, by Irolic ........ Mr Jackson

1826 b. c. Allerdale (aft. Perseverance) by Mr Richards

1828 b. f. by Frolic, or Enville.........

1829 b. f. by Frolic (dead) .............. IIr Wood

185 I b. f. by Neptune (son of Tiresias)......

1852 b. f. Rosina, by Frolic............ Sir J. Boswell

1853 b. c. Eolus, by Corinthian......... Ld Eglinton

$1854 \mathrm{ch}$. f. by ditto................

1853 b. c. by Liverpool.............. MIr W ood

1856

by The Earl..............

\section{PALAIS ROYAL,}

Bred at IIampton Court in 1820, got by Blucher, her dam by Election, out of Rubens' dam, by Alexander-llightllyer.

1829 b. c. (Young Sultan) by Sultan......)

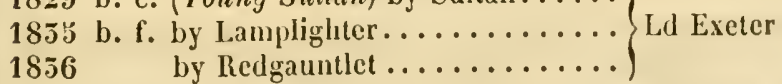

She was used as a hack from 1829 to 34 .

\section{PALATINE (sister to Arachne)}

Bred by Mr Hoch.dswortil, in 1820, got by Filho da Puta, her dan, Treasure, by Camillus-IIyacinthus-Flora. vol. IV. 
1850 br. l. Litlle Zan, by Reveller....... Ir Paterson 1855 b. f. Isa, by The Colonel.......... Sir J. D. King 185.4 br. f. Grapeskin, by Chateau Margaux Mr A. F. Berkeley

Missed in 1829, 51,52 , and 55 .

P'ALIIYRA, by Sorcerer (Vol. III, p. 294) was shot in 1832; her ch. c. by Bobadil, foaled in 1851, was called Porto Bello, and was sent to Russia in May 185 .

\section{PAN MARE,}

Bred by Mr Kellermans, in 1821, out of Vale Royal.

1850 br. f. by Sir Gilbert...........

1831 b. f. by Cannon Ball............ Hr Fryatt

$1855 \mathrm{ch}$. f. by Hawker .............

1856

$$
\text { by Pickpocket............... }
$$

\section{PANTILEA,}

Bred by MI WATt, in 1821, got by Comus, or Blacklock, her dam, Manuella, by Dick Andrews - Mandane.

1827 br. c. Kassan, by Whisker ....... Ld Queensberry

1828 b. c. Panopes, by Tramp..........

1829 b. c. by Middleton............... Mr Maberly

1830 dead foal..................

1851 b. f. Jane, by Iliddleton (put to the stud) Mr R. Wilson

1852 bl. c. Cyrus, by Camel............ IIr E. Peel

185.5 br. c. Solicitor, by Wrangler........ Mr W. Edwards

1854 missed to Albany.............. Mr R. Wilson

18.5 : b. f. by Sir Benjamin ........... Mr E. Peel

\section{PARADIGI,}

Bred by Mr Payse, in 1819, got by Partisan, out of Bizarre, by l'eruvian - Violante, by John Bull.

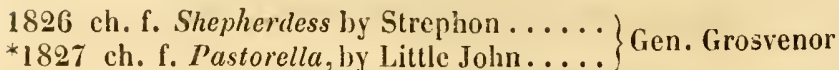

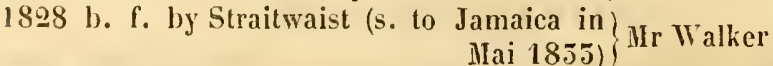

" l'astorelta had a coll by Sarpedon in 1854, and wis not covered that year. 


\section{1}

1829 ch. f. Stately, by Straitwaist (put to the )

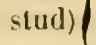

1850 b. c. Superb, by ditto........... Sir G. Heathcote

18.51 b. c. by Figaro ..............

1855 b. f. by Sir Hercules............

In 1852 missed to Chateau Margaux, 55 to Reveller, 34 to Filho da Puta.

\section{PARTIIENESSA,}

Bred by Lord Fitzwiluan, in 1821, got by Cervantes, her dam, Marianne, by Sorcerer, out of 'Thomasina.

1829 b. c. by Ilumphrey Clinker.........

1850 b. f. by Sandbeck..............

1851 br. f. by Chateau Wargaux, or Sand- Ld Fitzwilliam beck (s.to Germany in $185 \%$, covered by Gaberlunzic)...............

1853 b. c. Mr IV ags, by Langar......... Col. Peel

18.54 b. c. by Langar............... J.d Fitzwillian

Missed to Langar in 1852 and 56 , to Mulatto in 55.

\section{PARTISAN MARE,}

Bred by Col. Wrusor, in 1820, her dam, Spotless's dim. by Trumpator - Ilighlyer - Otheothëa.

1829 c. by Merlin ............... Mr Wilson

1850 b. c. Epicure, by Chateau Margaux... Mr G. Walker

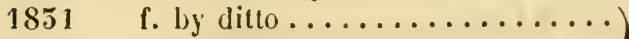

1832 by dilto $\ldots \ldots \ldots \ldots \ldots \ldots \ldots$ Mr Wilson

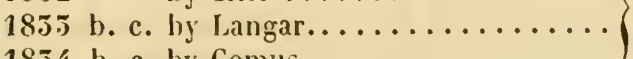

1854 b. c. by Comus...............

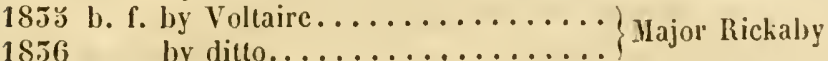

PURTISAN HARE,

Bred by the Duke of Grifros, in 1821, her dam. Pawn, sister 10

Penelope, by Trumpator-Prunella, by llighflyer-Promise.

1825 b. f. by Reveller. . . . . . . . . . . Sir J. Beresford

$18: 6$ c. by llagistrate (died a foal).....

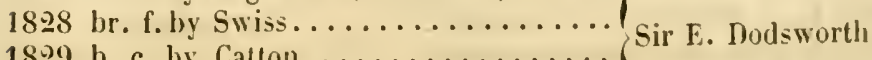

1829 b. c. by Catton..............

18.31 b. c. by Chateau IIargaux......... 
1855 ch. f. by Comus.............. IIis Majesty 1854 ch.f. Epaulette, by The Colonel..... Mr Greville

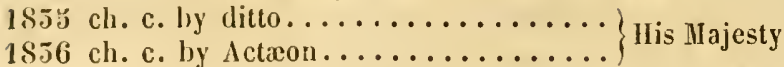

Not Covered in 1826, missed in 50 and 52.

\section{PARTISAN MARE,}

Bred by , in 1822, her dam, sister to Troubadour, by Dick Andrews, out of Donna Clara.

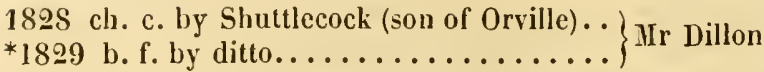

* Thismare is Mr Moon's. She produced in $1855 \mathrm{~b}$. f.by Gaberlunzie, and was covered by him in 55 , having missed in 54 and 55 .

\section{PARTISAN MARE,}

Bred by Lord Clarendon, in 1824, her dam, Donna Clara, by Cesario, out of Nimble, by Florizel-Rantipole, by BlankJoan, by Regulus.

1850 b. f. by Partisan...............

1851 ch. c. by Middleton (died a foal)......

1852 b. c. by Lamplighter.............

1855 b. f. by Chateau Margaux.......... Ld Lowther

1854 b. f. by Lapdog. . . . . . . . . . . .

1855 b. f. by leveller..............

1856

by $\operatorname{Logic} . \ldots \ldots \ldots \ldots \ldots \ldots$

\section{PARTISAN MARE (sister to Godolphin)}

Bred by Lord G. H. Cavendisu, in 1825, her dam, Ridicule, by Shuttle, out of sister to Oatlands, by Dungannon.

1828 br. f. by Nimrod (son of Whalebone). . Mr King 1829 br. c. by Whalebone........... Ld Chesterfield

1851 b. f. by ditto (s. to Germany)........ Mr King

1854 br. f. by Chateau Margaux......... Mr Mangles Missed in 1850,52 , and 55 .

\section{PARTISAN MARE (sister to Godolphin)}

Bred by Lord G. H. Cavendish, in 1824, as above.

1855 b. c. Makianna, by Belzoni ........ L Ld Jichfield

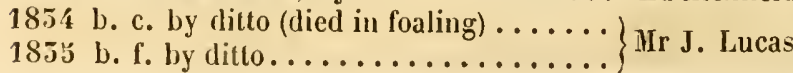




\section{3}

\section{P'ARTISAN IIARE.}

Bred by Mr Wrxnex, in 1824, out of Silvertail, by Gohanna.

1829 c. by Centanr ................... Mr Wilson

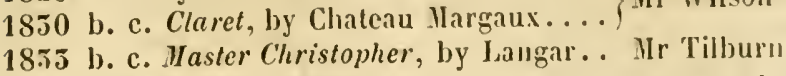

Missed in 1951 and 52, and suld to go abroad.

\section{PARTISAN MARE,}

Bred by IIr Batsos, in 1824, her dam, Jessy, by Totteridge, ont of Cracker, by llighllyer.

1829 b. c. Peter Pindar, by Middleton (s. to

Germany Oct. 1852)

1851 ch. f. by Criclieter ............

185 . bl. f. by Velocipede............ Mr Osbaldeslon

1855 b. c. Ebberston, by ditto.........

18.54 b. f. by ditto ...............

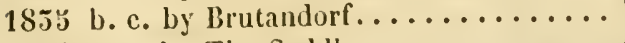

1836 by The Saddler............

Slipped twins by Emilius in 1828, missed to Middleton in 50.

\section{PARTISAN MIRE (sister to Contriver)}

Bred by Lord Lowtuer, in 1829 , her dam, sister to Schente, by Tiresias-Laphazard-Precipitate.

1955 ch. c. by Aaron................. Ir Forth

1854 barren..................................... Mr R. Watlins, jun.

$1836 \mathrm{ch}$. f. by IIokee Pokee...........

PASITIIEA,

Bred by Mr TAYLor, in 1825, got by Rubens, out of Jamia. by

Gohanna-Certhia, by Woodpecker.

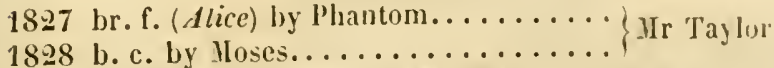

$1829 \mathrm{ch}$. f. Miss Badsley, by Middleton (put) Mr Digrs

1851 br. f. Myrina, by Whalebone....... . . Mr Taylor
1852 c. by Gaberlunzic (died a foal) ....

Not covered in 1829 : shot in May 1852 , having brolien her leg.

\section{P.STA,}

Bred by his Royal Higlıness the Duke of Jokk, in 1819, got by Sejmour, or Whalebone, out of Sagana, by Sorcerer.

20. 


\section{4}

1825 b. c. Crumpet (aft. Kean) by Rasping.

ISะ6 b.. โ. by Anacreou............

1828 1. с. by (irey Comus............

1829 bl. c. Pilton, by Grey Mliddleham....

1850 dead colt, by Reveller........... MLr W. Ley

1832 b. f. Medea, by Mancluke .........

185.4 br. c. loy Camcl...............

1858 b. c. by Elephant.............

18.56 by llarry............

Not covered in $1825,24,26$, or 30 ; barren in 35.

\section{PAS'TL (sister to Langar),}

Bred by Lord Lowtuek, in 1825, got by Selim, her dam by Walton, out of Sorcerer's dam, by llomed.

1828 ch. f. Flourish, by Partisau........

1829 ch. c. by ditto (dead). . . . . . . . .

$1850 \mathrm{ch}$. c. Prompter, by ditto ............. Ld Low ther

1852 clı. c. by Emilius (dead) ................ Ld Lowther

1855 clı. f. by l'artisan (s. to Spa)........

$183:$ dead c. by ditto...............

1856 ch. c. by lhowton ............ Mr Tattersal

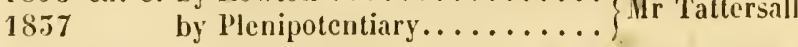

Missed to Partisan in 1851 and 54.

\section{PASTILLE,}

Bred by the Duke of Graftos, in 1819, got by Rubens, her dam, Parasol, by Potso's, out of Prunella.

1827 ch. c. by Emilius (died young) ......

1828 b. c. Eneas, by ditto (died at 4 yrs old)

1829 b. c. Ellipus, by ditto ............ D. of Grafton

1850 b. c. Egyptus, by Centaur (s.to France in 1S54)

1852 b. c. Egon, by Emilius ..........

185.5 b. f. Esmeralda, by Zinganee....... Mr W. Edwards

18.5 . slipped loal, by Emilius..........

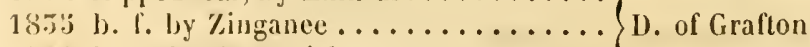

18.6 b. c. lyy st Patrick ............

Missed to Emilius in 1826 , to St Patrick in 1851.

\section{PAST1HE,}

Brerl hy the 1). of Rutuave, in 1822, got by Partisan, her dam, (Quadrille, by Selin-Canary-bird, by Whiskey, or Sorcerer. 
$18 \pi 1$ c. by Emilius (dicd young) $\ldots \ldots \ldots$ )

1859 b. c. by Whisker ............. Sir II. Wood

$185 j$ ch. c. Tournament, by Cctus.......

1856 by Cetus, or Rowtou..........

Barren in 185.1 and 53.

\section{PASTORA,}

Bred by IIr Sileplend, in 1817, got by Sir Oliver, her dam by Trumplator, out of Spinster, by Coriander-Dowager, by Jighllyer.

1852 b. ᄃ. by Canteen ............. D. of Buccleugh

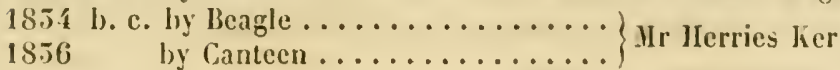

\section{PATIENCE,}

Bred by , in 1816, got by fyldener, her dam, fing Charles's dam, by Symmetry-Ilighlyer-Chrysolite.

1824 b. с. Job, by Spectre............ Ir Ormslyy Gore

1823 b. f. by ditto................... Ir Ijodenham

1827 b. c. Jasper, by ditto . . . . . . .

1828 b. с. The Judge, by The Duke.......

$18 j$ l ch. f. by Langar.............

1852 h. f. by The Juke.............. Mr Ormsby Gore

1854 ch. f. hy Langar..............

$18.55 \mathrm{ch}$. c. by The I)uke...........

1856 by Otdport.............

Had wins in 1825 which died; barren in 1829,50 , and 55.

\section{P.IT'TY PRIMROSE,}

Bred by Loril Fitzwiluay, in 1850, got by Confelerate, her dam, Sybil, by Interpreter, out of Galatea, by Amadis.

18.54 br. c. by Mr Best's Tinker.........

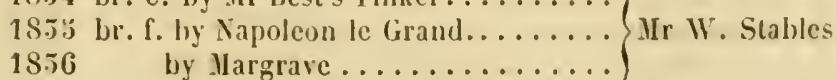

by Margrave.............

\section{P'IULIXE,}

Bred by

, in 1826, got by Hoses, her dam, (Yuadrille, by Selim - Cauary-bird, ctc. 
1852 b. f. Guevion, by Tramp .........

1854 ch. c. Glider, by Velocipede........ Mr Walker

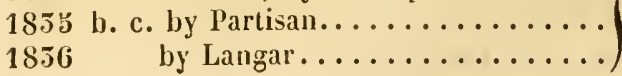

PAWN, sister to Penelope (Vol. III, p. 500) had only one foal since the last Stud-Book (viz. : 1855, ch. f. by Saracen) and she was shot in 1834 .

\section{PAWN JUNIOR,}

Bred by the Duke of Graftor, in 1817, got by Waxy, her dam, Pawn, by Trumpator, out of Prunella.

1825 b. c. Dervise, by Merlin ..........

1824 br. c. Deemon, by Amadis.........

1823 br. c. Druid, by Merlin (sent to India in 1829)

1826 b. c. Deacon, by Centaur........ D. of Gra fton

1829 b. f. Destiny, by ditto...........

1830 b. c. Divan, by Sultan ..........

*1831 b. f. Dublin, by St Patrick........

1852 b. f. by Sultan ..............

1855 br. f. by Saracen.............

1854 b. c. by Sultan.............. Ld Exeter

1835 b. c. by Taurus............... . . . . of Grafton

Missed in Centaur in 1827, slipped foal in 28.

* Produced, in $18 j 6$, ch. c. by Langar, her first foal.

\section{PAYNATOR MARE,}

Bred by Lord Cancisce, in 1809 , her dam, sister to Primrose, by Beningbrough, out of the Drone mare, sister to Constitution.

1816 b. c. Ganthorpe, by Henderskelf..... Major Bower

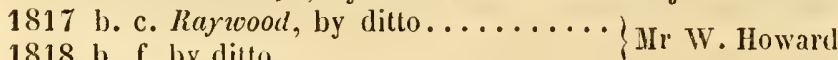

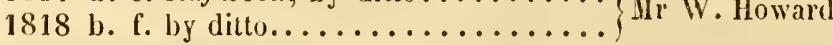

1820 br. c. by ditto ..............

1821 b. c. by ditto ................ L Larlisle

1822

by $\operatorname{ditto} \ldots \ldots \ldots \ldots \ldots \ldots$

1826 br. f. (Electra) by Abjer.......... Mr Martindale

1827 b. c. Kangaroo, by Whisker........ Ir Skipsey

1850 b. f. Clara Mowbray, by his grey Ara- Mr Attwood
bian (put to the stud) 
$\left.\begin{array}{l}1832 \text { b. c. Adrian, by his chesnut Arabian. } \\ 1835 \text { b. c. Valentinian, by Argantes...... }\end{array}\right\}$ IIr Attwood

No living produce in 1828,29 , or 51 ; barren in 54 and 53.

THE PAYNATOR MARE, out of sister to Zodiac (Vol. III, p. 503), was destroyed in the Autumn of 1851 ; her b. c. by Catton, in 1825, was called Scarbrough, and covered in Scotland.

\section{PEA BLOSSOII,}

Bred by , in 1806, got by Don Quixote, her dam by Pipator, out of a sister to Snow, by Slope-Florizel-Stride's dam.

1810 ch. f. Sweet-pea, by Selim......... Major Wilson 1811 ch. c. Mustapha, by ditto......... D. of Rutland 1812 b. f. by Walton...............

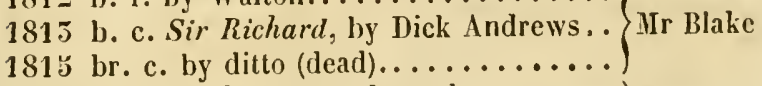

1816 b. f. Dahlia, by Haphazard........ MIr Farrall

1818 b. f. by ditto...............

1819 b. c. by ditto................

1820 ch. c. by Rubens (broke his leg and

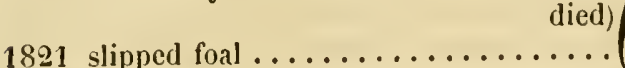

1829 by Orville............... Mr IIunter

1825 b. f. Marrowfat, by ditto..........

1824 c. by Gustavus..............

1825 f. Blossom, by ditto............

1826

\section{PEGGY,}

Bred by

, in 1822, got by Bourbon, out of IIasquerade, by Buzzard.

1826 b. c. by Blacklock.............. Jur J. Croft

1829

1851

1854

1853

1856

f. by Jock (died young)......... Mir J. Croft

f. by Catton, or Langar......... Ld Scarbrough

b. f. by Calton.................

gr. f. by Gustavus................. II Ilunter

Hissed to Catton in 1850 and 51. 
PENDULUII MARE,

Bred by Mr Weatueruex, in 1826, her dam by Shuttle-Drone -Y. Marske-Gentle Kitty, by Silvio.

18.50 b. f. Miss Percy, by Nigel (s. to Hun-

1852 b. f. by ditto (put to the stud)...... gary in 1854) NIr Kinder

1854 b.c. The Bothschafter, by Prime Hinister

1853 b. c. Perpetual Motion, by Sir Hercules $\}$ Mr Weatherley

\section{PENURY (sister to Pelican)}

Bred by Lord Sugo, in 1822, got by Oiseau, her dam, Miss Aide, by Sir Peter, out of sister to Tickle Toby, by Alfred.

1831 b. f. Peg-leg, by Edmund..........)

1852 b. f. by Cain................ Gen. Yates

1834 ch. f. by Bolivar (Blacklock) ........

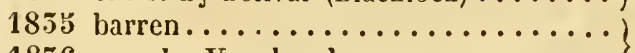

1856 by Vagabond............

\section{PERA (sister to Galata)}

Bred by I.ord Exeter, in 1826, got by Sultan, her dam, Advance, by Pioneer-Buzzard-sister to Champion, by Pot 80 's.

18.32 b.c. Ibrahim, by Emilius (s. to Germany)

185.5 b. c. Menas, by $\operatorname{ditto...........~}$

Hissed to Emilius in 1854, to Lapdog in $5 \breve{3}$, and sent to Virginia in 55 , covered by Defence.

PERI,

Bred by Lord Egremoxt, in 1822, got by Wanderer, her dam, Thalestris, by Alexander, out of Rival, by Sir Peter.

1826 bl. c. Sir IIercules, by Whalebone...

1829 b. f. Lady Racket, by Starch........

1830 br. f. Fashion, by ditto (s. to Germany Ld Langford

in 1855$)$

1831 br. f. Summer-hill by Whalebone....

18.52 cl. f. Crotchet, by Roller (put to the stud) Mr T. O. Powlett 
1853 b. c. by Starch (s. to Virginia). . . . . Mr Corbin

$1853 \mathrm{ch}$. f. by The Colonel........... Ilis Majesty

Missed in 1827 and 28 - to Irish Drone in 1834, to The Colonel in 1856. - Sir llercules was bred by IIr Jang, who sold the inare to Lord Langford in 1828.

\section{PERUVIANA,}

Bred by Sir T. Mostri, in 1812, got by Peruvian, her daun by Y. Woodpecker-Trentham-1)ecember.

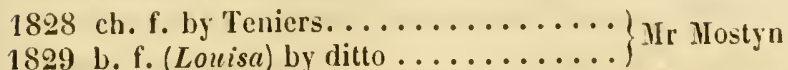

1850 ch. f. Georgiana, by ditto.......... Mr Scarisbrick

1851 b. c. Tom Jones, by ditto ......... Sir R. W. Bulkeley

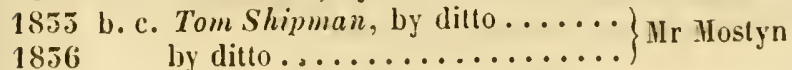

Missed to Teniers in 1852, 54, and 55.

PET,

Bred by Mr liawussox, in 1826, got by Gainsborough, her dam by Topsy 'Turry, out of Agnes, by Shuttle-Highflyer.

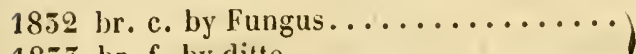

1853 br. f. by ditto .............. u ur Rawlinson

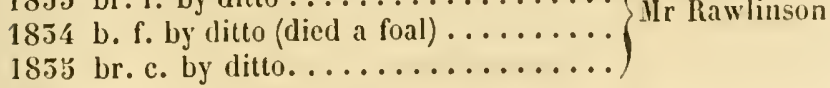

\section{PETER LELY MARE,}

Bred by Mr Best, in 1826, out of Merlin's dam. by DelpiniTipple Cider, by King Fergus.

1850 b. c. by Y. Phantom ............

1851 b. c. by Blacklock ..............

1852 b. c. by Tinker............... Mr Best

1856 b. f. by Voltaire ..............

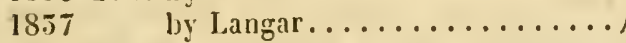

Barren in 1853, 54, and 53

\section{PETER LELI MARE,}

Bred by IJ. Garportu, in 1826, her dam (Sharpset': (lam) by Comus, out of Marciana, by Stamford. 


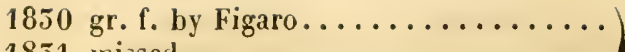

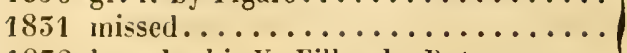

1852 b. c. by his Y. Filho da Puta ....... Mr Garforth

1855 missed to ditto $\ldots \ldots \ldots \ldots \ldots \ldots \ldots$

1854 gr. f. by ditto ...............

1853 gr. f. by Brutandorf ........... Mr Horsley

1856 by St Nicholas.................... Hr Horsley

\section{PETUARIA (Young)}

Bred by , in 1818, got by Rainbow, her dam, Petuaria, by Orville, out of Mandane, by Pot8o's-Y. Camilla.

1824 b. c. IIarvey, by Dandy ........... Mr Hunter, Dublin

1826 b. c. Smutta, by Oiseau, or Blacklock Mr Ridsdale

1827 ro. c. Leopard, by Theodore........ IIr Petre

1829 b. f. Dorabella, by Whisker (put to the)

1850 b. c. Cock-a-hoop, by Comus....... stud)

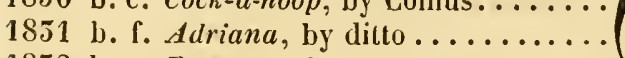

1852 b. с. Bamfylde, by Tramp ......... Hr Ridsdale

1855 br. c. by ditto, or Comus.........

1854 slipped a f. by Tramp...........

1855 b. f. by ditto ...............

1836 by Mulatto, or Starch........

Missed to Dandy in 1825, in 28 to Tramp.

\section{PETULANCE,}

Bred by Lord Orford, in 1829, got by Partisan, out of Stays, by Whalebone-Bobtail-Catherine, by Woodpecker.

1854 c. by Perchance, or Grand Duke...)

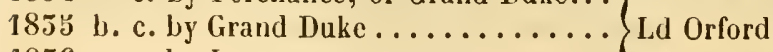

1856 by Jangar.............

\section{I'EWET,}

Bred by Mr DAx, in 1820, got by Bustard (son of Castrel) her dain, Calendulæ, by Camerton, out of Snowdrop, by Highland Fling.

1828 c. by Rubens (died the day of foaling)

1829 ch. f. by Middleton ............ Mr Day

- This Middleton mare produced in $185 \bar{j}$ Mr Mott's b. c.by Memnon, and has since been sent to Germany. 
1850 br. l. by Luzborough............

1851 b. c. by Godolphin, or liurgundy ....

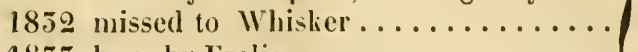

1855 b. c. by Frolic ................. J Mr Day

1854 br. c. by Ninırod (son of 'T'opssy T'urvy)

185 ' ch. f. by ditto ..............

1856 b. c. by Burgundy............

1837 by Saracen $\ldots \ldots \ldots \ldots \ldots \ldots$

PHANTOM, by IIambletonian (Tol. III, p. 507). Her two last produce should stand thus :-

$\left.\begin{array}{r}1828 \text { br. f. (The Witch) by Whalebone (s. to } \\ \text { Baron Biel in 185: } 3 \text {, covered by Taurus) }\end{array}\right\}$ Exeter

1850 b. c. Cowdray, by Sultan ......... Ld Tavistock

\section{PHANTOM MARE,}

Iired at Ilampton-Court, in 1815, her dam, sister to Election, by

Gohanna, out of Chesuut Skim, by Woodpecker. - Herod.

$\left.\begin{array}{r}1825 \text { b. c. (Sir John) by Little Johu (sent to } \\ \text { Swan River) }\end{array}\right\}$ Ld Egremont

1826 b. c. Frederick, by ditto . . . . . .

1827 b. f. Frederica, by ditto..........

1828 b. f. by Moses................. Ir Gratwicke

1850 b.f. The Margravine, by Little John. .

1852 b. c. by Emilius..............

1856 by Ilokee Pokee...........

Missed to Moses in 1829, to Little Joln in $\mathbf{5 1}$, to Helenus in $\mathbf{5 5}$,

barren also in 54, and slipped f. foal by The Colonel in 35.

\section{PHAN'TOM MARE,}

Bred by Mr Hallet, in 1S16, her dam, by Orerton, out of Gratitude's dam, by Walnut-Ruler-Piracantha, by Matchem.

1822 b. c. by X Y Z..............

1825 1). c. Billy Watson (aft. IIartlepool) by

1824 b. f. by Catton (s. to Van Dienan's

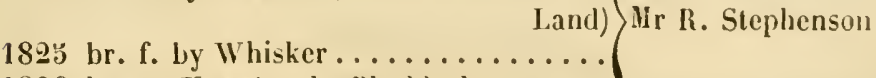

1826 br. c. Vollaire, by Blacklock.......

1827 br. f. Fisher Lass, by Osmond . . . . . .

1829 b. f. Variella, by Blacklock .........

Yol. IV. 
"1851 b. f. by Blacklock ............

1852 b. с. by Velocipede (dead)..........

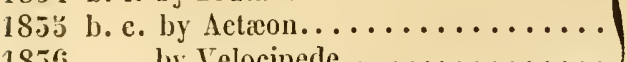

1856 by Velocipede.............

Missed in 1828 and 30.

* This mare is in Mr Cookes's stud. She had a b.f.by Jack Spigot, in 1835, and was covered by Langar.

\section{PHANTON MARE (sister to Cobweb)}

Bred by Lord Jensex, in 1820, her dam, Filagree, by Soothsayer, out of Web, by Waxy-l'enelope.

1823 missed to Anticipation...........

1826 br. e. by Filho da l'uta (dead) ......

1827 br. e. Donegani, by Tramp........

1828 b. e. by Master Ilenry (sent abroad) ..

1829 b. f. by ditto ...............

1850 ch. c. Ishmael, by Sultan ........

1851 ch. f. Flycatcher, by Godolphin....... Ld Jersey

1852 b. e. Ibrahim, by Sultan (s. to France in 1855$)$

1855 ch. f. Odessa, by ditlo..........

$1854 \mathrm{ch} . \mathrm{f}$. by Prian ...............

1855 b. с. by Cetus................

1856 c. by Cain ................

1857 by Gleneoc.............

\section{PHANTOII MARE,}

Bred by Mr H. Enwards, in , her dam by Pericles, out of Mary, by Sir Peter-Diomed-Desdemona, by Marske.

1851 h. f. (Misnomer) by Merlin ........ Gen. Walpole

185.5 ch. c. Mendizabal, by Merlin, or Mer-)

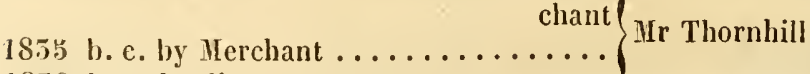

1856 b. c. by ditto ...............

This mare never ran. She was not covered in 1851 or 55.

\section{PHANTOM MARE,}

Bred by Lord Foley, in 1825, her dam, Breeze, by Soothsayer, out of Blowing, by Buzzard-Pot8o's - Maid-of-all-IVort. 
1829 b. f. by Master IIenry ........... Mr C. Stanley 1832 ch.c. Denhall, by Battledore........ Sir T. Stanley

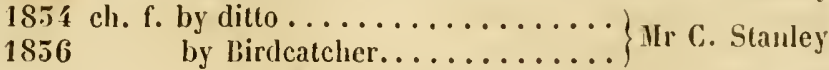

Barren in the intermediate years.

\section{PIIANTOII MARE,}

Bred by Sir Tatrox Srkes, in 182y, her dam, sister to Consul, by Camillus - Shuttle-Eliza, by llightĩyer-Augusta, by Eelipse.

1852 b. f. by Chateau Margaux (died a foal)

1853 gr. c. by Victory (son of Waterloo)...

1854 gr. c. by Comus................................. Tatton Sykes

1855 gr. c. by ditto...............

\section{PHANTOM MARE,}

Bred by MIr Ноцввоок, in 1829, her dam, Striking, Beanty, by Sorcerer-Beningbrough-Sir Peter.

185.5 b. f. by Camel................

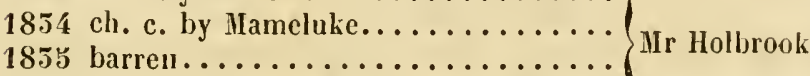

1856

by Camel................

\section{PHANTOM MARE,}

Bred by Mr HoLbrook, in 1850, her dam, Miss Skim, by Skim, out of Striking Beauty.

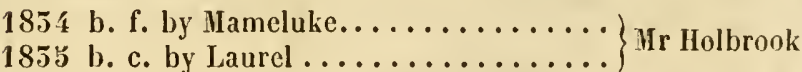

\section{PIIANTOII MARE (bay)}

Bred by Mr Farrall, in 1850, her dam, Gift, by Waterloo, out of Ralphina, by Buzzard-Dungannon-IIeinel.

1855 ch. f. by Erymus...............

1854 ch. f. by Tarrare.......................... Mr Farrall

1853

1856

c. by ditto.

GIFT, the dam of this mare, has had no foal since 1851.- Vol. III, p. 146.

\section{PHANTOIL MARE,}

Bred by Mr Tibirs, in 1817, her dam, Petronilla, by Sir Peter, out of Iris, by Brush- Ilerod. 


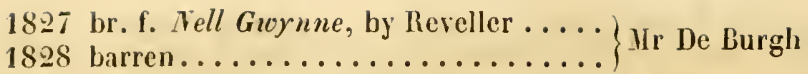

1829 br. c. Banqueter, by Reveller....... Ir Cosby

$18.50 \mathrm{br} . \mathrm{c}$. The Braro, by ditto.........

1851 b. c. by a son of Smolensko........

1852 br. c. Jacopo, by Magnum Bonum.... Hr De Burgh

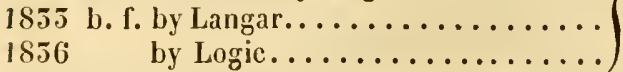

\section{YOUNG PHANTOU IIARE,}

Bred by Mr T. 0. Powletr, in 1827, her dam, Jack Spigot's dam,

by Sorcerer-Precipitate-Ilighflyer-Tiffany, by Eclipse.

1851 bl.c.Jack-and-all-Jack, by Jack Spigot Mr 'T. O. Powlett

1852 b. c. by Aetxon (dead) ............ Mr J. Orde

1855 b. c. Aleppo, by Mulatto........... Mr Leedham

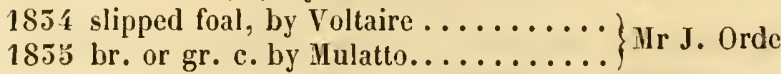

\section{PHEASANT,}

Bred by Mr Oruser Gone, in 1821, got by Bustard (son of Castrel) out of Plaything, by shuttle.

1825 b. c. by Iledley (died a foal) .........

1826 barren....................

1827 c. by Rowlston (died a foal)......

1828 b. f. Titlark, by Tramp...........

*1899 b. f. Swift, by Sir Oliver.......... IIr Ormsby Gore

1850 b. c. Petrel, by Waxy Pope........

1851 b. c. Dottrel, by The Duke........

1852 by ditto

1853 b. f. Miss Sheridan, by Langar ...... Mr Weatherley

185 i b. c. by Shakspeare ............. Col. Anson

Sent to Berlin in 1855 , covered by Priam.

* Sivirt produced a br. f. by The Duke, in 1854, and was sold to Prince Esterhazy. She was barren in 1855.

\section{PIIOEBÉ,}

Bred by Mr Waters, in 1817 , got by Castrel, her dam by Alexander, out of Iry, by Woodpecker-Trentham-Cunegonde.

1824 ch. c. Phœbus, by Milo

1826 ch. f. Delia, by ditto ............ Id Derby 


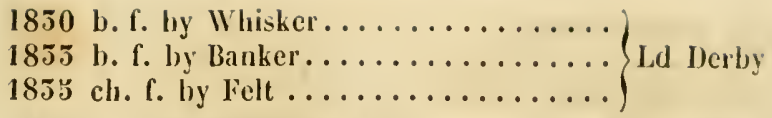

Missed in 1823, 99, 51,32 , and 54 ; cast foal in 1827 aud 28.

\section{I'IGIYY,}

Bred by the Duke of Graftor, in 1822, got by Election, her dam, Pawn, by Trumpator, out of I'runella, by Highllycr.

1828 ch. c. (Exile) by Enilius...........

1852 br. c. by Whisker .............. Mr W. Chifuey

1853

1856

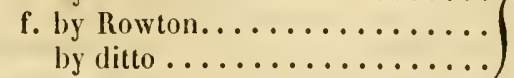

Ilissed in 1899 and 55 , slipped foal in 50 and 54.

\section{PIILLICO (sister to Godolphin)}

Bred by Lord G. H. Cavexpisi, in 1822, got by l'artisan, her dam, Ridicule, by Shuttle, out of sister to Oatlands, by Dungannoll.

1899 ch. c. by Phantom............. Mr J. Wilson

1850 b. f. by ditto............... Mr Grant

1852 br. c. Lucifer, by Lottery.......... Ir Forth

1854 b. c. by Peter Lely ............. Mr Grant

1855 ch. f. by ditto .............. Mr Tattersall

No foal in 1851 or 53 ; died in 1853 .

\section{PINCUSHION,}

Bred by Sir C. Buxbury, in 1820, got by Sol'cerer, or Williamson's Ditto, her dam, by Skyscraper-Dragon-Fidget's dain, by Matchem.

1828 ch. c. Friseur, by Blacklock....... Mr Robinson

1830 b. c. Emery, by Stainborough....... Sir S. Graham

1851 b. f. Teedle, by ditto ............ Ld Tavistock

1852 br. c. Profligate, by Filho da Puta.... Ir E. Peel

1835 ch. c. Idiot, by Bedlanite......... Mr Gardnor

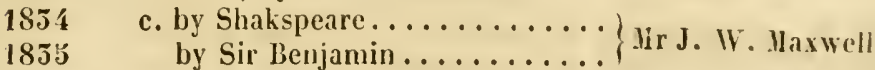

\section{PINTAIL,}

Bred by IIr Rcsu, in 1824, got by Pioneer, out of Ringtail. 
$18 \tilde{0} 0$ br. c. by Zealot (dead)...........

1851 b. c. Mulligatawny, by Turtle.......

1852 b. c. by Master Ileury (dead)........

18.5.j gr. f. Amy, by Minos ............ Mr Corbet

1854 gr. c. Blue Peter, by ditto.........

1853 b. c. by Sir IIercules ...........

18.56

\section{PIONEER MARE,}

Bred by the Duke of Grafron, in 1811, her dam, Historia's dam, by Pot8o's, out of Racket, by Herod.

1822 b. f. by Cannon Ball ............ Col. Gilbert 1826 b. f. by a half bred horse ......... Mr Wakefield

1828 b. f. by Goldfinder (half-bred) ......
Died in 1829, in foal to Sir Gray.

\section{PIONEER MARE (sister to Carthago)}

Bred by Mr Rush, in 1820, her dam, Reserve, by Waxy, out of Lady Janc, by Sir Peter.

1826 b. f. by Comus...............

1827 b. f. by Whalebone...........

1828 ch. f. (Miss Rush) by Phantom......

$18 \pm 9$ b. f. by Centaur . . . . . . . . . .

*18.50 br. f. (Mine) by Emilius .........

1851 missed to Partisan................ Mr Rush

18.52 b. f. by Jenkins, or Phantom .......

$18.5 \mathrm{j}$ ch. c. Perkins, by Jenkins........

18.54 br. c. lyy ditto..............

1855 c. by ditto (died young) ........

18.56 hy Camel...............

* Mine produced a b.c. by Count Porro in 1853.

\section{PIONEER MARE (My Aunt)}

Bred by Mr Rusn, in 1820, her dam, Discord, by Popinjay, out of Briseis.

1827 b. c. Caradoc, by Whalebone....... IIr Benson

1828 ch. f. My Cousin, by Anticipation.... Mr Thornes

1829 ch. f. by Wamba (died 5 days old)...

1850 ch. c. by ditto................... Mr L. Charlton

1851 clı. c. by ditto (died 3 months old)... 


\section{4}

1855 barren........................... Wrilson

18.54 br. c. by Shakespeare............

$183:$ f. by Shakspeare (died a foal)..... Mr Gapp

18.56 Ly The Grand Duke......... Mr Holbrook

\section{YOUNG PIPYLINA,}

Bred by Mr IIuvter, in 1822, got by Orville, her dam, l'ipylina,

by Sir Peter, out of Rally, by 'Trumpator.

1826 dead c. by Gustavus . . . . . . . . .

$1827 \mathrm{gr}$. c. by ditto (s. abroad) .........

$1829 \mathrm{gr} . \mathrm{c} .($ The Ghost) by ditto .........

$1850 \mathrm{gr}$. c. Forester, by ditto...........

1851 gr. c. (Cecil) by ditto............ Mr Hunter

1852 b. f. by ditto...............

185.5 b. f. by l'artisan.............

185 b. c. by ditto...............

1856 by Gustarus.............

Barren in 1828 ; not covered in 55.

IIQUET, by Sorcerer (Vol. III, p. 514), was barren in 1852, 34, and 55 ; her last two produce were:-

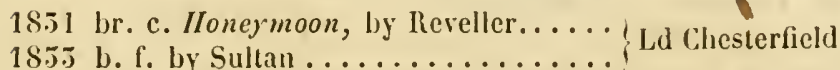

PLEDGE, by Waxy (Vol. III, p. 515) missed in 1852 and 55 , and died; her b. c. by Lottery was sent to Brussels in 18.5.

\section{POLLY OI.ITER,}

Bred by Mr Laxgord Brooke, in 1818, got by Sir Oliver, her dam by IIambletonian, out of Constantia, by Sir Peter-Mungo.

1824 br. f. by Ambo............... Mr Brooke

1823 gr. f. by The Marshal........... IIr Davenport

1828 br. f. Sarah, by Tramp........... Ir G. Ogden

1829 b. c. by Brutandorf............. Ir Giffard

1851 cast foal, by Bay Maltou..........

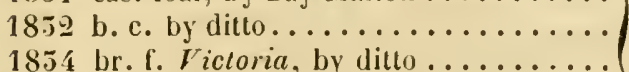

1854 br. f. Vicloria, by dilto ......... JIr Boardman

1853 ch. c. Tightboy, by ditto.........

1856 b. f. Possession, by ditto.........

18.37 by ditto.............

Barren iu $1826,27,50$, and 5.5 . 
POLYG.IR IIARE,

Bred by Mr Towsow, in 1850, out of Tranby's dahn, by Orville - Miss Grimstone, by Weasel-Aneaster-Damaseus Arabian.

1834 ch. f. by II umphrey Clinker........ IIr Gapp

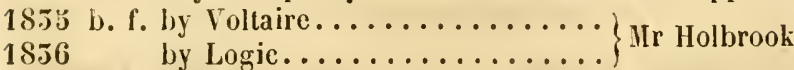

\section{POLYMNIA,}

Bred by Lord Rossuore, in 1816, got by IIusician, her dan, Promise, sister to Partisan, by Walton, out of Parasol.

1821 b. c. by Blucher ...............

1829 b. f. by Vandyke Junior (s. to France)

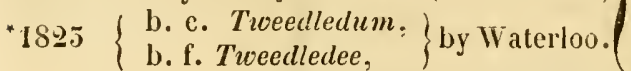

1824 gr. c. by IIr N. II. Smith's Arabian... Mr Curteis

1827 b. e. by Carbon...............

$1828 \mathrm{br}$. f. by ditto...............

1850 b. c. by heveller...............

1832 b. f. Mogmouse, by Mameluke....... Ir Bloss

1855 b. c. Paganini, by Phantom........

1853 br. c. Blaise, by Lamplighter........ IIr Curteis

1857 by Merehant............

Missed in 1825 and 29, not covered in 26, 30,53 , or 35.

* The filly never sucked, but was fed on cow's milk, and said to thrive better than the cult.

\section{POHONA,}

Bred by Lord Lowtren, in 181\%, got by Vespasian, her dam by Walton, out of Y. Giantess (Sorcerer's dam) by Diomed.

1821 ch. c. by Selim (died a day old)..... Mr L. Charlton 182.; ch. f. Rhapsorly, by Woful (died in 1827) Mr R. Benson

1824 b. f. by Manfred (died young) ....... Mr L. Charlton

1826 bl. c. Pippin, by Tiresias......... Ld Lowther

1827 b. c. Bud (aft. Raby) by ditto........ Ir Forth

1828 ch. c. by ditto (s. to Germany, and (Mr Grant

1829 b. f. by Partisan (s. to Baron Biel).... Ir Forth

18.50 b. c. Maple, by ditto..............

1851 b. f. by ditto...................

$1852 \mathrm{ch}$. c. by Truffle............... Ld Lowther

18.5 .5 b. c. by Partisan............... 
1834 b. f. by Partisan............... Mr Osbaldeston

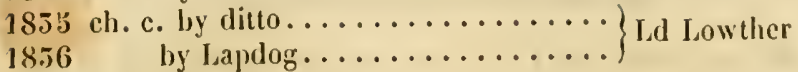

In 1822 missed to Castrel, 23 to Smolensko.

POSTIUUMA,

Bred by Mr Cussirs, in 1819, got by Orville, out of Medora, by Selim-Sir IIarry-Volunteer.

1826 cl. c. Post Obit, by Woful......... D. of Grafton

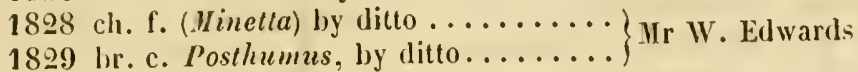

1830 gr. f. Fanny Grey, by Waterloo (put to $\}$ Id Che stud) $\}$ Chesterfield

1851 br. c. Paris, by ditto........... Jd Orford

1853 ch. f. Post IIaste, by The Colonel.... IIr E. Peel

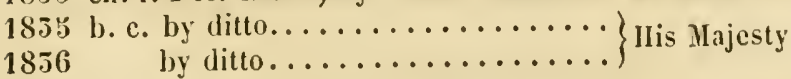

In 1852 missed to Waterloo, in 34 to The Colonel. - All the above foals, from 1829 inclusive, were bred at Hampton Court.

\section{POULTON MARE,}

Bred by Mr R. Joves, in 1825, her dam, Clearwell Lass, by Sorcerer, out of Miss Brocket.

1852 br. c. Old Joe, by Sailor...........

1854 c. by Swap (dead)............. Mr Bevan

1855

1856

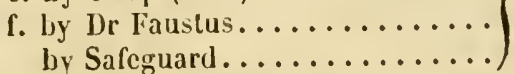

\section{PRIIA DONNA,}

Bred by , in 1821, got by Soothsayer, her dam,

Tippitywitchet, by Waxy, out of IIare, by Sweetbriar.

1827 b. c. (Donzelli) by Bustard, or Orville Mr Cooper

1828 ch. c. Moloch, by Muley........... Mr Sowerby

1850 ch. f. Malibran, by ditto.......... Sir L. Glyn

1852 br. c. De Beriot, by Longwaist...... Mr Biggs

1855 f. by aluley ............... II Nowell

1854 b. f. by ditto ............... Mr E. Peel

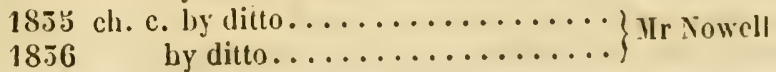

1856 


\section{PRIME MINISTER MIARE (Wagtail)}

Bred by Mr Yaretrgh, in 1818, her dam by Orville, out of Miss Grimstone, by Weasel-Ancaster-Damascus Arabian-Sampson.

1825 br. c. $H \cdot A d a m$, by Tramp........

1824 br. c. Laurel, by Blacklock........

$1823 \mathrm{br}$. f. Belinda, by ditto.........

1826 br. c. Pilgrim, by ditto .........

1827 b. c. by Figaro (died a foal) ........

1899 b. c. The Lion, by Brutandorf ....... Nlajor Yarburgh

1850 br. c. by Lottery ..............

1851 b. f. Omnibus, by Velocipede......

1855 b. f. by Lottery..............

183 b. f. by Brutandorf ............

1856 by Voltaire..............

In 1828 missed to Waverley, 52 to Tramp, $\mathbf{3} 4$ to Dr Syutax.

PRIME MINISTER MARE,

Bred by Sir Makk Srkes, in 1819, her dam by Shuttle, out of Eliza, by Ilighllyer-Augusta, by Eclipse.

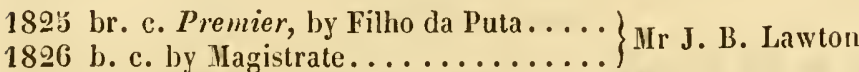

1827 b. c. Pedestrian, by Tramp......... Mr W. Scott

1828 br. c. Jonas, by Filho............ Mr Thistlewood

1822 br. f. Counterpart, by Duplicate (put to

1850 b. f. Pagoda, by Polygar..........

18.31 br. c. IIunchback, by Cain........ Mr J. H. Peel

1852 br. c. Sambo, by Edmund .......... Mr Bristow

1855 br. c. Count $D^{\prime}$ Orsay, by Dr Faustus. . Mr Phillipson

1854 b. c. by ditto................

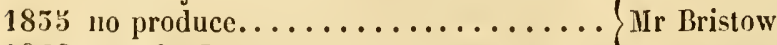

1856 by Dr Faustus............

\section{PRIME MINISTER MARE,}

Bred by Mr Srkes, in 1820, her dam, Lady Ern, by Stanford, out of sister to Repeator, by Trumpator-Demirep, by Highflyer.

182 : b. c. by Sir Walton............

1826 b. f. Beneft, by Oiseau ......... Mr Gully

1828 b. c. (Incerne) by Swiss..........

1850 br. c. Glenlee, by Emilius.......... Mr Turner 
f. by Emilius...............

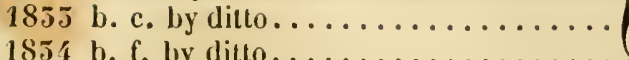

185 b. f. by St Patrick.............

1856 f. by Enilius..............

Slipped foal in 1829 and 32.

PRIHETTE,

Bred by Mr Kraptox, in 1820, got by l'rime Minister, her dam, Miss Paul, by Sir Paul, out of Mliss Dunnington, by ShuttleMiss Grimstone.

1824 b. c. by Blacklock............. Major Cuninghame

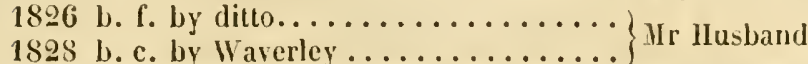

1829 b. c. by Physician, by Brutandorf.... Mr Skipsey

1850 b. c. by Figaro...............

1851 b. f. Phobé, by Comus (sent abroad) . Mr llusband

1852 b. f. by Columbus (died a foal) ......

$\left.\begin{array}{l}1853 \text { b. c. Morrison, by ditto, or Tramp... } \\ 1856 \\ \text { by brutandorf .............. }\end{array}\right\}$ MIr E. Peel

Missed in 1825, 27, 34, and 55.

\section{PRIIIGENA,}

Bred by Mr Aтrwood, in 1898, got by his grey Arabian, lier dam by $\Lambda$ bjer, out of The Duchess, by Cardinal York.

1852 ch. c. by his chesnut $\Lambda$ rabian (dead).

1855 ch. f. by Mr Astley's Black Turk, or

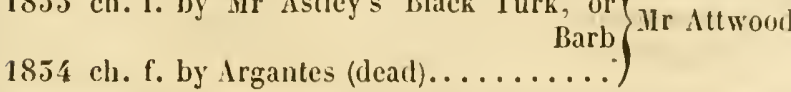

\section{PRIHLLA,}

Bred by Mr Ilumbe, in 1827, got by Cervantes, her dam, Cowslip. by Cockfighter.

1854 ch. f. by Count Porro........... Irr Tattersall

1853 ch. c. by Buzzard. ............ Mr Etwall

Sent to Virginia in $185 \%$, covered by Shakspeare.

\section{PRINCESS,}

Bred by Lord Stratnyone, in 1820, got by Comus, her dau, Remembrance, by Sir Solomon, out of Quecu Hab. 
$182: 3$ b. f. by Whisker (died a yearling).... Mr Bryan Salvin 1826 b. c. Principe, by ditto........... Mr Riddell

1827 ch. c. Bijan, by Blacklock ........ Mr Ridsdale

$1828 \mathrm{br}$. c. by Doctor Syntax (died young).. Mr Bryan Salvin

1829 ch. c. Prince, by Figaro .................... Ridsdale

18.50 ch. c. Emperor, by ditto.......... Mr Ridsdale

18.51 b. f. Isabella, by Lottery . . . . . . .

18.52 ch. f. by Tramp.................... Mr Salvin

1855 br. f. by lirutandorf(died immediately)

1854 cast foal, by Velocipede...........

$185: 5$ ch. c. by Actæon............. Mr Allen

1856 by Jerry.............

\section{PRINCESS JEMIMA.}

Bred by Mr Atkissos, in 1815, got by Remembrancer, her dam by Ormond-Anvil-Queen Mab, by Eclipse.

1820 br. c. (Marshal Blucher) by Walton.. Mr J. Smith 1829 b. c. by Ebor................

1825 l. f. Gin, by Juniper ....................... Wh hitelock

$1824 \mathrm{gr}$. f. by Grey Walton $\ldots \ldots \ldots \ldots \ldots . .$.
1823 br. f. by Reveller (died 4 yrs old $\ldots . .$.

1827 b. f. by Wanton, or Swis.......... Mr Painter

1828 ch. f. by Wanton (died a foal)....... Mr Thompson

1850 b. c. by Y. Whisker (bro. to Catterick)

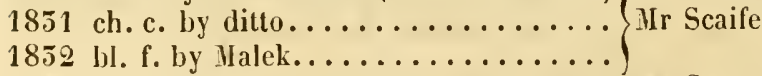

18.5.5 ๒. с. by Waverley.................. Spalding

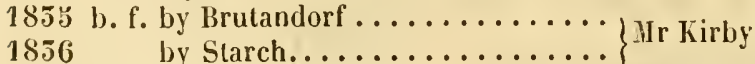

In 1821 missed to Walton, 26 and 29 to Wanton, and 54 to Lottery.

\section{PRINCESS ROYAL,}

Bred by Sir T. Mostyx, in 1818, got by Castrel, her dam, Queen of Diamonds, by Diamond-Sir Peter-Lucy, by Florizel.

$1826 \mathrm{ch}$. f. by Whisker (died young)..... Sir T. Mostyn 1828 b. f. Her Highness, by Hoses (put to the stud)

1829 b. f. by Banker ............... Mr Mostyn

1850 br.c. Prince Llewellyn, by Waxy Pope

18.1 b. f. Queen Bess, by Chateau Margaux 


\section{3}

1852 br. f. Queen of Trumps, by Velocipede

1856 by Zinganee............... Ir Iostyu

In 1827 missed to Tramp, 53 to Battledore, 5 i to Velocipede, and 3 's to Cliateau Margaux.

\section{PRINCESS ROYAL,}

Bred by Ir W. Eowanns, in 1827, got by Middleton, her dlam by Orville, out of Spinetta, by Trumpator-IIerod, etc.

1851 b. c. Royal William, by Royal Oak...)

1855 b. c. by l'atron (died a yearling) ..... Mr W. Edwards

1854 ch. f. Princess, by Augustus........

Missed to Royal Oak in 1852, and is dead.

\section{I'RINCESS VICTORIA,}

Bred by the Duke of Retravi, in 1827, got by Hiddleton, out of Adeline, by Soothsayer-Elizabeth, by Orville-l'ennytrumpet, by Trumpator.

1851 c. by a son of Walton (Ld Lowther's) Mr W. Edwards

1855 ch. c. The Shah, by Sultau......... Ir J. Day

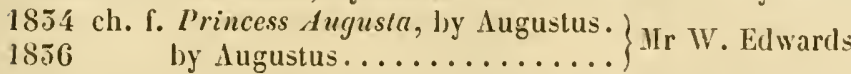

In 1852 missed to Royal Oak, in 35 to Taurus.

\section{PROBLEM,}

Bred by the Duke of Graftor, in 1825, got by Ilerin, her dam, Pawn, by Trumpator, out of Prunella.

1829 br. f. by Centaur (put to the stud).... D. of Grafton 1830 ch. c. Master Walter, by ditto ....... M. W. Edwards

•1851 ch. f. by Emilius ...............

1852 b. f. by ditto .................

1833 b. f. by Zingance.............. D. of Grafton

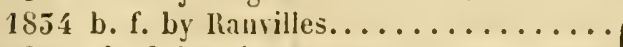

1853 ch. f. by Zinganee.............

$18 \tilde{6} 6$ by Taurus..............

* This Emilius mare produced ch. f. by Ranvilles, in 1854, missed to Cain in 55 , and was covered by Si Patrick.

VOL. 15 . 


\section{PRODIGIOUS,}

Bred by lord Hortgomerie, in 1814, got by Caleb Quotem. her dam, Fair Forester, by Alexander-Sir Peter-IIaid of Ely.

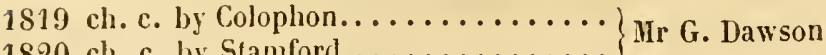

1822 ch. f. by the l'adan Aram Arabian ...

$182 \jmath$ b. c. by Derwent.............

1823 b. c. by ditto ..............

1826 b. f. by ditto...............

1827 b. f. by ditto ................

* 1828 b. f. The Gude Wife o' Tulloshill, by

1829 br. f. by Outcry............... Mr G. Dawson

$1850 \mathrm{gr}$. c. by ditto ..............

1852 b. f. by Caccia Piatti...........

1855 b. f. Fair Jane, by Jerry...........

1854 br. c. Bonny Jack, by Jack Spigot....

$18.5: 3$ b. c. Brecongill, by Brutandorf.....

1856 by Jerry.............

In 1824 missed to Champignon, 51 to Cleveland.

* This mare produced a br. c.by Percy, in 185.j, and was covered by him in 1855 , having missed to him in 54 and 55 .

\section{PROFILE,}

Bred by Mr Radclype, in 1825, got by Rubens, her dam, Effie

Deans, by Ashton, out of Harriet, by Sir Harry-Dorimant.

1853 b. c. Martinet, by The Coloneł....... Mr Radclyfle

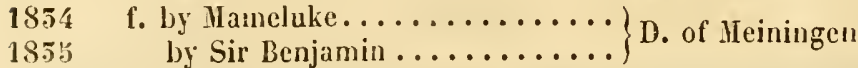

\section{PROSERPINE,}

Bred by Mr Joussox, in 1822, got by Ihadamanthus, her dam,

by Sir Peter, out of Eaton Lass, by PotSo's-Highflyer-Snap.

1850 br. c. Smedley, by Filho da Puta.....

1852 ch. c. Jupiter, by Langar..........

1855 b. c. Lucifer, by Peter Lely........ Mr Johnson

1854 br. f. by Olympus............. 1855 ch. c. by Langar. . . . . . . .

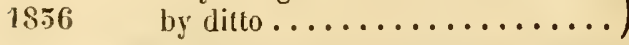

Her foals of 1828,29 , and 51 , died. 


\section{PROSODY,}

Bred by Mr R. W. Walker, in 1818, got by Don Cossack, out of Ietre, by Waxy-Woodbine, by Woodpecker.

1850 b. f. Prosody (aft. S'pondee) by Reveller 1851 br. c. Baronet, by Sir lluldibrand....

1852 not covered last season ...........

185.5 b. f. by Mamelulie............... Mr Taunton

1854 bl. c. Mustapha, by Rajah ........

1855 ch. c. by Firman..............

1856 by Wrangler (son of Rajah)....

\section{PROSPECTIVE (sister to Rowton)}

Bred by Lord Sugo, in 1814, got by Oiseau, out of Katherina, by Woful-Landscape, by Rubens.

1829 b. f. by Lottery (died a foal) ........

1830 ch. c. Clinker, by Humphrey Clinker. Mr Allen

1835 b. f. Promise, by Mulatto.........

Missed to Lottery in 1851 and 52 , and died in 55.

\section{PRUDE,}

Bred by Mr Boperhax, in 1822, got by Phantom, her dam by Stamford, grandam by Hambletonian, out of Violet, by Shark.

1852 br. c. Worcester, by Edmund....... Mr Thorne

1855 b. f. La Valiere, by Cain .........

1854 br. f. Faustina, by Doctor Faustus... Jur Bristow

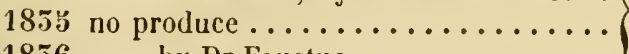

1856 by Dr Faustus . . . . . . . . .

\section{PRUDENCE (sister to Pledge)}

Bred by the Duke of Graftox, in 1811, got by Waxy, her dam, Prunella, by llighflyer, out of Promise, by Snap.

1814 f. by Thunderbolt (died a yearling). 1815 b. c. by Sorcerer (died a foal)....... 1817 ch. f. Rozena, by Haphazard....... 1818 b. c. Reginald, by ditto (sent to India). 1819 b. c. by ditto (died a foal)......... D. of Grafton

1821 b. f. Rebecca, by Soothsayer........ 1822 ch. c. Rufus, by Election (sold to the East India Company in 1828) 
1S24 b. c. Roderick, by Rubens ........

1826 br. c. Ronald, by Centaur..........

1827 br. c. Rupert, by Emilius..........

*1829 br. f. Ruth, by Merlin .......... D. of Grafton

1851 cl. f. by Sultan...............

1854 br. c. Rubicon, by ditto...........

1856 by Merchant $6 \ldots \ldots \ldots \ldots$.

In 1816 missed to Selim, 20 to Soothsayer, 2.5, 25, and 52 to

Merlin, 28 to Emilius, 50 to Centaur, 55 to Sultan, and 53 to Reveller.

* Rutu is in the D. of Portland's stud. She missed in 185.5 and 54, produced a b. f. by Zinganee in 1855 , slipped foal in 56 , and is covered by Glencoe.

\section{PRUDISII,}

Bred by Mr Thoвnнlu, in 1824, got by Merlin, her dam, I'rue, by Trumpator-Woodpecker-Trentham.

1829 b. c. Abelard, by Master Henry ..... )

1850 b. c. Abel (aft. Newcastle) by ditto... Mr L. Charlton

1851 b.c. by Pollio.................

$1852 \mathrm{br}$. c. by Emilius.............. Ld Chesterfield

1855 b. f. by ditto................ Mr L. Charlton

1854 b. f. by ditto (foaled in Holstein). .... 1). of Holstein

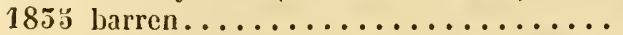

1856 by Rowton............. Count Szechenyi

She was sold to the D. of Holstein in July 1853, in foal to Emilius, and brought back in 54 ; resold and sent to Hungary in 1955 .

\section{PUCELLE,}

Bred by Lord Surfecd, in 1S21, got by Muley, out of Medora, by Selim-Sir Harry-Voluntecr.

1829 cl. f. by Woful. ............

1850 ch. f. by Whisker.............

1851 produce lyy ditto (died a foal) .........

1852 b. c. by Memnon (s. to Baron Biel in

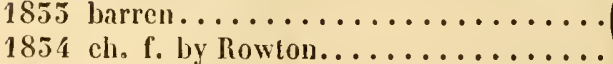

185 s ch. f. by ditto ...............

1856

by Cetus ................ 


\section{7}

PUFF,

Bred at Ilampton Court, in 1825, got by Waterloo, her dam, Blowing, by Buzzard-Pot8o's-Maid-of-all-Work, by Ilighllyer.

1829 br. c. Captain Wattle, by Champion.. Mr Nanney

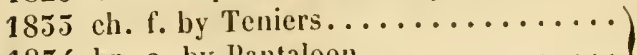

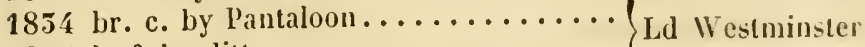

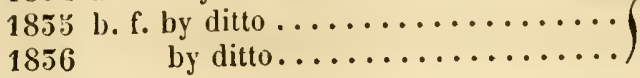

She had other foals before $\mathbf{1 8 5 5}$, but they all died joung.

\section{PURITY,}

Bred by Mr J. Crort, in 1822, got by Octavian, her lam, Heli Cat, by Cerberus, out of Cutty Sark, by IIaphazard-Brillante, by Whiskey.

1850 ch. f. by Jock . . . . . . . . . . .

1852 b. c. by Jerry ...............

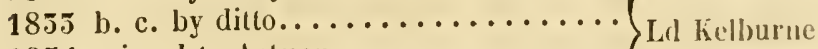

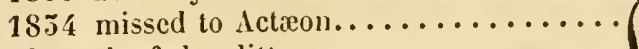

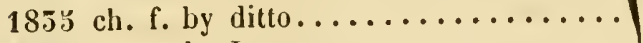

1856

by Jerry $\ldots \ldots \ldots \ldots \ldots \ldots$

\section{QUADRILLE (sister to Fandango)}

Bred by Mr Golnıve, in 18Is, got by Selim, her dam, Canary. bird, by Whiskey, or Sorcerer, out of Canary, by CorianderIliss Green, by IIighllyer-Harriet, by Matchem.

1821 ch. f. Katherine, by Soothsayer...... D. of Rutlanul

1822 b. f. Pastime, by Partisan.......... Mr F. Craven

1825 b. f. Louisa, by Orville.......... Mr Mytton

1824 b. c. Monops, by IIanfed .............. Mr Greville

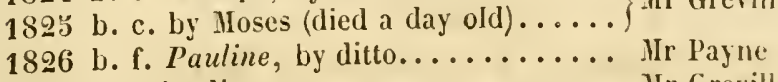

1827 by ditto ............... Ir Greville

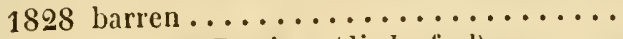

1829 b. c. by Partisan (dierl a foal)....... IIr Payne

1850 b. f. Caroline, by Partisan (died young)

1851 b. f. Cotillon, by ditto...........

1852 ch. c. Curtius, by Emilius.........

1855 ch. f. Copere, by Velocipede........ Ir G. Walher

1854 b.c. Capulet, by loung l'hantom.....

185 . b. f. by partisan .............

1856

by Emilius............... 


\section{QUADRON (sister to Mulatto)}

Bred by Lord Fitzwiluan, in 1826, got by Cattoll, out of Desdemona, by Orville - Fanny, by Sir Peter-Diomed.

1852 b. c. by Confederate (died in Dec. 1854 )

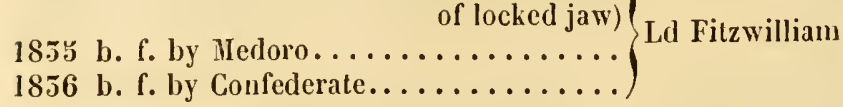

In 1855 missed to Confederate, 54 to Mambrino.

\section{QUEEN BATHSIIEBA,}

Bred by Sir D. II. Blair, in 1826, got by Prime Minister, her dam, Maria, by Trissy-Caleb Quotem-Anna Bullen.

1854 br. f. Palestine, by Y. Blacklock.....)

185 b. br. $f$. by Jerry............... Ld Eglinton

1856

c. by Priam...............

QUEEN COIL (Vol. III, p. 527) slipped foal in 1855 and 54 , and missed in 53 ; her two last produce should stand thus :-

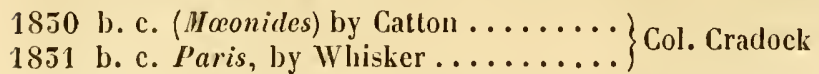

\section{QUEEN OF HEARTS (sister to Mouche)}

Bred by Mr Tuonvhile, in 1926, got by Emilius, out of Mercy, by IIerlin-Shoveler, by Scud-Goosander.

1853 br. c. by Bustard..............

1854 missed to Muley................. IIr Nowell

1855 br. f. by ditto $\ldots \ldots \ldots \ldots \ldots \ldots \ldots \ldots \ldots$
$1856 \quad$ by ditto $\ldots \ldots \ldots \ldots \ldots \ldots$ IIr Nowell

\section{QUICKLY,}

Bred by Earl Fitzwiluiar, in 1816, got by Mowbray, out of Sally, by Sir Peter-Diomed-Desdemona, by Marslie.

182 c. by a lialf-bred horse.......... Mr Reeves

1824 b. f. by The Laird.............. Mr Petre

1826 c. by Phantom.............. Mr Wilson

1827 c. by Cameleopard............. Mr Gell

1928 br. c. Doncaster, by Blacklock (not)

$1850 \quad$ c. by Polygar................ Mr Cholmley

Since 1850 she has had three foals by half-bred horses. 
RACIIEL RUYSCII,

Bred by Mr West, in 1824, got by Rubens, her dam by Cesario, out of Miss Holt, by Buzzard-Camilla, by Ilighllyer.

1851 br. c. Smuggler, by Slaver ........ Mr West

1855 b. f. Polly Peachum, by Shaver. ..... Mr Corbet

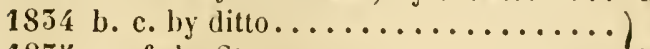

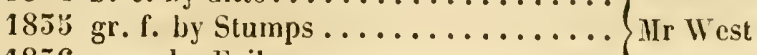

1856

by Exile $\ldots \ldots \ldots \ldots \ldots \ldots$.

\section{RACIIEL,}

Bred by Mr Kraptor, in 1825, got by $\Lambda$ madis, her dan by thon Cossack, out of Nitre, by Precipitate.

1827 ch. f. by Peter Lely ...........

1828 b. f. by ditto ................

1829 b. c. Ecclefechan, by Wau-lau-jah....

$1852 \mathrm{ch}$. c. Queensberry, by Monreith..... Mr Wilkin

1855 ch. f. by Corinthian..............

1854 ch. c. Abraham Newland, by Malek..

1853 b. c. Everton, by Liverpool........

1856 by Corinthian.............

Barren in 1850 and 51 .

\section{RACHEL,}

Bred by his Royal Highness the Duke of York, in 1825, got by

Whalcbone, out of Iloses' dam, by Gohanna.

1850 b. c. by Partisan .............. Ld Tavistock

1851 ch. f. Chataigne, by Emilius........ Id Orford

1852 ch. f. Adana, by Sultan ......... II Yates

1855 ch. c. by ditto............... Ld Westminster

1854 b. c. by ditto ............... Ld Chesterfield

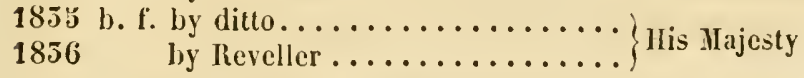

All the above foals were bred at IIampton Court.

\section{RACKET (sister to Bustard)}

Bred by Lord Staurond, in 1814, got by Castrel, her dam, Miss Ilap, by Shutlle-sister to Haphazard, by Sir Peter.

1822 b. f. by Filho da Puta, or Magistrate.. Hr Rogers

1825 ch. c. by Sam ............... MIr JIytton 


\section{0}

1824 ch. c. Battlerlore, by Sir Oliver...... Sir T. Stanley 1823 b. c. Rioter, by Reveller.......... Ld Ailesbury 1826 b. c. Marcus (aft. Bay Malton) by Filho da Puta $\}$ Ir Boardman

1827 b. f. Lucretia, by Sir Oliver........ Hl Robiuson

$1828 \mathrm{ch}$. c. by Rowlston (died 2 days old).. Mr Gore

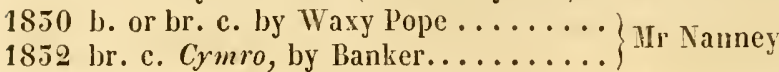

1833 b. f. by Ilerbert Lacey ........... Mr Billingtou

In 1829 missed to Sir Oliver, 51 to Bravo, barren in 55 and 54.

\section{RAHONA,}

Bred by Mr Bamp, in 1827, got by Whisker, her dam, Lady Cramfeazer, by Stamford, out of Mrs Barnet, by Waxy.

1852 ch. f. Chance, by Patron .......... Ir Marson

1855 b. c. by ditto (broke his back) ......

1854 cast foal, by Augustus ........... Id Exeter

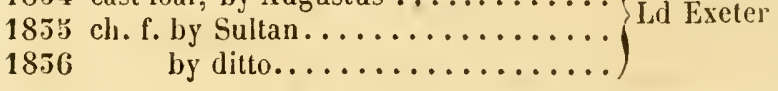

\section{YOUNG RANTIPOLE (bay)}

Bred by Mr NoweLL, in 182\%, got by Orville, or Ivanhoe, her dam, Rantipole, by Selim, out of Sweetheart, by Volunteer.

1852 b. f. by Longwaist (s. to Germany)... . Mr Nowell

1853 b. c. Sam, by Muley ............ Ir Buckley

1834 c. by Bustard (died 5 weeks old)...

1853 br. c. by Nuley............... Wr Nowell

1856

TIIE RAPHAEL MARE, out of Tiny (Vol. III, p. 351) was sent to Germany, as were Blythe and Sir Thomas.-Add the name Kislar Aga to her bl.c. by Reveller, foaled in 1851 .

\section{RAPHAEL MARE.}

Bred by Mr Pelrse, in 1821, her dam, sister to Rosette, by Beningbrough, out of Rosamond, by Tandem.

1824 b. c. by Grey Walton ........... 1823 and 26 cast foal, by St Patrick.................... Sir E. Dodsworth

1827 ch. c. by ditto.

1828 ch. c. by ditto................ 


\section{1}

1899 b. c. Wildboy, by Whisker........

1850 dead f. by Chateau Margaux ....... Sir E. Dodsworth

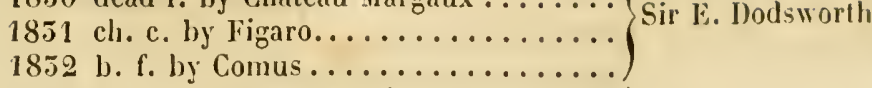

1855 b. c. Grotesque, by dilto (s. to Sweden)

1854 missed to Swiss ................

in 1850) Mr Dyson

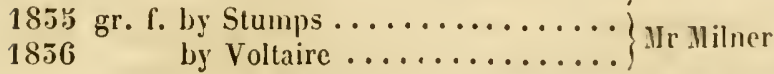

\section{RARITY,}

Bred by Mr Rawersos, in 1822, got by Anticipation, her dam by Williamson's Ditlo, out of Agnes, by Shuttle.

1829 ch. f. by Rubens..............

1855 ch. c. Maro, by Middleton.......... Mr S. L. Fox

1854 ch. f. by ditto .............. Mr Tattersall

1856 by Oppidan .............

She missed to Fungus in 1851 , and was ridden in that and the following year.

\section{REACTION,}

Bred by , in 1829, got by Truffle, her dam lyy Blacklock, out of IIusidora, by Meteor-Higliflyer.

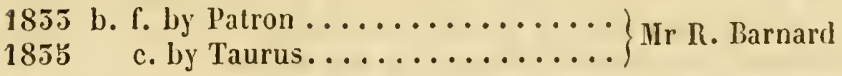

IIissed to Emilianus in 1854, and was sold to go to Germany in 53 with her foal, and covered by Buzzard.

\section{RECOVERY,}

Bred by Mr BeLL, in 1809, got by Hyacinthus, her dam by Orerton-Katherine, by Highflyer-Sincerity, by Matchem.

1816 b. c. by Fitz-Teazle............. Mr Milner

1818 b. f. by ditto................

1819 b. c. by ditto (died a yearling) .......

$1820 \mathrm{ch}$. c. by ditlo . . . . . . . . . . . Mr Bell

1821 ch. c. by ditto...............

1825 b. c. by ditto.................

1826 b. c. by $\operatorname{ditto} \ldots \ldots \ldots \ldots \ldots \ldots \ldots$. . . 
1852 b. f. Miss Ball, by Palmerin .........
1855 ch. f. by Record .............. Mr W. Simpson

1856 by Lot (son of Brutandorf).....

Barren in 1824 and 23.

\section{RECTORY,}

Bred by Lord Egrenost, in 1818, got by Octavius, her dam, Catherine, by Woodpecker-Camilla, by Trentham.

182.5 b. f. Incest, by Little John.........

1824 b. c. Grampus, by Whalebone (s. to Van)

Dieman's Land in 1829)

$1823 \quad \mathrm{f}$. by ditto............... Ld Egremont

1827 b. c. (Shark) by ditto (broke his leg in running in 1852 , and was shot)

1829 b. f. by ditto (died young).........

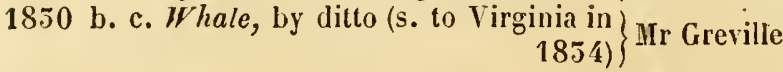

1851 b. c. Jonas, by ditto (s. to France, 1855)

1852 b. f. by Greyleg (s. to Germany)......

1854 b. f. by Chateau Margaux .......... Ld Egremont

1835 b. c. by dillo.................

Barren in 1822, 26, and 28, and missed to Chateau Margaux in 55.

\section{REMEMBRANCE,}

Bred by Lord Strathmore, in 1805, got by Sir Solomon, her dam, Queen Mab, sister to Mercury, by Eclipse-Tartar-Mogal - Sweepstakes-sister to Sloven, by Bay Bolton.

1813 ch. f. by Wizard ............ Mr Barker

1817 br. f. by Smolensko (sent abroad).... . Mr S. Reed 1818 ch. c. by Ardrossan (s. to Germany in 1 1822) Mr C. Marson

1819 b. c. Baron Bowes, by Woful....... Mr W. Peirse

1820 ch. f. Princess, by Comus.......... Mr Salvin

1821 ch. f. Bigottini, by Bigot.......... Ld Londonderry

1822 b. c. by Ardrossan (died a foal) $\ldots .\left\{\begin{array}{c}\text { The Trustees of Ld } \\ \text { Strathmore }\end{array}\right.$

182.5 b. f. Decision, by Magistrate....... . Ir Darnell

1824 b. f. hy Tramp................ Mr Kemp

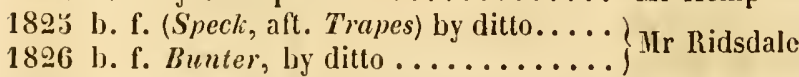

1827 ch. f. (Matilda) by Whisker........ The Trustees 
1831 b. f. Oblivion, by Jerry (put to the stud)

1852 b. c. by Actxon............... Mr Bowes

Not cosered in 1815 , missed to Sorcerer in 15 , to Ilambletonian in 16, to Chateau Margaux in 50 -ller produce of 1828 and 29 died as soon as fualed, and she died late in the year 1852.

THE REMEMBRANCER MARE, out of Mary (Vol. H1, [) 55\%) missed in 1852 and 55 , died in 1855.

\section{REMEMBRANCER HARE (sister to Penedick)}

Bred by Lord Stratumore, in 1812, her dam, Beatrice, by Sir

l'eter, out of l'yrrha, by Matchem-Duchess, by Whitenose.

1820 b. c. by Walton................

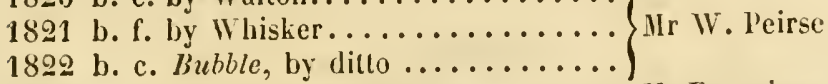

1823 b. c. Cromarty, by Catton.......... Mr Farquharson

1823 b. f. Whisk, by Whisker .........

1826 b. f. by Wanton (son of Woful)...... Mr W. Peirse

1827 b. f. by Swiss (died a yearling) ......

1828 br. f. Syma, by ditto (s. to Germany). D. of Richmond

1829 ch. f. by Middleton ............ Ld Tavistock

1850 ch. f. by ditto .............. IIr Dunkley

1851 b. f. by Royal Oak................

1854 slipped twins, by an Arabian ...... Ld Taristock

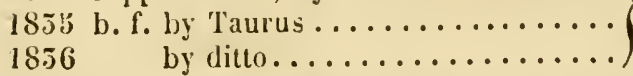

In 1819 missed to Leopold, in 24 to Whisker, harren also in 52 and 53 .

\section{REPOSADA,}

Bred by Lord Fitzwillial, in 1817, got by Amadis, her dall,

Orvillina, sister to Orville, by Beningbrough, out of Erelina.

1825 b. c. by Abjer (died 2 (lays old) .....

$182 y$ b. f. by Cameleopard.............

1826 b. f. Ly Tranp ................

1827 slipped foal, by ditto ........... Ld Fitzwilliam

1828 b. f. (The Gourd) by Champignon (dam

of Scarbrough, by Catton)

1829 b. c. by Comus................

1852 br. f. by ditto (s. to America in 1853) 


\section{4}

1853 b. c. Sepoy, by Iulatto.......... D. of Richmond

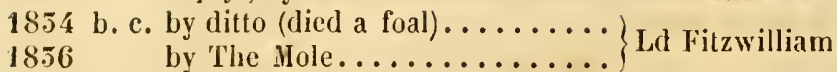

Missed in 1824, 50, 31, and 55 .

REVELLER MARE (black)

Bred by Sir Joln Beresford, in 1824, her dam, Lisette, by

Hambletonian, out of Constantia, by Walnut-Contessina.

1852 b. c. Ariel, by Actæon............

1853 br. c. Rover, by Waverley............

1854 b. c. Wrestler, by ditto.......... D. of Leeds

1855 missed to Aetæon

1856

by Langar................

RHODA,

Bred by the Duke of Rotusd, in 1815, got by Asparagus, her dan, Rosabella, by Whiskey-Diomed-Harriet, by Matchem.

1821 dead twins, by Anacreon.......... Mr Turner

$1825\left\{\begin{array}{c}\text { br. c. } \\ \text { f. (dead) }\end{array}\right\}$ by Smolensko ........

1824 b. c. by Merlin ..............

1826 b. c. Oatlands, by Tiresias.........

1827 br. c. by Comus ................

1828 b. c. Roadster, by $\Pi^{\circ}$ Adam..........

1829 ch. f. by Partisan .............. Mr Rush

1830 dead $f$. by ditto ..............

185 I slipped foal, by ditto...........

1852 b. c. by Jenkins, or Partisan........

1854 slipped twins, by Camel..........

1856 slipped foal, by Jenkins . . . . . . .

1857 by Laurel ..............

Missed in $1822,25,55$, and 55 .

\section{RHODA (Young)}

Bred by Mr Watson, in 1815 , got by Walton, her dam by Trumpator, out of Cinderella, by Dungannon-Cinderwench.

1818 br. c. by Y. Whiskey ...........

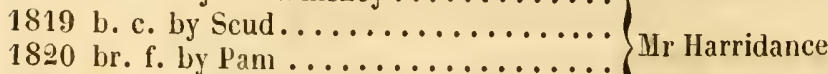

1822 b. c. Ripton, by Fungus............ 
${ }^{*} 1825$ b. f. Maldonia, by Fungus.........

1825 b. e. by Lory.................. Mr R. Wilson

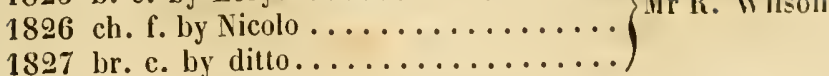

1827 br. c. by ditto................... Sir G. Heatheot

1829 b. f. The Peri, by Stainborough..... Mr R. Wilson

1850 b. c. $Y$. Rapid, by ditto..........

1831 br. c. by ditto................ Col. Peel

1852 br. c. (Ruinous) by Filho da P'uta....

1853 by Sir Benjamin ...........

Barren in 1821 and 24 ; missed to ljedlamite in 55 , to Stainborough in 54 .

- Maldovia produced a ch. c.by Velocipede in 1855 , a ch.f.by Defence in 56, missed to Nimrod in 54, was not covered that year, and was covered by Saracen in 36.

+ Sumine produced a b. f. by The Colonel, in 1855.

\section{RHODACANTHA,}

Bred by Mr Peirse, in 1820, got by Comus, out of Lisette, by Hambletonian-Constantia, by Walnut.

1826 ch. c. Rossignol, by St Patrick ......

1827 ch. c. Redstart, by Whisker........

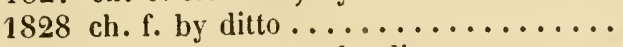

1829 b. c. W'interfield, by ditto . . . . . . .

1830 br. c. Lot, by Lottery ............

1851 ch. c. by Whisker (foaled dead)......

1852 ch. f. Marianne, by Malek......... D. of Leeds

1855 missed to Malek................

1854 b. c. Lothario, by Lottery..........

1853 gr. c. Vertumnus, by Actæon, or Velocipede

1837 by Mulatto..............

\section{RIDOTTO,}

Bred by Mr Culapmax, in 1825, got by Reveller, her dam by Watton, out of Goosander, by Hambletonian.

1829 b. f. Saraband, by Tramp.........

*1850 b. f. Risk, by Lottery...........

1851 b. f. Harmonia, by ditto .......... Ir Ridsdale

1832 b. f. by Tramp (s. to Germany in 1855)

1855 br. c. Galliard, by ditto ..........

1854 slipped a filly, by Tramp..........)

* Risk dropped down dead in her exercise when 2 yrs old.

VOL. IV. 
1855 br. f. by Tramp...............

1856

18.57

$$
\begin{aligned}
& \text { c. by Brutandorf } \ldots \ldots \ldots \ldots \ldots \text {. Mir Ridsdale } \\
& \text { by Velocipede.............. }
\end{aligned}
$$

\section{RINGLET,}

Bred by Lord Fitzwiluan, in 1826 , got by Whisker, her dam,

Clinkerina, by Clinker, out of Pewet, by Tandem-Termagant.

1850 b. c. by Confederate .............

1851 b.c. by Mulatto.................

1852 b. f. by Confederate..............

182.5 b. f. by Mulatto (dicd a foal)........ Ld Fitzwilliam

1854 bl. c. by Sandbeck (shot a yearling). .

1855 b. c. by $A$ madis ................

1856 missed to Hedoro ..............

\section{RIVULET,}

Bred by General Levisox Gower, in 1815, got by Rubens, her dam, sister to Champion, by Pot8o's-Iighflyer-Cypher.

1819 ch. c. King Fisher, by IIaphazard ... Gen. Grosvenor

$1820 \mathrm{ch}$. or ro. c. Hazard, by ditto ....... Major Wilson

1821 b. f. by ditto ................

1822 ch. c. by IIedley ..............

182.5 b. c. Waterman, by Woful (s. to P'russia in 1826)

1825 b. c. Neptune, by Tiresias .......... Ld Lowther

1829 ch. f. Mersey, by Partisan..........

1850 ch. f. Deva, by ditto............

1851 ch. c. Rhodanus, by ditto..........

In 1824 missed to Orville, 27 to Tiresias, 52 to Partisan, and was sold to go abroad, covered by him.

\section{RONIP,}

Bred by , in 1813, got by Selim, her dam by Remem-

brancer, out of Athe, by Y. Narske-sister to Fleacatcher.

1823 br. c. (Chew Bacon) by Merlin .......

*1826 b. f. (Galopade) by Reveller.........

1827 br. c. (Ivanhoe) by Noses .......... Mr Rush

1829 br. f. Rattle, by Whalebone.........

1851 b. c. Rebel, by ditto..............

* Galopade was sold to Baron Biel, and sent to Germany in 1855, covered by Alpheus. 
1855 b. f. Taglioni, by Camcl..........

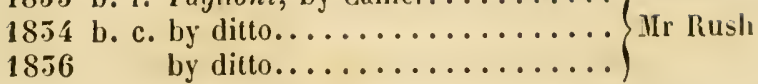

by ditto................

Yissed in $1824,28,30,52$, and $3: 5$.

\section{ROSALBA,}

Bred by Lord Derbx, in 1811, got by Milo, lier dam, sister to Rubens, by Buzzard-Alexander-Highnlyer, etc.

1818 b. c. by Thunderbolt (dead).........

1819 bl. c. by Rinaldo ..............

1821 gr. c. Grildrig, by Camillus ........

1822 b. c. by Rinaldo (dead)............

1825 ch. c. Prussian, by Blucher ........

1824 b. c. Murillo, by Magistrate.........

1825 cast a filly, by Tramp........... Ld Derby

$1826 \mathrm{ch}$. f. by Magistrate.............

1897 ch. f. Roseleaf, by Whisker.........

1829 b. c. by ditto................

1851 br. c. by ditto...............

1852 ch. f. Verbena, by Velocipede.......

1855 ch. c. by Felt $\ldots \ldots \ldots \ldots \ldots \ldots \ldots$

1856 by Velocipede .............

Not covered in 1852 ; barren in 1828,50 , and 54 .

\section{ROSALIA,}

Bred by Mr Nowel, in 182:, got by Walton, her dam, Rosanne, by Dick Andrews, out of Rosette, by Beningbrough-liosamond, by Tandenı.

1852 cast foal, by Longwaist.......... Ir Nowell

$1835 \mathrm{br}$. c. Mulberry, by Muley ........ Mr W. Edwards

1854 bl. c. by ditto.............. Ld Chesterfield

1855

\section{ROSALIND,}

Bred by Lord Fitzwilun, in 1811, got by Orville, her dam, Minstrel, by Sir Peter, out of Matron, by Florizel.

1816 ch. c. Esplandion, by Imadis...... I.d Fitzwilliam 
1817 br. c. Cacalier, by Amadis ........

1818 br. c. by ditto ....

$\left.\begin{array}{l}1819 \text { ch. f. hy Leopold } \\ 1820 \text { ch. f. by ditto ..... }\end{array}\right\}$ sent abroad..... Mr Lambton

1821 cli. c. Royalist, by ditto...........

1822 b. c. by Whisker.............. I. of Buckingham

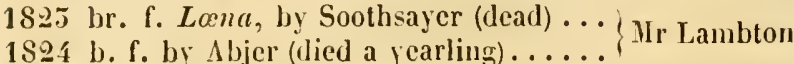

1823 b. c. by Dunsinane............. Col. Cradock

1329 h. c. by his grcy Arabian (dead) ......

1850 b. c. Ariosto, by his chesnut Arabian.

1851 gr. f. Orinda, by his grey Arabian ... Mr Attwood

1852 ch. f. Cleone, by his chesnut Arabian.

1855 ch. or ro. f. by his grey Arabian.....

Barren in $1826,27,28,54$, and 55 .

\section{ROSALIS,}

Bred by Mr HaLL, in 1824, got by Blacklock, her dam by Cerberus, out of Merlin's dam, by Delpini-Tipple Cider.

1851 b. f. by Tinker (put to the stud) ..... Ir Jackson

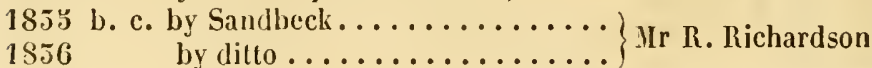

ROSAMEL, by Rubens (Vol. III, p. 542) was shot in 1834, having produced that year a b. f. by Longwaist. She missed in 1852 and 53.

\section{ROSAMOND,}

Bred by $\mathrm{Mr}$ Prise, in 1815, got by Grimaldi, her dam, Octavia, by

Pegasus, out of Gawlicy, by Ilighflyer-Giantess, by Matchem.

1852 b. c. by Doctor Eady (dead)........

1855 b. c. by Idle Boy .............

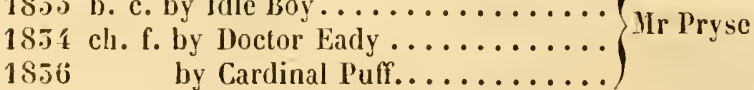

She was hunted prior to 18.51, and missed to Doctor Eady in $185 \mathrm{~s}$.

\section{ROSANNE,}

Bred by Mr Pernse, in 1811, got by Dick Andrews, her dam, Rosette, by Beningbrough, out of Rosamond, by Tandem.

$1 \$ 18$ b. f. Rose, by Rubens ............ Mr Dundas

1820 b. c. (Carnicul) by Comus (died in 1825) Mr Pcirse 
1821 br. c. Rosario, by Walton......... Mr Lambtou

1822 b. c. by Ebor................ Mr Peirse

1824 b. f. by Grey Walton (died 2 yrs old).
1823 br. f. Rosalia, by Walton........... J. Shelley

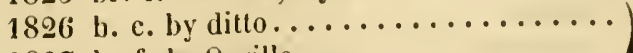

1897 b. f. by Orville...............

1829 f. by Muley.............. Mr Nowelt

1850 b. f. by ditto (put to the stud) .......

1831 br. c. Lansilowne, by ditto........

1852 b. f. by Longwaist.............

In 1819 missed to Rubens, 25 to Ebor, 55 to Longwaist, and died in March 1854 , in foal to Bustard.

\section{ROSARY,}

Bred by Mr Crouprox, in 1826, got by Tramp, or Waverley, her dam, Rosa Mundi, by Wizard, or Warrior, out of Rosamond, by Buzzard.

1832 br. c. Forwarl, by Cadiz .......... Mr S. Herbert

1854 f. by Camel (dead) ........... Ilr Finch

1835 br. c. by Buzzard ............. Hr llerberi

1836 by Sultan.

\section{ROSEBUD (first called Runnymede)}

Bred by Mr G.ıTes, in 1828, got by Little John, her dam by

Whalebone, out of Ransom, by Sir Peter-Shift, by Sweetbriar -

Sıар.

1854 br. c. by Lamplighter........... Mr Tattersall

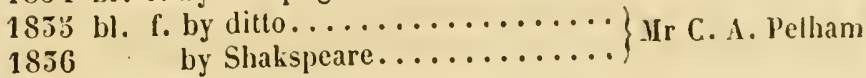

\section{ROSELEAF,}

Bred by Lord DeneY, in 1827, got by Whisker, out of Rosalla, by Milo-sister to Rubens.

1854 ch. f. Sweetbriar, by Langar .......

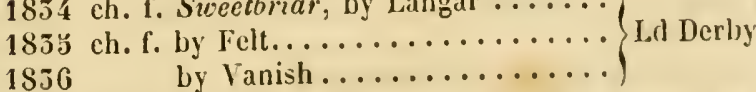

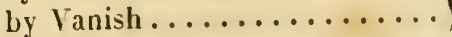

\section{ROTTERDAM,}

Bred by Colonel Wissor, in 1817, got by Juniper, her dam, Spotless, by Walton-Trumpator-Highlyer. 


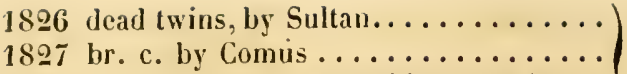

1898 b. c. Chapman, by Emilius (s. to Baron Col. Wilson

Biel)

1829 br. c. (Ilawker) by ditto ..........

18.55 ch. f. by Oscar, or Bedlamite .......

185 马

1856

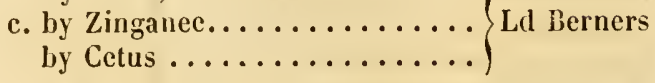

Missed to Emilius in 1850, 51, and 32.

\section{ROWENA (sister to Wouvermans)}

Bred by Mr Новамт, in 1816, got by Rubens, her dam, Brightonia, by Gohanna, out of Nutmeg, by Sir P'eter-Nimble.

1825 b. c. Amateur, by Abjer........... Mr L. Charlton

1825 b. I. by Smolensko (dead).............. MIr R. Wilson

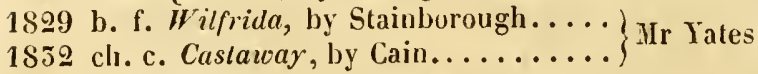

1834 br. or b. f. Castaside, by Mameluke, or Gen. Yates

1853 br. or bl. c. by Camcl (dead)......... Mr C. II. Turner, 1856 slipped twins, by Camel......... jun

Barren in 1822, 27, 28, 50, 51, and 55.

\section{ROWENA (sister to Reginald)}

Bred by the Duke of Graftox, in 1817, got by Haphazard, her dam, Prudence, by Waxy, out of Prunella, by Highllyer.

1822 ch. c. Nigel, by Election ..........

1825 ch. c. Norman, by Abjer ...........

1824 ch. f. Novice, by Selim........... D. of Grafton

1825 gr. c. Nutmeg, by Skim (sent to India in 18.9)

1326 b.c. Vortigern, by Emilius......... Mr Greville

1827 ch. f. by Merlin...............

1829 ch. f. Blanche of Deven, by Bedlamite

$1851 \mathrm{ch}$. c. by Leviathan (died a foal) ...... I Ld Chesterfield

18.52 ch. c. Willess. by Wamba..........

185.5 ch. f. by Langar..............

Barren iil 1828 and 50 , and is dlead. 
RUBENS MARL,

Bred by Mr Lucas, in 1815, out of Guildford Nan, by Guildford (son of IIightlyer) -Justice-I'arsley, by' Pot8o's-Lady Bolingbroke, by Squirrel.

1817 ch. c. by 4 rchduke............. Mr Lucas

1818 b. f. Augusta, by Woful.......... Ld Exeter

1819 was used as a hunter............. Hr I Lucas

1820 ch. f. by Marshal Blucher.........

1821 b. f. lyy a common horse..........

1823 b. f. by ditto (died 2 yrs old) .......

1824 b. c. by Captain Candid (sent to India

in 1828)

1826 ch. c. Patron, by l'artisan .........

1827 ch. c. Red Rover, by Middleton ..... I Id Excter

1829 ch. f. by llouutebank ...........

1851 b. f. by Partisan...............

1852 b. f. by Emilius ...............

18.54 ch. c. by Redgauntlet............

1853 b. f. by ditto

1836

by Reveller..............

Barren in 1825,28 , and 50 ; in 55 missed to Redgauntlet.

RUBENS MaRE, Xarifa's dam (Vol. III, p. 547), had no foal after 1851, and is dead; her last produce should stand thus :1831 br. c. Valet, by Bizarre.......... Mr Greville

\section{RUBENS MLARE,}

Bred by Mr Prese Prise, in 1816, her dam, llistake, by Waxy, out of Woodect, by IIentor-Blacaria, by Herod.

182.5 f. by Grimaldi (died a foal) ......

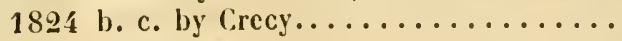

1827 b. c. by lledley (dead)............

1828 ch. c. by Rereller..............

1829 b. c. by Cardinal Puff (dead) ....... HI Pryse

1851

1852 b. f. by Cardinal luff (died in physic).

185.5 ch. f. by Idle Boy ..............

1854 ch. c. by Dr Eady . . . . . . . . .

1856

by Fungus................

Ilissed in 1825 and 50 , slipped twins in 26 , not covered in 5 亿. 


\section{RUBENS MARE,}

Bred by His Royal Highness the Duke of York, in 1S17, her dam, Queen of Hearts, by Sorcerer, out of Tooee, by Buzzard.

1821 b. c. by Vandyke Junior.......... Mr Crowther

1825 b. f. hy Ardrossan ............ Ld Kelburne

1824

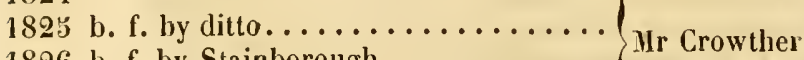

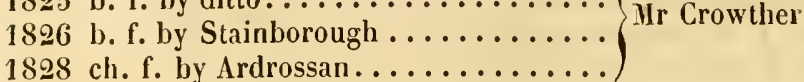

1830 ch. c. by Tremaine (Ardrossan) dead. Mr F. Lumley

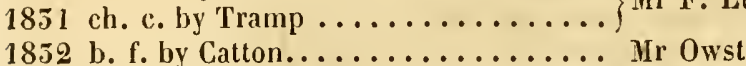

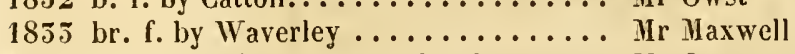

1855 ch. f. by his Young Blacklock....... Mr Owst

1856 by ditto.............. Col. Thompson

Missed in 1827,29 , and 54.

\section{RUBENS MARE,}

Bred by Mr Haffexdev, in 1817, her dam, Slipper, by Precipitate, out of Catherine, sister to Colibri, by WoodpeckerCamilla.

1821 b. c. by Shuttle Pope (sold for a stallion) when 2 years old, and unbroke)

$1825\left\{\begin{array}{c}\text { b. f. } \\ \text { c. (dead) }\end{array}\right\}$ by ditto.......... MIr Haffenden
1824 b. f. Lady's Slipper, by Waxy Pope ...

1825 b. c. by Anticipation............. Sir C. Morgan

1827 ch. f. Lady Blanche, by Bobadil (put) Hr Corbet

1829 ch. c. Count Robinson, by Waxy Pope Sir M. Wood

*1851 b. f. Lady Bell, by ditto..........

1855 b. f. by Bobadil (died a yearling)..... Mr Haffenden

Not covered in 1821 or 25 ; missed in 1828, 52, and 54 ; slipped foal in 1850 .

* Lady Bell produced a b. f. by lain in 1855, and was covered by Bobadil.

\section{RUBENS MARE (bay)}

Bred by

, in 1817, her dam by Sorcerer, out of Drowsy, by Drone. 


\section{3}

1828 ch. c. by Duport .............

1829 b. f. by ditto ................

1850 c. by Don Cossack (dead)......... Mr Slack

1852 b. c. hy IIussar (son of Whisker).....

1854 b. c. by Nigel ...............

Barren in 1851,55 , and 55 , and destroyed.

\section{RUBENS IIARE,}

Bred by the Duke of Rutuan, in 1819, her dam, Pennytrumpel.

by Trumpator-Y. Camilla, sister to Colibri, by Woodpecker.

$1825 \mathrm{ch}$. f. hy Waxy Pope (sent to France). M. de La Bastide

1824 b. f. by Smolensko (died in 1828 in foaling)

$182 y$ ch. c. Bugle, by Tiresias.........

1827 br. c. by ditto............... Id Lowther

*182S h. f. Guitar, by Jartisan .........

1829 br. c. by ditto (s. to Germany).......

1850 ch. f. Iurdy Gurdy, by ditto (ditto) ..

1851 b. f. by ditto (ditto) ............

Nissed to Tiresias in 1825, to Partisan in 52 and 53 , and sold to Baron Bicl in 55, covered by Partisan.

* Guitar missed in 1855,54 , and 55 , and was not covered in 55 .

\section{RUBENS MARE,}

Bred by Lord Bervers, in 1819, her dam, Spotless, by WaltonTrumpator-Highflyer - Otheothëa, by Otho.

1828 ch. f. by Abjer .............. Col. Wilson

$1852 \mathrm{ch}$. c. by Osear (son of Juniper) .....

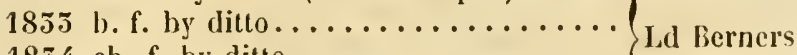

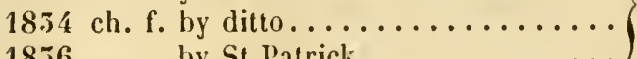

1856

by St Patrick.............

Barren in $1829,50,51$, and 55.

\section{RUBENS MARE,}

Bred by Lord Bersers, in 1819 , her dam, Tippilywitchet, by Waxy, out of Hare, by Sweethriar-Justice-Chymist-South.

189 b. f. by Woful................

$1826 \mathrm{ch}$. c. by IIazard (son of Haphazard).. Col. Wilson

1827 ch. c. (Recorery) by Emilius ........ 
182s ch. f. (Camarine) by Juniper.......

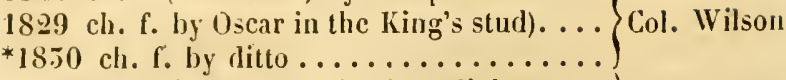

+18.51 cl. f. May-rlay, by Lamplighter ...

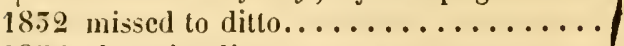

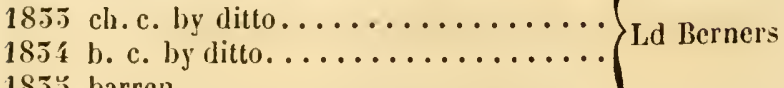

183 barren...................

18.56 by Lamplighter...........

- This Oscal mare produced a ch. f. by Lamplighter, in 1855, and was coveresl by Taurus.

$+M_{A Y-D A Y}$ broke ber leg in rumning for the Oaks 18j4, and was shot.

\section{RUBENS MARE (sister to Pastille)}

Bred by the Duke of Graftos, in 1820, out of Parasol.

1825 b. c. Cigar, by Skim (sent to India in

1828)

1826 missed to Phantom ............

1827 ch. f. Citron, by Centaur .........

1828 b. f. Catalpa, by ditto ..........

*1899 ch. f. by ditto ............... D. of Grafton

1850 b. f. Cymbal, by Emilius (put to the

1851 ch. c. by ditto (died 6 wecks old)....

1852 b. c. Clarionet, by ditto ..........

1855 b. c. Calmuck, by Zinganec.........

1854 b. f. by Reveller .............. Ld Exeter

Died in the autumn of 1854 , in foal to Revcller.

- This mare was sent to France in the autumn of 1854 ; she had one foal previously, which died.

\section{RUBENS MARE,}

Bred by Sir W. W. WYns, in 1821, out of the hipped mare, by Neteor-Petrowna, by Sir Peter.

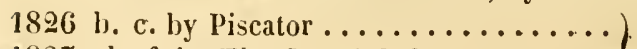

1827 ch. f. by The Grand Duke.............. Sir W. W. Wynn

1828 br. $\{$. by Piscator..............

1829 b. c. Uenry Masterton, by Master IIenry

1850 clı. c. by Waxy Pope............

18.1 br. c. Y. Tarragon, by Tarragon....

18.52 ch. c. Jacob Faithful, by Velocipede.

185.5 b. f. ly Filho da Puta ........... Sir T. Stanley

185 . missed to fielt . . . . . . . . . .

$185: 5$.. e. by Brutandorf . . . . . . . . .

1856 by Birdcatcher............ 


\section{RUBENS IIARE.}

lired by Mr Flemixg, in , her dam, Miss Jig, by Partisan. out of Jest, hy Waxy-Scotia, ly Delpini-King liergus.

1855 b. f. Foresail, by Sailor...........

1854 b. c. by Swap .............. Ir Allies

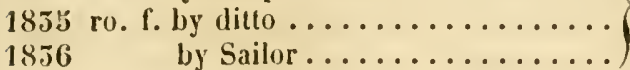

$$
\text { by Sailor. }
$$

\section{RUBENS MARE,}

Bred by IIr Dcxdas, in 1826, her dam, llelen, by AuticipationStamford-Merryfield's dam, by Star.

1852 b. f. Fame, by Trumpeter........

1853 not covered last year................ Hr llayward

1854 br. f. IIemlock, by Brownlock.......

1855 by ditto ........................ Baron

Henlock and the Rubens mare were sold to Baron Biel in the spring of $185 \mathrm{~s}$.

\section{RUBY,}

Bred by Mr Rawlissor, in 1823, got by Rubens, out of Revenge's dam, by Williamson's Ditto-Agnes, by Shuttle-llighllyer.

$1855 \mathrm{br}$. f. by Fungus, or the Barber .....

1854 b. c. Chadlington, by Fungus...... Ir Rawlinson

1855 b. f. by ditto................

1856

by ditto.

\section{ST PATRICK MARE,}

Bred by ur Jaeces, in 1826, her dam, Comedy, hy Consus Star-Y. Marske.

1852 ch. f. IIanta, by IIemnon.........

1854 b. c. Dirmid, by ditto............ Mr S. L. Fox

1853 foal, by $\Lambda$ ctaon (died) ...........

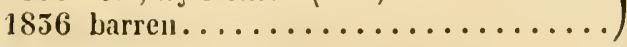

\section{ST PATRICK MARE,}

Bred by Lord Kelberxe, in 1828, her dain, Blue-stocking. by John Bull-Pipator-Plıœnomenon-Calliope. 
1855 ch. c. by Actron. . . . . . . . . .

1854 ch. c. by Albany (son of Smolensko) ...

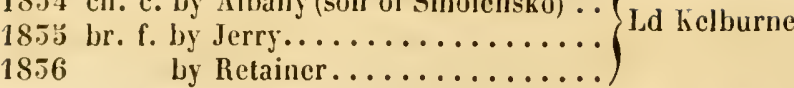

\section{SAI, (sister to Sam)}

Bred by Mr Trosxnill, in 1816, got by Scud, her dam, Hyale, by Phœnomenon, out of Rally, by Trumpator-Fancy.

1825 ch. f. Miss Tree, by Merlin........ Mr Tarleton

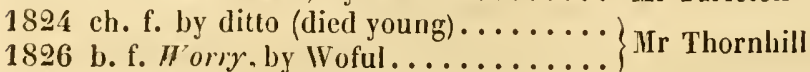

1827 b. c. by Emilius............... Ld Sefton

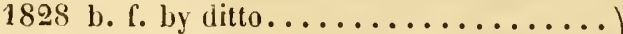

1829 b. c. by ditto (s. to Germany).......

1850 b. f. by ditto ................ Mr Thornhill

18.31

1852 b. f. by Merlin ...............

Missed in 1825,35 , and 54 ; slipped foal in 55.

SAL,

Bred by Mr Нерwовти, in 1825, got by Reveller, her dam, Terpsichore, by Woful, out of Nitre, by Precipitate.

1899 b. c. Carlton, by Catton.......... Mr W. Scott

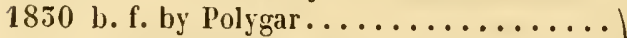

1831 b. c. loy Velocipede.............

1852 b. f. by Lottery ...............

1835 b. f. by Catton............... Mr MIuscroft

1834 b. c. by Voltaire

1853 b. f. by Langar

18.56

by Voltaire

SALAMANCA, by Sir Solomon(Vol. III, p.535) died in 1850 . Romeo was a br. not a gr.c.

\section{SALVADORA,}

Bred by Sir R. K. Dick, in 1820, got by Outery, out of Bella, by Beningbrough-Peterea, by Sir Peter.

1826 ch. f. by Orlando (died young) ...... 18 \} Sir R. K. Dick

1829 br. c. Pouch.box, by Canteen...... Capt. Rous 


\section{7}

1850 br. c. Smoter, by Osmond (s. to Ger-)

1851 ch. f. Miss Emily, by Actaon......

1852 b. f. by Brunswick.............. Sir Wm. Scott

1855 ch. c. Matadore, ly Caccia Piatti ..... Sr Watson
1854 ch. f. La Somnambula, by Brunswick Mr W.

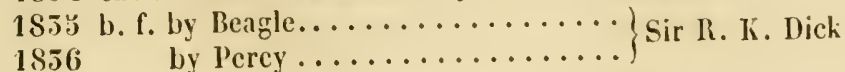

Missed in $182 y$ and 28.

\section{SAM MARE,}

Bred by Mr Thorxulz, in 1826, her dam, Morel, by Sorcerer, out of Ilornby Lass, by liuzzard.

1850 br. c. Dromelary, by Camel....... Sir M. Wood

1851 missed.................. Mr W. Chifney

18.92 br. f. by Whisker............. Sir M. Wood

$1855 \mathrm{ch}$. c. by Emilianus ............ D. of Cleveland

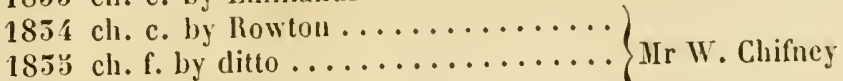

1856

\section{S.INCHO MARE,}

Bred by Lord FoLey, in 1810, out of a sister to Miss Furey and Chippenlaam, by Trumpator.

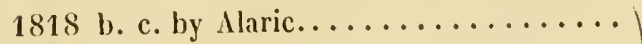

IS19 b. f. by ditto...............

1820 clı. c. by pharamond (dlead)........

1821 b. f. by Clavelino .............

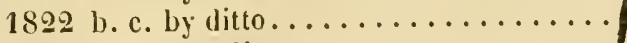

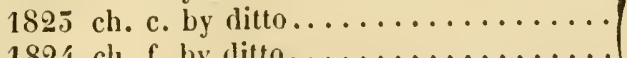

1824 ch. f. by ditto ...............

1826 br.f. Theresa, by Whalebone(s. to Baron
Bicl in 1850, in Coal to Nigel) Mr Sowerby

1827 br. f. Jannette, by Abjer (s. to Sweden

in 1851)

1829 ch. c. Castilian, by ditto ........

1850 ch. c. Catalonian, by Skiff ........

$1852 \mathrm{ch}$. f. Calalina, by ditto (s. to France

in 1854)

1855 br. f. Corumna, by ditto .........

Missed in 1825, 28, 51 , and 54 , and was shot.

YOL. 1Y. 
The S.INGHO MARE, out of Blacklock's dam (Vol. III, p. 535) produced a ch. f. by Velocipede, in 1832, and was shot.

\section{SANDBECK MARE,}

lired by Lord Fitzwiliay, in 1850, her dam, Parthenessa, by Cervantes, out of Marianne, by Sorcerer.

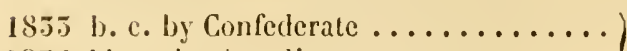

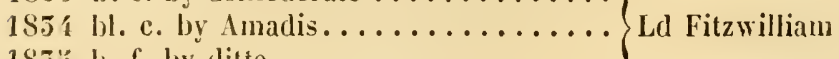

$185:$ お. f. by ditto..............

Missed to The Hole in 1856 , and put into the hunting stable.

\section{SARAII (sister to Swiss)}

Bred by Mr W. Peinse, in 1825, got by Whisker, out of sister to Corduroy, by Shuttle - Larly Sarah, by Fidget.

1850 br. c. Thrf-cutter, by Lottery........ Mr Coleman

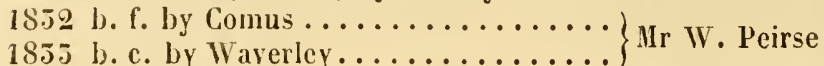

1854 b. c. by ditto............... Mr J. Day

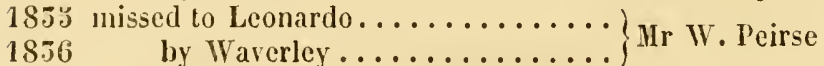

\section{SARSAPARILLA,}

Bred by Mr Panter, in 1821, got by Milo, out of Leviathan's dau, by Windle-Anvil-Suap.

1828 clı. f. Sassafras, by Champion......)

1829 br. c. Decoction, by Gulliver ....... Hr J. Painter

1850 br. c. Mezereon, by Lottery.......

$195.5 \mathrm{ch}$. c. by Memmon............. Mr Price

Missed in 1851,52 , and died in 55.

\section{SATURNIA,}

Bred by Mr Itrwoon, in 1828, got lyy his grey Arabian, her dam, Loretta, by Whitworth, out of Esther, by Shuttle.

1851 gr. c. by Hr Astley's black Turk or Barb

$1852 \mathrm{gr}$. . Tritouia, by his chesnut Arabian

1855 ch. c. by ditto................ Mr Attwood

1854 gr. f. by Warerley (dead)..........

185 \% J. f. by $\operatorname{Argantes} . . \ldots \ldots \ldots \ldots \ldots \ldots)$ 


\section{SC.INDNI.}

Bred by Lord lowtmen, in 1822, got by Selim, liel dam by IIaphazard-Precipitate-Colibri, by Woodpeclier.

1828 b. f. by Comus ............... Mr Cooper

1829 b. c. Sir Benjamin Backite (aft. Sir. Wr Forth

1851 b. c. Intriguer, by Reveller ........ Mr W. Edwards

1855 clı. f. Whisper, by The Colonel ..... I.I Uxbrilge

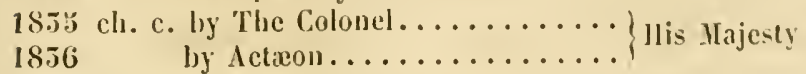

She had no foal in 1850,52 , or 51 .

\section{SCARPA,}

Bred by Mr Parre, in 1818, got by Crispin, lıer dam, Bizarre,

by l'eruvian, out of Violante, hy John Bull-sister to Skyscraper.

1826 ch. c. Geoffrey C'rayon, by Rubens... MIr T. Ml. Goodlake

*1827 ch. f. Carmine, by ditto.......... Mr Molony

1828 ओ. c. Africanus, by Emilius......... Mr T. Thornhill

1850 gr. f. Mouse Deer, by the Arab Buckfoot) Sir X. Wood

(died Mlay 185j)

1851 ch. f. Cornelia, by Emilius.................... Tr Thornhill

18.52 b. f. Lepida, by ditto..........
In 1829 slipued a filly by Rubens, 185.5 by Fungus, and died suddenly in March 1855 .

* Casuse produced in 1854 a b. c. ly Jameluke, missed to A grecable in 1855 , and was sent to Germany in 1855 , covered by Camel.

\section{SCIIEDAII,}

Bred by Lord liensers, in 1816, got by Juniper, lier dam, Spotless, by Walton-Trumpator-llighflyer.

1828 br. c. Whiskey, by Tiresias ........ Col. Wilson

$18 \pi$. br. c. by Oscar.............. Ld Berners

1853

1834

1856

f. by Zingance.................... Ld Orford

by Taurus, or Clearwell......

Dead foal in 1897; missed in $26,29,50,51$, and $5: 5$.

SCHEHE,

Bred by Jord Lowruen, in 18פ5, got by Tiresias, out of Scandal': dam, ly Haphazard-Precipitate-Colibri.

1829 br. f. (Intrigue) by Partisan .......

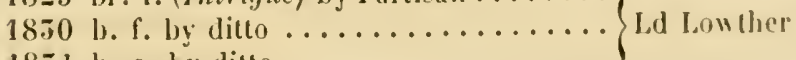

1851 b. c. ly $\operatorname{ditto} \ldots \ldots \ldots \ldots \ldots \ldots \ldots$ 

1852 b. c. Trickster, by Partisan .........
185.5 b. f. Coquine, by ditto (put to the stud) Missed to Mameluke in 1834, and sold to Baron Biel.

\section{SCHUHLA (sister to Varna)}

Bred by Lord Exeter, in 1827, got by Sultan, out of Bess, by Waxy - Vixen, by Pot8o's.

1855 b. c. by Zinganec.............. IIr Greatrex

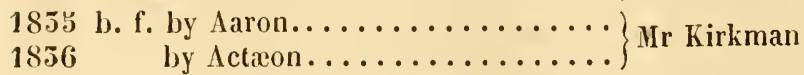

Sent to the United States in 1855 , with her foal.

\section{SCOTCH KATE,}

Bred by Mr T. Hutcursos, in 1825, got by Viscount, her dam by Remembrancer, out of Mary, by Y. Marske.

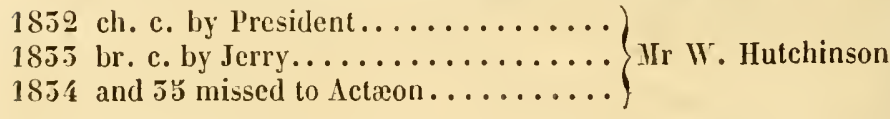

\section{SCRATCH (sister to Scandal)}

Bred by Lord Lowtiler, in 1820, got by Selim, her dam by Haphazard-Precipitate-Colibri, by Woodpecker-Camilla.

1825 b. f. Scribe, by Woful...........

1826 br. f. by Tiresias ............... Ld Lowther

1827 br. f. by ditto (died in 1829 , on the pas-
sage to Ircland)

1828 ch. c. Tam o' Shanter, by ditto ..... Mr Williamson

1829 ch. f. Scufne, by Partisan. . . . . . . . )

1850 br. f. Scurry, by ditto ........... Ld Lowther

18.31 slipped foal, by ditto..........

1852 ch. c. Splinter, by ditto . . . . . . . Sir II. Wood

1853 br. f. Skilful, by ditto ........... Mr W. Scott

1834 br. f. Claw, by ditto............ Mr J. Day

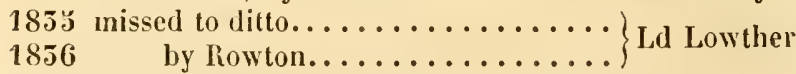

\section{SCRIBE,}

Bred by Lord Lowther, in 1825, got by Woful, out of Scratch. 1899 b. f. Sprat, by Partisan.......... Mr Williamson 


\section{1}

1850 br. f. Only That, by Partisan (put to the stud) $_{\}}$Sir J. Gerard

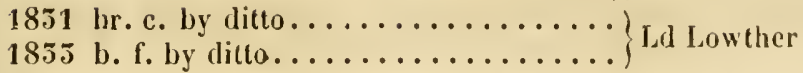

Missed to Partisan in 1832 and 54 , and sent to llungary in October 1834 , covered by Partisan.

\section{SCUD MARE (the chesnut sister to Sailor)}

Bred by Mr ThовмнцL, in 1820, her dam, Goosander, by llambletonian, out of Rally, by Trumpator.

1825 ch. f. by Merlin.............. Mr Thornhill

1827 ch. c. Cloudesley, by ditto (s. to D. of Holstein in 1852)

1828 slipped foal, by Werlin..........

1829 b. c. (Cupid) by Emilias ...........

1850 ch. c. by ditto (died young) ......... Mr Roberts

1851 b. c. (Althorp) by ditto (s. abroad, 1856)

-1852 ch. f. by Whisker.............

1855 missed to Partisan.............

1854 slipped foal. by the Colonel ........

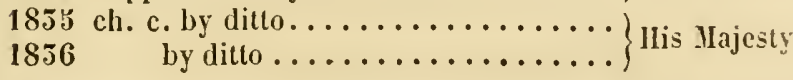

SCUD (or PIONEER) MIARE,

Bred by Mr GoLdrrg, in 1820, her dam, Canary-bird, by Whiskey, or Sorcerer, out of Canary, by Coriander.

1824 ch. c. (IIawk's Eye) by IIerlin ...... Ld Anson

1823 b. c. by ditto (died young)......... IIr Thornhill

1827 b. c. Error, by ditto............

*1828 b. f. Echo, by ditto ............

1829 ch. c. Vertigo, by ditto.........

1850 b. c. Imbar, by ditto........... Ir Forth

1851 b. f. (Nimble) by ditto............

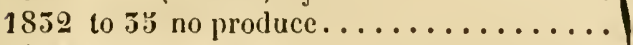

1836 by Hokee Pokee............

* Echo produced, in 1855 , a b. c. ly Gaberlunzie, and was covered by IIoliee Pokee. 


\section{SCUFFLE,}

Bred by Lord Lowther, in 1829, got by Partisan, out of Seratch, by Selim - Haplazard-Precipitate.

185 4 b. f. by Lapdog................ Ld Lowther

185.3 ch. c. by Reveller..............

1856 by Lapdog............. Mr Tattersall

\section{SCURRY (sister to Scuflle)}

Bred by Lord Lowther, in 1850, as above.

1854 b. f. by Cetus ............... IIr Williamson

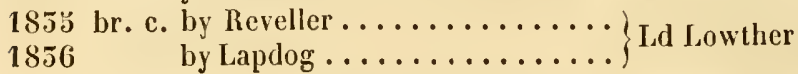

\section{SEA-BREEZE,}

Bred by Col. Yates, in 1824, got by Paulowitz, her dam, Zephyretta, by Iledley, out of Momentilla's dam, by Diomed.

1855 br. c. by Edmund.............

1854 br. f. by ditto ................. Hr C. II. Turner,

1855 br. f. by Camel............. jun.

1856

by ditto................

\section{SEA-MEIV (sister to Sailor)}

Bred by Mr Tnorvill, in 1815, got by Scud, her dam, Goosander, by Hambletonian, out of Rally, by Trumpator.

1820 b. c. by Rubens.............. Ir Thormhill

*1821 b. c. Myrmidon, by Partisan.......

1822 b. c. Fanst, by Merlin........... Mr Greville

1823 ch. f. by ditto ..............

1824 ch. f. Martha, by ditto .......... Mr Forth

1825 b. c. (Mercury) by ditto........... Mr Thornhill

1827 br. e. St Nicholas, by Emilius ...... Mr F. Richardson

1851 b. c. Unicorn, by ditto......... Mr Grant

1852 b. c. by ditto............... Sir I. Glyn

1835 b. c. by $\operatorname{ditt} 0 \ldots \ldots \ldots \ldots \ldots \ldots \ldots$. IIr Shackel

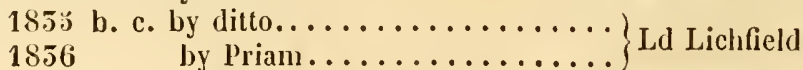

Missed in $1826,28,29,50$, and 54 .

* Myrmdon was sent to France in 1824, brought back to Englandin March 1820 , and sold the same month to the Prussian Government. 
SELIII MARE, Clara (Vol. III, p. 565) has had no produce from 1832 to 53 inclusive. - Massaroni was sold to Baron Biel, and the Reveller filly sent to Germany.

\section{SELIM MIARE,}

Bred by Lord Exeter, in 1821, her dam by Golumpus, out of sister to Beningbrough, by King Fergus-Ilerod.

1826 b. c. by Centaur...............

1827 br. c. lyy ditto (died 2 years old)..... Mr R. Prince

1828 c. by ditto...............

1829 b. f. by Camel (s. to Germany 1852)..

1850 br. c. by ditto ...............

1851 f. by Tiresias............ D. of Portland

1852 slipped foal, by ditto $\ldots \ldots \ldots \ldots \ldots$

1853 b. f. by Banker (died young)........

1835 missed to Gustavus, and sold ......

\section{SELIII IIARE (chesnut)}

Bred by Mr Sabler, in 1829, her dam, Euryone, by Witchcraft, out of Fair Ellen, by the Wellesley Grey Arabian.

1828 br. f. Euryone, by Reveller (put to the

1899 br. Europa by ditto................

1831 b. c. Defensive, by Defence........ Mr Sadler

1852 ch. c. (Safeguard) by ditto..........

1854 ch. c. Defender, by ditto..........

1853 br. c. by ditto .................

Missed to Middleton in 1850 , slipped foal by Iefence in $\mathbf{5 5}$.

\section{SELIM MARE (chesnut)}

Bred by Mr Crockrond, in 1824, her dam by lipator, out of Qucen Mab.

1829

18.51

c. by Camel ...............

$1852 \mathrm{ch}$. c. by Patron .............. L L Lynedoch

1853 missed to ditto .................. ( 1854 ch. f. by Partisan (s. to Berlin 1853).. Lowther

1835 ch. f. by ditto ...............

Sent to Germany in 183 , covered by Rowton. 


\section{4}

\section{SELINA,}

Bred by II R Royal Highness the Duke of Yonk, in 1822, got by Phantom, her dam, Rosalina, by Giles, out of Iademoiselle, by Diomed.

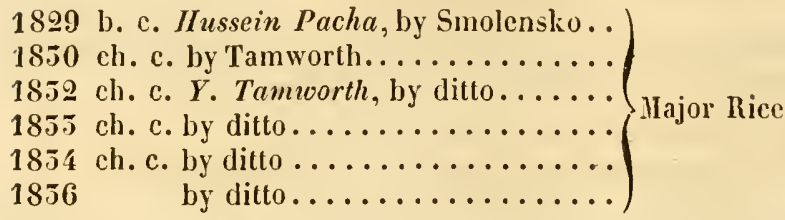

Missed to Tamworth in 1851 and 55.

\section{SELINA,}

Bred by Mr BeLL, in 1822, got by Fitz-Teazle, out of Rally, sister to Rover, by Hyacinthus-Overton-Katherine, by Ilighflyer.

*1829 b. f. by Arbutus..............

1851 br. c. Lot, by Brutandorf................. Mr Bell

1855 b. f. by Humphrey Clinker..........

1856 by Clinker (son of ditto).......

* This Arbutus mare prodnced a b. e. by Sandbeck in 1855, and died soon after; the foal was brought up by hand.

\section{SEI.INA,}

Bred by Mr R. Wilsor, in 1826, got by Nícolo, her dam by Shuttle, out of sister to Dowager, by Hambletonian-Goldenlocks.

1850 ch. f. Solitaire, by Emilius ........

1852 b. c. The Philosopher, by Filho da Puta Mr Frewen

(s. to D. of Ilolstein in 1853)

1854 ch. c. by Augustus.................... Hr WV. Cooper

185 b ch. c. by Cain ...............

Not covered in 1850 or 52.

\section{SELIIA,}

Bred by IIr Haxkey Smitn, in 1817, got by Selim, her dam by Kill Devil, out of Juliana, by Crop-Alexis-Boxer's dam, by Blank-Snip-Parker's Lady Thigh. 
1824 b. c. Soldan, by Vandyke Junior .... IIr W. Fox

1826 b. c. Scheik, by his Lordship's Arabian 1827 b. c. by IIoses (died a foal) ........ 1829 ch. c. (The General) by Whisker.... 1850 b. f. by St latrick (died a foal)..... L Ld Orford

1851 b. f. by ditto (s. to Germany in 1855 , covered by $\Lambda$ corni)

1852 ch. c. by ditto..............

185.3 b. c. by Emilius (died a foal) ........

183 z̆ cl. c. Lara, by Cain............. Ld Stradbroke

In 1825 missed to Hacduff, 28 to Perchance, 34 to St Patrick.

\section{SENTIMENT,}

Bred by Mr Killaro, in 1822, got by Sclim, out of Louisa, by l'egasus-Nelly, by Postmaster.

1826 ch. c. Toaster, by Reveller .........

1827 ch. f. Tipss, by ditto.................. Hir Dilly

1852 ch. f. Termagant, by Luzborough ...)

1853 ch. c. Tyrant, by ditto.......... MIr Wreford

1834 br. f. by Wrangler (Rajah) ...........

1853 b. c. by Defence ...............

Missed to Moses in 1828, to Lapdog in 29 , to Luzborough in 51 , and sold to Count Szechenyi in 1855 , corered by Defence.

\section{SERENADE,}

Bred by Mr Nowect, in 1824, got by Rainbow, her dam, Scheherazade, by Selim, out of Gipsy, by Trumpator-Herod.

1851 b. c. hy Redgauntlet (cut at 2 yrs old) 1852 slipped foal, by Emilius ...........

1853 br. c. hy Oswestry ............ Ir Trelawny

1854 slipped foal, by Hindostan.........

1853 b. f. by ditto................

1856

by ditto................

\section{SIIERRY,}

Bred by Mr S. Pearce, in 182:, got by Pendulum, out of Caroline, by Trombone. 
1829 br. c. March, by Eryx ..........

1850 br. f. by ditto ................. Mr S. Pearce

1851 b. f. by ditto (put to the stud) ....... Mr S. Pearce

Covered by Eryx in 1852, and sold to go abroad.

\section{SHOE-HORN (sister to Shoe-strings)}

Bred by Sir Jony Cope, in 1816, got by Teddy-the-Grinder, her dam, Roxana, by Alexander, out of l'rincess, by Eclipse.

1825 b. c. by Cannon-ball, or Interpreter. . Mr Forth 1824 ch. c. Granby, by Cannon-ball ....... Mr Petre

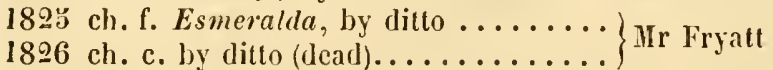

1827 b. c. Bullet, by ditto............

1828 ch. f. Mopsa, by ditto...........

*1850 b. f. Monica, hy Lottery ..........

1851 ch. c. Shot, by Velocipede ........

1852 missed to ditto.............. Ir Wormald

1855 ch. f. Zelinda, by ditto .........

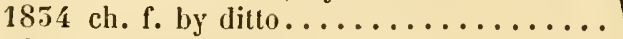

185 s ch. f. by ditto..............

* Movica produced a ch. c.by Yelocipede, in 1855, and wascovered again by him.

\section{SHORTWAIST,}

Bred by Mr WALKER, in 1825, got by Interpreter, out of Nancy, by Dick Andrews - Spitfire, by Beningbrough.

1829 b. c. by Partisan............. Mr Walker

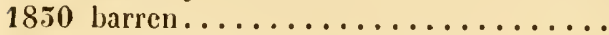

1851 ch. c. by partisar.............. MIr Forth

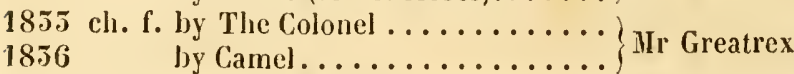

If she had any foals between 1855 and 56 , they are dead.

\section{SHOVELER (sister to Sailor)}

Bred by $11 \mathrm{r}$ Tuorxule, in 1816, got by Scud, her dam, Goosander, by Hambletonian-lially, by Trumpator. 


\section{7}

1822 b. f. Mercy, by Merlin...........

1824 b. f. Mulebird, by ditto..........

1825 l. c. Magnet, by ditto............ Hr Thornhill

1827 b. c. Captice, by Cervantes (s. to France)

1828 b. f. Eamoig, by Emilius..........

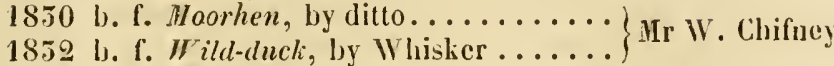

1855 ५. c. Eric, by Emilius . . . . . . .

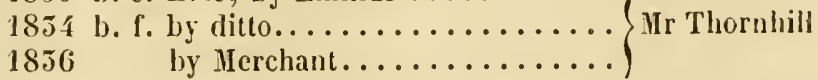

Missed in $1825,26,29,31$, and $5 \%$.

\section{SHOW LASS,}

Bred by Lord Exerer, in 1850, got by Mountebank, her dam, by Woful, out of Zealot's dam, by Rubens.

18.54 b. c. by Walthamstow ..........

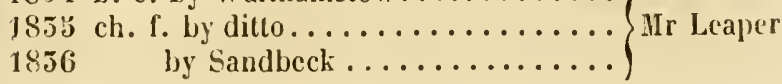

\section{THE SIHEWV,}

Bred by $\quad$, in 182y, got by Master llenry, her dam, by Precipitate-Highflyer-Dragon's dam, by Spectator.

1831 c. by St Patrick............. Mr R. Prince

$1852 \mathrm{ch}, \mathrm{c}$. Jat, by ditto............ Mr Greville

1855 b. f. by Zingance............. Mr R. Prince

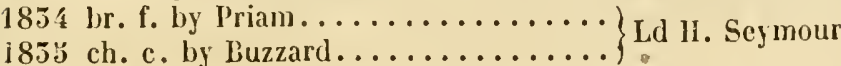

She was sent to France in 1834, with her foal.

SIIUTTLE MARE, Lady of the Vale's dam (Vol. III, p. 370 ) died in 1854. Her produce of 1829 died a foal, and she had only one foal after 1830 -viz. :

1852 ch. c. Actconides, by Actæon....... Sir J. Boswell

\section{SHUTTLE MARE (sister to Corduroy)}

Bred by Sir Williax Gerard, in 1812, her dam, Lady Sarah, by

Fidget-Alfred-Magnolia, by Marske-Babraham-Sedbury .

1816 b. c. Ly Walton (dead) ................... Mr Weirse

1817 b. f. by ditto................

1818 b. f. by Smolensho............ Mr James 
1819 ch. c. by Woful............. Mr W. Peirse

1820 ch. c. Crab, by Leopold........... Mr Frankland

1821 b. c. Swiss, by Whisker.......... Col. Cradock

1822 b. c. by ditto (died 2 yrs old) ........ Mr W. Peirse

182.3 b. c. King Calton, by Catton........ Ld Kennedy

1824 b. f. by Whisker (died 2 months old). Mr W. Peirse

1825 br. f. Sarah, by ditto........... Ld Kennedy

1823 b. c. Prince Eugene, by ditto...... 1827 br. f. by W W. Peirse

1828 ch. f. Suke, by ditto............ Mr Thompson

ch. f. by ditto (foaled in December)...

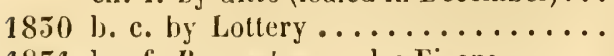

1851 br. f. Benevolence, by Figaro .......

1832 ch. f. by Comus.................... Mr W. Peirse

1855 b. f. Elizabeth, by Waverley.......

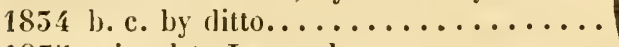

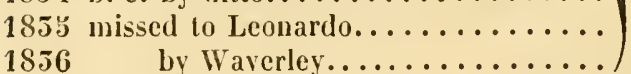

* This December filly produced a b.f. by Waverley, in 1855 , and was covered by him in 55 , having missed in 54 and 35.

\section{SHUTTLE MARE (sister to Pope)}

Bred by Mr Barlow, in , her dam by Oberou, out of The

Engraver's dam, by Stride-sister to Sharper, by Ranthos.

1821 br. c. Elephant, by Filho da Puta....

182.3 br. f. Elephanta, by ditto (s. to France)

1824 br. c. Bhurtpore, by Jagistrate (s. to

the Duke of Lucca in 1829) Mr Ilouldsworth

1823 br. f. by Fillo da Puta...........

1826 br. f. by Sherwood, or Hagistrate....

1899 b. f. The Fiend, by Whisker........

1850 br. f. Eagle, by Lottery .......... Mr Moss

1851 b. c. Emigrant, by Figaro (s. to Ger-) Mr IIouldsworth

1852 b. f. MIadame de Jeck, by Langar.... Mr Spalding

1853 br. f. by Vanish............. Mr Houldsworth

1834 b. c. by ditto............... Mr W. Houldsworth

Missed in 1822, 27, and 28 .

SHUTTLE MARE (sister to Pope)

Bred by Mr BarLow, in 1815, as above. 
1820 b. c. Stratherne, by Whisker......

1821 b.c.Baugundee(aft. Moredun) by Bustier

1822 b. c. (dead) by Prime Hinister....... Sir 1). Moncrieffe

1824 b. c. Strathay, by ditto.........

1823 b. c. Nararino, by Whisker.........
1826 b. c. by l'rime Minister........... Ir Nowell

*1827 b. f. Jay, by Slufller............ Mr Wagstall

1829 br. f. $L^{\prime}$ Amitié, by Muley.......... Mr S. llay

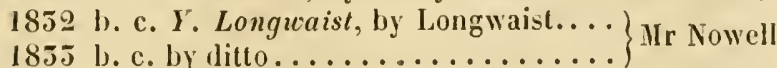

Missed to Muley in 1828,50 , and 51 , to Longwaist in 54 , and is dead.

- Jay produced a b. f. Lottery in 1852 , which died, and slie was sent to Germany in $185 \tilde{5}$, with a colt foal by St Nicholas.

\section{SIGII,}

Bred by Colonel Yates, in 1825, got by Guy Mannering. out of Zephyretta, by lledley-Diomed-Imperator.

1828 gr. f. Sylph, by Swap ..........

1829 ch. f. Sensitive, by Cain......... Mr Yates

1851 b. c. I"illiam, by Edmund.........

1852 ch.c. Sylvan, by Cain........... Col. Pecl

1853 b. c. Edward, by Edmund.......... Mr Yates

1834 b. f. by Mamcluke.............. Mr Tattersall

1856 by Oppidan............ Ld Southampton

Missed in 1850 ; in 55 cast twins by Cain.

\section{SIGNORINA,}

Bred by Mr Nanney, in 1822, got by Champion, her dam by

Williamson's Ditto, grandam by Star, out of Baron Nile's dam,

by $\mathbf{I}$. Marske.

1829 b. c. by Banker..............

1850 br. f. by Filho da Puta..........

1851 missed to Camel..............

1852 br. c. Friar Tuck, by Emilius....... Mr Nanney

1855 br. f. by Flexible.............

$1854 \mathrm{ch}$. c. by Priam ..............

1835 ch. f. by Belzoni..............

She won the cup at Wrexham, in October 1828 , when in foal with the Banker colt.

VOL. IV. 


\section{0}

\section{SILVERTAIL,}

Bred by Lord Egrenovt, in 1815, got by Gohamma, her dam by Orville, out of Selim's dam, by Alexander-llighllyer.

1821 ch. c. (Shadow) by Ihantom (sent to the

1894 ch. f. by Partisan................ Mr Wyndham

1823 ch.f. Uryanda, by Tiresias(s. to Ireland)

1826 br. c. Augur, by ditto........... Ld Southampton

1827 br.f. Hiss IIenry, ly ditto (s. to France)

1828 h. f. by ditto................ Mr Wyndham

1829 br. c. Argent, by Whalebone (s. to Virginia in 1854)

1850 b. c. by Lottery (died young) .......

1851 dead colt, by Velocipede.......... Mr Wilson

1855 ch. f. by Comus ................

1854 ch. c. Quiclisilver, by Velocipede....

1853 1. e. by Voltaire..............

18.56 by Mulatto..............

Missed in 1822, 25, and 52.

SIR ANIJREIV IIARE (a twin)

Pired lyy Mlr Watr, in 1815, her dam, Tuneful, by Trumpator, out of Sea-fowl, by Woodpecker.

$181 ;$ b. c. Apollo, by Whitworth ........ Mr Yales

1818 b. f. by Macbeth (not trained)....... Mr L. Carter

1819 b. c. by Leopold (died) ...........

1820 b. f. by ditto (nol trained)......... IIr T. l'eirse

1821 b. c. by Whisker (died) ...........

1822 b. c. Vapid, by Octavian .......... Mr Metcalfe

1825 b. f. by Don Juan.............. Mr L. Carter

1825 br. c. Tiploe, by Percy............

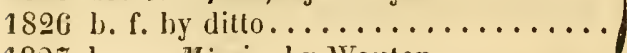

1827 br. c. Mimic, by Wauton ..........

1829 b. f. Myrtle, by ditto ........... Mr ILetcalle

1850 l. f. by Whisker (died 2 yrs old).....

1851 br. c. Monitor, by Wanton.........

1856

by Physician ..............

Missed in 1824, 52, 53, 54, and 55 ; not coveredin 27.

SIR DAVID MARE,

Bred by Lord Beinaven, in 1815, her dam by Hambletonian, out of Lady Mary's dam, by Highflyer-Mlarskic. 


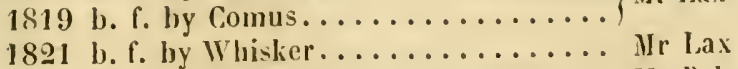

1825 b. c. lyy Don Juan................ IIr Palmer

182 亿 b. f. by Grey Malton.............. Wr llamilton

1826 br. f. ly Smolensko................ Wr Mlarley

1828 b. f. (Braithwaite, aft. The T'utor) by $\}$ St Mratrick ) Mrarrison

1830 br. f. Palm-flower, by Jerry.........
1831 br. f. Mar-flower, by ditto.......... Mrarley

1852 b. c. The Stag, by Actieon.......... IIr G. Crompton

1834 b. f. by Actæon ................ Mr Jlarley

1833 c. by ditto................

Missed to St Patrick in 1829, to Jerry in 36 , missed also in 18.5.

\section{SIR IIARRY DINSDALE WIARE,}

Bred by Mr Lase, in 1820, her dam, Orelia, by Eagle, out of Roxy.

by Alcxander-Winifred, by Justice-Cygnet-Cartouch.

1825 br. c. by Muley...............

1826 br. f. ly ditto (put to the stud) ......

1827 missed to Herlin..............

1828 br. c. Hypocrite, by Rector.........

1829 br. c. by ditto ...............

1830 b. c. by ditto ................. Mr Lane

1831 b. f. by ditto...................

1852 br. f. by ditto ................

1853 b. c. by ditto.................

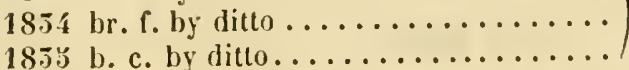

\section{SIR OLIVER MIARE,}

Bred by Mr Yates, in 1816, her dam, Miss Blanchard, by Hanble-

tonian, out of Lady Sarah, by Fidget-NIfred-Magnolia.

1820 ch. c. Demon, by Magic..........

1821 ch. f. hy Friday ................. Jr E. Peel

1822 by Paulowitz $\ldots \ldots \ldots \ldots \ldots \ldots$. 18.3 br. c. Nemo, by ditto..........

182.5 br. c. Nemo, by ditt............. Mr Thompson 18.31 ch. f. Miss Kershaw, by Predictor (s.) $\begin{array}{r}\text { abroad) } \\ \text { Ld Chesterfield }\end{array}$

18.52 ch. f. by ditto ...............

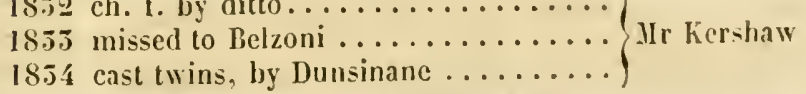




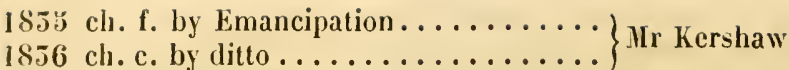

She was put to common horses from 1825 to 30 .

\section{SIR OLIVER MARE,}

Bred by , her dam by Hambletonian, out of Constantia, by Sir Peter.

1829 bl. c. Eugene Aram, by Banker.....

1850 c. by ditto (died a day old).......

1851 ch. f. by Teniers (died a yearling) ...

1852 b. c. by Lottery (died 2 days old) .... S Sir T. Stanley

1855 ch. c. by Teniers .............

1854 missed to Recovery .............

1856 by Pantaloon, or Joceline.....

\section{SKIMI MARE,}

Bred by Mr Batson, in 1825, her dam, Jessy, by Totteridge, out of Cracker, by Highflyer, out of Nutcracker, by Matchem.

1850 br. c. by Partisan ..............

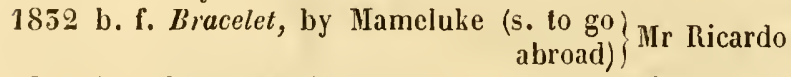

1853 b. c. by Hameluke............. Mr Hall

185 í b. f. by ditto (s. to Ireland).........

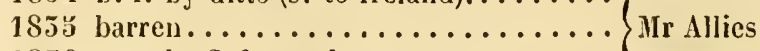

1856

by Safeguard.

\section{SKIM MARE,}

Bred by Mr BruhL, in 1827, her dam, Grey IIclen, by TeasdaleElection-Fair Helen, by Hambletonian.

1851 b. c. Little Rover, by Cydnus....... Mr I Lambden

$1852 \mathrm{gr}$. f. by a half-bred horse......... Ir Greenhill

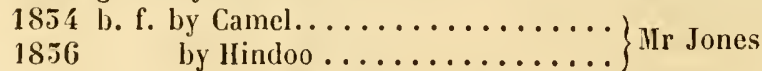

Missed in 1855 and 55.

\section{SLIGIIT,}

Bred by MIr Vansitrant, in 1816, got by Selim, her dan by Pot8o's, out of Editha, by Herod-Elfrida, by Snap.

1822 b. f. by Amadis (died a foal) .........
182.5 b. c. by Whisker (s. to Florence) ... M 
1824 b. f. by $A$ bjer...............

$1823 \mathrm{ch}$. f. by Conqueror.............

I8 26 b. f. Magawiska, by Whisker.......

1828 ch. c. Rubini, by St latrick ........

1829 ch. c. Sepoy, by Polygar.......... Mr Vansittart

1830 b. f. Rafle, by Lottery ...........

1852 b. c. by Catton................

1854 b. c. by ditto .................

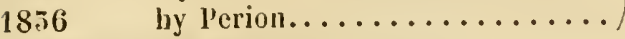

Missed to St Patrick in 1827, to Lottery in 51, to St Nichulis in 35 , to I'erion in 53.

\section{SLIGO MARE,}

Bred by Lord Lichfield, in 1829, her dam, Ina, by Smolensko, grandam, Morgiana, by Coriander, out of Fairy, by Highllyer.

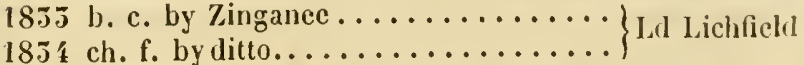

Was killed in 1353, in foal to Emancipation.

Bred by

\section{SMASH,}

in 1822, got by Soothsayer, her dam, Mary, by Gohanna.

1829 ch. f. Rosine, by Rossini.......... IIr Corbet

1850 not covered last year ............

1851 ro. c. Ramsbury, by Buckfoot........ IIr T. Smith

1854 ch. f. Spree, by The Colonel........ IIr Fo.

185 ch.c. by Shortwaist............. Ir Smith

1850

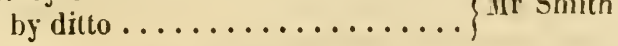

Not covered in 1829 or 51 ; missed to The Colonel in 53.

\section{SIIOLENSKO MARE (bay)}

Bred by Sir C. Buneury, in 1819, her dam, Comical's dam, by Skyscraper - Dragon-Fidget's dan, by IIatchem.

1822 b. c. (Ranger) by Partisan.......... Ir Wilson $1824 \mathrm{ch}$. c. Kit, by Mlerlin (sent to India in 1829 ) Ld Orford

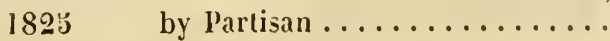

1826 br. c. Chiron, by Centaur..........

1829 b. f. IIenny, by Stainborough.... . . . Ld Chesterlield 
1850 dead foal, by Stainborough.......

1851 br. f. by ditto ............... Mr R. Wilson

1855 ch. f. by Bedlamite ............

1854 b. f. by Albany ............. Sir M. Wood

1853 foal by Grey Comus (since dead) ..... Mr Robinson

Nissed in $18 \geq 8$ and 52 .

\section{SHOLENSKO MALE,}

Bred by , in 1817 , her dam by Sir PeterMambrino-Marigold, by Ilerod.

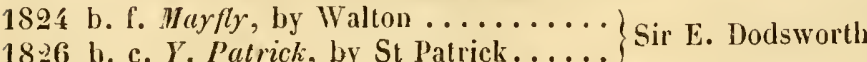

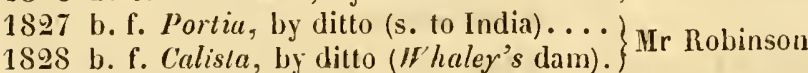

1852 br. f. by Battledore............. Mr Legh

1855 br. c. Blackburn, by Peter Lely......

1834 br. f. by Filho .............. Mr W. Turner

18.5 br. c. by Recovery.............

Missed in 1829,50 , and 51.

\section{SHOLENSKO MARE,}

Bred by Mr Gascolgne, in 1818, her dam, Miss Canuon, by Orville, out of Cannon-ball's dam, by Weathercock - Cora.

1822 gr. f. Camillina, by Camillus ....... Mr Uppleby

1824 b. c. by Cervantes............... Mr Gully

1823 b. c. by ditto............... Mr Potter

1826 bl. c. by Blacklock............. Sir T'. Stanley

1827 slipped twins, by Figaro ......... Mr Potter

1828 b. c. Tetotum, by Lottery.......... Mr Clifton

$1829 \mathrm{br}$. c. by ditto (died in foaling) ......

1850 b. c. by ditto (died a week old)......

1851 b. f. by ditto ................

1852 b. f. Miss Potter, by ditto ......... Mrs Potter

$1834 \mathrm{hr}$. c. by ditto (died a foal) ........

185 . b. c. by Ilumphrey Clinker.........

1856 by The Saddler ............

Barren in 1823 and 55.

\section{SHOLENSKO MURE,}

Bred by Sir Tatron Srkes, in 1821, her dam, sister to Orphan, by Camillus -Gabriel-Legacy, by King Fergus. 
1829 b. f. by Y. Phantom ...........

$1850 \mathrm{gr} . \mathrm{f}$. by ditto ..............

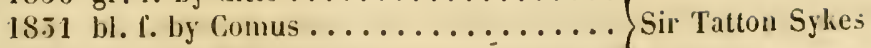

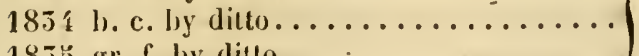

$1855 \mathrm{gr}$. f. by ditto ...............

In 1852 missed to Velocipede, 55 to Y. Phantom.

\section{SIIOLENSKO MARE,}

Bred by Mr R. Wisson, iu 1824, her dam by Shutte, out of sister to Dowager, by Hambletonian.

1828 b. c. by Negociator ............. Mr Lacey

1829 br. c. Ludlow, by Fillo da Puta (s. Mr Beardswortlı abroad)

Cast foal in 1855, missed in 52 and 54 , and sold.

- Mnselle produced a f.by Filho, or Vanish, in 1855.

+ Sister lo Ludiow produced a b. c. by Filho, or Vanish, in 1855.

\section{SIIOLENSKO MARE,}

Bred by Hr Clanke, in 1821 or 22, her dam, l'atience, by Buzzard, out of Gipsy, by Trumpator.

1829 f. by Figaro . . . . . . . . . . . Mr Foril

$\left.\begin{array}{r}1855 \text { b. c. Chance, by Lottery (s. to France } \\ \text { in 185:) }\end{array}\right\}$ Mr Kirby

Missed in 1851, 52, and sent to Germany in 55, covered by Lottery.

\section{SHOLENSKO MMRE,}

Bred by Mr R. Wrisor, in 1824, her dam, Lady Ern, by Stamford, out of sister to Repeater, by 'Trumpator-Demirep, by IIighllyer.

1828 br. f. Wedloch, by Figaro.........

1829 bl. f. Kitty Fisher, by Bravo........

1850 bl. f. by lilho da Pula............

1851 bl. c. Sir William, by Camel ...... Jr Nanney

18.52 slipped foal . . . . . . . . . . . . . .

1854 b. f. by St l'atrick.............

183 br. c. by Belzoni.............. 


\section{S.IOLENSKO MARE,}

Bred by JIr R. Wilsox, in 1824, her dam, Zoraida, by Don Quixote, out of Lady Cow, by Joln Bull.

IS28 ch. c. Miletus, by Middleton........ Mr Maberly

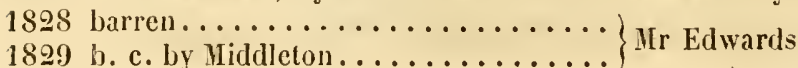

1850 ch. f. by ditto .............

1851 bl. f. lyy Stainborough ................ Mr R. Wilson

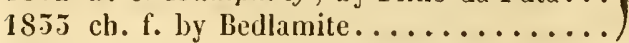

18.54 b. с. by Albany ............... J Mr Gapp

1836 by The Grand Duke.........

\section{SHOLENSKO MARE,}

Bred by Mr HoLbrook, in 1S2S, her dam, Brunette, by Waxy, out of Charcoal, by Sir Peter.

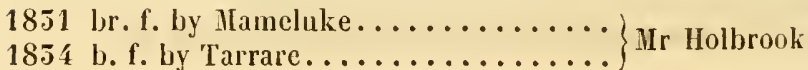

THE SMOLT,

Bred by Mr Ballue, in 1818, got by Viscount or Stamlord, uut of Penclope, by Shuttle-Lady Sarah, by Fidget-Alfred.

1825 b. c. by Whitworth (died a yearling)..

1824 br. c. Zingaro, by Champignon......

182 ว cl. c. Taures, by Ardrossan........

1828 br. f. Salmonia, by Cleveland........ IIr Baillic

1829 ch. c. by ditto..............

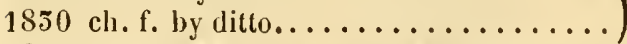

1851 br. c. (Mellerstein, aft. Masetto) by Co- Hr G. Cromptou

1836 by Physician ............ Mr Dawson

Missed in $1826,27,53,54$, and $5: 5$; not covered in 51 .

SNAIL,

Bred by Dr Wizus, in , got by Y. Warter, her dam, Elizabeth, by Beningbrough, out of Miss Judy, by Alfred-Ianilla, by Goldfinder.

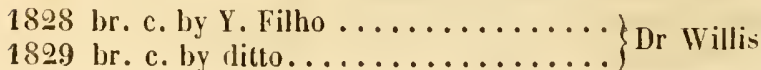




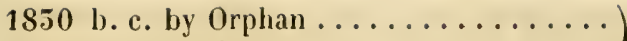

1831 b. c. by ditto ................

1852 b. f. by Mountebank............. Dr Willis

1853 l. f. by ditto (dead)..............

1854 ch. c. hy Y. Haphazard............

Dead.

SNARE,

Bred by Mr Thorrmite, in 1816, got by Scud, out of Prophetess, hy Sorcerer-Pamela, by Whiskey-Diomed.

1826 bl. c. Wrangler, by Rajah..........

1828 b. f. Wrilna, by Smolensko (s. to D. of IIolstein, 1854)

1829 br. f. Wire, by Sir JIuldibrand...... $1850 \mathrm{ch}$ or ro. c. W'onford, by Grey Middleham NIr Wrefort

1851 ओ. с. Warrener, by Sir Huldibrand.. 1853 br. f. Weasel, by Luzborough ....... 1854 br. c. by Brownlock............ 185: dead produce $\ldots \ldots \ldots \ldots \ldots \ldots \ldots$ 1856 by Defence

\section{SNOWBALL,}

Bred by Mr Rupsbale, in 1819, got by Prime Ninister, her dam,

Vesta, by Delpini, out of Faith, by Pacolet-Atalanta.

182.5 bl. f. hy Walton (died 2 days old).... 1824 br. f. Brownlock (aft. Nivalis) by Blacklock

1825 missed to Jack Spigot ............ Mr J. Smith

1826 b. f. by ditto (died 2 yrs old, in the possession of his Majesty)

1827 gr. f'. by St Patrick (died a yearling)...

1828 gr. c. M. Bish, by Lottery ......... Ld Cleveland

$1829 \mathrm{gr}$. c. by ditto...............

18.50 slipped foal, by Jerry ..........

1831 br. f. Zillah, by ditto........... Mr J. Smith

1852 b. f. Margarette, by $\Lambda$ ctæon........

185.5 br. c. Plunder, by Jerry........... Sir J. Boswell

1854 missed to Jerry ...............

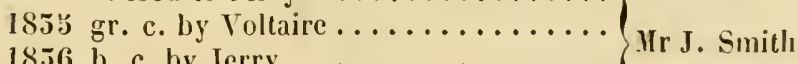

1856 b. c. by Jerry $\ldots \ldots \ldots \ldots \ldots \ldots \ldots$

18.57 by Physician 


\section{8}

SNOWDROP, by llighland Fling (Vol. III, p. 391) was shot in 1852. Galantine, her last produce, was sent to Germany in 1852, and Smilax died the same year, leaving no produce.

\section{SOLA,}

Bred by Mr Wriphall, in 1822, got by Partisan, out of sister to Caroline, by Whalebone-Marianne, by Mufti.

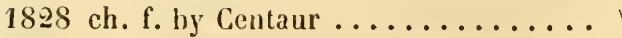

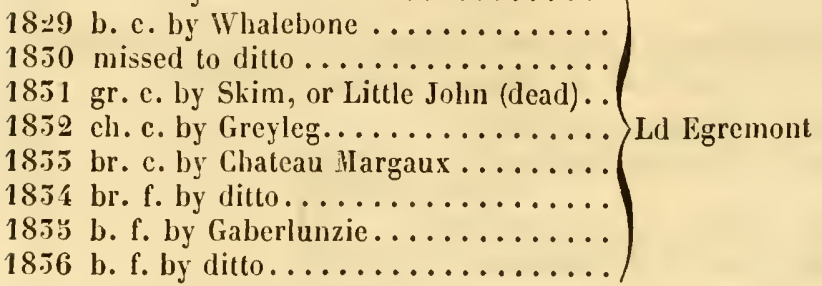

SONTAG,

Bred by the D. of Graftox, in 1825, got by Woful, her dam, Miracle, by Soothsayer, out of Merrymaid, by Buzzard-Highflyer.

1851 b. f. by St Patrick............. Mr Jas. Weatherby

1832 b. c. The Brigand, by Emilius...... . Mr Barrett

1833 b. c. Zingaro, by Zinganec.........

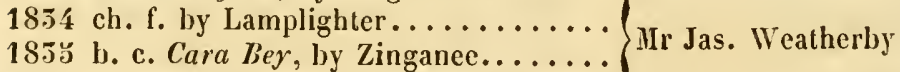

1856 by Rowton.............

\section{SOOTHSAYER IIRE,}

Bred by Lord FoLer, in 1816, her dam, Hiss Eliza Teazle, by Sir Peter, out of Eliza, by Highflyer-Augusta, by Eclipse - Herod.

1821 f. by Blucher.............. Ld Foley

1822 br. f. by Waterloo (dead) .......... Mr Nowell

1825 b. c. by ditto (s. to Germany in 1826) Mr Lambton

1894 b. c. by Rainbow.............. Mr Payne

1826 b. c. (Ofellus) by Orville.......... Ir Irby

1827

1828

18.30

18.54 c. by Bustard ................ f. by Muley...............

185.5 foal, hy ditto (died) $\ldots \ldots \ldots \ldots$ In Nowell

b. f. by Longwaist .............

b. f. ly ditto...

Barren in $1825,29,51,52,55$, and 56 . 


\section{SOOTHSAYER MARE,}

Bred by Mr Goodssox, in 181\%, her dam by Buzzard, out of sister to Bangtail, by Ilighflyer-Cahtherine, by Y. Marske-Gentle Kitty, by Silvio.

1822 ro. c. by Quizzer............. Ld Bridgwater

1824 ro. f. Miss Craven, by Mr Lowe .....

1825 ro.f. by Whalebone (killed when a foal) Mr Gates

1826 ro. f. Jung frau, by Skim.........

1829 ch. c. The IIermit, by Mr Lowe ..... D. of Richmond

1850 ro. f. Baleine, by Whalebone....... D. of Richmond

1831 ro.f. (Bodice) by ditto........... Sir M. Woor

1852 ro.f. Constantia, by Camel ......... Mr Suwerby

1855 ch. c. by Wrangler ............ Mr R. Wilson

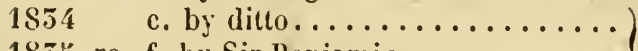

1855 ro. f. by Sir Beujamin........... Ld Chesterfield

$1856 \quad$ by Zingance............

SOOTHSAYER MARE, sister to Meg Merrilies (Vol. III, p. 395) produced a ch. f. by Luzborough, in 1852, was barren in 55 , and sold to the $\mathrm{D}$. of Holstein.

\section{SOOTISA YER MARE,}

lired at Hampton Court, in 1822, her dam, Cobbëa, by Skyscraper - Woodpecker-Heinel, by Squirrel.

1827 b. f. by Master Henry . . . . . . . .

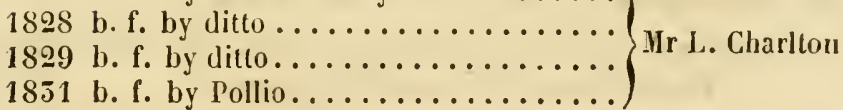

1852 b. c. by ditto ..................

1855 ro. c. Khylan, by Harlequin (the $\Lambda$ rab)

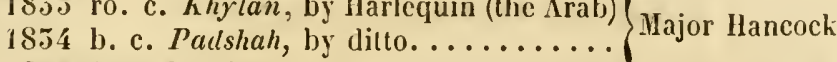

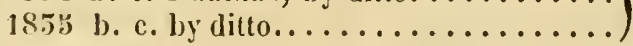

Barren in 1850 and 56 .

\section{SOOTHSAYER MARE (Oracle)}

Bred by Lord Sramford, in 1820, her dam, Miss Ilap, by Shuttle, out of the sister to IIaphazard, by Sir Peter.

1828 b. c. by Tramp (dead)............ Mr G. Clark

1829 b. c. Bassetlato, by Catton......... Ld Mountcharles 
1850 b. f. by Tramp $\ldots \ldots \ldots \ldots \ldots \ldots . . . \ldots \ldots$
$* 18.51$ b. f. Blue Bell, by Duplicate ('Tomes's) Mr G. Clark

Died in 1832.

* Buve Bral produced, in 1855 , Sir T. Stanley's b. c. Young Crutch, by Crutch, and was covered by Rowion.

\section{SOOTHSAYER MARE,}

Bred by Lord Lowtner, in 1815, her dam, Miss Furey, by 'Trunpator-Mark Anthony-Signora, by Snap.

1825 ch. c. by Andrew ............ Mr Fleming

1824 b. f. by Whalebone .............

1851 dead foal, by Hal.............

1832 b. f. by ditto (died 5 yrs old)....... Mr Burgess

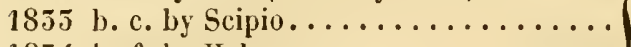

1854 b. f. by IIal................

Not covered in the intermediate years, and missed to Scipio in 53.

\section{SOOTHSAYER HARE,}

Bred by Mr P'AGE, in 1822, her dam by liubens, out of Pointers' dam, by Woodpecker-Gohanna's dam, by Ilerod.

1829 bl. f. Olga, by Paulowitz (s. to Ger-)

1851 ch. c. Alexis, by Leviathan......... Ld Chesterfield

1832 ch. c. by Enilius..............

1854 ch. c. by l'riam...............

Barren in 1828 and 50 , missed to Zinganee in 55 , and was sold to Baron Biel in 54, covered by Priam.

\section{SORCERER MARE (sister to Bourbon)}

Bred by Sir Jouv Snellex, in 1810, her dam by PrecipitateHighflyer-Tiffany, by Eclipse-Y. Hag, by Skim.

1814 f. by Gamenut (died a foal)....... Mr Peirse

1818 br. c. Jack Spigot, by Ardrossan, or

$1820 \mathrm{gr}$. f. by Whisker...............

1825 br. c. Gazebo, by Ardrossan........ Mr Orde Powlett

1824 gr. c. Saucy Dick, by ditto.........

1827 gr. f. by Y. Phantom.............

1829 slipped foal, by Partisan........... 
1850 b. c. by Y. Phanton ..............

$1851 \mathrm{gr}$. c. (Isaac) by Figaro............. 3 .

1855 gr. f. The Ladly Berners, by Lamplighter

Missed in 1815, 16, 19, 21, 22, 25, 28, 26, 32, 54, and 53.

\section{SORCERER HARE,}

Bred by Sir T. C. Bunbury, in 1817, her dam, Tawny, by Mentor, out of Jemima, by Satellite-Waxy's dam, by Iterod-Lisette, by Snap-Miss Windsor, by the Godolphin Arabian.

1822 b. f. by Phantom..............

1824 b. c. Maresfield, by $\Lambda n t a r \ldots \ldots \ldots \ldots$ Sir J. Shelley passage to Virginia)

*1828 b. f. Sweetlips, by Emilius ........ Mr Williamson 1852 br. f. Symmetry, by Phantom ...... Gen. Grosvenor

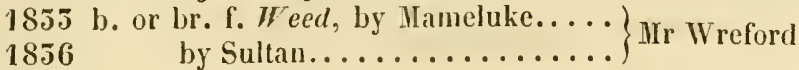

Barren in 1925, 25, 27, 29, and 55; slipped twins in 1850 and 54 ; had a dead foal in 1831.

- Sweetlips produced, in $185 \tilde{5}, \mathrm{Mr}$ Greville's b. c. Lieutenant, by The Colonel, and has since been sent abroad.

\section{SOUVENIR,}

Bred by Mr Nowell, in 1824, got by Orville, her dam, Dulcamara, by Waxy, out of Witchery, by Sorcerer-Cobbëa, by Skyseraper.

$1853 \quad$ c. by Brutandorf ............

1854 missed to Tiresias............. D. of Portland

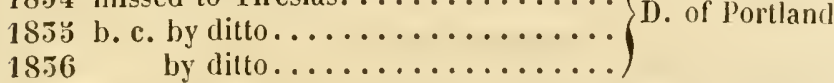

SPAE-IVIFE,

Bred by Sir Maxwell, in 1825, got loy Soothsayer, her dan, Culty Sark, by Haphazard-Brillante, by Whiskey-Diomed.

1850 ch. f. Roy's Wife, by Ben Ledi......

1851 ch. f. by ditto...................... Sir Waxwell

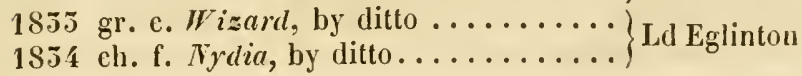

vol. IV. 


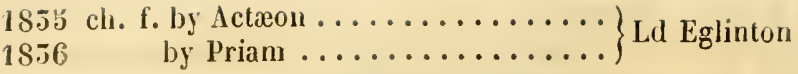

\section{SPAVINA,}

Bred by Lord Orford, in 182y, got by Orville, her dam, Mirandola, by Haphazard, out of Allegretta.

1829 b. f. by Camel.

1850 not covered last year.

1851.b. f. Bashful, by St Patrick (s. to America in 1835)

1852 b. f. by Lamplighter............ Ld Orford

185 s ch. c. by Langar...............

$1854 \quad$ c. by Catton.................

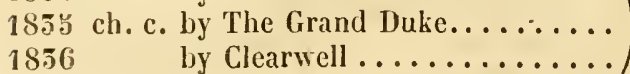

\section{SPERMACETI,}

Bred by Lord Egresont, in 1820, got by Whalebone, out of sister to Wanderer, hy Gohanna-Catherine, by Woodpecker.

1828 b. c. by Reveller.

1899 b. f. (Sublime) by Partisan (put to the His Majesty

1851 b.c.Stradbally, by Reveller, or Waterloo Mr Cosby

1855 b. f. Calantha, by Sultan .......... Col. Peel

1854 b. f. by Sultan ..............

1855 b. c. by The Colonel ........................... Hajesty

1856 by Prianı.............

Missed to Waterloo in 1827 and 50, to Emilius in 52.

\section{SPINNING JENNY,}

Bred by Major WiLsov, in 1814, got by Juniper, her dam by Oscar, out of Dairy-maid, by Diomed-Nelly, by Conductor.

1822 br. c. Bull Dog, by Tiresias ........

1825 ch. c. by $\Lambda$ bjer ..............

1824 b. f. by Woful................

182. c. by Tiresias (dead) ...........

1827 foal, by Centaur (dead) .......... Col. Wilson

1828 br. f. by Tiresias...............

1829 ch. c. by Emilius ..............

1852 b. c. by Lamplighter............ 


\section{3}

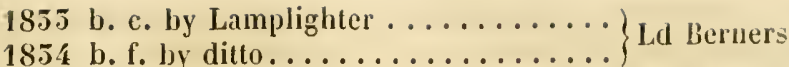

$185 y \quad$ c. by ditto............... Mr Seager, of Ger-

1856 by ditto............. many

In 1826 missed to Reveller, 50 to Truflle, 5 I to Lamplighter.

\section{SPOTLESS (sister to Bolter)}

Bred by Major WiLsov, in 1809, got by Walton, her dam by Trum pator-Jighflyer-Otheothëa, by Otho-Snap-Regulus.

1816 ch. f. Schedam, by Juniper .......

1817 b. f. Rotterdam, by ditto .........

1818 ch. f. by ditto..............

1819 ch. f. by Rubens . . . . . . . . . .

1820 bl. f. Black Daphne, by Juniper.....

1821 b. c. Augur, by Interpreter........

1825 b. c. Lamplighter, by Merlin...... Col. Wilson

1824 b. f. Cinderella, by dilto.........

$1825 \mathrm{gr} . \mathrm{c}$. by Y. Gohanna

1826 ch. f. by Hazard (son of Haphazard)..

1827 ch. c. Ringleader, by Nlerlin.......

1850 ch. f. Malvina, by Oscar.........

1854 br. c. by ditto...............

1856 by son of Comus and Rotterdan.

Barren in 1822, 29, 52, and 55; her produce of 1828 and 51 died.

\section{SPRAT,}

Bred by Lord Lowtnes, in 1829, got by Partisan, her dam, Scribe, by Woful, out of Scratch, by Selin.

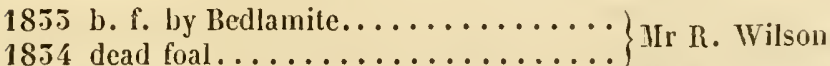

1855 b. f. by Shakspeare ............. Mr WV. Goodwin

Sent abroad in 1855 , covered by Taurus.

\section{SPRINGE (sister to Snare)}

Bred by Mr Tновинц, in 1818, got by Scud, her dam, P'ophetess, by Sorcerer, out of Pamela, by IThiskey-Lais.

1825 ch. c. Sine quâ non, by Tenjers.....

1826 c. by Tramp (died 24 hours old) ....

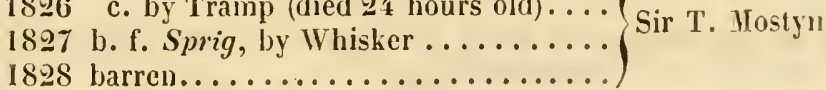


$1829 \mathrm{ch}$. f. Minialure, by Teniers........ Mr Armitstead

1850 b. f. Lauretla, by Lottery ......... Ir France

1851 b. f. by Peter Lely............. Ir Armitstead

1855 f. by Bravo...............

1854 c. by Felt (died).............. Mr R. J. Mostyn

1853 c. by Sir Gray..............

\section{SPRITE,}

Bred by Mr Northey, in 1819, got by Phantom, out of Ralphina, by Buzzard-Dungannon-Squirrel.

1826 b. f. by Waterloo............. Ld Mountcharles

1827 b. c. Vanloo, by ditto........... His Majesty

1828 br. c. Brussels, by ditlo..........

1850 b. f. Elfin, by Cain........................

Died in 1852 , in foal to Edmund.

\section{SQUIB,}

Bred by Mr Yates, in 1820, got by Soothsayer, her dam, Berenice, by Alexander, out of Brunette, by Amaranthus-Mayfly.

1828 b. f. Blitz, by Paulowitz..........

1829 barren.........................

1850 br. f. Eclat, by Edmund........... Mr Yates

1851 b. f. Eliza, by ditto ............ IIr Yate

1852 ch. c. Willfire, by Cain

1855 br. c. by Edmund (dead) . . . . . . . .

1854 b. f. by Mamcluke .............

1855 br. c. by Sir Hercules................ Mr Tattersall

1856 missed to Actæon ............

The STAMFORD MARE, out of Miss Buckle (Vol. III, p. 406) produced a c. by Lambtonian, in 1852, and has had no thoroughbred foal since; her f. by Filho, in 1851, was called Mrs Scarlet. She produced b. c. Toughskin, by Toughstick, in 1854, and was not covered that year.

\section{STAMFORD HARE,}

Bred by Mr G. Dawsor, in 1816, her dam by Weasel-AlfredWakeficld Lady. 


\section{5}

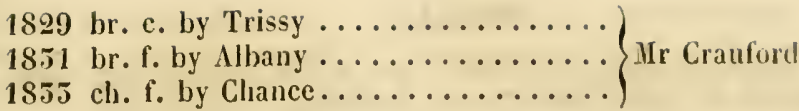

\section{STAMFORD MARE,}

Bred by , in , her dam, Lady of the Iake, by Beningbrough, out of Thatchella.

1854 br. c. by Albany (son of Smolensko).. )

185 . b. c. by Beagle ............... Sir J. Roswell

1856

by l'hysician

\section{STAMFORD MARE (Euanthe)}

Bred by Mr Nicholsor, in 1816, her dain by Restless, out of Viscount's dam, by Bordeaux-Prophet-Saltram's lam, by Snap.

1821 ch. c. Spring-kell, by Epperston.....)

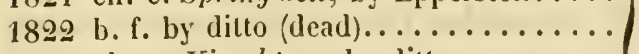

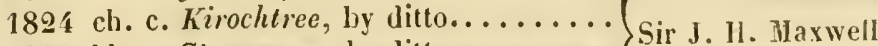

182 's bl. c. Glenamour, by ditto ........

1826 bl. f. by ditto ...............

1829 b. c. Logic, by ditto (died in 1834) ...

1951 br. c. Cant, by Canteen .......... D. of Buccleugh

1855 slipped twins, by ditto ..........

In 1828 missed to Epperston, 50, 52, and 54, to Canteen, and was shot。

\section{STAYS,}

Bred by Lord Egrenort, in 1821, got by Whalebone. her dam by

Bobtail, out of Catherine, sister to Colibri, by Woodpecker.

1827 br. f. Murwari, by Manac..........

1828 ch. f. by Perchance......................... Orford

1829 b. f. Petulance, by Partisan .......
$1850 \mathrm{ch}$. f. by Sultan.............. Ir Ricardo

1851 b. c. Airy, by St Patrick..........

1852 b. f. Bodice, by Langar ......... I.d Orford

1855 ch. c. (Jacliet) by Iangar. ..........

1854 b. f. by Jerry ...............

Not covered in 185 1 -staked herself in $5: 5$, and died. 


\section{STELLA,}

Bred by Lord Stamfonn, in 1808, got by Sir Oliver, her dam, Scotilla, by Anvil, out of Scota, by Eclipse-Hcrod, etc.

1816 b. c. Magician, by Soothsayer ......

1818 b. c. Peter Lely, by Rubens ........ Ld Stamford

1820 b. f. Fury, by Soothsaycr. .........

*1822 b. f. Linnet, by Bustard (Castrcl).... Sir J. G. Egerton

1824 b. f. Lark, by liubens............ Mr Mytton

1826 b. c. Independence, by Filho da Puta,

1828 b. c. Colwick, by Filho da Puta......

or Sherwood Mr Beardsworth

1850 b. f. Caroline, by Chateau Margaux .. Mr Walker

1855 b. f. Midsummer, by Filho da Puta...

1855 b. c. by Filho, or Vanish.......... Mr Lacey

Missed in $1817,21,25,25,27,29,51,52$, and 54 , and in 1819 slipped foal.

Mr lacey bred all the foals from 1824 inclusive.

* Linnet is dead, and had only one foal, viz.in 1828, Col. Peel'sb. f. Linnet, by Paulowitz.

\section{STRA WBERRY,}

Bred by Mr 'Гномаs Srkes, in 1825, got by Soothsayer, her dam by Timothy - Beningbrough-Expectation.

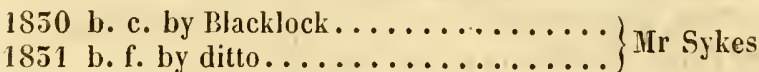

$1852 \mathrm{ch}$. f. by Tramp (died)..................... Mr S. Templeman

\section{STRIKING BEAUTY,}

Bred by Lord Staweld, in 1814, got by Sorcerer, her danı by Bcninglorough-Sir Peter-Miss Gunpowder.

1818 ch. c. by Juniper ............. 1820 b. c. by Crim. Con. (sent to India) ... 1821 ch. f. Miss Skim, by Skim ........ 1822 br. f. by Sampson (dam of Fury, by

1824 dark gr. c. by Skim................ Mr Holbrook

1823 b. c. by Mr Lowe.

1827 br. c. by Riclard (sent to France). 1829 b. f. by Phantom .............. 1850 b. c. by ditto.................. 1851 c. by Mamcluke. 


\section{7}

1855 br. f. by Camel............. IIr Theolsald

1854 b. f. by Iamcluke.............. Mr Ilolbrook

1853 b. f. by Camel. .

Barren in 1819, 25, 26, and 52.

STRUMPET, by Hambletonian (Vol. III, p. 415) died in 1852, barren-her last produce should stand thus :-

1829 br. f. Cecilia, by Filho (s. to Germany) Mr W. II. Stanley

The sister to Cecilia, foaled in 1826 , produced a b.c.by Memnon in $185 \overline{5}$, and died the next year.

\section{SUKE (sister to Swiss)}

Bred by $\mathrm{Mr}$ W. PeIrse, in 1828, got by Whisker, her dam by Shuttle, out of Lady Sarah, by Fidget-NIfred-Magnolia, by Marske.

1853 ch. f. Kate, by Frolic

1834 b. c. by Harry (dead) ............ Mr P. Thomson

1855 b. c. by Musquito (s. of Master Henry)

1856 by ditto.................

\section{SULTANA (sister to Sultan)}

Bred by $\mathrm{Mr}$ CвоскғовD, in 1820, got by Selim, her daun, Bacchante by Williamson's Ditto, out of sister to Calomel, by IIercury.

1828 b. c. Turk, by Phantom .......... Mr Payne

1829 br. f. Corset, by Whalebone (in Mr Mr Ilouldsworth Houldsworth's stud)

1850 ch. f. by Partisan............ Mis Majesty

1851 b. f. Begum, by ditto........... Ld Stradbroke

1834 b. c. (dead) ................. His Mlajesty

Missed to Waterloo in 1852 , to The Colonel in 1855 and $5 \%$, to Actron and The Colonel in 56.

\section{SULTAN JIARE (sister to Cactus)}

Bred by Lord Exeter, in 1829, her dam, The Duchess of York, by Waxy, out of Moses' dam.

1854 missed to Chateau Margaux........

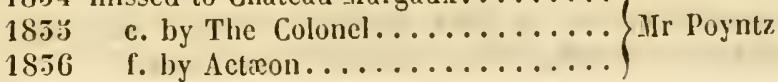




\section{8}

\section{SUITANESS,}

Bred by Mr Burtow, in 1829, got by Saladin, her dam, Kate, by Catton, out of Miss Garforth, by Walton-IIyacinthus-Zara, by Delpini.

18.5 .5 ch. c. by Velocipede...........

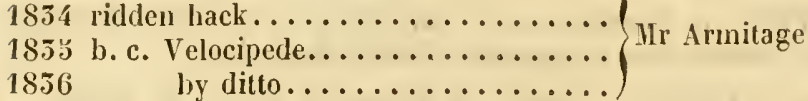

SUNFLOWER, by Castrel (Vol. III, p. 414) has had no foal from 1832 to 36 inclusive. - Add the name Miss Chester, to her ch. f. by Whisker.

\section{SURPRISE (brown)}

Bred by Mr Thorrnill, in 1822, got by Scud, her dam, * Manfreda, by Williamson's Ditto, out of 'Tawny, by Nentor.

1827 br. c. (Agreeable) by Emilius........ Mr Thornhill 1828 br. f. by ditto ............... Mr W. Chifney

1829 b. c. Pounce, by Merlin ......... Mr Biggs

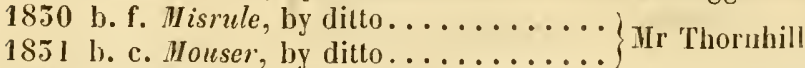

1852 b. f. (Nosegay) by Emilius......... Ld Chesterfield

1855 missed to Emilius . . . . . . . . . .

1854 b. f. by Rowton (died a foal)....... Mr Thornhill

183 b. c. by St Patrick .............

* Manfreda died in 1825 , having had only one other foal, which died young.

\section{YOUNG SIVEET-PEA,}

Bred by Mr Nowell, in 1825, got by Godolphin, out of Sweetpea, by Selim-Pea-blossom, by Dón Quixote-Pipator.

1829 ch. f. by Muley...... . . . . . . . . Mr Nowell

1851 b. c. Tarick, by dillo ........... Mr E. Peel

1852 b. c. by Longwaist............. Mr Greatrex

1854 b. с. by Muley.............. Mr I'age

18.5 .5
18.56

Missed in 1850 and 55.

\section{SYBII,}

Bred by Lord Fitzwilual, in 1822, got by Interpreter, her dam, Galatea, by Amadis, out of Paulina, by Sir Peter. 
1827 b. f. by Cervantes...............

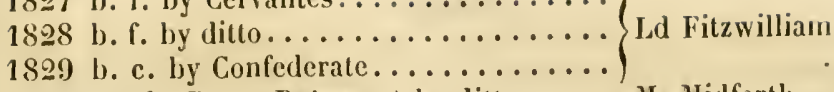

1830 ch. f. (Patty Primrose) by ditto ..... Mr Midforth

1832 ch. c. The General, by ditto......... Mr Marson

1854 b. f. by Bachelor (son of Colebs).....)

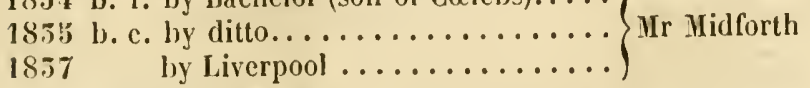

Missed in 1851, 55, and $\mathbf{3 6}$.

\section{SYCORAX (sister to Soothsayer)}

Bred by Mr JADIs, in 1815, got by Soreerer, her dam, Goldenlocks, by Delpini, out of Violet, by Shark-Syphon, etc.

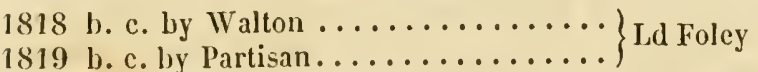

1820 br. f. by Pioneer.............. Gen. Grosvenor

1822 bl. f. by Waterloo.............. Mr W. Cooper

1826 b. c. Glenfinlas, by Moses........... Mr Greville

1827 c. by ditto (died in foaling).......

1828 ch. f. Barbara Bell, by Middleton.... Mr Kymer

1829 b. f. by ditto ................

1850 b. f. by ditto.................

1851 ch. c. IIernani, by Mameluke......

1833 b. c. by Emilius (died a foal)........ Mr Greville

$1854 \mathrm{ch}$. c. Laocoon, by Priam..................
$1856 \quad$ by Priam, or Zinganee

Missed in 1821, 24, 25, and 32 ; slipped foal in 23 and 53.

\section{SYLPII,}

Bred by Mr R. Griffitis, in 1824, got by Spectre, her dam, Fanny Legh, by Castrel, out of Miss Hap, by Shuttle.

1829 b. c. Soubahdur, by Champignon .... Mr Griffiths

1850 b. f. by Master IIenry............. Mr E. Jones

18.31 br. c. Rambler, by Reveller.......

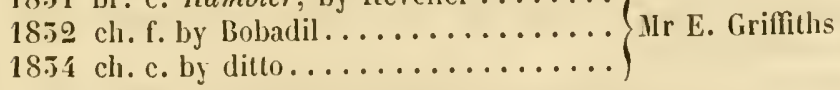

Barren in 1855, not covered in 54.

The TEDDY TIIE GRINDER MARE, Recruit's dam (Vol. III, p. 417) missed in 1855, 34, and 53.-. Idd the name (Conscript) to her b. c. by Whalebone, in 1828; he was sent abroad in 53. 
TEARS,

Bred by Mr R. Wissov, in 1825, got by Woful, out of Miss Stephenson, by Scud, or Sorcerer-sister to Petworth, by Precipitate-Woodpecker.

18.32 b. f. by Defence .............

1855 ch. c. by ditto.............. Mr Sadler

$1854 \quad$ f. by ditto ..............

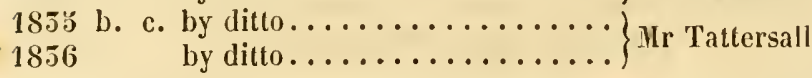

Sold to Col. Hampton, of Charleston, South Carolina, in 185\%. with her foal.

THALESTRIS,

Bred by Mr Glossop, in 1809, got by Alexander, her dam, Rival, by Sir Peter, out of Hornet, by Drone-Manilla, by Goldfinder - Mr Goodricke's Old England mare.

1815 br. c. by Dick Andrews.......... D. of Rutland

1817 b. f. (IIrs Bang) by Soothsayer....

1819 c. by Y. Gohanna............

1820 br. c. by Wanderer..............

1822 b. f. (Peri) by ditto .............

1825 bl. f. Ogress, by Octavius .........

1824 br. c. Nimrod, by Whalebonc.......

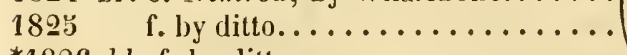

*1826 bl. f. by ditto............... Ld Egremont

1827 bl. c. (Egremont) by ditto (sent to the Swan River by Mr Henty)

+1828 bl. f. $(I d a)$ by ditto.............

1831 ch. f. Petworth Lass, by Whalebone, or

Little John

1832 c. by Gaberlunzie (died the next day)

1854 gr. f. by Chatcau Margaux, or Skim..

Missed to Whalebone in 1829 and 50 , to Chateau Margaux in 55 ,

to Chateau Margaux and Gaberlunzie in 53 ; she was covered

by Whalebone for the last time on the 2 nd of April 1850 , and

by little John on the 10 th-the latter stallion died immediately after covering her.

N. B. Thalestriswas trained after, as well as before, producing her first foal.

* This mare produced in $1850 \mathrm{a}$ b. c. Monarch, and was sold to Baron Biel, and sent to Germany in 1831 .

T lda was sent 10 America in 1855 , with a b. c. foal by Gaberlunzie, and covered by Loggic.

5 Petworth Lass was sent to Germany in 1855 , covered by Logie and Shakspeare. 


\section{THIELES,}

lired by the Duke of Poktuaxn, in 1824, got by Tiresias, her dan. Ambiguity, by Election, or Blucher, out of Castrel's dam, by Alexander-llighllyer-Alfred.

1851 b. f. hy Figaro (died young) ........
1855 b. c. by The Colonel... . . . . . . .

1854 missed to Vanish.............. 1). of Portland

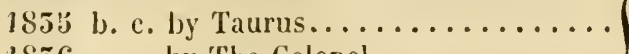

1856 by the Colonel ............

\section{THEODOSIA,}

Bred by IIr ELLis, in 1895, got by Tramp, her dam, Harrict, by Stripling, out of Maniac, by Shuttle.

1851 f. by Catton (died 2 days old).....

1852 b. f. hy ditto (not trained, and put to Mr Milnes

1835 f. by ditto (died 2 days old).......

1854 b. c. Lincoln, by Negotiator........ Capt. Taylor

1855 dead c. by ditto............. Mr Milnes

\section{TIIEOPIIANIA,}

Bred by Mr Gascuigie, in 1825, got by Walton, her dam, Truila, by Sorcerer-Weathercock-Cora, by Matchem.

1832 b. f. by Langar (died a yearling)..... IIr Murray $185 j$ ch. f. Bobbinet, by Bobadil ......... Ir Rawlings

1854 b. c. by Barclay, by Stumps........

$1855 \mathrm{gr}$. c. by ditto................ Mr Murray

18.56 by Cain

\section{THERESA PANZA,}

Bred by Sir Jonx Brxa, in 1818, got by Cerrantes, her dam, Gadabout, by Orville, out of Mlinstrel, by Sir Peter.

1824 ch. f. Contrition, by Tramp........

1826 br. c. Niger, by Blachlock.......... Sir J. Byng

1828 br. c. by Horisco (ran in Ireland)....

1830 b. f. Weeper, by Woful (put to the

1852 ch. c. Wilful Will, by Langar....... Stud) Ld Chesterfield 
1855 b. f. Wish, by Sultan ...........

1854 ch. c. Well-a-day by Priam............... Ld Chesterfield

1856 by Zinganee, or Priam.......

Missed in 1827 and 29 , in 51 to Leviathan, in 55 to Trainp.

\section{THUNDERBOLT MARE,}

Bred by IIr Kinex, in 181 , her dam by Precipitate, out of Tag, by Trentham.

1824 ch. c. Linton Loch, by Blacklock.... Mr Rolling

182: by ditto $\ldots \ldots \ldots \ldots \ldots \ldots \ldots \ldots \ldots \ldots \ldots \ldots$ Hr Hall

1855 br. f. Lady Fanny, by St Nicholas...)

1834 b. c. St John, by ditto ........... Mr J. Briskham

1856 c. by ditto...............

Had a half-bred foal in 1829 , cast foal in 50 -her produce of 27 and 51 died young; she missed in 52 and 53.

\section{THUNDERBOLT MARE (black)}

Bred by Mr Owst, in 1816, her dam (foaled in 1812) by Orville, out of Marvel's dam, by St George-Ethe, by Y. NarskeSerina, by Goldfinder.

1820 b. f. by a half-bred horse .........

1822 b. f. by Chorus .............. Mr Owst

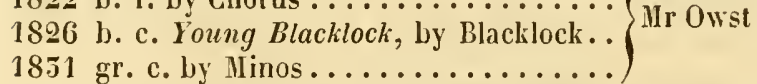

1855 bl. c. Black Hambleton, by St Nicholas Mr S. L. Fox

1836 by Kexby, brother to Catton..... Mr Owst

Barren in $1821,25,24,27,28,32,54$, and 55 -slipped foal in 29-not covered in 24 or 29 . - This mare was the only produce of the Orville mare, which was killed by accident in the Spring of $181 \%$.

\section{THUNDERBOLT MARE,}

Bred by Lord Wharichiffe, in , her dam, Ionkey's dam, by

Sir Peter, out of sister to Tickle Toby, by Alfred-- Herod.

1852 b. f. Lady Di, by Actæon.........

1854 bl. c. by ditto .............. D. of Leeds 1856

by Physician, or Starch.......

Missed in 1851,55 , and 55 . 
THRA,

Bred by the Duke of Graftos, in 1821, got by Soothsajer, or Castrel, her dam, Pope Joan, sister to Pledge, by Waxy.

1827 b. f. Trinket, by Tramp... . . . . . . Mr Dilly

1828 ch. c. Traveller, by ditto ......... IIr Giffard

1829 b. c. Peradventuce, by Lottery......

1850 ch. f. (Miss Middleton) by Middleton..

1851 ch. f. Festival, by Reveller.........

1852 ch. c. Turban, by Sultan .......... Ir ILouldsworth

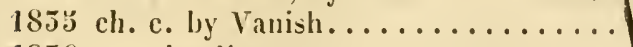

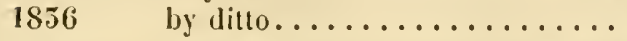

Ulissed to Vanish in 1853 and 54.

TIB,

Bred by Lord Stico, in 1826, got by Langar, her dam, Wilful, sister to Wire, by Waxy, out of I'enelope.

1852 ch. f. by The Duke (s. to Germany) ..

1853 dead c. by ditto...............

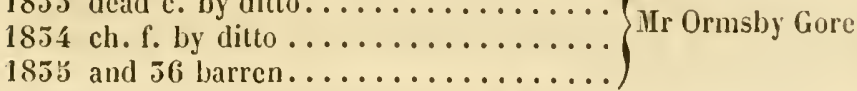

Sold to Baron de Maltzahn.

TINTORETTO,

Bred by Mr Twanlex, in 1825, got by Rubens, out of Belinda, by Beningbrough.

1828 ch. f. by Figaro (dead) .......... 18 . Mr Twamley

1832 b. c. Bassano, by Filho da Puta .... L Ld Lichfield

1853 b. f. Matilda, by Memnon......... .

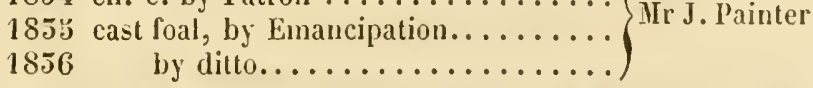

Not covered in 1829, missed to Reveller in 31 .

\section{TIPPET,}

Bred by Mr Peinse, in 1828, got by Swiss, her dam, Wagtail, by

Y. Woodpecker, out of Lady Cow, by John Bull.

1852 ch. c. by Jerry ............. Mr Spalding 


\section{4}

1855 b. c. by ditto............... Mr Spalding

185.5 missed to Brutandorf...........

18.56

by $\operatorname{Logic} \ldots \ldots \ldots \ldots \ldots \ldots$

TIPPITYWITCIIET, by Waxy (Vol. III, p. 422) has had only one foal since the last Volume was published, viz. :-

1832 b. f. (Plaything) by Lamplighter......
1856 by son of Comus and Rotterdam. Ld Berners

Ard the name Corkscrew to her ch. c. by Scud, in 1825.

\section{TIRESIAS MARE (sister to Scheme)}

Bred by Lord Lowther, in 1826, her dam by Haphazard-Precipitate.

1829 ch. f. by Partisan............. Mr Forth

1850 ch. c. Contriver, by ditto ........ Sir M. Wood

1851 ch. f. by ditto.............. Ld Lowther

sold to the Duke of Holstein in Feb. 1852, in foal to Partisan,repurchased by Mr Tattersall, in 1854, and sold in that year to Mr Avery, of Virginia.

\section{TIRESIAS MIARE,}

bred by Lord Lowther, in 1826, out of Turban's dam, by Hambletonian-Marcia, by Coriander-Faith.

18.50 b. f. by Albany (put to the stud) ....

1851 b. f. by Wrangler (put to the stud)... Mr R. Wilson 18.2 gr. c. by Albany

\section{Sent to Sardinia in 1853.}

\section{TIRESIAS MARE,}

Bred by Lord Lowriler, in 1826, her dam, Scratch, by SelimHaphazard-Precipitate-Colibri, by Woodpecker.

1830 b. f. Tigress, by Skiff...........

1855 br. c. by Y. Emilius (bro. to Mouche) .

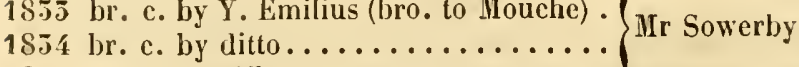

1855 b. f. by Skiff ................

Missed to Skiff in 1851 and 52. 


\section{TIRESIAS MIARE,}

Bred by Lord Lowtuen, in 1823, her dam, Bizarre, by Permian, out of Violante, by John Bull.

1850 ch. c. by St Patrick............

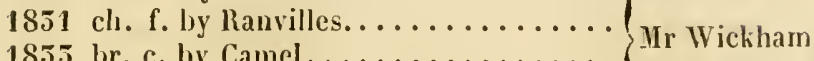

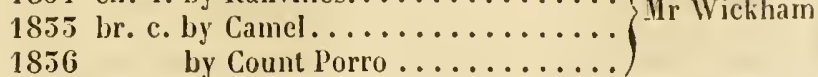

Not covered in 1851,55 , or 54 .

\section{TIRESIAS MARE (sister to Bullogo)}

Bred by Lord Berners, in 1828, her dam, Spinning Jenny, by Juniper-grandam by Oscar, out of Dairymaid, by Diomed.

1852 b. f. by Lamplighter............

1855 b. f. by ditto ............... Ld Berners

1855 b. f. by ditto ..............

Sold to the Duke of Ilolstein in 1855 , with her foal.

\section{TONTINE,}

Bred by the Duke of Graftox, in 1822, got by Election, oul of Pope Joan, by Waxy-Prunella, by Jlighllyer.

1827 ch. f. Finance, by Merlin........ 1828 ch. c. by ditto..............

1829 ch. c. Falconer, by ditto . . . . . . .

1850 ch. c. Fillalgo, by Sultan .........

1851 ch. f. by St Patrick............ D. of Grafton

1852 ch. f. Florin, by Sultan ..........

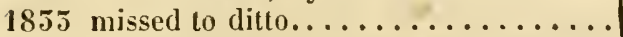

1854 b. f. by Shakspcare...........

1855 b. c. by rlitto................

1856

by Taurus................

\section{TOPSY-TURVY IIARE,}

Bred by Mr Rawlixsox, in 1818, her dam, Agnes, by ShuttleHighflyer-Goldfinder-Lady Bolingbroke.

1826 b. f. Pet, by Gainsborough........

1827 br. f. Mrs Brown, by Spectre (s. to Mr Rawlinson

Baron de Maltzahn in 1856) 
1828 b. c. The Barber, by Shaver (s. to D. of Holstein in 1852)

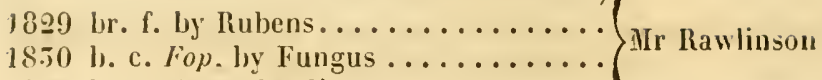

1851 b. c. Bryn, by ditto

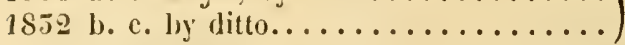

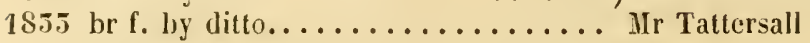

1854 b. c. by lliddleton.............

1853 b. f. by St Nicholas............ Mr Beilby Thompson 18.56

by ditto $\ldots \ldots \ldots \ldots \ldots \ldots \ldots$

\section{TORELLI,}

Bred by Mr WATr, in 1815, got by Cerberus, her dam, IIiss Cranfield, by Sir Peter-Pegasus-Mendoza's dans.

1824 b. c. Talma, by Filho da Puta .....

1825 br. c. Terror, by Magistrate (s. to France

$1827 \mathrm{ch}$. c. Thoresby, by Sluerw in 1855$)$

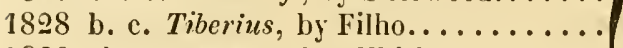

1829 ch. c. Trident, by Whisker.........

1850 ch. c. Titus, by Truffle............... Mr Houldsworth

1852 ch. f. Tullia, by Emilius...........

1855 b. f. Tarquinia, by ditto (put to the stud)

1855 ch. c. lyy Sultan.............

1856 by Beagle.............

Barren in 1826, missed to Reveller in 51, to Vanish iı 54.

$$
\text { TOSO, }
$$

Bred by Mr NowelL, in 1824, got by Rainbow, her dam, Brown Duchess, by Orville, out of Sagana, by Sorcerer.

1852 b. c. by Skiff (dead)............

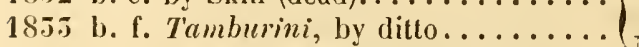

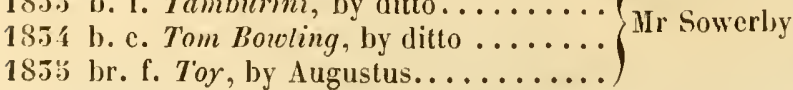

\section{TRAFALGAR MARE,}

Bred by Lord Grosveror, in 1910, her dam, Iusidora, sister to

Meteora, by Meteor, out of Maid-of-all-work, by Ilighllyer.

1818 b. c. by Walton............... H. R. II. D. of York 


\section{7}

1819 b. c. by Selim (dead)........... D. of Rutland

1820 ch. c. Ypsilanti, by Selim.........

*1829 l,r. C. IIylula, by Interpreter .......

1898 ch. f. by Don Juau, brother to Gustavus Sir W. Milner (s. to Russia in 185:)

1851 ch. c. Currency, by Velocipede......

1854 ch. f. by dillo................

Missed in all the intermediate years, and was shot in 1854.

* Hyzda was hunted till 185.j, produced a bl. f. Smoke, by Vanish, in 1854, missed in 55, and was covered that year by Vanish.

\section{TRAGEDY,}

Bred by Lord Fitzwilliar, in 1821, got by Smolensko. out of Desdemona, by Orville-Fanny, by Sir Yeter.

1826 slipped a f. by Tiresias...........

1851 b. c. by Catton (s. to Germany, 1853) .

1852 slipped a c. by Sultan.......... D. of Portland

1834 b. c. by Calton $\ldots \ldots \ldots \ldots \ldots \ldots$.

1835 b. f. by Priam (dead) $\ldots \ldots \ldots \ldots \ldots$

1836 by ditto ................

Not corered between 1826 and 30 , missed to Catton in 5.5.

\section{TRAMP MARE,}

Bred by IIr G. Rosrssox, in 1821, her dam by Hambletonian, out of Vesta, by Delpini-Faith, by Pacolet.

1896 ch. c. Bolivar, by Blacklock........

*1829 b. f. Bounce, by lirutaudorf ........ Mr G. Robinson

1831 br. f. Brunetle, by Lottery..........

1852 b. c. Burlinglon, by Brutandorf..... Mr S. Herbert

1834 b. c. Bulbridlye, by IIumphrey Clinlier

1857 by llulatto................. Gr Robinsou

Barren in $1827,28,50,55,55$, and 56 .

* Bouxce was sold to go to Baden, in the summer of 185a, will a b. f. by Humphrey Clinker, aud covered by Iulatto.

\section{TRAMP MARE,}

Bred by Mr Rasses, in 182.5, her dam by IIambletonian, grandau by IIuby-Carbuncle - Bashful's dam, by Doge.

1851

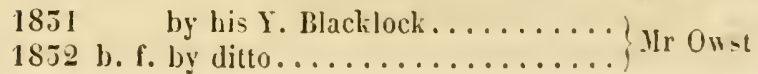


1835 br. c. Keyingham, by ditto........ Mr Kirby

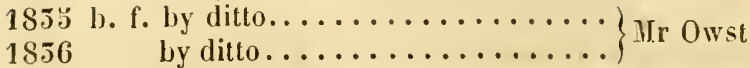

Missed in 1828, 29, 50, and 54.

TRAMP MARE (sister to Barefoot)

Bred by Mr Crouptos, in 1821, her dam, Rosamond, by Buzzard, out of Roseberry, by Phonomenon.

1S5I b. f. by Blacklock (put to the stud)....

1832 dead foal, by Grey Walton ........ Sir Tatton Sykes

1853 b. f. by Y. Phantom .............

The mare was sent to Germany in 18.5 .5$.

\section{TRAMP MARE,}

Bred by Mr Moss, in 18פ6, her dam by Sancho, out of Blacklock's dam, by Coriander-Highflyer.

1851 ch. f. by Velocipede ............

1832 missed to Columbus............. Mr JIoss

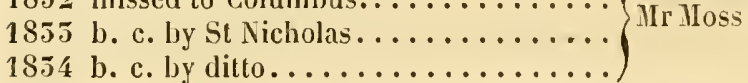

1853 by ditto.............. Ir Etches

\section{TRAMP MARE,}

Bred by Lord Licufield, in 1826 , her dam, Prue, by Trumpator -Woodpecker-sister to Driver, by Trentham.

1851 ch. c. Gale Middleton, by Middleton..)

1855 b. c. by Albany................ Ir Forth

1856 by Hokee Pokee...........

\section{TRAMPOLINE,}

Bred by Lord Jersey, in 1825, got by Tramp, out of $\mathrm{W} \mathrm{cb}$, by Waxy-Penclope, by Trumpator.

1850 b. c. Glenmore, by Phantom .......

1851 ch. c. Glencoe, by Sultan ..........

1852 ch. c. Silenus, by Reveller.........

1855 ch. c. by Reveller (died a yearling) ... Ld Jersey

1854 c. by Priam (died 5 days old).....

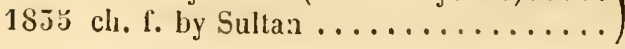




\section{TRAPES (late Speck)}

Bred by the Trestees of Lord Stratinore, in 1825, got by Tramp, out of Remembrance, by Sir Solomon-(Queen Iab, by Eclipse.

1852 b. с. by Lottery.............. In Champion

1854 b. с. by ditto (dead)............ Mr Thornhill

18.5 .3 clı. c. by St Patrick............

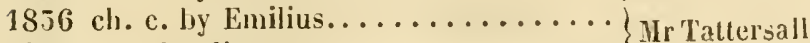

1857

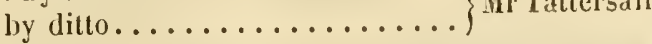

TREASURE, by Camillus (Tol. III, p. 426) produced a ch. c. by Langar, in 1855 , missed to him in 54 , and was shot.

\section{TRICK,}

Bred by Lord Exeter, in 1829, got by Mountebank, her dam, Augusta, by Woful-Rubens-Guildford Nan.

$18.5 \mathrm{ch}$. f. by Sultan

1854 missed to Chateau Margaux.........

1853

1856

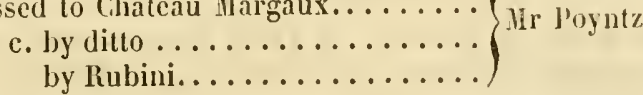

\section{TRICTRAC,}

Bred by the Duke of Grafror, in 1814, got by Dick Indrews, out of Pope Joan, by Waxy-Prunella.

1821 ch. f. Double Entendre, by Comus... Mr L. Fox

1822 br. c. Backgammon, by Walton..... Ld Orford

1825 b. c. (The Constable) by Hagistrate (s.) Mir Wilson

to Ireland, and there called Oueensberry)

1825 br. c. Punster, by Tiresias (died young)

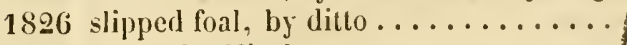

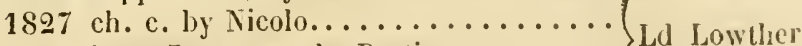

1829 b. c. Lazarone, by Partisan.........

1851 b. c. (Rioter) by Reveller...........

1855 ch. c. by Partisan (dead) . . . . . . . .

18.54 cl. c. Domino, by ditlo........... Mr Sowerby

18.56

by Rowlon................ Ld Lowther

Mlissed to Vandylie Junior in 1824, to Partisan in 2S, 30, and 52. 


\section{TRINKET,}

Bred by Mr Wormatd, in 1824, got by Grey Walton, her dam, Crazy Jane, by Remembrancer-Beningbrough-Fraxinella.

1855 gr. c. by Velocipede ............ Mr Armitage

1854 f. by The Lion.............. Mr Wormald

\section{Died in 1854.}

\section{TRINKET,}

Bred by Mr DitLy, in 1827, got by Tramp, her dam, Tiara, by Soothsayer, or Gastrel, out of l'ope Joan, by Waxy.

1851 b. f. by Catton................. Mr W. Scott

1855 ch. c. Lander, by Langar......... Ld Derby

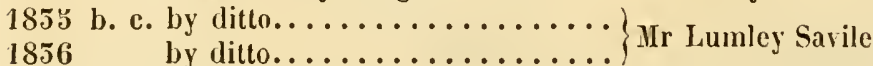

Barren in 1852 and 54.

\section{TURQ̨UOISE,}

Bred by the Duke of Graftox, in 182\%, got by Selim, her dam, Pope Joan, by Waxy, out of Prunella, by Highlyer.

1831 ch. c. Ulick, by St Patrick ........

1852 br. c. by Lamplighter........... D. of Grafton

1855 missed to Zinganee.............

1854 br. c. Ruby, by Reveller.......... Ir Sowerby

1855 br. f. by ditto $\ldots \ldots \ldots \ldots \ldots \ldots \ldots \ldots \ldots \ldots \ldots$ D. of Grafton

TWATTY (sister to Spaniel)

Bred by Lord EgRenoxt, in 1819, got by Whalebone, her dam by Canopus-Y. Woodpecker.

1826 f. by Centaur............. Mr Wyndham

1827 br. f. (Little Gift) by ditto......... Mr Gully

1828 c. by ditto.............. Mr Wyndham

1829 by ditto ............. Ld Wharncliffe

1850 br. c. by Catton................ Hr Cooper

1831 br. c. Cockatoo, by Stainborough .... Gen. Grosvenor

1852 br. f. by Fillo da Puta (s. to Ireland). Mr H. Haxwell

1853 br.c. The Nosegay Boy, by Bedlamite IIr Weatherley 
185 b. f. by Sir Benjamin Sent to Belgium in 1855 , covered by Logic.

\section{THE TWINKLE,}

Bred by Mr Peirse, in 1821, got by Waltol, her dam by Orville. out of Lisette, by Ilambletonian - Constantia, by Walnut.

1826 b. c. "Irinkle, by Whisker........

1827 f. by Tramp (died a foal)........

1828 br. f. by Whisker..............

1899 b. c. by Catton............... Sir C. Monk

1850 b. f. by Chateau Margaux..........

1852 br. f. by Fillo................

1854 br. f. by Caccia Piatti...........

185 b. c. hy a country horse..........

Not covèred in 1830 or 52 .

\section{VALENCIA,}

Bred by Mr livaptov, in 1823, got by Cervantes, her dain by Camillus-Precipitate-Mendoza's dam, by Paymaster.

1852 b. c. by Androgeus ............. Mr Gully

1855 b. c. by ditto............... Mr Wood

1854 br. c. by Voltaire ............. Ld Chesterfield

1853 b. c. Wilibert, by Waverley ........ Mr Cookes

1856 by Liverpool ............ Mr Dyson

\section{VALENTINE,}

Bred by Mr Jaues, in 182.5, got by Soothsayer, her dam by Sir David, out of Miss Cranfield, by Sir Peter-Pegasus.

1852 ch. c. by Figaro............... Mr W. Cooper

1855 missed to Velocipede............ Mr Smallwood

1854 ch. c. by ditto................ Mr E. Peel

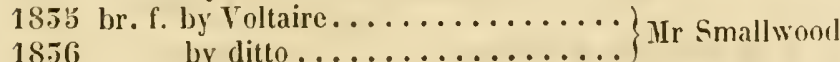

\section{VALVE,}

Bred by Lord Stigo, in 1820, got by Bob Booty, out of Wire, sister to Whalebone, etc. by Waxy, out of Penelope.

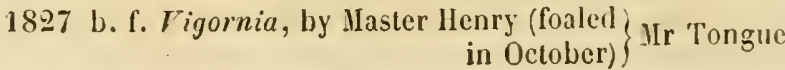




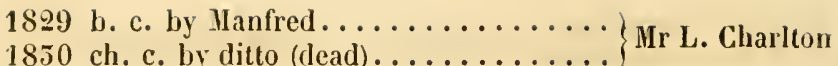

1851 br. f. Pussy, by Pollio.............
1852 br. f. Griselda, by ditto.........

1853 br. f. Frances, hy Emilius .........

1854 br. c. The Steamer, by ditto........ Mr F. R. Price

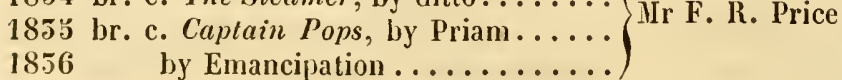
by Emancipation .

VAN,

Bred by Lord Sulgo, in 1829, got by Ivanhoe, out of Wire, sister to Whalcbone, by Waxy-Penelope.

1855 ch. c. by Velocipede...........

1854 ch. c. by ditto.................

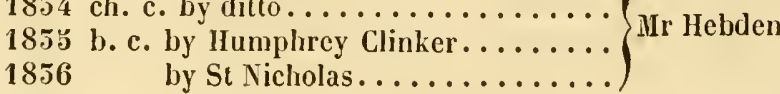

\section{VANESSA,}

Bred by , in 1828, got by Gulliver, out of Quail, by Gohanna-Certhia, by Woodpecker-Trentham.

1855 b. c. by Gustavus .............

1854 b. f. by ditto.....................

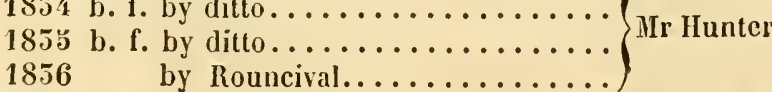

by Rouncival..............

\section{VARENNES (sister to Quadrille)}

Bred by Mr Gol.Divg, in 1818, got by Selim, her dam, Canarybird, by Whiskey, or Sorcerer, out of Canary, sister to Ilippocampus, by Coriander-Miss Green, by Highflyer-Harrict, by Hatchem.

1824 b. f. Brocard, by Whalebone (put to the stud) $\{$ Ld Verulam

1823 b. f. by ditto (ran at Florenec)....... Mr Payne

1827 b. c. Albert, by Waterloo, or IIoses (died rising 4 yrs old)

1828 b. c. Vestris, by Whalcbone........

1851 ch. f. (Eslar) by Mameluke........ Ld Verulam

1852 b. c. Perrot, by Emilius............

1855 b. c. by ditto...............

185 4 b. f. by ditto ............... 
1855

1856

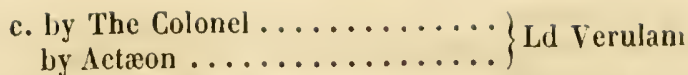

In 1825 missed to Partisan, 26 to Waterloo, 29 to Merlin, 50 to Moses.

VARIS,

Bred by Mr Alies, in 1828, got by Lottery, or Arbutus, out of

Wyndham's dam, by Blacklock-Cerberus-Miss Cranfield.

1835 b. f. by Victory (son of Waterloo)....

1854 b. f. by Humphrey Clinker......... Mr Allen

1855 ch. f. by ditto...............

1856 by Clinker............. Ir F. Wilson

\section{VARIELLA (sister to Voltaire)}

Bred by $\mathrm{Mr}$ Stepievsox, in 1829, got by Blacklock, her dam by Phantom-Overton-Walnut.

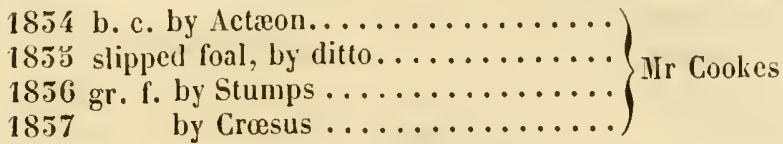

\section{VARIETY,}

Bred by Mr Cinampios, in 1816, got by Selim, or Soothsayer, her dam, Sprite, by Bobtail, out of Catherine, by Woodpecker.

1821 b.c. Miller of Mansfield, by Filho da Puta 1822 b. f. Maid of Hansfield, by ditto.....

1823 ch. c. His Worship, by Hagistrate....

1825 b. f. Mansfield Lass, by Filho......

1826 b. c. by Sherwood, or Magistrate....

1828 b. f. Circassian, by Sultan iput to the Mr Ilouldsworth

1829 b. f. Persian, by Whisker put to the

1851 b. f. Georgian, by Buzzard stud

1852 b. c. Caliph, by Sultan...........

1853 ch. c. Vathek, by ditto...........

1855 c. by ditto................

Barren in 1824, 27, 50, and 54 .

\section{VARNA,}

Bred by Lord Exeter, in 1826, got by Sultan, her dam, Bess, by Waxy, out of Vixen, by Pot8o's-Cypher, by Squirrel. 


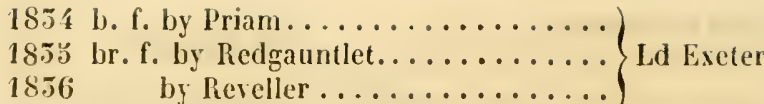

\section{VARNISII,}

Bred by the Duke of Graftox, in 1819, got by Rubens, her dam, Vestal, by Walton, out of Dabchick, by PotSo's.

1823 b. f. by Whalebone (put to the stud)..

1826 b. c. by ditto (s. to India in 1829)..... Mr Combe
1827 b. f. by Moses (died young)........

1828 b. f. Miss Dulwich, by Richard......

1829 b. c. Dainty Davie, by ditto . . . . . .

1851 b.c. Master Charles, by ditto........ Mr Ramsay

1852 b. f. by Richard (died young).......

1855 b. f. by a half-bred horse..........

$1854 \mathrm{gr}$. f. by a cart-horse............

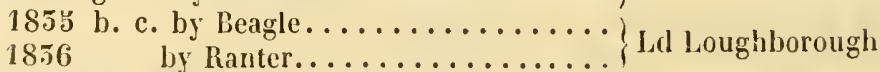

\section{VAULTRESS,}

Bred by the Duke of Portland, in 1819, got by Walton, her dam

by Election, out of Fair IJelen, by IIambletonian.

1826 gr. f. Flirt, by Blacklock.......... Mr Irby

1827 bl.c.Pilgrim(aft.Arlington)by Waverley Mr Arnold

1828 gr. f. Lady Fractious, by Comus.....

1829 b. c. The Rhodian, by Blacklock (s. to Mr Petre Germany)

1852 gr. c. Vaulter, by Reveller.........

1855 gr. c. Somerset, by ditto (s. to Ger- Mr Shard many)

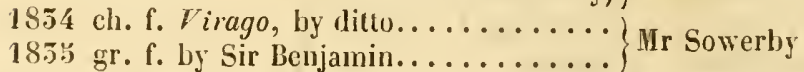

Missed in 1825 and 51 , slipped foal in 1850.

VELL (sister to Varnish)

Bred hy the Duke of Graftox, in 1820, got by Rubens, her dam, Vestal, by Walton, out of Dabchick.

1826 ch. f. Heloise, by Woful (died young). Mr Dilly 
1827 slipped foal to Partisan ..........

1828 b. c. by Comus (died a yearling).....

1829 br. f. Smolensko...................... Stonehewer

1850 ch. f. Chantilly, by Gustavus .......

1831 b. f. by Reveller ..............

1852 b. f. by ditto................

Sold to Baron Biel, covered by Mameluke.

\section{VENUS,}

Bred by Mr Bexsox, in 1823, got by North Star (son of Y. Daffodil) her dam, Gift, by Y. Gohanna, out of Y. Wthe, hy Sir Peter.

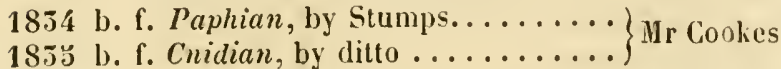

\section{IERBENA,}

Bred by Lord Verulay, in 1821, got by Comus, her dam, laurelleaf, by Stamford-sister to Druid, by Pot8o's.

1855 b. f. by Reveller.............. Mr Marsh

1854 br. c. Ich Dien, by Camel ........ Mr Phillimore

Missed to Count Porro in 185\%, and sold to go abroad.

THE VERMIN MARE, Eliza's dam (Vol. III, p. 454) died in 1834, having missed in 1852 and 53 . - IIer last produce should stand thus :-

1851 b. c. Worlaby Baylock, by Blacklock.. Mr llesseltine

\section{VERSATILITY,}

Bred by Mr Fletcher, in 1826, got by Blacklock, her dam,

Arabella, by Williamson's Ditto, out of Esther, by Shuttle.

1852 b. c. Weathercock, by Whisker.....

1855 b. c. Waverer, by Emilius ........

1854 f. by Lapdog (died a foal)........ Col. Peel

1855 f. by Defence ..............

1836 bl.f. by Camel ...............

VEST 1 ,

Bred by Mr Jouxsox, in 1825, got by Governor, her dam by Sir Peter. out of Eaton Lass, by P'ot8o's. 
1850 b. c. by Filho................

1851 b. c. Smedley Lely, by Peter Lely....

1832 b. f. Venus, by Langar........... Mr Johnson

1853 and 54 barren.................. Johnson

1855 br. f. by Olympus.............

1856 by Langar.............

Y. VESTA,

Bred by Mr Mlasox, in 1825, got by Egremont, out of Vesta, by Delpini-Faith, by Pacolet.

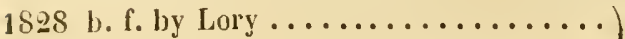

1829 b. f. by Ben Ledi ..............

18.51 b. c. by Lottery (died 5 days old).... J Ir Mason

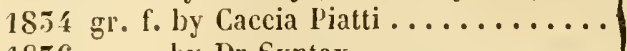

1856 by Dr Syntax............

Missed in 1850, 52, 55, and 53.

\section{VICARAGE,}

Bred by Lord Egrenoxt, in 1818, got by Octavius, her dam liy Election, out of sister to Skysweeper, by Highflyer.

1822 c. by Whalebone (killed by accident) when a foal)

1825 b. f. Mignonette, by ditto......... Ld Egremont

1823 b. f. (Petworth) by ditto s. to the Swan

1826 b. f. (Merino) by ditto River

1829 b. f. Runnymede, by ditto (s. to Baron ?

Biel in 1855 , covered by The Colonel) $\}$ Ir Dockeray

1850 b. c. by ditto (died a foal) .........

1851 b. f. by ditto (s. to Germany in 1855 , covered by Logic)

1854 b. c. by Chateau Margaux (dead)...... Ld Egremont

1833 b. f. by ditto................

1856 b. c. by $A$ corn...............

Slipped foal in 1827 ; barren in $24,28,52$, and 55 .

\section{VICTOIRE,}

Bred by Mr G. Walker, in 1828, got by Whisker, her dam, Vourneen, by Sorcerer, out of Tooee, by Buzzard-Violet, by Shark.

1854 b. c. Michael, by Emilius......... Ld Uxbridge

$1853 \mathrm{~s}$ ch. c. by ditto............. Mr Thornhill 
VICTORI.I,

Bred by Sir R. K. Dick, in 1826, got by Tramp, her tam, Bellit,

by Beningbrough, out of l'eterea, by Sir l'cter.

1952 b. c. Welcome, by Luzborough ......

1854 b. c. by Sultan.................. Mr Wreford

1856

Barren in 185.5 and 53.

\section{VICTORINE,}

Bred by Mr Corset, in 1816, got by Llaphazard, her dam, l'hantasmagoria, by Precipitate, out of Cerberus' dam, by Herod.

1826 c. by Rubens (died a foal)........ Mr I. Day

1828 b. f. by Figaro............... Sir 11 . IVood

1829 ch. f. hy Burgundy............ Mr l. Day

1850 ch. f. by ditto, or Langar........ Sir M. Wood

185.5 by Defence ............. D. of Holstein

1834 b. c. by Mloses................. Hr Tattersall

1857

by Glencoe ................ Whr Tattersall

Had no foal till 1826 , missed in $27,51,52,55$, and 56 ; she was sent to IIolstein in 1832 , in foal to Defence, and brought back, in foal to Moses.

\section{VILLOSA,}

Bred by Mr Walkoex, in 1826, got by Mr Attwood's chesnut Arabian, her dam by Phantom, out of Maresfield's dam, by Sorcerer.

1851 ch. f. Isse, by his grey $A \mathrm{rab}$ (put to the

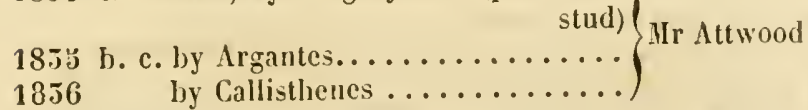

Barren in 1852, and used as a hack till 34.

VITELLINA, by Comus (Vol. III, p. 4.59) produced a c. by Emilius, in 1852, and was sold to the D. of Holstein that year, in foal to Camel.

\section{ULRICA,}

IHed by Mr Cookes, in 1830, got by Sherwood, her dant, Miss Wentworth, by Cervantes. 
18.54 b. c. Winford, by Stumps.........

$185 \mathrm{z}$ gr. f. Almeria, by ditto ..........

1857

by Velocipede.

\section{ULTIMA,}

Bred by Mr Elurs, in 1822, got by Bourbon, her dam, Elba, sister to St Helena, by Stripling-Maniac, by Shuttle.

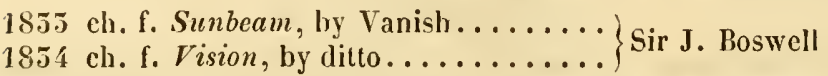

1853 missed to ditto $18 \ldots \ldots \ldots \ldots \ldots \ldots \ldots$ Mr Martin

VOURNEEN by Sorcerer (Vol. III, p. 440) missed in 1854, and was shot. IIer last produce should stand thus :-

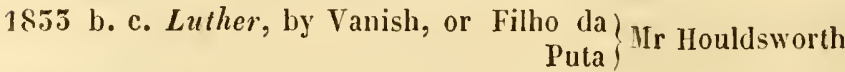

\section{URGANDA,}

Bred by Lord Deriy, in 1821, got by Milo, her dam by Sorcerer, grandam by Sir Solomon-Y. Marske.

1829 br. f. by Whisker.............

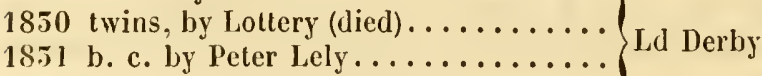

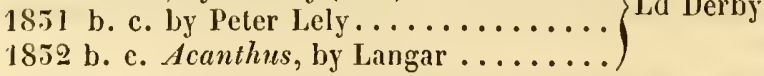

1854 b. c. by Felt.............. Ld Stanley

1853 b. c. by Humphrey Clinker........ W. Wr Boardman

1856

In 1827 missed to Friend Ned, 28 to General Mina, barren also in

55, and sent to the United States in Aug. 1855, with her foal.

\section{URSULA,}

Bred by Lord Fitzwillan, in 1818, got by Cervantes, her dam,

Fanny, by Sir Peter-Diomed-Destlemona, by Marslie.

1824 ch. c. (Englemere) by Catton....... Sir J. Byng

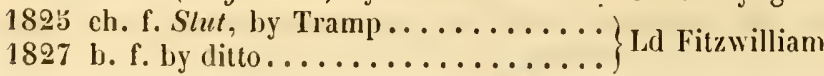


Missed in $1826,28,29,50,51,52$, and 5.5 , and died in 56 , two hours after foaling.

\section{USQUEB BAUGII MARE (sister to King of the Valley)}

Bred by Mr Sader, in , her lam by Devi Sing-Lily of the Valley, by Windlestone.

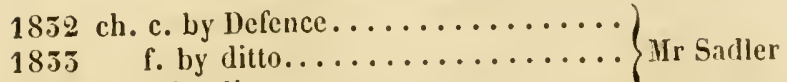

185 b. b. c. by ditto................

1856

by Windeliffe............ Ld Dorchester

\section{USOUEB.AUGII MARE (sister to Popinjay)}

Bred by Mr SAdere, in 1821, her dam, Atalanta, by Walton, out of sister to Tup, by Javelin-Flavia, by P'lunder-Snap.

$1832 \mathrm{ch}$. f. by Defence..............

1855 ch. c. by ditto $\ldots \ldots \ldots \ldots \ldots \ldots \ldots$ Ir Sadler

1854 ch. f. by Fitz-Walton ............

1853 missed to Defence.............

\section{WAGTAIL,}

Bred by Lord Dersy, in 1829, got by Whisker, her dam by Sorcerer, grandam by Sir Solomon-Young Marske.

1834 b. c. by Felt...............

1836

by Vanish

WAGTAIL, by Y. Woodpecker (Vol. III, p. 442) was sent to

Vienna in 1852, covered by Velocipede. ller last produce in

England, was

1852 br. c. Lottu, by Lottery........... Ir Marson

The WALTON MARE, out of Y. Giantess (Vol. III, p. 442) missed in 1852, had a b. f. by Falcon in 5.5, and was shot in November $185 \tilde{\jmath}$, at Tickhill.

The WALTON HARE, out of Y. Noisctle (Vol. IIl, p. 445) was gored by a bullock in 1852 and killed. being then in foal to Catton. 


\section{WALTON MARE,}

Bred by Mr Harrison, in 1815, her dam, Helen, by IIambletonian, out of Susan, by Overton-Drowsy, ly Dronc.

1820 ch. c. Momus (aft. Sir Roger) by Comus)

1821 b. c. Sovereign, by ditto.......... Mr Harrison

1823 b. f. by Alexander.............

1826 b. f. by Columbus............. Hr Armstrong

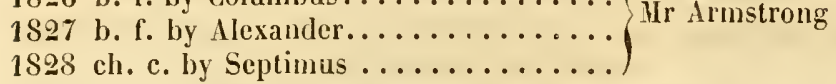

1850 br. c. Primus, by Alexander....... 18 IIr Allen

1852 ch. c. by ditto............. Mr J. Etty

1854 ch, c. Zumalacarregui, by Ilumphrey

185 b br. c. by ditto...............

1856 by $\operatorname{Tramp} \ldots \ldots \ldots \ldots \ldots$ Mr Mllen

In 1829 missed to Septimus, in $5 j$ to Iottery.

\section{WALTON MARE,}

Bred by Mr Rinneus, in 1816, her dam by Shuttle-Delpini-Tuherose, by Herod-Grey Starling, by Starling.

1821 b. c. Caccia Piatti, by Whisker.....

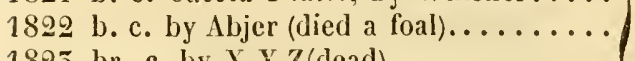

1825 br. c. by X Y Z(dead)........... IIr Riddell

1824 b. f. Contruband, by Soothsayer.....

182 ' br. c. Ben Lomond, by Blacklock ....

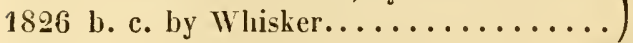

1850 b. f. by Albany ................ Hr R. IVilsou

18.52 b. f. hy Filho da Puta, or Albany ..... Mr R. Wilson

1855 b. c. by Bedlamite (s. abroad in 1855) Col. Peel

1855 by Sir Benjamin..........

Missed in 1827, 28, 51 , and 54.

\section{WILTON MIRE,}

Bred by Mr Cholmelex, in 1819, her dam, Lady Eri, by Stam-

ford, out of sister to Repeator, by Trumpator-Demirep.

182.; bl. c. Old Swan, by Amadis ........

1824 br. c. by Blacklock .............

182 b. c. Shadrack, by Cannon-ball...... Mr Platel

1826 missed to Smyrna ..............

1827 dead colt, by ditto............ 
1828 b. f. by Nolens Volens (dead) .......

1829 b. f. by Smyrna...............

1850 b. l. by ditto.............. Hr Pearse

18.51 ch. f. by Orphan (son of Coniac).....

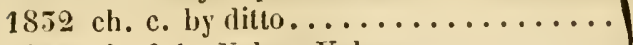

18.5 ch. f. by Nolens Volens..........

WALTON MARE (sister to Wrangler) Vol. III, p. 447, was sent to France in 1851 , in foal to Emilius.

\section{WALTON MARE,}

Bred by Mr Raine, in 1821, her dam, Victress, by GovernorIlambletonian--Lopcatcher, by Y. Marslie.

1827 b. c. by Swiss............... Mr Jaques

$182 S$ br. c. Speculation, by Whisker...... Mr J. Smith

1829 missed to Swiss ...............

1850 b. f. by ditto........................ Mr Jaques

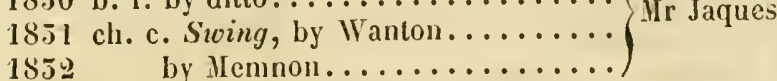

1855 b. c. Fernex, by Voltaire............. Sir G. Heathcote

1855 b. א. by The Colonel ...........

\section{WALTON MARE ( $Y$. Johannah Southcote)}

Bred by Ir Nowez, in 1826 , got by Walton, her dam, Johaunah Southcote, by Beninglorough, out of Lavinia, by Pipator.

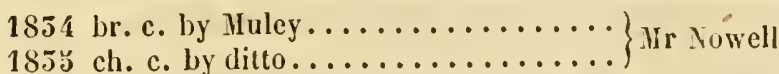
Missed to Longwaist in $185 \overline{5}$, to Muley in 56 .

\section{WALTON MARE,}

Bred by Mr LeE, in 1822, out of Pasta's dam, by Lucli's-all, grandam by PotSo's, out of Maid-of-all-Work.

1826 b. c. by Blacklock............ Mlr Lee

1829 b. c. Nowbray IIill, by ditto....... Mr Gardiner

1855 br. f. by Voltaire .............. Mr Lee

1837 by The Hole ............ Mr Allen

Missed in $1827,28,54,55$, and 56 ; not covered in 29,50 , or 51 . 
WALTON MARE,

Bred by Ir Fergusos, in 1825 , her dam by shuttle, grandan by Oberon-Phœnomenon-Calliope, by Slouch .

1850 bl. c. by Jerry .

1851 b. f. by Cricketer................

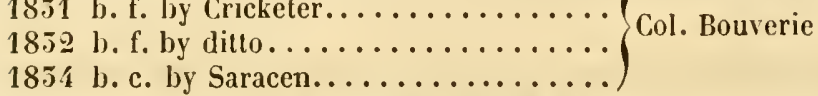

Missed in 1855 and 53.

WALTZ,

Bred by the Duke of Graftos, in 1829, got by Election, out of Penelope, by Trumpator-Prunella, by Highflyer.

1826 b. f. (IIorisca) by Morisco......... D. of Grafton 1827 ch. c. (Waltram) by ditto......... Ld Cleveland 1829 cl. c. Ali Baba, by Sultan......... Mr Nevill

1830 dead foal, by Tarrare .......... Mr Theobald

183 I b. f. by Mameluke............... Mr Champion

1852 b. c. Torvaldo, by Camel........... Sir R. W. Bulkeley

1855 b. f. by Mameluke.............. Mr Champion

1854 b. f. by Camel ................

1855 b. f. by Laurel ............... Mr Theobald

1856

\section{WANDERER MARE (Isabel)}

Bred by Lord Egrenort, in 1824, her dam, Caroline, by Whalebone-Marianne, by IIufti-Haria, by Telemachus.

1829 b. c. by Woful

1850 b. f. by Emilius (dead) ................... Hr Newton

183 I b. c. (Ulysses) by ditto............ Ld Lowther

1852 b. c. Hobby, by Merlin ........... Mr G. Walker

18.54 ch. f. by Langar .............. IIr J. Rogers

Covered by Buzzard in 1854, and sold to go abroad.

WANDERER MARE (Colly, aft. Annie)

Rred by Lord Egrenovr, in 1827, out of Caroline, as above.

1851 ch. c. by Greyleg............. Ld Egremont

18.52 b. f. (dead) by ditto............ Mr Hunter, Dublin 


\section{3}

1853 ch. c. Attacké, by Roller, or Chateau

Margaux Ir Hunter, Dublin

1854 b. f. Ayesha, by Dronc............
1855 b. c. Archer, by Y. Blacklock......

\section{IVASIHERIVOMAN (sister to Nectar)}

Bred by Mr Axpewew, in 1818, got by Walton, lier dam, L'Iluile de Venus, by Whiskey - PotSo's - Maid-of-all-work, by llighllyer.

1825 br. f. (Novelty) by Blacklock ....... Mr IIorsley

1896 lir. c. Wedenblock, by ditto........ Mr Watt

1852 b. c. Dante, by his ch. Mrabian.....

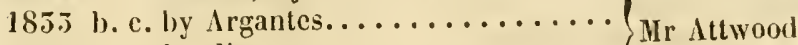

18.5 .3 b. c. by ditto $\ldots \ldots \ldots \ldots \ldots \ldots$

1856 by Alhassan $\ldots \ldots \ldots \ldots \ldots \ldots$.

Hlissed in $1824,25,27,50,51$, and 54 ; in 28 slipped foal, by Lottery.

WASP, by Gohauna (Vol. III, p. 450) was shot in 1855, having missed to Gaberlunzic in that and the three preceding years.

\section{WATERLOO MARE (black)}

Bred by , in 1826, her dam, Prize, sister to 'remium, by Aladdin, out of Moses' dam, by Gohanna.

1852 b. c. Dromio, by Shakspeare ....... Sir M. Wood

18.34

1856

c. by Bedlamite.............. Mr Cooper

by Shakspeare............ Mr Frewen

Missed to Shakspearc in 1855 , not covered in 34 .

\section{W ATERLOO MARE,}

Bred by Mr Suard, in 1821, her dam, Legacy, by Beningbrough, out of Roxana, by Sir Peter-Tulip, by Damper-Eclipse.

1828 b. c. Bacchanal, by Reveller ......

1829 b. c. by Cadiz...............

1830 b. c. by ditto (dead)........... Jur larquharson

1851 barren

1854

1853

c. by Cadiz.

c. by ditto.

\section{WAVERLEY MARE (black)}

Bred by Mr MAsox, in 1825, her dain, Lancashire Mitch, by Ir Teazle, out of the Yellow mare, by Taudern-Perdita. 
18.55 b. f. by Tramp.............. Ir Hesseltine

1854 f. by Velocipede ............

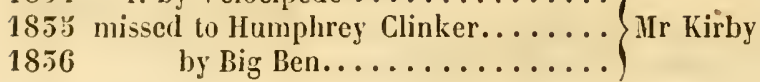

\section{WAVERLEY MARE,}

Bred by Mr Yates, in 1828, her dam (Garcia's dam) by Shuttle, out of Katherine, by Delpiai-Paymaster-Le Sang.

1852 br. f. by Filho da Puta (s. abroad) ....

1855 b. f. by ditto .................

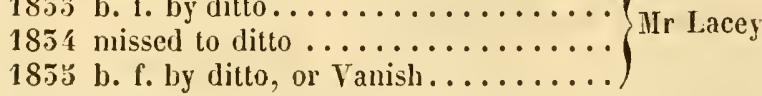

\section{WAXY MARE,}

Bred by Mr Robsox, in 1815 or 16 , her dam, sister to Erictho, by

Sorcerer, out of sister to Rockingham, by Highflyer-Purity.

1822 ch. f. by Carlton.............. IIr J. Day

1825 br. c. IIercules, by ditto .......... 1824 br. c. Tarquin, by Smolensko..... Mr Shard

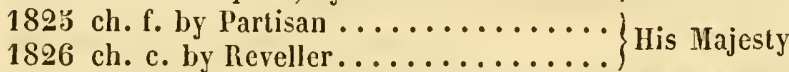

${ }^{*} 1827$ b. f. Norna, by Waterloo ........ Mr Wickham

1828 br. f. by Waterloo............

1829 b. c. by ditto ...............

1850 b. c. by Gustavus...............

1851 gr. f. by Phantom, or Dunsinane.....

1852 missed to Nigel............. Mr Wigram

1835 b. f. by Mameluke.............

1854 b. f. by Nigel ................

1855 ch. f. by ditto ...............

1856 by ditto...............

- Noris produced, in 1851, Mr. Wickham's ch. f. (Robinetta) by Robin Hood, and was afterwards trained again.

\section{WAXY MARE,}

Bred by Mr Payne, in 181:, her dam, Bizarre, by Peruvian, out of Violante, by John Bull-sister to Skyscraper, by Highflyer.

1821 br. c. Ecarté, by King of Diamonds .. Mr Bartley 182.5 br. f. Castrellina, by Castrel........ M. L. Charlton 1824 b. f. by Catton................ Mr Newton 


\section{5}

1825 b. f. Lady Irane, by Reveller.......

1826 b. c. Timour, by Tramp.........

*1827 b. f. Tartarina, by ditto........

1828 br. c. Sir John, by ditto..........

1829 b. f. Lady Stafford, by Comus.......

1850 b. f. Curiosity, by Figaro......... Mr A. Bower

1851 not covered last year.

1852 two b. fillies, by Langar........

1855 missed to ditto..............

1854 ๒. с. Lord Stafford, by ditto .......

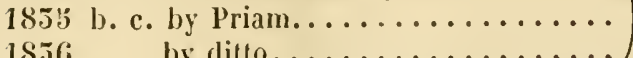

1856

by $\operatorname{ditt} 0 \ldots \ldots \ldots \ldots \ldots \ldots \ldots$

- Tartarixa produced in 185.5 a b.f. by Comus.

\section{II $\mathrm{AXY}$ MARE,}

- Bred by Sir G. Hearncote, in 1813 , her dam by Bobtail, oul of Paulora, by Dungannon.

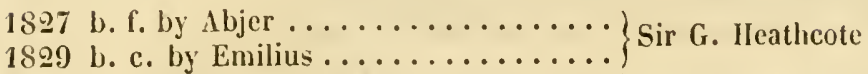

1852 b. c. by Camel, or Middleton ......

1854 gr. f. by Falcon............... Mr F. Lumley

1856 by Langar .............

In 1828 missed to Blacklock, 50 to Figaro, 51 to Godolphin; barren also in 55 and 55 .

WAXY MARE,

Bred by Lord Jenser, about 1818 , her dam by Walton, grandam by Sancho. out of Ringtail.

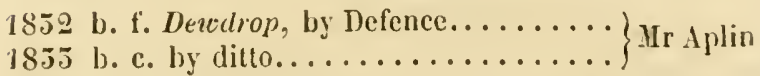

She had some half-bred foals prior to 1852 , and has been barren since 1855.

\section{WAXY MARE (Duchess of York)}

Bred by H. R. II. the Duke of York, in 1817, her dam, Moses' dam, by Gohanna, out of Grey Skim, by Woodpecker-Herod.

1821 b. c. by Soothsayer (sent to Brazil).... H. R. Il. the D. of 1822 b. f. Dahlia, by Phantom ......... Tork 
182.5 b. c. by Mlerlin (s. to the Netherlands)

189 . b. c. Dandelion, by ditto.......... H. R. H. the D. of

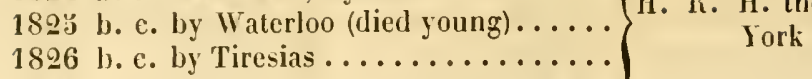

1827 by Waterloo.............

1829 b. f. by Sultan ...............

1830 br. c. Cactus, by ditto............

1851 b. f. Mimosa, by ditto............

1852 b. f. by ditto (put to the stud) ...... Ld Excter

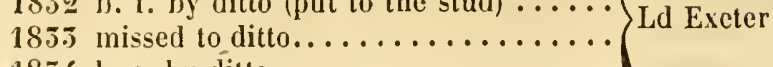

1854 b. c. by ditto.................

1853 b. c. by ditto

1856 by ditto

\section{WHALEBONE MARE (black)}

Bred by Lord Egrenont, in 1816, her dam, Ransom, by Sir Peter, out of Shift, by Sweetbriar-Susan, by Snap.

1824 br. c. Vulcan, by Oetavius (her first foal)

1828 b. f. Runnymede (aft. Rosebud) by Little

John Mr Gates

1850 dead colt, by ditto..............

1852 br. c. Mohair, by Camel .......... Sir S. Graham

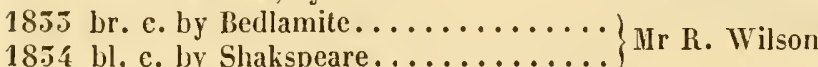

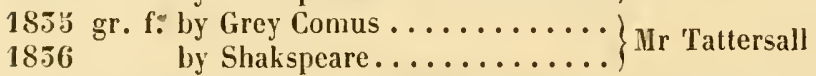

Barren in 1829-between 1824 and 28 she had two colts by Little

John, which died, and one of the years she was not covered.

\section{WHALEBONE MARE,}

Bred by Mr Coune, in 1824, her dam by Seymour, out of a sister to Parisot, by Sir Peter-Deceit, by Tandem-Perdita, by Herod.

1829 ch. c. by Bizarre.............. Mr Combe

1830 br. c. by ditto............... Mr M. Gray

1831 b. c. by Reveller.............. IIr Combe

1855 ch. c. Fusileer, by The Colonel...... Col. Westenra

1854 br. c. by Reveller..............

185 a b. f. by The Colonel............. Mr Combe

1856

by Rubini 


\section{7}

\section{IVILALEBONE MARE (Letty)}

Bred by Lord Egremovr, in 1826, out of Gift, by Y. Gohannasister to Grazier, by Sir Peter.

1829 gr. c. loy Greyleg..............

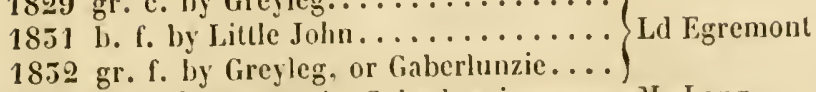

1855 b. c. Sladeland, by Gaberluszie ..... Mr Lang

She was covered by Gaberlunzie March 5, 1851, and by Grejleg April 28 ; missed in 1850 and 54.

\section{WIALEBONE MARE (sister to Spermaceti)}

Bred by Lord Egrenorr, in 1828, her dam, sister to Wanderer, by Gohanna, out of Catherine, by Woodpecher.

1852 b. c. Cajeput, by Emilius.......... Ld lichfield

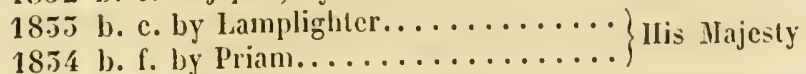

The mare is dead-her last foal was sold to Col. llampton, of South Carolina.

\section{WHALEBONE MARE,}

Bred by , in 1828, her dam, Rose, by Alexander the Great-Worthy-Hare's dam, by Justice.

1855 bl. f. by Defence.............

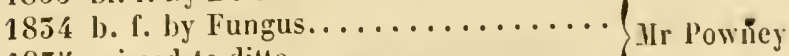

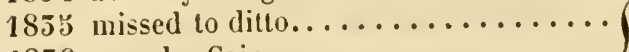

1856

by Cain.

\section{WHALEBONE MARE,}

Bred by Mr R. W. WALKer, in 1826, her dam, Wegenkorb, by Partisan, out of Wowski, by Mentor-Waxy's dam, by Herod.

1851 ch. c. by Defence............. Mr I. Sadler

1852 bl. c. Braco, by ditto............ Mr S. L. Fox

1855 ch. c. by The Coloncl............ Mr ILowes

1854 b. c. by Fungus................ Mr Rawlinson

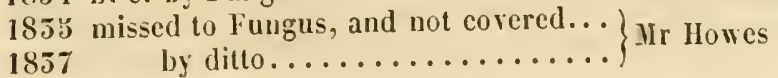

\section{WHIMSEY,}

Bred by Lord Lowruer, in 1829, got by Partisan, her dam, Bizarre, by Peruvian, out of Violante, by John Bull-IIighflyer. VoL. IV. 
185 á b. f. by sligo.................

1853 br. f. by Priam................ Ld Lichfield 1836

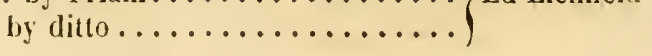

\section{WHISK,}

Bred hy Mr W. l'eirse, in 1823, got by Whisker, her dam, sister

to Benedick, by Remembrancer, out of Beatrice, by Sir Peter.

185.5 b. c. Cimetar, by Manreluke .......

1854 b. f. by Fungus............... Mr Biggs

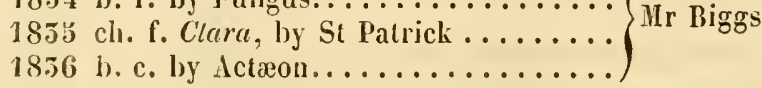

\section{WHISKER MARE,}

Bred by Mr Crosptor, in 1820, her dam, Tramp's dan, by Gohanna, out of Fraxinella, by Trentham.

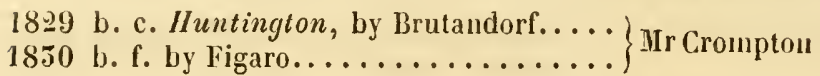

1831 by Blacklock

1855 ch. c. Rattle, by Langar...........

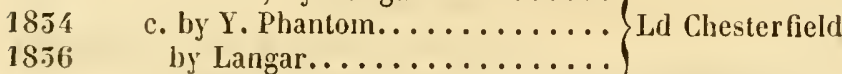

Barren in 1852 and 55.

\section{WHISKER MARE,}

Bred by Mr Orde Powletr, in 1820, her dam, sister to Bourbon,

by Sorcerer-Precipitate-Ilighflyer-Tiffany, by Eclipse.

1824 c. by Ardrossan.............. Mr R. Oswald 1825 b. c. Auchencruive, by Monrcill......

1829 gr. f. by Figaro.............. Mr T. 0. Powlett

1850 b. f. by Lottery (dead)...........

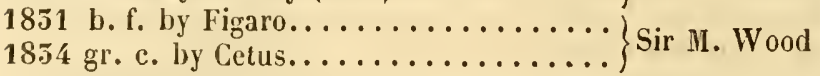

Slipped foal in 1827, missed in 26 and 28 , to Sultan in 52 and $\mathbf{5 5}$,

barren also in $5 \dot{5}$, and sent to Germany, covered by Cetus.

\section{WHISKER MARE (sister to Catterick)}

Bred by Mr Kaye, in 1821, her dam by Bay Trophonius, grandam by Slope, out of Lardella, by Y. Marske. 
1826 br. f. by Blacklock (s. to Denmark) ..

1828 b. f. by Doctor Syntax.......... IIr Kaye

1829 ch. f. Ellerton Lass, by Wanton.....

$1830 \mathrm{ch}$. c. by ditto ...............

1852 b. f. by Lottery (sold abroad)......

185.4 b. f. by ditto............... Mr Kirby

1855 b. f. by Brutandorf $\ldots \ldots \ldots \ldots \ldots \ldots \ldots$

Barren in 1827 and 55, slipped foal by Canto (her brother) in 51.

\section{IVIIISKER MARE,}

Bred by Lord Darungtov, in 1829, her dam, Castrelia, by Castrel, out of Madrigal, by Sir Peter-Pot80`s.

1828 b. c. Emilianus, by Emilius........

*1829 ch. f. Emiliana, by ditto ........ Hr If. Chifney

1850 missed to Camel..............

1851 br. c. Shilelagh, by St Patrick (s. to Hungary, 185j)

1855 ch. c. by Emilius.............

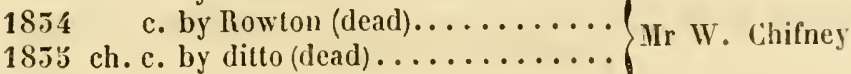

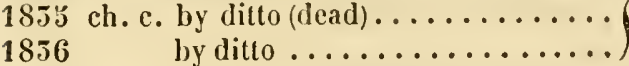

* Emiliana produced, in 1855, a ch. c. by Rowton, and was covereal again by him.

\section{WIISKER MARE,}

Bred by Mr WATt, in 1822, her dam, Mandane, by Pot80's, out of Y. Camilla, by Woodpecker.

1828 b. c. (Liverpool) by Tramp........ Mr Watt

1829 b. c. Manchester, by Blacklock...... Sir II. Wood

1850 b. c. Whirlpool, by ditto.........

1851 b. f. by ditto (died rising 1 year oid) .

1852 b. f. Waresti, by Sultan.......... MIr Wreford

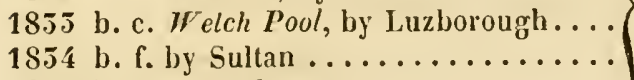

185 b. c. by Defence.............

18.56 missed to Camel............ Mr Tattersall

\section{WHISKER MARE (sister to Whisk)}

Bred by IIr W. Pesrse, in 1821, her dam, sister to Benedick, by Remembrancer, out of Beatrice, by Sir Peter. 
1828 b. c. Hofer, by Swiss ............. Mr Cookes

1829 b. f. Dragletail, by Tramp (s. to Ger- $\begin{array}{r}\text { many, 1856) } \\ \text { Mr Biggs }\end{array}$

Died at Ticklill, and had no foal except the above.

\section{WHISKER MARE,}

Bred by Mr R. Stepnevsox, in 182̈, her dam, Voltaire's dam, by Phantom-Overton-W Walnut-Ruler.

1850 br. c. Rousseau, by Blacklock.......

1851 br. c. by ditto (shot 2 yrs old) ......

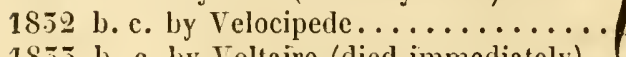

1855 b. c. by Voltaire (died immediately)..
1854 b. c. by Brutandorf............... Mr R. Stephenson

185.5 missed to Voltaire.............

18.56 b. c. by ditto $\ldots \ldots \ldots \ldots \ldots \ldots$

$18 \pi 7$ by Physician.............

\section{WIIISKER MARE (sister to Coulon)}

Bred by Mr Watr, in 1826, her dam, Miss Cranfield, by Sir Peter -Pegasus-Paymaster.

1850 ch. c. by BlackIock............

1831 b. c. Bubastes, by ditto................

1852 ch. f. Mansel Otz, by ditto ....... Mr Watt

185.5 b. f. by Brutandorf............

1834 b. f. by ditto................

1855 b. c. by Humphrey Clinker.........

Sold to go abroad in 1853, in foal to Belshazzar.

\section{WHISKER MARE,}

Bred by the Duke of LeEDs, in 1828, her dam by Dick Andrews, out of Gammer Gurton, by Pharamond.

1854 br. c. Mustee, by Lottery, or Mulatto. Mr J. Day $\left.\begin{array}{ll}1855 & \text { ch. f. by Medoro } \ldots \ldots \ldots \ldots \ldots \ldots \ldots\end{array}\right\}$ Mr J. Bawe

\section{WHISKER MARE (bay)}

Bred by Mr Ros, in , her dam, by Orville, out of Otterington's dam.

1823 b. f. The Nun, by Blacklock. . . . . . . Mr Armitage 
1826 ch. f. Melrose, loy Pilgarlic .........

* 1827 b. f. by Octavius ................ Ld Scarbrough

1852 br. f. Miss Milner, by Malek (put to

1852 br. F. Mre stud) Mr S. King

1834 b. c. Vernal, by $\Lambda$ ctxon...........

1856 by Langar.

Mr L. Savile

- This Octavius mare produced, in 1851. Mr Robinson's b. c. Aratus, by Catton, missed in 1852, and was sent to America in that year, covered by Catton.

\section{WHISKER MARE (sister to Memnou)}

Bred by Mr WArt, in 182-8, her dam, Manuella, by Dick Indrews, out of Mandane, by PotSo's.

1852 b. f. by Brutandorf .............

1855 missed to Falcon ..............

$1854 \mathrm{gr}$. c. by ditto .................. Whatt

1853 ch. f. by Margrave ............

1856

by ditto.

\section{WHISKER MARE,}

Bred by Mr Swarr, in 1826, her dam, Wathcote Lass, by Remembrancer, out of Gratitude's dam, by Walnut.

1850 br. c. by Doctor Syntax........... Mr T. Jackson

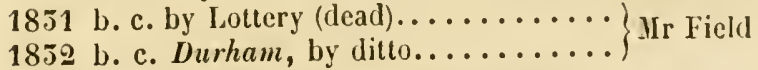

1855 by St Nicholas............

Sold to go abroad in 1855 , in foal to Count Porro.

\section{WHISKER MIRE,}

Bred by Mr Fergusos, in 1825, her dam, Lady Paul, by Sir Paul, out of Brown Javelin, by Javelin-Y. IIaiden, by IlighAlyer.

1835 b. c. by Jerry (dead)...........

1854 b. f. by detaon............. D. of leeds

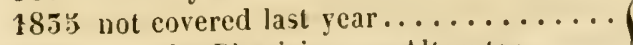

18.56

by Physician, or Alteruter...... 
IVIIISKER MARE,

Bred by Ir T. O. Powletr, in 1827, her dam by Ebor, out of Swinton's dam, by Shuttle-Delpini-Tuberose, by Herod.

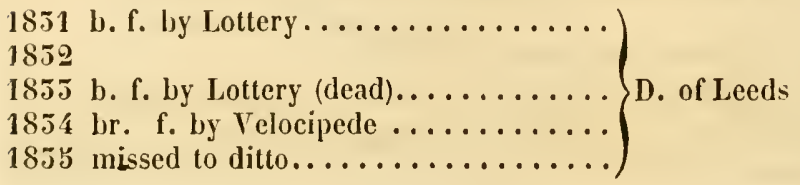

\section{WHISKER MARE (sister to Redstart)}

Bred by the Duke of Leens, in 1828, her dam, Rhodacantha, by Comus, out of Lisette, by Hambletonian.

1855 ch. f. by Cbampion.............

1854 missed to Actron............. D, of Leeds

185 b. f. Barbarina, by Brutandorf......

1856 by Physician

\section{WHISKER MARE,}

Bred by Mr Gascolgne, in 1827, her dam, Louisa, by Orville, out of Thomasina, by Timothy-Violet, by Shark.

1855 c. by Lottery (died soon after fualing)

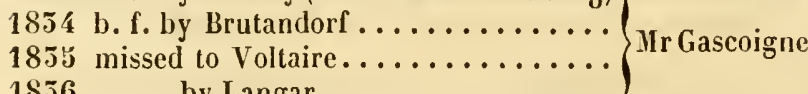

18.36

by Langar. .

\section{WHISKER MARE,}

Bred by Sir Charles Morck, in 1828, her dam, The Twinkle, by Walton-Orville-Lisette, by Ilambletonian.

1852 missed to Filho da Puta......... Sir C. Monck

1853 br. c. Belsay, by Comus.......... Gen. Grosvenor

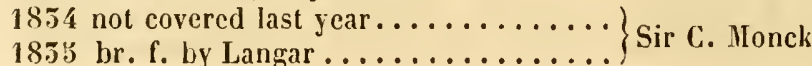

\section{WIISKER MARE,}

Bred by Jord Derey, in 1829, her dam, Urganda, by Milo, grandam by Sorcerer-Sir Solomon-Y. Harske. 
1854 br. f.

b. c. (died) by Zinganee ........ Mr I:. Marshall

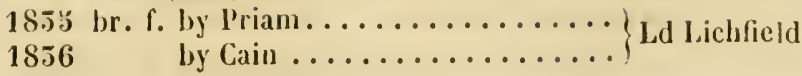

\section{WIIITELOCK MARE,}

Bred by Ur Tatron Srkes, in 1815 , her dam, Leicester Lass, lyy Young Imperator, grandam by Alexander, out of Kiss-my-lady.

1826 b. c. by President .

1899 b. c. by Young Phantom..........

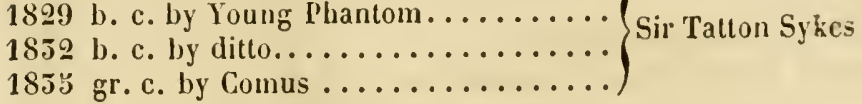

She was hunted till 1823 ; missed to Dinmont in 27, to Lottery in 28 , to Comus in 30 and 51 ; barren also in 5.5 and 54 .

\section{WIIITE ROSE,}

Bred by Capt. AYre, in 1819 , got by Comus, her dain by Whitworth, out of Torchbearer's dam, by St George-Pontac.

1823 b. c. by St Patrick (died 4 days old).. Dr Lloyd

1826 ch. c. Thatcher, by Blacklock........ Mr Loy

1827 ch. f. by Welbeck ............. Dr Lloyd

1829 br. c. Lelero, by Otho ............. Mr Loy

1850 br. c. by Coronation (dead) ........ Dr Lloyd

1832 b. c. by Waverley.............. Wr Lloy

Not covered in 1827 ; barren in 18.51 .

The WIIITWORTI MARE, her dam by Worliny, (Vol. III, p. 46.5) produced b. f. by Bob Logic in 185.5. and is dead.

\section{WHITWORTH MARE,}

Bred by Mr Sinafro, in 1816, her dam by Beningbrough, or Zechariah, out of Lady Charlotte, by Buzzard-Calash, by IIerod.

1823 gr. c. Lillle Johnny Myers, by Viscount IIr Jopliu

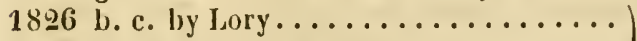

1828 ch. c. by Doctor Syntax...........

1829 ch. f. by Ben Ledi (died a week old). . Jir Shafto

1850 br. c. by Chateau Margaux (dead)....

1851 ch. c. by Agricola ...............

1852 b. c. by Waverley............. 
$1853 \mathrm{hr}$. f. by Waverley ............

1853 b. f. by Stotforth............. Mr Shafto

18.56

by Physician ..............

Ilad a half-bred f. in 1823, and not covered; missed in 27 and 54.

\section{WIIIZGIG,}

Bred by the Duke of Grafton, in 1819, got by Rubens, out of Penelope, by Trumpator-Prunella, by Highflyer .

1825 br. c. Omen, by Orville..........

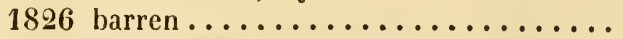

1827 b. c. Orbit, by Centaur ..........

*1828 b. f. Oxygen, by Emilius (put to the

1829 slipped foal, by ditto ......... stud)

$1830 \mathrm{br}$. f. Octave, by ditto (put to the stud) D. of Graftor

1851 ch. c. Olympic, by Reveller.........

1852 br. c. Orpheus, by Emilius (s. to Ger-

1835 ch. f. by Reveller

1856 by Taurus $\ldots \ldots \ldots \ldots \ldots \ldots$.

In 1833 missed to Emilius, in $\mathbf{3 4}$ to Reveller.

* OxYGEN produced in $1855 \mathrm{ch}$. c. by Zinganee, and was covered by St Patrick.

\section{WIDGEON,}

Bred by , in 1827, got by Whisker, her dam by

Dick Andrews, out of Desdemona, by Sir Peter-IIeroine, by Phœnomenon.

1832 ch. f. by Bedlamite (died in foaling)...

1855 b. c. Wingfield, by ditto...........

1854 br. f. by Cadland.....................

Sold to Baron de Maltzahn, March 1856.

\section{WILLIAMSON'S DITTO MARE,}

Bred by Sir Mark Mastermax Sykes, in 1810, her dam, Young, Rachel, by Volunteer, out of Rachel, sister to Maid-of-all-Work. $\left.\begin{array}{r}1815 \text { gr.c. Acceptance (afterwards (Jack Tar) } \\ \text { by Camillus (sent to India) }\end{array}\right\}$ Mr Acred

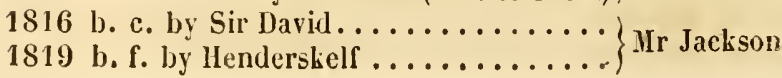




\section{5}

1820 eh. e. General Mina, by Camillus....

1821 ch. e. by Cestrian (died 5 yrs old)....

1822 br. c. (Liberator) by Blueher (sent to

Ireland)

1825 b. f. by Bustard ...............

\$824 b. e. Joceline, by Catton (s. to Baron

Biel in 1854 or 55$)$ Sir 'T. S. M. Stanley

*1825 br. f. by Tramp...............

1826 slipped foal, by ditto...........

+ 1827 ch. f. Augustina, by Tramp......

1828 br. c. Frankenstein, by Manfred.....

1829 ch. c. by Catton..............

1850 b. f. by Lollery...............

1851 ch. c. by Battledore.............

* This Tramp mare has harl one foal, viz. : 185.5 a br. f. Lena, by Battledore, and she was covered by him in 1855 .

+ Avgustixa prodnced a ch. c. by Ballledore in 1854; missed in 185.5 and 55 , and was covered in 55 by Pantaioon.

Barren in 1852 and 55 , and died in 5.5.

\section{WILLIA HSON'S DITTO MARE,}

Bred by Mr Rawlisson, in 1812, her dam, Agnes, by ShuttleHighflyer - Goldfinder-Lady Bolingbroke, by Squirrel.

1821 b. f. Reality, by Anticipation ......

1822 ch. f. Rarity, by ditto...........

1825 ro.f. Resemblance, by Gainsborough (s.

to France in 1829)

$182 \%$ ch. f. Ruby, by Rubens...........

1827 b. e. Rattler, by Spectre.......... IIr Rawlinson

1829 br. f. by Spectre, or Rubens........

1850 b. c. Revenge, by Fungus .........

1851 br. f. Resolute, by ditto (put to the stud)

1852 b. f. Reliance, by ditto..........

1855 b. c. Retaliator, by ditto.........

Not covered in 1823 , missed in 26 and 28 , and died in 55 , three weeks after foaling.

\section{WILLIAISON'S BITTO MARE,}

Bred by Mr N. II. Smiтh, in 1822, her dam by Trumpator, out of Countess, by Sir Peter.

1825 gr. f. (Zoolfah) by his Arabian....... IIr N. II. Smith

1826 b. c. by Nicolo. j 
1827 by his Arabian............ Mr N. H. Smith 1828 b. f. by Gulliver.............. Mr Lyne Stephens 1899 br. c. (Typhon) by Truffle......... D. of Rutland 1852 ch. c. Claremont, by Partisan .......

1854 br. f. by l'riam.............. Ld Chesterfield 1856

by Colwick..............

Missed in 1855 to Emilius, and in 55 to Zingance.

\section{WILLIAMSON'S DITTO IIARE,}

Bred by , in 1822, her dam, Antelope, by SorcererAnvil-Lily of the Valley, by Eclipse.

1827 f. by Figaro.............. Ld Verulam

1828 b. c. Ticket, by Lottery...........

1850 ch. f. Tiara, by Waxy Pope............... Mr Ormsby Gore

1852 ch. f. by The Duke ............

185.5 ch. c. King Lear, by Bedlamite ..... Mr Sowerby

18.54 c. by Shakspeare ........... Mr R. Wilson

1855 by ditto.

Barren in 1829 and 51.

THE WINDLE MARE, Leviathan's dam (Vol. III, p. 467) produced, in 1852, br. f. Orphan, by Belzoni (put to the stud) and died a few hours after foaling her.

\section{WINGS,}

Bred by Gen. Grosvexor, in 1822, got by The Flyer, her dam, Oleander, by Sir David-Whiskey-Grey Dorimant.

1828 slipped foal, by Phantom..........

1829 ch. c. by Middleton............... Ir S. Stonehewer

1830 b. c. by Partisan...............

1851 b. c. Pigeon, by Reveller......... Gen. Grosvenor 1852 by Camel

185.5 b. f. by Brutandorf............. Mr Greatrex

1854 b. c. Carazan, by Camel.......... Ld Stradbroke 1856 ch. c. by Actieon.............. His IIajesty

Missed to Camel in 1853.

TIIE WITCI,

IBred at Ilampton Court, in 1821, got by Soothsayer, her dam, sister to Ilospitality, by Election, out of Selim's dam. 


\section{7}

1827 ch. f. The Fairy, by Ennilius........

1828 ch. c. Cripplegate, by IIorisco...... Ir S. Stonehewer

1829 b. c. by Middleton.............

1850 ch. c. Toby, by 'Tarrare (s. to India)...)

1851 b. 1. (Goblin) by Enilius.......... D. of Richmond

1852 b. c. Conjuror, by Camel..........

1855 b. f. Gipsy, by Sir Hercules........ Mr Hendrick

1854 b. c. Wizard, by Y. Blacklock.......

The last three foals were bred in Ireland.

\section{WOFUL IIARE (Fairy)}

Bred by Mr Wissor, in 1819, her dam, Gousalvi's dam, lyy Remembrancer, out of Charmer, by Phœnomenon-Fitz-herod.

1824 ch. f. Sprite (aft. Countess) by Comus $1825 \quad c$. by ditto (died a foal) $\ldots \ldots \ldots \ldots$

1826 b. f. Bonnet Pet, by St Patrick ......

1827 br. c. Shamrock, by ditto......... Sir F.. Dodsworth

1828 b. f. by ditto................

1829 b. c. by Catton..............

1850 b. f. Charmer, by Swiss ..........

1851 br. f. by Velocipede ............ Mr Carter

1853 twins, by Langar (dead)..........

1834 ch. f. by ditto................ Mr G. Clark

$1853 \mathrm{ch}$. c. by ditto..............

Missed to Langar in 1832 , to Vauish in 56 .

\section{WOFUL MARE,}

Bred by Lord Exeter, in 1825 , her dam (Zealot's dam) by Rubers, out of Guildford Nan, by Guildford.

1827 b. f. by Phantom.............

$182 S$ b. f. Terapia, by Sultan (put tothe stud)

1829 ch. c. by Mountebank...........

1830 b. f. (Shoulass) by ditto .......... Ld Exeter

1851 ch. c. Lumber, by Sultan ........

185.5 ch. f. by Redgauntlet ...........

Barren in 1852,54 , and 55 .

\section{WOFUL, HARE,}

Brel by Lord Bersers, in 182:3, her dam, Camarine's dam, by Rubens, out of 'Tippitywitchet. 


\section{8}

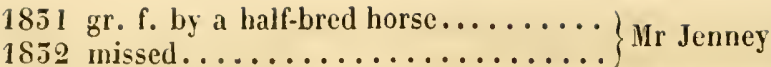

1853 ch. f. Lauly Day, by St Hubert...... Mr R. Prince

1854 ch. f. by ditto $\ldots \ldots \ldots \ldots \ldots \ldots \ldots$

1853

1856

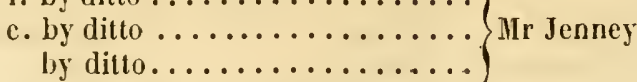

WOFUI, MARE,

Bred by Mr Bergess, in 1824, her dam, Allegretta, by Trumpator, out of Y. Camilla.

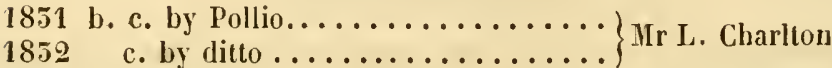

1855 b. f. by ditto (s. to Berlin in 1855) . . Ld Chesterfield

1854 b. f. Iecuba, by Priam........... M. Greville

1855 missed to Zingance...........

WOFUL MARE,

Bred by Lord Exeter, in 1824, her dam, Phantom, by Hambletonian-Precipitate-Ilighflyer-Tiffany, by Eclipse.

1829 ch. f. by Figaro...............

1850 c. by ditto............... Mr Harmer

1851 f. by ditto.............

1852 br. c. Green Leighton, by ditto...... Mr R. Wilson

1854 b. f. by Shakspeare............ Ld Rivers

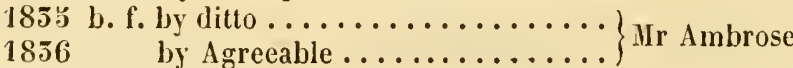

WOFUL MARE,

Bred by Mr Ilanuoxd, in 1825, her dam by Selim, out of Pipylina, by Sir Peter.

1829 ch. c. by Truflie

$1830 \mathrm{ch}$. f. by Tarrare.

1851 br. f. Stockwell Lass, by ditto (s. to

Germany in 1853) Mr Theobald

1852 b. f. by Mameluke.............

1853

f. by Camel................

1834 b. c. by Laurel ...............

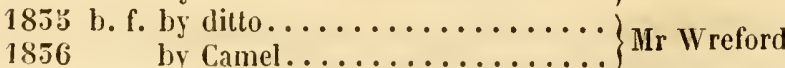




\section{9}

\section{WOFUL MARE,}

Bred loy Lord Exeter, in 1825, out of Ilolbein's dam, by Golumpus.

$\left.\begin{array}{r}1850 \text { br. f. loy Sultan (sent to Jamaica in } \\ \text { May 1855) }\end{array}\right\}$ Mr Grant

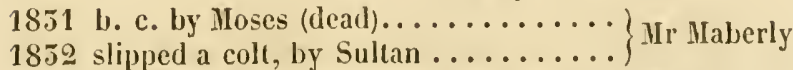

1855 ch. c. by Bedlainite............. Mr Ilills

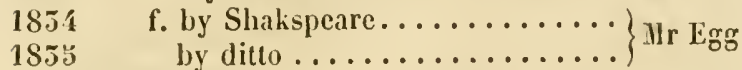

\section{WOFUL MARE,}

Bred by , in 1828, her dam by Eeningbrough, out of sister to Star, by Jlighflyer.

1854 b. f. by Segrave..............

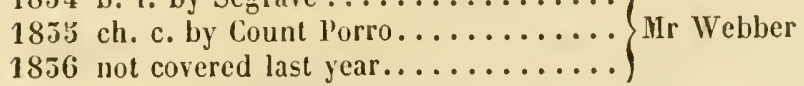

\section{WOKINGHAM MARE,}

Bred by Mr Frasptov, in 1824, her dam by *Grey Lop, grandam, Fushlight, by Woodpecker-Bobtail, by Eclipse.

1851 br. c. Lincroft, by Figaro.........

1852 b. c. Tormentor, by ditto .........

1855 missed to Lottery .............. MIr Jas. Jackson

1854 b. f. by Waverley .............

1853 b. c. by William the Fourth .......

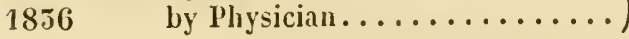

* Grey Lop was bred by Sir J. Rous, in 1791, and was own brotber to Juliana, by Crop.

\section{WOODBINE (sister to Fortuna)}

Bred by Mr Warsov, in 1817, got by Comus, her dam by Patriot

-Phœnomenon-Czarina, by Babraham Blank-Melpomene.

1825 b. f. Jessamine (aft. Cream) by Catton)

1826 b. c. by 'Tramp (killed by' accident)... Hr Darnell

$1828 \mathrm{ch}$. c. by ditto...............

1851 ch. c. by Peter Lely............................... Derby

rol. IV. 


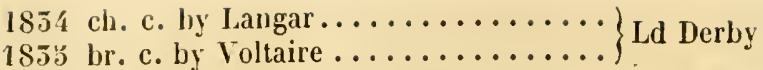

In 1824 missed to Vandyke Junior, 25 to Whisker, 27 to Cerrantes; barren also in 1852 .

\section{WORRY,}

Bred by Mr ThonхнiLl, in 1826, got by Woful, her dam, Sal, by Scud, out of Hyale.

* 1851 b. f. by Emilius............... Mr Thornhill 1852 b. c. Teddy the Tiler, by ditto...... Ld Chesterficld

Sent to France in 1855, with a ch. c. foal, by Emilius, and stinted to him again.

* This mare produced a b. c. by Shakspeare, in 1835 .

\section{WORTHLESS,}

Bred by Mr Wart, in 1822, got by Walton, her dam, Altisidora, by Dick Andrews, out of Mandane.

1827 b. c. Apuntador, by Blacklock .......

1828 b. c. by Champignon (s. to Vienna in Mr Watt 1850)

1829 b. f. Beauty, by Catton........... Mr Beardsworth

1830 b. c. by Blacklock............. Mr Grant

1851 b. c. Haldon, by ditto........... Sir L. Glyn

1852 ch. c. Operator, by Emilius ........

1854 ch. f. by Peter Lely ............. Mr Grant

1855 missed to ditto..............

1856 by Priam............. Ld Chesterfield

\section{WORTHY MARE,}

Bred by Lord Queevsenrry, or Mr Rossor, in 180\%, her dam, Tiny, by Sir Peter, out of Wren, by Woodpecker.

1809 b. c. by Sancho (died)..................

1815 b. c. Clarendon, by Orville......... Mr Brandling

1816 b. c. Corncreali (aft. Fitz-Langton) by $\begin{aligned} & \text { Langton } \\ & \text { Mr Young }\end{aligned}$

1817 b. c. (died) by ditto............. Mr Wiley

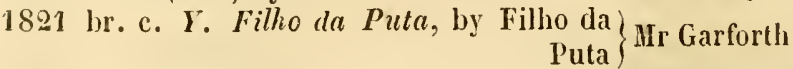

1825 ch. c. by Tramp.............. Mr Jas. Croft 


\subsection{1}

1825 br. f. Kathleen, by Blacklock........ Ir Crosier

1826 b. f. Ceresina, by ditto........... Mr Wiley

Missed in the intermediate years, and died in 1951.

N. B. The produce of this mare were incompletely given in Vol. II. She was the property of Mr. Wiley, of Bransby, during the whole period of her breeding.

\section{XARIFA,}

Bred by Mr PAGE, in 1826, got by Ioses, her dam by Rulsens, out of Pointer's dam, by Woodpecker-Gohanna's dam, by Herod.

1852 b. f. Chatterbox, by Filho da Puta.... Mr W. Edwards

1855 ch. f. by bedlamite ........... Mr R. Wilson

1854 b. f. by Shakspeare ............

1855 b. f. by Grey Comus ............ His Majesty

18.36

by Rubini ...............

\section{YNYSYMAENGWYN,}

A chesnut mare, bred by Mr V. Coneet, foaled in 1819, got by Fitz-James, her dam by Sascnagh, grandam by Meteor, out of Mr Corbet's grey Highflyer mare, sister to the dam of Superior.

1828 ch. c. by Eryx............... Ir Corbet

1850 ch. f. Mountain Maid, by Zealot..... Gen. Grosicum

1851 b. c. Algerine, by Mameluke.......

$185 j$ ch. f. Themesis, by Middleton........

$1854 \mathrm{ch}$. c. by Minos............... Mr Corbet

1855 ch. c. by ditto ...............

1856 by Bizarre..............

Mlissed in 1829 and 52.

\section{YORKSHIRE LASS,}

Bred by Mr Witsox, in 1826, got by Cerrantes, out of the Juggler's dam, by Pipator-Delpini - Tubcrose, by Herod.

1850 gr. c. by Gustavus.............

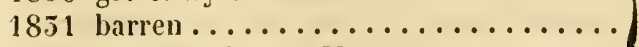

185 ○ b. f. by Chateau Margaux...........

1855 ch. f. by Langar .............. Mr W ilson

1834 ch. c. by Velocipede............

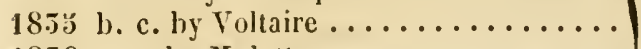

1856

by Mulatto 


\section{ZAFRA,}

Bred by the Duke of Graftos, in 1819.

1850 ch. c. by Moses..............

1851 ch. c. Robinson Crusoé, by Helenus .. D. of Richmond

1852

185 by ditto f. by Gaberlunzic..

\section{ZAIRE,}

Bred by Mr Caldey, in 1819, got by Selim, out of Zephyretta, by Hedley - Momentilla's dam, by Diomed.

1823 foal, by Moonraker (died young) .... . Mr Henty

1826 b. f. Keepsake, by Memorandum..... Gen. Grosvenor

1827

by Skim................

1828 ch. c. Crutch, by Little John ...... . Capt. Rous

1850 gr. f. Violante, by Gustavus........ Mr A. Vansittart

1832 b. f. by Nonplus.

1854 b. f. by Tarrare .............. M. Greatrex

1856 ch. f. by The Colonel...

1857

by Plenipotentiary ..........

Missed in $1829,5 \mathrm{I}, 55$, and 5 5.

\section{ZARINA,}

Bred by Lord Lichfietd, in 1827, got by Morisco, her dam, Ina, by Smolensko, out of Morgiana, by Coriander.

$1851 \mathrm{ch}$. f. by Sligo (put to the stud)...... Ld Lichfield

1832 ch. f. by Peter Lely............. Mr Mott

1854 b. c. by Cadland ..................

1855 ch. f. by Emancipation ........................... Mr F. Marshall

\section{ZEAL,}

Bred by the Duke of Graftox, in 1818, got by Partisan, her dam,

Zaida, by Sir Peter, out of Alexina, by King Fergus-Lardella.

1824 br. f. Arab, by Woful............

1825 gr. c. Archer, by Skim (s. to India)...

1826 b. c. Artist, by Emilius .......... D. of Grafton

1827 br. c. Abbot, by ditto ............

1828 b. c. Almoner, by ditto............ 


\section{3}

1829 missed to Emilius

1850 br. f. by Centaur (s. to Mr Lichtwald,

1851 br. or bl. c. by Lamplighter........ D. of Grafton

1835 b. c. Alumnus, by Saracen.........

1834 br. f. Algebra, by Ranvilles ........

1856 slipped foal by ditto............

Mlissed in 1829,52 , and 53.

\section{ZELINDA,}

Bred by Mr C. DAY, in 1825, got by Rubens, her dam, 'Zuleiha, by Gohanna, out of Trinidada, by Y. Woodpecker.

\section{9}

c. by Truffle.

1850 and 51 barren $\ldots \ldots \ldots \ldots \ldots \ldots \ldots$

1852 b. f. Boletas, by Fungus...........

1835 ch. c. Zeno, by Middleton................ IIr Day

185 b. c. by Sir IIercules............

1856 missed to Cain.......................

\section{ZELINE,}

Bred by Mr $\Lambda$ Lexarder, in 1825 , got by Viscount, her dam, Albuera, by Ilambletonian, or Beningbrough-Constantia, by Walnut-Contessina, by Y. Marske.

1851 br. f. by Luzborough ...........

1832 gr. f. Mariana, by Maresfield....... Mr G. I). Tyler

$18 \% 6$ by Actæon.

In 1850 missed to Tarrarc, 55 to Chateau Margaux, and not covered that year.

\section{ZENOBIA,}

Bred by Lord Verulas, in 1828, got by Wialebune, out of Venom, by Rubens-Spitfire, by Beningbrough-Y. Sir Peter (son of Doge).

$1852 \mathrm{ch}$. f. Kathleen, by Phantom (s. to Baron)

1834 ch. f. La IJéprisée, by Velocipede.... S Sir G. Iteathcote

Barren in 1855 and 5 . 


\section{$35 \cdot 4$}

\section{ZEPHYRINA,}

Bred by Lord G. H. Cavendish, in 1819, got by Middlethorpe, out of Pagoda, by Sir Peter-Rupce, by Coriander.

$1825 \mathrm{ch}$. f. by Magistrate (died a foal) .....

1826 ch. f. Kalmia, by ditto..........

1827 b. c. by Sherwood.............. IIr W. Charlton

1829 b. c. Rokeby, by Abjer ...........

1831 b. f. Iannah, by Tramp..........

$1852 \mathrm{ch}$. f. by Langar.............. Ir Giffard

1855 b. f. by Filho da Puta...........

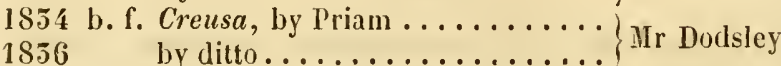

Slipped foal in 1824, missed in 28, 50 , and 55 .

\section{ZILLAI,}

Bred by Lord Licifield, in 1827, got by Whisker, her dam, Elizabeth, by Orville, out of Pciny-trumpet, by Trumpator-I. Camilla.

1855 b. f. by Lamplighter............

18.54 b. f. Cassandra, by Priam.........

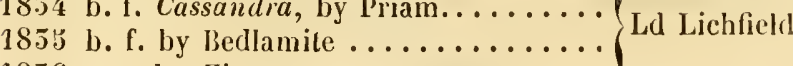

1856 by Zinganee............

\section{ZINC,}

Bred by the Duke of Graftox, in 182., got by Woful, out of Zaida, by Sir Peter.

1827 br. f. Emerald, by Emilius (s. to Sweden in 1851)

*1828 br. f. by Aljer.

1829 bl. c. Ebony, by Truffle

1850 b. c. Emir, by Centaur

1851 br. f. by Sultan (died 6 weeks old) ... D. of Grafton

† 1852 br. c. Emu, by Cannon Ball,or Buzzard

1855 slipped foal, by Bedlamite.........

1834 br. f. by Shakspeare (s. to Germany)..

1853 b. f. by ditto

1856 b. c. by Merchant.

* This Abjer mare produced, in 1855, Mr Pettit's b. f. Prudence, by Wiseacre, had a dead foal in 1854 , uissed in 1855 , and was covered that year, by Wiseacre.

+ Emu was foaled on the 50th March-his dam wascovered by Cannon Ball on the 8 th of April, and by Buzzard on the 28 th. 


\section{$35 \%$}

$Z \operatorname{IRZ} \Lambda$,

Bred by $11 \mathrm{r}$ Ckonptos, in 1822, got by Amadis, or Blacklock, out of Rosamond, by Buzzard.

1828 ch. c. Edie Ochiltree, by Tramp.....)

1829 ch. c. by Whisker.............. Mr G. Crompton

1851 b. c. by Figaro ................... Ir Broenenberg

1852 br. f. by Tramp ............. Mr Broenenberg

1853 br. c. by St Nicholas.............

1854 ch. c. by ditto (died a foal) ......... Mr G. Crompton

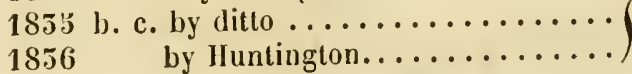

\section{ZOÉ,}

Bred by Lord Lowtrer, in 1823, got by Orville, out of Nina, by Selim-Pennytrumpet-Y. Camilla.

1850 ch. c. Zeluco, by Bobadil .......... Mr MEolony

1851 b. c. Old Bill, by Waxy Pope ........ Mr Mills

1852 b. f. Amarlillo, by Bobadil..........

1855 ch. f. by ditto (died a yearling) ......

1854 missed......................... IIraffenden

1853 br. f. by Bobadil ...............

1856 by $\operatorname{ditto} \ldots \ldots \ldots \ldots \ldots \ldots \ldots$

\section{ZORA (sister to Azor)}

Bred by Mr PAYNe, in 1812, got by Selim, out of Zoraida, by Don Quixote-Lady Cow, by John Bull.

1817 ch. f. by Woful (sent abroad) ....... Mr Rush

1818 ch. f. Isidora, by Blucher.......... Mr Kingston

1819 ch. C. Flora M'Ivor, by ditto........ Hr T. Waller

1820 br. c. by dillo............... Ld Suffield

1821 br. c. by ditlo................

1825

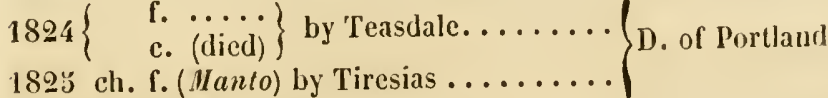

c. by ditto (died a yearling)......

1826 ch. c. by ditto ...............

$1827 \mathrm{ch}$. c. Jonathan, by ditto ......... Mr Yates

1828 b. f. Susanna, by Swap......... 


\section{6}

1829 ch. c. Non Compos, by Bedlamile....)

1830 ch. c. Nonsense, by ditto ......... Col Pecl

1855 ch. f. IIoax, by ditto............

Missed in 1822, 51, 52, 54, 55, and 56 .

\section{ZULEIKA,}

Bred by Lord Egremost, in 1810, got by Gohanna, her dam, Trinidada, by Young Woodpecker, out of Platina, by MercuryHerod-Y. Hag, by Skim-Crab-Childers, etc.

1820 ch. c. Ontario (aft. Melainpus) by Sooth-

1821 ch. c. IIelenus, by ditto ............ Mr C. Day

1825 b. c. Panic, by Spectre............ Mr Hills

1824 b. f. (Passion, aft. Ariel) by ditto....

1823 ch. f. Zelinda, by Rubens..........

1826 ch. c. Zeuxis, by ditto ...........

1827 b. f. by Spectre.............. Hr C. Day

1829 br. f. Milcah, by Middleton.........

1851 ch. f. by Mameluke (dead)..........

1853 ch. f. by Velocipede............

Missed to Spectre in 1829, to Luzborough in 50 , to Whisker in 52 , and died July 7,1855 . 


\section{AP PEN D IX.}

\section{$\Lambda$ DELAIDE,}

Bred by Ir GuLLi, in 1829, got by Lottery, out of Stotforth's dam, by Sir Paul-Brown Javelin, by Javelin.

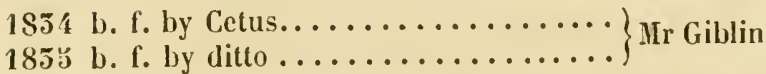

185 วั

To the ALEXANDER MARE, out of Comedy (Vol. III, p. 7) add :

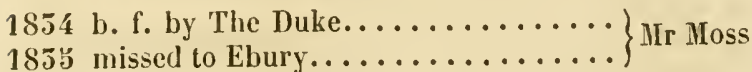

\section{AHADINE,}

Bred by Mr SerJentson, in 1818, got by Whitelock, her dam by Sorcerer, out of Brown Charlotte, by Highflyer-sister to

Soldier, by Eclipse.

1822 gr. c. Lycastus, by Minos......... I,d Normanby

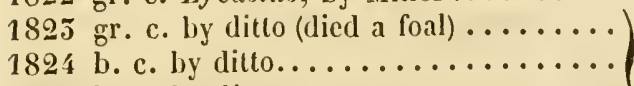

1825 b. c. by ditto................. Hr IIaythorne

1826 br. c. Wandering Boy, by Oiscau....

1827 slipped a filly, by Theodore ........

$1828 \mathrm{ch}$. c. by ditto................. Ir J. Scott

1829 b. c. by ditto (died a yearling).......

1850 b. c. Ploughboy, by $\Lambda$ rbutus, or Theo- Mr S. Reed dore

1851 b. c. by Theodore.............

1852 b. f. by Tramp, or Grey Walton (put to IIr Ilaythorne the stud)

185.5 ch. f. by St Nicholas........... Mr W. Stanley

1854 b. f. by llumphrey Clinker.........

1855 missed to Clinker.............. IIr Haythorne

1856 by St Nicholas, or The Dube... 


\section{AHADIS IIARE,}

Bred by Lord Fitzwiluan, in 1821, her dam, Valentina, by Smolenstio, out of Shepherdess, by Shuttle.

1832 br. f. by Sandbeck ............ Ld Fitzwilliam

1853 br. f. by Mulatto (dead) . . . . . . . . Mr Dickinson

\section{AMIML (sister to Augustus)}

Bred by Lord ExeTER, in 1850, got by Sultan, out of Augusta, by Woful.

1853 b. f. by Redgauntlet.............
1856 b. f. by Reveller ........... Exeter

\section{ARDROSSAN IIARE,}

Bred by Mr Gascoigne, in 1822, her dam, Shepherdess. by Shuttle-Buzzard-Amn of the Forest, by King Fergus.

1854 br. f. by Merman .............

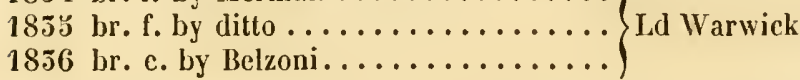

AVON LASS (Vol. III, p. 22) has had only one foal since 1852 , viz : -

1854 br. c. by Camel,.............. Mr Grover

\section{BENEFIT,}

Bred Mr Gulwy, in 1826, got by Oiseau, her dam by Prime Minister, oul of Lady Ern, by Stamford.

1855 ch. f. Goldfinch, by Mameluke...... Mr S. Herbert

1854 and 58 missed............. MIr C. Finch

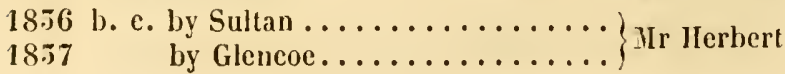

\section{BESSY BEILAII,}

Bred by Mr Krva, in 182\%, got by Filho da Puta, out of Lunatic, by l'rime Minister.

1854 b. c. by Lottery ............. Sir II. Wood

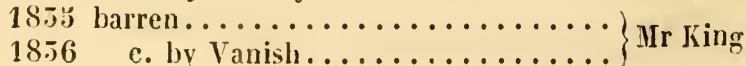




\section{9}

BLSK.

lired by in 1824 , got by Whalebone, her dası, Car, by IIaphazard, out of sister to Bourbon. by Sorcerer.

1853 b. c. by Tranby ..............

1856 b. f. by Cain ................ Mr I. Day 1857 by Nimrod (Topsy Turvy) ......

\section{CANNON BALl, MARE (sister to Cadwal)}

Bred by Mr Bradsiaw, in 1822 or 25 , her dam by Williamson's Ditto-Woodpecker-Syphon-Nlatchem.

1828 gr. c. Speculator, hy Lottery....... Ld Derby

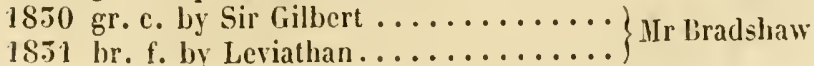

To CASTRELLA (Vol. III, p. 64) add

1852 br. f. Slime, by Picton .......... Ld Sligo

Ilso the name Broadsword to lier br. c. by Whisker, foaled in 1825.

To CAT, by Stamford (Vol. III, p. 64) add

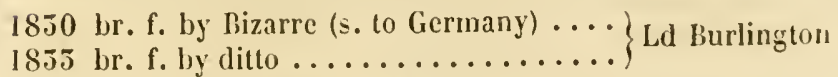

Missed in 18.34, and is dead.

\section{CENTAUR MARE (Recotery)}

lired by IIr BATsox, in 1827, out of Jlarrict (Plenipotentiary's dam) by Pericles-Selim-l'ipylina.

1854

1855

18.56

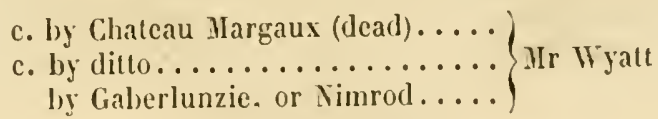

\section{CHAMPIGNON MARE,}

A grey mare, out of an Arabian mare.

1851 ch. f. by Actæon ............. Ld Elcho

185: c. by Osman (an Arab) ......... Ld Lynedoch 


\section{CHROMATICA,}

Bred by , in , got by Stamford, herdam,

Pecunia, by Pensioner, out of Monica, by Sir Peter-Y. Marske

- Matchem.

1850 b. c. by Canteen.............. Sir J. Boswell

1855 b. c. by Conservator........... Ld Eglinton

\section{CITRON,}

Bred by the Duke of Grafros, in 1827, got by Centaur, her dam, sister to Pastille, by Rubens, out of Parasol.

1834 b. c. by The Colonel...........

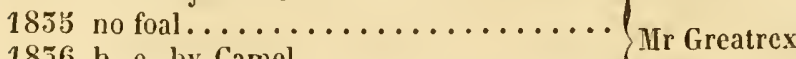

1856 b. с. by Camel ...............

COIIUS MLRE,

Bred by Lord WнавNchiffe, in 1828, her dam, Scandal, by Selim-Haphazard-Precipitate-Colibri.

1855 f. by Shakspeare (dead) ........

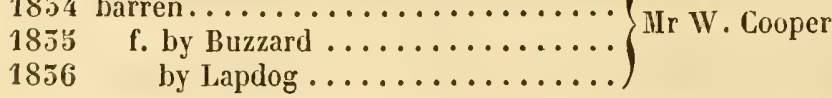

\section{CONCILIATION,}

Bred by the Duke of Ricunond, in 1828, got by Moses, her dam, The Duchess, by Cardinal York, out of Miss Nancy.

1854 b. f. by Mameluke..............

1855 b. c. by Tranby ................... of Richmond

1856 ch. c. by Rubini ..............

\section{CREDULITY,}

Bred by Capt. Berkeley, in 1827, got by Phantom, her dam,

Clarinet, by Camillus, out of sister to Alonzo, by Pegasus.

$\left.\begin{array}{l}185 \text { b. ch. Superstition, by Dunsinane .... } \\ 1856 \text { b. c. Irvinite, by Hindoo ......... }\end{array}\right\}$ Mr Bruhl 


\section{1}

\section{CELANDINE,}

Bred by Mr Careless, in 1826, got by Rubens, out of Geraldine, by Walton-Arquebusade, by Sancho.

18.53

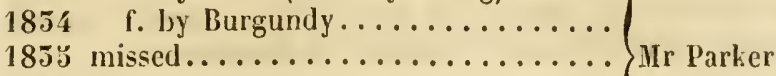

c. by Filho (died a yearling) ......

$1856 \mathrm{ch}$. c. by Belzoni.

1837

by Saracen.

\section{CLINKER IIARE,}

Bred by Mr 'Tıвits, in 1819, out of Shutle Pope's dam, by Oberon-Stride.

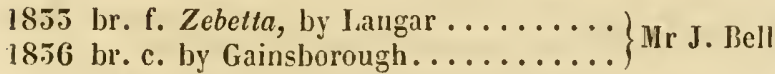

Missed to Voltaire in 1851 , to Gainsborough in 53.

\section{DABCHICK,}

Bred by Mr Beecuex, in 182J, got by Anticipation, her dam, Atalanta, by Walton, out of sister to Tup, by Javelin.

1852 br. f. by Belzoni..............

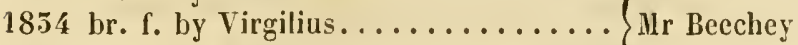

1856

by Windcliffe.............

She had no foal in 1855 or 35 .

\section{DECEPTION,}

lired by Lord Exerer, in 1850, got by Mountebank, out of Advance.

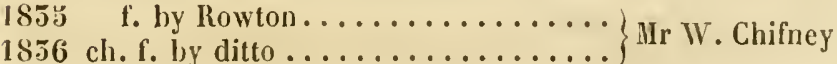

DICK ANDREWS MARE, Rector's dam (Tol. IH, p. 105') add1952 ch. f. by Rector............... Mr Lane 1854 br. f. Lucetta, by Sarpedon........ Gen. Grosvenor

The mare is dead.

DINAH,

Bred by Mr Gascolgve, in 1828, got by Champignon, ler dam, Louisa, by Orville, out of Thomasina, by Timothy.

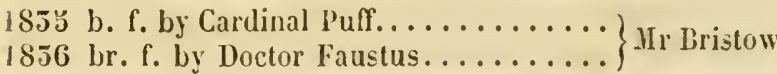


To DODO (Vol. III, p. 484) add-

185 \& b. f. by Oppidan.............. Sir C. Knightley

DON JUAN MARE,

Bred by Mr Carter, in $\mathbf{1 8 2 5}$, her dam by Sir Andrew, out of 'Tuneful, by Trumpator-Seafowl, by Woodpecker.

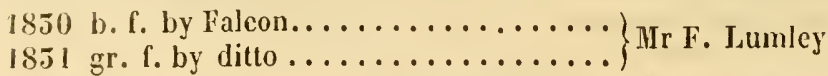

1852 gr. f. by ditto ............. Mr S. King

1855 b. f. by ditto ............... Mr F. Lumley

Cast twins in 1854 and 55 , and sold.

THE DUCHESS, by Cardinal York (Vol. III, p. 112) slipped foal in 1852, and missed in 1855, 54, 35, and 56.

\section{YOUNG DUCHESS,}

fhed by Mr Sispsox, in 1824, got by Constable, her dam, Lady Abbess, sister to The Duchess, by Cardinal York.

185.5 b. c. by Peter Lely ............. Mr Scarisbrick

18.54 b. c. by Lottery .............. Mrr Jas. Lea

Sent to the United States in 1854 , in foal to Recovery.

To ENCHANTRESS, by Sorcerer (Tol. III, p. 126) add :1855 b.c. The Vivaude, by Reveller, or Pacha. Ld Conyngham

\section{EUCHARIS,}

Bred by Sir A. Dow, in 1814, got by Remembrancer, her dam by Hambletonian, grandan by Trumpator, out of Cinderella.

1827 br. c. by Fitz-Orville............

1828 b. c. by Recorder ............. Col. Johnston

1899 l. c. by Fitz-Orville.............

1851 c. by ditto (dead) . . . . . . . . . . .

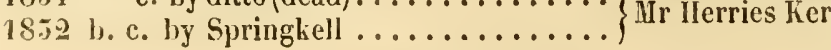

Dead.

\section{EURYONE,}

Bred hy Hr SadLer, in 1828, got by Reveller, her dam by Selim, out of Euryone, by Witcheraft.

1853 br. f. by Defence.............
$1856 \quad$ c. hy ditto $\ldots \ldots \ldots \ldots \ldots \ldots$ 
FAME, by Caleb Quotem (Vol. III, p. 151) has had no foal since 1851 .

\section{FENELIA,}

Bred by Ir Adeane, in 1824, got by Woful, her dam by Wallon. grandan by Sorcerer, out of Medora's dam.

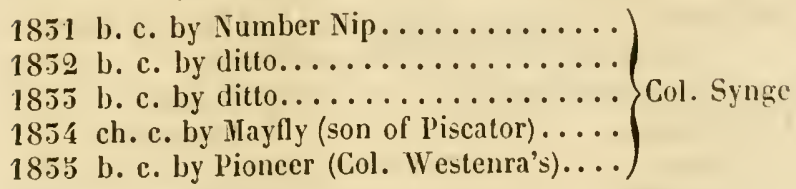

FICKLE,

Bred by Lord Lowther, in 1826 , got by Smolensko, her dam by Orville, out of Lisette, by Hambletonian-Constantia, by Walnut.

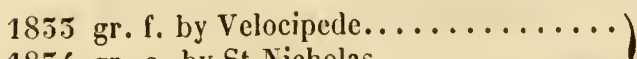

1854 gr. c. by St Nicholas........... Ur Osbaldeston

1855 b. f. by Brutandorf $\ldots \ldots \ldots \ldots \ldots \ldots$
1856

\section{FYLDENER IIARE (Dalcea)}

Bred by Mr Bradery, in , her dain, Duchess of Limls, by Junius, out of Arriana, by Y. Eclipse.

1828 bl. c. by Skyrocket (dead)......... Sir F. Lawley

185 b. f. by Bedlamite.............. Mr B. Thompson

1855 b. c. by Monarch............... Sir F. Lawley

1853

by a son of Cannon Ball ........

GOSSIP, by Walton (Vol. III, p. 135).--Her last two produce should stand thus :-

1850 b. f. Tabby, by Manfred ......... Mr L. Charlton

1851 b. f. Termagant, by Pollio ......... Mr Worley

GRAMARIE, by Sorcerer (Vol. III, p. 137). - Iler last produce should stand thus :-

$\left.\begin{array}{r}1831 \text { bl. f. Enchantress, by Reveller (s.to Spa } \\ \text { in } 1854 \text { ) }\end{array}\right\}$ Mr Hott

\section{GRISELDA.}

Bred by MIr H. Dawsov, in 1827. got by Lory, her dam by Remembrancer, out of Mary - by Y. Marske. 
18.51 br. c. Wy Mr Astley's BI. Turk, or Barb Mr Attwood 1852 br. f. Euphemia, by do. (put to the stud)

1855 ch. or sorrel f. by Argantes........ Hr C. Peck $185:$ by Voltaire ................. Mr C. Pech

\section{IIAMBLETONIAN MARE,}

Bred by Mr Rasxes, in 1810 , her dam foaled in 1799 , by Iluby Carbuncle-Bashful's dam, by Doge.

1817 b. c. by a half-bred lorse ......... Mr Raines

1819 b. f. by dineas............... Mr Simpson

1821 b. c. by ditto.................

1825 ch. f. by Tramp .............. Mr Raines

1824 b. c. by Thornton.............

1827 ch. c. by Y.Phantom............ Hr Owst

Barren in $1816,18,20,22,25$, and $26:$ died in 27 , with her foal.

\section{IIARRIET,}

Bred by Dr Wrutrs, in 1825, got by Cannon Ball, her dan, Snail, by Y. Warter, out of Elizabeth, by Beningbrough.

1852 f. by Nigel (dead)............. Mr Firth

1853 trained, and not covered..........

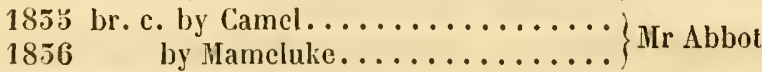

To II WWKLEAD, by the Borac Arabian (Vol. III, p. 170) add1832 b. c. Chevalier, by Jerry.......... Mr R. Walker

\section{HEART'S-EASE,}

Bred by IIr Cinspion, in 1829, got by Fitho da Puta, out of Rough Robin's dam, by Langton.

18.54 b. f. by Lottery .............

1855 missed to ditto ............. MIr Kirby

1836 c. by Starch ..............

Sold to Baron de Maltzahn in the Spring of 1856.

IIENRIETTA,

Bred by Mr J. Surn, in 1831, got by Jerry, out of Chat, by Quiz.

185.5 ch. c. by Actron................

1856 b. c. by Y. Longwaist............. Mr J. Smith

1857

by Memnon Junior. 


\section{HER HIGINESS.}

Bred by Sir T. Mostyx, in 1828, got by Moses, her dam, I'rincess Royal, by Castrel, out of Queen of Diamonds.

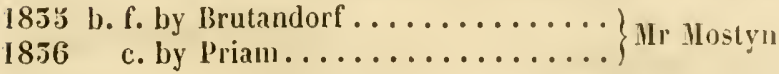

\section{HONEY-IIOON,}

Bred by Lord Westursster, in 1850 , got by Filho da Puta, wut of Ilybla.

1853 br. c. by Pantaloon (dearl)..........

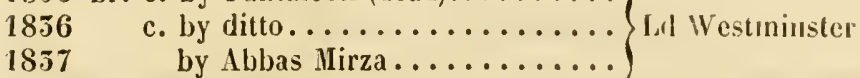

by Abbas Mirza............

I'II SURE HE SIIAN'T (Vol. III, page 184́) produced, in 185.5, a b. f. by Y. Corrector, and died that year. The Whisker mare out of her had no produce in 1832,55 , or 54 .

INFANT LYRA (Vol. III, p. 183) had dead foals in 1855,54 , 55 , and 56.

\section{JOANNA,}

Bred by Lord Jersey, in 1850, got by Sultan, her dam, Fillagree, by Soothsayer, out of Web, by Waxy-Penelope.

1853

18.36

1857

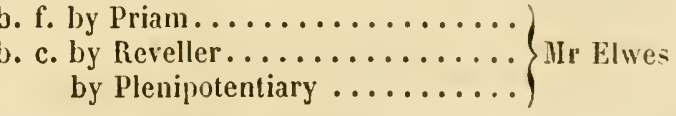

To JOHANNA, by Selim (Vol. III, p. 186) adel-

I852 b. c. by Sandbeck.............. Mr Platel

1853 b. c. Redshank, by ditto .......... Mr Bird

She had uo produce after 1935.

\section{JULIANA,}

Bred by IIr Durdas, in 1825, got by Pyramus, out of Euphrasia, by Rubens-Witch of Endor. by Sorcerer.

1831 b. c. Aurungzebe, by Flexible ......

1832 br. c. by ditto ............... Ur Bach

18.54

$185 \%$

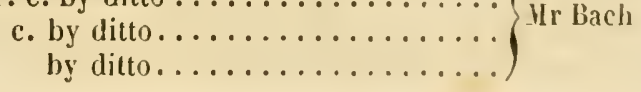




\section{JULIET,}

Bred by Lord Westminster, in 1819, got by Thunderbolt, out of Meteora, by Meteor.

18

1828 b. f. by Negotiator .............

1850 b. c. by Sir Gilbert.............

1852 b. c. by Y. Juniper............. Mr Wright, Thoby

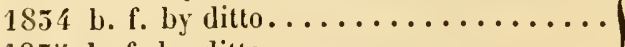

185 b. f. by ditto ...............

\section{JUNIPER MARE (Sabina)}

Bred by Mr Varsittart, in 1813, got by Juniper, out of Selima, by Selim-PotSo's-Editha, by Herod.

1828 br. c. Birkenhead, by Smolensko .... Mr F. R. Price

1830 b. f. Spitpost, by Mameluke........ Mr Williamson 1855

by Grey Comus............

Barren in 1852,55 , and 54 .

\section{LARK (sister to Peter Lely)}

Bred by Mr Lacer, in 1824, got by Rubens, out of Stella, by Sir Oliver.

1828 b. c. by Banker.

1850 b. c. Lindley (aft. Discount) by Banker

$1851 \mathrm{ch}$. f. by Belzoni (put to the stud) ...

1834 br. f. by dillo ................ Nir Gearey

1855 ch. c. by ditto.

1836

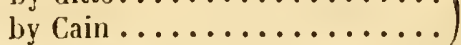

Missed in 1829, 32, and 35.

LARK,

Bred by II. R. H. the D. of YoRK, in 1822, got by Partisan, her dam, Brown Duchess, by Orville, out of Sagana.

1828 f. by Blacklock (s. to Germany)....

1850 f. by Wrangler ...............

1851 c. by ditto............... Ld Huntingfield

185 2 b. f. (Frisk) by Filho da Puta (s. to

Germany, 1855) 


\section{7}

\section{LASSIE,}

Bred by Mr Powelt, in 1819, got by Smolensko, her dam, Itighland Lass, by Ilighland Fling, out of Iris, by Brush.

1823 b. c. by Skim

1826 b. c. by Tancred..

1829 bl. c. by Wrangler..............

1830 b. c. (dead) by Middleton (Orville).... Mr Powell

1851 by a half-bred horse.........

1852 bl. c. by Wrangler .............

1853 b. f. by ditto.................

\section{LEAD (sister to Rowton)}

Bred by Lord Stigo, in 1825, got by Oiseau. out of Katherina, by Woful-Landscape, by Rubens.

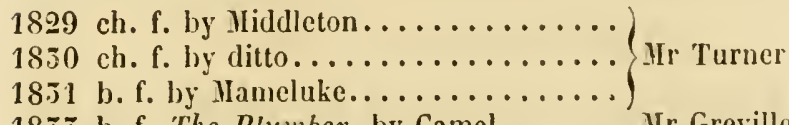

18.5.3 b. f. The Plumber, by Camel........ Mr Greville

1856 b. c. by ditto..................

To LEGACY, by Beningbrough (Vol. III, p. 206) add1852 b. f. by Reveller............. Mr Shard

\section{LEMON-SQUEEZER,}

Bred by Mr Wellesley, in 1807 , got by the Wellesley Grey Arabian, her dam, Orange-squeezer, by Highllyer, out of Iopsqueezer, by IIatchem.

1812 gr. f. Lemonade, by Brigliadoro.....

181.5 gr. f. by Sorcerer...............

1814 br. f. by Patriot.................

$181 \mathrm{~g}$ br. c. by Dick Andrews ........... IIr E. F. Welles

1816 b. f. by 1 pplegarth.............

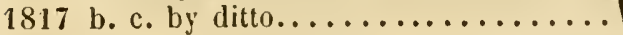

1818 gr. c. by ditto................

L'ORIENT MARE,

Bred by Mr IIerRIck, in 1815, her dam by Newbyth (son of Hagng) grandam by Dungannon, out of Brown Charlotte. 
1821 ch. c. Ferdinand, by Friday........ Ld Ailesbury 1850 b. f. by Golumpus.............. II Ilerrick 1851 br. c. Billinge, by Belzoni......... Sir J. Gerard

Died in 1852.

\section{L'ORIENT MARE,}

Bred by Mr Herrick, in 1815, her dam, Brown Bess, by Sir Peter, out of Brown Charlotte, by Highflyer.

1852 br. c. by Welbeck.............

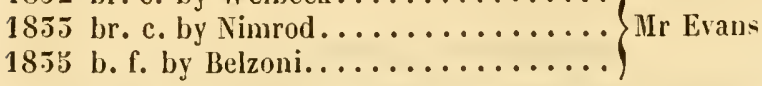

Missed to Fungus in 1854 , died in 1836.

\section{LILLA (first called Little Duchess)}

Bred by Mr Criтchley, in 1827, got by Bobadil, her dam, Aglaia, by Fyldener, grandam by Walton, out of Gipsy, by Guildford.

185.5 br. c. by his Robin Hood .........

1854 ch. f. by ditto ...............

1855 barren................. IIr Golden

1856 ch. c. by Robin Hood............

18.77 by Mulatto.............

To LITTLE RED RIDING IIOOD (Vol. III, p. 212) add-1855 ch. c. by Peter Lely............ Mr Ackers

She has had no other foals since 1831 .

LOCKET,

Bred by Mr Kraptov, in 1825, got by Blacklock, her dam, Miss Paul, by Sir Paul, out of Miss Dunnington, by Shuttle.

1851 f. by Lamplighter . . . . . . . . . Ir Roberts

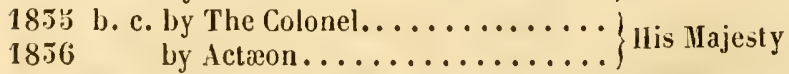

Had a dead foal in 1852, not covered in $\mathbf{5 3}$. 


\section{OTTERY MLARE (Leda)}

Bred by IIr Dicksos, in 1828, her dam. Harphan Lass, by Camillus, out of Statira, by Beningbrough.

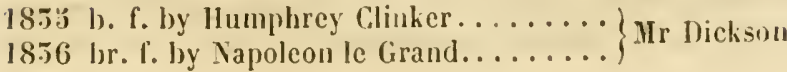

\section{LOTTERY MARE,}

bred loy , in 1829 or 50 , her dam by Ebor, out of Copeland's dam.

$18.54 \mathrm{ch}$. f. by Parlington............

185: slipped foal, by Velocipede ........ Mr (zibles

19.56 ch. c. by ditto...............

18.57 by Physician............

The LUCK'S-ALL MARE, l'asta's dam (Vol. IIl, p. 493) was not put to horse for several years after 1851 , and is dead.

\section{LUCK'S-ALL MARE,}

Bred by Mr Graberv, in 1818, her dam by Devi Sing-Blemish - Comet-Everlasting, by Eclipse.

1829 ch. f. by a half-bred horse........

1831 br. f. ly Amadis...............

1832 b. c. by Lovemore.............. IJd Stratharon

1855 ch. f. by ditto...............

185 ' by Confederate $\ldots \ldots \ldots \ldots$.

\section{MICIUUFF MIRE,}

Bred by Mr HaLL, in 1825, her dam by Cerherus. out of ILerlin's dam, hy Delpini-Tipple Cyder, by King Fergus.

1831 b. c. by Brutandorf..............

1852 b. c. by ditto $\ldots \ldots \ldots \ldots \ldots \ldots$

185.5 not covered last year............. Mr Janby

18.54 b. f. by Bachelor (son of Cœlebs).....

1835 br. f. by Yapoleon le Grand ........

1856

by ditto................ 
To MADAME SAQ̨U, by Remembrancer (Vol. III, p. 220) add-

1852 b. f. by Lottery (died a foal) ........ Mr Kirby

185.5 b. c. by ditto................ Mr L. Heseltine

The mare died in $\mathbf{1 8 5 5}$, immediately after foaling, and the foal was brought up by hand.

\section{MADCAP,}

Bred by Mr Mavtor, in 1820, got by Dinmont, her dam by Star, out of Moorpoult, by Y. Marske-Son of Omar - Whitenose.

1824 ch. f. Haidee, by Smyrna.........

1826 ch. f. Palmflower, by ditto.........

1827 ch. f. by Cannon Ball ............ Mr Platel

1829 ch. f. by Y. Filho (son of Miniature)...)

1850 b. c. by a half-bred horse.......... Mr Fisher

1851 b. c. by Pacha....................... Mr Fisher

1853 ch. c. Demetrius, by Defence....... MIr Sadler

1854 br. f. by Brownlock ............

1855 br. f. by ditto .................... Mr Williams

1857

by Defence..............

Barren in 1825, 28, 52, and 56.

MAID OF ALL WORK,

Bred by Mr Fergusox, in 1819, got by Octavian, her dam by Pipator, grandam by Dragon, out of Queen Mab.

1832 ch. c. Freckle, by Swiss...........

1835 b. f. by Chance................... Ir Crawford

1854 ch. c. by Albany.............

\section{MLARIORA,}

Bred by Lord Exerer, in 1828, got by Sultan, out of Miss Catton by Golumpus-Lucy Gray, by Timothy.

1854 ch. c. by Redgauntlet ...........

185 . missed to ditto...................... Ld Exeter

1856 b. c. by Reveller.............

To MARPHISA, by Haphazard (Vol. III, p. 2.52, add-

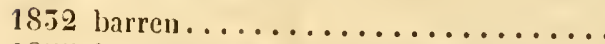

1855 bl. f. Priestess, by Voltaire........ 


\section{1}

MATIDA, by Ambrosio (Vol. III, 1. 255) was barren in 1852 , and shot in the December of that year.-Add the name Lubentia to her ch. f. by Reveller in 1851 .

\section{MELODY,}

Bred by Mr Bersov, in 1826, got by Bustard, her dam by Black Sultan.

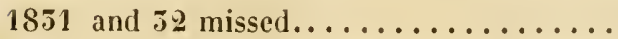

1853 and 54 picked foals by Lottery .....

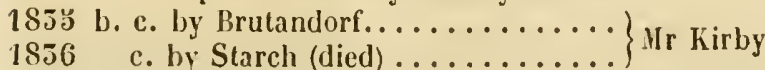

\section{MELROSE,}

Bred by Lord Scanenocen, in 1826, got by Pilgarlic, her dam by Whisker-Orville-Otterington's dam.

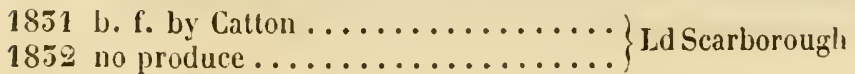

1855 ch. f. Xenoclea, by Catton.........

1854 gr. f. Judy Callaghan, by Falcon ..... Mr Fox, of Drif$1855 \mathrm{ch}$. f. by Napoleon le Grand ........ field.

\section{MERLIN MIARE (sister to Mufti)}

Bred by IIr Nowelt, in 1824, her dam, by Dick Andrews, out of Donna Clara, by Cesario.

1830 ch. f. Magma, by Tamworth....... Mr Davies

1851 b. f. by ditto............... Major Rice

1855 ch. c. by ditto............... Major Stretton

Missed in 1829 , had a half-bred foal in $\mathbf{3 4}$, and died.

To MIRACLE, by Soothsayer (Vol. III, p. 245), add1852 b. f. by Harry............... Mr White

Also the name Prodigy to her ch. f. by Woful, in 1827.

\section{MISCHANCE,}

A Chesnut mare, foaled in 1829 , got by Merlin, her dam by Ilaphazard, out of Web.

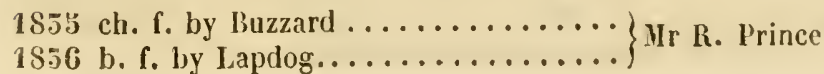




\section{MISS GILES,}

Bred by Mr Cattle. in $185 \mathrm{I}$, got by Lottery, out of Arcot Lass. by Irdrossan.

185 'b. f. by Humphrey Clinker

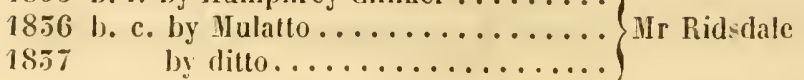

\section{MIISS ROBSON,}

Bred by , in 1821, got by Woful, out of sister

to Erichtho, by Sorcerer-sister to Rockingham, by Highflyer.

1827 b. f. by Sir Edward............. Mr Hellier

1828 b. c. Blue Ruin, by Patshull....... Mr Roberts

1829 b. c. by Banker ..............

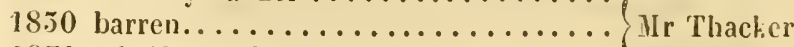

18.51 a half-bred foal. ..............

\section{MISS WITCH,}

Bred by Mr Nowelt, in 1850, got by Muley, out of Y. Caprice, by Waxy-Caprice, by Walton.

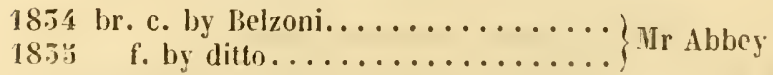

\section{MRS FRY,}

Bred by Mr Wilsox, in 1823, got by Walton, out of Vourneen, by Sorcerer-Tooee, by Buzzard.

1827 cl. f. (The Balkan) by Blacklock .....

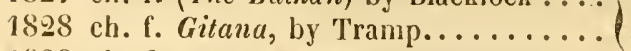

1829 ch. f. (Wallfower) by Whisker................ Wr W. Fox

18.50 b. f. by Lottery ................

1851 ch. I. Mrs Oakley, by Blacklock ..... Mr S. L. Fox

$\left.\begin{array}{l}185.5 \\ 1854 \\ 18 . \text { b. f. by ditto... }\end{array}\right\}$ (dead) ......... Mr Hejwood

$1855 \mathrm{ch}$. f. by Parlington............

\section{IIOPSY,}

Bred by Ur Petre, in 1828, got by Comus, out of Juliana, by Gohanua-Platina, by Mercury. 


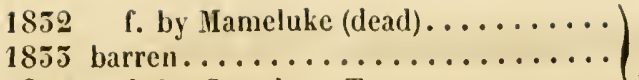

1854 f. by Camel, or Tarrare......... Mr Wright

1855

f. by Count Porro $\ldots \ldots \ldots \ldots \ldots \ldots$
by ditto $\ldots \ldots \ldots \ldots \ldots$

1856

\section{MOGGY (sister to Scarecrow)}

Bred by Lord Egremoxr, in $181 \mathrm{~s}$, got by Canopus, out of Margaretta, by Sir Peter.

1824 b. c. Rockingham, by Regent...... Mr Howard

1826 br. c. Iloward, by Wortlyy (s. to Ireland) Mr llaberly

1828 br. f. Fidelity, by ditto..........

1855 b. c. by Pendulum, or Vicar (son of Mr Howard Muley)

Barren in 1850,51 , and 54 -not covered in 54 -her produce of 1829 and 52 died young.

\section{MORGIANA,}

Bred by Lord Surfield, in 1820, got by Muley, out of Miss

Stephenson, by Scud, or Sorcerer-sister to Petworth, by Precipitate.

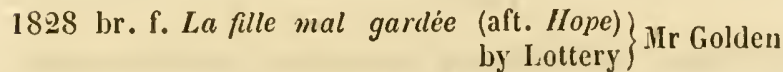

1850 br. f. Lafitte, by Chateau Margaux (s. to $\}$ Mr Cookes

1851 bI. c. London, by Chateau Margaux. . Mr IIarson

1852 br. c. (Sheet Anchor) by Lottery .... Mr Golden

She died in 1835, having had a stone thrown at her about a month before her time of foaling.

\section{NIVALIS,}

Bred by Mr Joux Surn, in 1824, got by Blacklock, her dam, Snowball, by Prime Minister, out of Vesta, by Delpini.

1850 br. f. by Figaro...............

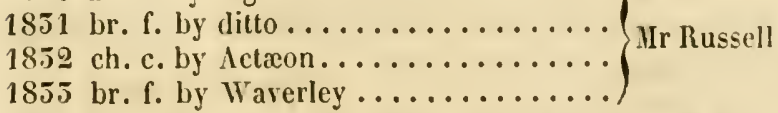

Barren in 1829 ; sold in 1855 , to go abroarl. vol. Ir. 
To NUT BROWN MAID (Vol. III, p. 279) add :-1953 f. by Percy ............... Mr Collingwood

Her foal of 1855 died, and that of 34 was by a half-bred horse.

\section{OCEANA,}

Bred by Mr Osbalnestor, in 1816, got by Cerberus, out of Doctor Syntax's dam, by Beningbrough-Jenny Mole.

1822 ch. f. Gertrude, by $\Lambda$ madis. ........ Col. Lautour 1825 ch. f. Miss Gift, by Glowworm...... Mr Innapton

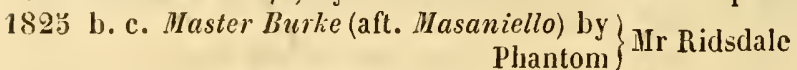

1826 b. f. by Peter Lely (dead) .......... Mr Knapton 1827 ch. f. Susanna, by Figaro..........

1828 b. c. Caspian (aft. Crispin) by Lottery Mr Ridsdale (s. to France in October 1854)

1850 b. c. Pacific, by Lottery ........... Mr E. Peel

1851 b. c. by Y. Phantom............. Mr Osbaldeston

1852 ch. f. by Palmerin.............. Mr Clark

1855 ch. c. Righton, by ditto ......... Mr Marson

\section{OLIVE,}

Bred by Mr Ogbex, in 1828, got by Tarragon, out of Despatch.

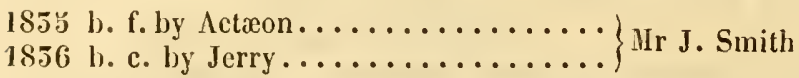

To OLIVERA, by Sir Oliver (Vol. III, p. 282), add1851 slipped foal, by Bonassus..........

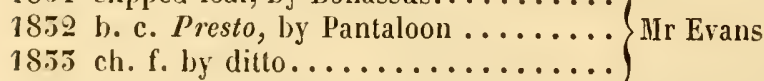

Barren in 1854 and 55 , and shot.

ONLY TIIAT, Bred by Lord Lowruen, in 1850, got by Partisan, out of Scribe.

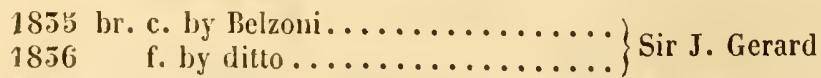
1857 by Dr Syntax.............. Mr Tattersall 
ORVILJE MARE,

Bred by Mr Duxsley, in 1812, her dam, Miss Grimstone, by Weasel-Ancaster-Damascus Arabian-Sampson.

1817 b. f. by Langton ..............

1818 b. f. by J'rime Minister (Laurel's dami) Jr Yarburglt

1819 b. f. by Catton. ................

1820 br. c. by Prime Minister..........

$1824 \mathrm{gr}$. c. by a brother to Gustavus .... J J Townrow

182 b. c. by Blacklock............. Mr Townron

1826 br. c. Tranby, by ditto........... Mr Ridsdale

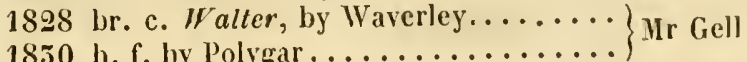

1851 b. f. Charlotte, by Androgeus....... Ir Boulton

1852

1854 b. f. Miss Bowe, by Catton .........

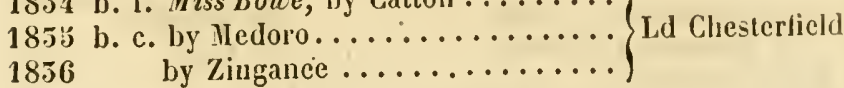

Barren in 1821, 22, 25, 27, and 29 .

ORVILLE MARE out of Lisctte (Vol. III, p. 289). Hissed in 1832 , produced a gr. f. by Partisan in 1835, and was sold.

To the ORVILLE MARE, dam by Pipator (Vol. III, p. 287) add--

1852 br. f. by Percy (s. to Ancrica) .......
1855 b. c. by Duplicate..............

Died in 1854.

\section{GREY ORVILLE MARE,}

Bred by Mr Batmax, in 182., her dam by St George, grandam by Itambletonian, great grandam by Delpini, out of Black-eve's Susan, by Alfred, etc. ctc.

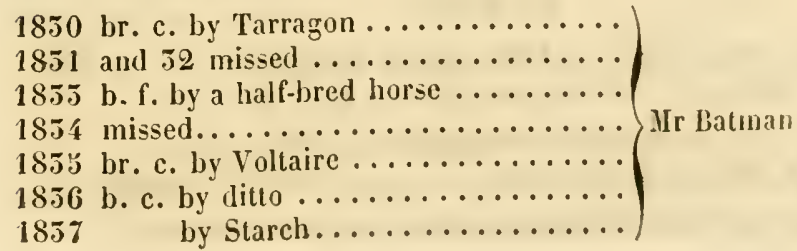


OXYGEN,

Bred by the Duke of Grafrov, in 1828, got by Emilius, out of Whizgig.

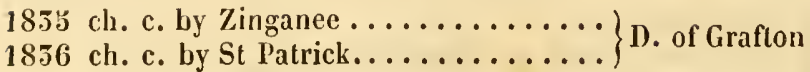

PAULINA, by Orville (Vol. III, p. 500). Missed in 1852, 35, 54, slipped $t w i n$ fillies by Emancipation in 55, and was shot.

\section{PRIMROSE,}

Bred by Mr Humbe, in 1821, got by Comus, out of Cowslip, by Cockfighter.

1826 b. f. Aconite, by Tramp.......... Mr Armitage

1829 b. f. Mabooba, by Saladin .......... Mr Legh

1830 br. c. by Lottery ..............

1852 br. c. by ditto .......................... Turner

$1854 \mathrm{ch}$. f. by Recovery ............

$1855 \mathrm{ch}$. c. by ditto.

Barren in 1851 and 55.

\section{PROSELYTE MARE,}

Bred by , in , her dam by Orion, out of Harriet, by Delpini.

1851 br. f. by Y. Sancho.

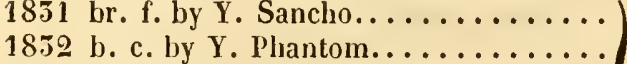

1855 b. c. by $\operatorname{ditto} \ldots \ldots \ldots \ldots \ldots \ldots \ldots \ldots \ldots \ldots \ldots \ldots \ldots$ Mr Hill, Sledmere

1855 ch. f. by Napoleon-le-Grand........

1856

by ditto.................

\section{RIGMAROL,}

Bred at Hampton Court, in 1822, got by Soothsayer, out of Rantipole, by Selim-Sweetheart, by Volunteer.

1830 br. c. Louis-d'or, by Luzborough.... Mr Goddard

1854 ch. f. Otè, by Doctor Eady ......... Mr H. J. Smith

She has had only these two foals, to 1856 inclusive. 
To ROSA MUNDI (Vol. III, p. 345) add-

$1852 \mathrm{ch}$. c. Tennis-ball, by Battledore ....
$1836 \quad$ by Olympus, or Recovery ....

She was barren in $1851,55,51$, and 53 .

\section{ROSE,}

Bred by Mr Peirse, in 1818 , got by Rubens, out of Rosinne, ly Dick Andrews.

1822 ch. f. by Pyramus (dead) . . . . . .

1826 ch. f. Rosebud, by Robin Adair (s. to Mr Dundas the Swan River)

by ditto..................

1828 br. c. by Figaro ............... Ir Beardsworth

1831 br. c. by Velocipede.............. Mr Thompson

1832 ch. c. Peter Simple, by Velocipede... Sir T. Stanley

1855 ch. c. by IIerbert Lacy............ Ir R. Halsall

Died in the autumn of 1855 , in foal to Felt.

\section{ROSALIND,}

Bred by Mr Mort, in 1829, got by Paulowitz, oul of Isidora, by Blucher-Zora, by Zclim-Zoraida, by Don Quixote.

1854 b. c. Premier, by Priam...........

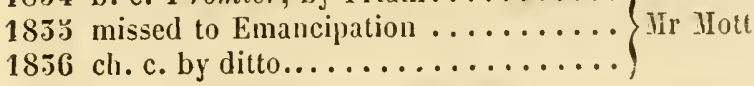

\section{RUBENS ILARE (sister to Wouvermans)}

A chesnut mare, foaled in 1814, her dam, Brightonia, by Gohamia, out of Nutmeg, by Sir Peter.

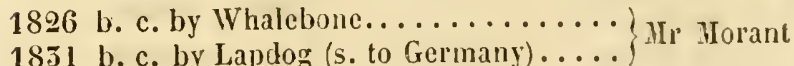

1835 b. f. by Reveller.............. IIr Shard

She had no other produce that lived.

\section{RUFINA (sister to Velocipede)}

Bred by $\mathrm{Mr}$ Moss, in 1S21, got by Blacklock, her dain by Sorcerer, out of Virgin, by Sir Peter. 
$1829 \mathrm{ch}$. c. by Figaro, or Bravo (died a foal)

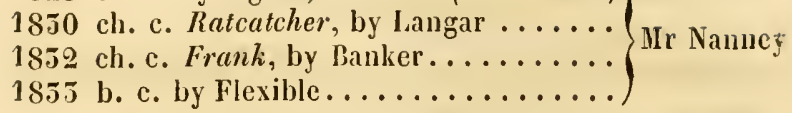

Missed in 1851 , and died in 55 , soon after foaling.

\section{SAFFI,}

Bred by Mr S. Bartard, in 1818, got by a son of Dick Andrews (which was out of Lord Lowther's Barb mare) her dam by Totterilge, out of sister to Marianne, by Mufti-Maria, by Telamachus.

1822 b. c. by Woful .............. Mr Elwes

1824 b. f. Barbary, by Ardrossan (died, leav-)

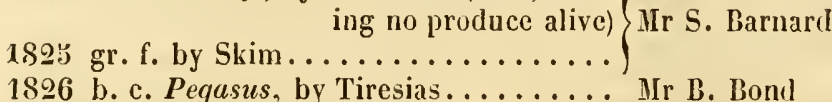

1826 b. c. Pegasus, by Tiresias........... Mr B. Bond

1899 b. f. Banquet, by Truffle .........

1855 br. c. Barbary, by Jenkins........ Mr Batson

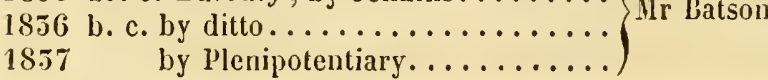

Mlissed in $1825,27,28,50,51,52,54$, and 53 .

To SANCHO MARE, out of Miss Furey (Vol. III, p. 536) add1852 gr. f. Wicket, by Stumps ........ Mr Mott

\section{SARAH,}

Bred by Mr Tarletov, in 1824, got by Whisker, out of Jenny Wren, by Young Woodpecker.

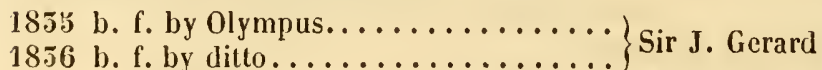

1857 by Dr Syntax........... Mr Tattersall

To the SELIM MARE, sister to Ottoman (Vol. III, p. 565) add1852 ch. f. by Belzoni (put to the stud).... Mr Eyke 1854 ch. f. by Patron ............... Mr F. Marshall

Barren in 1855 ; died in 1853 . 


\section{9}

\section{SEILII MARE,}

Bred by Mr R. Wilsos, in 1821, her dam, Miss stephenson, by Scud, or Sorcerer, out of sister to Petworth, by Precipitate.

1829 b. e. Sparrowhawk, by Buzzard ..... Ifr Tarleton

1851 br. c. Amurath, by Whisker ...... D. of Cleveland

1832 by Guerilta

SELINA, by Delpini (Vol. III, p. 565). Iler last two produce should stand thus :-

1850 b. f. Falernia, by Chateau Margaux Gen. Grosvenor (put to the stud)

1851 gr. c. Arthingworth, by Reveller..... Ld A. Conyngham

1852 barren

\section{SESSIONS,}

Bred by Lord Egrerovt, in 1826, got by Wanderer, her dam by Orville, out of the Alexander mare, dam of Selim and Rubens.

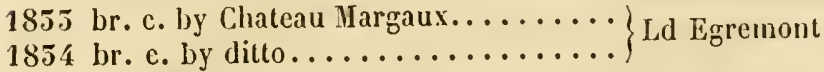

Missed in 1835, and sold to Mr Boardman, of the United States. in 1855 , stinted to Nimrod (son of Whalebone).

\section{SEVIGLIA,}

Bred by Mr HAFFexDex, in 182S, got by Figaro, her dam, sister

to Arbutus, by Walton-Wizard-Lisette, by Ilambletonian.

1852 c. by Gustavus.............. Mr Gillsy

1855 b. c. Snowball, by Alington ........ Mr Walker

1854 f. by Gustavus..............

1855 f. by ditto............... Mr Gilby

1856 a half-bred foal .............

SEVILLA,

Bred by Mr Garforti, in 1850, got by Figaro, out of Lepanto's dam, by Comus-Marciana, by Stamford.

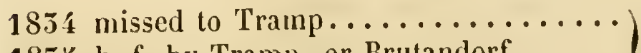

1853 b. f. by Tramp, or Brutandorf ...... Mr Ridsdale

1856

1837

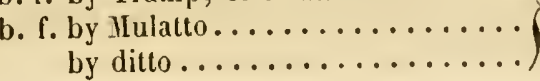




\section{0 \\ SHEL.DRAKE (sister to Sailor)}

Bred by II Tновмнlц, in 1821, got by Scud, out of Goosander.

*1826 ch. f. (Miss Windsor) by Woful.... . . Ir Thornhill 1827 ch. c. (Jack Tar) by Emilius........ Ld Cleveland

1828 b. c. by ditto ................

1899 ch. c. by ditto (dead) ............ Ir Thornhill

$1852 \mathrm{ch}$. f. by Merlin (s. to Baron de Maltzahn 1856, in foal to Emilius)

Missed to Merlin in 1850 and 51 ; sold in 52.

* Miss Windsor produeed, in 1850, Mr W. Edwards's ch. f. by Waterloo, or Middleton, had adead foal in 31 , missed in 32 , an 1 was shot in 5.5 with her foal, which was deformed.

\section{SHUTTLE MARE,}

Bred by Mr JADIs, in 1812, her dam, sister to Dowager, by Hambletonian, out of Goldenlocks, by Delpini.

1816 ch. f. by Comus (dead) .......... Burnard

1818 b. c. Shuttlecock, by Orville ......... Mr J. Day

1819 b. c. by Smolensko............. D. of Rutland

1820 b. f. Sempstress, by Williamson's Ditto. Col. Lowther

1821 ch. c. by ditto ................ Mr IIankey Smith

18 29 b. c. by ditto.................

182.5 ch. f. by Smolensko, or Chilton Junior.

1824 b. f. by Smolensko................Ir R. Wilson

$182 \mathrm{~b}$ b. f. (Bassilia) by ditto...........

1826 ch. f. (Selina ) by Nicolo . . . . . . . . . )

1827 ch. c. Suffolk Punch, by Wrangler... Hr Batson

1828 ch. c. Adain Brock, by Blacklock.... Ir Pettit

1829 br. c. by Stainborough .......... Ir R. $\Lambda$. Fellowes

1850 br. c. by ditto ................

1851 ch. c. by Wrangler.............

1852 b. c. by Filho da Puta........... JIr R. Wilson

1855 b. c. by Wrangler.............

18.54 f. loy ditto................

185:5 by Sir Benjamin ...........

\section{SIR CHARLES MARE (chesnut)}

Bred by Mr Costeer, of Iluggat, in 1816, got by Black Sir Charles, out of The Engraver's dam, by Stride - sister to Sharper, by Ranthos. 


\section{1}

$1824 \mathrm{ch}$. f. by Richmond............ Ir Atkinson 1823 ch. c. by ditto (s. to India).......... Mr Scouswar 1827 ch. c. by ditto (s. to Russia).......... Mr Athinson 1829 b. c. The Bishop, by ditto (s. to Ger-) Mr Nicholson

1851 b. f. by Borodino ............. Mr $\Lambda$ tkinson $1852 \mathrm{gr}$. f. by Y. Camillus............. 1853 gr. c. by ditto............. Mr IIarshall

Missed in 1826 and 50 , and died in 55.

To SIR PAUL MARE (Lady Paul) Vol. III, p. 581, add1852 b. c. Democracy, by Tramp........ Mr Gully She had no living produce after 52 , and is dead.

\section{SKIMI MARE,}

Bred by Lord EGrenoxt, in 1850, out of Centaur's danı, by Orville-Alexander.

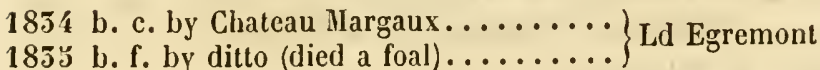

\section{SPECIE,}

Bred by Mr Tronvelel, in 1821, got by Scud, out of Quail. 1828 b. f. Manca, by Merlin........... Mr Thornhill 1852 ch. c. The Rustic, by ditto........ Mr Etwall 1855 b. f. by St Patrick.............. 1856 missed by Rowton............ Gen. Grosvenor 1857 by Count Porro.............

Missed to Emilius in 1827, to Merlin in 29 and 51 , and had no produce that lived, in 55 or 34.

\section{SPINETTE,}

Bred by Mr Godpard, in 1818, got by Haphazard, out of Spinetta, by Trumpator-Herod-Snap.

1826 b. f. by Champion (son of Poulton) ...

1899 b. f. Maid of Cadiz, by Cadiz....... Mr Farquharson

1853 b. c. by ditto................

Cast foal in 1851 ; barren in $52,55,54$, and died in 56 . 


\section{2}

\section{ST GEORGE IIARE,}

Bred by Mr Batuax, in 1814, her dam by Hambletonian, grandam (Bachelor and IIeta's grandam) by Delpini, out of Blackeje'd Susan, by Alfred-Herminta, by Panton's Ilermit, etc.

1820 b. f. (died young) by a half-bred horse 1823 br. f. by Grey Orville ...........

1826 gr. c. by Sir Christopher .........

1828 b. c. by Dinmont.............. IIr Batınan

1829 gr. c. by Blucher.............

1850 gr. c. by Grey Orville...........

1851 b. c. (died young) by Figaro........

1852 b. с. by Tramp.................... Rr. Johuson

1854 b. c. by a half-bred horse.......... Mr Batman

Missed in all the intermediate years.

\section{STREA HLET,}

Bred by Lord Lowther, in 1828, got by Tiresias, her dam, Bizarre, by Peruvian, out of Violante.

1855 bl. f. by Velocipede............

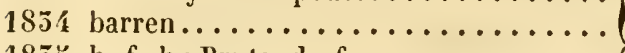

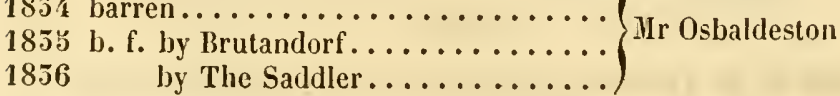

\section{SULTAN MARE,}

Bred by Lord Clarendox, in 1929 , her dam, Antiope, by Whalebone, out of Amazon, by Driver.

1855 b. f. by The Colonel............ D. of Richmond

1856

1857

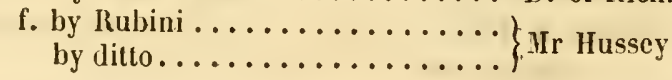

\section{SWISS MARE,}

Bred by Mr Parkinsos, in 1850, her dam by Reveller, grandam by Waxy, out of Elve, sister to Hagic, by Sorcerer.

1854 br. f. by Voltaire.

185 b. f. by The Lion. . . . . . . . . . .

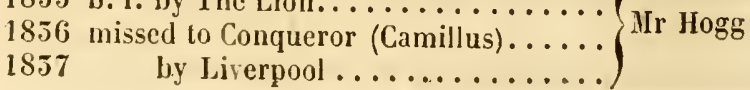




\section{3}

TESANE,

Bred by Mr S. L. Fox, in 1850 , got by Whisker, out of Iady of the Tees, by Octarian.

1855 b. f. by Medoro................ Mr S. L. Fox

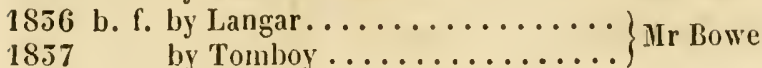

\section{TITTLE 'TATTLE,}

Bred by Mr Chartor, in 1825, got by Sam, her dam, Gossip, by Walton, out of Hesperia, by Cesario-Ilind, by Sir leter.

1851 c. by Langar (dead)....................... JIr J. Stevens

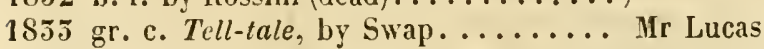

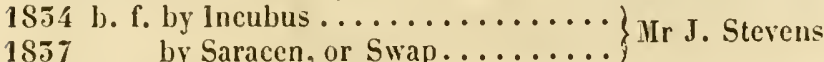

Not covered in 1854 , missed to Defence in 56 .

\section{TRAMPINA,}

Bred by Mr Upplerr, in 1825, got by Tramp, her dam, Harriet, by Delpini, etc.

$1852 \mathrm{ch}$. f. by Velocipede............

$1855 \mathrm{ch} . \mathrm{c}$. (died a foal) by Actacon.......

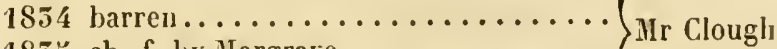

$1853 \mathrm{ch}$. f. by Margrare..............

1856 b. c. by Voltaire.

1857

by Voltaire................

TRAMP MARE,

Bred by Lord Fitzwillau, in 1826, her dam, Reposada, by Amadis, out of Orvillina.

1851 br. c. Johnny W'atson, by Comus.... Mr Ridsdale 1852 b. f. by Brutandorf.............. Mr Watmough

TRAVELLER MARE,

Bred by Mr Garfortu, in 1818, her dam by llambletonian, out of Vesta. 


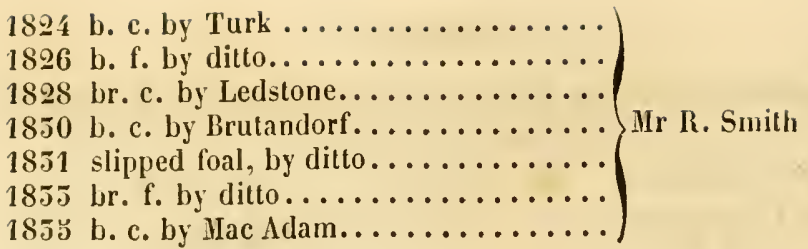

\section{TREDR1LLE,}

Bred by the Duke of Grafton, in 1815 , got by Walton, her dam,

Pope Joan, by Waxy, out of Prunella, by Highflyer-Promise.

1819 ch. f. by Bourbon (died 2 days old)... D. of. Grafton

1821 ch. f. by Soothsayer.............

1822 ch. f. Medina, by Selim ..........

1825 ch. c. The Moslem, by ditto........

1825 br. f. Manille, by Orville ..........

1826 ch. c. Ombre, by Phantom (s. to India

1828 b. c. by Whalebone (sold to D. of Holin 1829) Ld Verulam stein in 1852)

1829 ch. c. Basto, by Truffle..........

1850 b. c. Little Cassino, by Sultan.......

1851 b. c. by Reveller.............

1854 ch. c. by Mamcluke............ Mr Theobald.

Not covered in 1819 ; missed in 1824, 27, and 52.

\section{TRULLA,}

Bred by Mr Gascoigne, in $181 \mathrm{y}$, got by Sorcerer, her dan by Weathercock, out of Cora, by Matchem-Turk-Cub.

1821 bl. f. Elizabeth, by Walton........

1825 b. f. Theophania, by ditto.........

1824 b. f. Trull, by Tramp (s. to Hungary in 1829$)$

1825 bl. c. (Cupid) by Whisker......... Mr Gascoigne

1827 br. c. Rex, by Figaro ...........

1828 b. f. Diana, by Catton ...........

1829 b. f. Tuberose, by St Patrick (s. to Germany in 1854, covered by Brutandorf)

1851 br. c. by Lottery (sent abroad in 1854) Mr Greatrex

1856 by Lapdog. ............ Mr Mills

Hissed in $1820,22,26,50$, and 52, 55. 


\section{5}

\section{VEIJET,}

Bred by Lord Stago, in 1825, got by Oiseal, out of 11 ire.

1828 ch. c. Bras de Fer, by Langar..... . Ld Sligu

$1829 \mathrm{ch}$. f. Brazil, by Iranhoe.........

1850 br. f. Brace, by Master Robert......

1851 ch. c. Bran, by II umphrey Clinker... I Id Sligo

1852 b. c. by Langar (died)

1855 f. by Picton (dead).............

1854 b. f. by Picton...............

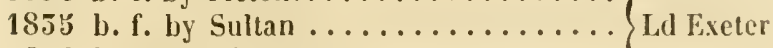

1856 b. с. by ditto...............

\section{WALTON MARE,}

Foaled in 1825, her dam by Wizard, out of Remembrance, by Sir Solomon-queen Mab, by Eclipse.

$1828 \mathrm{ch}$. c. Will-o'the-Wrall, by Marmion. IIr S. Fox

1829 b. f. Lady Clapham, by Jack Spigot... J Jr J. WV. Young
1850 b. c. by ditio (died 4 days old) .......

1851 slipped foal, by Velocipede........

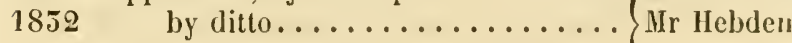

1855 b. f. by St Nicholas.............

1854 b. f. by ditto..............

Sent to America in 1854, with her foal, and covered by St Nicholas.

\section{IVALTON MIRE,}

Bred by Mr ADesie, in , her dam by Sorcerer, out of Medora's dam, by Sil IIarry.

1824 f. Fenella, by Woful......... Col. Synge

1825 f. by Woful, or Tiresias ........

1826 c. by Tiresias................

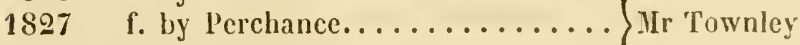

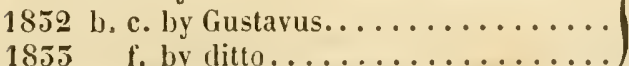

She is supposed to have been harren in the intermediate years, and is gone to Russia.

\section{W ANDERER MARE,}

Bred by Mr Gates, in 1825, out of Colset, hy WhalebonePrincess, by Sir Peter-Dungannon. 


\section{6}

1829 gr. c. Friar Tuck, by Little John .... Ir Gates

18.52 br. or ro. c. by Camel........... Mr Knight

\section{WHALEBONE MARE (Fiddle-Faddle)}

Bred by Lord Egrenowr, in 1822, her dam by Teddy-the-Grinder, out of sister to Wanderer, by Gohanna-Catherine.

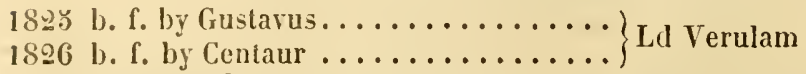

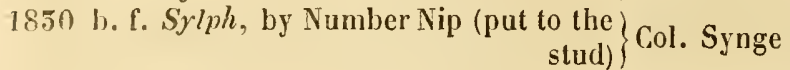

Died in Ireland in 1850 , soon after foaling.

\section{WHALEBONE MARE,}

Bred by Mr Conee, in 182\%, her dam, Varnish, by Rubens, out of Vestal, by Waiton-Dabchick, by PotSo's.

1855 ch. c. Sherry, by Revelter......... Mr W. Edwards

185.3 b. c. by The Colonel ............ Mr Wythen Jones

Barren in 1854, and found dead in the field, Sept. 5 5.

WHALEBONE MARE,

Bred by Lord Egremont, in 1828, her dam, Harpalice, by Gohanna, out of Amazon, by Driver.

1854 b. c. by Gaberlunzie (brolic his leg when a foal)

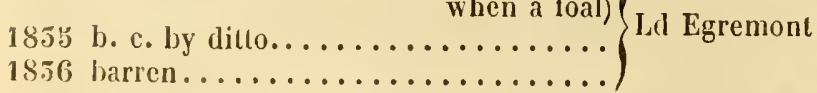

\section{WHISKER MALE,}

Bred by Mr Foluanbe, in 1827, her dam by Walton, out of Young Noisette, by Diomed-Noisette, by Squirrel.

$1851 \mathrm{ch}$. f. by Velocipede ............ Mr Foljambe

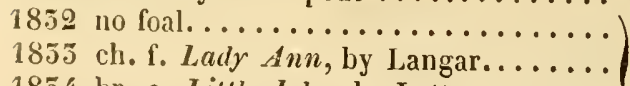

1854 br. c. Little John, by Lottery......... HIr G. Clark

$185:$ ch. f. by Langar

18.56

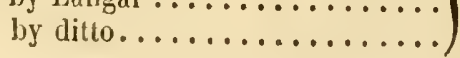




\section{7}

\section{WIIISKER IIARE,}

Bred by the Duke of LeEDs, in 1827, her dam by Dick Nullew: out of Gammer Gurton, by l'haramond-America, by IIarske.

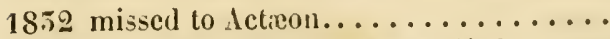

1855 br. c. by his Robin Ilood (died a year-

ling)

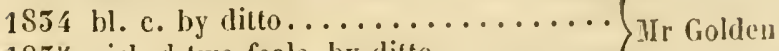

1855 picked two foals, by ditto .........

1856 bl. c. by llargrave.............

1837

by Mulatto.

\section{WHISKER MARE (sister to Maria)}

Bred by the Trostees of Lord Stratimone, in 1828, her dam,

Giloside Fairy, by Hermes.

1852 ch. c. by Velocipede........... Capt. Taylor

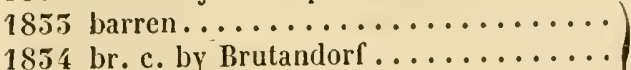

1855 b. f. by Tramp ............. Ir Clough

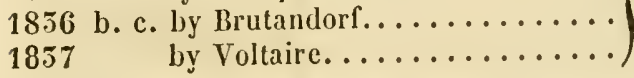

\section{WIIISKER MARE,}

Bred by Ir Ferguson, in 1822, her dam by Pipator-I)ragonQueen Mab.

1854 ch. f. Golden-drop, by Actæon......

1835 ch. f. by Gainsborough.......... IIr J. Bell

1856 b. f. by ditto, or Physician.......

\section{TVISHFUL,}

Bred by Mr Rrav, in 1817, got by Walton, her dam by Beningbrough, out of Blacklock's dam, by Coriander-Wildgoose, by Hightlyer.

1828 b. f. by Smolensko ................. Mr Theobald

1829 bl. f. by ditto ...............

1830 ch. c. by Duport. . . . . . . . .

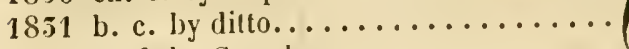

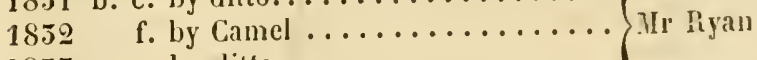

1855

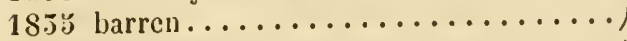

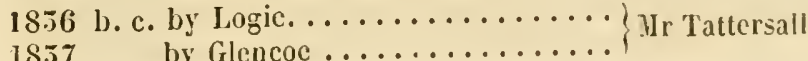




\section{8}

\section{YOUNG WRYNECK,}

Bred by Mr fiscoigne, in 1809, got by Stamford, her dam, Wryneck, by Beningbrough, out of Miss Tippet, by Morwick Ball.

1816 ch. f. by Cerrantes............

1817 b. f. Twist, by $\Lambda$ madis ........... Ld Fitzwilliam

1818 ch. c. by Petronius.............

1819 ch. f. Miss Wentworth, by Cervantes. M. Reed

The first three of the above foals were, by mistake, put down in the Second Volume as the produce of Old Wryneck. Spring-cantle was foaled in 1820. Y. Wryneck had some other produce, of which the Publishers have no account.

\section{YOUNG WHISKEY MARE,}

Bred by Mr Harridance, in 1818, her dam by Sir Peter, nut of IIornet, by Drone.

1826 b. f. by Truth..............

1827 br. c. by Tiresias .............

1850 br. c. by Tarrare ............. Mr Harridance

1852 br. c. by Fillo da Puta................

1853 b. f. by Tantivy (killed 2 years old)...

The mare has since been used as a hack.

\section{LADY CAROLINE,}

Bred by Sir C. Bunbury, in 1820, got by Partisan, her dam, sister to Orange-llower, by Trumpator-Orange-bud.

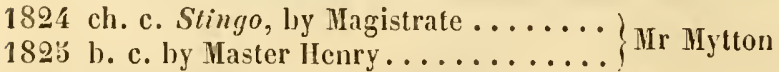

1826 b. f. Caroline, by Filho da Puta ..... Mr Ormsby Gore

1827 b. f. by Banker...............

1828 ch. f. Reseda, by Rubens.......... Sir R. W. Bulkeley

1829 foal by Pacha (died young).........

1850 not covered last ycar...........

1851 ch. f. by Claude Lorraine.........

1852 b. c. Fillelio, by Stumps.......... Mr Corbet Smith

1853 ch. c. by Belzoni... b. . ...........

1856 ch. c. by ditto . . . . . . . . . .

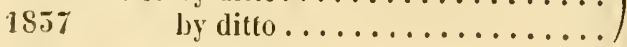




\section{9}

Pedigrees of some IIorses and Mares whose dams will not be found anong the Brood Hares in this Volume.

ARGANTES, Mr Attwood's, a chesnut horse, foaled in 1827, got by his chesnut Arabian, his dam ly Bellerophon, grandam, sister to the dams of Herlin and 'The Colonel, by Delpini, out of Tipple Cyder.

BARTOLOZZI, now IIr Scarisbrick's, by Tramp, out of Witch, sister to Arbutus. - Produced a b. c. by Velocipede, in 185j, and has had no foal since.

BLOODIIOUND, a chesnut colt, foaled in $185 \%$, by Beagle. out of The Charmer, foaled in 1824, by Y. Gohanna, out of Elvira, by Orville.-She was not covered in 185 .

BROCAllD, by Bobadil, out of Miss Brocket.-Produced in 1855 , Mr F. Craven's br. i. Sinallwaist, by Shortwaist (son of Whalebone) and missed in 1854 and $5 \%$.

CHANCE, a chesnut horse, foaled in Scotland in 1819, and got by King Corney, out of Ruby, by Interloper-Logie o'BuchanFortitude-Lady Bolingbroke, by Squirrel. - King Corney was got by Rob the Ranter, out of the above-named Logie o'Buchan mare.

COALITION, Mr Houldsworth's, foaled in 1824, got by Hagistrate, his dam by Filho da Puta, out of Catherine.

CORA, bred by the D. de Guiche, in France, in 1824, got by Truffle, out of Ilelen, by Whiskey.-She produced, in 1854 , b. c: Job, by Middleton (her first foal), slipped foal in 38 , and was sold to Baron de Maltzahn in the spring of I856, in foal to The Colonel.

COUNTESS, by Comus (p. 547).-l'roduced a ch. c. by Defence, in 1855, in the stud of Ld Egremont, slipped twins in 36, and was sold in Feb. 56 to Baron de Maltzahn, covered by Nonsense.

ENCHANTIENT, a bay mare, foaled in 18.50, got by Merlin, dam by Pliantom, out of Spell.-She was sent to Germany. The Phantom mare was also the dam of Mazourke, by Little John, foaled in 1826.

HARRIETTE WILSON, foaled in 1824, got by Manfred, out of Sybil, by Sorcerer.-P'roduced, in 185.5, IIr Bestow's ch. c. Abercrombie, by Advance; slipped foal in 5 is and 53 , and dierl early in 56 , in foal.

HAWK'S-EIE, a grey colt, foaled in 18.5. gat by Jajor Yarburgh's IIAdam, his dam by lottery-Cottingham-Harrison's Trumpator.- He is the only foal of his dam. 
LOCKINGTON, a bay horse, foaled in 1850 , got by Y. Phantom, his dam, foaled in 1818, by Cerberus-Diana, by Kill Devil.

MANBY (Mr C. A. Pelham's) foaled in 1824, got by Colossus, her dam by Bucephalus, out of Vicissitude.

MARY IIORNER, a brown mare, bred by Mr J. Wheatley, in 1850, got by Ld Kielburne's Pirate, her dam by HetmanPalmflower, by Dent's Sir Charles.-Slie produced, in 1855, Ir Ilorner's bl. c. Solomon, by Waverley.

NIMROD, foaled in 1825, got by Phantom, his dam by Sir Solomon, out of Attraction.

QUEEN OF HEARTS, got by Tagus, dam by Hambletonian, out of Mulespinner.

SCHOOL-GIRL, by Tancred, out of Waxy Lass.-Produced b. c. by Middleton, in 1852, missed in 52,55 , and was sold.

SCORNFUL, by Woful, dam by Haphazard, produced, in 1828 , a b. f. by Moriseo, which is now in Mr Theobald's stud, and had, in 1854, a b. f. by Camel. - Scornful was afterwards sent abroad.

SNEAKER, a brown mare, foaled in 1830 , got by Camel, dam by Soothsayer, out of Hare.

STAPLETON, a brown horse, bred by Mr Hiles, in 1828, got by Strephon, out of sister to Vulcan, by Thunderbolt--Cranberry.

SUSAN, by Walton, dam by Sancho, now in Mr Beilby Thompson's stud.-Was put to horse for several years, and proved barren till 1834, when she had a colt by a cart horse, and the next year she had a colt by St Nicholas. Both the colts are dead.

TOHIY WHITEHEAD, a bay horse, foaled in 1852, got by Mameluke, out of Puzzle, by Whisker, who missed in 1855 , and was sent to Germany.

VIOLA, Sir J. Boswell's, a brown mare, foaled in 1852, got by Canteen, out of Sal, sister to Jerry, who died soon after foaling her.

WELLINGTON, a bay horse, foaled in 1825, got by Cannon-ball, out of Psyche.

THE YOUNG CIIANCELLOR, a chesnut horse, foaled in 1831, got by 'Tiresias, out of Quæ (foaled Dec. 18I7) by Quiz, out of Grey Duchess.

YOUNG WARTER, bred by Dr Willis, got by Warter, his dam, sister to Minimus, by Dungannon. 


\section{IRISH UARES.}

\section{AGNES,}

Foaled in

, got by Recordon, out of Rhoda. by Commodore.

1850 b. f. by Starch...............

1851 missed....................

1852 ch. c. by Milo (son of Langar) dead... Y yr Flood

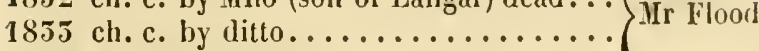

1854 ch. c. by ditto ...............
1855 b. f.

AMBER,

Bred by Mr Hovlosworti, in 1817, got by Ambo, her dam. Stamfordia, by Stamford-Legacy, by King Fergus.

1823 bl. f. by a half-lored horse.........

1826 bl. c. Seedling, by an Irish horse.....

1827 b. f. by ditto................ Major Hamilton

1829 b. c. by Y. Castrel.............

1850 b. f. Mertin, by ditto ...........

1851 ch. c. by Zealot............... Mr Ilontgomery

1852 ch. c. Arab, by ditto............

1853 ch. c. Ascot, by Peterborough....... Ir Pottinger

1854 bl. f. Ariel, by Lapwing..........

\section{AIIIDON,}

Bred by Mr Moxtgomery, in 1850, got by Starch, her dam, Anonymous, by Captain Candid, out of Holbein's dam, by Golumpus.

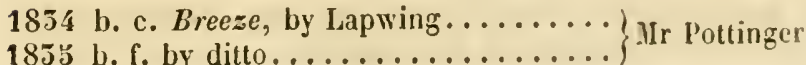


ANGELICA,

Bred at Hampton-Court, in 1819, got by Sir Harry Dimsdale, out of Lady Grey, by Stamford-Bordeaux.

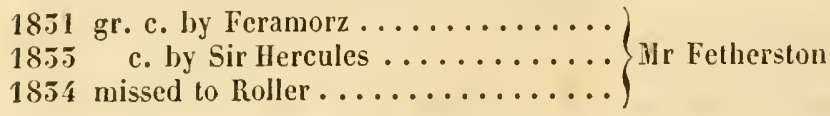

ANNA,

Bred by Mr Batrersby, in 1810, got by Jerry Sncak, out of Patty.

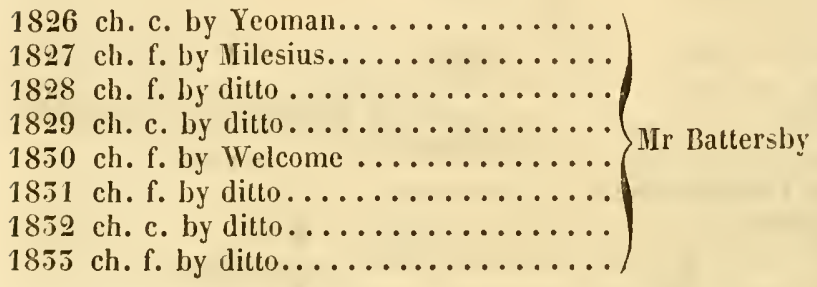

ANONYMOUS,

Bred by Lord Exeter, in 1824, got by Captain Candid, out of Holbein's dam, by Golumpus-sister to Beningbrough, by King Fergus.

1828 b. c. The Adjutant, by Middleton ...

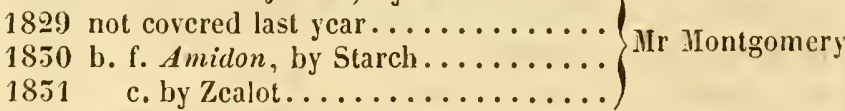

1852

1855 b. c. by ditto.............. IIr A. Montgomery 1854 b. f. by Zealot................

\section{BERESINA,}

Got by Smolensko, dam by Saucho.

1851 b. c. Corsair, by Dandy ..........

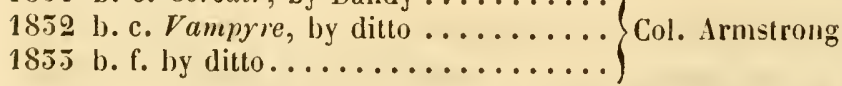

\section{BITTERN,}

Bred by Lord Sugo, in 1826, got by the Sligo Waxy, out of Bigottini, by 'Thunderbolt. 


\section{3}

1852 br. c. St Patrick, by Mlcaston.......)

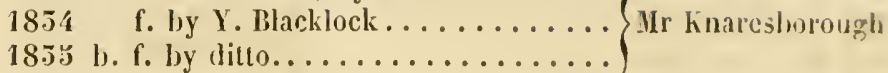

Missed in 1851 and 55.

\section{BLANCHE,}

Bred by Mr Yates, in 1827, got by Filho da Pula. her dam, Iatdy of the Lake, by Sorcerer.

1851 h. f. by Bedlamitc.

1852 ch. c. by Speculation..............

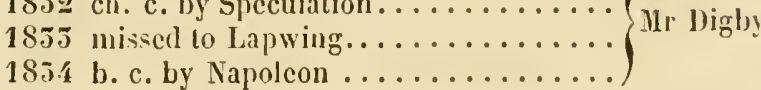

BLECHER HARE,

Bred by Sir T. Stanley, in 1822, her dam, Cowslip, by Alexander.

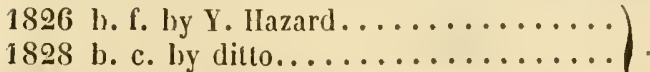

1850 b. f. by ditto................ Capt. Gombell

1852 b. c. Bowman, by Sagiltarius......

1854 b. c. Archer, by dilto............

Missed in $1827,29,31$, and 55 .

\section{BOB BOOTY MIMRE (sister to Narcissus)}

Bred by Mr Bowes Dalr, in , her dam by Commodure, out of Y. Lalage, by Chocolate.

1851 ch. f. by Humphrey Climker, or Dun-) licllin

1852 br. c. hy Humphrey Clinker, or Pala- Mr Blake dour

1855 b. c. by Dunkellin, or Jamian de Lacy

\section{BOB BOOTY WNRE,}

Bred by , in , her dam, dVilful, sister te II ire, by Waxy.

$1850 \mathrm{ch}$. f. Cheat, hy Roller.......... Ld Cilanmorris 
1852 ch. c. by Economist ............

$\left.\begin{array}{l}1855 \text { ch. f. by ditto } \ldots \ldots \ldots \ldots \ldots \ldots \ldots \ldots \\ 1854 \text { br. f. by ditto } \ldots \ldots \ldots \ldots \ldots \ldots \ldots \\ 1853 \text { ch. f. by Y. Blacklock } \ldots \ldots \ldots \ldots \ldots\end{array}\right\}$ Ir Graydon

\section{BRANDY BET,}

Bred by Ir Montgonery, in 1829, got by Canteen, out of Bigottini, by Thunderbolt.

1854 ch. f. Cruiskeen, by Sir Hercules......
1853 b. f. by Y. Blacklock........... Hilltown

\section{BRUNETTE,}

Foaled in 1S28, got by O'Fy, out of Lissy, by Swordsman.

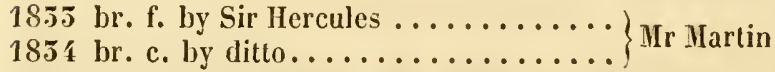

1853 br. c. by Y. Blacklock..........

\section{CLARI,}

Bred by Mr HouldswortI, in 1824, got by Smolensko, her dam by Precipitate-Highflyer-Juno, by Spectator.

1829 b. f. Clara, by Filho da Puta........ Mr Houldsworth

1850 missed to Lottery.............

1851 b. f. by Y. Blacklock.............

1852 cl. c. Cripple, by Speculation ('Tramp). Mr R. Borrowes

1855 b. c. Crocus, by Signal, or Y. Blacklock

1854 b. c. Cordial, by Y. Blacklock.......)

\section{CONELY (sister to Carouser)}

Bred by Mr Соск, in 1822, got by Comus, her dam, Anticipation, by Beningbrough, out of Expectation, by Herod.

1826 b. f. Bavarian, by Tramp ........ Mr Ridsdalc 1828 b. f. by Lottery ................ Mr G. Crompton ${ }^{*} 1850$ br. f. Chrystal, by Chatcau Margaux. 1852 br. c. The General, by Picton, or Eco-

185.5 c. by Picton.................. Mr Noke

Yissed in 1827, 29, and 51 .

* Chirpstal produced, in 1855, Mr Molt's ch.c. by Emancipation. 
CORA, by Master Bagot (Vol. III, p. : 16 ) had a dead foal in 1855 , and missed in 54 and 55 . Her last produce should stand thus :-

1852 ch. f. Dust, by Giles .......... Ld Sligo

\section{CUIRASS,}

Bred by Lord Sugo, in 1825, got by Oiseau, out of Castanci. 1827 b. c. Elk, by Waxy Pope......... Mr Maher 1829 ch. f. Eel, by Langar ........... 1831 b. f. Elm, by Starch......... Sligo

1852 b. c. Emu, by Picton............ 1856

Missed in 1828 and 50 , not corered in 55.

\section{DANDIZETTE,}

Bred by Mr Blake, in 1817, got by Waxy Pope, out of Lcitrim Clib.

1822 ch. f. by Bob Booty.............

1825 ch. c. Beppo, by ditto ............

1824 b. c. Liberator, by Feramorz.......

1850 br. c. Dunkellin....................... Mr M. Blake

1851 b. c. by Humphrey Clinker.........

1852 br. f. Redmantle, by ditto.........

1855 ch. c. Galway, by ditto...........

She had no produce between 1824 and 50 .

\section{DANDY MARE (sister to Ballysax)}

Her dam, Moll Anthony, by Commodore.

1850 b. c. Peace Haker, by Drone ........

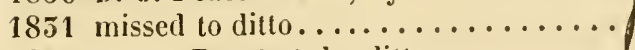

1852 gr. c. Barefoot, by ditto......... Hr E. Kelly,

1855 ch. f. by Philip the First..........

1854 ch. f. Lightning, by Drone........
1855 gr. c. Friar, by ditto..........

\section{DISCOVERY,}

Bred by Mr Batsox, in 1826, gol by Tiresias, her dam, Harrict, by Pericles-Selim-Pipylina, by Sir I'eter. 
1852 ch. c. Giraffe, by Giles..........

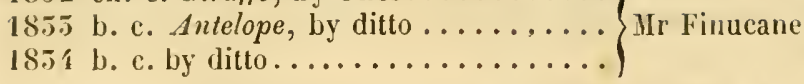

\section{DREAM,}

Bred ly Lord Sugo, in 1827, got by Waxy Pope, out of Cora, by Master Bagot.

1850 b. f. Drowsy, by Master Robert .....

1851 b. c. by Humphrey Clinker (died a foal) Mr R. Borrowes

1852 b. c. Dawdle, by Giles ...........

1854 b. f. Dolt, by Picton............

Missed in 1855 and 55 .

\section{DRUDGE,}

Bred by Mr UAxon, in 1828, got by Waxy Pope, out of Cora, by Master Bagot.

1853 b. f. Kitchen Maid, by Picton....... Mr Disney

18.54 missed.

\section{DUCKLING (sister to Lapdog)}

Bred by Lord EqREnort, in 1820, got by Whalebone, her dam by Canopus-Y. IVoodpecker.

1828 b. c. Duxbury, by Phantom ....... Mr Standish

1852 br. f. by Alcaston (dead) .........

1855 b. f. by Mayfly .............. IIr Hunter

1854 b. f. by The Distingué...........

She had no foal in $1829,50,51$, or 55 .

\section{ELIGHE (now called Sharavogues)}

Bred by Lord Egrenoxt, in 1815, got by Teddy the Grinder, her dam, Young Amazon, by Gohanna, out of Amazon, by Driver.

* 1827 b. f. Zillah (aft. Barebones) by Bonassus )

(Orville) Mr Lang

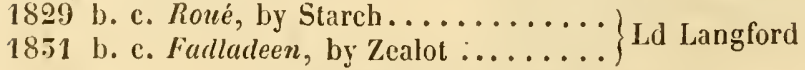

1854 br. or ro. c. by Sir Hercules........ Mr G. Clark

Missed to Waxy Pope in 1828, to Starch in 1850,52 , and 55.

* Barebones produced in 1851 Mr Lang's f. Zinc, by Zealot, and missed in 18.52 and 5.5 . 


\section{7}

\section{FAITHLESS,}

Got by Bob Booty, out of Lady Sarah, by Champion.

$1827 \mathrm{ch}$. f. by Feramorz............

$1828 \mathrm{ch}$. f. by ditto ...............

1829 ch. c. by Iranhoc . . . . . . . . .

1850 b. f. by Giles (died young)......... Hr H. J. Blake

1855 ch. c. by Ilumphrey Clinker, or Dun-

kellin

1854 by Dunkellin...........

Barren in 1851 and 52.

FANNY,

Got by Champion, out of Patty, by 'Tugg.

1828 ch. c. Paul Clifford, by Recordon, or

fioller

1829 ch. f. Polly Trashery, by Roller..... Mr Mansfield

1855 br. c. Salmagundi, by a son of Castrel

1854 b. f. by Napoleon...............

Missed in $1850,51,52$, and 55 .

\section{FANNY,}

Got by Regent, out of Rhoda, the dam of Caprice.

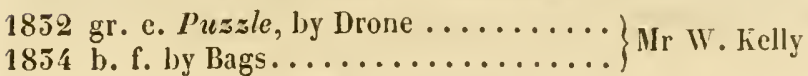

Missed in 1851 and 55.

FERAMORZ MARE,

Bred by Mr Blake, in 1828, out of Napoleon's dam. by Waxy Pope-Lady Sarah, by Champion.

1832 br. c. by IIumphrey Clinker.......)

1855 b. f. by ditto................ Mr Blake

1854

by Dunkellin.............

\section{FLIGITT (chesnut)}

Bred by Mr B. DaLy, in 1809, got by the Irish Escape, out of Young Heroine, by Bagot-Heroine (Tom Turf's dam) by Herod (son of Cade) Snap-sister to Regulus.

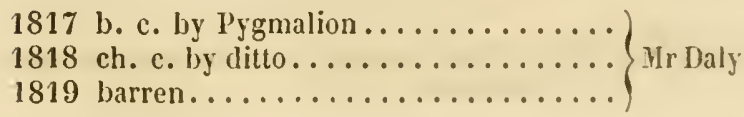

VOL. IV. 


\section{8}

1820 ch. c. Klepper, by Bob Booty ......

1821 ch. f. by ditto..............

182.5 ch. f. Guiccioli, by ditto.........

1824 ch. f. Reine Canziani, by ditto.....

182 : ch. c. by ditto................ Mr M. Blake

1826 cl. c. by ditto.................

$1829 \mathrm{ch}$. f. by byron ..............

${ }^{*}$ i $\$ 50$ b. f. Beda, by Camelopard .......

$1851 \mathrm{ch}$. f. Iy Humphrey Clinker ........

1852 ch. f. Fly, by ditto ............ Mr Mansfield

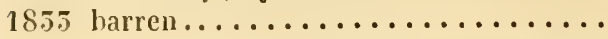

1854 by paladour ...........

"BEDa produced in $1854 \mathrm{Mr}$ Mlansfield's b. f. Bud, by Y. Blacklock, and missed to him in 1855 .

\section{FLORA MIAC IYOR,}

(iot by Master Robert, dam by Fitz-Emily, out of The Painter's dam, by Trimmer.

$1851 \mathrm{gr}$. f. by Giles................

1852 ch. f. IIelena, by Napoleon........ Mr Flood

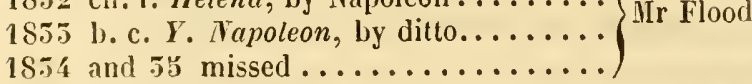

FOAII,

Bred by Lord Sugo, in 1825, got by Langar, her dam, Steam, by Waxy Pope, out of Miss Staveley, by Shuttle-DroneMatchem.

1851 b. c. Spume, by Roller .......... Ld Sligo

1852 ch. f. Duchess, by Y. Blacklock ..... Mr Watts

1855 ch. c. Schemer, by Speculation (son of $)$ Tramp) $M$ Ir Studdert

1854 ch. c. Spec, by ditto........... Mr Watts

$1855 \mathrm{ch}$. f. by ditto.............. Mr Studdert

1857 by The Distingué ................ Mr Studdert

FOLI,Y by Waxy (Vol. III, p. 520), missed in 1852 and 55 , and is dead. 


\section{FRIENDSIIP,}

Bred by IIr Gore, in , got by Goodall, out of firicully by Tom 'Tug-Creamchecks, by Lennox.

1827 c. by Oak Jack (killed) ........

1828 br. f. by Roller...............

1829 ch. f. by Ivanhoe.............

1850 b. с. by Roller ............... Jr Malıer

1855 b. c. Mulgrave, by Sir IIercules .....

18.54 b. c. by Lapwing .............

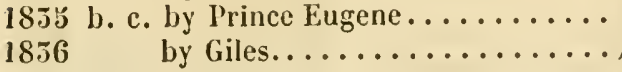

\section{GAUNTLET MARE,}

A Bay Mare, foaled in 1818, her dam, Polly l'eachum, by Sichedoni (son of Pot8o's) grandam by Commodore-Irish Jass, by Turnip (son of Pot8o's-a sister to Mother Bunch, by Mercury.

1897 b. c. Pioneer, by Roller.......... Capt. Forbes

1850 b. f. Cleopatra, by ditto.......... Mr Sherlock

1852 f. Maid of Arlee, by Napoleon.....

1855 f. by Roller (died a foal)............. Ir Sherlock

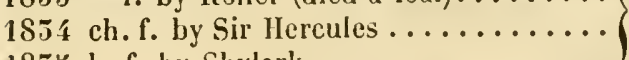

1855 b. f. by Skylark...............

Barren in 1828, 29, and 51 .

\section{GLANCE,}

Bred by Lord Suigo, in 1828, got by Waxy Pope, out of Glohe. by Quiz, out of Paleface, by Y. Woodpectier.

1832 ch. c. by Speculation ........... Mr Maher

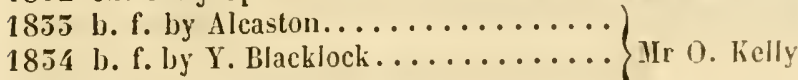

1855 b. f. by De Vere.............

\section{GUICCIOI.I,}

Bred by Mr BLAke, in 1825, got by Bob Booty, out of lilight, by Escape.

1829 ch. f. The Bijou, by Roller........

$18.50 \mathrm{ch}$. c. Northumberland, by ditto (broke Ir IInnter his leg in the paddoeli) 
1831 b. f. by Master Robert...........

1852 ch. c. by Roller (dead)............

1855 ch. c. Birdcatcher, by Sir Ilercules... M. Hunter

1854 missed to ditto...............

1855 ch. c. George, by Roller...........

She ran as late as Nov. 1828, and won the Mares' Plate in Sept. 1850 , at the Curragh.

\section{HELEN,}

Bred by Mr R. Wilson, in 1828, got by Blacklock, her dam, Helena, by Rubens, out of Sprightly, by Whiskey-Romance, by Gouty.

1854 b. f. by Lapwing (dead)...........

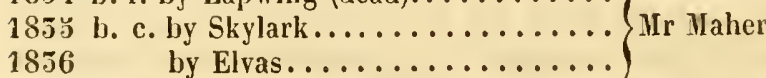

\section{IENRIETTA,}

Got by Master Robert, dam by Queensberry, out of Remnant, by Trumpator.

1852 b.f. by Speculation.............

1855 br. c. Honesty, by Sir Hercules....... IIr J. Kelly

1854 ch. c. by ditto...............

\section{HOLLYHOCK MARE (sister to Hesperus)}

Bred by Sir N. Lorrus, in 1826, out of Rally, by Waxy.

1851 br. e. Wrangler, by Wrangler ...... Mr Watts 1852 b. f. by Giles.................. Mr Hunter

Missed in 1855, 54, and 55.

HOLLYHOCK MARE (sister to Bachelor)

Bred by , in , her dam, Spinster, by Shuttle.

1899 b. c. Shamrock, by Roller ......... Mr Maher

$1852 \mathrm{gr}$. f. by Speculation............ Mr Davis

Died in 1855. 


\section{HUMBLG,}

Bred by H. R. II. the Duke of YoRK, in 1821, got by Hedley, or Seymour, out of Gramarie, by Sorcerer.

1826 c. by MIoses............... II ].. Herbert

1827 ch. f. The Woman of Endor, by ditto (put to the stud) $\}$ Mr Montgomery

1830 br. f. The Washerwoman, by Stareh.

1851 b. f. by Zealot................

1832 ch. e. The Squire, by Zealot........

1855 b. f. by Sir IIercules ............

1854 b. f. by Drone............... Mr Osborme

183 br. f. by Y. Blacklock ...........

In 1828 and 29 missed to Mildleton.

To JANNETTE, by Camillus (Vol. III, p. g.2.j) ad 1826 ch. e. Moynalta, by Regent ...... L. I Inson

\section{ILDEGARDA (sister to Napoleon)}

Bred by Mr Blake, in 182:, got by Bob Booty, her tam by Waxy Pope, out of Lady Sarah.

1850 b. c. by Dunkellin.............. Mr Blake

1831 b. c. Thump, by Humphrey Clinker. Col. Westenra

$1852 \mathrm{ch}$. f. by ditto .............. Ir Blake

$1835 \mathrm{ch}$. f. by ditto ............. Ir Kirwau

\section{KISS,}

Bred by Lord Rossmore, in 1827, got by Waxy pope, her diun by Champion (son of Pot8o's out of Brown lanyy, by .Havimin.

1852 gr. f. Whim, by Drone..

1853 ch. f. Sweetlips, by Roller.........

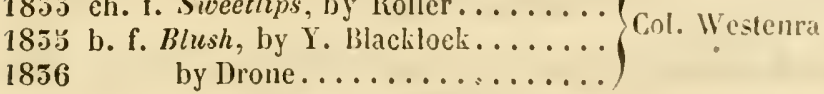

Missed in 18.51 and 54 .

\section{KITTEN.}

Bred by Lord Surgo, in 1816, got by his Waxy. her daun by Master Bagot-Woodpecker-sister to Jimiper. by Snap. 


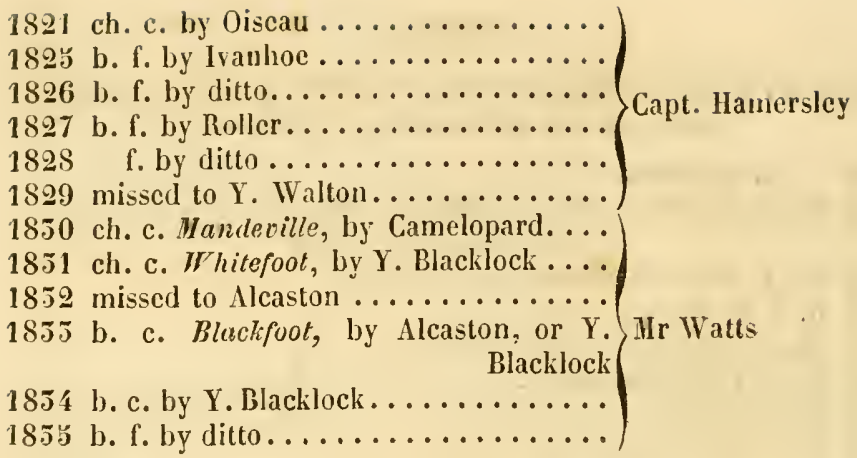

\section{I.ADY HERON,}

Bred by Mr Kelcy, in 1827, got by Langar, her dam, Stork, by Oiseau, out of Miss Staveley.

1831 gr. f. by Drone ..............

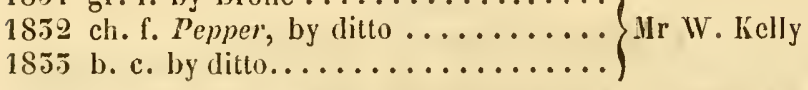

Missed in 1854, and is dead.

LANGAR MARE,

Bred by , in , her dam, The Trout, by Rainbow, out of Wilful, by Waxy.

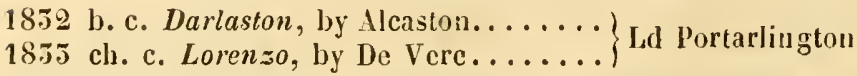

Missed in 1834, and is dead.

LANGAR MARE,

Bred by Mr Btake, in 1826, her dam, Mother Bunch, by Dicki Andrews.

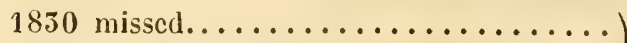

1851 b. f. by Humphrey Clinker ..........

1852 b. c. by ditto............... Mr Blake

185.5 ch. c. by ditto..................

1854 by Paladour, or Damian de Lacy. 


\section{LILIAS,}

Bred by IIr Kirwas, in 1822, got by Bob Booty, out of Penelope,

by Sivordsman.

1828 ch. c. Johnny, by Recordon .......

1829 missed to Roller

$1850 \mathrm{ch}$. c. ditto........ died soon after

1851 ch. c. by Master Robert foaling Jr R. Hunter

1852 ch. c. by Roller (dead)...........

$1855 \mathrm{ch}$. f. by ditto..............

1854 ch. f. by The Distinguc..........

\section{LIZZY,}

Bred by Mr Kinwar, in , got by Bob Booty, out of Jissy, by Swordsman.

1851 br. c. Stiff, by Starch...........

1854 br. f. by Sir IIercules............ IIr nighy.

1835 ch. c. by Y. Blacklock...........

Missed in 1850,52 , and 55.

\section{LOTTERY,}

Bred by Mr Caldwell, in , got by Legacy, out of Tell Spriggins, by Master Bagot-Nannetle, by Old Bagot.

1850 ch. f. by Noble.

1851 missed to Starch...................

$1852 \mathrm{ch}$. c. Jungle Admee, by Roller ..... Ld llillown

1855 ch. c. Cushneiche, by ditto........

1834 b. c. Bedershin, by De Vere.......

\section{MINES,}

Bred by Mr DAY, in 1823, got by Spectre, out of Calendulat.

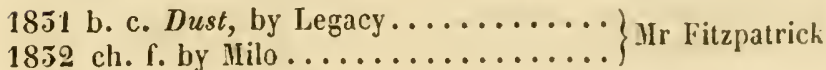

Mlissed in $185 \%$ and 54 .

MANSFIELD I.ISS,

Bred by Mr Hoclosworta, in 1825, got by Fillı da Puta, her dam, Variety, by Selim, or Soothsayer, out of Sprite, by Bobtail. 
1852 missed to Ilumphrey Clinker........

18.5 ch. f. Sporting Mag, by Y. Hazard... Capt. Gambell

1854 b. c. Dart, by Sagittarius..........

MARGARET, by Hambletonian (continued from Vol. III, p. 228).

1852 b. f. Eva, by Camelopard.......... Ir Farrall

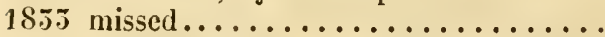

1834 by Paladour.

\section{MARY ANNE,}

Bred by , in , got by Waxy Pope, her dam,

Witch, by Sorcerer-Precipitate-Diddler's dam, by Highflyer.

1850 dead foal.

1831 b. c. Sir Giles, by Legacy, or Giles...

1832 ch. c. Zero, by Speculation.......... IIr Flood

183.5 bl. f. Water Witch, by Sir Hercules..

1854 b. f. by Drone................

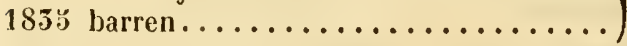

\section{IASTER GOODALL MARE,}

Bred by Mr Iluster, in 1822, out of Parthenope.

1829 ch. c. Mir $z a$, by Roller.

1850 missed to Starch.............. Mr Watts

1831 b. c. Needle, by ditto ...........

1832 b. f. by Starch, or Alcaston ........ IIr Henrick

1855 b. f. by Alcaston...............

\section{MERLIN,}

Bred by Major Hasiltox, in $\mathbf{1 8 3 0}$, got by Y. Castrel, her dam, Amber, by Ambo.

1853 missed to Zealot............. NIajor Hamilton

1854 b. c. Decanter, by Lapwing. . . . . . . Hr Pottinger

1853 by ditto............... Dr Wilson

To MISS BOHFORD (Vol. III, p. כ้28) add

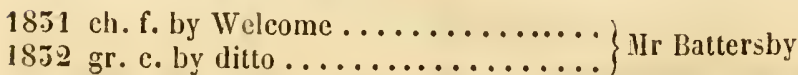

Died in 1855, in foal to Sir Hercules. 
MISS FOOTE,

Bred by Mr KIRwav, in 1829, got by Oiscau, her dam, Lissy, by Swordsman-Tug-Bagot.

1852 ch. f. Cinclerella, by Drone....... Mr W. Kelly
$1855 \mathrm{gr}$. c. IIarrington, by ditto.......

Hissed in 1850,51 , and 54 .

\section{MISS PRATT,}

Bred by Sir J. Brra, in 1825, got by Blacklock, out of Gadabout, lyy Orville.

1852 b. c. by Sagittarius, or Y. Hazard....

1835 ch. f. Quiver, by Sagittarius, or Noble. Capt. Gambell

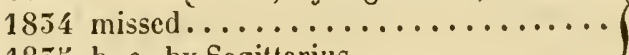

1855 b. c. by Sagittarius.............

\section{MIOROCCO,}

Bred by Lord Rossrone, in 1819, got by Friday, out of Josephina, by Poulton.

Missed in $1851,53,54$, and 55.

\section{NABOCKLISII IILRE,}

Bred by Lord Crenonve, in 1825, her dam, Niss Tooley, by Teddy-the-Grinder, out of Lady Jane, by Sir Peter.

1850 ch. c. Beagle, by Roller ..........

1831 gr. c. by Robert..............

1832 ch. f. by Economist................. Ir Ferguson

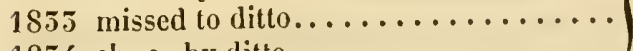

1854 ch. c. by ditto ...............

\section{NUTILAID,}

Bred by Lord Rossuore, in 1824, got by Friday, her dam. Trefoil, by Oiseau, out of Wilful. 
1851 b. c. Fracture, by Homer

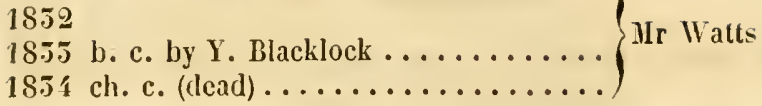

OVERSEER MARE,

Bred by Mr J. KeLLY, in , her dam by Fitz-Emily .

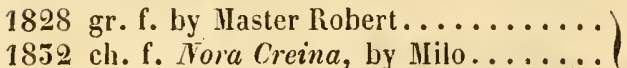

1852 ch. f. Nora Creina, by Milo........ Mr J. Kelly

$1854 \mathrm{ch}$. c. by ditto................

Missed in 1829, 50, and 51 .

PARTHENOPE, by Walton (Vol. III, p. 551) produced a b. f. by

Y. Blacklock, in 1852, and a b. c. by Giles (son of Rainbow) in 1834 , and died that year.

\section{PASTA,}

Bred by Mr LeE, in 1825, got by Catton, dam by Luck's-all Pot80's - Maid-ol-all-Work, by Highflyer.

1829 ch. c. Tancredi, by Roller........

1852 gr. c. Argirio, by Roller, or Drone ..

1855 ch. c. Romeo, by Sir Hercules ....... Id Milltown

1855 b. c. Ersce, by Y. Blacklock, or Alcaston)

Missed in 1850 and 51.

\section{PENANCE (sister to Penitence* ${ }^{*}$}

Bred by Lord Stigo, in 1825, got by Oiseau, out of Miss Aide.

1851 b. c. Comet, by Lumber. . . . . . . . .

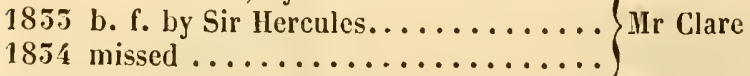

* Penitence produced, in 1829, Lord Clanmorris's b. c. by O'Fy, and missed the four following years.

\section{PENINSULA,}

Bred by Lord Stigo, in 1821, got by Navigator, out of Miss Aide, by Sir Peter. 


\section{7}

1850 ch. c. Newbrook, by Roller........ Id Clanmorri.

$1852 \mathrm{ch}$. f. by Economist............ M J Ilunter

$1854 \mathrm{ch}$. f. by ditto.............. Mr Ilunter

Yissed in $185 \mathrm{I}, 55$, and 55.

PERDIT $\Lambda$,

Bred by Lord Sugo, in 1850, got by Langar, out of Delenda, by Gohanna.

1854 b. c. by Y. Blacklock...........

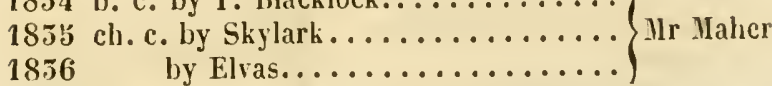

\section{YIIANTOM MARE,}

Her dam, Defiance, by Gauntlet, out of Glauvina, by Dungannol.

1826 b. c. Sagittarius, by Centaur.......
1828 ch. f. Philomel, by Y. Hazard...... Gapt. Gambell

Missed in 1827; died in 1850.

PIILOIIEL,

Bred by Capt. Gameelt, in 1828, as above.

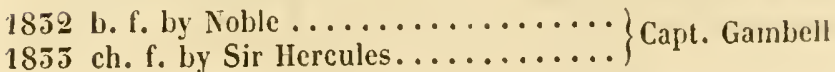

\section{PLELAD (sister to Ildegarda)}

Bred by Mr BuAKe, in 1827, got by Bob Booty, out of Napoleon's dam, by Waxy Pope.

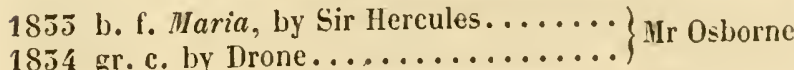

\section{POWLETT,}

Bred by Mr Orde Powlets, in 1826, got hy Jack Spigot, her dam, Miss Fanny, by Walton.

1852 bl. c. Patch, by Starch, or Manfred...

1855 ch. f. Penitent, by Sir Ilercules..... MIr R. Borrowes

1854 missed to ditto..............

1855

by Y. Blacklock............ 


\section{8}

To PRIZE, by Aladdin (Vol. III, p. 555) add

1854 ch. c. by Tureoman............ Mr Walkington

\section{QUEENSBERRY MARE,}

Bred by Lord Sulgo, in , her dam, Remnant, sister to Rattle, by Trumpator, out of Faney, by Florizel.

1825 ch. c. Saladin, by Master Robert.....

1827 b. f. Ilenrietta, by ditto.............

1829 b. f. by Ivanhoe................

1830 gr. c. New Fashion, by Drone.......

1852 b. c. Orphan, by Milo, or Alcaston...)

Missed in 1826 and 51 , and is dead.

RATTLE,

Got by Bonassus, out of Moll Rowe, by Walton-WaxyHighflyer.

1829 br. c. by Viseount............

1851 b. f. by Zealot ............... Mr Savage

1852 br. f. by Mount Loftus ...........

1853 missed to Zealot...............

\section{RECLUSE (sister to Rasselas)}

Bred by Lord Egremont, in 1828, gol by Wanderer, her dam by Orville, out of Selim's dam, by Alexander.

1852 b. f. Zora, by Nimrod (Whalebone)...

1855 bl. e. Recherché, by Chateau Margaux. MIr J. R. Hunter

1834 and 55 missed...............

RECORDON, or ROLLER MIARE,

Her dam, Moll Anthony, by Commodore.

$1851 \mathrm{ch}$. f. by Drone...............

$1852 \mathrm{ch}$. f. by Sir Hercules................ Ir E. Kelly

1853 br. c. IIonest Ned, by ditto . . . . . . . .

1854 b. c. by ditto................. Mr Disney 


\section{ROXANA,}

Bred by Mr Mac Donnele, in got by Buffer, her dam, Plenty, by Bagot, out of Ilarvest, by Richmond.

1820 ch. f. Enchantress, by Overseer .....)

1821 ch. f. Romp, by Oliver Cromwell .... Mr M M Donnell

1850 b. c. by Master Robert (dead)....... .

She missed from 1821 to 50.

\section{SALLY,}

Bred by , in got by Recordon, out of sister to Pirouette, by Young Eagle-Parisot, by Sir Peter.

1851 b. e. Tiby-tight, by Recordon....... Ld Portarlington

1852 ch. c. Budget, by Drone, or Billy the Beau )

1855 bl. f. Crim. Con. by Sir Hercules .... Mr Daris

$1854 \operatorname{missed} . \ldots \ldots \ldots \ldots \ldots \ldots \ldots \ldots$

\section{SIR WALTER RALEIGII MARE,}

Bred by Lord Crenorve, in 1817, her dam, Miss Tooley, by Teddy, out of Lady Jane, sister to Ilermione, by Sir PeterPaulina, by Florizel.

${ }^{*} 1820$ gr. c. Chanter (aft. Skeleton) by Master

1825 gr. c. Drone, by ditto ............

1824 gr. f. by ditto.................... Kr Kelly

$1826 \mathrm{gr}$. f. Mary Anne, by ditto.........

1827 gr. c. Regulator, by ditto .........

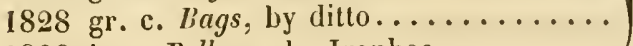

1829 b. c. Bellows, lyy Ivanhoe

$1852 \mathrm{ch}$. c. The Major, by Speculation....
1834 f. by Sir Ilercules, or Napoleon.... Telly

Mlissed in 1822, $23,50,51$, and 5.5 .

* Sezetor was sold to Mr Riley, and sent to New South Wales.

\section{SKYLARK,}

Bred by Mr Trendergast, in , got by Musician, out of sister to Pirouette, by Y. Eagle-Yarisot, by Sir Peter. vol. $w$. 
1820 b. c. Giles, by Rainbow...........

1821 b. f. by Olympus...............

1824 b. f. by ditto................ Mr Daxon

1893 b. f. by Oliver Cromwell ..........

1826 b. c. Skylark, by Waxy Pope .......

1829 b. c. Westport, by Langar.......... Ld Sligo

1850 b. f. Lodola, by Y. Hollyhock....... Ld Milltown

1853 c. by Starch............... Ld Clanmorris

1854 b. f. by Economist ............. Mr Ferguson

Missed in $1827,28,51$, and 32.

SMOKE,

Bred by Lord Sugo, in 1822, got by Oiseau, out of Steam.

1828 ch. f. by Dunkellin (foaled late in Aug.)

1850 b. f. by ditto................

1851 ch. c. by Humphrey Clinker........ Mr M. J. Blake

1852 ch. f. Soot, by ditto.............

1855 b. c. by ditto, or Paladour..........

\section{SHOLENSKO MARE,}

Bred by Mr Lax, in 1826, out of Mayflower's dam, by Sir DavidHambletonian-Ilightlyer.

1850 c. by Noble (dead)............

$1851 \mathrm{br}$. c. by Bank of Ireland .............

1852 b. c. by ditto (dead).............. Mr Allingham

1854 b. c. by Fitz-Orville (i)on's)........

1856 by ditto...............

Missed in 1855 and 55 .

\section{STAYS (sister to Starch)}

Bred by Lord Sugo, in 1827, got by Waxy Pope, out of Mliss Staveley, by Shuttle-Drone.

1850 b. f. Eve, by Banker............

1852 b. c. Leinsier, by Munster .........

1855 b. f. by Sir Hercules............. Mr Ruthven

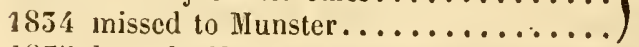

1853 b. c. by Young Blacklock.......... Mr Pottinger 
STEAI, by Waxy Pope (Vol. III, p. 357) has had only one foal since 1831 , viz.-

1855 ch. f. by Y. Blacklock........... Mr IIolmes

\section{STORK,}

Bred by Lord Sugo, in 1822, got by Oiseau, out of Miss Staveley.

1826 b. c. by Master Robert (dead). $1827 \mathrm{ch}$. f. Lady Heron, by Langar (put to

1828 missed to Master Robert ...........

1829 b. c. by Ivanhoe (dead) . . . . . . . Jir Kelly

1830 b. f. by Drone................

1831 gr. c. Seagull, by ditto..........

1832 b. c. Curlero, by ditto

1853 b. c. by ditto

1834 c. by ditto

\section{STREAM (sister to Steam)}

Bred by Lord Stigo, in 1823, got by Waxy Pope, her dam. Miss Staveley, by Shuttle-Drone-Matchem.

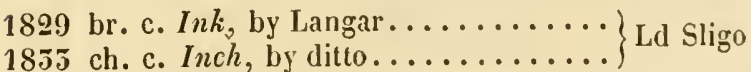

Missed in 1850, 51, and 54; slipped foal in 52 .

\section{TIPSY,}

Bred at Hampton Court, in 1818, got by Election, her dam, Hirtlr, by Trumpator, out of Hoity-toity, by Ilighflyer.

1825 b. f. Y. Tipsy, by Master Robert..... Mr Watts

1829 b. f. by IIollyhock............

1830 b. f. by ditto ............... Sir N. Loftus

1832 b. f. by Giles.................

1855

by Spot.

\section{Y. TIPSY,}

Foaled in 1893 , as above.

1829 b. c. by Friar (son of Spartacus) ..... IIr Flood 
1850 br. c. Wait-a-while, by Carlow .....

1851 missed to Y. Blacklock.............

1852 br. f. Wait-still, by Alcaston....... Mr Fitzpatrick

1855 b. c. by Napoleon............. Mr Fitzpatrick

1834 b. f. by ditto...............

185 b. c. by Y. Blacklock............

\section{TRINKET,}

Bred by Lord Egremovt, in 1827, got by Whaleboue, her dam, Y. Amazon, by Gohanna, out of Amazon, by Driver.

1855 b. c. Locket, by Y. Blacklock.......

1854 b. c. by Drone ............... NIr Naher

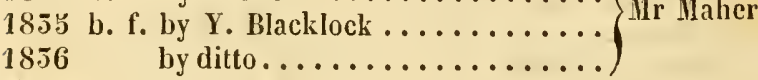

\section{TROU'T,}

Bred by Lord Sugo, in 1819, got by Rainbow, out of Wilful.

1829 b. c. by Roller, or Ivanhoe ........

1850 b. f. by Camelopard.............. IIr Naher

1831 ch. f. Luck's-all, by Y. Blacklock.....
1832 ch c. by Speculation ............

Died in 1852.

\section{VANITY,}

Bred by

, in ; got by Bonassus, out of Violante, by Cockfighter.

1829 ch. f. by Viscount.............

1851 b. f. by Mount Loftus.......... Mr Savage

1852 b. c. by ditto...............

Missed in 1850 and 55.

VAMP,

Bred by Lord Sulgo, in 1825, got by Langar, out of Wire, sister

to Whalebone, by Waxy-Penelope, by Trumpator-Prunella,

by Ilighffyer.

1851 b. c. Vanguard, by Roller........ Mr Maher

1852 missed to Y. Blacklock.......... Mr Studdert 
1855 b. c. Varlet, by Speculation........

1834 br. c. by Y. Blacklock ............ Mr Haher

1855 b. f. by Drone................

Sent to the United States, in 1855, with her foal, and covered by Speculation.

\section{VAT,}

Bred by Lord Sugo, in 1826, got by Langar, out of Wire.

1855 br. c. Wedge, by Picton..........

1854 b. c. Windfall, by Fitz-Orville (Don's).

1855 b.f. Wrinkle, by Picton, or Fitz-Orville Col. Westenra

1856 b. c. by Drone.

1857

by Priam.

VERMILION (Vol. III, p. 538 ) produced, in 1S52, a b. f. Routine, by Picton, and died in 55 .

\section{VESPERS (sister to Hesperus)}

Foaled in 1825, got by IIollyhock, out of Rally, by Waxy.

1850 b. c. Ballyroan, by Roller, or Snout. Ld Portarlington

1851 b. f. by Naster Robert............

1832 b. c. Confusion, by Starch ......... Ir Lang

1855 b. c. Natty Bumpo, by Sir Hercules... Mr Lang

\section{URGANDA,}

Bred by Mr Wrxohsu, in 1825, got by Tiresias, out of Silvertail, by Golianna-Orville - Selim's dam, by Mlexander.

1853 ch. c. Enterprise, by Speculation.... Ir Ion Studdert

$\left.\begin{array}{l}1854 \\ 1855 \\ \text { ch. f. f. by ditto } \ldots \ldots \ldots \ldots \ldots \ldots \ldots \ldots\end{array}\right\}$ Mr Hunter

1856 ch. f. Elm, by Roller ............ Ir studdert 1857

To the WVAXY POPE ILARE, Levanter's dam (Tol. III, p, 5.54) add-

1852 br. c. St Laurence, by H. Clinker.... Ir R. Burlie

1853 ch. f. by ditto ............... Ir IIolmes 


\section{WAXY POPE MARE,}

Bred by Mr Krrwan, in , her dam, Penelope, by Swordsman.

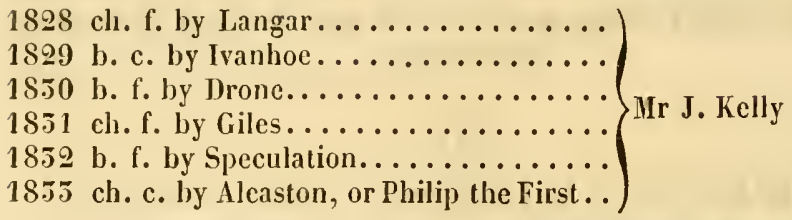

WAXY POPE MARE (Y. Penelope)

Bred by Mr Krnwan, in , own sister to the preceding.

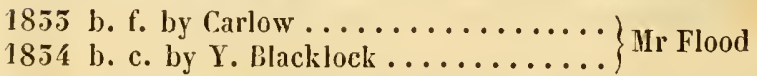

To the WAXY POPE MARE (Napoleon's dam) Vol. III, p. 555, add -

1852 b. c. by Humphrcy Clinker (dead) ...)

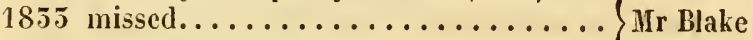

1854 f. by Dunkellin.............

WEGENKORB,

Bred by Sir C. Bunbury, in 1822, got by Partisan, out of Wowski,

(Smolensko's dam) by Mentor-Waxy's dam, by Herod.

1826 br. f. by Whalebone........... Mr R. W. Walker

1828 ch.c. Cinnamon, by lliddleton......

1829 missed to ditto...................

1851 b. f. Catchpenny, by Zcalot ........

1852 ch. f. Clove, by Zealot............

1853
1854 b. or by. c. by Alcaston.............

\section{WHALEBONE MARE (sister to Recruit)}

Bred by Lord Egremont, in 1826, her dam by Teddy-the-Grinder -Gohanna-Catherine, by Woodpecker.

1831 b. c. by Mlaster Robert (dead) ....... Mr Montgomery

$18 j 2$ b. c. Cadet, by Roller............ Mr Haher 


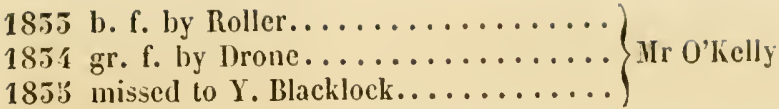

To WIRE, Vol. III, p. 540 , add-

1832 bl. f. Vinegar, by Picton (put to the

1855 b. c. Verulam, by Lottery ......... UIr IIolmes

1854 ch. c. Vigo, by Y. Blacklock........

1853 ch. f. by ditto

1856 bl. c. by ditto

\section{WOMAN OF ENDOR,}

Bred by Mr Montgonery, in 1827, got by Mloses, her dam, Ilumbug, by IIedley, or Seymour, out of Gramaric.

1852 ch. c. Crescent, by Zealot.........

1855 ch. f. Cashmere, by Peterborough....

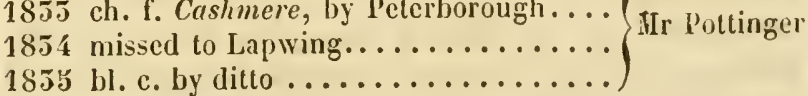

\section{ZILLAH,}

Bred by Lord Grosvevor, in 1895, got by Blacklock, out of Zadora.

1850 br. c. Fillalgo, by Filho da Puta.....

1831 b. f. by Legacy...............

1852 br. c. The Colonel, by Alcaston......

1855 missed to ditto.............. Ir Watts

1854 br. c. by ditto ................

1853 br. c. by ditto ...............

1856 b. c. by Skylark ..............

ZOE,

Bred by MIr Kinwas, in 1820, got by Recordon, her dam, Nanny, by Commodore, out of Mary Gray, by Triar.

1850 foal by a half-bred horse.......... Mr Savage

1851 b. f. Dame Quickly, by Nount Loftus (put to the stud)

1852 missed to ditto............... Mr pottinger

185.5 ch. c. IIotspur, by Roller .........

18.54 b. c. Hamlet, by Lapwing..........

1855 missed to ditto.............. 
Pedigrees of some Irish Stallions, not included in the preceding List.

BANK OF IRELAND, bred by Mr Kirwan, in 1825, got by Recordon, out of Penelope, by Swordsman.

BOXER was got by Tom Bagot (first called Dancer) his dam by Richmond, out of the Banister mare.

BRIEN BORU, a bay horse, bred by Mr Fransey, of Kilbride, got by Mr Kirwan's Slug, his dam by Swindler, out of Rusticoat, by Chanticleer.

YOUNG MUSICIAN, got by Old IIusician, his dam (bred by Lord Rossmore) got by Rainbow, grandam by Boxer, out of Black Deuce, by 'Trumpator-Peeress, by Herod-Promise, by Snap. 


\section{ADDENDA, \&c.}

To Vol. 11I. - consistingalmostentively of names given, since publication, to horses whose alams it has not been necessary to re-print in the present Volume.

Page in Vol. III.

Foaled in

14 Dominie Skelp, by Champignon, out of Anna Bullen . . . 1824

55 Myrrha, by Malek, ont of Bessy, who has had no foal since)

37 b. f. (not colt) Ella, by Eppersion, out of Bit of Tartan . 1829

59 Criterion, by Renovator, out of Canidia . . . . . . . 1825

61 Alington, by Gustarus, out of Canvas (s. to France in 1852) 1826

70 Mirabeau, (late Eyebrow) by Whisker, dam by Cervantes. 1829

96 Conservator (late Parson Harvey) by Phantom, dam by 1823

108 Hohenlohe, by Flibbertigibbet, dam by Don Cossack . . . . 1826

156 Hardwicke, by Polygar, out of Mystery . . . . . . . 1829

168 Catherine, by Huley, out of Harriet, by Selim . . . . . 1829

180 Jack-a-Lantern, by Champignnon, dam by Jack-a-Lantern. 1829

185 Bachelor, by Cœelebs, ont of Jessy . . . . . . . . 1820

194 Prior, by Truth, out of Jesebel . . . . . . . . 1851.

189 Exchange, by Swap, out of Isidora . . . . . . . 1828

201 Zannah, by Whalebone, out of Læna . . . . . . 1825

209 Ronilo, b. c. by Nicolo, out of Lezinka. . . . . . . . 1827

218 Lupin, by Velocipede, out of Lupino. . . . . . . . . 1851 251 Sierra (D. of Richmond's) by Wamba, dam by Marmion $) 1850$

257 Annelle, by Phantom, out of Meg Merrilies. . . . . . 1826

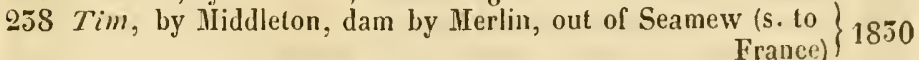

242 Lady Bee (late Miss Maria) by Count Porro . . . . . . . 1828

Katinka, sister to ditto ........... 1850

$2: 51$ Billesden was foaled in 1826, not 27

264 Pestilence (aft. The Mayor) by Cleveland, out of Mrs Barnet. 1829

268 Araminta, by IIarmodius, out of Momentilla . . . . 1824

275 Queen MIab, by Truffle, out of Naughty Girl (s. to Russia 1850

286 Sally Maggs. by Catton, out of Orphan ........ 1828

286 Lady Ormond, by Oetarian, out of King David's dam (now)

in the Bretloy s(ud)

292 Y. Juniper (Col. Lowther's) brother to Oscar . . . . . 1818

296 Cinderella, by Gaberlunzie, out of Partial . . . . . 1850 
502 Coronet, brother to Jubilee, by Catton . . . . . 1825

305 Cis, sister to Cain, by Paulowitz . . . . . . . . . . 1829

525 Freney, by Roller, ouf of Promise . . . . . . . 1850

552 Red Rose, by Rubens, out of Reality (s. to Prussia in Oct. 1829

55ㅇ John o'Gaunt, brother to Gamebny . . . . . . . . 1825

569 Physician, by Dr. Syntax, out of Molyneux's dam. . . . 1827

580 Sans Souci, by Blacklock, dam by Sir Malagigi . . . . . 182:5

581 Ferdinand, by Cervantes, out of sister to Otho . . . . . 182 i

586 Herrier, by Merlin, dam by Skim . . . . . . . . . 1899

596 Queen of Hearts, sister to Baltic, by Borodino . . . . . 1827

598 Hector, by Master Henry, out of Sorceress . . . . . . 1829

401 Wildfire (late Mystery) by Marmion . . . . . . . . 1818

404 The last produce of Regent's dam was a filly, and was sold to Count Bassewitz

407 Glantivy, by Spectre, out of Prude's dam . . . . . . 1828

428 Z ora, b. f. by Catton, out of Trotinda ......... 1852

429 St Hubert, by Williamson's Ditto, out of sister to Repeater. 1822 Despair, by Woful, out of Saracen's dam . . . . . 1830 The produce of Saracen's dam, in 1827 , was a b. f. and did not die young.

450 Catlap, by Catton, out of Turnip . . . . . . . . 1829

444 To the last Walton mare's produce add-

b. f. Mrs Waylett, by Y. Filho da Puta . . . . . . . 1829

464 The Duke (late Wellington) by Champion, dam by Wil-) liamson's Ditto 1818

465 Crocodile, by Camel out of Witchery (s. to France) . . 1850

469 Schedule, by Octavian, out of Lady Sarah's dam (she produced in 1855 Ld Chesterfield's br. c. $A$, by Voltaire, and was sold to the King of Prussia in that year, covered by Priam) ...................... Snacks (aft. Norna) by Reveller, out of Cornelian's dam, by Woful (the Woful mare was sent to Germany)

470 Tristram Shandy, by Belzoni, dam by Woful . . . . 1851

472 Romer, by Reveller, out of Woodbine . . . . . . . 1850

470 Marian, by Blucher, out of Zadora . . . . . . . . . 1824

478 Parenthesis, by Master Henry, out of Zoraida. . . . . 1826

Bucellas, by Burgundy, out of Y. Zuleika . . . . . . 1829

481 Casket, by Moonraker, out of Cabinet . . . . . . . 1825

482 Clarinet was got by Camillus

484 Donna Clara was got by Cesario

488 Ambassador, ch. c. by Marshal Blucher, out of Geraldine. 1827

491 Lady . Jersey (Mr Allen's) by Theodore, dam by Haphazard. 1830

493 Bamboozlc, by Welbeck, out of Lily of the Valley . . . 1851

500 Billy Lack-a-day, by Spectre, out of IIorgiana . . . . . . 1824 
TO THS VOLIE.

Most of the names which follow, have been gicen in the progress of the work, and too late to appear in their proper places, but the whole are included in the general Index.

Page

lioaled in

4. Beggarman, by Zinganee, out of Adeline....... 1853

10 Antigone, ch. f. by Augustus, out of Amoret. . . . . 1856

15 Spectre, by Firman, out of Apparition. . . . . . . 1855

17 Arsenic, by The Colonel, out of Arsena. . . . . . . 1855

20 Ticliet, by Lottery, out of Baleine. . . . . . . 1853

22 Arrillerator, ch. c. by Velocipede, out of Barbara. . . . 1856

57 Grisi, by Emilius, out of Bravura. . . . . . . . 1855

45 Beggar-my-neighbour, by Partisan, out of Bustle. . . . 1854

79 Oberon, by Velocipede, out of Dahlia. . . . . . . 1855

95 Toga, by Sultan, out of Dulcinea. . . . . . . 1855

102 Phantom Minor, by Phantom, out of Klizabeth. . . . . 1851

147 Mushroom, by Fingus, ont of Jewess. . . . . . . 1834

164 Concealment, by Royal Oak, out of Leeway. . . . . 1835

171 Fury, by Tramp, out of Lunacy. . . . . . . . 1852

205 Juliet, by Rowlston, out of Miss O'Neil. . . . . . 1827

205 Mordecai, by Lottery, out of Miss Thomasina. . . . . . 1855

221 Strength, b. c. by Sir Hercules, out of Occina. . . . . 1836

227 O'Connell, b. c. by Y. Emilius, dam by Orville, out of 1856 Sprightly

252 Donna Maria, by Partisan, out of Donna Clara. . . . . 182

240 Carew, by Tramp, or Comus, out of Y. Petuaria. . . . 1855

256 Puce, by Rowton, out of l'ucelle. . . . . . . 1834

269 Rosette, by Lamplighter, out of Rosebud. . . . . . . 1855

278 Stafford, by Memnon, out of Sarsaparilla. . . . . . 1853

282 Fanchon, by Lapdog, out of Scuffle. . . . . . . 185

291 The Hind, by Actacon, dam by Sir David. . . . . . . . 1831

507 Quicksilver, by Memnon, out of the sister to Cecilia, foaled 1835

in 1826$\} 1855$

515 Delee, by Camel, dam by Tiresias. . . . . . . . . . $185 j$
516 Thalia, light b. or dun f. by Augustus, out of Toso. . . 1856

535 Detonator, brother to Dewdrop......... 1835

550 Agamemnon, by Langar, out of Woodbine. . . . . . 1854

378 Brato, by Flexible, out of Rufina. . . . . . . 185j 


\section{BIONDETTA,}

Bred by Mr Prexdergast, in 1819. got by Rainbow, out of Jamnelle, by Camillus-Helen, by Delpini.

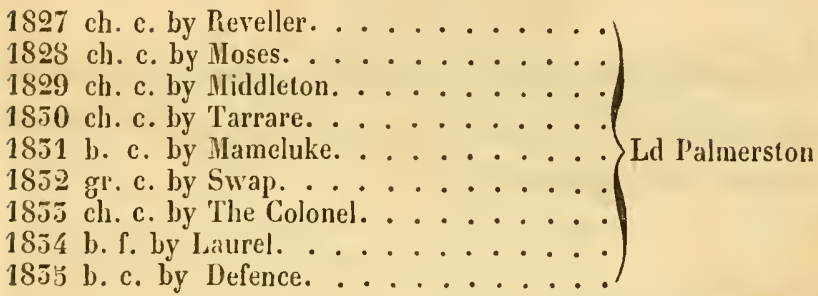

Sent to France in 1835 , covered by Count Porro.

To DUENNA, by Sir David (Vol. III, p. 405) add-

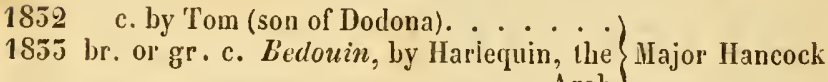

1854 Arab)

by ditto. .......... Ld Donoughmore

To the SHUT'TLE MIARE, Henwick's dam (Vol. III, p. 575) add-

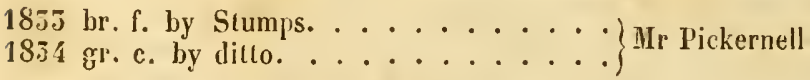

Missed to Stumps in 1855 , and died.

GREGSON was got by Evander, his dam by a brother to Recruit, out of Cygnet, by Buzzard-Didapper, by IIerod.

LAUD, a bay horse, foaled in 1850 , got by Zealot, his dam lyy Canterbury out of Herrythought, by Totteridge. - Ilis dan produced also a filly by Maresfield in 1852.

V]RGIN14 (Mir 'Houldsworth's) foaled in 1851, by Figaro or Buzzard, out of Treasure, by Camillus, produced a filly by Filho da Puta in 1835 , and a filly by Filho in 1830 .

52 Castrellina died, in 1855 , in foaling twins by Physician, which died a!so.

65 last line, read Henrietta, "loy Jerry," not "Humphrey Clinker."

85 Desdemona was shot in Jan. 1836 , being worn out. 
98 to Elfrid's produce add:

$1853 \mathrm{br}$. f. by Cliateau Margaux.

12'5 Galatea's foal of 185 : was by The Colonel, or Tranlyy.

128 In the pedigree of Gingarella it appears to the Editor there must be a cross omitted, as he is informed the Cardoch mare died only a short time ago, and therefore could not have been out of Georgiant, who was foaled in 1771.

204 Nliss P'atrick's ch. P. by Velocipede, was foaled in 1853; she was barren in 1852.

215 Mushroom. by Dick Andrews, is shol.

2i:3 Queen Bathsheba's foal of 1850 , the Editor is since informed, is a b. f. by Priam.

290 Silvertail's produce in 185.5 was a ch. $\int$. by Comus, or Emilius.

510 To the filly by Whalebone, out of Thalestris, foaled in 182:3. add the name Bang-up; she was barren till 18.76 , when she produced Mr J. Scoll's b. f. Miss Flareaway, by Mulatto, and was covered by Velocipede.

511 line 6 from the botton, for Contrition, "by Tramp," read " by Tiresias."

519 the first produce of Trapes was :

1851 b. c. Gaudaloupe, by Velocipede.

553 Tartarina's b. f. by Comus, was foaled in 1854 .

540 Dragletail produced, in 18.55, a b. f. by Sherwool.

5.13 Augustina's ch. c. by Battledore, was foaled in 18.55 ; she missed in 1854 .

571 Since this page was printed, the Editor has received a fuller pedigree of Melody; she was bred hy Mr Wadlow, got by Bustard, her dam by Black Sultan, grandam, sister to Nosegay. by Warrior. out of Cecilia, by Beninghrough.

THE END. 




\section{$250-100000$}

$1-120161$

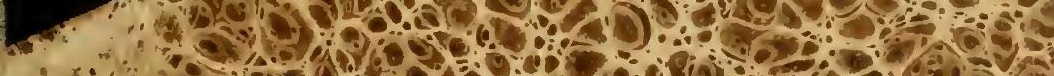

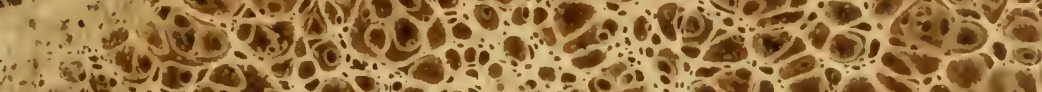

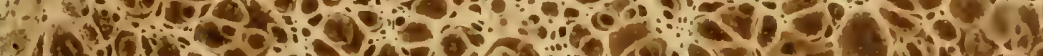

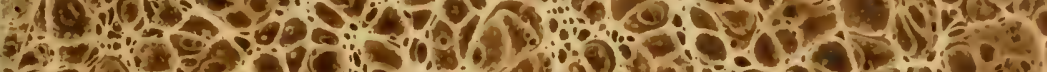
1.

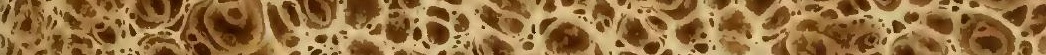

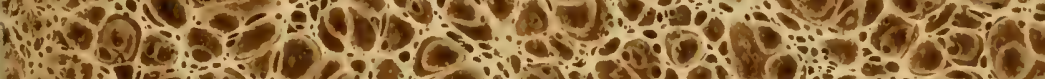

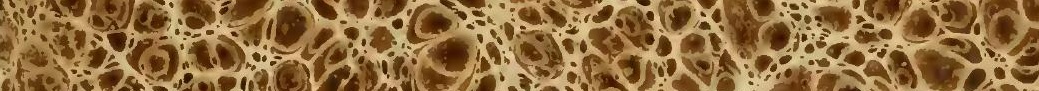

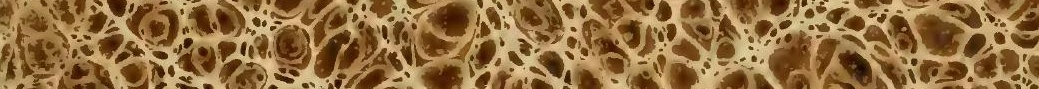

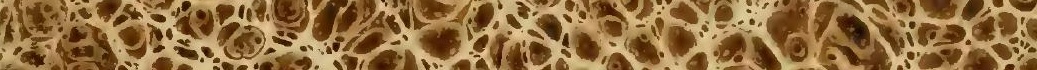

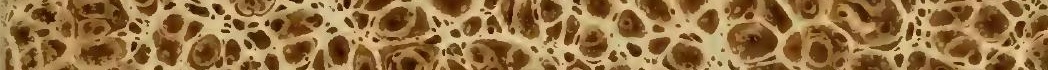

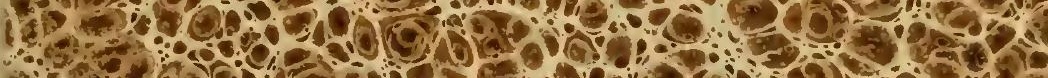

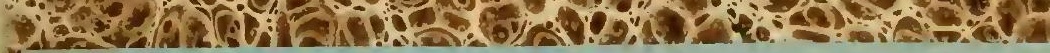

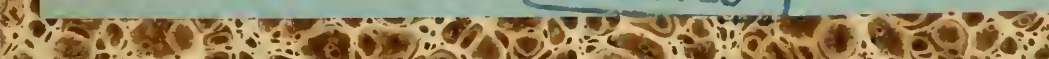

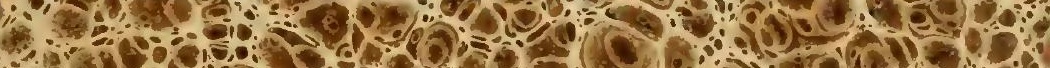

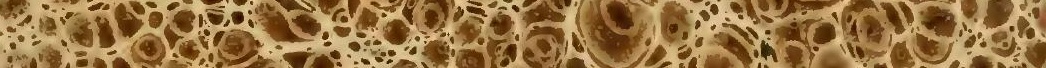
Hi=

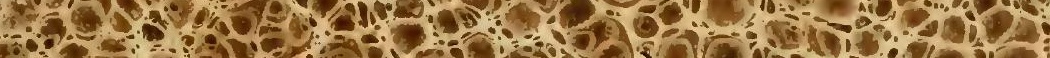

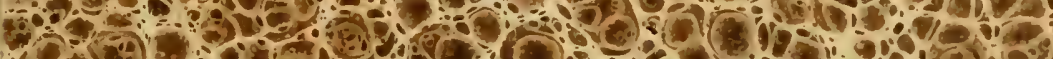

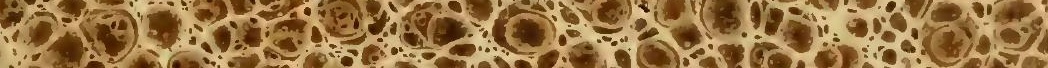

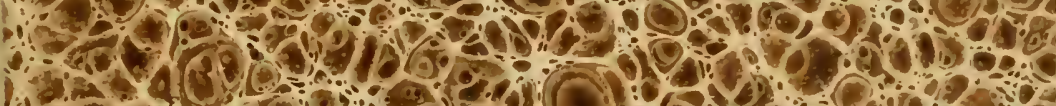

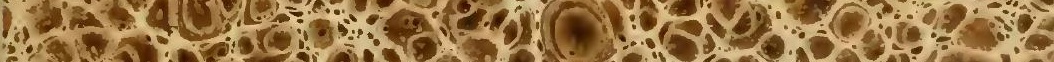

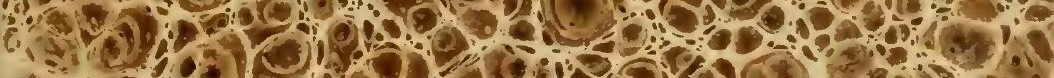

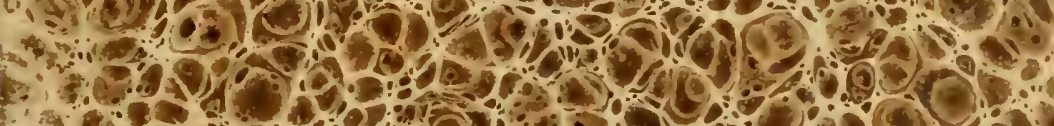

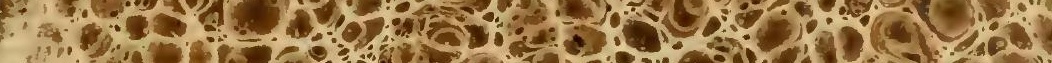
a i. 


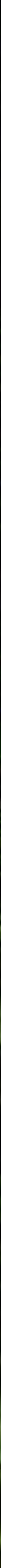

